Florida International University FIU Digital Commons

$6-27-2013$

\title{
Funtional Near Infrared Spectroscopy Study of Language, Joint Attention and Motor Skills
}

Ujwal Chaudhary

Florida International University, uchau001@fiu.edu

DOI: $10.25148 /$ etd.FI13080901

Follow this and additional works at: https:// digitalcommons.fiu.edu/etd

Part of the Bioelectrical and Neuroengineering Commons, Bioimaging and Biomedical Optics Commons, and the Biomedical Devices and Instrumentation Commons

\section{Recommended Citation}

Chaudhary, Ujwal, "Funtional Near Infrared Spectroscopy Study of Language, Joint Attention and Motor Skills" (2013). FIU Electronic Theses and Dissertations. 929.

https://digitalcommons.fiu.edu/etd/929 


\section{FLORIDA INTERNATIONAL UNIVERSITY}

Miami, Florida

\section{FUNCTIONAL NEAR INFRARED SPECTROSCOPY STUDY OF LANGUAGE, JOINT ATTENTION AND MOTOR SKILLS}

A dissertation submitted in partial fulfillment of

the requirements for the degree of

DOCTOR OF PHILOSOPHY

in

BIOMEDICAL ENGINEERING

by

Ujwal Chaudhary 
To: Dean Amir Mirmiran

College of Engineering and Computing

This dissertation, written by Ujwal Chaudhary, and entitled Funtional Near Infrared Spectroscopy Study of Language, Joint Attention and Motor Skills, having been approved in respect to style and intellectual content, is referred to you for judgment.

We have read this dissertation and recommend that it be approved.

Anthony McGoron

Armando Barreto

Wei-Chiang Lin

Anuradha Godavarty, Major Professor

Date of Defense: June 27, 2013.

The dissertation of Ujwal Chaudhary is approved.

Dean Amir Mirmiran

College of Engineering and Computing

Dean Lakshmi N. Reddi

University Graduate School

Florida International University, 2013 


\section{ACKNOWLEDGMENTS}

I am perpetually grateful to Dr. Anuradha Godavarty who has been an exceptional mentor for me. Her stringent yet patient guidance has molded me into a high quality researcher. She invested a lot of his time in teaching me technical writing and presentation of scientific work. Her patient yet persistent guidance has taught me to never give up on my goals and the value of critical thinking. She is a big part of the success of this dissertation and all my future accomplishments.

I am grateful to be surrounded by so many amazing people who have helped and supported me throughout this process. The past and present member of optical imaging laboratory Dr. Banghe Zhu, Bryant Thompson, Jean Gonzalez, Joe DeCerce, Manuela Roman, Rad Akhter, Rigoberto Roche, Dr. Sarah Erickson, Sergio Martinez, Michael Hall and Dr. Young-Jin Jung were very supportive throughout my dissertation. Dr. Leonard Elbaum helped me understand the intricacies of physical therapy and made my resolve of serving the humanity even more strong. My dissertation committee members Dr. Anthony McGoron, Dr. Armando Barreto and Dr. Wei-Chiang Lin have provided insight and guidance toward my dissertation. I would like to acknowledge the support of the University Graduate School for providing me with the Dissertation Evidence Acquisition and Dissertation Year Fellowship. I would also like to acknowledge MBRSRISE and Kauffman award for providing fund for my research.

I would like to acknowledge my wife, Kavita chaudhary, my father, my mother, my closest friend, Jaimit Parikh and Rupak Dua for providing tremendous support and encouragement. 


\section{ABSTRACT OF THE DISSERTATION \\ FUNCTIONAL NEAR INFRARED SPECTROSCOPY STUDY OF LANGUAGE, JOINT ATTENTION AND MOTOR SKILLS}

by

Ujwal Chaudhary

Florida International University, 2013

Miami, Florida

Professor Anuradha Godavarty, Major Professor

Near infrared spectroscopy (NIRS) is an emerging non-invasive optical neuro imaging technique that monitors the hemodynamic response to brain activation with ms-scale temporal resolution and sub-cm spatial resolution. The overall goal of my dissertation was to develop and apply NIRS towards investigation of neurological response to language, joint attention and planning and execution of motor skills in healthy adults. Language studies were performed to investigate the hemodynamic response, synchrony and dominance feature of the frontal and fronto-temporal cortex of healthy adults in response to language reception and expression. The mathematical model developed based on granger causality explicated the directional flow of information during the processing of language stimuli by the fronto-temporal cortex. Joint attention and planning/ execution of motor skill studies were performed to investigate the hemodynamic response, synchrony and dominance feature of the frontal cortex of healthy adults and in children (5-8 years old) with autism (for joint attention studies) and individuals with cerebral palsy (for planning/execution of motor skills studies). The joint attention studies on healthy adults showed differences in activation as well as intensity and phase dependent connectivity in 
the frontal cortex during joint attention in comparison to rest. The joint attention studies on typically developing children showed differences in frontal cortical activation in comparison to that in children with autism. The planning and execution of motor skills studies on healthy adults and individuals with cerebral palsy (CP) showed difference in the frontal cortical dominance, that is, bilateral and ipsilateral dominance, respectively. The planning and execution of motor skills studies also demonstrated the plastic and learning behavior of brain wherein correlation was found between the relative change in total hemoglobin in the frontal cortex and the kinematics of the activity performed by the participants. Thus, during my dissertation the NIRS neuroimaging technique was successfully implemented to investigate the neurological response of language, joint attention and planning and execution of motor skills in healthy adults as well as preliminarily on children with autism and individuals with cerebral palsy. These NIRS studies have long-term potential for the design of early stage interventions in children with autism and customized rehabilitation in individuals with cerebral palsy. 


\section{TABLE OF CONTENTS}

CHAPTER PAGE

CHAPTER 1. Introduction............................................................................. 1

CHAPTER 2. Background: Near Infrared Spectroscopy of Brain ............................ 9

2.1 Near infrared spectroscopy of brain: principle, theory and advantages ............... 9

2.1.1 Principle of near infrared spectroscopy of brain.......................................... 9

2.1.2 Mathematical modeling of light in brain .................................................. 13

2.1.2.1 Modified Beer-Lambert Law ............................................................... 13

2.1.2.2 The diffusion approximation to radiative transport equation................... 18

2.1.3 NIRS measurement techniques ............................................................ 20

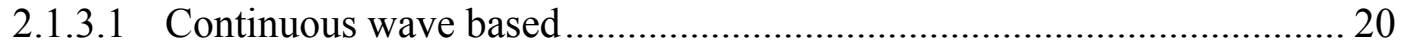

2.1.3.2 Frequency-Domain based.................................................................. 21

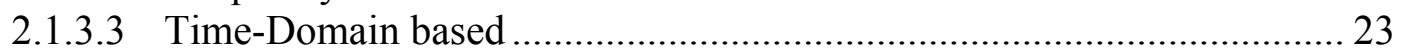

2.1.4 Advantages of Near infrared spectroscopy of brain .................................... 24

2.2 Near infrared spectroscopy of brain: Instrumentation......................................... 26

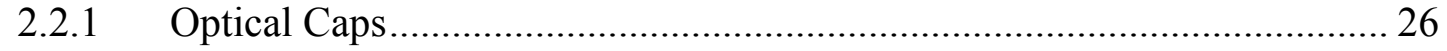

2.2.2 NIRS imaging system ...................................................................... 30

2.2.2.1 Commercial NIRS system................................................................. 30

2.2.2.2 In-house developed NIRS system ........................................................ 32

2.2.3 NIR light source ............................................................................ 33

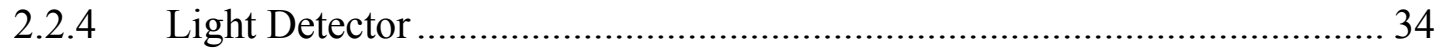

2.3 Near infrared spectroscopy of brain: signal analysis ....................................... 36

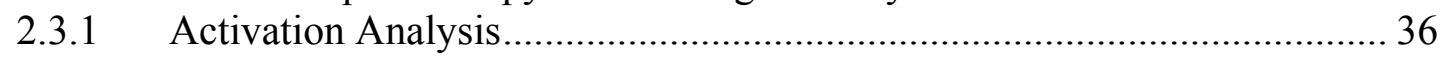

2.3.2 Connectivity Analysis .............................................................................. 37

2.3.2.1 Anatomical/Structural Connectivity....................................................... 39

2.3.2.2 Functional Connectivity ...................................................................... 40

2.3.2.3 Effective Connectivity......................................................................... 41

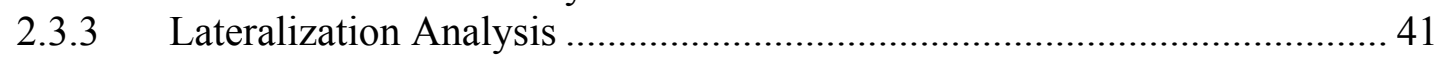

2.4 Applications of NIRS during my doctoral work ............................................... 43

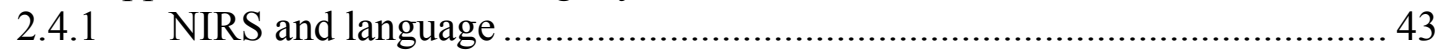

2.4.2 NIRS and Joint attention........................................................................ 44

2.4.3 NIRS and planning and execution of motor skill stimuli ........................... 45

CHAPTER 3. Near Infrared Spectroscopy Instrumentation ...................................... 48

3.1 Frequency domain based NIRS system: Commercial ...................................... 48

3.2 Continuous wave single wavelength based NIRS system: In-house developed 51

3.2.1 Single wavelength laser diode based source system..................................... 51

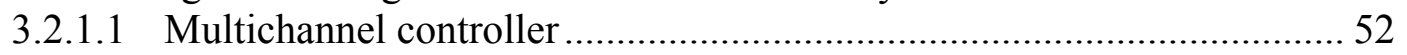

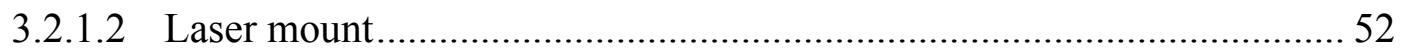

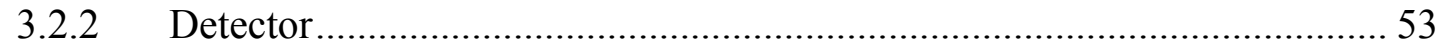

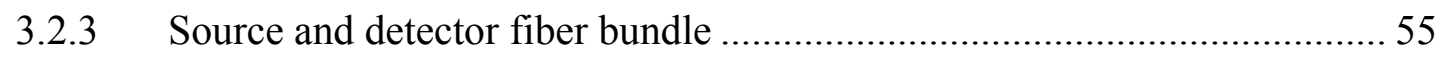

3.2.4 Working principle of single wavelength based NIRS system ..................... 56 
3.3 Continuous wave dual wavelength based NIRS system: In- house developed.. 59

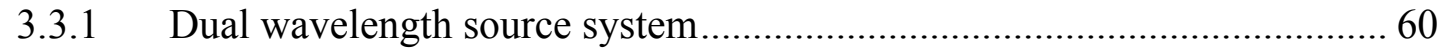

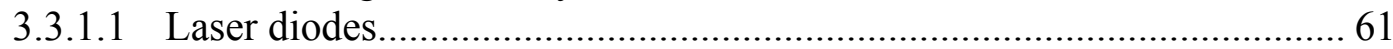

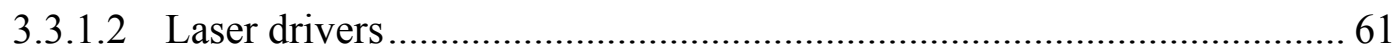

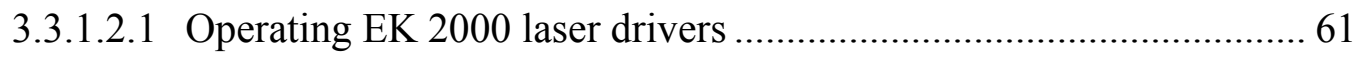

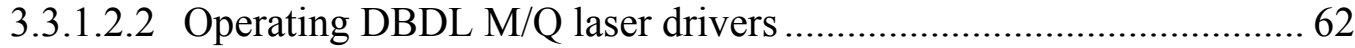

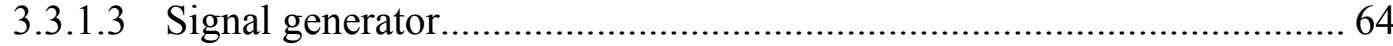

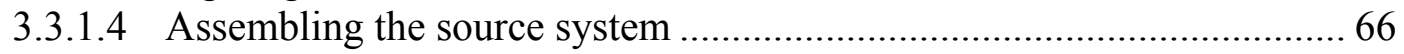

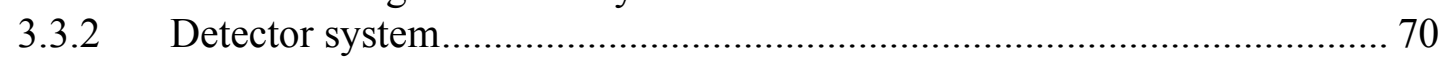

3.3.3 Integration of source and detector systems .............................................. 71

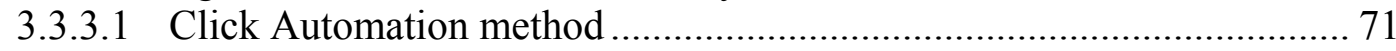

3.3.3.2 External trigger through USB based signal generator............................. 72

3.3.3.3 Signal generator................................................................................... 72

3.4 Testing of dual wavelength NIRS system ....................................................... 73

3.4.1 Modulating $690 \mathrm{~nm}$ wavelength of light keeping $830 \mathrm{~nm}$ wavelength of light OFF .................................................................................................... 74

3.4.2 Modulating $830 \mathrm{~nm}$ wavelength of light keeping $690 \mathrm{~nm}$ wavelength of

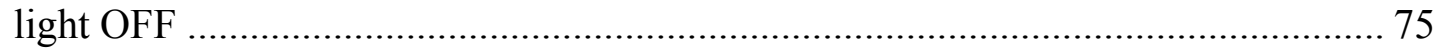

3.4.3 Modulating both $830 \mathrm{~nm}$ and $690 \mathrm{~nm}$ wavelength of light......................... 77

CHAPTER 4. Near Infrared Spectroscopy Data Analysis....................................... 79

4.1 Extraction and format of NIRS data from imagent .......................................... 79

4.2 Extraction and format of NIRS data from In-house developed single wavelength

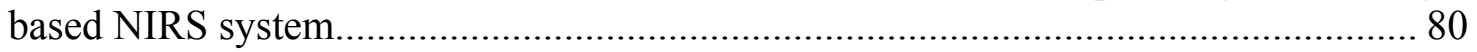

4.3 Extraction and format of nirs data from In-house developed dual wavelength

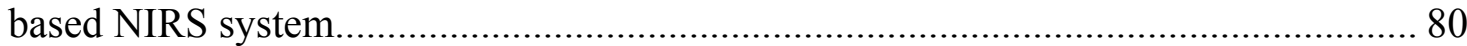

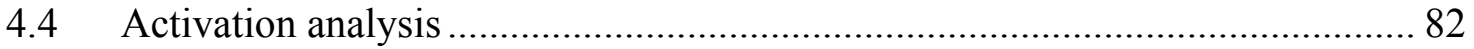

4.5 Functional connectivity analysis ................................................................ 90

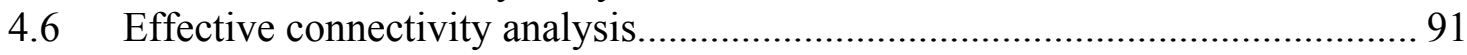

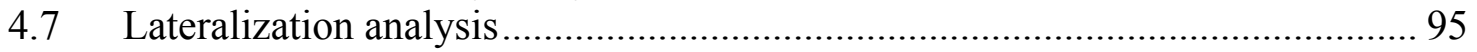

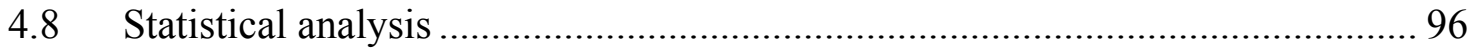

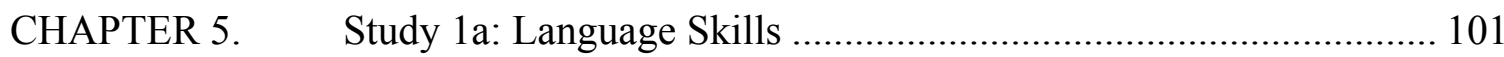

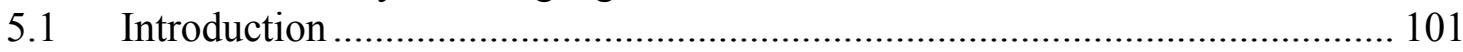

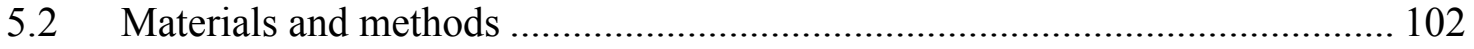

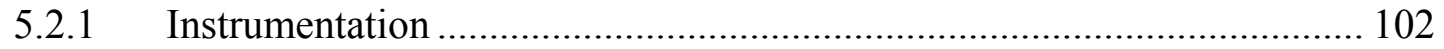

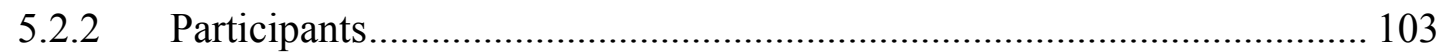

5.2.3 Stimulus and Procedure …………………….......................................... 103

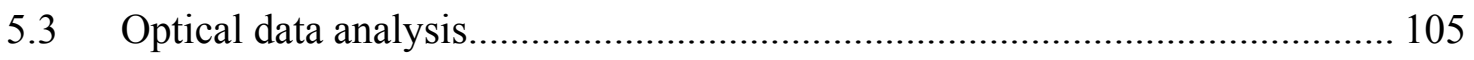

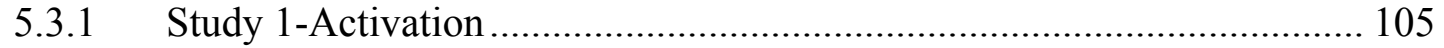

5.3.2 Study 2- Connectivity ......................................................................... 105

5.3.3 Study 3- Cortical Lateralization............................................................... 106

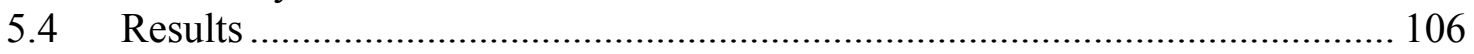

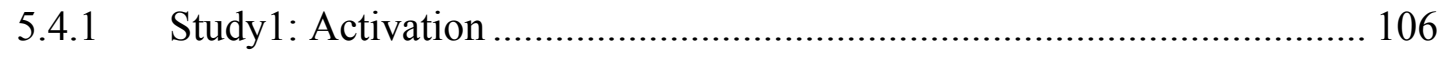

5.4.2 Study 2: Connectivity ........................................................................... 109 


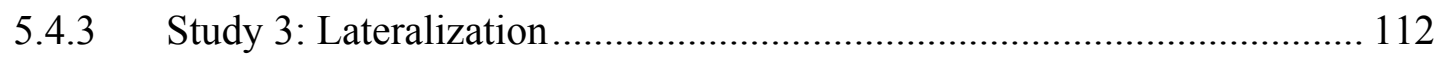

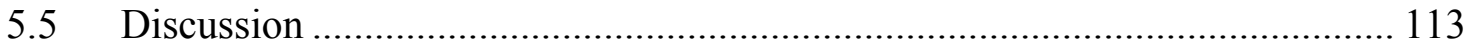

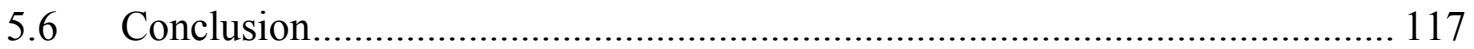

CHAPTER 6. Study 1b: NIRS and Language Skills in Fronto-Temporal Cortex. 119

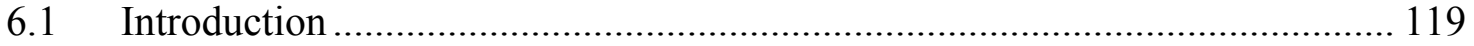

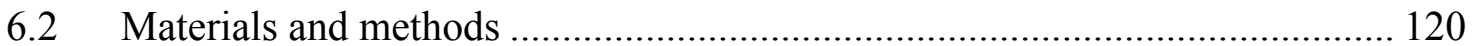

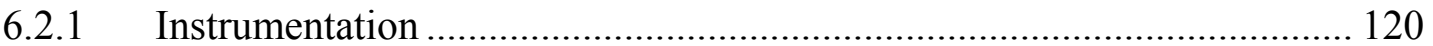

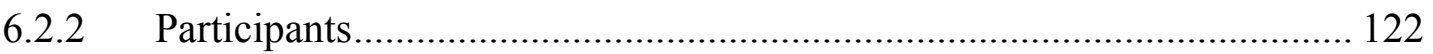

6.2.3 Experimental design and protocol ......................................................... 122

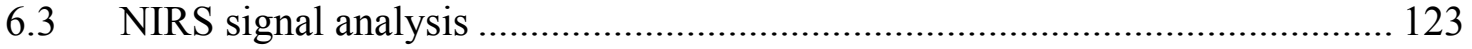

6.3.1 Activation and Lateralization Study ................................................... 126

6.3.2 Functional Connectivity Study ……………….................................. 128

6.3.3 Effective Connectivity Study .......................................................... 128

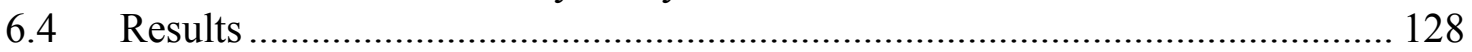

6.4.1 Study A: Word Expression Paradigm ................................................... 128

6.4.1.1 Activation and Lateralization Study................................................. 128

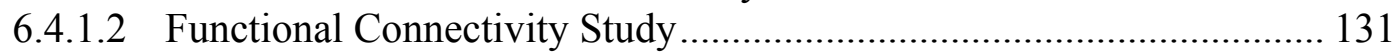

6.4.1.3 Effective Connectivity Study ............................................................. 133

6.4.1.4 Effective Connectivity Study ........................................................ 140

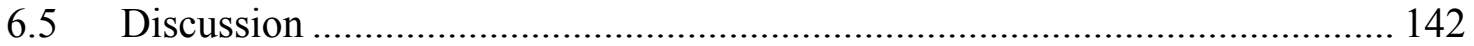

6.5.1 Study A: Work Expression Paradigm .................................................. 145

6.5.1.1 Functional connectivity .................................................................... 145

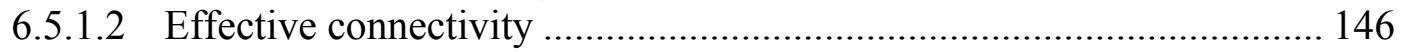

6.5.2 Study 2: Word Reception Paradigm ..................................................... 148

6.5.2.1 Functional connectivity ................................................................ 148

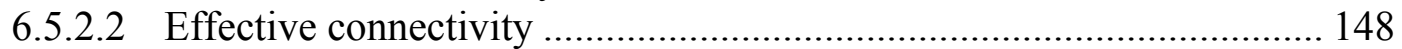

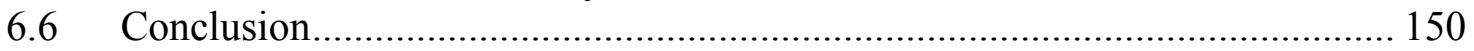

CHAPTER 7. Study 2a: NIRS and Joint Attention of Frontal Cortex in Healthy

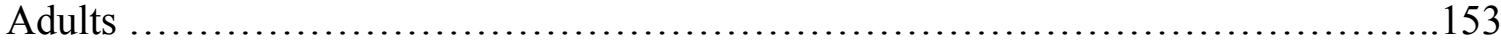

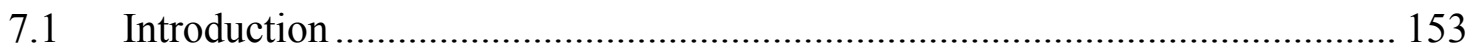

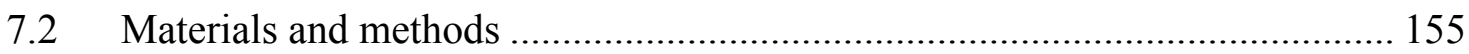

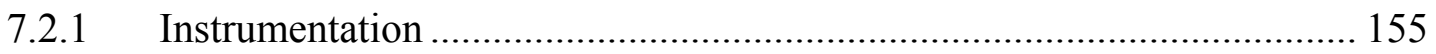

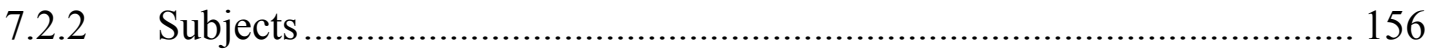

7.2.3 Experimental design and task ............................................................ 156

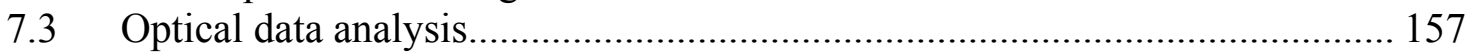

7.3.1 Study 1: Connectivity and cortical lateralization using intensity data...... 158

7.3.2 Study 2: Connectivity using time dependent phase data ……………....... 160

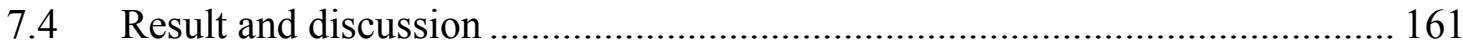

7.4.1 Study 1: Connectivity and cortical lateralization using intensity data...... 161

7.4.2 Study 2: Connectivity using time dependent phase data ……………....... 170

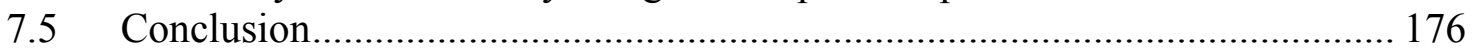


CHAPTER 8. Study 2b: NIRS and Joint Attention of Frontal Cortex in Children 179

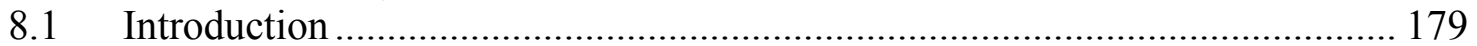

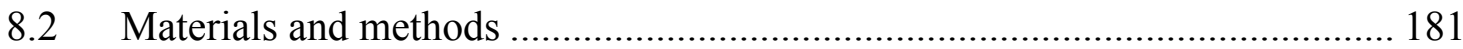

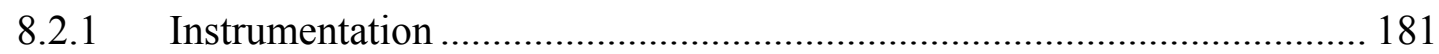

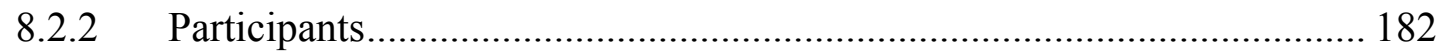

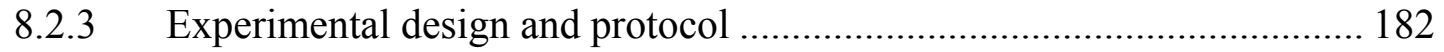

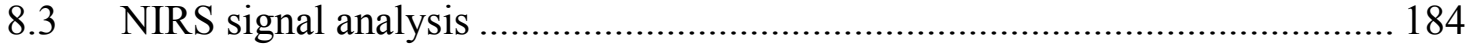

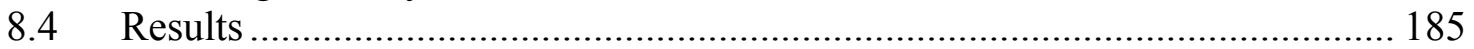

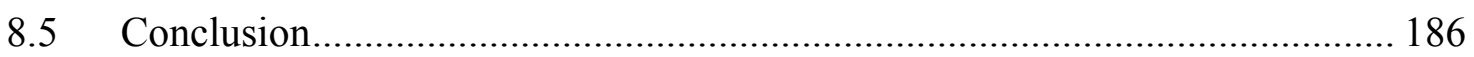

CHAPTER 9. Study 3a: Investigation of Planning and Execution in Individuals with

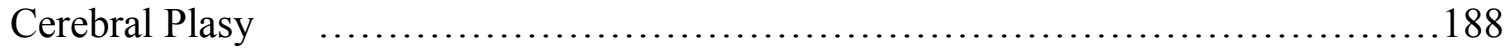

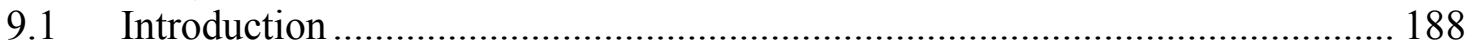

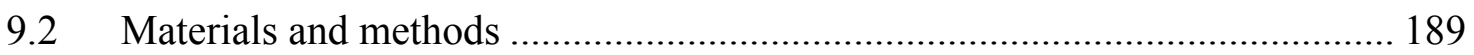

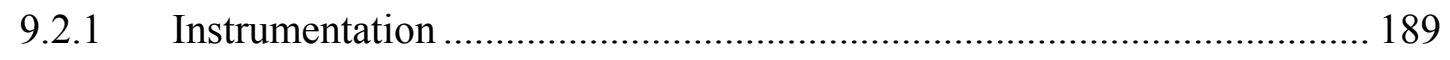

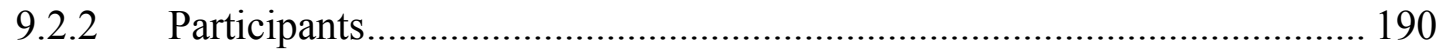

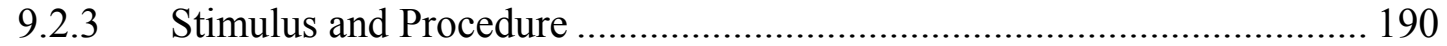

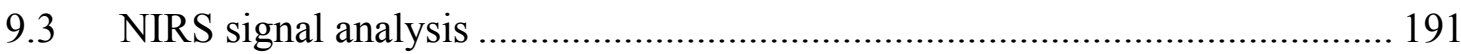

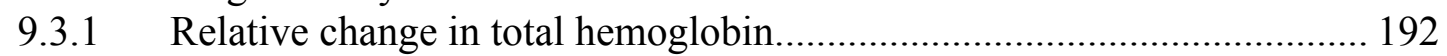

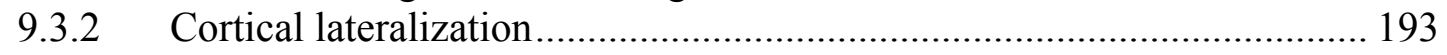

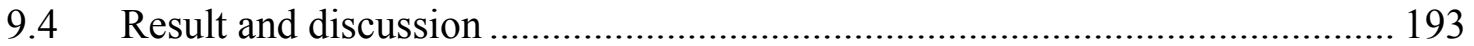

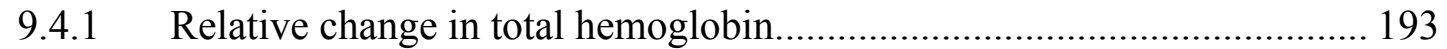

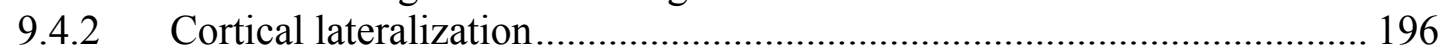

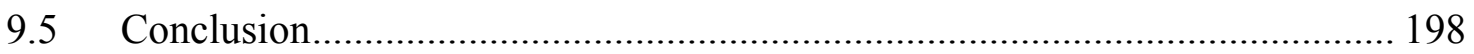

CHAPTER 10. Study 3b: Motor Skill Investigation of Planning and Execution in

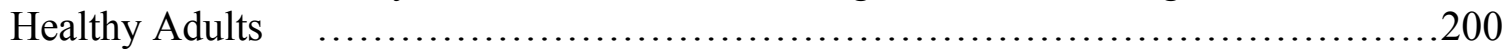

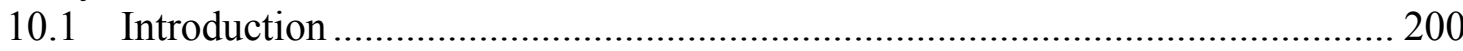

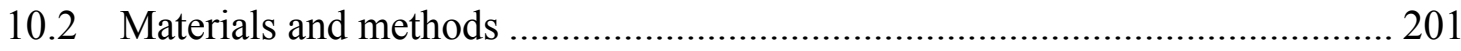

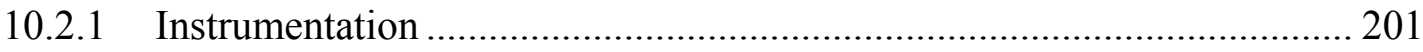

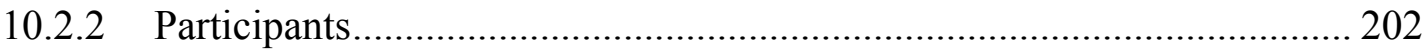

10.2.3 Experimental design and protocol ................................................... 202

10.2.3.1 Paradigm 1: Dot placement - Coarser motor control ........................ 202

10.2.3.2 Paradigm 2: Dot placement - Finer motor control .......................... 203

10.2.3.3 Paradigm 3: Ball throws ............................................................... 204

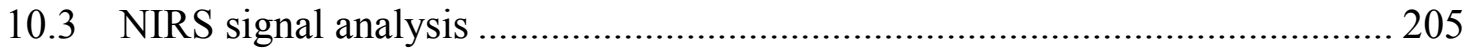

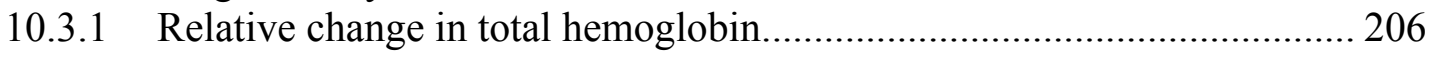

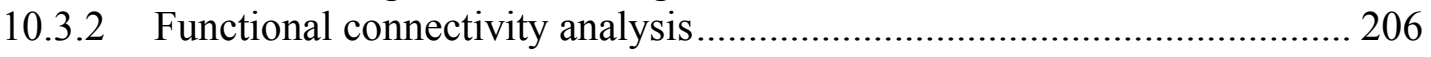

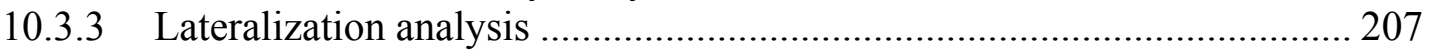

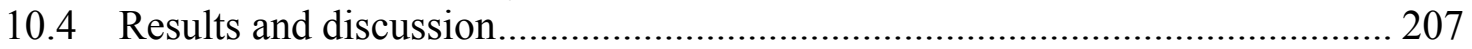

10.4.1 Paradigm1: Dot placement - coarser motor control .............................. 207

10.4.1.1 Relative change in HbT and Lateralization results......................... 207

10.4.1.2 Functional connectivity results.................................................... 212

10.4.2 Dot placement - coarser motor control............................................. 213

10.4.2.1 Relative change in $\mathrm{HbT}$ and Lateralization results......................... 213 


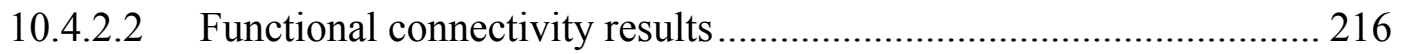

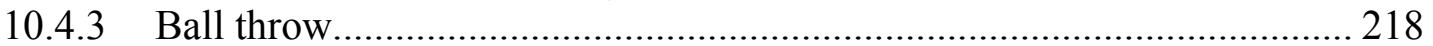

10.4.3.1 Relative change in $\mathrm{HbT}$ and Lateralization results........................... 218

10.4.3.2 Functional connectivity results....................................................... 220

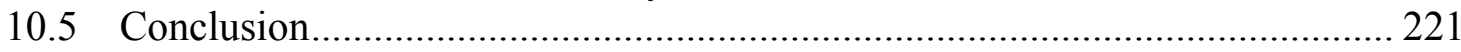

CHAPTER 11. Study 3c: Simultaneous NIRS and Kinematics Study of Planning and Execution of Motor Skills (Single Wavelength Study) ................................................ 224

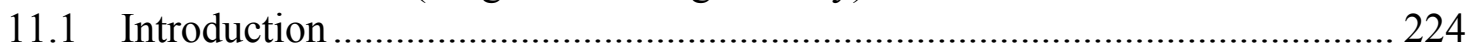

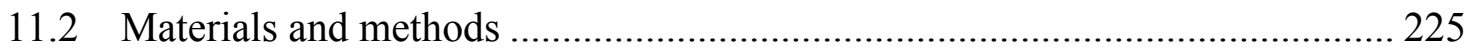

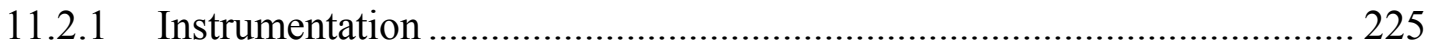

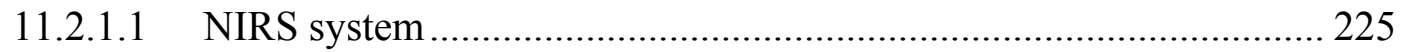

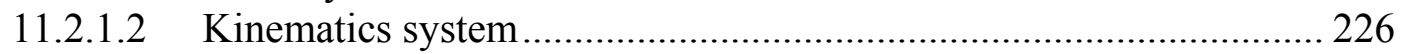

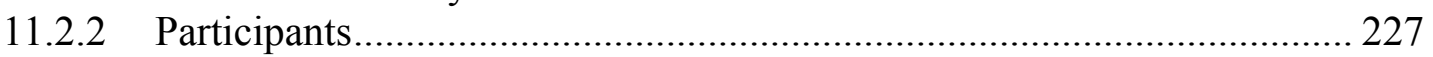

11.2.3 Experimental design and protocol ....................................................... 227

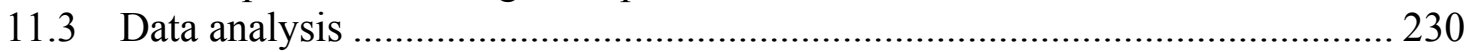

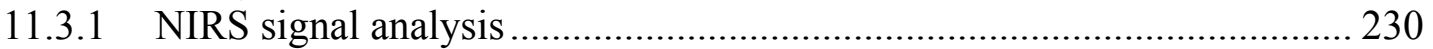

11.3.1.1 Relative change in total hemoglobin .............................................. 230

11.3.1.2 Functional Connectivity Analysis .................................................... 231

11.3.1.3 Cortical lateralization .................................................................... 232

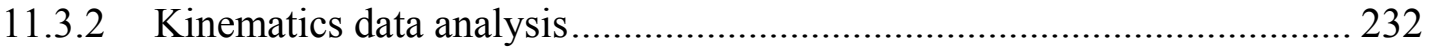

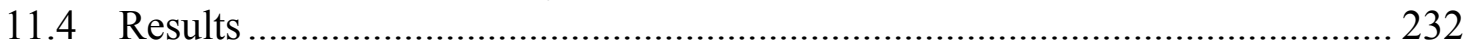

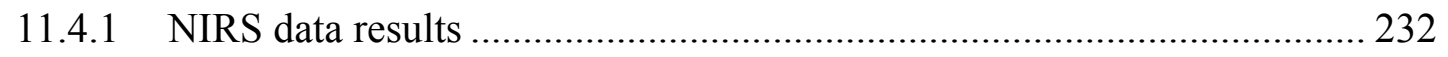

11.4.1.1 Relative change in HbT .............................................................. 232

11.4.1.2 Functional Connectivity ................................................................. 235

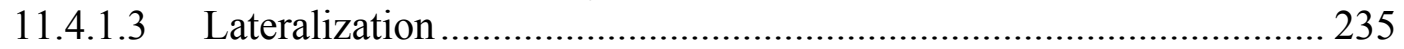

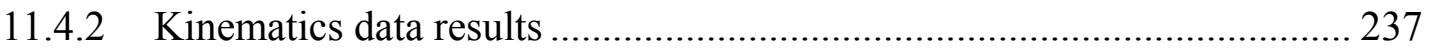

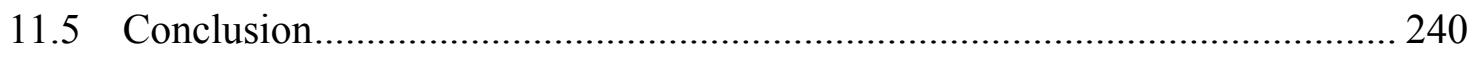

CHAPTER 12. Study 3d: Hemodynamic Response Measurement of Simultaneous NIRS and Kinematics Study of Planning and Execution of Motor Skills ...................... 242

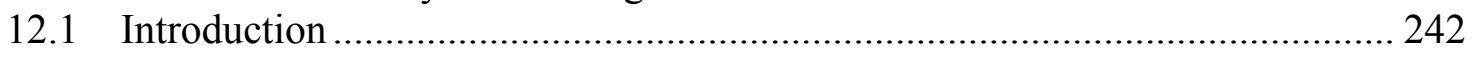

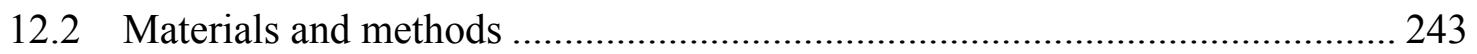

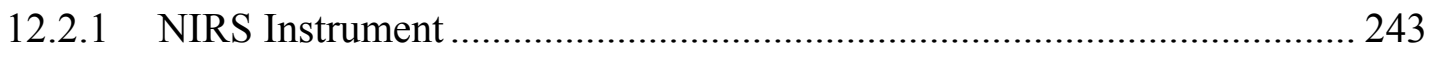

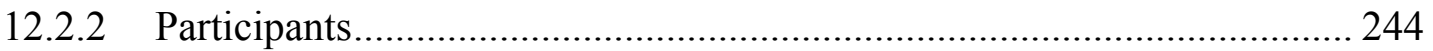

12.2.3 Experimental Design and Stimulus........................................................ 244

12.2.3.1 Paradigm 1: Target touching with finger......................................... 245

12.2.3.2 Paradigm 2: Target touching with pen ............................................ 246

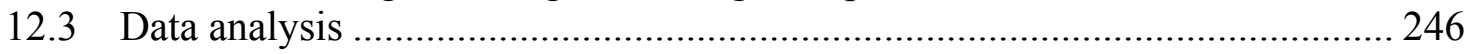

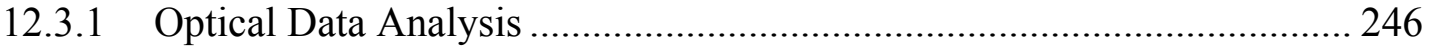

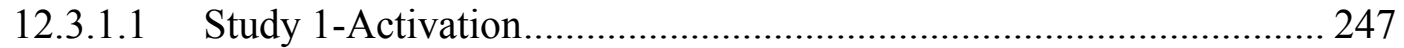

12.3.1.2 Study 2- Connectivity ........................................................................ 247

12.3.1.3 Study 3- Cortical Lateralization ...................................................... 247

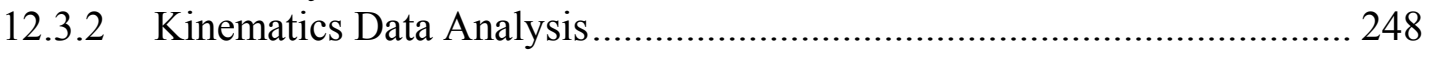

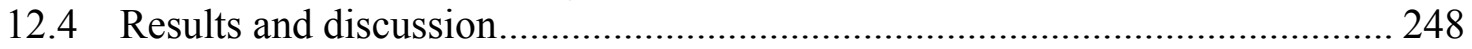


12.4.1 Paradigm 1: Target touching with finger ................................................ 248

12.4.1.1 Study1: Activation..................................................................... 248

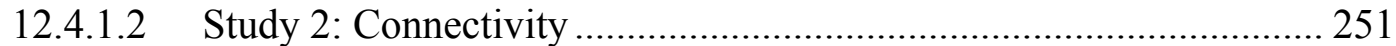

12.4.1.3 Study 3: Cortical Lateralization......................................................... 253

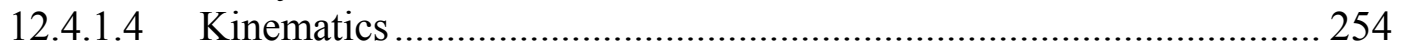

12.4.2 Paradigm 2: Target touching with pen................................................. 256

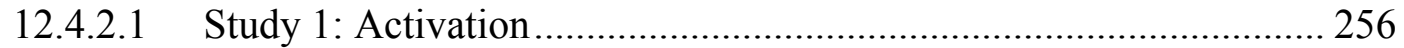

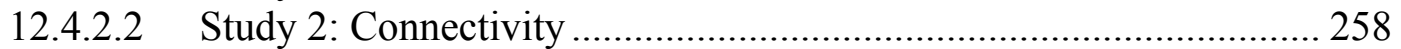

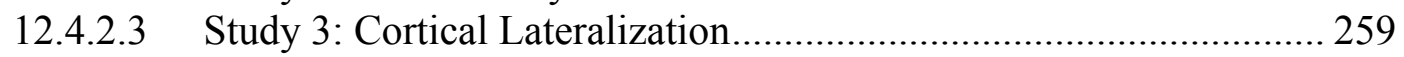

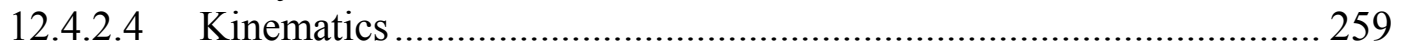

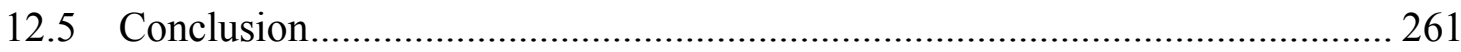

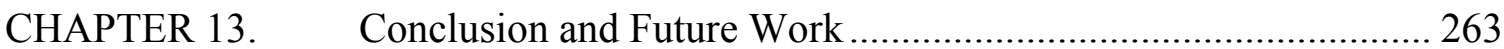

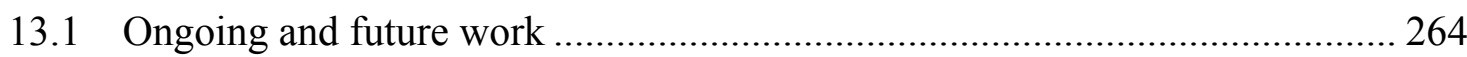

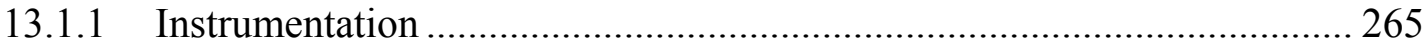

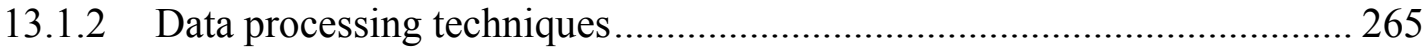

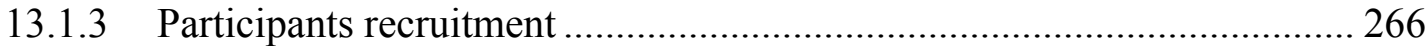

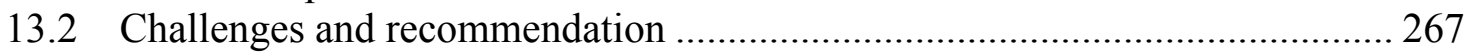

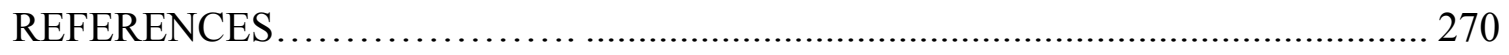

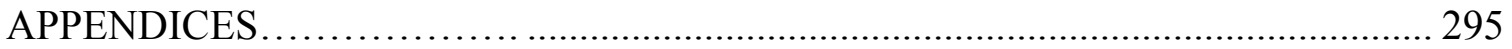

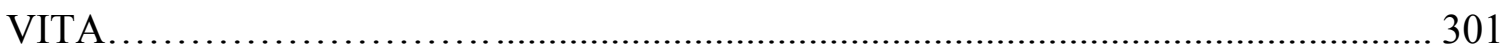




\section{LIST OF TABLES}

TABLE

PAGE

Table 1.1 : Optical probes developed by different researchers for functional brain

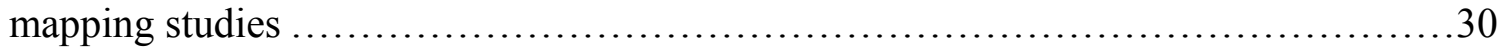

Table 1.2: List of commercially available NIRS systems (table modified and adapted from Wolf et al., 2007). In the table CW stands for Continuous wave, FD stands for frequency domain and TD stands for time domain.............................. 32

Table 1.3: List of recently developed in-house NIRS imaging systems (table modified and adapted from Wolf et al., 2007). In the table CW stands for Continuous wave, FD stands for frequency domain and TD stands for time domain. CCD stands for Charged

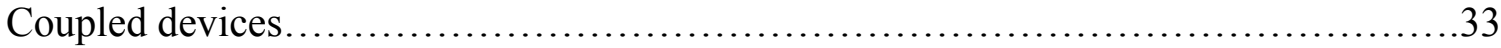

Table 4.1: Different types of biological signals and their frequency range (modified and adapted from Boas \& Franceschini, 2004). 89

Table 1.4: The p-values of the paired t-test performed using the average across all participants $\mathrm{HbO}, \mathrm{HbR}$ and $\mathrm{HbT}$ data of a particular region with the every single channel data of that particular region at $1 \%$ level of significance for word expression paradigm. A $p$-value $\geq 0.01$ indicates no significant difference between the regional data and each individual channel data.

Table 1.5: The p-value of the paired t-test performed using the average across all participants $\mathrm{HbO}, \mathrm{HbR}$ and $\mathrm{HbT}$ data of a particular region with the every single channel data of that particular region at $1 \%$ level of significance for word reception paradigm. A p-value $\geq 0.01$ indicates no significant difference between the regional data and each individual channel data.

Table 1.6: Zero-order correlation coefficient for changes in $\mathrm{HbO}$ (Oxy-Hemoglobin), HbR (Deoxy-Hemoglobin), and HbT (Total Hemoglobin) data (averaged across all participants), for each symmetric channel pair (left and right), across the three stimuli Joint (J), Non joint (NJ) and Rest (R). The correlation coefficients range from -1 to +1 , with -1 is strong negative correlation, +1 is strong positive correlation, and zero is no correlation 165

Table 1.7: Correlation coefficients for Inter stimuli (Joint (J) vs. Non Joint, Joint (J) vs. Rest (R) and Non Joint (NJ) vs. Rest (R)) zero order correlation analysis using $\mathrm{HbT}$ (Total Hemoglobin) data (averaged across all participants) for all channels. The correlation coefficients range from -1 to +1 , with -1 is strong negative correlation, +1 is strong positive correlation, and zero is no correlation 166

Table 1.8: Correlation coefficients for relative phase shift at $830 \mathrm{~nm}$ and relative phase shift at $690 \mathrm{~nm}$ (averaged across all participants), across the three stimuli Joint (J), Non joint (NJ) and Rest (R) and for each symmetric channel pair. The correlation coefficients 
range from -1 to +1 , with -1 is strong negative correlation, +1 is strong positive correlation, and zero is no correlation...................................... 171

Table 1.9: Pre frontal cortical lateralization or dominance in response to planning and execution of motor skill task and rest task in controls and adult (s) with cerebral palsy.

Table 1.10: Correlation value between left vs. right pre and anterior frontal channel, when the participants performed the dot placement coarser motor control paradigm using their dominant and non-dominant hand.

Table 1.11: Correlation value between left vs. right pre and anterior frontal channel, when the participants performed the dot placement finer motor control paradigm using their dominant and non-dominant hand 216

Table 1.12: Correlation value between left vs. right pre and anterior frontal channel, when the participants performed the ball throws paradigm.

Table 1.13: Pre frontal cortical lateralization or dominance (averaged across all participants) in response to planning and execution of motor skill task and rest task in healthy adults 237

Table 1.14: The results of paired t-test statistical analysis at $\mathrm{p}<0.05$ to determine the differences in activation (in terms of $\mathrm{HbO}, \mathrm{HbR}$, and $\mathrm{HbT}$ ) between target touching with finger (TT1) vs. Rest for the both the task performed using (A) dominant hand and (B) non-dominant hand. 251

Table 1.15: The results of paired t-test statistical analysis at $\mathrm{p}<0.05$ to determine the differences in activation (in terms of $\mathrm{HbO}, \mathrm{HbR}$, and $\mathrm{HbT}$ ) between target touching with pen (TTP) vs. Rest for the both the task performed using (A) dominant hand and (B) nondominant hand. 258

Table 1.16: The results of quantitative change in (A) $\mathrm{HbO}$, (B) $\mathrm{HbR}$ and (C) $\mathrm{HbT}$ during the target touching task performed by participants with finger and with pen using their dominant and non-dominant hand across trial 1 and trial 2. 261 


\section{LIST OF FIGURES}

FIGURE

PAGE

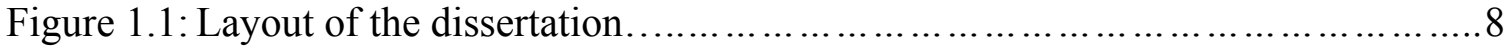

Figure 1.2: Absorption spectra for de-oxy hemoglobin $(\mathrm{Hb} / \mathrm{HbR})$, oxy hemoglobin $(\mathrm{HbO} 2 / \mathrm{HbO})$, and water $(\mathrm{H} 2 \mathrm{O})$ in the near-infrared range. The isosbestic point near 800 $\mathrm{nm}$ is the point where the absorptivity of $\mathrm{HbR}$ and $\mathrm{HbO}$ are equal (modified and adapted from Palmer \& Williams, 1974; Wray et al., 1988).................................11

Figure 1.2: Spatial sensitivity profile of light propagation in the layered model of a highly scattering brain media obtained using Monte Carlo simulation. (Modified and adapted

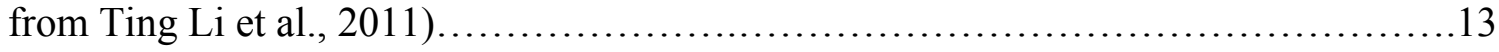

Figure 1.3: Schematic representation of continuous wave $(\mathrm{CW})$ technique. The figure depicts the transport of light through a multilayered model created to mimic different layers in the brain. In $\mathrm{CW}$ technique, light of initial intensity $\mathbf{I}_{\mathbf{0}}$ is incident onto the surface of the scalp and the transmitted attenuated light of intensity $\mathbf{I}_{\mathbf{1}}$ is measured at a distance $\mathrm{d}$ from the incident point (the multilayered brain model depicting the attenuation of light in brain is modified and adapted from Ting Li et al., 2011)...................21

Figure 1.4: Schematic representation of frequency domain technique. The figure depicts the transport of light through a multilayered model created to mimic different layers in the brain. In the $\mathrm{CW}$ technique, light of initial intensity $\mathbf{I}_{\mathbf{0}}$ is incident onto the surface of the scalp and the attenuation in intensity $\mathbf{I}_{\mathbf{1}}$ and phase shift $\Phi$ in the transmitted light is measured at a distance $\mathrm{d}$ from the incident point (the multilayered brain model depicting the attenuation of light in brain is modified and adapted from Ting Li et al., 2011)......22

Figure 1.5: Schematic representation of time domain technique. The figure depicts the transport of light through a multilayered model created to mimic different layers in the brain. In the time-domain technique, light of initial intensity $\mathbf{I}_{\mathbf{0}}$ is incident onto the surface of the scalp and the change in temporal distribution of picosecond pulse of light is measured at a distance $\mathrm{d}$ from the incident point (the multilayered brain model depicting the attenuation of light in brain is modified and adapted from Ting Li et al., 2011)......24

Figure 1.6: Temporal and spatial resolution comparison of different neuro imaging modalities (Figure adapted from Strangman et al., 2002).........................26

Figure 1.7: Hemodynamic response function, HRF plotted against time (in seconds). The plot shows the HRF as three tasks were being performed by the participants............37

Figure 1.8: Anatomical/Structural connectivity showing the anatomical links between different units in the brain (http://www.scholarpedia.org/article/Brain_connectivity).....39

Figure 1.9: Functional connectivity showing the temporal correlation among the activity of different neurons (http://www.scholarpedia.org/article/Brain_connectivity). 
Figure 1.10: Effective connectivity showing the causal or the directional interaction between distinct units within a nervous system (http://www.scholarpedia.org/article/Brain_connectivity)... 41

Figure 1.3: Frequency domain based optical imager, Imagent (ISS, Inc., Champaign, IL). The red circles depict the detectors and the yellow rectangles depict the sources. The Imagent was provided by Brain Institute at MCH (Miami Children's Hospital). 49

Figure 1.4: Block diagram of the different components used to develop the single wavelength ICCD based NIRS system. .51

Figure 1.5: The source of the Gen-2 imager is driven by the multichannel controller that is a combination laser driver (LD) and thermoelectric cooler driver (TECD), which allows for the control of up to 8 independent laser mounts.........................52

Figure 1.6: (A) The PI-Max II ICCD camera (from Princeton instruments) used for the detection of low intensity light, (B) the ICCD camera within the enclosure for the NIRS system, (C) Optics employed in conjunction with PI-Max II, such as lens tubes and other lens adapters 53

Figure 1.7: Winview interface showing the mode in which ICCD camera is run, the region highlighted in red is a drop down menu which enables selecting different timing mode. The single wavelength ICCD based NIRS system was run in "Free" run mode as shown in highlighted red area..................................................... 54

Figure 1.8: The developed single wavelength ICCD based NIRS system................56

Figure 1.9: Winview interface showing the set exposure time (the area highlighted in red) of CCD camera. The area highlighted in green shows the mode in which the intensifier is run and the also the gain of the intensifier. As shown in this Figure the exposure time of the camera was set as $0.2 \mathrm{sec}$, the intensifier was run in shutter mode and the gain of the intensifier for this particular data acquisition duration was 100 (abu) .................58

Figure 1.10: Block diagram of the different components used to develop the dual wavelength ICCD based NIRS system. .60

Figure 1.11: (A) EK 2000 laser driver to drive A pin style laser diodes. (B) DBDL M/Q to drive $\mathrm{C}$ type laser diodes. (C) Schematic diagram of working of laser drivers (figure adapted from manual of EK 2000 laser driver). (D) LDS2 9 V DC power supply from Thorlabs .63

Figure 1.12: (A) NI PCIe-6341X Series Data Acquisition card, (B) NI SHC68-68-EPM cable and (C) NI BNC-2110 a shielded connector block with serves as the output terminal of the signal

Figure 1.13: Labview interface showing the different input parameter, (A) Channel parameter, (B)Timing parameter, (C) Buffer parameters, (D) Generated waveform and (E) Array to set the frequency and the waveform shape. 66 
Figure 1.14: Block diagram of the dual wavelength source system

Figure 1.15: The power supply module with eight 9V DC power supplies to power eight laser driver boards.

Figure 1.16: The assembled dual wavelength source system. (A) The front panel, (B) Set of four Laser drivers for $830 \mathrm{~nm}$ laser diodes, (C) Set of four Laser drivers for $690 \mathrm{~nm}$ laser diodes and (D) The signal generator.

Figure 1.17: The front panel with female SMA connectors to optically couple (A) $830 \mathrm{~nm}$ and (B) $690 \mathrm{~nm}$ laser diodes to optical fiber.

Figure 1.18: The fully assembled dual wavelength source system. The bottom section is the power section and the top section is the laser systems section.....

Figure 1.19: Winview interface showing the mode in which ICCD camera is run, the region highlighted in red is a drop down menu which enables selecting different timing mode. The dual wavelength ICCD based NIRS system is run in External sync mode as shown in the highlighted red area. 70

Figure 1.20: Stem plot of the intensity acquired by the ICCD camera with $830 \mathrm{~nm}$ sources OFF and $690 \mathrm{~nm}$ sources ON. Here, the odd frames correspond to the $690 \mathrm{~nm}$ wavelength of light and even frames correspond to the $830 \mathrm{~nm}$ wavelength of light. .75

Figure 1.21: Stem plot of the intensity acquired by the ICCD camera with $830 \mathrm{~nm}$ sources ON and $690 \mathrm{~nm}$ sources OFF. Here, the odd frames correspond to the $690 \mathrm{~nm}$ wavelength of light and even frames correspond to the $830 \mathrm{~nm}$ wavelength of light. 76

Figure 1.22: Stem plot of the intensity acquired by the ICCD camera with alternating 830 $\mathrm{nm}$ sources and $690 \mathrm{~nm}$ sources. Here, the odd frames correspond to the $690 \mathrm{~nm}$ wavelength of light and even frames correspond to the $830 \mathrm{~nm}$ wavelength of light.....78

Figure 1.23: Intensity at $830 \mathrm{~nm}$ (in arbitrary units, abu), acquired using in-house developed system (described in section 3.3), plotted against time (in seconds). The figure depicts the change in the intensity at $830 \mathrm{~nm}$ across the five blocks of task (T) and rest (R) period 84

Figure 1.24: Intensity at $690 \mathrm{~nm}$ ((in arbitrary units, abu), acquired using in-house developed system (described in section 3.3), plotted against time (in seconds). The figure depicts the change in the intensity at $690 \mathrm{~nm}$ across the five blocks of task (T) and rest (R) period 84

Figure 1.25: Filtered intensity at $830 \mathrm{~nm}$ plotted against time. . The figure depicts the change in the filtered intensity at $830 \mathrm{~nm}$ across the five blocks of task (T) and rest (R) period 
Figure 1.26: Filtered intensity at $690 \mathrm{~nm}$ plotted against time. The figure depicts the change in the filtered intensity at $690 \mathrm{~nm}$ across the five blocks of task (T) and rest (R) period.

Figure 1.27: Normalized and filtered intensity at $830 \mathrm{~nm}$, plotted against time. The figure depicts the change in the normalized intensity at $830 \mathrm{~nm}$ across the five blocks of task (T) and rest $(\mathrm{R})$ period. .86

Figure 1.28: Normalized and filtered intensity at $690 \mathrm{~nm}$ plotted against time. The figure depicts the change in the normalized intensity at $690 \mathrm{~nm}$ across the five blocks of task (T) and rest $(\mathrm{R})$ period.

Figure 1.29: Change in optical density at $830 \mathrm{~nm}$ acquired plotted against time. The figure depicts the change in the optical density at $830 \mathrm{~nm}$ across the five blocks of task (T) and rest

period. 88

Figure 1.30: Change in optical density at $690 \mathrm{~nm}$ plotted against time. The figure depicts the change in the optical density at $690 \mathrm{~nm}$ across the five blocks of task (T) and rest (R) period.

Figure 1.31: Change in concentration of $\mathrm{HbO}, \mathrm{HbR}$ and $\mathrm{HbT}$ averaged across the five blocks of task $(\mathrm{T})$ and rest $(\mathrm{R})$ period, obtained after processing change in optical density at $830 \mathrm{~nm}$ and $690 \mathrm{~nm}$, plotted against time.

Figure 4.10: Flowchart depicting the implementation of granger causality algorithm for elucidating the effective connectivity across the cortical regions under investigation....94

Figure 1.32: Source-detector layout corresponding to the prefrontal (Fp) and the anterior frontal (AF) region of standard 10-10 (Hoshi et al, 1994) electrode placement system. The solid circles and hollow circles used in the figure represent detectors and sources respectively. The hollow squares represent the path between the sources and detectors (i.e. channels). All the even number channels correspond to the left frontal cortex, and the odd number channels correspond to the right frontal cortex 103

Figure 1.33: Averaged hemodynamic response function across all the participants (HRF) plotted against time (in seconds) for each channel (Ch. 1-8) in response to the languagebased stimuli. Each plot shows the HRF data for the block stimuli of Word (W), Jaw Movement (J), and Rest (R) performed by the participants. In each plot, the y-axis is the change in hemoglobin concentration, while x-axis is the time in seconds. In each plot, the red solid curve represents the change in $\mathrm{HbO}$ (oxy-hemoglobin), the blue dotted curve represents the change in $\mathrm{HbR}$ (deoxy-hemoglobin) and the black dash-dot curve represents the change in HbT (total hemoglobin). The hemodynamic changes from 0-30 sec corresponds to Word task, 30-60 sec to Jaw movement task, and 60-90 sec to Rest task. The numbers shown in square boxes on top of each plot corresponds to the number given to each source-detector combination or channel as shown in Figure 5.1.... 107 
Figure 1.34: Pictorial representation of the differences in $\mathrm{HRF}$ ( $\mathrm{HbO}, \mathrm{HbR}$, and $\mathrm{HbT}$ ) across the three stimuli (W, J, and R) in each channel, obtained from a three-paired t-test. The grey shaded channels represent significant differences between the two stimuli (W vs. J, W vs. R, and J vs. R) of interest for a given HRF data. The non-shaded white channels represent insignificant differences between the two stimuli of interest for a given HRF data. The solid black circles correspond to detectors and the hollow circles correspond to sources placed on the pre and anterior frontal region of brain as shown in Figure 5.1...109

Figure 1.35: Bar plot of the correlation coefficient obtained from the zero order correlation analyses study in response to language-based stimuli on the frontal cortex. The plot depicts the correlation coefficient values obtained from (A) $\mathrm{HbO}$, (B) $\mathrm{HbR}$, and (C) HbT data, across the three stimuli (Word, Jaw, and Rest) and left vs. right channel pairs (4 of them) in the pre-frontal (PF) and anterior frontal (AF) cortex..........................110

Figure 1.36: Inter stimuli (W vs. J, W vs. R and J vs. R) zero order correlation analysis using $\mathrm{HbT}$ data for all channels in the result using color code for the range of correlation values. As shown in the color code box correlation values in the range of 1.00 to 0.50 has been assigned red, 0.50 to 0.00 orange, 0.00 to -0.50 green and -0.50 to -1.00 blue. Using the source detector layout described in the Figure 1 each channel has been color coded, as described above, based on the correlation value obtained using HbT data for each stimuli pair i.e. W vs. J, W vs. R and J vs. R. ............................111

Figure 1.37: Cortical dominance representations using source detector layout as described in Figure 5.1 across all left vs. right channel pairs (4 of them) for each of the three stimuli (Word, Jaw, Rest) in the frontal cortex (averaged across all participants). The dominant channel pair (across the left and right cortex) is shaded grey........................113

Figure 1.38: A. Arrangement of the source-detector layout employed during the study. The layout follows the standard International 10-10 (Jurcak et al. 2007) electrode placement system. The red circles and black x-mark represent sources and detectors, respectively. The line represents the path between the sources and detectors (i.e. channels). B. The Brodmann's layout of the different regions in the brain. The rectangular highlighted area represents the area investigated during the study. The figure represents the Brodmann area corresponding to the 10-10 electrode placement system. The channels 6, 7 and 8 were placed in such a way that it encompasses the Brodmann area 44 and 45 which contains the Broca's region (called Anterior left (AL) in the figure), while the channel 1,2, and 3 were placed in such a way that it encompasses the Brodmann area 39 and 40 which contains the Wernicke's region (called Posterior left (PL) in the figure). The channels were correspondingly placed on the right side to form the right homologue (i.e. Anterior Right (AR) and Posterior Right (PR)) of the AL and PL, respectively........121

Figure 1.39: Hemodynamic change plotted against time (in seconds) for A. Anterior left $(\mathrm{AL})$ region, B. Anterior right $(\mathrm{AR})$ region, $\mathrm{C}$. Posterior left $(\mathrm{PL})$ region and $\mathrm{D}$. Posterior right $(\mathrm{PR})$ region in response to the word expression based stimuli. Each plot shows the 
averaged hemodynamic data across all participants and for each stimuli including Word Generation (W) (0-30 sec), Jaw Movement (J) (31-60 sec), and Rest (R) (61-90 sec)...129

Figure 1.40: Pictorial representation of the differences in data $(\mathrm{HbO}, \mathrm{HbR}$, and $\mathrm{HbT})$ across the three stimuli (W, J, and R) in each region, obtained from a three-paired t-test. The grey shaded channels represent significant differences between the two stimuli (W vs. J, W vs. R, and J vs. R) of interest for a given averaged (across all participants) hemodynamic data. The non-shaded white channels represent insignificant differences in the averaged hemodynamic data between the two stimuli of interest.................130

Figure 1.41: Lateralization diagram showing dominance (marked by grey) between left and right fronto-temporal regions during the three stimuli of the Word Expression Paradigm (obtained from the averaged $\mathrm{HbT}$ data across all participants). The four regions are - Left Anterior (LA), Right Anterior (RA), Left Posterior (LP), Right Posterior $(\mathrm{RP})$ 131

Figure 1.42: Pictorial representation of the result of functional connectivity analysis using zero order Pearson's correlation during word expression paradigm using average hemodynamic data across all participants 1: $\mathrm{HbO}, 2$ : $\mathrm{HbR}$ and 3: $\mathrm{HbT}$ data for (A) Word Generation, (B) Jaw Movement, and (C) Rest. The four regions under investigation are represented by four squares, where in the Anterior Left (AL) region contains the Broca's region; Anterior Right (AR) region contains the right homologue of AL region; the Posterior Left (PL) region contains the Wernicke's region; and the Posterior Right (PR) region contains the right homologue of PL region...

Figure 1.43: Pictorial representation of the causal and directional flow of information obtained from effective connectivity analysis (or granger causality analysis) during word expression paradigm using average hemodynamic data across all participants 1: $\mathrm{HbO}, 2$ : HbR and 3: HbT data, for (A) Word Generation, (B) Jaw Movement, and (C) Rest. The directional flow of information across the four regions (AL, PL, AR, and PR) is shown using thickening and thinning of arrows, where in the thick arrow shows strong flow of information and thin arrows shows weak flow of information 135

Figure 1.44: Averaged hemodynamic changes (across all participants) plotted against time (in seconds) for A. Anterior left (AL) region, B. Anterior right (AR) region, C. Posterior left $(\mathrm{PL})$ region and $\mathrm{D}$. Posterior right $(\mathrm{PR})$ region in response to the word reception based stimuli. Each plot shows the averaged hemodynamic data for the block stimuli of Forward Audio task (FA), Reverse Audio task (RA), and Rest (R) performed by

Figure 1.45: Pictorial representation of the differences in hemodynamic data $(\mathrm{HbO}, \mathrm{HbR}$, and $\mathrm{HbT}$ ) across the three stimuli (FA, RA, and R) in each region, obtained from a threepaired t-test. The grey shaded channels represent significant differences between the two stimuli (FA vs. RA, FA vs. R, and RA vs. R) of interest for a given averaged hemodynamic data (across all participants). The non-shaded white channels represent 
insignificant differences between the two stimuli of interest for a given averaged hemodynamic data.......................................................... 137

Figure 1.46: Lateralization diagram showing dominance (marked by grey) between left and right fronto-temporal regions during the three stimuli of the word expression paradigm obtained from averaged $\mathrm{HbT}$ data (across all participants). The four regions are - Left Anterior (LA), Right Anterior (RA), Left Posterior (LP), Right Posterior (RP)...138

Figure 1.47: Pictorial representation of the result of functional connectivity analysis using zero order Pearson's correlation during word reception paradigm using average hemodynamic data across all participants 1: $\mathrm{HbO}, 2$ : $\mathrm{HbR}$ and 3: $\mathrm{HbT}$ data, for (A) Forward Audio, (B) Reverse Audio, and (C) Rest stimuli..........................139

Figure 1.48: Pictorial representation of the causal and directional flow of information obtained from effective connectivity analysis (or granger causality analysis) during word reception paradigm using average hemodynamic data across all participants 1: $\mathrm{HbO}, 2$ : $\mathrm{HbR}$ and 3: HbT data, for (A) Forward Audio, (B) Reverse Audio, and (C) Rest. The directional flow of information between the regions is shown using thickening and thinning of arrows, where in the thick arrow shows strong flow of information and thin arrows shows weak flow of information.

Figure 1.49: Source-detector layout corresponding to the prefrontal and the anterior frontal brain region of the standard 10-20 electrode placement system.........................155

Figure 1.2: Hemodynamic response function (HRF) (averaged across all participants) in terms of $\mathrm{HbO}$ (Oxy-Hemoglobin) with respect to time, plotted for each 30-sec stimuli ( $\mathrm{J}$, $\mathrm{NJ}$, and R) and for each symmetric channel pair (left and right) on the same plot......162

Figure 1.3: Hemodynamic response function (HRF) (averaged across all participants) in terms of HbR (deoxy-Hemoglobin) with respect to time, plotted for each 30-sec stimuli $(\mathrm{J}, \mathrm{NJ}$, and $\mathrm{R}$ ) and for each symmetric channel pair (left and right) on the same plot....163

Figure 1.4: Hemodynamic response function (HRF) (averaged across all participants) in terms of HbR (Deoxy-Hemoglobin) with respect to time, plotted for each 30-sec stimuli (J, NJ, and R) and for each symmetric channel pair (left and right) on the same plot...164

Figure 1.5: Zero-order correlation bar plots for (A) HbO (Oxy-Hemoglobin), (B) HbR (Deoxy-Hemoglobin), and (C) HbT (Total Hemoglobin) data (averaged across all participants), for each symmetric channel pair (left and right) and across the three stimuli (J, NJ and R) 167

Figure 1.6: Cortical lateralization bar plot for each symmetric channel pair (left and right) and across the three stimuli ( $\mathrm{J}, \mathrm{NJ}$ and R). Laterality index value $(\mathrm{L})>0.25$ indicates left cortical lateralization, $\mathrm{L}<-0.25$ indicates right cortical lateralization and $-0.25<\mathrm{L}<$ 0.25 indicates no dominance or bilateral activation... 
Figure 1.7: Relative phase shift (at $830 \mathrm{~nm}$ ) (averaged across all participants) across the three stimuli (J, NJ and R) for each symmetric channel pair (left and right).............172

Figure 1.8: Relative phase shift (at $690 \mathrm{~nm}$ ) (averaged across all participants) across the three stimuli (J, NJ and R) for each symmetric channel pair (left and right).... 173

Figure 1.9: Zero-order correlation bar plots for (A) Relative phase shift at $830 \mathrm{~nm}$ and (B) Relative phase shift at $690 \mathrm{~nm}$, (averaged across all participants) across the three stimuli ( $\mathrm{J}, \mathrm{NJ}$ and R) and for each symmetric channel pair, where PF is pre-frontal and AF is anterior frontal cortex 174

Figure 1.50: Source-detector layout on the prefrontal and the anterior frontal brain region of the brain, in accordance to the standard 10-20 electrode placement system, during the NIRS joint attention studies

Figure 1.51: Participant with the optical cap during the brain imaging study. The source and detector fibers were placed in the frontal cortex 182

Figure 1.52: (A) Joint (J) attention stimulus, movement of the gaze of the presenter (lady in the center of cartoon) is congruent with the presentation of the moving object (the cartoon near the edge of square, here it is Johnny bravo). (B) Non Joint attention (NJ) stimulus, movement of the gaze of the presenter (lady in the center of cartoon) is incongruent with the presentation of the moving object (the cartoon near the edge of square, here it is pink cartoon character)

Figure 1.53: (A) Activation plots depicting changes in hemodynamic response (in terms of $\mathrm{HbO}, \mathrm{HbR}$, and $\mathrm{HbT}(\mathrm{HbO}$ - oxy-hemoglobin, HbR - deoxy-hemoglobin and $\mathrm{HbT}$ total hemoglobin)) during J, NJ and R in a Typically Developing (TD) Children. In the Figure, L corresponds to left frontal cortex and $\mathrm{R}$ to correspond to the right frontal cortex. (B) Activation plots depicting changes in hemodynamic response (in terms of $\mathrm{HbO}, \mathrm{HbR}$, and $\mathrm{HbT}(\mathrm{HbO}$ - oxy-hemoglobin, $\mathrm{HbR}$ - deoxy-hemoglobin and $\mathrm{HbT}$ - total hemoglobin)) during $\mathrm{J}, \mathrm{NJ}$ and $\mathrm{R}$ in a Child with autism. In the Figure, L corresponds to left frontal cortex and $\mathrm{R}$ to correspond to the right frontal cortex 186

Figure 1.54: Source detector placement is same as the 10-20 system of electrode placement for the pre frontal region of the brain. Each source detector pair is called channel. Fp1 and Fp2 represent the detectors placed on the left and right prefrontal, respectively. Fpz represent the source placed on the mid pre frontal...................................189

Figure 1.55: Average change in total hemoglobin across all participants (HbT) as a function of time across the source detector combination Fp1-Fpz (plot in dashed black) and Fp2-Fpz (plot in solid red). The quantified change in HbT calculated by summing the change in $\mathrm{HbT}$ for the central $20 \mathrm{sec}$ period of the ball throw task and rest period is shown as thick dotted black line and thick solid red line across the source detector 
combination Fp1-Fpz and Fp2-Fpz, respectively. The first 30 sec represent the change in HbT during ball throw task (BT) and the last $30 \mathrm{sec}$ represent Rest period (R)........194

Figure 1.56: Cortical lateralization/dominance map during task and rest for all the participant type. In the map the highlighted rectangular box represent the prefrontal region under investigation and the thick line denotes the dominant side. A - Dominance map for the right handed control. B - Dominance map of the left handed control. CDominance map of the right handed individuals with CP. D- Dominance map of the left handed individuals with CP...................................................... 198

Figure 1.57: Source detector layout corresponding to the 10-10 electrode placement system showing the placement of source (solid circle) and detector (hollow circle) on the pre and anterior frontal region of brain. Each source detector pair is called channel. Fp1 and Fp2 represent the detector placed on the left and right prefrontal, respectively. Fpz represent the source placed on the mid pre frontal. AF3 and AF4 represent the detector placed on the left and right anterior frontal, respectively. AFz represent the source placed

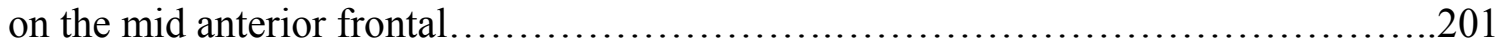

Figure 1.58: Change in total hemoglobin ( $\mathrm{HbT})$ (averaged across all participants) as a function of time for the source detector combination Fp1-Fpz (plot in solid red), Fp2-Fpz (plot in dashed black), AF3-AFz (plot in dash-dot green) and AF4-AFz (plot in dotted blue) during dot placement coarser motor control paradigm. In the plot, the y-axis is the relative change in $\mathrm{HbT}$, while $\mathrm{x}$-axis is the time in seconds. The relative change in $\mathrm{HbT}$ from 0-30 sec corresponds to dot placement task (DP-C), 30-60 sec to rest period (R1), 60-90 sec to dot placement with balance task (DP-CB) and 90-120 sec to second rest period (R2). A - Depicts the relative change in $\mathrm{HbT}$ when the participants performed the tasks via their dominant hand. B - Depicts the relative change in $\mathrm{HbT}$ when the participants performed the tasks via their non-dominant hand.......................209

Figure 1.59: Bar plot depicting the quantified relative change in $\mathrm{HbT}$ across different source-detector pairs for the dot placement coarser motor control paradigm performed by the participants via their dominant and non-dominant hand. In each plot the relative change in $\mathrm{HbT}$ across a particular source-detector pair is shown by a bar plot as well as a quantitative value calculated by averaging the central $20 \mathrm{sec}$ of the relative change in $\mathrm{HbT}$ during a particular stimulus, where (A) includes task (Dot placement coarser motor control: DP-C) and rest (R1) using dominant hand, (B) includes task (Dot placement coarser motor control with beaker: DP-CB) and rest (R2) using dominant hand, (C) includes task (DP-C) and rest (R1) using non-dominant hand, and (D) includes task (DP$\mathrm{CB}$ ) and rest (R2) using non-dominant hand. In each plot the bar in black and grey represents the quantitative relative change in HbT during task (DP-C or DP-CB) and rest period (R1 and R2) respectively. The results from lateralization analysis are also shown in Figure 10.3 by using lines of different style and thickness connecting a particular source and detector pair. In Figure 10.3 the thick dash line drawn to represent the prefrontal channels, denotes left dominance.

.211

Figure 1.60: Change in total hemoglobin (HbT) (averaged across all participants) as a function of time across the source detector combination Fp1-Fpz (plot in solid red), Fp2- 
Fpz (plot in dashed black), AF3-AFz (plot in dash-dot green) and AF4-AFz (plot in dotted blue) during dot placement finer motor control paradigm. In the plot, the y-axis is the change in $\mathrm{HbT}$, while $\mathrm{x}$-axis is the time in seconds. The change in $\mathrm{HbT}$ from $0-30 \mathrm{sec}$ corresponds to dot placement task (DP-F), 30-60 sec to rest period (R1), 60-90 sec to dot placement with balance task (DP-FB) and 90-120 sec to second rest period (R2), when using the (A) dominant hand, and (B) non-dominant hand........................214

Figure 1.61: Bar plot depicting the quantified relative change in $\mathrm{HbT}$ across different source -detector pairs for the dot placement finer motor control paradigm performed by the participants via their dominant and non-dominant hand. In each plot the relative change in HbT across a particular source-detector pair is shown by a bar plot as well as a quantitative value calculated by averaging the central $20 \mathrm{sec}$ of the relative change in HbT during a particular stimulus, where (A) includes task (Dot placement finer motor control: DP-F) and rest (R1) using dominant hand, (B) includes task (Dot placement coarser finer control with beaker: DP-FB) and rest (R2) using dominant hand, (C) includes task (DP-F) and rest (R1) using non-dominant hand, and (D) includes task (DPFB) and rest (R2) using non-dominant hand. In each plot the bar in black and grey represents the quantitative relative change in HbT during task (DP-F or DP-FB) and rest period (R1 and R2) respectively. The results from lateralization analysis are also shown in Figure 10.5 by using lines of different style and thickness connecting a particular source and detector pair. In Figure 10.5 the thin line drawn to represent the channels denotes bilateral dominance................................................... 215

Figure 1.62: Change in total hemoglobin (HbT) (averaged across all participants) as a function of time across the source detector combination Fp1-Fpz (plot in solid red), Fp2Fpz (plot in dashed black), AF3-AFz (plot in dash-dot green) and AF4-AFz (plot in dotted blue) during ball throws paradigm. In the plot, the y-axis is the relative change in $\mathrm{HbT}$, while $\mathrm{x}$-axis is the time in seconds. The relative change in $\mathrm{HbT}$ from $0-30 \mathrm{sec}$ corresponds to ball throws using dominant hand task (BTD), 30-60 sec to rest period (R1), 60-90 sec to ball throws using non-dominant hand task (BTND) and 90-120 sec to

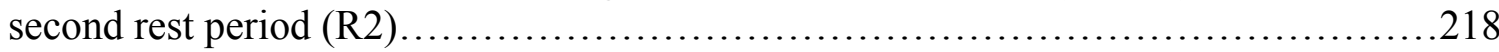

Figure 1.63: Bar plots depicting the quantified change in $\mathrm{HbT}$ across different source detector pairs for the ball throws paradigm. In each plot the relative change in HbT across a particular source-detector pair is shown by a bar plot as well as a quantitative value calculated by averaging the central $20 \mathrm{sec}$ of the relative change in HbT during a particular stimulus where (A) includes task (Ball throw with dominant hand: BTD) and rest (R1) and (B) includes task (Ball throw with non-dominant hand: BTND) and rest period (R2). The results from lateralization analysis are also shown in Figure 10.7 by using lines of different style and thickness connecting a particular source and detector pair. In Figure 10.7 the thin line drawn to connect channels denotes bilateral activation or no dominance across any channels............................................. 219

Figure 1.64: Source detector layout corresponding to the 10-20 electrode placement system showing the placement of source (hollow circle) and detector (solid circle) on the pre and anterior frontal region of brain. Each source detector pair is called channel. Fp1 
and Fp2 represent the detector placed on the left and right prefrontal, respectively. Fpz represent the source placed on the mid pre frontal................................ 226

Figure 1.65: The complete experimental setup of the simultaneous kinematics and NIRS system. The set-up shows the participant sitting in front of motor capture system with the optical probes on his head connected to NIRS system...........................228

Figure 1.66: Visual 3-D representation of retroreflective markers placed on the arms of the participants (shown by yellow rectangle) and on the A4 sheet (shown by white square) .229

Figure 1.67: The A4 sheet showing circle drawn on its four corners with retro-reflective marker placed in the center of each circle. (A)- shows the target touching pattern performed by participants using their dominant hand, and (B)- shows the target touching pattern performed by participants using their non-dominant hand.

229

Figure 1.68: Change in total hemoglobin ( $\mathrm{HbT})$, averaged across all participants, as a function of time across the source detector combination Fp1-Fpz (plot in solid red line) correspond to left pre-frontal channel and Fp2-Fpz (Plot in dashed black line) correspond to right pre-frontal channel. The numerical value shown in each 30 second period (with the label corr. val.) is the correlation coefficient between left and right pre frontal region of the brain

Figure 1.69: Block wise change in total hemoglobin ( $\mathrm{HbT})$ as a function of time across the source detector combination Fp1-Fpz (plot in solid red), Fp2-Fpz (plot in dashed black). In the plot, the $\mathrm{y}$-axis is the change in HbT, while $\mathrm{x}$-axis is the time in seconds. In each block $\mathrm{T}$ denotes the target touching task period and $\mathrm{R}$ the rest period. A- Random block wise plot from the group of participants among whom the change in HbT decreases as block progresses. B - Random block wise plot from the group of participants among whom the change in $\mathrm{HbT}$ increases as block progresses 234

Figure 1.70: Quantified bar plot of the block wise change in HbT plotted in Figure 6. A and $\mathrm{B}$ - Depict the quantitative relative change in $\mathrm{HbT}$ across the left and right pre frontal region of brain, respectively, for plot 11.6A. C and D - Depict the quantitative relative change in $\mathrm{HbT}$ across the left and right pre frontal region of brain, respectively, for plot $11.6 \mathrm{~B}$ 236

Figure 1.71: Bar plot showing the average number of touches, along with the standard deviation, performed by each participant using their left and right hand. .238

Figure 1.72: Bar plot showing the number of touches performed by the randomly chosen participant, for whom the change in HbT was plotted in Figure 11.6, block wise. A Depicts the number of touches performed by the participant for whom the change in $\mathrm{HbT}$ was plotted in Figure 11.6A. B- Depicts the number of touches performed by the participant for whom the change in $\mathrm{HbT}$ was plotted in Figure 11.6B.................238

Figure 1.73: Source-detector layout corresponding to the prefrontal and the anterior frontal region of standard 10-20 electrode placement system. The solid circles and 
hollow circles used in the figure represent sources and detectors respectively. The dashed line represents the path between the sources and detectors (i.e. channels). All the odd number channels correspond to the left frontal cortex, and the even number channels correspond to the right frontal cortex..... .244

Figure 1.74: The A4 sheet showing circle drawn on its four corners, showing the target touching pattern performed by participants using their (A) dominant hand, and (B) nondominant hand. .245

Figure 1.75: Hemodynamic response function (HRF), averaged across all participants, plotted against time (in seconds) for each channel (Ch. 1-4) in response to the planning and execution of motor skill-based stimuli performed by participants using their dominant hand's index finger. Each plot shows the HRF data for the block stimuli of target touching task (T), and Rest (R) performed by the participants. The hemodynamic changes from 0-30 sec correspond to target touching task and 30-60 sec to Rest task. The numbers shown in square boxes on top of each plot corresponds to the number given to each source-detector combination or channel as shown in Figure 12.1 ..................249

Figure 1.4: Hemodynamic response function (HRF), averaged across all participants, plotted against time (in seconds) for each channel (Ch. 1-4) in response to the planning and execution of motor skill -based stimuli performed by participants using their nondominant hand's index finger. The details of the figure are same as described in caption of Figure 12.3. .250

Figure 1.5: Bar plot of the correlation coefficient obtained from the zero order correlation analyses study in response to planning and execution of motor skill-based stimuli performed by participants using their dominant hand's index finger. The plot depicts the correlation coefficient values obtained from (A) $\mathrm{HbO},(\mathrm{B}) \mathrm{HbR}$, and (C) HbT data, across the two stimuli (target touching and Rest) and left vs. right channel pairs ( 2 of them) in the pre-frontal (PF) and anterior frontal (AF) cortex........................252

Figure 1.6: Bar plot of the correlation coefficient obtained from the zero order correlation analyses study in response to planning and execution of motor skill-based stimuli performed by participants using their dominant hand's index finger. The details of the figure are same as described in the caption of Figure 12.5 ....................253

Figure 1.7: Cortical dominance representations using source detector layout as described in Figure 12.1 across all left vs. right channel pairs ( 2 of them) for each of the two stimuli (target touching and rest) in the frontal cortex. The figure depicts the lateralization when the participants perform the target touching task with (A) dominant hand, and (B) nondominant hand. The dominant channel pair (across the left and right cortex) is highlighted

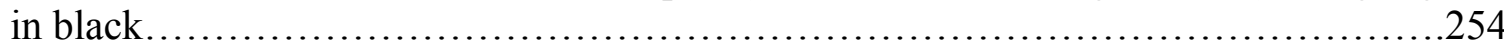

Figure 1.8: Bar plot showing the average number of touches (along with the standard deviation) during the target touching task performed by each participant using their dominant and non-dominant hand's index finger during (A) Trial 1 and (B) Trial 2...255 
Figure 1.9: Hemodynamic response function (HRF), averaged across all participants, plotted against time (in seconds) for each channel (Ch. 1-4) in response to the planning and execution-based stimuli performed by participants holding pen with their dominant hand. The details of the figure are same as described in caption of Figure 12.3 .......256

Figure 1.10: Hemodynamic response function (HRF), averaged across all participants, plotted against time (in seconds) for each channel (Ch. 1-4) in response to the planning and execution-based stimuli performed by participants holding pen with their nondominant hand. The details of the figure are same as described in caption of Figure

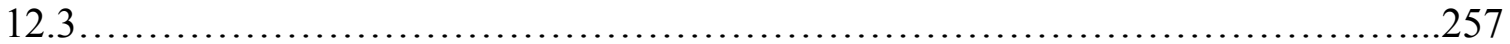

Figure 1.11: Bar plot of the correlation coefficient obtained from the zero order correlation analyses study in response to planning and execution of motor skill-based stimuli performed by participants holding pen with their dominant hand. The details of the figure are same as described in caption of Figure 12.5 .........................258

Figure 1.12: Bar plot of the correlation coefficient obtained from the zero order correlation analyses study in response to planning and execution of motor skill-based stimuli performed by participants holding pen with their non-dominant hand. The details of the figure are same as described in caption of Figure 12.5 ..........................259

Figure 1.13: Bar plot showing the average number of touches along with the standard deviation during the target touching task performed by each participant while holding pen with their dominant and non-dominant hand during (A) Trial 1 and (B) Trial 2........260 


\section{LIST OF SYMBOLS}

$\mathrm{I}_{0} \quad$ Initial Intensity

$\varepsilon_{\mathrm{HbO}}^{\lambda 1}$ Absorption coefficient of oxy hemoglobin at wavelength 1

$\varepsilon_{\mathrm{HbO}}^{\lambda 2}$ Absorption coefficient of oxy hemoglobin at wavelength 2

$\varepsilon_{\mathrm{HbR}}^{\lambda 1} \quad$ Absorption coefficient of de-oxy hemoglobin at wavelength 1

$\varepsilon_{\mathrm{HbR}}^{\lambda 2}$ Absorption coefficient of de-oxy hemoglobin at wavelength 2

$\triangle O D \lambda_{1}$ Change in optical density at wavelength 1

$\triangle O D \lambda_{2}$ Change in optical density at wavelength 2

$\mu_{\mathrm{a}} \quad$ Absorption coefficient

$\mu_{\mathrm{s}} \quad$ Scattering coefficient

$\mu_{\mathrm{s}}$ ' Reduced scattering coefficient

$A(t, \lambda)$ Attenuation of $\lambda$ wavelength light in given media at time $t$

c Speed of light

$\mathrm{C}(\lambda)$ Concentration of chromophore in the media at $\lambda$

d Pathlength

$g \quad$ Anisotropy factor

G Geometrical factor

I Intensity

mW mili Watt

$\varepsilon(\lambda)$ Molar extinction coefficient of a chromophore at $\lambda$

$\lambda \quad$ Wavelength

$\Phi \quad$ Fluence

$G(\lambda)$ Geometrical factor at $\lambda$.

$t \quad$ Time 


\section{LIST OF ACRONYMS AND ABBREVIATIONS}

AF Anterior Frontal

AL Anterior Left

AR Anterior Right

ASD Autism Spectrum Disorder

BA Brodmann Area

BIC Bayesian Information Criterion

BTD Ball Throw Dominant Hand

BTND Ball Throw Non-Dominant Hand

CP Cerebral Palsy

CW Continuous Wave

DP Dot Placement

DPF Differential Pathlength Factor

DTI Diffusion Tensor Imaging

EEG Electroencephalogram

FA Forward Audio

fMRI Functional Magnetic Resonance Imaging

HbO Oxygenated Hemoglobin

HbR Deoxygenated Hemoglobin

HbT Total Hemoglobin

HomER Hemodynamic Evoked Response

HRF Hemodynamic Response Function

ICCD Intensified Charged Coupled Devices

J Jaw Movement (Chapters 5 \& 6) 
J Joint Attention (Chapters $7 \& 8$ )

MRI Magnetic Resonance Imaging

NIR Near-Infrared

NIRS Near Infrared Spectroscopy

NJ Non-Joint Attention

OD Optical Density

PET Positron Emission Tomography

PF Pre Frontal

PL Posterior Left

PMT Photo-Multiplier Tube

PR Posterior Right

R Rest

RA Reverse Audio

RJA Responding To Joint Attention

RTE Radiative Transport Equation

SPECT Single Photon Emission Computed Tomography

TD Typically Developing

TT Target Touching

TTP Target Touching with pen

W Word Generation 


\section{CHAPTER 1. Introduction}

Near infrared spectroscopy (NIRS) is an emerging neuro-imaging modality which employs near infrared light in the range of $650 \mathrm{~nm}-900 \mathrm{~nm}$ to non-invasively investigate cerebral oxygenation changes in healthy and neurologically challenged adults and children (Strangman et al., 2002). During the NIRS of brain NIR light is shone onto the surface of the head, through an optode, and as the scattered and attenuated NIR light exits the head after travelling through the cortical region of the brain, it is detected by a detector optode placed at a certain distance from the source optode. The detected attenuated light intensity is then used to quantify the change in oxy hemoglobin $(\mathrm{HbO})$, de-oxy hemoglobin $(\mathrm{HbR})$ and/or total hemoglobin $(\mathrm{HbT})$, which is an indication of brain activity (Cope et al., 1987). Thus NIRS provides functional information, i.e., change in oxy/de-oxy hemoglobin concentration, total hemoglobin concentration, oxygen saturation of blood and the rate of blood flow in the brain. There are several neuro-imaging modalities which are currently in use, including electroencephalography (EEG), magneto electroencephalography (MEG), positron emission tomography (PET) and functional magnetic resonance imaging $(f \mathrm{MRI})$. EEG and MEG measure the electrical activity of the brain, fMRI provides information regarding the hemodynamic activity of the brain and PET provides information regarding the glucose metabolism in the brain. EEG has high temporal resolution but limited spatial resolution. $f$ MRI and PET have high spatial resolution but poor temporal resolution, which makes them highly susceptible to movement artifacts as the participants are required to be still in the claustrophobic imaging environment (Strangman et al., 2002). In comparison to these imaging modalities, NIRS can measure hemodynamic activity in the brain non-invasively without 
the use of any harmful radiation, and with excellent temporal sensitivity as well as reasonable spatial sensitivity (Strangman et al., 2002). NIRS is also relatively robust to movement artifacts thereby enabling the researchers to investigate the brain's response to routine human activity (Strangman et al., 2002). Thus the NIRS neuro-imaging modality provides the neuro-imaging community an ability to investigate the functional activity in the moving subject which cannot be performed using fMRI (as the subjects are required to stay stationary in the claustrophobic environment during the imaging).

\subsection{Objective and Significance}

The objective of my doctoral work was to apply near-infrared spectroscopy for the functional imaging of the brain in response to various stimuli, categorized as 1) Language studies, 2) Joint attention studies, and 3) Planning and execution of motor skills stimuli studies.

Initially, I developed a NIRS system by modifying and adapting the intensified charged couple device (ICCD) based optical mammography system developed in the Optical Imaging Laboratory (OIL) at Florida International University (FIU) to enable NIRS of brain in response to different stimuli. I also employed a commercial near-infrared optical imaging system; known as Imagent, which employs photomultiplier tubes (PMTs) based detectors to perform NIRS of brain in response to different stimuli. The ICCD based imaging system has an advantage over PMTs based imaging system wherein the ICCD based system is not limited by the numbers of detectors. Thus the main advantage of the ICCD based NIRS system is that the number of detectors in ICCD based NIRS instrument can be easily increased by increasing the number of detector fiber bundles 
which collects and send light to the face of the ICCD camera. The number of detectors in the NIRS system which I developed can be increased up to 8, and in future work it can be increased up to 16. The first version of the developed ICCD based NIRS system employed a $785 \mathrm{~nm}$ wavelength single source system (as described in section 3.2). The $785 \mathrm{~nm}$ wavelength of light is close to the isosbestic point; the point at which the absorption spectra of oxy and de-oxy hemoglobin intersect. Thus the optical measurement taken at this wavelength provides the change in total hemoglobin concentration independent of the oxygen saturation content of hemoglobin (Chance et al., 1988). The single wavelength ICCD based NIRS system was further modified to perform dual wavelength NIRS of the brain (as described in section 3.3) to elucidate a change in the concentration of oxy, de-oxy and total hemoglobin concentration. The dual source based NIRS employs $830 \mathrm{~nm}$ and $690 \mathrm{~nm}$ of light to acquire optical measurements at two different wavelengths of light, which can be further processed to determine changes in oxy, de-oxy and total hemoglobin concentration. The optical data acquired using the NIRS system was post processed using custom developed software. During the process of development of a data processing algorithm a mathematical model based on the granger causality (Goebel et al. 2003) approach was employed to explicate the directional flow of information in the cortical regions of the brain.

The reason and the significance of performing the above mentioned language, joint attention and planning and execution of motor skills studies are as described below.

1)Language studies - These studies were performed on healthy adults using a commercial NIRS system, Imagent. The application of language studies is in the field of 
epilepsy. In the field of epilepsy, surgical resection of epileptogenic tissue successfully alleviates seizures in the majority of patients with medically resistant partial epilepsy. Pre-surgical evaluation is carried out to map critical regions of the brain controlling motor and language functions such that these regions can be preserved during surgery. Eloquent cortical regions can be documented noninvasively by imaging techniques such as PET or $f$ MRI, but they are limited by inadequate temporal resolution. Furthermore, since these techniques require an awake and cooperative patient, they cannot be readily applied to the pediatric population. Mapping is therefore generally performed by electrically stimulating the cortex via subdurally implanted electrodes. Although highly effective, this technique incurs a potential, albeit small risk for cortical tissue injury. The use of NIRS for mapping language in pediatric epilepsy patients will prove effective over current functional neuro-imaging technqiues that are challenging to work with awake children during active stimulus. In addition to epileptic populations, the NIRS approach involving activation and connectivity analyses has the potential to be applied to schizophrenic and aphasic populations, where abnormality in language processing is found. Thus, even before applying the NIRS technique in the field of epilepsy research it was used to investigate the underlying neurological response of language in the frontal as well as fronto-temporal regions of brain in healthy adults, as described in chapters 5 and 6 respectively. A mathematical model based on granger causality was also developed to investigate the directional flow of information in the language region of the brain, as described in chapter 6 .

2) Joint attention studies - These studies were performed on healthy adults and typically developing children as well as children with autism using the Imagent 
commercial NIRS system. The application of joint attention studies is in the field of autism. Joint Attention is the process of sharing one's experience of observing an object or event, by following gaze or pointing gestures. One of the primary characteristics of children with autism is that they display robust levels of impairments in joint attention development from as early as 12 to 18 months of age (Osterling et al., 2002; Swettenham et al., 1998). Recently, NIRS was applied for the first time at FIU-OIL (Zhu et al., 2009) to understand the frontal activation in healthy adults in response to joint attention skill and also to demonstrate the feasibility of NIRS towards autism research. The work was based on that done by other researchers to investigate joint attention experience in adults using fMRI (Williams et al., 2005). Hence, based on the paradigm used in the earlier fMRI study, NIRS was employed to study the hemodynamic responses to joint attention experience (using responding to joint attention, or RJA-based stimuli) in the frontal cortex of normal adults (Zhu et al., 2009). This work demonstrated (Zhu et al., 2009) successful application of NIRS towards autism research, thereby setting the stage for the future extensive NIRS study on typically developing children and children with autism in response to joint attention skill based paradigm. Herein NIRS was used to investigate the underlying neurological response of frontal cortex in healthy adults as well as typically developing children and children with autism, as described in chapters 7 and 8 respectively.

3)Planning and execution of motor skill studies - These studies were performed on healthy adults and individuals with cerebral palsy using an in-house developed single wavelength based NIRS system. Studies were also performed on healthy adults by combining the in-house developed dual wavelength based NIRS system with a 
kinematics system to simultaneously investigate the hemodynamics in the frontal region of the brain and kinematics of hand movement. The application of planning and execution of motor studies is in the field of cerebral palsy. In the field of cerebral palsy (CP) research, several advanced neuroimaging techniques have been used to investigate and explicate the etiological basis of CP. Structural neuroimaging techniques, such as MRI (Griffiths et al., 2010; Horridge et al., 2011; Kułak et al., 2007; Kułak et al., 2008; Sööt et al., 2008), DTI (Fariaa et al., 2011; Rha et al., 2011; Yoshida et al., 2011) and CT (Accardo et al., 2009), have been applied to explicate the probable cause of CP because of structural changes in the brain due to brain mal development and/or lesion. Functional neuroimaging techniques, such as fMRI (Burton et al., 2009; Schwilling et al., 2012; Sutcliffe et al., 2009; Wingert et al., 2010) and PET/SPECT (Batista et al., 2007; Lee et al., 2007; Wong et al., 2006), have been employed to elucidate the functional change in the brain associated with activation of brain pre- and post tasks performed by individuals with CP. Electrophysiological neuroimaging techniques, such as EEG/MEG (Fitzgerald et al., 2007; Garfinkle \& Shevell, 2001; Garfinkle \& Shevell, 2009; Pires et al., 2011; Rinaldis et al., 2010), have been used to investigate the neural and temporal dynamics of different tasks performed by individuals with CP. Despite the different neuroimaging research performed in the field of $\mathrm{CP}$, the current understanding of the relationships between neural plasticity, neural activation, and specific motor activities are quite limited in individuals with CP. To date, none of the functional neuroimaging techniques have been employed to investigate the functional change in the brain of individuals with $\mathrm{CP}$ during a task as these techniques (i.e. fMRI and PET/SPECT) require the participants to be stationary in a claustrophobic environment. Hence it is challenging to investigate the 
motor response of the individuals with $\mathrm{CP}$ during an active stimulus using the current functional neuro imaging modalities. These several advantages of the NIRS neuroimaging technique makes it a reliable tool to investigate the cerebral hemodynamics change in individuals with cerebral palsy in response to complex tasks. Thus the NIRS neuroimaging technique was first used to investigate the planning and execution of motor skills in the frontal cortex of healthy adults and individuals with $\mathrm{CP}$ as described in chapters 9 and 10. The planning and execution of motor skills was also combined with kinematics to simultaneously investigate the brain hemodynamics and hand kinematics in healthy adults as described in chapters 11 and 12 .

Thus the goal of my doctoral work was to apply the NIRS neuroimaging technique towards the extensive investigation of neurological response of language, joint attention and planning and execution of motor skills in healthy adults. Preliminary studies on joint attention and planning and execution of motor skill stimuli were also performed to investigate the neurological response in children with autism and individuals with cerebral palsy, respectively. The significance of a NIRS study of joint attention skills is that it has the potential to explicate the neural basis of autism, which has the potential of early intervention in diagnosis of children with autism. The significance of NIRS study in the planning and execution of motor skills is that it can be used by a physical therapist to design a custom rehabilitation regime to help individuals with cerebral palsy achieve maximum functional independence via training to improve their motor abilities. The layout of the dissertation is as shown below in Figure 1.1. 


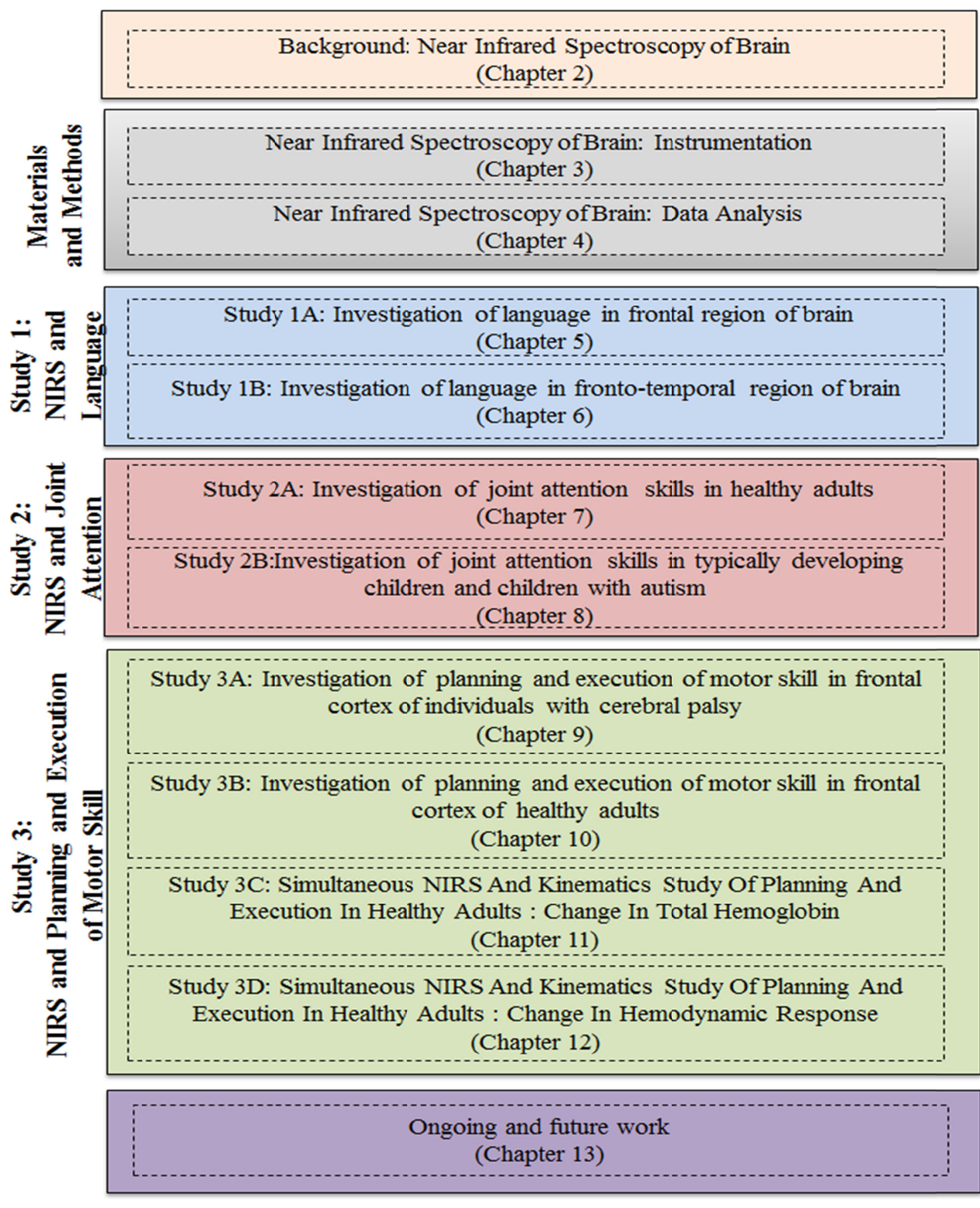

Figure 1.1: Layout of the dissertation 


\section{CHAPTER 2. Background: Near Infrared Spectroscopy of Brain}

2.1 Near infrared spectroscopy of brain: principle, theory and advantages

In 1977 Jobsis discovered that the functional activity of the brain could be studied using light in the near infrared range (Jobsis, 1997). This discovery lead to the development of a new technology for monitoring functional brain activity based on the absorption and scattering properties of near-infrared (NIR) light, known as near infrared spectroscopy (NIRS). NIRS uses diffuse NIR light to perform either spectroscopy or imaging depending upon the number of optodes (term used to denote the optical electrode i.e. the source and detector) used to probe the region of brain under investigation. If fewer number of optodes are used to monitor the cerebral oxygenation change from the region of interest and the signal obtained is displayed in terms of a waveform then it is called near infrared spectroscopy (NIRS). On the other hand if greater numbers of optodes are used and the signal obtained is reconstructed to display in terms of an image then it is called diffuse optical imaging (DOI). NIRS of brain acquires cerebral oxygenation and hemodynamics (change in concentration of oxy (HbO), de-oxy (HbR) and total hemoglobin ( $\mathrm{HbT})$ concentration) information from the brain after travelling in and out through the scalp and the highly scattering brain tissue (Cope at al., 1987; Strangman et al., 2002).

\subsubsection{Principle of near infrared spectroscopy of brain}

NIRS monitors the hemodynamic response to brain activation, on the basis that the neuronal activation is tightly coupled to vascular response, giving rise to the term 'neurovascular coupling' (Heeger et al., 1999; Heeger et al., 2000; Hess et al., 2000). 
The neuronal activation in response to stimuli is followed by secretion of neurotransmitters, change in the size of surrounding cells, vasodilation or constriction, change in the cerebral blood flow and change in oxygenation state of the blood in the brain (Palmer \& Williams, 1974; Wray et al., 1988). NIRS measures these vascular responses of the cerebral blood vessels, i.e. the change in cerebral blood flow and the change in oxygenation state of blood in the brain using near infrared light (NIR). The human brain consists of different light absorbing species, known as chromophores, such as water, cytochrome $\mathrm{c}$ oxidase and hemoglobin. The brain is opaque to the visible range of light as this is strongly absorbed by water and tissues present in the brain, but there exists a NIR range of light from $650-950 \mathrm{~nm}$ where the brain is not totally opaque. In the NIR range light absorption by water and tissues of the brain change drastically, and thus the only dominant chromophores present in the brain are cytochrome $\mathrm{c}$ oxidase and hemoglobin as shown in Figure 2.1. Cytochrome c oxidase and hemoglobin are vital for continued aerobic metabolism in the brain. The content of hemoglobin within the brain tissue is approximately $600 \mathrm{mg} / 100 \mathrm{mg}$ tissue which is several times the concentration of cytochrome oxidase. Thus the dominant chromophore present in the brain during NIR imaging is hemoglobin, which accounts for the majority of light attenuation in the brain (McCormick et al., 1992). When NIR light enters the brain the photons get absorbed and scattered by the dominant chromophore in the brain (which in the case of NIR range of light is hemoglobin). Here, the scattering event of photon dominates the absorption event, making optical transport of light in the brain to be scattering dominant. 


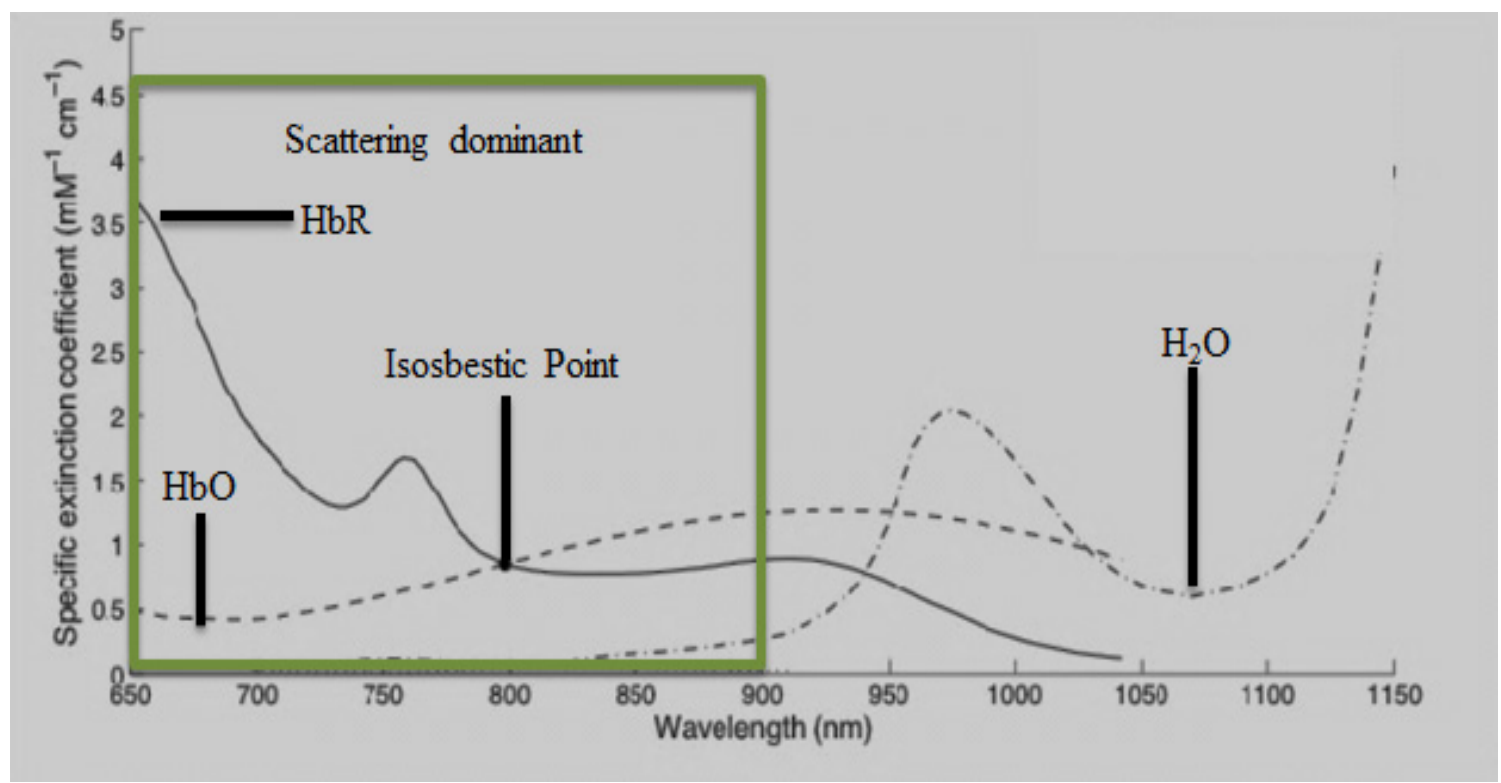

Figure 2.1: Absorption spectra for de-oxy hemoglobin (Hb/HbR), oxy hemoglobin $\left(\mathrm{HbO}_{2} / \mathrm{HbO}\right)$, and water $\left(\mathrm{H}_{2} \mathrm{O}\right)$ in the near-infrared range. The isosbestic point near 800 $n m$ is the point where the absorptivity of $\mathrm{HbR}$ and $\mathrm{HbO}$ are equal (modified and adapted from Palmer \& Williams, 1974; Wray et al., 1988).

In summary, near-infrared spectroscopy (NIRS) of brain employs NIR (near infrared) light in the range of $650-900 \mathrm{~nm}$ (which corresponds to $\sim 2 \mathrm{eV}$ photons) as there exists an optical window within which the light is minimally absorbed and preferentially scattered by the dominant chromophores oxy- $(\mathrm{HbO})$ and deoxy-hemoglobin $(\mathrm{HbR})$ present in the brain (as shown in Figure 2.1) (Benaron et al., 1993; Hebden et al., 1997; McCormick et al., 1992). The blood components of oxy- (HbO) and deoxy-hemoglobin (HbR) exhibit low absorption and enhanced scattering in the NIR range (McCormick et al., 1992; Palmer \& Williams, 1974; Wray et al., 1988), thus allowing deeper tissue penetration of NIR light and quantification of oxy- and deoxy-hemoglobin concentrations in tissue. Two different wavelengths of NIR light are used to quantify the concentration of $\mathrm{HbO}$ and $\mathrm{HbR}$ in the brain. Researchers have shown that the NIR light of wavelength close to 
$690 \mathrm{~nm}$ and $830 \mathrm{~nm}$ provide dominant information about $\mathrm{HbR}$ and $\mathrm{HbO}$, respectively (Boas \& Franceschini, 2004). Along with this there also exists an isosbestic point, close to $800 \mathrm{~nm}$ (Chance et al., 1988; Palmer \& Williams, 1974; Wray et al., 1988), at which the absorption spectra of $\mathrm{HbR}$ and $\mathrm{HbO}$ overlap thus providing information about change in total hemoglobin concentration $(\mathrm{HbT})$ independent of the oxygen saturation content of hemoglobin. Alternatively, the change in $\mathrm{HbT}$ is also calculated by summing up the changes in the concentrations of $\mathrm{HbO}$ and $\mathrm{HbR}$.

During NIRS of the brain, NIR light is shone onto the surface of head, via optical fibers (also termed as optodes), and as the scattered and attenuated signal exits the head after travelling through the cortical regions, it is detected by a detector optical fiber (or detector optode) placed at a certain distance from the source optode (as shown in Figure 2.2 (Ting Li et al., 2011)). The detected attenuated light intensity is further processed to quantify the change in $\mathrm{HbO}, \mathrm{HbR}$ and/or $\mathrm{HbT}$, which is an indication of brain activity. The light transport model used to describe the attenuation of light as it passes through the highly scattering brain media is described in the following section (section 2.1.2) 


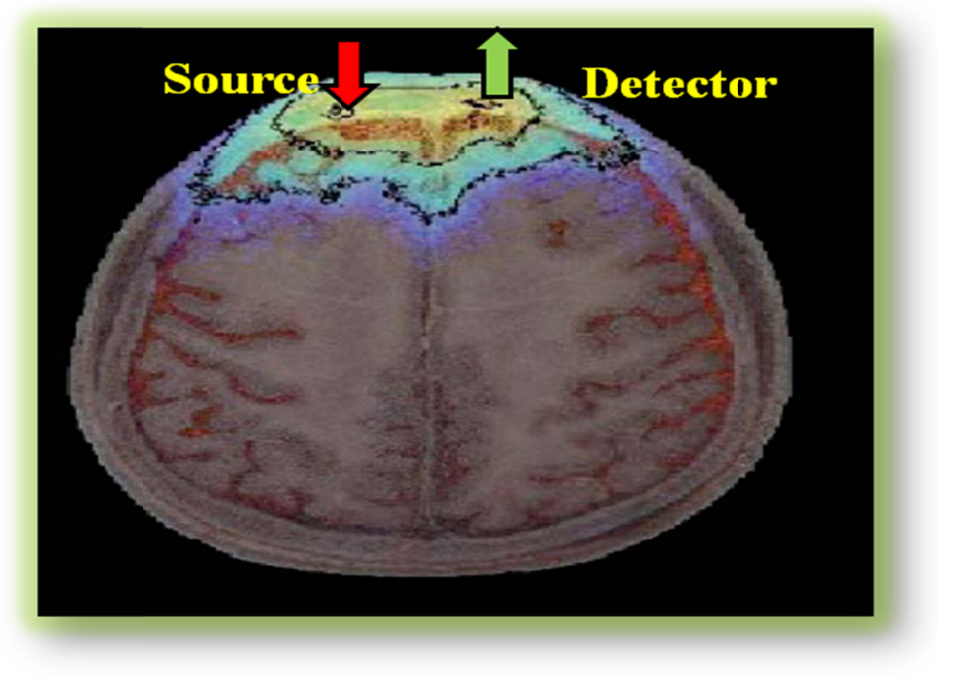

Figure 2.2: Spatial sensitivity profile of light propagation in the layered model of a highly scattering brain media obtained using Monte Carlo simulation. (Modified and adapted from Ting Li et al., 2011)

\subsubsection{Mathematical modeling of light in brain}

The quantification of detected attenuated light intensity from the brain is carried out using a model which describes the transport of light through a dominantly scattering medium. Light transport through the brain is modeled using either the Modified Beer Lambert's law or the diffusion equation approach. The modified Beer Lambert's law is widely used to model the propagation of light inside the brain as it facilitates real time online monitoring of light propagation.

\subsubsection{Modified Beer-Lambert Law}

The transport of light in an absorbing media is given by the Beer-Lambert law which describes the attenuation of light at given a wavelength when it passes through a media 
containing chromophores. The Beer's law is described using the expression shown in Eq.

2.1 .

$A(t, \lambda)=\log \left(\frac{I(t, \lambda)}{I_{0}(t, \lambda)}\right)=\varepsilon(\lambda) \cdot C(\lambda) \cdot d(\lambda)$

where in Eq.2.1, $A(t, \lambda)$ is the attenuation (or optical density) of $\lambda$ wavelength light when it passes through a given media at time $t . \mathrm{I}(t, \lambda)$ is the intensity of $\lambda$ wavelength light transmitted out of the given media at time $t$, and $\mathrm{I}_{0}(t, \lambda)$ is the initial intensity of $\lambda$ wavelength light incident on a media at time t. $\varepsilon(\lambda)$ is the molar extinction coefficient of a chromophore at $\lambda$ of wavelength light, as shown in Fig. 2.1, expressed in $\mathrm{mM}^{-1} \mathrm{~cm}^{-1}$. $C(\lambda)$ is the concentration of chromophore in the media at $\lambda$ of wavelength light and $d(\lambda)$ is the direct path length of a photon from the emitting to the receiving optode placed on the media at $\lambda$ of wavelength light, i.e. the geometrical distance or the inter optode distance.

The Beer-Lambert law holds true only for a purely absorbing media and the equation falls apart if there is scattering in the media. Hence the Beer-Lambert law in its standard form cannot be applied to describe the transport of light through a highly scattering media, such as brain. Therefore the Beer-Lambert law is modified to describe the attenuation of NIR light due to scattering and absorption by a chromophore in the brain. The modified Beer-Lambert law takes into account the attenuation due to scattering in the media and the changes in the path length of the photon due to its scattering. In a highly scattering media such as the brain, the path length of the photon is greater than the geometrical distance between the optodes because of the scattering of the photon in the media. Delpy et al. (1988) defined a scaling factor to correct for the path-length; the differential path- 
length factor (DPF). Thus the modified Beer-Lambert law, after accounting for the scattering event, is described by the expression shown in Eq. 2.2.

$A(t, \lambda)=\log \left(\frac{I(t, \lambda)}{I_{0}(t, \lambda)}\right)=\varepsilon(\lambda) \cdot C(\lambda) \cdot d(\lambda) \cdot D P F(\lambda)+G(\lambda)$

where $\operatorname{DPF}(\lambda)$ is the differential path length factor at $\lambda$ of wavelength light as defined by Delpy et al. (1988) to correct for the path-length in scattering media. The differential pathlength factor or scaling factor depends on the number of scattering events that occur. The DPF is a function of the scattering coefficient (it increases with increasing scattering coefficient), the anisotropy g, the absorption coefficient (it decreases with increasing absorption coefficient), and the geometry of the medium. It is included in the BeerLambert law to describe attenuation in a scattering medium (Matcher \& Cooper, 1994). The DPF is considered approximately constant for a given tissue, since the measured difference in attenuation is small compared with the large constant background attenuation in the tissue. $G(\lambda)$ is an unknown term representing scattering coefficient of the tissue together with the geometry of the optodes, i.e. the scattering losses. Therefore an absolute calculation of chromophore concentration cannot be derived from Eq. 2.2. This equation (Eq. 2.2)can only be used to calculate changes in concentration of chromophores by calculating changes in optical density (i.e. $A(t, \lambda)$ ) at two different wavelengths of light in order to cancel out the $G$ term, assuming the same value of $G(\lambda)$ for all chromophores in the medium (Matcher \& Cooper, 1994). Thus the quantitative changes in the concentration of chromophores can be derived assuming that DPF and $d$ remain constant during the measuring period. The changes in concentration of 
chromophores can be calculated by taking NIRS measurement at different wavelengths of light corresponding to the chromophore and writing the modified Beer-Lambert at different wavelengths of light and then solving the equations by matrix inversion.

To quantify the change in concentration of $\mathrm{HbO}$ and $\mathrm{HbR}, 830 \mathrm{~nm}$ and $690 \mathrm{~nm}$ of light is used as these wavelengths of light are sensitive to changes in concentration of $\mathrm{HbO}$ and HbR, respectively. Then modified Beer-Lambert is written at two different wavelengths of light as shown in Eq. 2.3 and 2.4, respectively.

$$
\begin{aligned}
& \Delta O D \lambda_{1}=\varepsilon_{H b R}^{\lambda 1} * L *[H b R]+\varepsilon_{H b O}^{\lambda 1} * L *[H b O] \\
& \Delta O D \lambda_{2}=\varepsilon_{H b R}^{\lambda 2} * L *[H b R]+\varepsilon_{H b O}^{\lambda 2} * L *[H b O]
\end{aligned}
$$

The change in optical density calculated using Eq. 2.3 and 2.4 is then used to calculate the changes in concentration of $\mathrm{HbO}$ and $\mathrm{HbR}$ as shown in Eq. 2.5 and 2.6, respectively.

$$
\begin{gathered}
\Delta[H b O]=\frac{\Delta O D \lambda_{1} \cdot \varepsilon_{H b R}^{\lambda 1}-\Delta O D \lambda_{2} \cdot \varepsilon_{H b R}^{\lambda 2}}{\left(\varepsilon_{H b O}^{\lambda 1} \cdot \varepsilon_{H b R}^{21}-\varepsilon_{H b R}^{\lambda 1} \cdot \varepsilon_{H b O}^{\lambda 2}\right) \cdot L} \\
\Delta[H b R]=\frac{\Delta O D \lambda_{1} \cdot \varepsilon_{H b O}\left(\lambda_{2}\right)-\Delta O D \lambda_{2} \cdot \varepsilon_{H b O}^{\lambda 1}}{\left(\varepsilon_{H b R}^{\lambda 1} \cdot \varepsilon_{H b O}^{\lambda 2}-\varepsilon_{H b O}^{\lambda 1} \cdot \varepsilon_{H b R}^{\lambda 2}\right) \cdot L}
\end{gathered}
$$

$\mathrm{HbT}$ is obtained by adding the values of $\mathrm{HbO}$ and $\mathrm{HbR}$.

$$
\Delta[H b T]=\Delta[H b O]+\Delta[H b R]
$$

where in Eq. 2.3, 2.4, 2.5, 2.6 and 2.7, $\Delta[\mathrm{HbO}], \Delta[\mathrm{HbR}] \& \Delta[\mathrm{HbT}]$ are changes in concentration of $\mathrm{HbO}$ (oxy-hemoglobin), HbR (de-oxy hemoglobin) and HbT (total hemoglobin), respectively. $\triangle O D \lambda_{1}$ and $\triangle O D \lambda_{2}$ are changes in optical density at wavelength $1(830 \mathrm{~nm}) \&$ wavelength $2(690 \mathrm{~nm})$, respectively. $\varepsilon_{\mathrm{HbR}}^{\lambda 1}$ and $\varepsilon_{\mathrm{HbR}}^{\lambda 2}$ is the 
absorption coefficient of $\mathrm{HbR}$ at wavelength $1(830 \mathrm{~nm})$ \& wavelength $2(690 \mathrm{~nm})$, respectively; and $\varepsilon_{\mathrm{HbO}}^{\lambda 1}$ and $\varepsilon_{\mathrm{HbO}}^{\lambda 2}$ is absorption coefficient of $\mathrm{HbO}$ at wavelength 1 (830 $\mathrm{nm})$ \& wavelength $2(690 \mathrm{~nm})$, respectively. L is the effective average path length of light through the tissue experiencing the absorption change. $\mathrm{L}$ is obtained from the product of the actual source-detector separation and differential pathlength factor (DPF). The source-detector separation is measurable along the surface of the head, and the extinction coefficient for a given chromophore can be looked up in tables (Strangman et al., 2002). The differential pathlength factor is either measured (with a time-domain or frequency domain instrument) or it is estimated as the distance between source and detector (for a continuous wave measurement) (Strangman et al., 2002).

If the wavelength of light used in NIRS instrument is close to the isosbestic point (described in section 2.1) then the optical density at the isosbestic point is equal to the change in concentration of $\mathrm{HbT}$, as shown in Eq. 2.8.

$\begin{array}{ll}\Delta O D_{\text {Isosbestic point }}=\Delta[H b T] & \text { Eq. } 2.8\end{array}$

where in Eq. 2.8, $\triangle O D_{\text {Isosbestic point }}$ is the change in optical density calculated using the modified Beer Lambert law at the wavelength of light close to the isosbestic point and $\Delta[H b T]$ is the change in total hemoglobin concentration. Equation 2.8 provides the change in $\mathrm{HbT}$ concentration without giving any information about oxygen saturation of blood in the brain. 


\subsubsection{The diffusion approximation to radiative transport equation}

A more descriptive model of light propagation in tissue is given by the radiative transport equation (RTE), which is restricted to interactions between photons themselves and is derived by considering changes in energy flow due to incoming, outgoing, absorbed and emitted photons within an infinitesimal volume $\mathrm{dV}$ in the medium (energy balance). Exact solutions for the RTE exist for simple cases such as isotropic scattering in simple geometries. The RTE is simplified and applied to light-tissue interactions and transport modeling by making a diffusion approximation, which states that the model is applicable in cases of dominant scattering in the medium. The diffusion approximation to the radiative transport equation to model light transport in the tissue is described below.

The diffusion equation (Pogue et al., 1994) to model the transport of light through a highly scattering media such as brain is given by an equation as shown in Eq. 2.9 for continuous wave based NIRS measurements.

$\nabla \cdot D(\boldsymbol{r}) \nabla \emptyset(\boldsymbol{r}, t)-v \mu_{a}(\boldsymbol{r}) \emptyset(\boldsymbol{r}, t)+v S(\boldsymbol{r}, t)=\frac{\partial \emptyset(\boldsymbol{r}, t)}{\partial t}$

where $\emptyset(\boldsymbol{r}, t)$ is photon fluence rate through the tissue i.e. the rate of incidence of the number of photons on an imaginary small sphere from all directions, divided by the cross-sectional area of that sphere. The term $v$ is the speed of the light in the media and $\mu_{a}$ is the absorption coefficient of light in the tissue $\left(\mathrm{cm}^{-1}\right)$, which is the reciprocal of the distance travelled by a photon before an absorption event occurs. $S(\boldsymbol{r}, t)$ denotes the isotropic source that provides the number of photons emitted at a position $\boldsymbol{r}$ and time $\mathrm{t}$ 
per unit volume per unit time. The term $D$ in Eq. 2.9 is known as the photon diffusion coefficient $\left(\mathrm{cm}^{2} / \mathrm{sec}\right)$ and is defined using the expression shown in Eq. 2.10.

$D=\frac{v}{3\left(\mu^{\prime}{ }_{s}+\mu_{a}\right)}$

where $\mu_{s}^{\prime}$ is the reduced scattering coefficient of the media $\left(\mathrm{cm}^{-1}\right)$ which is defined using the expression shown in Eq. 2.11.

$\mu_{s}^{\prime}=(1-g) \mu_{s}$

where $\mu_{s}$ is scattering coefficient of light in the media $\left(\mathrm{cm}^{-1}\right)$, which is the reciprocal of the distance travelled by the photon before a scattering event occurs, and $\mathrm{g}$ is the anisotropy factor which defines the scattering direction of the photon in the media. The anisotropy factor is defined using the expression shown in Eq. 2.12.

$g=\langle\cos \theta>$

where $\theta$ is the angle associated with a typical single scattering event in the media. In human biological tissue the value for anisotropy is $\sim 0.9$, which explains the forward scattering nature of light in the tissue.

In a highly scattering media such as the brain, since the scattering of photons dominates the absorption of the photon, Eq.2.10 can be written as shown in Eq.2.13.

$D=\frac{v}{3\left(\mu^{\prime}{ }_{s}\right)}$ 
The forward solution of the diffusion equation is usually obtained by assuming the region of brain under investigation to be a semi-infinite media with spatially varying optical properties of the tissue using finite-difference, finite-element and Monte Carlo methods.

\subsubsection{NIRS measurement techniques}

The main target of the near infrared spectroscopic measurements is to detect the change in the absorption and/or scattering of light as the light propagates through the cortical regions of the brain (Cope et al., 1987). These absorption changes are predominantly due to the dominant chromophore present in the brain, i.e., the two different states of the hemoglobin in the blood, $\mathrm{HbO}$ and $\mathrm{HbR}$, changes in which are indicate brain activity (Cope et al., 1987). Three different optical measurement techniques can be employed to detect changes in $\mathrm{HbO}$ and $\mathrm{HbR}$. These include: continuous wave, frequency domain and time domain measurements.

\subsubsection{Continuous wave based measurement technique}

In continuous-wave (CW) systems, light is shone continuously at constant amplitude, or modulated at frequencies not higher than a few tens of kilohertz (which provides straylight rejection). CW systems measure only the amplitude decay of the incident light (as shown in Figure 2.3) (Boas \& Franceschini, 2004; Strangman et al., 2002). The main advantages of CW NIRS are the sampling rate, the size of the instrument, weight, simplicity and cost, which makes CW-based NIRS ideal for bedside monitoring. However, CW-based NIRS has a few disadvantages, including the penetration depth and the difficulty to separate absorption and scattering effects. Since CW-based technique cannot give distinct information about the absorption and scattering coefficients, it cannot 
be used to measure the absolute changes in $\mathrm{HbO}$ and $\mathrm{HbR}$ concentrations. It can only be used for oxygenation trend monitoring. $\mathrm{CW}$ systems have been extensively used to investigate cerebral oxygenation changes in healthy and neurologically challenged adults and children (a few example references: Nioka et al., 1997; Siegel et al., 1999).

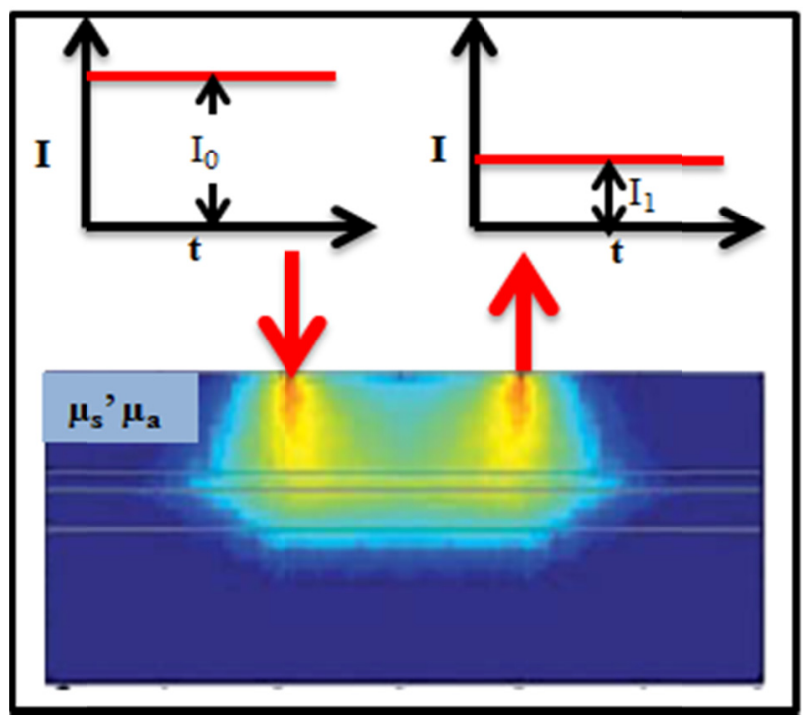

Figure 2.3: Schematic representation of continuous wave $(\mathrm{CW})$ technique. The figure depicts the transport of light through a multilayered model created to mimic different layers in the brain. In $C W$ technique, light of initial intensity $\mathbf{I}_{\mathbf{0}}$ is incident onto the surface of the scalp and the transmitted attenuated light of intensity $\mathbf{I}_{\mathbf{1}}$ is measured at a distance $d$ from the incident point (the multilayered brain model depicting the attenuation of light in brain is modified and adapted from Ting Li et al., 2011).

\subsubsection{Frequency-Domain based measurement technique}

In frequency domain (FD) systems, an amplitude modulated light source is shone into the head and the amplitude decay and phase shift of the detected light is recorded, which provides information about the absorption and scattering properties of tissue (Ntziachristos et al., 1999) (as shown in Figure 2.4). The frequency domain systems 
perform measurements of changes in intensity, phase shift, and modulation using either (1) a single wavelength and a fixed interoptode distance, (2) multiple wavelengths and a fixed inter-optode distance, or (3) a single wavelength and multiple inter-optode distances (Delpy \& Cope, 1997). The main advantages of frequency domain systems are the sampling rate and the relative accurate separation of absorption and scattering effects. A limitation of this technique is that the radio frequency modulated light cannot exceed 200 $\mathrm{MHz}$, because a linear relationship between phase shift and path-length no longer applies above $200 \mathrm{MHz}$ (Arridge et al., 1992).

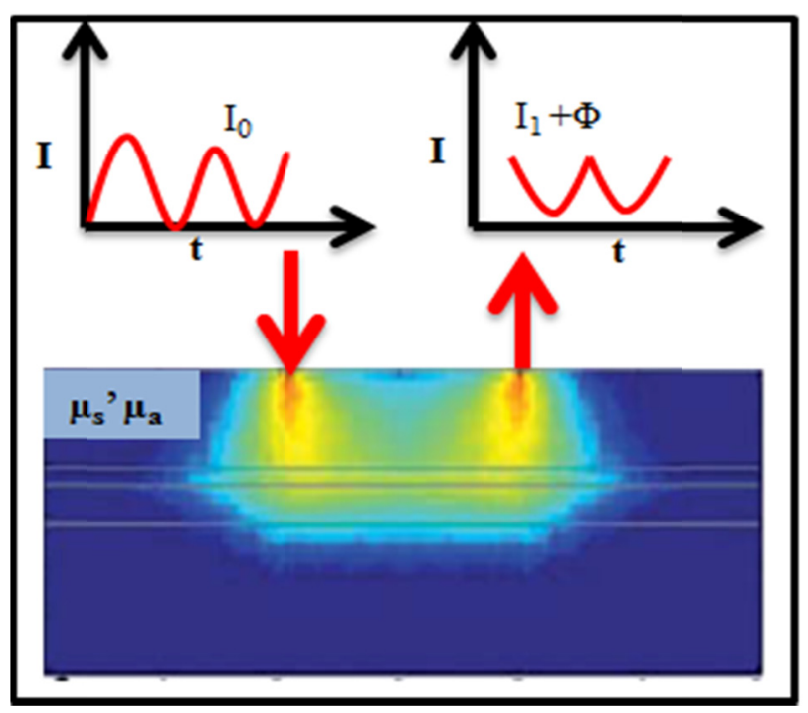

Figure 2.4: Schematic representation of frequency domain technique. The figure depicts the transport of light through a multilayered model created to mimic different layers in the brain. In the $C W$ technique, light of initial intensity $\mathbf{I}_{\mathbf{0}}$ is incident onto the surface of the scalp and the attenuation in intensity $\mathbf{I}_{\mathbf{1}}$ and phase shift $\Phi$ in the transmitted light is measured at a distance $d$ from the incident point (the multilayered brain model depicting the attenuation of light in brain is modified and adapted from Ting Li et al., 2011).

Frequency domain system has been extensively used to investigate cerebral oxygenation in adults and children (a few example references: Franceschini et al., 2000; Gratton et al., 1997; Pogue et al., 1994). 


\subsubsection{Time-Domain based measurement techniques}

In time domain (TD) system, picosecond pulses of light are incident onto the brain and the output light is analyzed to determine the tissue absorption and scattering across different layers of head (Strangman et al., 2002) (as shown in Figure 2.5). Time domain systems have advantages of good spatial resolution, greater depth penetration and the most accurate determination of absorption and scattering (Strangman et al., 2002). However, there are several disadvantages including the sampling rate, instrument size, instrument weight, necessity for cooling (during imaging studies), lack of stabilization, and the cost. Time-domain systems have been used to study cerebral oxygenation and hemorrhage in neonates (Benaron et al., 1993; Chance et al., 1988; Hebden et al., 1997).

Several commercial and in-house NIRS systems have been developed using the continuous wave, frequency domain and time domain measurement techniques described in this section. Depending on the measurement techniques employed, various research groups have developed appropriate NIRS instrumentation (as described in Section 2.2.2). Regardless of the measurement technique used to investigate the cerebral oxygenation, NIRS has several advantages over other functional neuroimaging techniques despite its disadvantages, which are described in following section (section 2.1.4). 


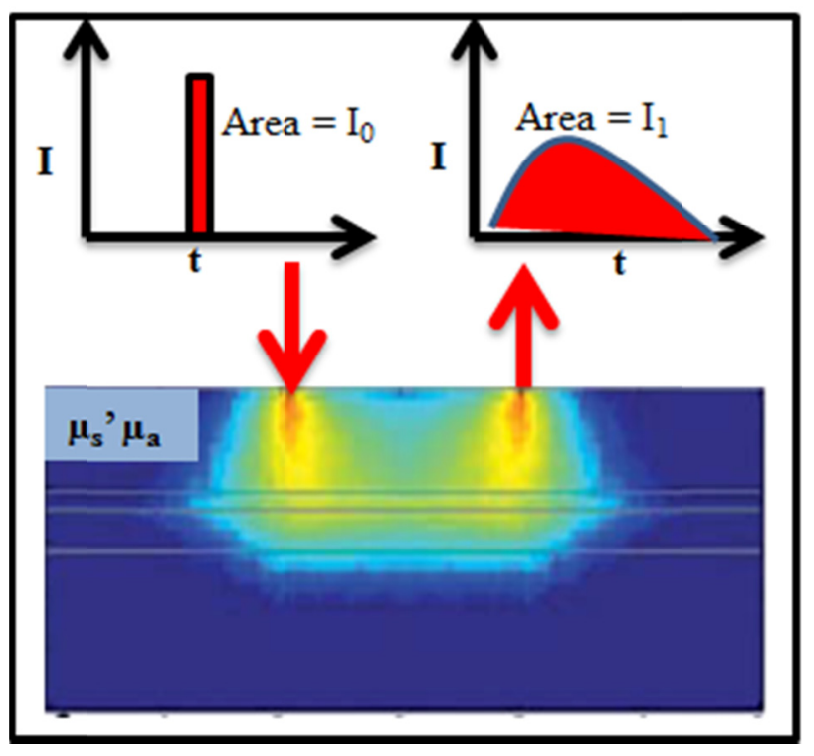

Figure 2.5: Schematic representation of time domain technique. The figure depicts the transport of light through a multilayered model created to mimic different layers in the brain. In the time-domain technique, light of initial intensity $\mathbf{I}_{\mathbf{0}}$ is incident onto the surface of the scalp and the change in temporal distribution of picosecond pulse of light is measured at a distance $d$ from the incident point (the multilayered brain model depicting the attenuation of light in brain is modified and adapted from Ting Li et al., 2011).

\subsubsection{Near infrared spectroscopy versus other neuro-imaging modalities}

NIRS is an emerging neuro-imaging modality which has been used reliably to monitor and investigate cerebral oxygenation changes in healthy and neurologically challenged adults and children (Strangman et al., 2002). There are several neuro-imaging modalities which are currently in use; hence it is required to compare the NIRS with other neuroimaging modalities. Different non-invasive neuro-imaging methods that are presently in use for examining functional brain activity, detect either the direct activation related to electrical activity of the brain (e.g. electroencephalography (EEG), magneto electroencephalography (MEG)) or the consequent hemodynamic response (functional magnetic resonance imaging $(f M R I))$. EEG is the oldest neuroimaging technique which 
measures the electrical activity of the brain, and this technique has high temporal resolution but poor spatial resolution. $f$ MRI provides information regarding the hemodynamic activity of the brain. It has high spatial resolution but poor temporal resolution and highly susceptible to movement artifacts as the subjects are required to be still in the claustrophobic imaging environment. In comparison to these imaging modalities, NIRS is the only neuroimaging modality that can measure the hemodynamic and metabolic responses associated with neuronal activity, as well as measure neuronal activity directly, as it can detect 'fast' signals in the order of 50-200 milliseconds (Franceschini et al, 2000), which is a more direct measure of the neuronal activity than the hemodynamic signals. Additionally, in comparison to the EEG, $f$ MRI, PET, or SPECT, NIRS can provide excellent temporal sensitivity as well as reasonable spatial sensitivity (Strangman et al., 2002) (as shown in Figure 2.6). NIRS is also relatively robust to movement artifacts thereby enabling the researchers to investigate the brain's response to routine human activity (Strangman et al., 2002). Thus providing the neuroimaging community an ability to investigate the functional activity in a moving subject which cannot be performed using fMRI (as the subjects are required to stay stationary in the claustrophobic environment during the imaging). The major limitation of NIRS is limited depth penetration of NIR light in the brain, which limits its capability to investigate hemodynamics only to the surface of the cerebral cortex. 


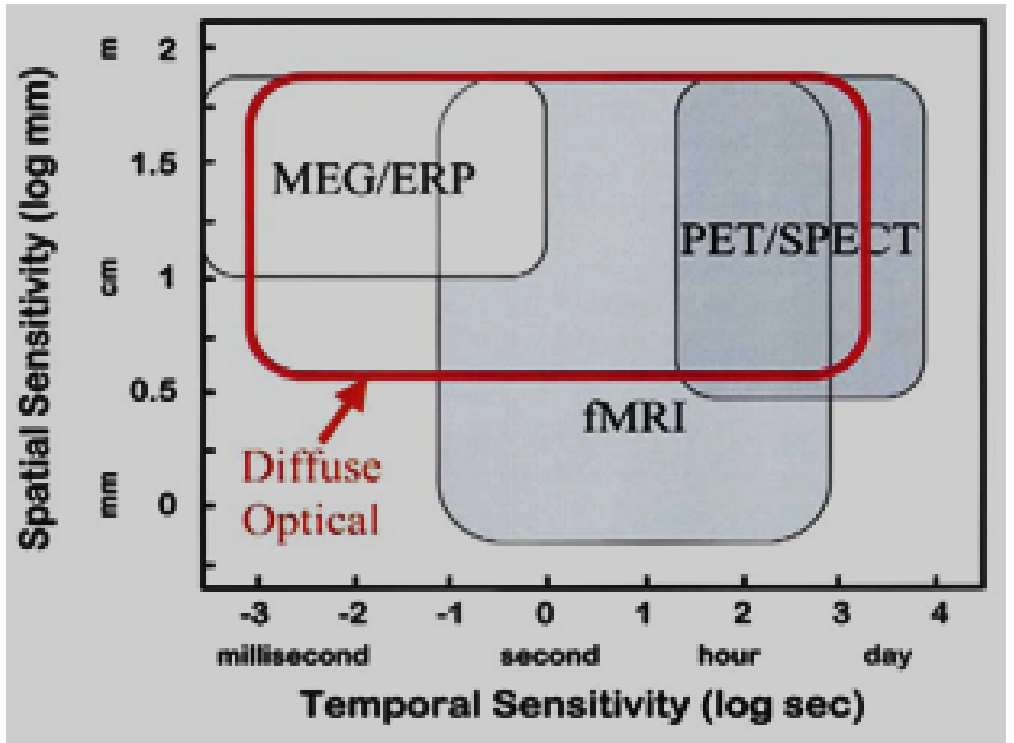

Figure 2.6: Temporal and spatial resolution comparison of different neuro imaging modalities (Figure adapted from Strangman et al., 2002).

\subsection{Near infrared spectroscopy of brain: Instrumentation}

Based on the techniques of continuous wave, frequency domain and time domain measurements different NIRS instruments have been developed by several researchers. These NIRS systems monitor the brain oxygenation and hemodynamic changes in response to stimuli or even during resting state from the surface of the human head. The NIRS instrument is optically coupled to the surface of the human head using source and detector fiber bundles, which are held by optical caps.

\subsubsection{Optical Caps}

Optical caps are indispensable for acquiring optical measurements from the human brain. Many researchers have developed different optical caps based on the specific application of functional brain mapping and the region of the brain imaged (as shown in Table 2.1). 


\begin{tabular}{|c|c|c|c|}
\hline No. & Figure of Optical probe & Application & Disadvantages \\
\hline 1) & & $\begin{array}{l}\text { Monitoring the } \\
\text { brain oxygenation } \\
\text { and hemodynamic } \\
\text { changes in } \\
\text { response to } \\
\text { stimulation in an } \\
\text { intensive care unit } \\
\text { (Hebden, 2003). }\end{array}$ & $\begin{array}{l}\text { 1) Applicable for } \\
\text { imaging only a } \\
\text { specific region of } \\
\text { the brain, and not } \\
\text { generalized for all } \\
\text { regions. } \\
\text { 2) Hair } \\
\text { interference } \\
\text { issues, although } \\
\text { not a major } \\
\text { problem in infants } \\
\text { (since they have } \\
\text { less hair). }\end{array}$ \\
\hline 2) & 2 & $\begin{array}{l}\text { Assessing the } \\
\text { changes in blood } \\
\text { volume and } \\
\text { oxygen saturation } \\
\text { in premature infant } \\
\text { brain using near } \\
\text { infrared light in } \\
\text { continuous mode } \\
\text { (Hintz et al., } \\
2001 \text { ). }\end{array}$ & $\begin{array}{l}\text { 1) Applicable to } \\
\text { specific region for } \\
\text { imaging, not } \\
\text { generalized for } \\
\text { any region. } \\
\text { 2) Uncomfortable } \\
\text { to infants, from } \\
\text { the high density } \\
\text { of probes in a } \\
\text { small area. }\end{array}$ \\
\hline 3) & & $\begin{array}{l}\text { Used to diagnose } \\
\text { cerebral diseases } \\
\text { and measure } \\
\text { functional changes } \\
\text { in the brain using } \\
\text { the optical } \\
\text { topographic } \\
\text { system by } \\
\text { Hitachi, Ltd. }\end{array}$ & $\begin{array}{l}\text { 1) Hair } \\
\text { interference could } \\
\text { be a prevalent } \\
\text { problem. } \\
\text { 2) Used only for } \\
\text { particular region, } \\
\text { so it is region } \\
\text { based } \\
\text { configuration. }\end{array}$ \\
\hline
\end{tabular}




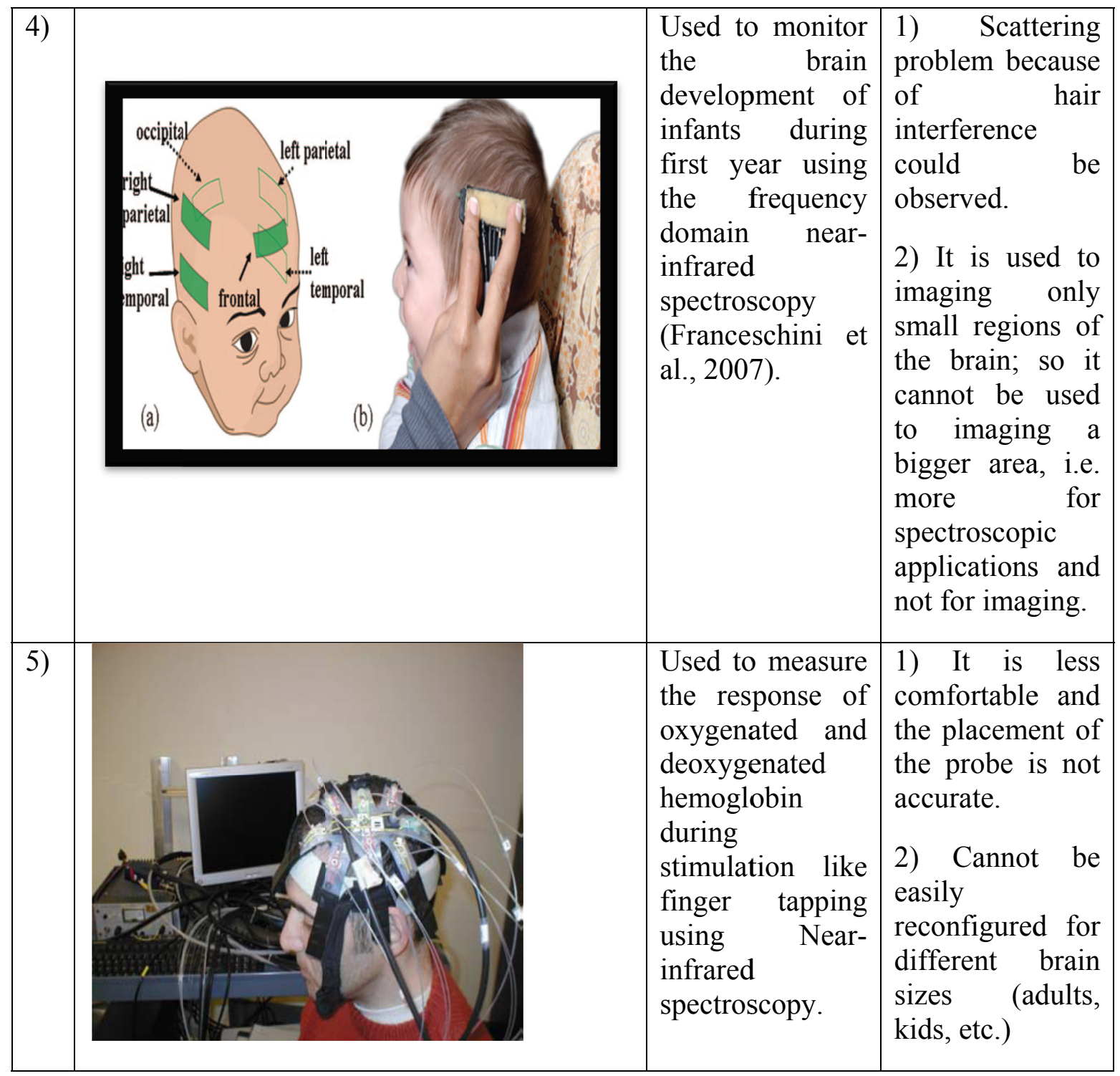

\begin{tabular}{|l|l|l|}
\hline 6$)$ & $\begin{array}{l}\text { Used to assay the } \\
\text { functional } \\
\text { information of the } \\
\text { brain of newborn } \\
\text { infant using near } \\
\text { infrared less light } \\
\text { comfortable and } \\
\text { the placement of } \\
\text { the probe is not } \\
\text { austin, 2007). }\end{array}$ \\
\end{tabular}




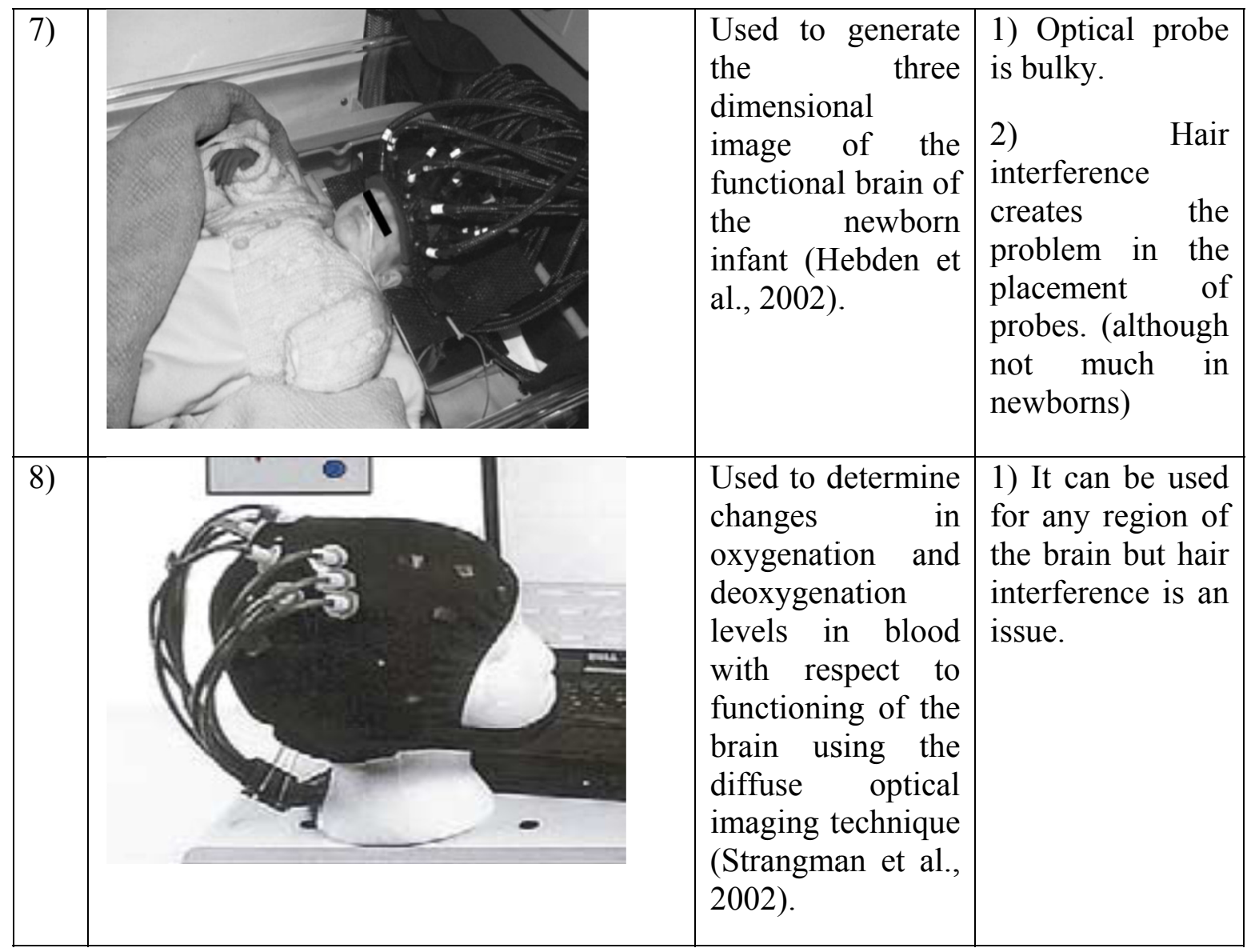

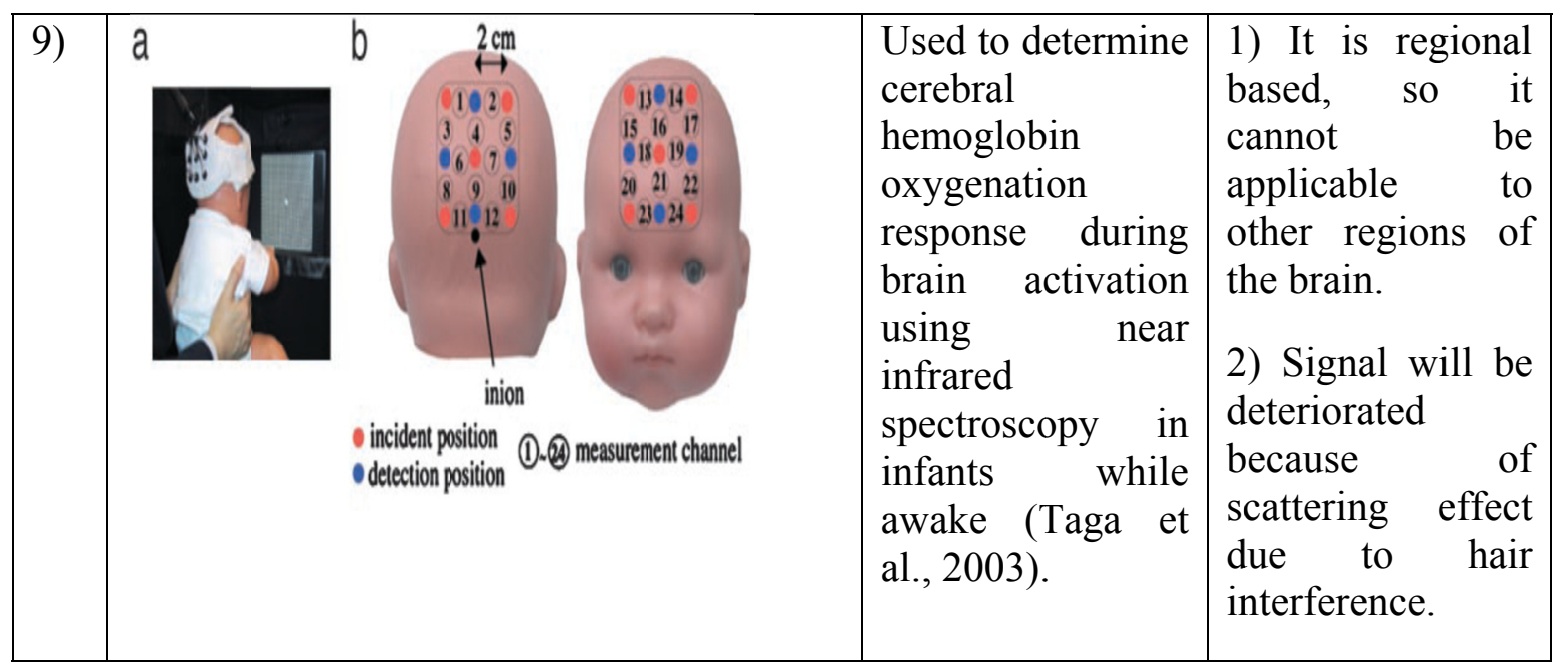

Table 2.1 : Optical probes developed by different researchers for functional brain mapping studies 
Table 2.1 depicts different optical caps that have been developed to acquire optical signals from the brain. As discussed in Table 2.1 these optical caps are either suitable for acquiring signals from specific regions of the brain or they suffer from hair interference during signal acquisition. Moreover, these caps are not commercially available as most of the time they are for proprietary use.

Different optical caps have been developed at FIU-OIL (Florida International University's Optical Imaging Laboratory) to probe different regions of the brain with minimal hair interference and easy placement on both pediatric and adult populations. The relevant optical caps developed will be described in sections 5 to 10 pertaining to the studies performed to investigate different regions of the brain. The optical caps described in this section serve as an interface between the head and NIRS instrument, which acquires optical signals from the surface of the head.

\subsubsection{NIRS imaging systems}

As described in section 2.1.3, several NIRS instruments have been developed commercially and in-house to date to acquire optical measurements from the surface of the head. These instruments have been developed on the principle of continuous wave, frequency domain, and/or time domain based measurement technique as described in section 2.1.3.

\subsubsection{Commercial NIRS imaging systems}

There are several commercially available NIRS instruments in the market. These instruments differ in terms of measurement techniques used and/or the number of 
channels provided by the manufacturer. The commercially available NIRS systems are shown in Table 2.2.

\begin{tabular}{|c|c|c|c|c|c|}
\hline $\begin{array}{l}\text { Sl. } \\
\text { No. }\end{array}$ & Instrument & Technique & $\begin{array}{c}\text { Number } \\
\text { of } \\
\text { channels }\end{array}$ & Company & Website \\
\hline 1. & Astem $\mathrm{Hb}-1$ & $\mathrm{CW}$ & 2 & Astem, Japan & $\frac{\text { www.astem- }}{\text { jp.com }}$ \\
\hline 2. & CerOx 3210F & $\mathrm{CW}$ & 2 & Ornim,Inc.USA & $\frac{\text { www.ornim.co }}{\underline{\mathrm{m}}}$ \\
\hline 3. & Dynat & $\mathrm{CW}$ & Up to 32 & NiRx, USA & www.nirx.net \\
\hline 4. & ETG-4000 & $\mathrm{CW}$ & 44 & Hitachi, Japan & $\begin{array}{l}\frac{\text { www.hitachim }}{\text { ed.com }} \\
\end{array}$ \\
\hline 5. & ETG-7000 & $\mathrm{CW}$ & 72 & Hitachi, Japan & $\frac{\text { www.hitachim }}{\text { ed.com }}$ \\
\hline 6. & $\begin{array}{l}\text { EQUANOX } \\
7600 \\
\end{array}$ & $\mathrm{CW}$ & 2 & Nonin, USA & $\frac{\text { www.nonin.co }}{\underline{\underline{m}}}$ \\
\hline 7. & $\begin{array}{l}\text { EQUANOX } \\
8004\end{array}$ & $\mathrm{CW}$ & 4 & Nonin, USA & $\frac{\text { www.nonin.co }}{\underline{\mathrm{m}}}$ \\
\hline 8. & $\begin{array}{c}\text { FORE-SIGHT } \\
2040 \\
\end{array}$ & $\mathrm{CW}$ & 2 & Casmed, USA & www.casmed.c \\
\hline 9. & Imagent & FD & Up to 128 & ISS, USA & www.iss.com \\
\hline 10. & Inspectra $\mathrm{StO}_{2}$ & $\mathrm{CW}$ & 4 & $\begin{array}{l}\text { Hutchinson, } \\
\text { USA }\end{array}$ & $\begin{array}{l}\text { www.htibiome } \\
\text { asurement.com }\end{array}$ \\
\hline 11. & INVOS 5100 & $\mathrm{CW}$ & $1-4$ & $\begin{array}{c}\text { Somanetics, } \\
\text { USA }\end{array}$ & $\frac{\text { www.somaneti }}{\text { cs.com }}$ \\
\hline 12. & NIMO & $\mathrm{CW}$ & 1 & NIROX, Italy & www.nirox.it \\
\hline 13. & NIRO 100 & $\mathrm{CW}$ & 2 & $\begin{array}{c}\text { Hamamatsu, } \\
\text { Japan }\end{array}$ & $\begin{array}{l}\text { www.hamamat } \\
\text { su.com }\end{array}$ \\
\hline 14. & NIRO 200 & $\mathrm{CW}$ & 8 & $\begin{array}{l}\text { Hamamatsu, } \\
\text { Japan }\end{array}$ & $\begin{array}{l}\text { www.hamamat } \\
\text { su.com }\end{array}$ \\
\hline 15. & NIRS 4/58 & $\mathrm{CW}$ & 4 or 58 & $\begin{array}{l}\text { TechEn, Inc., } \\
\text { USA }\end{array}$ & $\begin{array}{l}\text { www.nirsoptix } \\
\text { com }\end{array}$ \\
\hline 16. & nScan D1200 & $\mathrm{CW}$ & 16 to 32 & $\begin{array}{c}\text { Arquatis, } \\
\text { Switzerland }\end{array}$ & $\begin{array}{l}\frac{\text { www.arquatis. }}{\text { com }} \\
\end{array}$ \\
\hline 17. & nScan W1200 & $\begin{array}{l}\text { Wireless } \\
\text { CW }\end{array}$ & 16 & $\begin{array}{l}\text { Arquatis, } \\
\text { Switzerland }\end{array}$ & $\begin{array}{l}\frac{\text { www.arquatis. }}{\text { com }} \\
\end{array}$ \\
\hline 18. & ODISsey & $\mathrm{CW}$ & 2 & $\begin{array}{l}\text { Vioptix, Inc., } \\
\text { USA }\end{array}$ & $\frac{\text { www.vioptix.c }}{\text { om }}$ \\
\hline 19. & OM 220 & $\mathrm{CW}$ & 2 & $\begin{array}{c}\text { Shimadzu, } \\
\text { Japan }\end{array}$ & $\frac{\text { www.med.shi }}{\text { madzu.co.jp }}$ \\
\hline 20. & OMM 2001 & $\mathrm{CW}$ & 42 & $\begin{array}{c}\text { Shimadzu, } \\
\text { Japan }\end{array}$ & $\frac{\text { www.med.shi }}{\text { madzu.co.jp }}$ \\
\hline
\end{tabular}




\begin{tabular}{|c|c|c|c|c|c|}
\hline 21. & OMM 3000 & $\mathrm{CW}$ & 64 & $\begin{array}{c}\text { Shimadzu, } \\
\text { Japan }\end{array}$ & $\frac{\text { www.med.shi }}{\text { madzu.co.jp }}$ \\
\hline 22. & OxiplexTS & FD & 2 & ISS, USA & Www.iss.com \\
\hline 23. & $\mathrm{O} 2 \mathrm{C}$ & $\mathrm{CW}$ & 2 & LEA, Germany & www.lea.de \\
\hline 24. & PortaLite & $\begin{array}{c}\text { Wireless } \\
\text { CW }\end{array}$ & 1 & $\begin{array}{c}\text { Artinis, } \\
\text { Netherlands }\end{array}$ & $\frac{\text { www.artinis.co }}{\underline{\mathrm{m}}}$ \\
\hline 25. & TRS-20 & TD & 2 & $\begin{array}{c}\text { Hamamatsu, } \\
\text { Japan }\end{array}$ & $\frac{\text { www.hamamat }}{\text { su.com }}$ \\
\hline
\end{tabular}

Table 2.2: List of commercially available NIRS systems (table modified and adapted from Wolf et al., 2007). In the table CW stands for Continuous wave, FD stands for frequency domain and TD stands for time domain.

From Table 2.2 it can be seen that most of the commercially available NIRS instruments are based on the principle of continuous wave, while just 2 and 1 of them are based on frequency domain and time domain techniques, respectively. Most of the commercially available systems are relatively expensive and are limited by the number of channels. Hence NIRS systems have also been developed by several research groups in-house as listed in Table 2.3 of section 2.2.2.2.

\subsubsection{In-house developed NIRS imaging systems}

The in-house developed NIRS imaging systems are shown in Table 2.3.

\begin{tabular}{|c|c|c|c|c|}
\hline $\begin{array}{c}\text { Sl. } \\
\text { No. }\end{array}$ & $\begin{array}{c}\text { Author } \\
\text { (reference) }\end{array}$ & Technique & $\begin{array}{c}\text { Number of } \\
\text { channels }\end{array}$ & University or firm \\
\hline 1. & Akin, 2006 & CW & 16 & BonğaziÇi Univ., Turkey \\
\hline 2. & Boden, 2007 & CW & 22 & Charité, Germany \\
\hline 3. & Contini, 2006 & TD & 16 & Politecnico of Milan, Italy \\
\hline 4. & Cuccia, 2005 & CW & CCD & Irvine Univ., USA \\
\hline 5. & Culver, 2006 & CW & 300 & Washington Univ., USA \\
\hline 6. & Everdell, 2005 & CW & 20 & Univ. College London, UK \\
\hline 7. & Ho, 2007 & FD & 16 & Yonsei Univ., Japan \\
\hline 8. & $\begin{array}{c}\text { Kashyap, 2007 } \\
\text { CW }\end{array}$ & 64 & $\begin{array}{c}\text { Univ. of Texas, Arlington, } \\
\text { USA }\end{array}$ \\
\hline 9. & Kek, 2006 & CW & 64 & Hokkaido Univ., Japan \\
\hline 10. & $\begin{array}{c}\text { Leon-Carrion, } \\
2006\end{array}$ & CW & 16 & Drexel Univ., USA \\
\hline
\end{tabular}




\begin{tabular}{|c|c|c|c|c|}
\hline 11. & $\mathrm{Li}, 2005$ & $\mathrm{CW}$ & 16 & Southeast Univ., China \\
\hline 12. & Liebert, 2006 & $\mathrm{TD}$ & 16 & $\begin{array}{l}\text { Physikalisch Technische } \\
\text { Bundesaustalt, Germany }\end{array}$ \\
\hline 13. & Montcel, 2004 & $\mathrm{TD}$ & 8 & Strasbourg Univ., France \\
\hline 14. & $\begin{array}{l}\text { Muhlemann, } \\
2006 \\
\end{array}$ & $\mathrm{CW}$ & 16 & $\begin{array}{l}\text { Univ. Hospital Zurich, } \\
\text { Switzerland }\end{array}$ \\
\hline 15. & Nissila, 2005 & FD & 16 & Helsinki Univ., Finland \\
\hline 16. & Selb, 2006 & $\mathrm{TD}$ & 32 & Harvard Univ., USA \\
\hline 17. & Schmidt, 2000 & $\mathrm{TD}$ & 32 & Univ. College London, UK \\
\hline 18. & Schmitz, 2002 & $\mathrm{CW}$ & Variable & Columbia Univ., USA \\
\hline 19. & Ueda, 2005 & TD & 16 & Hamamatsu, Japan \\
\hline
\end{tabular}

Table 2.3: List of recently developed in-house NIRS imaging systems (table modified and adapted from Wolf et al., 2007). In the table CW stands for Continuous wave, FD stands for frequency domain and TD stands for time domain. CCD stands for Charged Coupled devices.

From Table 2.2 and Table 2.3, it can be seen that the in-house developed systems have relatively greater number of channels as compared to commercially available system, thereby providing the capability to investigate larger regions of the brain. The commercial and In-house developed NIRS use different types of light sources and detectors to investigate cerebral hemodynamics. Different types of sources and detectors used in NIRS systems are described in the following section (section 2.2.3).

\subsubsection{NIR light source}

The light source used in NIRS systems should be such that it provides a specific wavelength of light to quantify the change in the concentration of $\mathrm{HbO}$ and $\mathrm{HbR}$ in the cerebral cortex. The light sources available are:

1) White light with filter for NIR light - provides flexible and variable color resolution but it has low optical power per wavelength of light $(<5 \mathrm{~mW})$. 
2) Light emitting diode (LED) - provides the light in a $30 \mathrm{~nm}$ band surrounding the specific wavelength of light and the optical power of light is in low $(<1 \mathrm{~mW})$ to medium range $(30 \mathrm{~mW})$.

3) Laser diode - provides specific wavelength of light within a band of $\pm 1 \mathrm{~nm}$ and the optical power of light is in low $(<1 \mathrm{~mW})$ to high range $(500 \mathrm{~mW})$.

The factors considered in the selection light source are the discrete wavelength and optical power. Hence based on the properties of different light source described above, the widely used light source in the NIRS instrument for brain are laser diodes of specific wavelengths. The specific wavelength of the light which enters the brain depends on the tissue chromophores of interest (e.g. HbO, HbR, HbT). The light which exits the head after travelling the cortical region of brain is detected by appropriate detectors. Different types of detectors are used in NIRS instrument as described in following section (section 2.2.4).

\subsubsection{Light Detector}

The light detector used in the NIRS instrument should be sensitive to detect the extremely low light intensity which exits the head. The optical detectors that are presently in use are:

1) Silicon photodiodes (SPDs) - have low sensitivity but high dynamic range ( 100 $\mathrm{dB})$.

2) Avalanche photodiodes (APDs) - have high sensitivity and medium dynamic range $(\sim 60 \mathrm{~dB})$. 
3) Photomultiplier tubes (PMTs) - have very high sensitivity below $820 \mathrm{~nm}$ after which the sensitivity drops and medium dynamic range $(\sim 60 \mathrm{~dB})$.

4) Charge-coupled devices (CCDs) - have variable sensitivity which depends on the wavelength of light to be detected and dynamic range upto $60 \mathrm{~dB}$.

The type of detector used in NIRS instrument for brain depends upon the particulars of application. The neuroimaging application which requires fast data acquisition rate generally uses PMTs or APDs. The investigation of larger area of brain using NIRS requires greater number of detectors and since PMTs and APDs are expensive, increasing the area of investigation increases the number of detectors thereby increasing the cost and size of instrument. As compared to other types of detectors, i.e. SPDs, PMTs and APDs, although the speed of data acquisition of CCD is relatively slow it is not limited by the numbers of detectors. The number of detectors in a CCD based NIRS instrument can be easily increased by increasing the number of detector fiber bundle which collects and send light to the face of CCD camera.

Based on the advantages and disadvantages of different NIRS measurement techniques, sources and detectors (discussed in section 2.2), during my doctoral work I developed a CW based NIRS system which uses laser diode based source system and intensified charged coupled (ICCD) based detector. The details of the development of CW based NIRS system will be described in section 3.2.

The NIRS instruments described in this section, based on any of the measurement technique i.e. time domain, frequency domain and continuous wave, are employed to acquire optical signal non-invasively from the surface of head after the incident light has 
travelled through the cortical regions of brain. Thus the optical signal acquired contains information of the activity in the brain which must be processed to extract the information encoded in them. The different kind of brain's optical data processing and analysis is described in the following section (section 2.3).

\subsection{Near infrared spectroscopy of brain: signal analysis}

The acquired optical measurement using the NIRS instruments contains raw information about the attenuation of the near infrared (NIR) light as it exits the head after travelling through the cortical regions of brain. NIR light as it enters the cortical region of the brain is absorbed by the two dominant chromophores $(\mathrm{HbO}, \mathrm{HbR})$ present in the brain (Cope et al., 1987; Strangman et al., 2002). The percentage of light coming out of the brain is the function of the concentration these chromophores $(\mathrm{HbO}, \mathrm{HbR})$ present in the cortical region of interest. The modified Beer Lambert law, as described in section 2.1.2.2, is used to calculate the attenuation at two different wavelengths of light using Eq.2.5 and Eq. 2.6. The wavelength dependent attenuation of light is then solved mathematically (which will be described in section 4.1) to calculate the change in concentration of $\mathrm{HbO}, \mathrm{HbR}$ and $\mathrm{HbT}$. These hemodynamic changes can be used to elucidate activation (defined in section 2.3.1), functional connectivity (defined in section 2.3.2.2), effective connectivity (defined in section 2.3.2.3), and lateralization (defined in section 2.3.3) in the region of brain under investigation.

\subsubsection{Activation Analysis}

Activation analysis is performed to investigate the changes in relative concentration of $\mathrm{HbO}, \mathrm{HbR}$ and total hemoglobin $\mathrm{HbT}(=\mathrm{HbO}+\mathrm{HbR})$ as a function of time, in response to 
the stimulus being processed by the underlying cortical region. During activation analysis the optical data acquired from the surface of the head (intensity) is processed to compute the relative change in $\mathrm{HbO}, \mathrm{HbR}$ and $\mathrm{HbT}$ using the modified Beer Lambert's law described in section 2.1.2.2. The activation analysis elucidates the hemodynamic response of the cortical regions under investigation when the stimulus is being presented to the participants during the study. The activation analysis is represented by 1-D plot of the change in $\mathrm{HbO}, \mathrm{HbR}$ and $\mathrm{HbT}$ as a function of time (as shown in Figure 2.7). The details of activation analysis are described in section 4.1.

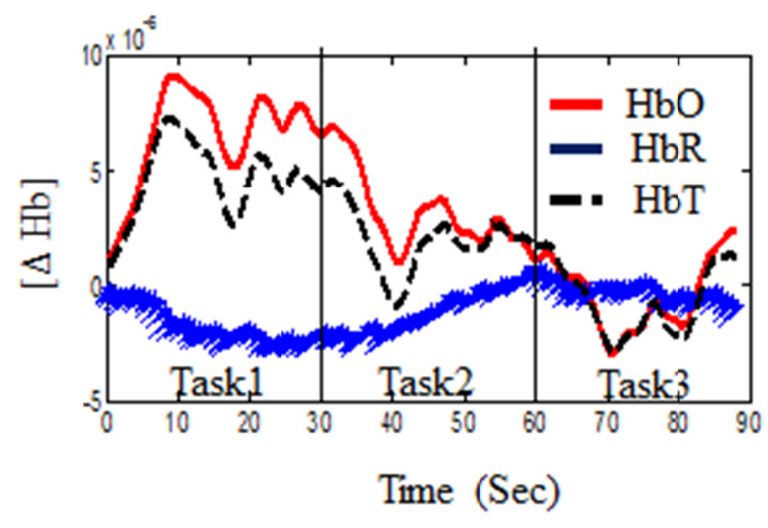

Figure 2.7: Hemodynamic response function, $H R F$ plotted against time (in seconds). The plot shows the HRF as three tasks were being performed by the participants.

\subsubsection{Connectivity Analysis}

Brain connectivity refers to the pattern of connection and interaction between units of brain. The unit here can correspond to a single neuron, assembly of neuron or a specific region of the brain. In the past, analysis were being performed to determine the assembly of neuron firing in coherent fashion (Aertsen et al., 1989, 1994; Gerstein \& Aertsen, 1985; Gerstein \& Perkel, 1969) which leads to the development of concepts of functional 
and effective connectivity (Aertsen et al., 1989). The connectivity pattern is formed by structural links such as synapses or fiber pathways which forms a distributed network wherein the different regions of the brain specializing in different function communicate with each other to process sensory input to provide a motor output (Friston, 1994; Swanson, 2003).

Depending on the method used to ascertain the connectivity pattern in the brain, the brain connectivity analysis can be divided into three categories:

(1) Anatomical connectivity which describe the anatomical links between different units in the brain (Lee et al., 2003).

(2) Functional connectivity which describes the temporal correlation among the activity of different neurons (Friston 1994; Horwitz 2003; Lee et al. 2003).

(3) Effective connectivity which describes the causal interaction between distinct units within a nervous system (Friston 1994; Horwitz 2003)

The distributed network in the brain or the connectivity pattern is established by synapses between neighboring neuron representing anatomical connectivity. The information flow in the established synapses or fiber pathways can be ascertained by the temporal correlation or coherence in the information flow representing functional connectivity (Friston 1994). The network of directional effects of one neural element over another can be elucidated by effective connectivity (Aertsen et al., 1989). Thus it can be stated that the neural activity in the brain is dependent on the connectivity in the brain. Hence the study of brain connectivity is vital to explicate the information processing pattern of the brain. 


\subsubsection{Anatomical/Structural Connectivity}

Structural connectivity refers to a network of synaptic connections linking sets of neurons or neuronal element (Lee et al., 2003). They form a distributed network in the brain defined by parameters like synaptic strength or efficiency. This physical pattern is relatively unwavering at shorter time scales (seconds to minutes). At longer time scales (hours to days), structural connectivity patterns are likely to undergo considerable morphological change and plasticity. Structural connectivity can only be determined using invasive tracing studies except DTI (diffuse tensor imaging), but DTI is limited by its insufficient spatial resolution. NIRS cannot be applied to determine the structural connectivity in the brain as the structural connectivity is not related to the hemodynamic response of the brain. A picture depicting the structural connectivity between different regions of the brain is shown in Figure 2.8.

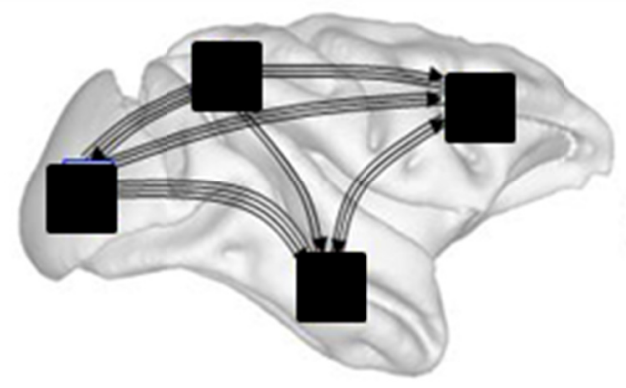

Figure 2.8: Anatomical/Structural connectivity showing the anatomical links between different units in the brain (http://www.scholarpedia.org/article/Brain connectivity). 


\subsubsection{Functional Connectivity}

Functional connectivity is fundamentally a statistical concept. Functional connectivity is defined as the (Friston 1994; Horwitz 2003; Lee et al. 2003) "temporal correlation between spatially remote neurophysiological events". Functional connectivity measures simultaneous coupling between two time series. It does not give us information on whether two units are connected or not i.e. it is agnostic to the directional or causal relationship between two time series as shown in the Figure 2.9 (http://www.scholarpedia.org/article/Brain_connectivity). Functional connectivity is often calculated between all elements of a system, regardless of whether these elements are connected by direct structural links. Unlike anatomical connectivity, functional connectivity is highly time-dependent. The details of functional connectivity analysis are described in section 4.2. NIRS has been applied in the past to study functional connectivity in the brain during resting state ( $\mathrm{Lu}$ et al. 2010; Zhang et al. 2010) and even during task (Chaudhary et al., 2011; Rykhlevskaia et al., 2006).

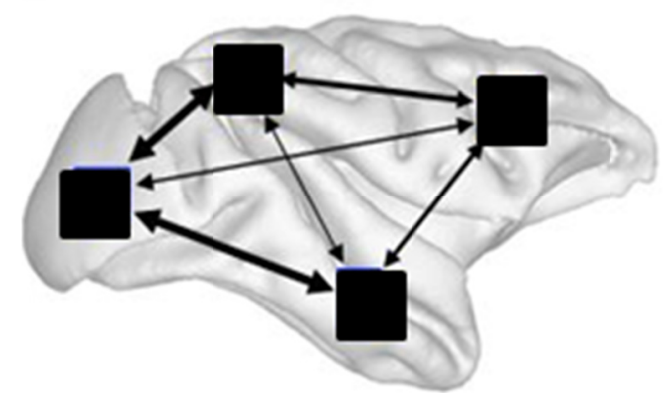

Figure 2.9: Functional connectivity showing the temporal correlation among the activity of different neurons (http://www.scholarpedia.org/article/Brain_connectivity). 


\subsubsection{Effective Connectivity}

Effective connectivity attempts to describe or make inferences about the direction of influence between regions (Friston 1994; Horwitz 2003) as shown in Figure 2.10 (http://www.scholarpedia.org/article/Brain_connectivity). Effective connectivity can be estimated using more detailed models and applying additional assumptions to calculated correlations or covariances to answer about the directional influences (Friston 1994; Horwitz 2003). The details of effective connectivity analysis are described in section 4.3. NIRS has been applied in the past to study the effective connectivity in the brain in response to stimuli (Rykhlevskaia et al., 2006).

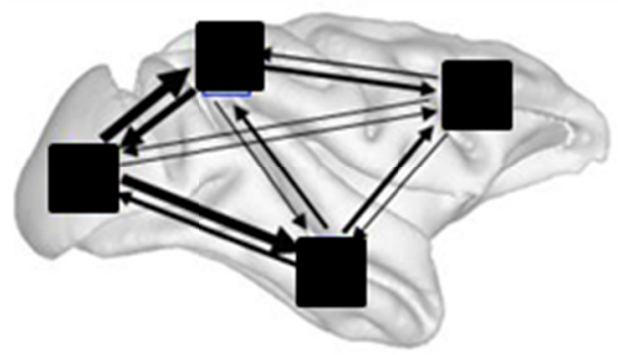

Figure 2.10: Effective connectivity showing the causal or the directional interaction between distinct units within a nervous system (http://www.scholarpedia.org/article/Brain_connectivity).

\subsubsection{Lateralization Analysis}

Lateralization analysis using NIRS is performed to understand the hemispherical dominance of the one cortical region over other during the task or even during the resting state of brain (Watanabe et al. 1998; Watson et al. 2004). The details of lateralization are 
described in section 4.4. NIRS has been applied in the past to investigate the cortical dominance during different tasks (Watanabe et al. 1998; Watson et al. 2004).

Thus based on NIRS neuroimaging technique described in this chapter, NIRS has been successfully used towards functional brain mapping in awake adults, infants and children as well in response to active stimuli (Lloyd-Fox et al., 2010; Strangman et al., 2002). Some of the many applications (sample references listed) of NIRS to neuroimaging include: (i) cerebral response to visual (Heekeren et al, 1997; Meek et al, 1995; Ruben et al, 1997), auditory (Sakatani et al, 1998) and somatosensory stimuli (Franceschini et al, 2003); (ii) cerebral response to motor system (Colier et al, 1999; Hirth et al, 1996; Schwartz et al, 2005) and language (Sato et al, 1999); and (iii) prevention and treatment of seizures (Adelson et al, 1999; Sokol et al, 2000; Steinhoff et al, 2005; Watanabe et al, 2000) and psychiatric concerns such as depression (Eschweiler et al, 2000; Matsuo et al, 2000; Okada et al, 1996), Alzheimer disease (Fallgatter et al, 1997; Hanlon et al, 1999; Hock et al, 1996) and schizophrenia (Fallgatter et al, 2006; Okada et al, 2004), as well as stroke rehabilitation (Chen et al, 2000; Nemoto et al, 2000; Saitou et al, 2000; Vernieri et al, 1999); (iv) detection of brain ischemia (Kuebler et al, 1998; Patel et al, 1998), necrosis, and hemorrhage (Yodh et al, 2003). During my doctoral work, NIRS was implemented to investigate the neural basis of language, joint attention and planning and execution of motor skills in healthy adults, as described below in section 2.4. 


\subsection{Applications of NIRS during my doctoral work}

The goal of my doctoral work was to apply NIRS towards the extensive investigation of neurological response of language, joint attention and planning and execution of motor skills in healthy adults. Joint attention and planning and execution of motor skill stimuli were also used preliminarily to investigate the neurological response in children with autism and individuals with cerebral palsy, respectively. The neuroimaging of language, joint attention and planning and execution of motor skills using NIRS is described in details in sections 2.4.1, 2.4.2 and 2.4.3, respectively.

\subsubsection{NIRS and language}

In the area of language development and brain activation/functionality using NIRS, researchers have performed studies using various experimental paradigms in normal adults and pediatric populations (Bortfeld et al, 2009; Herrmann et al, 2003; Hermann et al, 2006; Kuwabara et al, 2006; Sakatani et al, 1998). The studies were focused primarily in understanding the differences in the hemodynamic responses in different (typically frontal and temporal) regions of the brain in response to language-based stimuli (Herrmann et al, 2003; Hermann et al, 2006; Jayakar et al, 2005; Kubota et al, 2005; Kuwabara et al, 2006; Quaresima et al, 2002; Sakatani et al, 1998). In addition, a few researchers have analyzed the cortical lateralization and dominance features of the brain in different populations, based on their application focus (e.g. schizophrenia, epilepsy) (Gallagher et al, 2008; Hermann et al, 2003; Kennan et al, 2002; Kubota et al, 2005; Noguchi et al, 2002; Watanabe et al, 2000; Watson et al, 2004). All the language studies carried out so far using NIRS (Gallagher et al, 2008; Herrmann et al, 2003, Jayakar et al, 
2005; Kennan et al, 2002; Kubota et al, 2005; Kuwabara et al, 2006; Noguchi et al, 2002; Qaresima et al, 2002; Sakatani et al, 1998; Watanabe et al, 1998; Watson et al, 2004) have focused on the brain activity differences and/or cortical dominance. To the best of our knowledge none of these studies have investigated the functional and effective connectivity and/or lateralization in the language regions of brain simultaneously. Activation, functional and effective connectivity and lateralization analysis when performed simultaneously has the potential to elucidate the integration and synchrony between different region of motor cortex. Thus during my doctoral work NIRS was used to investigate the activation, functional and effective connectivity and/or lateralization in the language regions of brain simultaneously. The details of the study are described in chapter 5 and 6.

\subsubsection{NIRS and Joint attention}

In the field of autism research, NIRS has been applied in limited studies related to autism, where the focus of the research was the language processing abilities of adults with Persuasive Developmental Disorders, PDD) (Kuwabara et al., 2006) and also the language processing abilities in children and adults with autism (Kawakubo et al., 2009). Recently, NIRS was applied for the first time at FIU-OIL (Zhu et al., 2009) to understand the activation in the healthy adults in response to joint attention skill and also to demonstrate the feasibility of NIRS towards autism research. The Joint attention stimuli refer to the capacity of subject to coordinate attention with a social partner. The work was based on the work done by other researchers to investigate joint attention experience in adult using fMRI (Williams et al., 2005). Hence, based on the paradigm used in the 
earlier fMRI study, NIRS was employed at OIL to study the hemodynamic responses to joint attention experience (using responding to joint attention, or RJA-based stimuli) in the frontal cortex of normal adults (Zhu et al., 2009). This work demonstrated successful application of NIRS towards the autism research, thereby setting the stage for future extensive NIRS study on typically developing children and children with autism in response to joint attention skill based paradigm. Thus during my doctoral work NIRS was used to investigate the underlying neurological response of frontal cortex in healthy adults as well as preliminarily in typically developing children and children with autism. The details of the study are described in chapter 7 and 8 .

\subsubsection{NIRS and planning and execution of motor skill stimuli}

Individuals with cerebral palsy $(\mathrm{CP})$ often have motor impairment syndromes that occur as a result of a permanent, non-progressive lesion of the brain (Kułak et al., 2008). Several advanced neuroimaging techniques have been used to investigate and explicate the etiological basis of CP. Structural neuroimaging techniques, such as MRI (Griffiths et al., 2010; Horridge et al., 2011; Kułak et al., 2007a; Kułak et al., 2007b; Sööt et al., 2008), DTI (Fariaa et al., 2011; Rha et al., 2011; Yoshida et al., 2011) and CT (Accardo et al., 2004), have been applied to explicate the probable cause of CP because of structural change in the brain due to brain mal development and/or lesion. Functional neuroimaging techniques, such as fMRI (Burton et al., 2009; Schwilling et al., 2012; Sutcliffe et al., 2009; Wingert et al., 2010) and PET/SPECT (Batista et al., 2007; Lee et al., 2007; Wong et al., 2006), have been employed to elucidate the functional change in the brain associated with activation of brain pre- and post- tasks performed by individuals 
with CP. None of the above mentioned functional neuroimaging techniques have been employed to investigate the functional change in the brain of individuals with $\mathrm{CP}$ during a task as these techniques, $\mathrm{fMRI}$ and PET/SPECT, requires the participants to be stationary in a claustrophobic environment. The several advantages offered by NIRS neuroimaging technique, as described in section 2.1.4, makes NIRS technique a reliable tool to investigate the cerebral hemodynamics change in individuals with cerebral palsy in response to complex tasks during physical therapy regime of individuals with $\mathrm{CP}$. Individuals with $\mathrm{CP}$ who participate in physical therapy programs generally demonstrate some degree of decreased motor impairment and functional improvement, in spite of the permanent nature of their central nervous system (CNS) lesion. The literature strongly suggests that this improvement is due to plasticity of the viable portions of the CNS, and that plasticity is dependent, at least in part, on repeated activation of specific areas of the CNS. Therefore, activities to include in physical therapy programs for any given person with $\mathrm{CP}$, or for adults with acquired brain lesions, might best be chosen based on the magnitude, duration, and location of CNS activation they engender, with the assumption that these activations will facilitate plastic changes within the CNS that will ultimately yield the best possible functional outcomes (Kulak et al., 2007; Rha et al., 2011). Thus during my doctoral work, NIRS technique was initially used to perform a preliminary investigation of the planning and execution of motor skill tasks in individuals with cerebral palsy in comparison to the controls as described in chapter 9. This study was followed by an extensive investigation of the planning and execution of various motor skills stimuli in controls as described in chapter 10. The planning and execution of motor skill stimuli was further investigated using NIRS technique in conjunction with 
kinematics study to simultaneously investigate the response of brain and movement of hand during planning and execution of motor skill in healthy adults as described in chapters 11 and 12 . 


\section{CHAPTER 3. Near Infrared Spectroscopy Instrumentation}

Near infrared spectroscopy (NIRS) measures the attenuation of near infrared light after travelling through the cortical region of brain using NIRS instrument which measures attenuated light based on three different principle namely, continuous wave, frequency domain and time domain as described in chapter 2. During my doctoral work NIRS instrument which works on the principle of frequency domain and continuous wave were employed. The commercial frequency domain based NIRS system used for my studies is described in section 3.1. The continuous wave based NIRS system developed in-house and used for latter part of my studies is described in section 3.2 and 3.3.

\subsection{Frequency domain based NIRS system: Commercial}

A frequency-domain based optical imaging system, Imagent (ISS, Inc., Champaign, IL) (shown in Figure 3.1) was used to investigate the change in hemodynamic response of the cortical regions of the brain in response to different stimuli. Different studies which were performed using the Imagent are described in chapters 5, 6, 7 and 8 . The Imagent is a multi-channel system with 32 intensity-modulated laser diodes (16 emit $690 \mathrm{~nm}$ laser light, and the remaining 16 at $830 \mathrm{~nm}$ wavelength), and four gain-modulated photomultiplier tube (PMT) detectors that collect the optical signals at both the wavelengths separately. The light sources are electronically multiplexed at a frequency of $100 \mathrm{~Hz}$ (10 ms on-time per laser diode) to time-share the four optical detectors. The modulation frequency, which can be adjusted from $(100-300 \mathrm{MHz})$ was set at $110 \mathrm{MHz}$. This frequency was chosen due to the fact that it allows for maximum depth penetration of optical signals into the tissue (Gibson et al., 2005). The entire instrument is computer 
controlled and the frequency-domain measurements (DC, $\mathrm{AC}$, and phase shift) are obtained from different source-detector locations using a Fast-Fourier-Transform data acquisition card. The optical signals are acquired in real time using the software package (BOXY) of the instrument. BOXY(C) provides real-time information regarding signal to noise ratios of the four photomultiplier tubes used by the Imagent. On the Boxy software's interface, the signal from each PMT (photo multiplier tube) detector (each detector) is represented as a square with either green or yellow color. The coloring is based on the PMT's noise threshold level. If the signal's SNR (Signal to noise ratio) from a PMT is above the noise threshold it shows green and if the signal's SNR from a PMT is below the noise threshold it shows yellow. Since Imagent can operate in frequency domain it can provide optical signal in terms of change in DC, AC and phase shift.

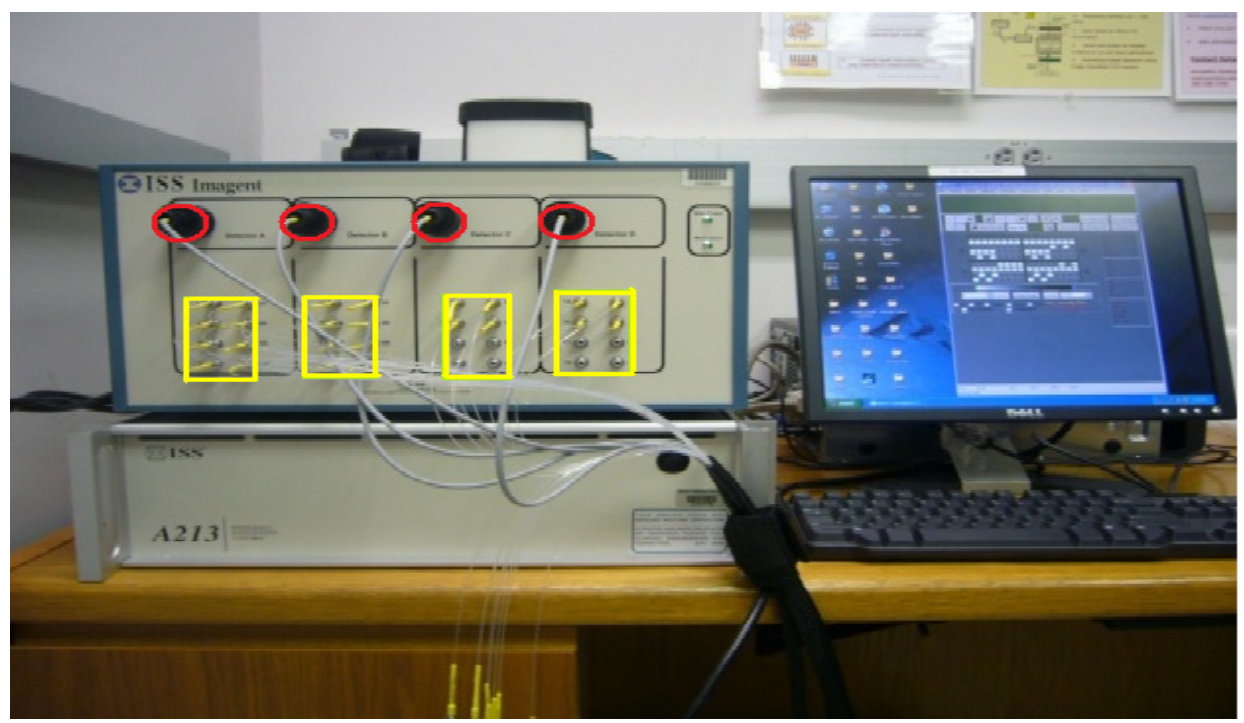

Figure 3.1: Frequency domain based optical imager, Imagent (ISS, Inc., Champaign, IL). The red circles depict the detectors and the yellow rectangles depict the sources. The Imagent was provided by Brain Institute at MCH (Miami Children's Hospital). 
The optical data acquired using the commercial NIRS instrument (Imagent) was processed to perform activation, functional connectivity and lateralization analysis as would be described in the chapters 5, 6, 7 and 8 .

The studies described in chapters $9,10,11,12$ and 13 were performed using in-house developed NIRS system. During my doctoral work I developed a NIRS system by modifying and adapting the intensified charged couple device (ICCD) based optical mammography system developed in our OIL. The ICCD based imaging system has an advantage over PMTs based imaging system wherein the ICCD based system is not limited by the numbers of detectors. The number of detectors in ICCD based NIRS instrument can be easily increased by increasing the number of detector fiber bundle which collects and send light to the face of ICCD camera. The initially developed ICCD based NIRS system was single wavelength system (as described in section 3.2), which can only provide the relative change in total hemoglobin concentration (when the single wavelength is around the isosbestic point) without elucidating the change in the oxygen saturation content of hemoglobin. The single wavelength ICCD based NIRS system was further modified to perform dual wavelength NIRS of the brain (as described in section 3.3) to elucidate the change in the $\mathrm{HbO}, \mathrm{HbR}$, and $\mathrm{HbT}$ concentrations. The in-house developed ICCD based NIRS system is described in the following sections (section 3.2single wavelength based NIRS system and section 3.3 - dual wavelength based NIRS system). 


\subsection{Continuous wave single wavelength based NIRS system: In-house developed}

The single wavelength NIRS system for brain imaging was developed by modifying and adapting the ICCD based optical mammography system developed in OIL. The major components of the developed NIRS system (as shown in Figure 3.2) are the single wavelength laser diode based source system, the intensified charge-coupled device based detector, source and detector fiber bundles described in section 3.2.1, 3.2.2 and 3.2.3 respectively. The working of the developed single wavelength based NIRS system is described in section 3.2.4.

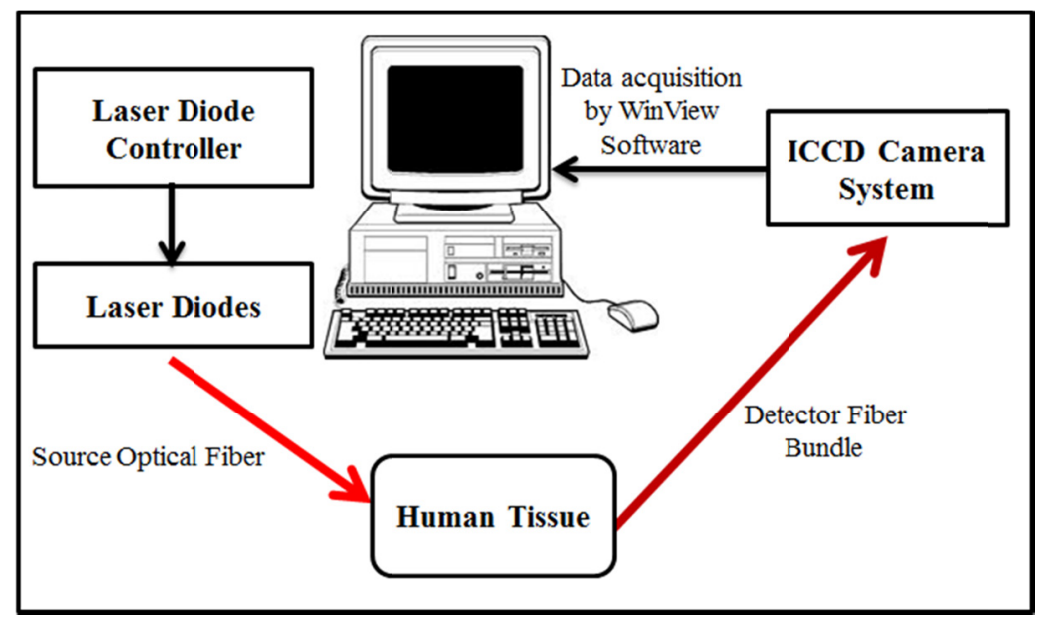

Figure 3.2: Block diagram of the different components used to develop the single wavelength ICCD based NIRS system.

\subsubsection{Single wavelength laser diode based source system}

The single wavelength source system is composed of two main components, the multichannel controller and the laser mount. The multichannel controller, described below in section 3.2.1.1, drives the laser mounts with an integrated laser diode driver and thermal electric cooler driver. The laser system is composed of six independent diode 
laser mounts, described in section 3.2.1.2, which are controlled by a multichannel controller.

\subsubsection{Multichannel controller}

The sources are driven and cooled by a Newport Irvine CA 92606, USA 8008 multichannel controller (as shown in Figure 3.3). The Newport 8008 features 8 independent channels and a large range of current values. These 8 independent channels were optimized individually to provide a homogenous source distribution. The developed source system has six sources leaving 2 channels for future expansion.

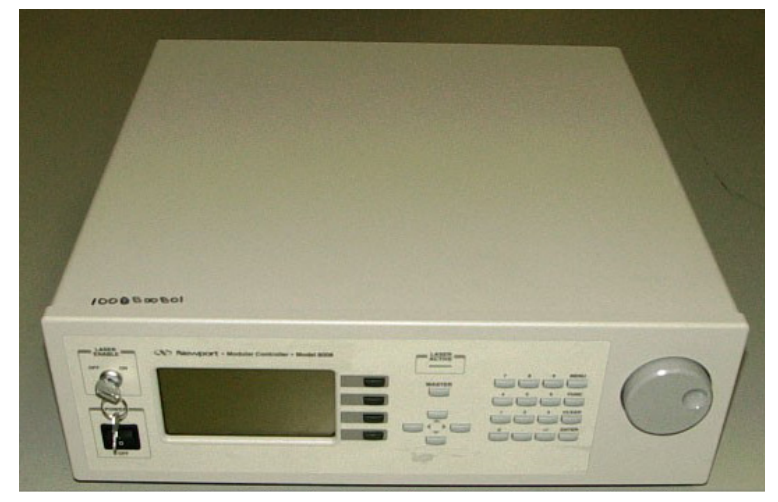

Figure 3.3: The source of the Gen-2 imager is driven by the multichannel controller that is a combination laser driver (LD) and thermoelectric cooler driver (TECD), which allows for the control of up to 8 independent laser mounts.

\subsubsection{Laser mount}

The TCLDM9 (Thorlabs, Newton, New Jersey, TCLDM9) is a sturdy temperature stable laser mount accepting a $785 \mathrm{~nm}$ laser diode. The diode employed (Thorlabs, Newton, New Jersey, 785P100) emits light at $785 \mathrm{~nm}$ and can produce up to $100 \mathrm{~mW}$ of optical power in CW. The laser mounts have female SMA connectors which serve as a 
coupling point with the optical fibers. A coupling point is a location where the laser diode emission and the optical fiber are attached on the same optical axis. The aspheric lens collimates the light from the laser diode onto the optical fiber and also maximizes the light entering into the optical fiber.

\subsubsection{Detector}

The detector is composed of the PI-Max II produced by Princeton Instruments (Figure 3.4) and the series of lenses, lens tube and a ring attachment for the optical fiber bundle. The PI-Max II produced by Princeton Instruments (Figure 3.4) offers a built-in intensifier which can be software controlled via Labview or Winview.

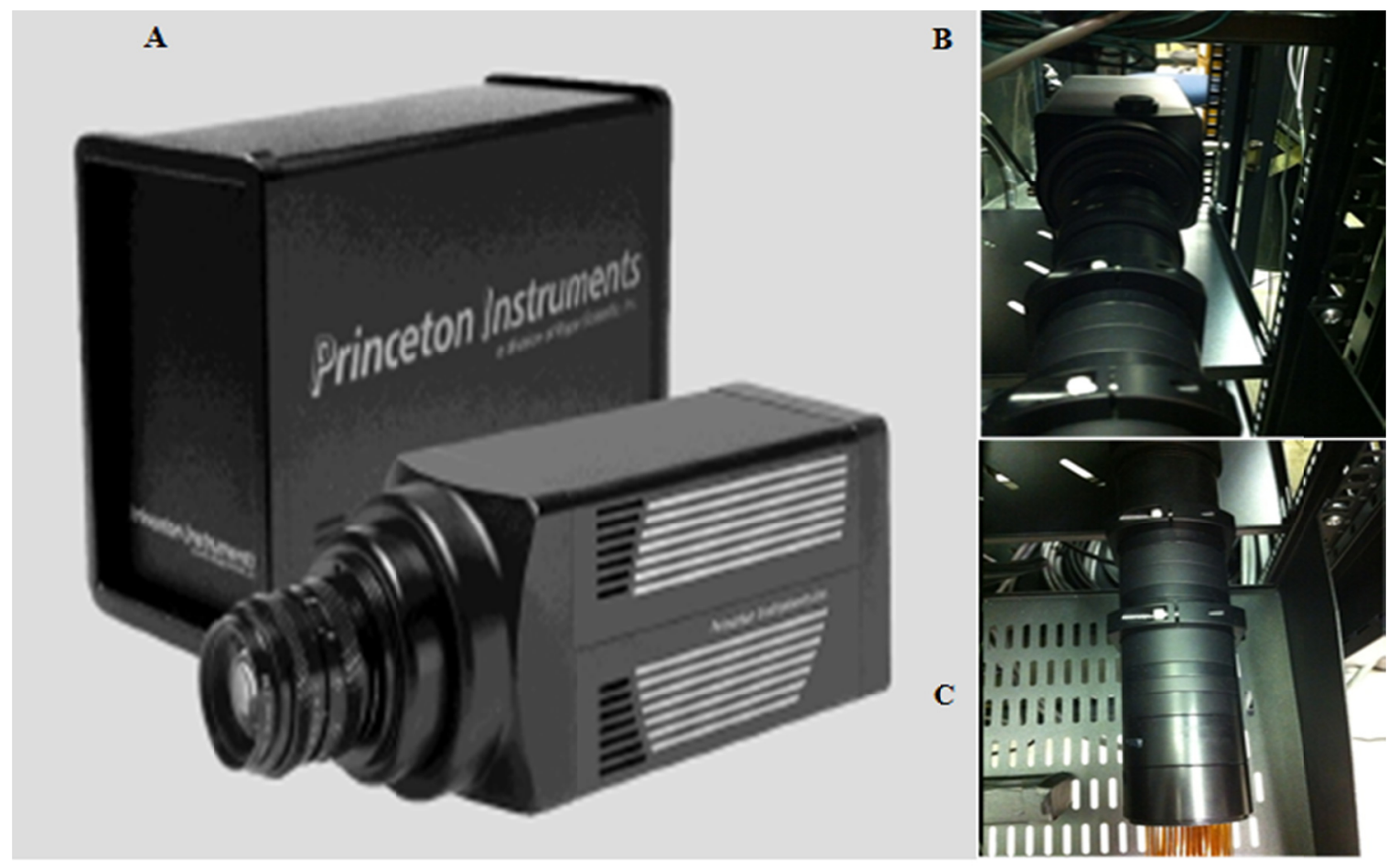

Figure 3.4: (A) The PI-Max II ICCD camera (from Princeton instruments) used for the detection of low intensity light, $(B)$ the ICCD camera within the enclosure for the NIRS system, (C) Optics employed in conjunction with PI-Max II, such as lens tubes and other lens adapters. 
The Winview interface showing the free run timing mode in which the ICCD camera is run to acquire the optical signal is shown in Figure 3.5. The ICCD camera collects the signal intensity (in a.b.u.) on the fiber bundle and saves the image as a file, which allows access to the optical signal. The probe fibers are attached to the camera via a series of lens tubes. The lens tubes or assembly are coupled to the ICCD camera via a Nikon focusing lens (Nikon Melville, NY US branch, Nikon $50 \mathrm{~mm} \mathrm{f/1.8D} \mathrm{AF)} \mathrm{that} \mathrm{is} \mathrm{C-}$ mounted to the ICCD camera.

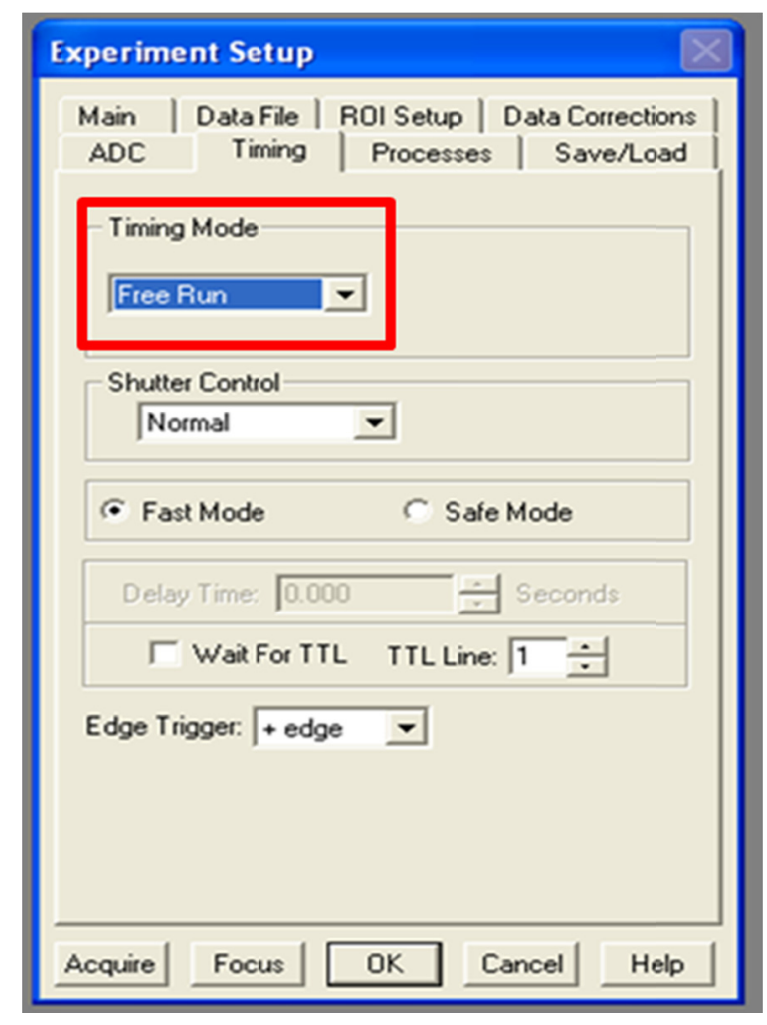

Figure 3.5: Winview interface showing the mode in which ICCD camera is run, the region highlighted in red is a drop down menu which enables selecting different timing mode. The single wavelength ICCD based NIRS system was run in "Free" run mode as shown in highlighted red area. 
The lens assembly for the ICCD camera focuses the image of the detector fiber bundle onto the ICCD of the camera (Figure 3.4). The distance between the camera's intensifier and the optical detection fiber bundles were adjusted based on Eq. 3.1 thin lens equation

$\frac{1}{S_{1}}+\frac{1}{S_{2}}=\frac{1}{f}$

where in Eq. 3.1, $f$ is the focus of the lens, $\mathrm{S} 1$ is the distance from the lens to the handheld probe and $\mathrm{S} 2$ is the distance from the lens to the image. The equation could be solved for either S1 $(67 \mathrm{~mm})$ or S2 $(106 \mathrm{~mm})$. However the distance between the surface of the camera and the image intensifier cannot be changed in the developed ICCD based NIRS system and must be added to S2.

The source and detector system described in section 3.2.1 and 3.2.2 shines light onto the brain and collects the attenuated light intensity from the brain, respectively via source and detector fiber respectively described in section 3.2.3 below.

\subsubsection{Source and detector fiber bundle}

Source fiber of core diameter of $400 \mathrm{~mm}$ and numerical aperture of 0.37 is used to deliver light to the surface of the head. The source fiber has spectral range of 400-2000 nm and is 72" in length. The attenuated light intensity, as it exits the head after travelling through the cortical regions is collected via an optode connected to the detector fiber bundle. The detector fiber bundles have $2.5 \mathrm{~mm}$ core diameter, 0.6 numerical aperture and are 72 " in length.

Thus the NIRS system developed using the three major components of NIRS as described above in section 3.2.1, 3.2.2 and 3.2.3, is shown in Figure 3.6, and this system was used 
to perform studies described in chapters 9,10 and 11 . The working principle of the developed NIRS system is described below in section 3.2.4.

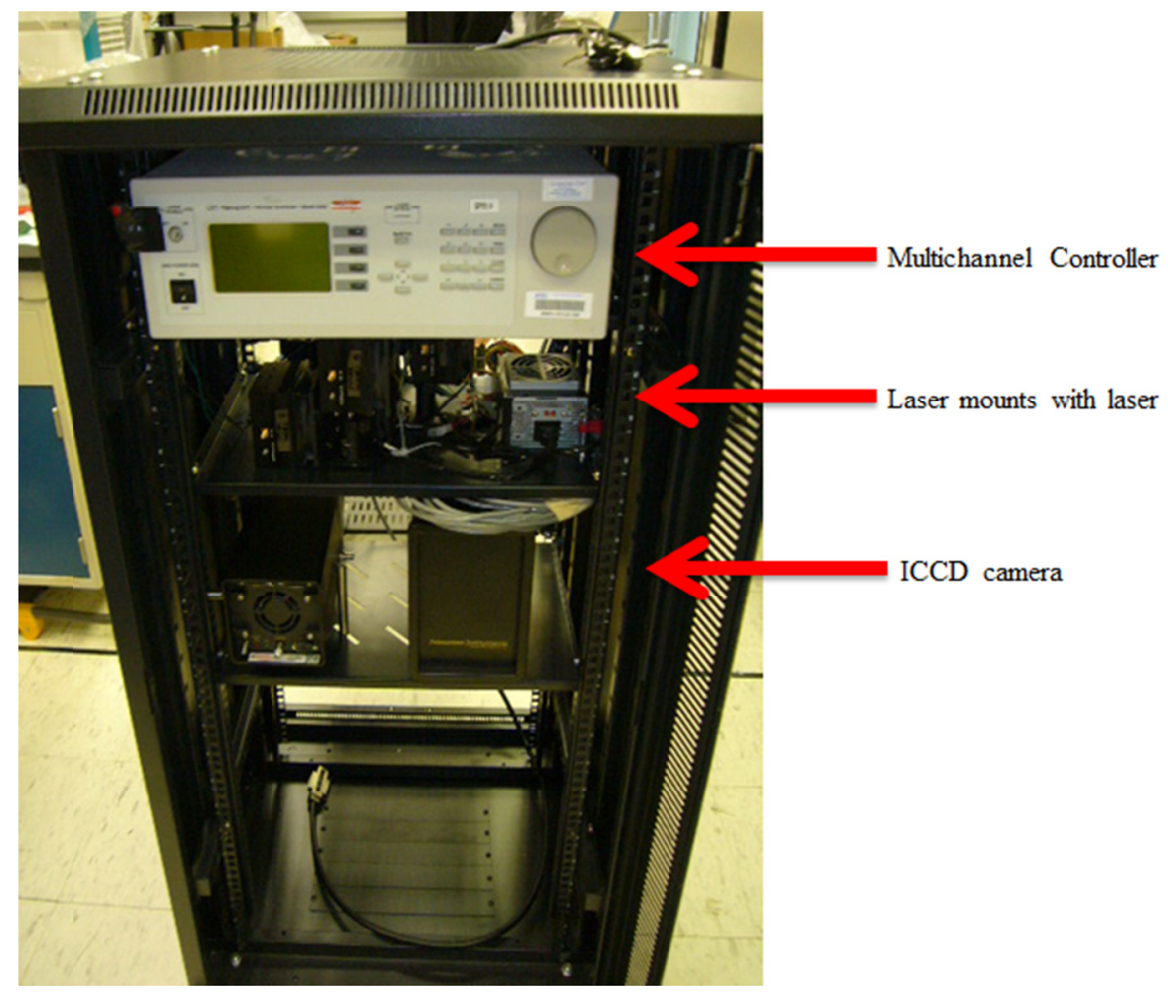

Figure 3.6: The developed single wavelength ICCD based NIRS system.

\subsubsection{Working principle of single wavelength based NIRS system}

The developed continuous-wave (CW) NIR spectroscopy (NIRS) system constituted of a laser diode-based source system, an intensified charge-coupled device (ICCD) based detector system, and a custom-developed optical cap containing source and the detector fiber bundles. The laser diode based source system consists of $785 \mathrm{~nm}$ laser light sources with variable optical power output (as described in section 3.2.1). The light from the source system is delivered to the region of the head under investigation via an optode connected to source fibers (as described in section 3.2.3). The attenuated light intensity, as it exits the head after travelling through the cortical regions is collected via an optode 
connected to detector fiber bundle (as described in section 3.2.3), depicted by the directional flow of signal in Figure 3.2. The detector fiber bundle delivers the light to an ICCD based detector system (as described in section 3.2.2), which is operated in shutter mode at after adjusting the value of the ICCD camera as shown in Figure 3.7. The ICCD camera is operated at an acquisition rate of $4.76 \mathrm{~Hz}$ determined by the formulas shown in Eq. 3.2 and Eq. 3.3.

$T_{I}=T_{e x}+T_{T I}$

where in Eq.3.2; $T_{I}$ is the total time taken to acquire a single image, $T_{e x}$ is the exposure time of the CCD camera and $T_{T I}$ is the time taken to transfer and integrate the charge on the CCD camera.

$I_{R}=\frac{1}{T_{I}}$

where in Eq. 3.3; $I_{R}$ is the acquisition rate of ICCD detector and $T_{I}$ is the total time taken to acquire a single image.

During the study (described in chapters 9,10 and 11) $\mathrm{T}_{\mathrm{ex}}$ was set at $0.2 \mathrm{sec}$ as shown in Figure 3.7 (optimized exposure determined after a series of experimental studies), $\mathrm{T}_{\mathrm{TI}}$ was found to be $0.01 \mathrm{sec}$ after a series of experimental studies. Hence upon plugging in the values of $\mathrm{T}_{\mathrm{ex}}$ and $\mathrm{T}_{\mathrm{TI}}$ in Eq. 3.2 the total time, $\mathrm{T}_{\mathrm{I}}$, taken to acquire a single image was calculated to be $0.21 \mathrm{sec}$. Thus the reciprocal of $\mathrm{T}_{\mathrm{I}}$, which is the acquisition rate given by Eq. 3.3, translates into an acquisition rate of $4.76 \mathrm{~Hz}$.

The optical signal acquired from the developed NIRS system is processed to elucidate the change in $\mathrm{HbT}$ concentration using mathematical equations described in (section 2.1.2.1). 


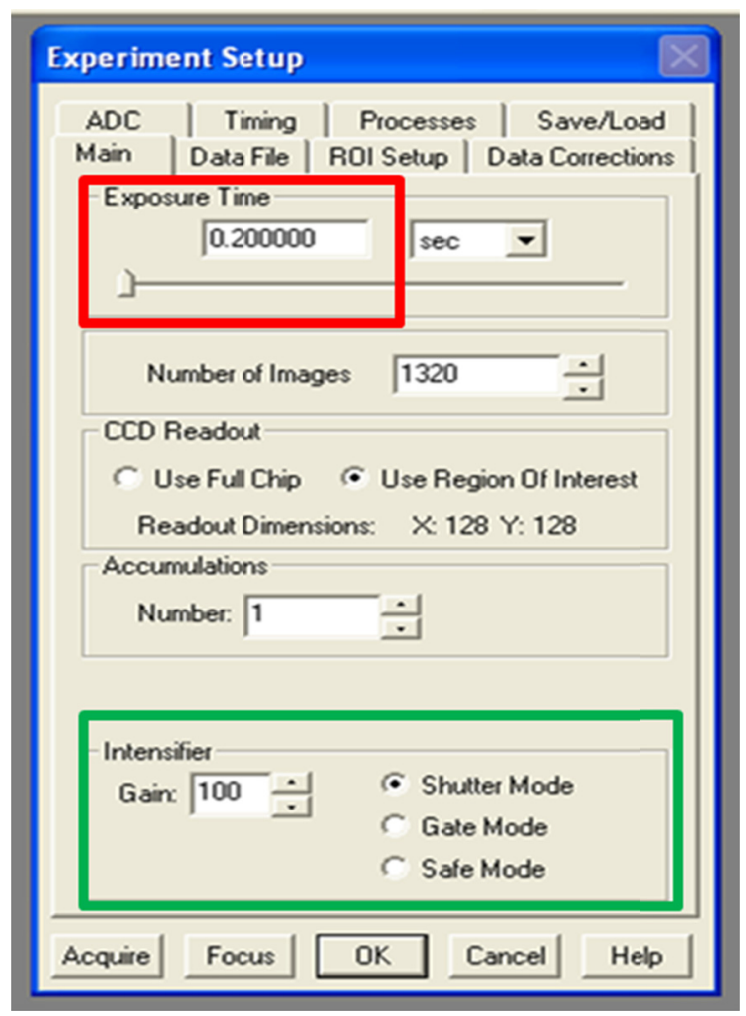

Figure 3.7: Winview interface showing the set exposure time (the area highlighted in red) of CCD camera. The area highlighted in green shows the mode in which the intensifier is run and the also the gain of the intensifier. As shown in this Figure the exposure time of the camera was set as $0.2 \mathrm{sec}$, the intensifier was run in shutter mode and the gain of the intensifier for this particular data acquisition duration was 100 (abu).

The single wavelength based NIRS system described above in section 3.2 uses $785 \mathrm{~nm}$ of NIR light, since the $785 \mathrm{~nm}$ is close to isosbestic point, and hence provides change in HbT concentration. Hence to elucidate the change in concentration of two different chromophores i.e. $\mathrm{HbO}$ and $\mathrm{HbR}$ (as described in section 2.1.1) there is a need to perform NIRS of brain using two different wavelengths of NIR light. Thus a dual wavelength based NIRS system was developed by designing and developing completely new source system while using the same ICCD camera (described in section 3.2.2) used for single 
wavelength based NIRS system. The design and development of dual wavelength based NIRS system is described below in section 3.3.

3.3 Continuous wave dual wavelength based NIRS system: In- house developed A dual source wavelength based NIRS system was developed in-house to perform NIRS of the brain at two different wavelengths of NIR light (830 nm and $690 \mathrm{~nm}$ ). NIRS of brain at two different of NIR light elucidate the change in concentration $\mathrm{HbO}, \mathrm{HbR}$ and $\mathrm{HbT}$ (as described in section 2.2.1) in the region of brain under investigation. The detailed block diagram depicting the building blocks or the components used and connection between different components is shown in Figure 3.8. The major components of the developed NIRS system (as shown in Figure 3.8) are the dual wavelength laser diode based source system, the intensified charge-coupled device based detector, source and detector fiber bundles. Different components used to develop dual wavelength based source system are described in section 3.3.1.

The ICCD camera based detector is same as the one used to develop single wavelength based NIRS system and the only difference is the mode in which the camera is operated to acquire dual wavelength data (as described in section 3.3.2). The source and detector fiber bundles used is the same as the one used in single wavelength based NIRS system as described in section 3.2.3. A signal generator described in section 3.3.3 is used to modulate the dual wavelength based source system. The same signal generator is also used to synchronize the source system and ICCD camera as described in section 3.3.5. Finally the developed dual wavelength NIRS system was tested (as described in section 
3.4) and used to perform functional brain imaging studies in response to different tasks as described in chapter 12 and 13.

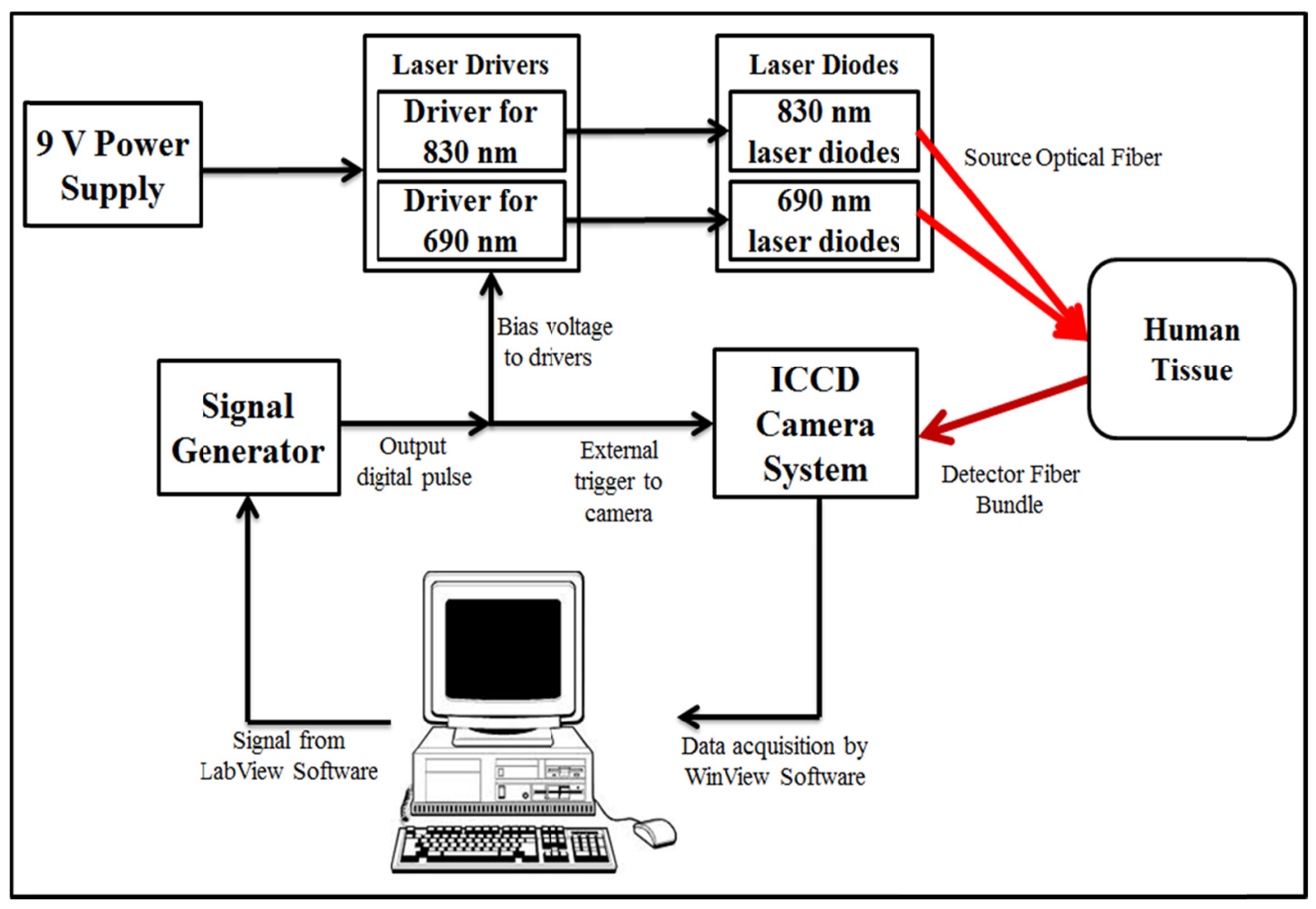

Figure 3.8: Block diagram of the different components used to develop the dual wavelength ICCD based NIRS system.

\subsubsection{Dual wavelength source system}

The components used to develop dual wavelength source system are; (i) laser diodes of $830 \mathrm{~nm}$ and $690 \mathrm{~nm}$ wavelength of NIR light, (ii) laser driver to provide the bias voltage needed to lase the laser diode, (iii) the power supply to supply the required voltage to laser driver, and (iv) the signal generator to provide the external modulation signal in order to modulate the two different laser diodes. 


\subsubsection{Laser diodes}

The laser diodes employed are of wavelength $830 \mathrm{~nm}$ and $690 \mathrm{~nm}$ to facilitate measuring changes in $\mathrm{HbO}$, and $\mathrm{HbR}$ concentrations. The $830 \mathrm{~nm}$ wavelength laser diode (part number - DL5032-001, acquired from Thorlabs, Newton, New Jersey, 785P100) has maximum optical power output of $30 \mathrm{~mW}$ and operates in $\mathrm{CW}$ mode. The $690 \mathrm{~nm}$ wavelength laser diode (part number - HL6738MG, acquired from Thorlabs, Newton, New Jersey, 785P100) has maximum optical power output of $50 \mathrm{~mW}$ and it also operates in $\mathrm{CW}$ mode. The laser diodes are mounted onto laser driver, as described in section 3.3.1.2 below, to provide the bias voltage needed to lase the laser diode.

\subsubsection{Laser drivers}

Two different kinds of laser driver were acquired to drive the two different kind of laser diode. To drive the $830 \mathrm{~nm}$ laser diodes, laser driver named EK 2000 (as shown in Figure 3.9A) was acquired from Thorlabs; and to drive the $690 \mathrm{~nm}$ laser diodes, laser driver named DBDL M/Q (as shown in Figure 3.9B) was acquired from Microlasers Inc. A 9 V DC power supply (as shown in Figure 3.9D) was connected to each laser drivers to provide the voltage needed to turn on the laser driver. The schematic layout of the connection between the laser diodes, laser driver and power supply is shown in Figure 3.9C and the steps followed to operate the laser drivers are described below in section 3.3.1.2.1 (for EK 2000) and 3.3.1.2.2 (for DBDL M/Q).

\subsection{Operating EK 2000 laser drivers}

The steps followed to operate the EK 2000 laser drivers are described below: 
Step 1: The current limit and photo diode current trimpot is set to zero by turning the trimpot several times in the anti-clockwise direction.

Step 2: The EK 2000 board is powered using a 9V DC power supply as shown in Figure 3.9D.

Step 3: The jumper is installed on the pin P10 to run the laser in continuous mode.

Step 4: The current limit trimpot is adjusted slowly while observing the voltage across the limit out pin to set the maximum operating current for the laser diodes. The relationship between the voltage observed and the operating current is $40 \mathrm{~mA} / \mathrm{V}$ i.e. if the max current needed for the laser diode is $80 \mathrm{~mA}$ then the voltage was set at $2 \mathrm{~V}$.

Step 5: A calibrated power meter is used to monitor the laser diode output while the photodiode current set trimpot is turned clockwise to obtain the desired operating power level.

Step 6: The external voltage (described in section 3.3.3) source is connected to P10 to modulate the laser diode. For the EK2000, an external voltage of $0 \mathrm{~V}$ means fully on and $2.5 \mathrm{~V}$ mean fully off. Hence, the voltage is kept between $(0-2.5 \mathrm{~V})$.

\subsection{Operating DBDL M/Q laser drivers}

The steps followed to operate the EK 2000 laser drivers are described below:

Step 1: The limit current and set current potentiometer is set to 0 by turning the trimpot clockwise several times. 
Step 2: The limit current to be supplied is calculated by looking at the value of the slope efficiency of the laser diode (as obtained from the laser diode's specification sheet). The power is then turned on and the limit current is set to the calculated value.

Step 3: The limit current (blue color) potentiometer is set by monitoring the output voltage between GND and $\mathrm{I}_{\mathrm{Mon}}$ pin. The output voltage should not exceed $0.7 \mathrm{~V}$. For optimum working condition the voltage should be set at $0.6 \mathrm{~V}$.

Step 4: After setting the limit current, the set current potentiometer is turned clockwise to turn on the laser diode. Note: Once the limit current is set to the operating current it should not be changed and only the set current trimpot should be changed in order to change the optical power of the diode.

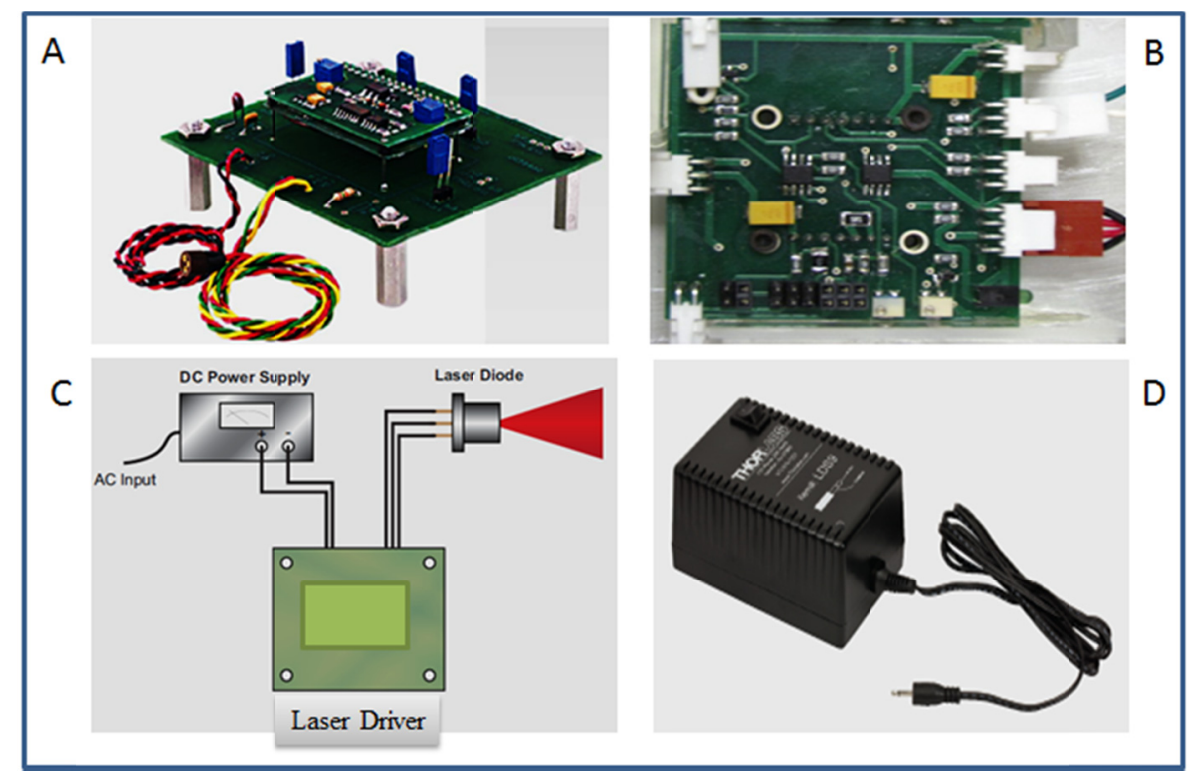

Figure 3.9: (A) EK 2000 laser driver to drive A pin style laser diodes. (B) $D B D L M / Q$ to drive $C$ type laser diodes. (C) Schematic diagram of working of laser drivers (figure adapted from manual of EK 2000 laser driver). (D) LDS2 9 V DC power supply from Thorlabs. 
Step 5: The external voltage (described in section 3.3.3) source is connected to $V_{\text {ext }}$ pin in order to modulate the laser diode. The external modulating voltage is supplied by following the relation $100 \mathrm{~mA} / \mathrm{V}$, which is dependent on the specification of the laser diode.

For EK 2000 laser driver, which is used to drive $830 \mathrm{~nm}$ laser diode, the operating voltage was set at $1.75 \mathrm{~V}$ and for DBDL laser driver, which is used to drive $690 \mathrm{~nm}$ laser diode, the operating voltage was set at $0.65 \mathrm{~V}$.

\subsubsection{Signal generator}

A NI PCIe-6341X Series Data Acquisition card (shown in Figure 3.10A) is used to generate digital pulse to modulate the two different wavelengths of laser diodes and also to synchronize the dual wavelength source system with the ICCD camera, as described in section 3.3.2. The NI PCIe-6341X Series Data Acquisition card is installed in one of the data card slots of the computer. A Labview supported NI-DAQmx driver software is also installed to drive the NI PCIe-6341X Series Data Acquisition card. The NI PCIe-6341X Series Data Acquisition card is connected to NI SHC68-68-EPM (shown in Figure 3.10 B) cable, which delivers the signal to a connector block NI BNC-2110 (shown in Figure $3.10 \mathrm{C})$ that has the output terminal for the desired signals. The flow of signal from the NI PCIe-6341X to the connector block NI BNC-2110 through NI SHC68-68-EPM cable is depicted by the directional arrow in Figure 3.10. 


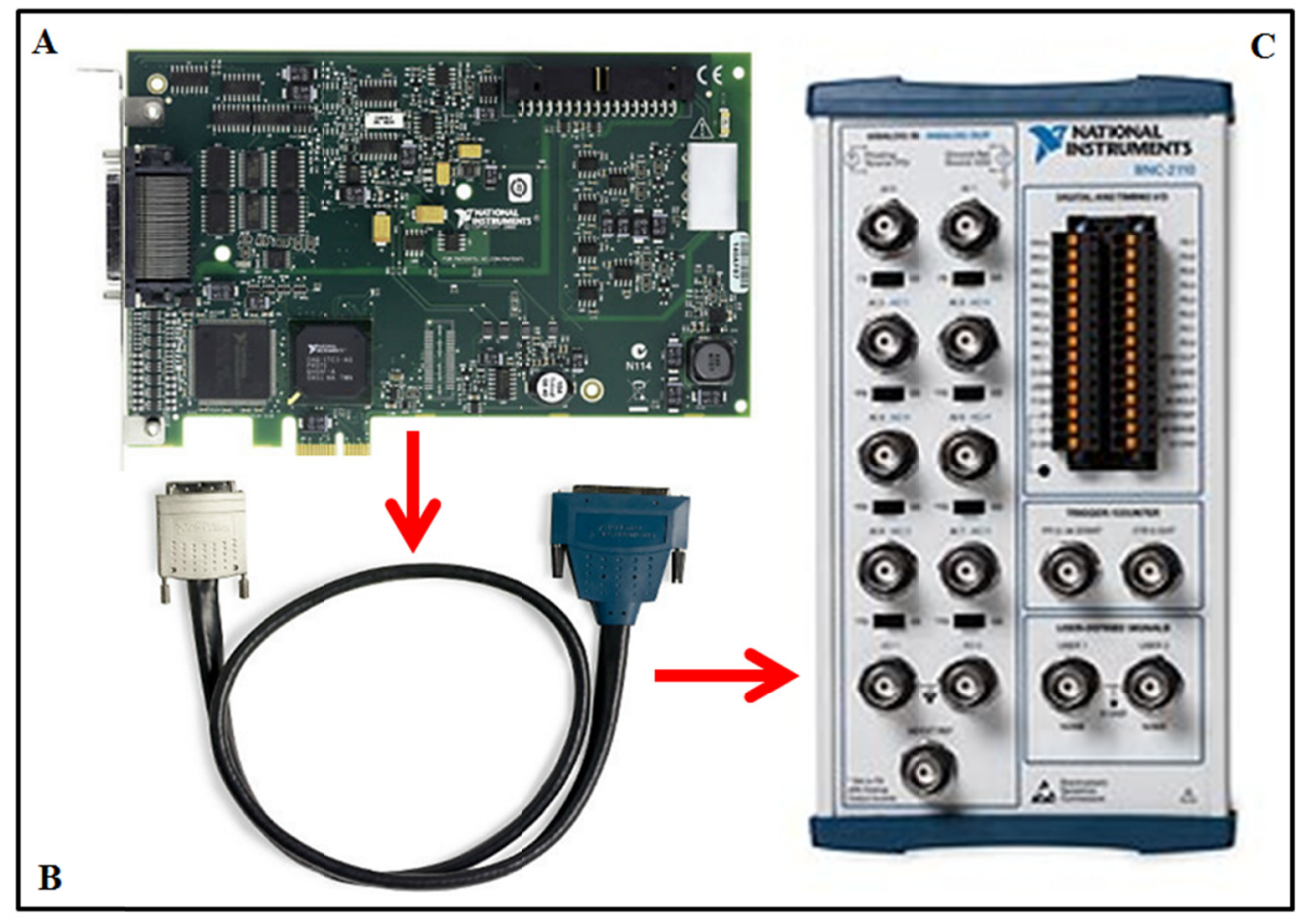

Figure 3.10: (A) NI PCIe-6341X Series Data Acquisition card, (B) NI SHC68-68-EPM cable and (C) NI BNC-2110 a shielded connector block with serves as the output terminal of the signal.

The graphical user interface to control the generation of signal by NI PCIe-6341X Series Data Acquisition card is a Labview based VI (virtual instrument). The VI used was developed by modifying and adapting the VI present in the online library of Labview. A screen shot of the VI used is shown in Figure 3.11, which depicts the input parameter needed to generate a required waveform. The yellow highlighted region (Figure 3.11A) shows a dropdown menu which help selects the number of output channels on the connector block. The green highlighted region (Figure 3.11B) shows the timing parameter which helps select the clock to generate the pulse. The blue highlighted region (Figure 3.11C) shows the buffer parameter which determines the sample size. The red highlighted region (Figure 3.11D) shows the waveform generated across the different 
channel. The black highlighted region (Figure 3.11E) determines the frequency of the waveform to be generated. Thus the VI has the provision to control the shape of the waveform, the number of waveforms, and the frequency of the waveform generated.

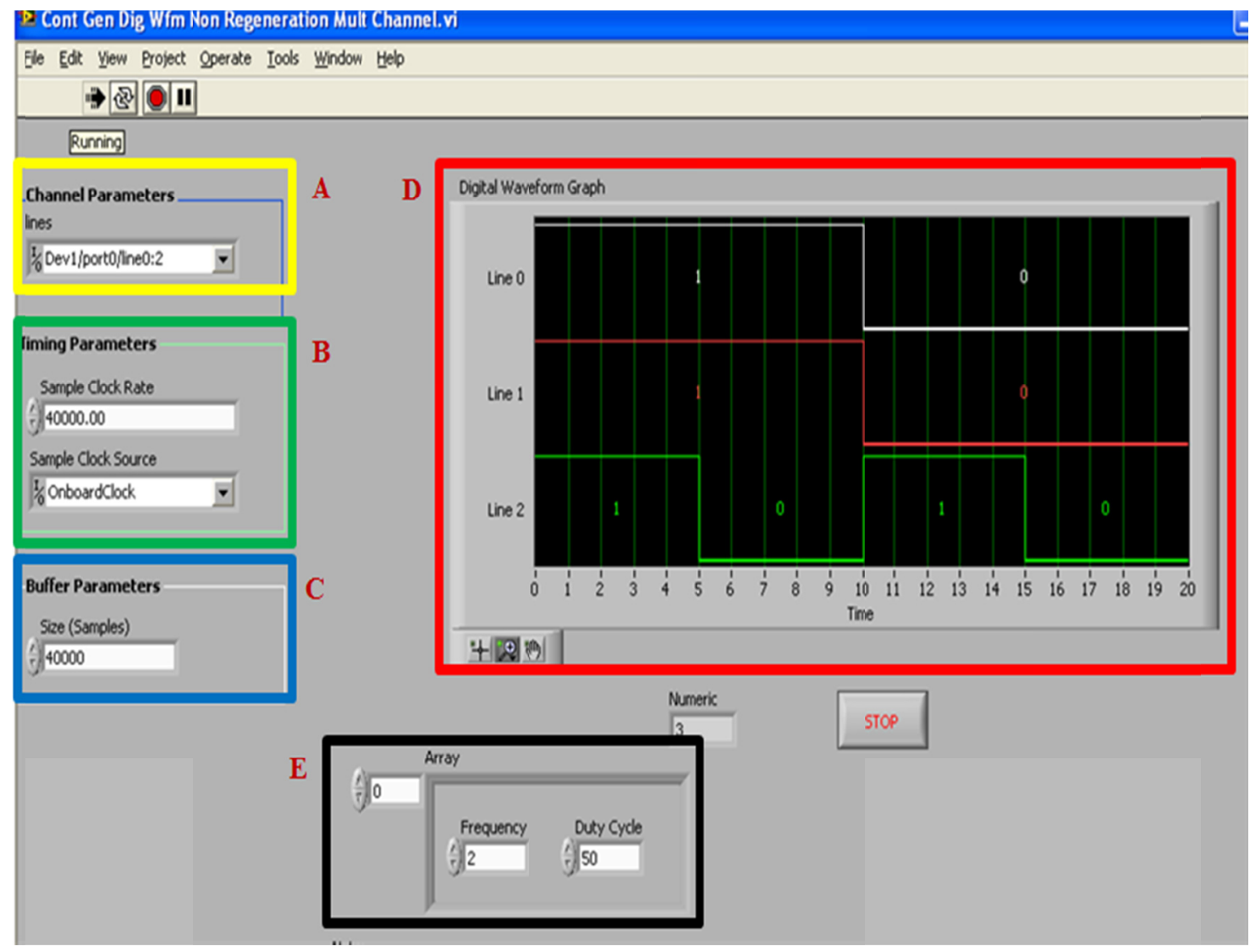

Figure 3.11: Labview interface showing the different input parameter, (A) Channel parameter, (B)Timing parameter, (C) Buffer parameters, (D) Generated waveform and (E) Array to set the frequency and the waveform shape.

\subsubsection{Assembling the source system}

Two metallic boxes of dimension $(40 \mathrm{~cm} \times 40 \mathrm{~cm} \times 10 \mathrm{~cm})$ were acquired to compactly assemble the components to develop the source system. The components acquired to develop the source system were four EK 2000 laser drivers, four DBDL M/Q laser drivers, signal generator, three $830 \mathrm{~nm}$ laser diode, three $690 \mathrm{~nm}$ laser diode, eight power 
supplies and female SMA connectors. The block diagram of the assembled source system is shown in Figure 3.12.

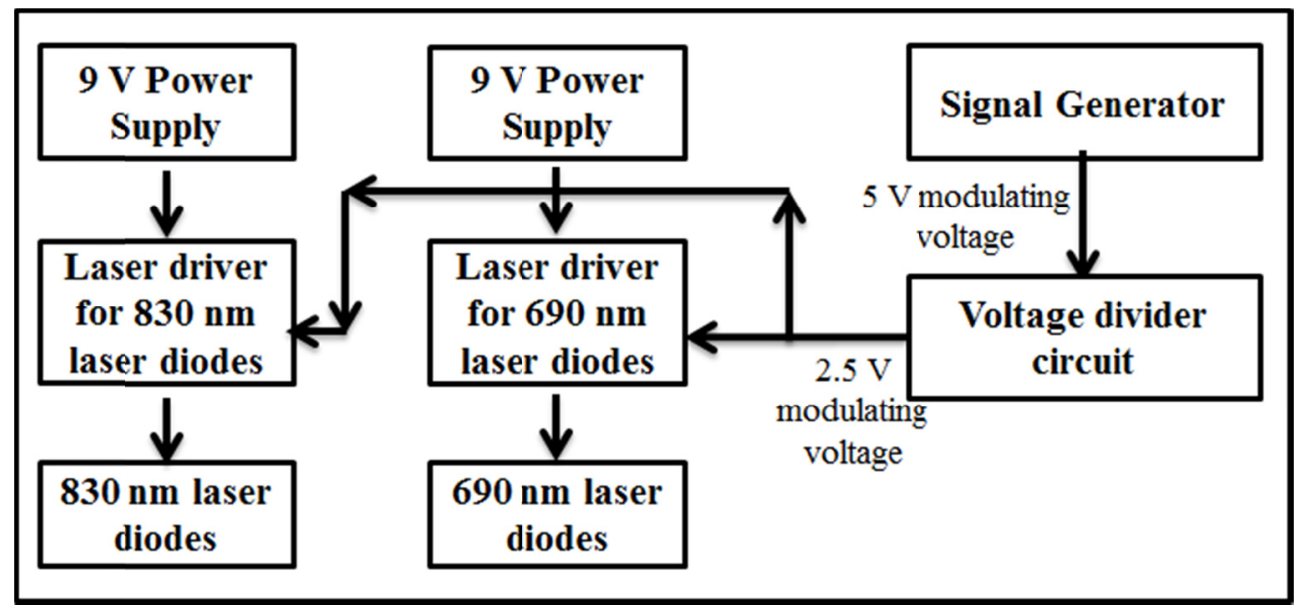

Figure 3.12: Block diagram of the dual wavelength source system.

One of the metallic box is made as a power module as shown in Figure 3.13, where in all the power supplies are kept to power the laser drivers.

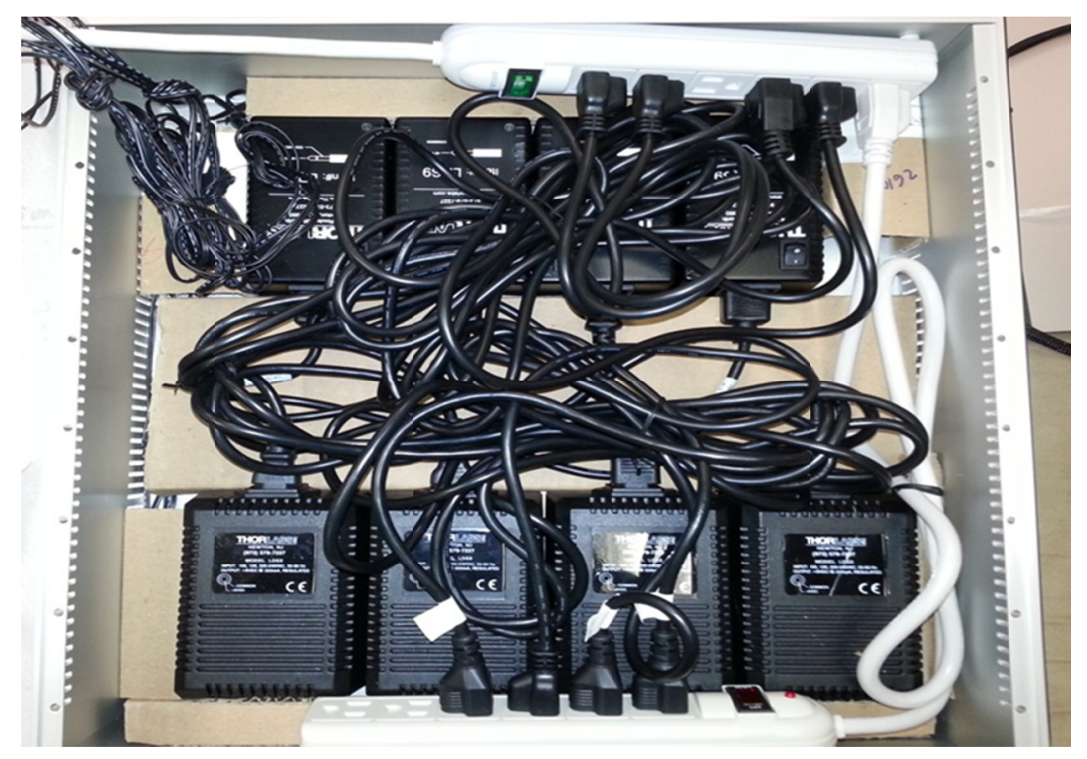

Figure 3.13: The power supply module with eight $9 \mathrm{~V} D C$ power supplies to power eight laser driver boards. 
All the power supplies are connected to an extension cable which is powered through regular wall power supply. The other metallic box is made as the laser source module which consists of set of front panel (shown in Figure 3.14A), four laser drivers for 830 $\mathrm{nm}$ laser diodes (shown in Figure 3.14B), four laser drivers for $690 \mathrm{~nm}$ laser diodes(shown in Figure 3.14C), and the signal generator (shown in Figure 3.14 D).

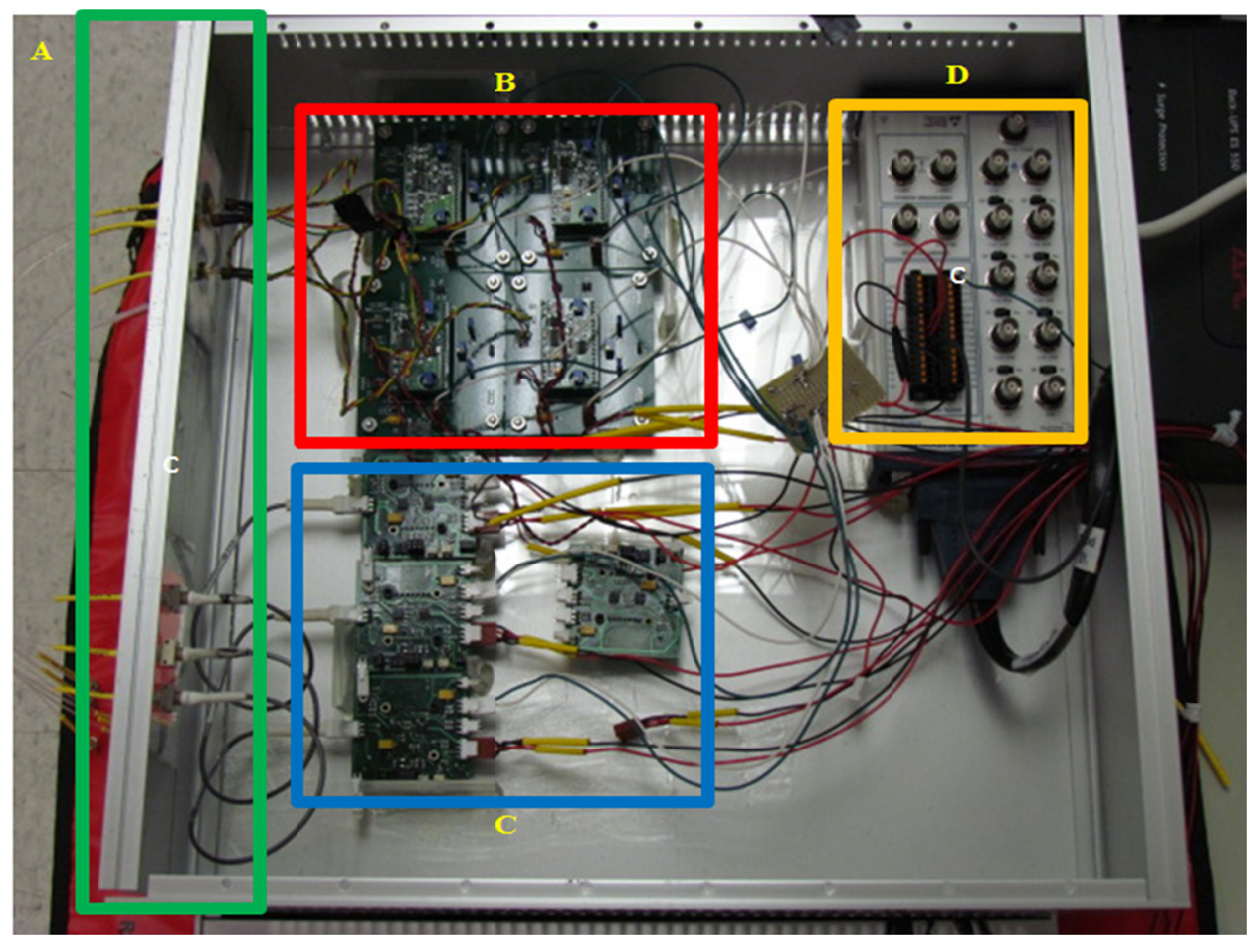

Figure 3.14: The assembled dual wavelength source system. (A) The front panel, (B) Set of four Laser drivers for $830 \mathrm{~nm}$ laser diodes, (C) Set of four Laser drivers for $690 \mathrm{~nm}$ laser diodes and (D) The signal generator.

The front panel, shown in Figure 3.15, consists of female SMA connectors to optically couple the laser diodes with the source fiber. The red region (shown in Figure 3.15A) shows the set of three female SMA connectors to optically couple the $830 \mathrm{~nm}$ laser diode to the optical fiber. The yellow region (shown in Figure 3.15B) shows the set of three 
female SMA connectors to optically couple the $690 \mathrm{~nm}$ laser diode to the optical fiber. The laser drivers for $830 \mathrm{~nm}$ and $690 \mathrm{~nm}$ laser diodes are operated as described in section 3.3.1.2.1 and 3.3.1.2.2, respectively. The laser drivers are modulated by supplying the external modulating voltage from the signal generator. Thus the dual wavelength source system was assembled and the finally developed and assembled dual wavelength source system is shown in Figure 3.16.

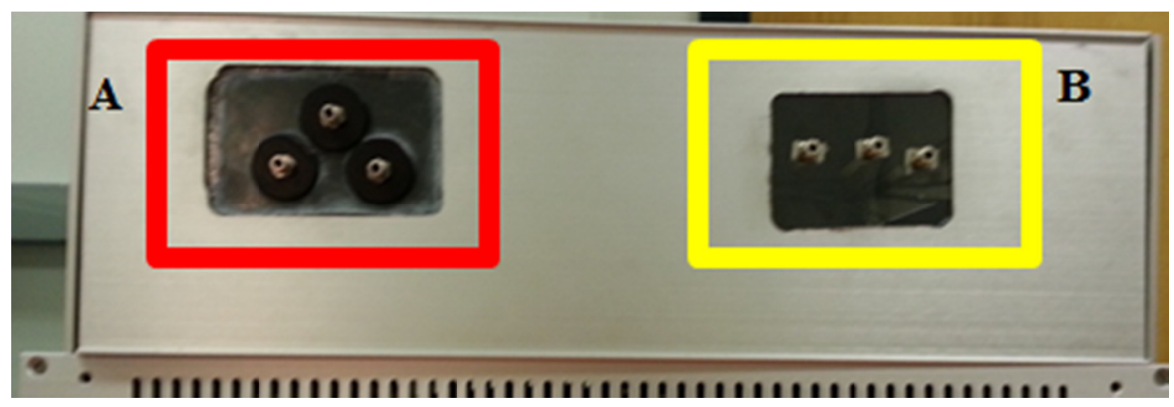

Figure 3.15: The front panel with female SMA connectors to optically couple (A) $830 \mathrm{~nm}$ and (B) $690 \mathrm{~nm}$ laser diodes to optical fiber.

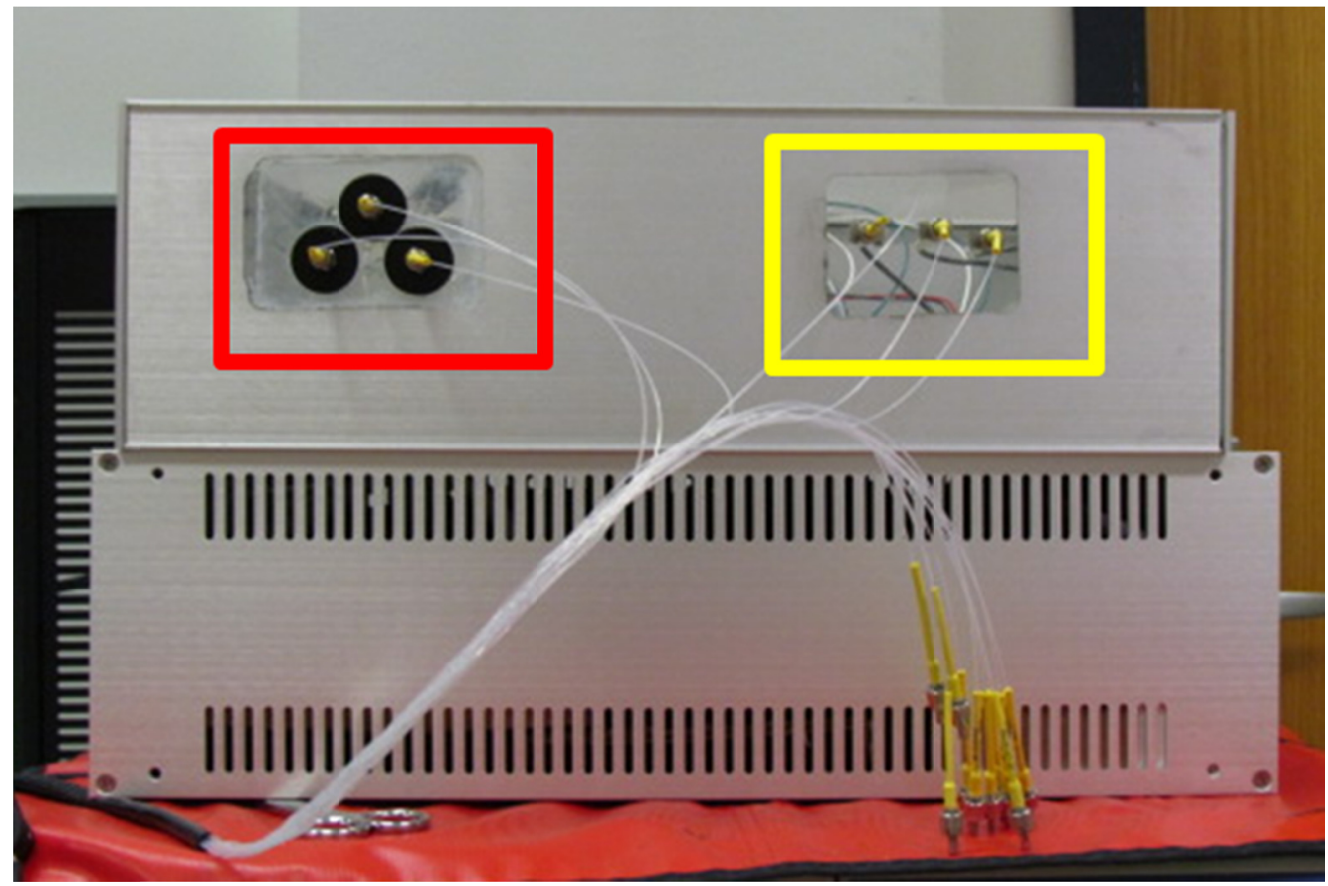

Figure 3.16: The fully assembled dual wavelength source system. The bottom section is the power section and the top section is the laser systems section. 


\subsubsection{Detector system}

The same PI-Max II ICCD camera from Princeton Instruments described in section 3.2.2, is used to develop the dual wavelength NIRS system. The only difference lies in the mode in which the camera is run, as shown in Figure 3.17. In the dual wavelength NIRS system two different wavelength of light modulate at a frequency set by the signal generator described in section 3.3.1.3. Hence the camera should also be modulated at a rate such that it turns on and off to acquire the optical signal from the two different wavelengths of light. The ICCD camera is turned on and off in sync with the turning on and off the two different sources by using the external sync mode of the camera. The time mode of the camera is set as external sync at the Winview interface of the camera in order to run the ICCD camera in external sync mode (as shown in Figure 3.17).

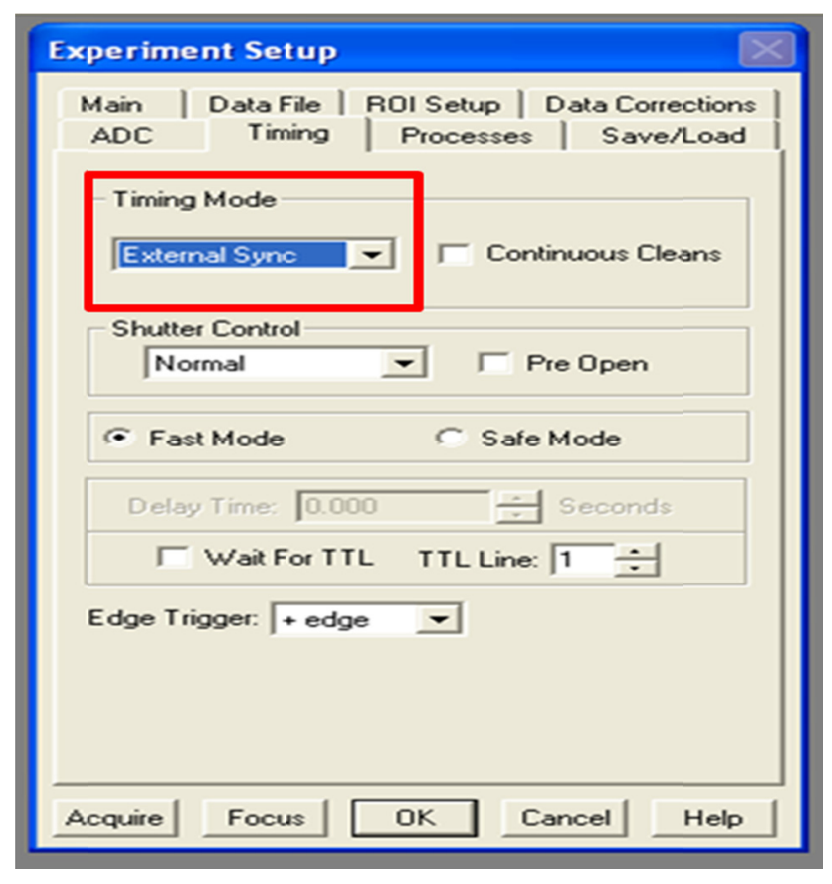

Figure 3.17: Winview interface showing the mode in which ICCD camera is run, the region highlighted in red is a drop down menu which enables selecting different timing mode. The dual wavelength ICCD based NIRS system is run in External sync mode as shown in the highlighted red area. 


\subsubsection{Integration of source and detector systems}

The dual wavelength source system is synchronized with the ICCD camera so that they turn on and off at the same time. The synchronization is necessary to correctly monitor the change in hemodynamic response of the tissue. In order to synchronize the source system with the ICCD camera system a trigger signal generated by the signal generator is used (as described in section 3.3.3.3). Various methods were attempted to synchronize the source system with the camera system before finalizing one method. These methods are described, in section 3.3.3.1, 3.3.3.2 and 3.3.3.3, below.

\subsubsection{Click Automation method}

In this method, a click automation software was used to synchronize the source system and the camera system. Click automation software belong to the class of the software which are being used to automatically click/perform multiple task at the same time. Hence this software was used to click the start button of both the source system and the camera system at the same time. The software claims to performs multiple clicks at the same it but its performance is computer dependent, slower the computer greater the delay time between two clicks and vice versa. Hence this was not a reliable method, and it was also observed that there was a delay time between two clicks which was difficult to

quantify. Thus this leads to the delay between the turning $\mathrm{ON}$ of the camera and the source system, thus defeating the purpose of syncing the two systems. 


\subsubsection{External trigger through USB based signal generator}

In this method, a USB based signal generator which uses the computer clock to generate waveform was used to provide external trigger to the camera in order to sync the source and camera system. By using this USB based signal generator the source system was synchronized with the camera system by running the camera in external trigger mode, as described in section 3.3.2. Whenever the camera saw the rising edge of the voltage it turned $\mathrm{ON}$ for the specified time and also one of the laser source was turned $\mathrm{ON}$ and other OFF and so on. The main limitation of this method was that it was unable to control multiple laser sources at the same time.

\subsubsection{Signal generator}

In this method external trigger mode of the ICCD camera is used, as described in section 3.3.2, to synchronize the source system with the camera system. A 5V DC signal generated by the signal generator described in section 3.3.3 is supplied to the external trigger terminal of the ICCD camera. The same signal generator supplies the required 2.5 $\mathrm{V}$ external modulating voltage to the laser diode drivers. In order to acquire the optical signals, the ICCD camera is set in external trigger acquire mode as shown in Figure 3.17 and the labview module is turned ON, as shown in Figure 3.11. The labview supplies the precise trigger signal to synchronize both the source and the camera system. The synchronized system was run several times to check the time delay and synchronization error and it was determined that there was no time delay or synchronization error. Also one of the major advantage of this method is that it can be used synchronize several channels of source system with the camera system. Hence in the finally developed NIRS 
system the external trigger mode of the ICCD camera is used to synchronize with the source system via an external trigger signal generated by the signal generator. The tests performed to check the modulation of two different wavelengths of laser diodes and its synchronization with the camera system is described in the following section (section $3.4)$.

\subsection{Testing of dual wavelength NIRS system}

The synchronized source and camera system was tested for the modulation of the laser diodes sources and its integration with the camera system by running a series of experiments. In the experiments, two source and four detector fibers (optodes) were connected to the source and camera system, respectively. The source and detector optodes were connected to a square probe with pin hole to snap in the tip of source and detector optodes. The distance between the source and detector optodes was kept at 3 $\mathrm{cm}$. The probe was placed on a wooden platform by maintaining a distance of $3 \mathrm{~cm}$ between the probe and the wooden platform. During the experiment the frequency of modulation of source system was set as $2 \mathrm{~Hz}$ and the acquisition rate of camera was set as $4 \mathrm{~Hz}$. The optical power of the $830 \mathrm{~nm}$ and $690 \mathrm{~nm}$ wavelength of light was tuned to 3.5 $\mathrm{mW}$ and the gain of the ICCD camera was set to $30(\mathrm{abu})$. The source and camera system is synchronized in such a way that the odd and even frame of optical data acquired by the ICCD camera corresponds to $690 \mathrm{~nm}$ and $830 \mathrm{~nm}$ wavelength of light, respectively. Three different experiments were performed (as described below in sections 3.4.1, 3.4.2 and 3.4.3) by turning one wavelength of light OFF and other ON and so on. The logic for this study was that if the source and camera systems had been synced properly then low 
signal will be seen in the alternate frame, for the source which was turned OFF, and high signal will be seen in the alternate frame, for the source which was turned ON. During the experiments optical signals were acquired by the ICCD camera for a total of $5 \mathrm{~min}$ and all the three experiments were repeated 5 times. Finally the change in intensity acquired by the ICCD camera was plotted as stem plot to depict the change in intensity of the acquired optical signal (as described in the sections below).

\subsubsection{Modulating $690 \mathrm{~nm}$ wavelength of light keeping $830 \mathrm{~nm}$ wavelength of light OFF} In this experiment $830 \mathrm{~nm}$ wavelength of light was turned OFF and only $690 \mathrm{~nm}$ wavelength of light was modulated at a frequency of $2 \mathrm{~Hz}$. The hypothesis for this experiment is that when $830 \mathrm{~nm}$ wavelength of light is turned OFF and only $690 \mathrm{~nm}$ wavelength of light is modulated at a frequency of $2 \mathrm{~Hz}$, the stem plot should show higher intensity of light at odd frames and low or no intensity of light at even frame. The stem plot corresponding to the change in intensity acquired during the experiment from the four different detector fiber bundle shows the same pattern. Hence, here the stem plot corresponding to one of the detector fiber bundle is shown in Figure 3.18. The Figure 3.18 shows higher intensity of light at odd frame and less or no intensity of light at even frames, thereby validating the turning ON and OFF $690 \mathrm{~nm}$ of light while the $830 \mathrm{~nm}$ was turned OFF. It is also evident from Figure 3.18 that the intensity of light acquired was very stable throughout the period of acquisition. The experiment was repeated five times to check the stability of modulation and data acquisition by the ICCD camera. The repeated studies gave similar results thereby confirming the precision in modulation of light source and data acquisition by ICCD camera as well as the stability of the system. 


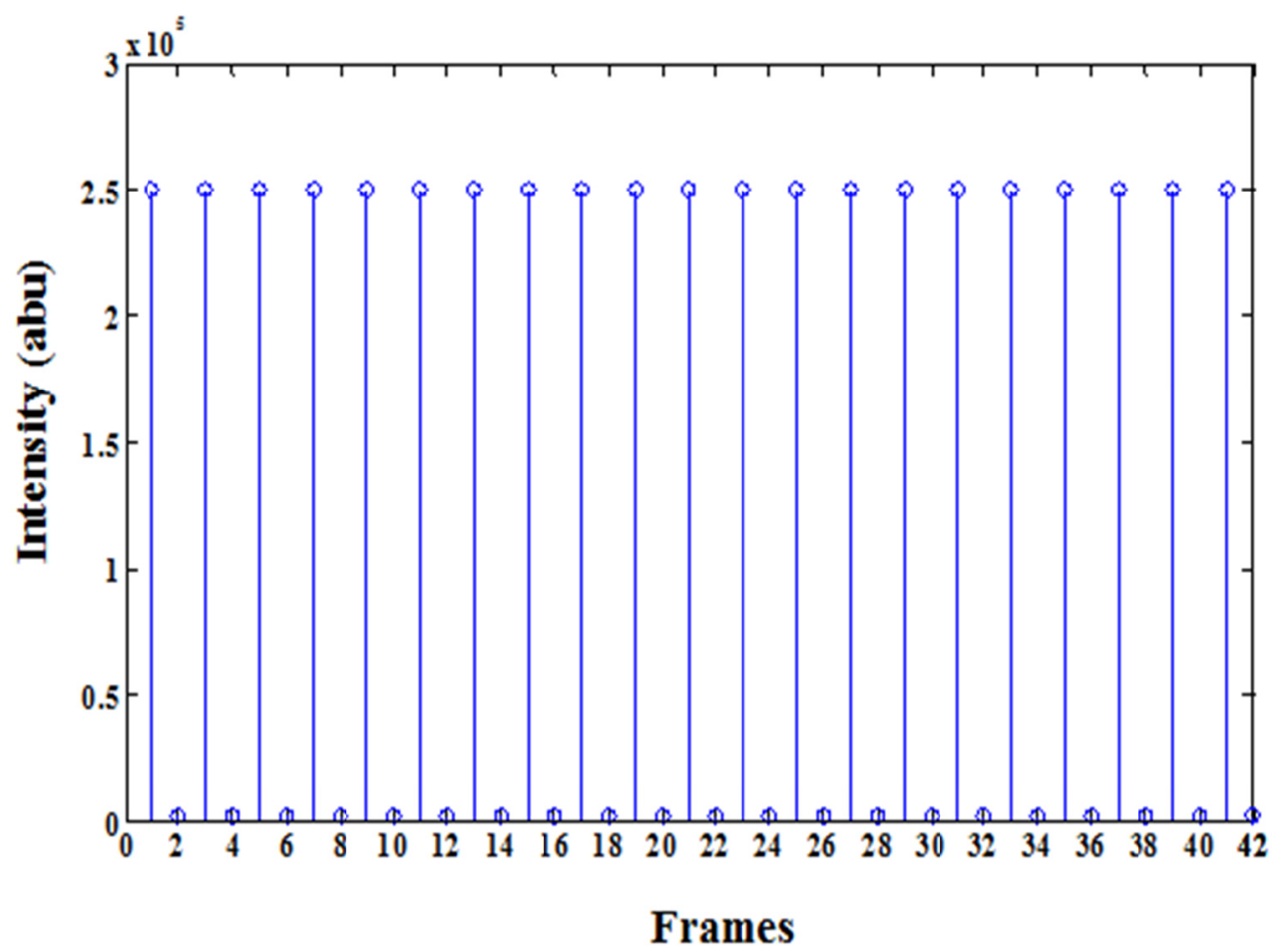

Figure 3.18: Stem plot of the intensity acquired by the ICCD camera with $830 \mathrm{~nm}$ sources OFF and $690 \mathrm{~nm}$ sources ON. Here, the odd frames correspond to the $690 \mathrm{~nm}$ wavelength of light and even frames correspond to the $830 \mathrm{~nm}$ wavelength of light.

\subsubsection{Modulating $830 \mathrm{~nm}$ wavelength of light keeping $690 \mathrm{~nm}$ wavelength of light OFF}

In this experiment $690 \mathrm{~nm}$ wavelength of light was turned OFF and only $830 \mathrm{~nm}$ wavelength of light was modulated at a frequency of $2 \mathrm{~Hz}$. The hypothesis for this experiment is that when $690 \mathrm{~nm}$ wavelength of light is turned OFF and only $830 \mathrm{~nm}$ wavelength of light is modulated at a frequency of $2 \mathrm{~Hz}$, the stem plot should show higher intensity of light at even frames and low or no intensity of light at odd frames. The stem plot corresponding to the changes in intensity acquired during the experiment from the four different detector fiber bundle shows the same pattern. Hence, here the stem plot corresponding to one of the detector fiber bundle is shown in Figure 3.19. The Figure 
3.19 shows higher intensity of light at even frame and less or no intensity of light at odd frame, thereby validating the turning ON and OFF $830 \mathrm{~nm}$ of light while the $690 \mathrm{~nm}$ was turned OFF.

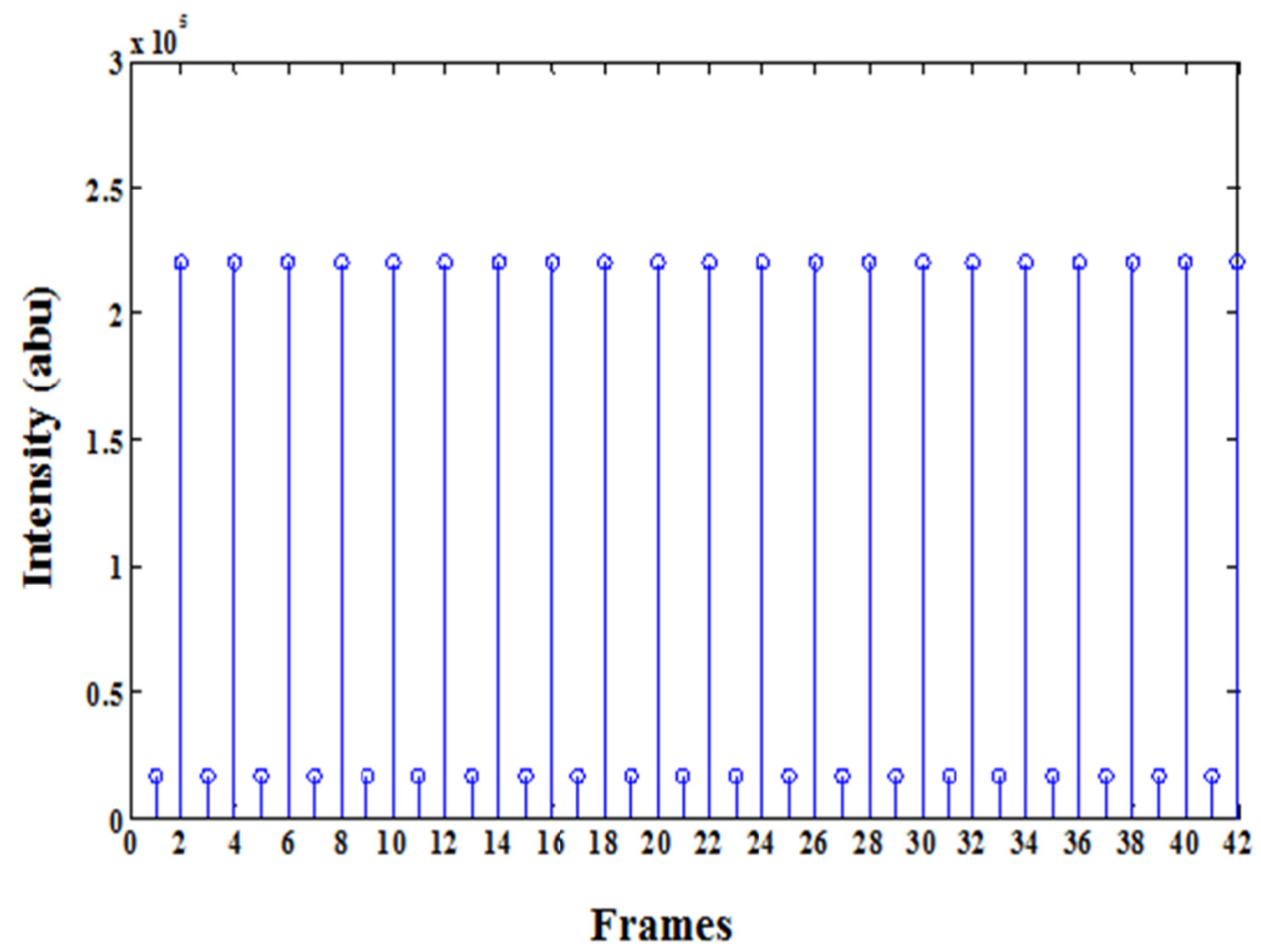

Figure 3.19: Stem plot of the intensity acquired by the ICCD camera with $830 \mathrm{~nm}$ sources $O N$ and $690 \mathrm{~nm}$ sources OFF. Here, the odd frames correspond to the $690 \mathrm{~nm}$ wavelength of light and even frames correspond to the $830 \mathrm{~nm}$ wavelength of light.

It is also evident from Figure 3.19 that the intensity of light acquired was very stable throughout the period of acquisition. The experiment was repeated five times to check the stability of modulation and data acquisition by the ICCD camera. The repeated studies gave similar results thereby confirming the precision in modulation of light source and data acquisition by ICCD camera as well as the stability of the system. 


\subsubsection{Modulating both $830 \mathrm{~nm}$ and $690 \mathrm{~nm}$ wavelength of light}

In this experiment $830 \mathrm{~nm}$ wavelength of light and $690 \mathrm{~nm}$ wavelength of light were modulated at a frequency of $2 \mathrm{~Hz}$. The hypothesis for this experiment is that the stem plot of the modulating $830 \mathrm{~nm}$ and $690 \mathrm{~nm}$ wavelength of light should be the sum of stem plot obtained from experiment described in section 3.4 .1 and 3.4.2. The stem plot corresponding to change in intensity acquired during this experiment from the four different detector fiber bundle shows the same pattern. Hence, the stem plot corresponding to one of the detector fiber bundle is shown in Figure 3.20. In the Figure 3.20 , the odd frame corresponds to change in intensity acquired to $690 \mathrm{~nm}$ and the even frame corresponds to the $830 \mathrm{~nm}$ wavelength of light.

The stem plot shown in Figure 3.20 is the sum of the stem plot shown in Figure 3.18 and 3.19 thereby confirming the modulation of light source. It is also evident from Figure 3.20 that the intensity of light acquired was very stable throughout the period of acquisition. The experiment was repeated five times to check the stability of modulation and data acquisition by the ICCD camera. The repeated studies gave similar results thereby confirming the precision in modulation of light source and data acquisition by ICCD camera as well as the stability of the system. 


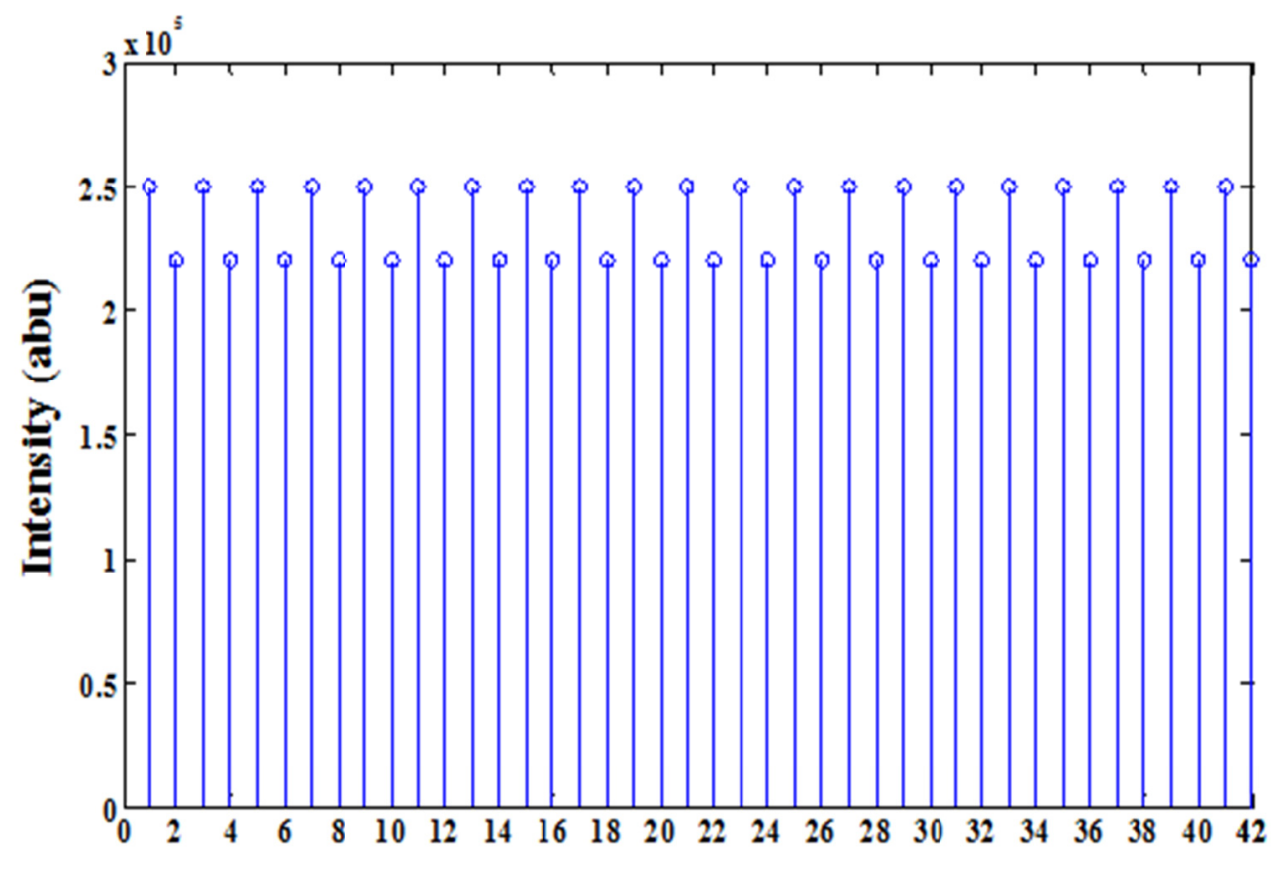

Frames

Figure 3.20: Stem plot of the intensity acquired by the ICCD camera with alternating 830 $\mathrm{nm}$ sources and $690 \mathrm{~nm}$ sources. Here, the odd frames correspond to the $690 \mathrm{~nm}$ wavelength of light and even frames correspond to the $830 \mathrm{~nm}$ wavelength of light.

Thus the experimental tests performed (as described in this section) proved the integration of source and detector systems, precise modulation of two different sources, and reliable optical signal acquisition by the ICCD camera as well as stability of the developed NIRS system. The developed dual wavelength NIRS system was used to perform different NIRS studies of the brain (as described in chapter 12). 


\section{CHAPTER 4. Near Infrared Spectroscopy Data Analysis}

The NIRS instruments described in Chapter 3 acquires the attenuated light as it exits the head after travelling through the cortical regions of the brain. The acquired attenuated light intensity contains information about the change in the hemodynamics in the region under investigation in response to stimuli or during rest. The different NIRS instruments described in chapter 3 acquires NIRS data in different formats due to the difference in instrumentation. In this chapter the format of data acquired by three different NIRS instrument described in chapter 3 and the data processing to elucidate the activation, functional connectivity, effective connectivity and lateralization have been described.

\subsection{Extraction and format of NIRS data from Imagent}

The commercial NIRS instrument (Imagent, ISS), described in section 3.1, is a frequency domain based system, which provides both the change in intensity and phase shift of the attenuated light, as it exits the head. The Imagent acquires data across the specified number of source and detector pairs (channels) used during the study and save it in a text file using the Boxy software of Imagent. The text file contains the change in intensity (DC), frequency (AC) and phase shift information with respect to time across the channels used during the study. The text file (.txt) is converted to Matlab file (.MAT) by using a program called Boxy2Homer to separate the $\mathrm{DC}, \mathrm{AC}$ and phase shift information for each channels with respect to time. During my doctoral work the change in intensity (DC) was primarily used to elucidate the activation, functional connectivity, effective connectivity and lateralization as described in section $4.3,4.4,4.5$ and 4.6 respectively. 
The phase shift data was also used to elucidate the change in relative phase shift and phase dependent connectivity as described in chapter 7.

4.2 Extraction and format of NIRS data from in-house developed single wavelength based NIRS system

The in-house developed single wavelength based NIRS system, described in section 3.2, is a continuous wave based system which acquires change in intensity of single wavelength of light via winView software. The winView software is used to control the ICCD camera which collects the light from the detector fiber bundle. The detector fiber bundle is coupled with ICCD camera via a C-mount which is seen as a bright spot of light encompassing particular area of pixels, specified by the diameter of detector fiber bundle, on the face of ICCD camera. The attenuated light intensity acquired by the ICCD camera corresponding to the number of detector fiber bundles coupled to the face of ICCD camera is saved as .SPE file. The .SPE file is converted to a .MAT file using a program named RoperASCII2MAT. The change in intensity data stored in .MAT file is then processed to elucidate relative change in total hemoglobin $(\mathrm{HbT})$, functional connectivity and lateralization as described in section 4.3, 4.4and 4.6 respectively.

\subsection{Extraction and format of NIRS data from in-house developed dual wavelength} based NIRS system

The in-house developed dual wavelength based NIRS system, described in section 3.3, is a continuous wave based system which acquires change in intensity at dual wavelength of light via WinView software. The ICCD camera acquires the change in intensity associated with the two different wavelengths of light in such a way that the odd frames 
corresponds to the $690 \mathrm{~nm}$ wavelength of light and even frames corresponds to $830 \mathrm{~nm}$ of light. Thus the data acquired and saved in .SPE format, as described in section 4.2, contains both the change in intensity associated with $830 \mathrm{~nm}$ and $690 \mathrm{~nm}$ wavelengths of light. The extracted change in intensity data from Imagent and in-house developed single or dual wavelength NIRS system was used to calculate the change in concentration of $\mathrm{HbO}, \mathrm{HbR}$ and $\mathrm{HbT}$ using modified Beer Lambert law as described below.

The modified Beer Lambert law (described in section 2.1.2.1), provides the change in optical density of wavelength of light $\lambda$ when it travels through a media containing $C$ concentration of light absorbing species. In the NIR range of light the dominant chromophores in the brain, as described in section 2.1.1, are $\mathrm{HbO}$ and HbR. Thus to quantify the change in concentration of $\mathrm{HbO}$ and $\mathrm{HbR}, 830 \mathrm{~nm}$ and $690 \mathrm{~nm}$ wavelength of light is used because of the maximum sensitivity of attenuation profile of $\mathrm{HbO}$ and $\mathrm{HbR}$ at these two wavelengths, respectively (described in section 2.1.1). The Eq. 2.7 of modified Beer Lambert law is used to calculate the change in optical density at $830 \mathrm{~nm}$ and $690 \mathrm{~nm}$ of light using Eq. 2.3 to 2.7, as described in section 2.1.2.1.

The change in intensity data from in-house developed single wavelength NIRS system was used to calculate the relative change in concentration of $\mathrm{HbT}$ using modified Beer Lambert. The in-house developed single wavelength based NIRS utilizes $785 \mathrm{~nm}$ of light which is close to isosbestic point (Chance at al., 1988) (described in section 2.1) thereby providing the information about the relative change in total hemoglobin $(\mathrm{HbT})$ as shown in Eq. 4.1.

$\Delta O D_{\text {Isosbestic point }}=\Delta[H b T]$

Eq. 4.1 
where in Eq. 4.1, $\triangle O D_{\text {Isosbestic point }}$ is the change in optical density calculated using modified Beer Lambert law at wavelength of light close to isosbestic point and $\Delta[\mathrm{HbT}]$ is change in total hemoglobin concentration. The Eq. 4.1 provides change in concentration of $\mathrm{HbT}$ without giving any information about oxygen saturation of blood in the brain.

This change in $\mathrm{HbO}, \mathrm{HbR}$ and $\mathrm{HbT}$ is used to elucidate the change in activation (described in section 4.4), functional connectivity (described in section 4.5), effective connectivity (described in section 4.6) and lateralization (described in section 4.7) in the brain..

\subsection{Activation analysis}

Activation analysis (as defined in section 2.3.1) is performed to investigate the change in relative concentration of $\mathrm{HbO}, \mathrm{HbR}$ and total hemoglobin $\mathrm{HbT}$ (sum of $\mathrm{HbO}$ and $\mathrm{HbR}$ ) as a function of time, in response to the stimulus being processed by the underlying cortical region. During my doctoral work, as described in chapter 3, two different NIRS systems (commercial system- Imagent and In-house developed system) were used to perform NIRS of brain. The optical data acquired using the commercial NIRS system (Imagent), as described in section 3.1, was processed using a HomER (Huppert et al., 2009) (Hemodynamic Evoked Response) software to obtain change in $\mathrm{HbO}$, $\mathrm{HbR}$ and $\mathrm{HbT}$, developed in the Photon Migration Lab (PMI) at Massachusetts General Hospital (MGH, Harvard, http://www.nmr.mgh.harvard.edu/PMI) (Huppert et al., 2009). While, the optical data acquired using in-house developed system was processed using custom code developed in Matlab. The change in intensity data acquired from the in-house developed single wavelength $(785 \mathrm{~nm})$ NIRS system is processed until the calculation of change in 
optical density that corresponds to the relative change in $\mathrm{HbT}$. While, the change in intensity data acquired from the in-house developed dual wavelength (690 and $830 \mathrm{~nm}$ ) NIRS system is processed to calculate the change in $\mathrm{HbO}, \mathrm{HbR}$ and $\mathrm{HbT}$. Thus, regardless of the difference in the format of optical data obtained from two different NIRS system, the steps followed to process the optical data acquired was similar.

The steps followed to convert the optical signals (intensity) to $\mathrm{HbO}, \mathrm{HbR}$ and $\mathrm{HbT}$ are shown below:

Step 1: The raw optical data acquired at $830 \mathrm{~nm}$ (as shown in Figure 4.1) and $690 \mathrm{~nm}$ (as shown in Figure 4.2) is filtered using a band pass filter $(0.3 \mathrm{~Hz}-0.0016 \mathrm{~Hz})$ to remove the signals arising from systemic physiology. The optical signals acquired from the surface of head is contaminated by systemic signals like heartbeat, respiratory signal and others as tabulated in Table 4.1 (Boas \& Franceschini, 2004). Hence there is a need to filter the hemodynamic optical signal of this contaminated signal which is done by employing a band pass filter of $0.3 \mathrm{~Hz}-0.0016 \mathrm{~Hz}$ (Boas \& Franceschini, 2004) based on the frequency range of other signals as shown in Table 4.1 (Boas \& Franceschini, 2004).. The filtered optical data acquired at $830 \mathrm{~nm}$ and $690 \mathrm{~nm}$ is shown in Figure 4.3 and 4.4 respectively. 


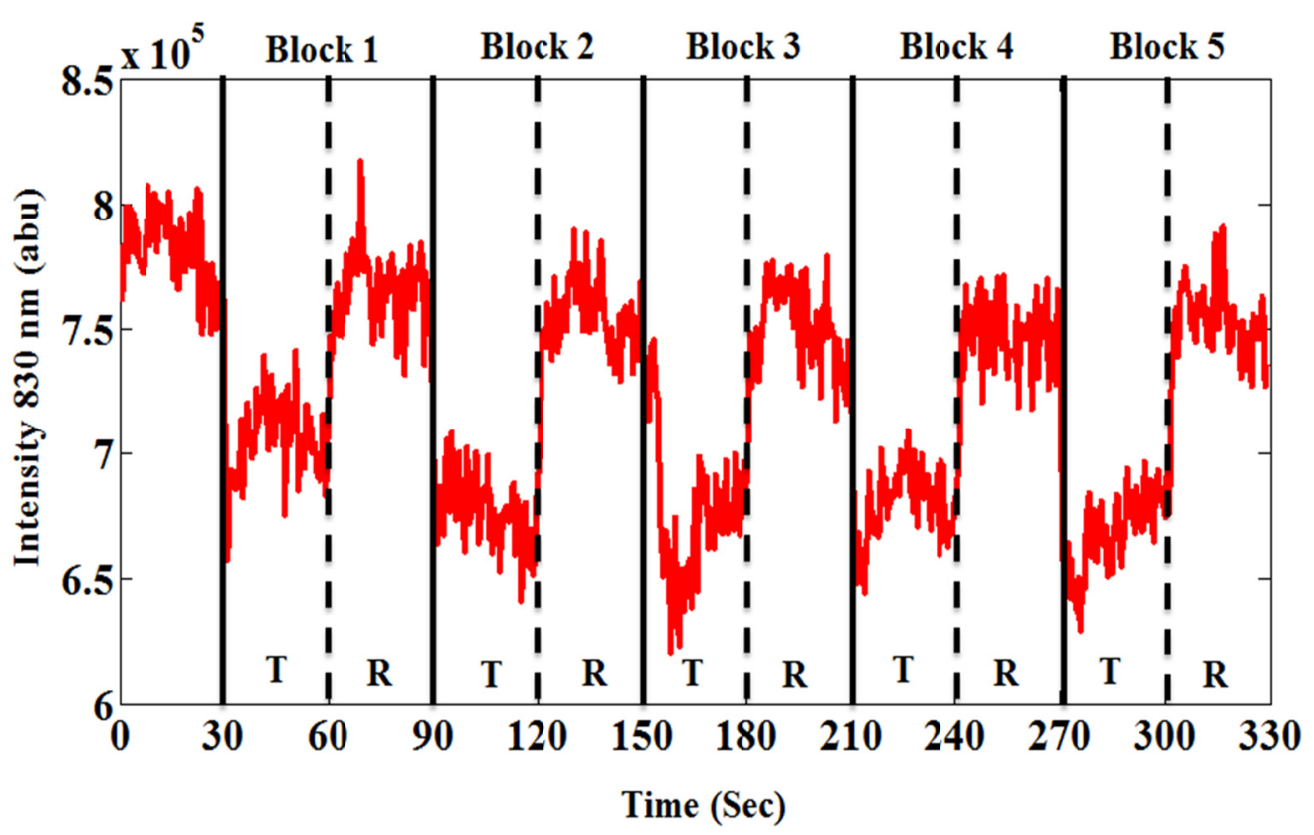

Figure 4.1: Intensity at $830 \mathrm{~nm}$ (in arbitrary units, abu), acquired using in-house developed system (described in section 3.3), plotted against time (in seconds). The figure depicts the change in the intensity at $830 \mathrm{~nm}$ across the five blocks of task $(T)$ and rest (R) period.

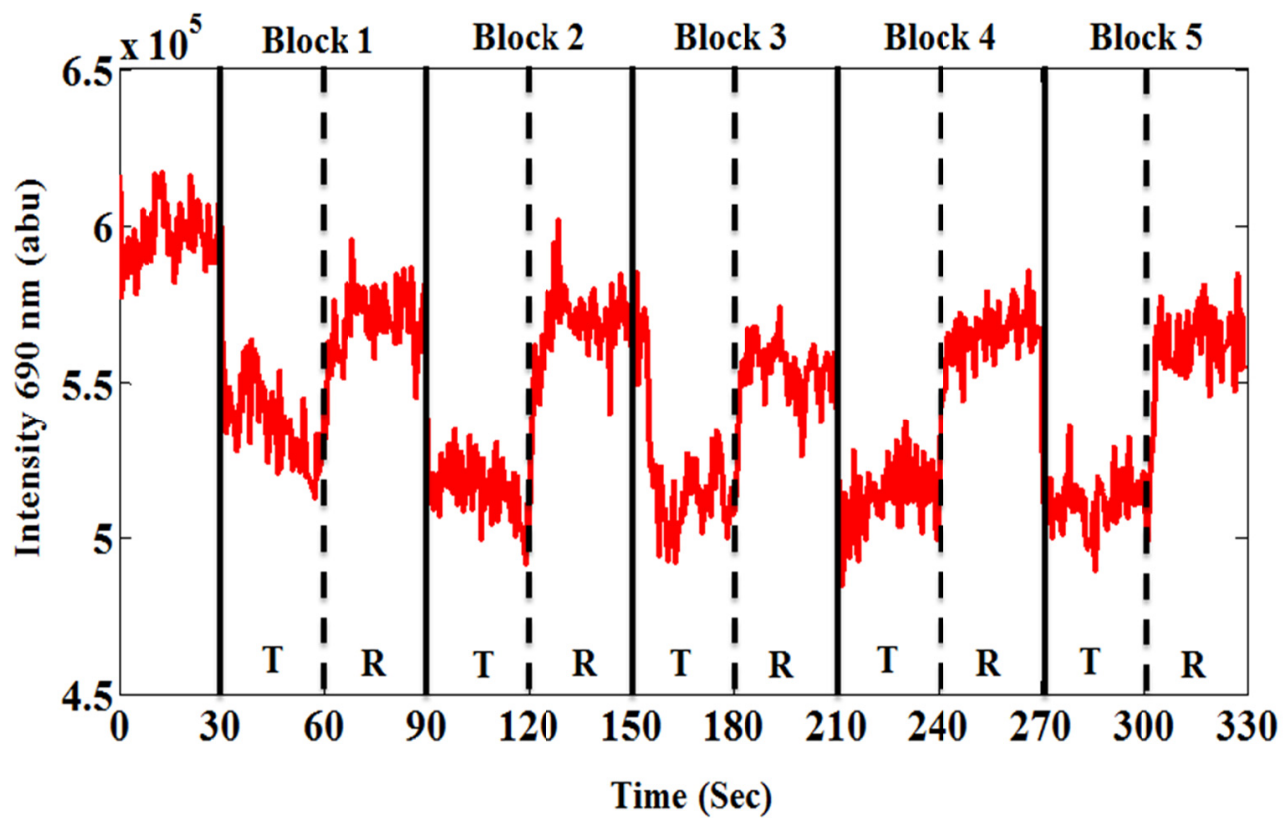

Figure 4.2: Intensity at $690 \mathrm{~nm}$ ((in arbitrary units, abu), acquired using in-house developed system (described in section 3.3), plotted against time (in seconds). The figure depicts the change in the intensity at $690 \mathrm{~nm}$ across the five blocks of task (T) and rest (R) period. 


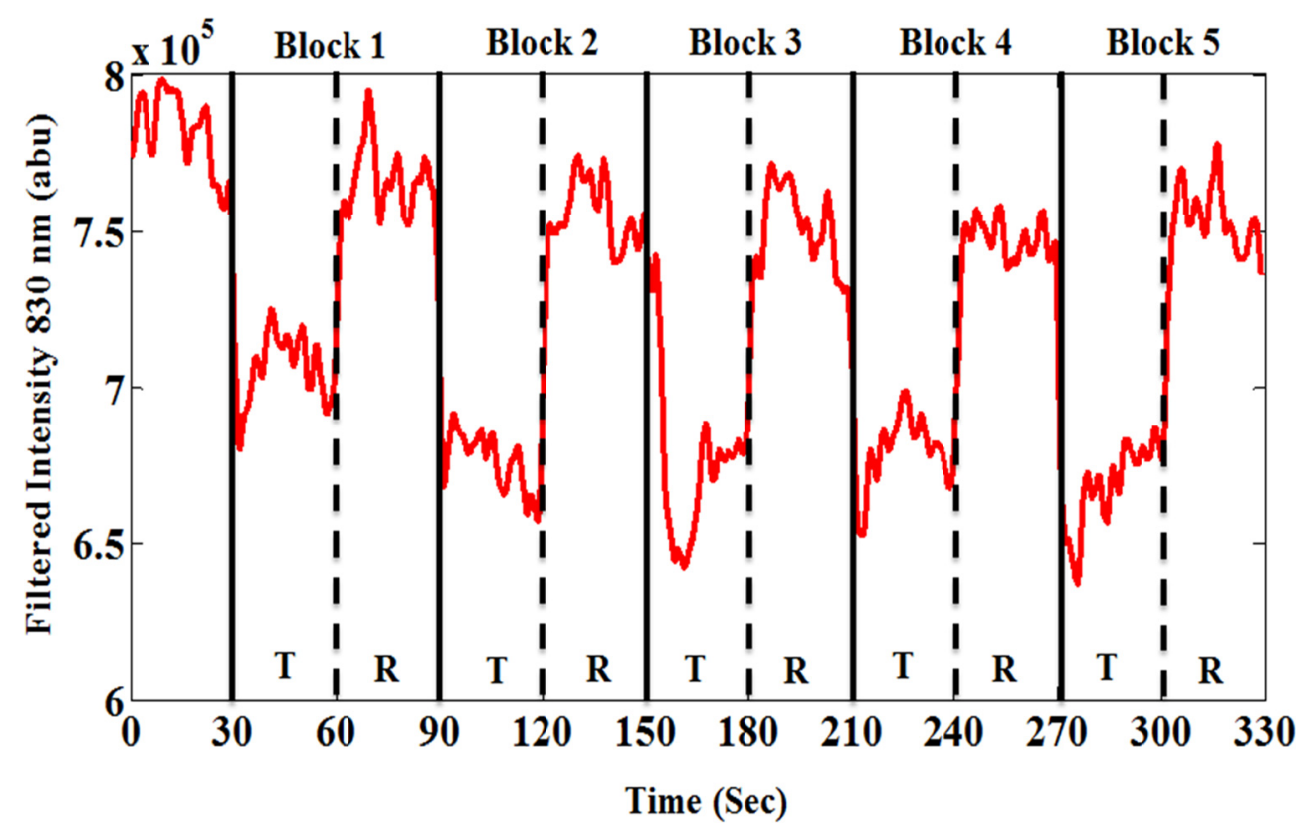

Figure 4.3: Filtered intensity at $830 \mathrm{~nm}$ plotted against time. . The figure depicts the change in the filtered intensity at $830 \mathrm{~nm}$ across the five blocks of task (T) and rest $(R)$ period.

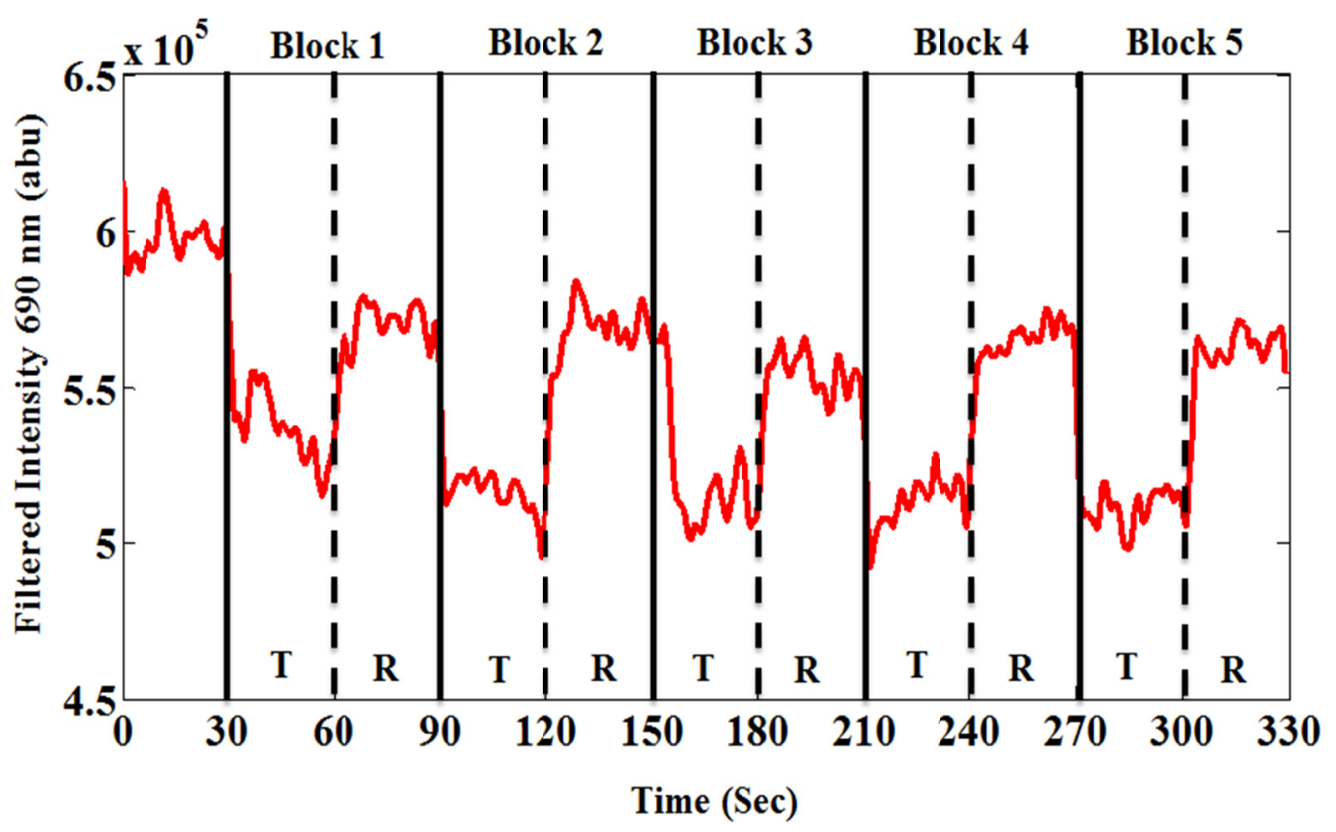

Figure 4.4: Filtered intensity at $690 \mathrm{~nm}$ plotted against time. The figure depicts the change in the filtered intensity at $690 \mathrm{~nm}$ across the five blocks of task (T) and rest (R) period. 
Step 2: The filtered optical data is further normalized to remove the instrument effect and provide the relative change in intensity by dividing the optical data by its mean, given by expression shown in Eq. 4.2.

$I_{N}(\lambda)=\frac{I(\lambda)}{I_{0}(\lambda)}$

where in Eq. 4.2, $I_{N}(\lambda)$ is normalized intensity data at wavelength $\lambda, I(\lambda)$ is raw intensity data at wavelength $\lambda$ and $I_{0}(\lambda)$ is the mean of the raw intensity data at wavelength $\lambda$ acquired during either task or rest period. The normalized intensity at 830 $\mathrm{nm}$ and $690 \mathrm{~nm}$ is shown in Figure 4.5 and 4.6, respectively.

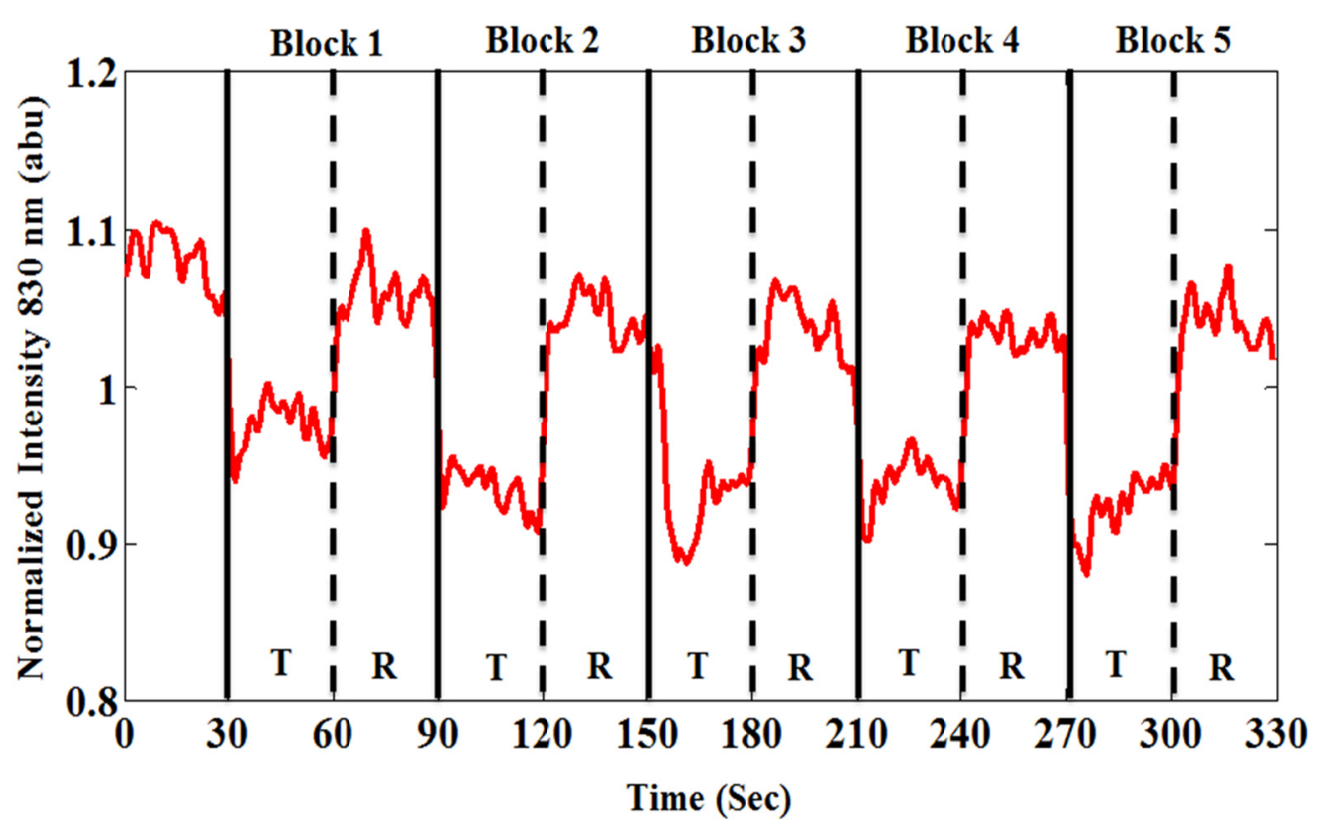

Figure 4.5: Normalized and filtered intensity at $830 \mathrm{~nm}$, plotted against time. The figure depicts the change in the normalized intensity at $830 \mathrm{~nm}$ across the five blocks of task (T) and rest $(R)$ period. 
Step 3: The normalized data is then used to calculate change in optical density for each wavelength, given by expression shown in Eq. 4.3.

$\Delta \mathrm{OD}(\lambda)=-\log \left(I_{N}(\lambda)\right)$

where in Eq. 4.3, $\Delta \mathrm{OD}$ is change optical density at wavelength $\lambda$ and $I_{N}(\lambda)$ is normalized intensity data at wavelength $\lambda$. The change in optical density at $830 \mathrm{~nm}$ and $690 \mathrm{~nm}$ is shown in Figure 4.7 and 4.8, respectively.

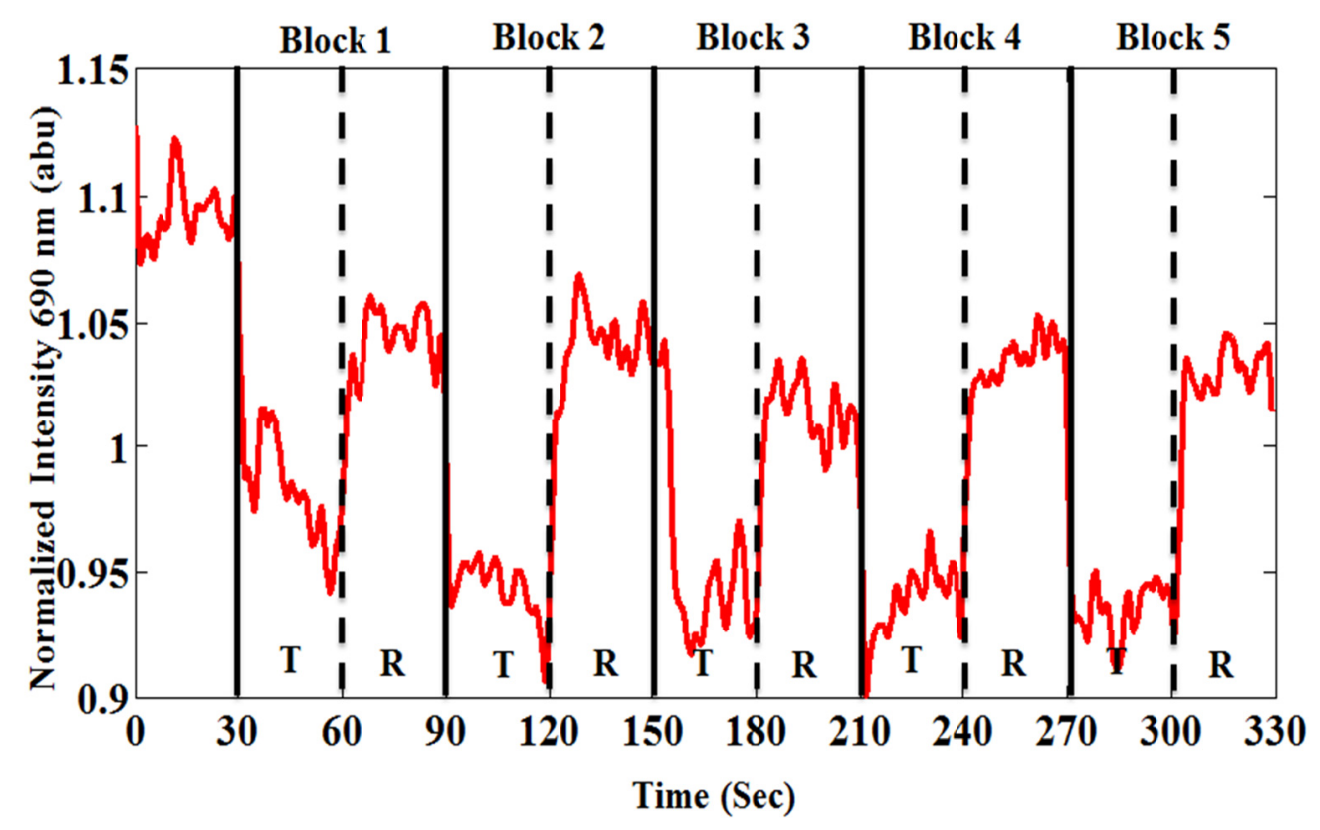

Figure 4.6: Normalized and filtered intensity at $690 \mathrm{~nm}$ plotted against time. The figure depicts the change in the normalized intensity at $690 \mathrm{~nm}$ across the five blocks of task (T) and rest $(R)$ period. 


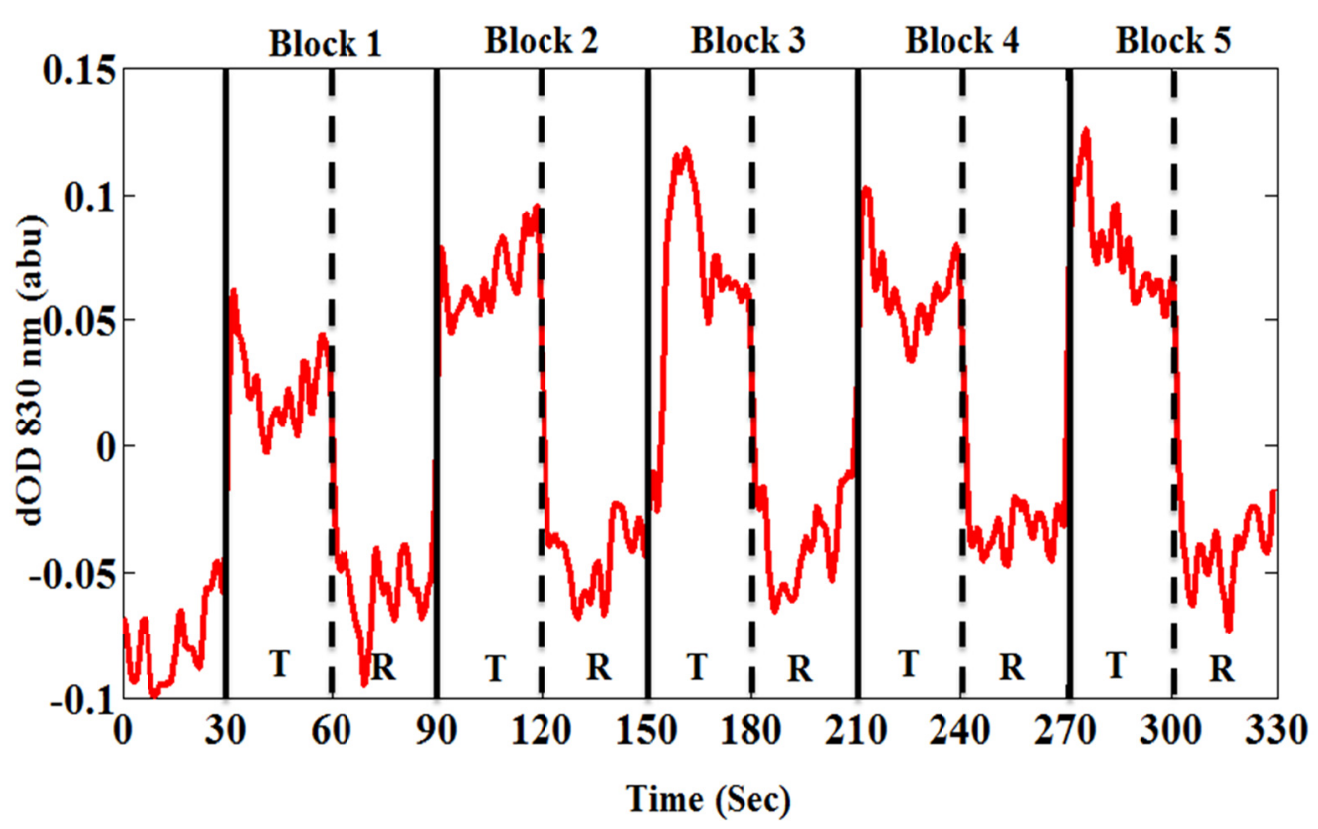

Figure 4.7: Change in optical density at $830 \mathrm{~nm}$ acquired plotted against time. The figure depicts the change in the optical density at $830 \mathrm{~nm}$ across the five blocks of task $(T)$ and rest $(R)$ period.

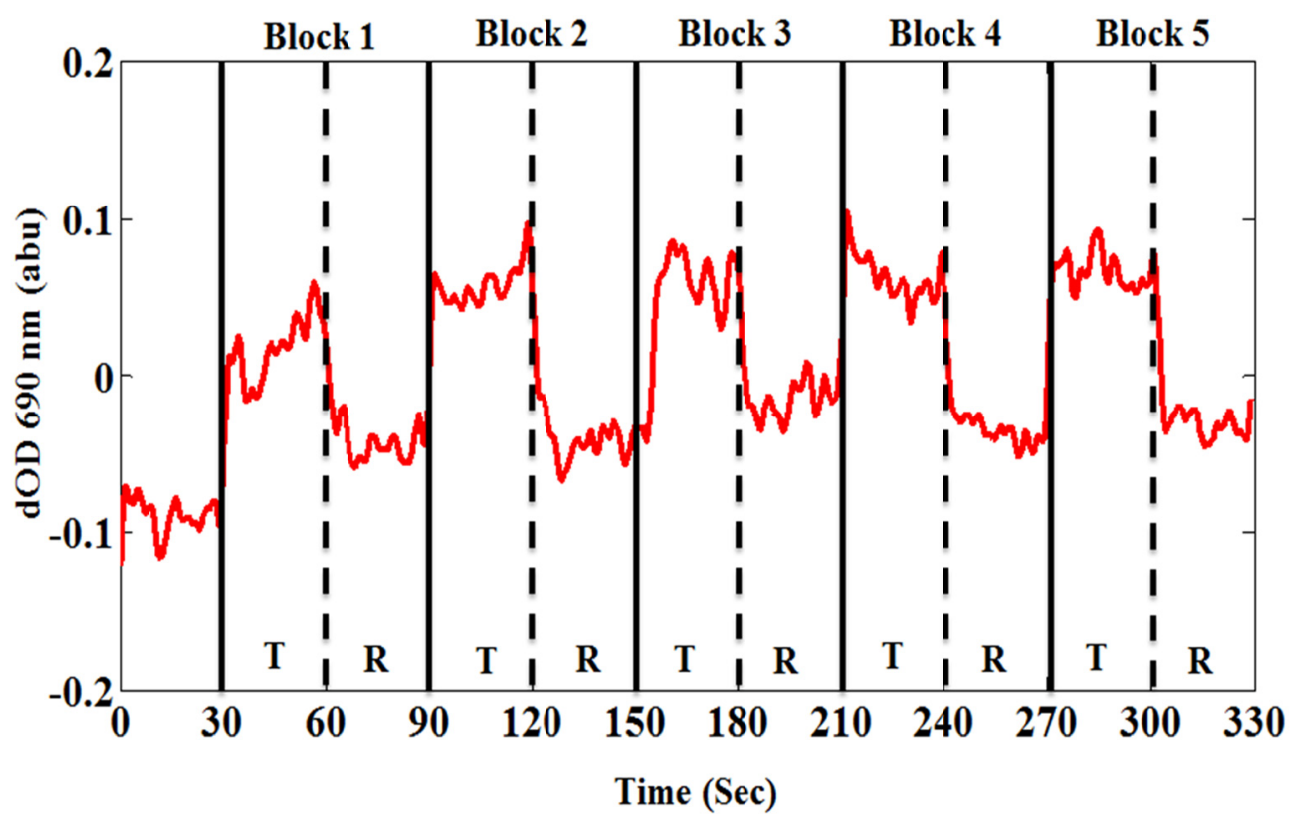

Figure 4.8: Change in optical density at $690 \mathrm{~nm}$ plotted against time. The figure depicts the change in the optical density at $690 \mathrm{~nm}$ across the five blocks of task (T) and rest $(R)$ period. 
Step 4: The dOD at $830 \mathrm{~nm}$ and $690 \mathrm{~nm}$ is then used to calculate changes in $\mathrm{HbO}, \mathrm{HbR}$ and $\mathrm{HbT}$ using Eq. 2.5, 2.6 and 2.7, respectively. The change in $\mathrm{HbO}, \mathrm{HbR}$ and $\mathrm{HbT}$ across the five blocks is further averaged to get the averaged change in $\mathrm{HbO}, \mathrm{HbR}$ and $\mathrm{HbT}$ as shown in Figure 4.9.

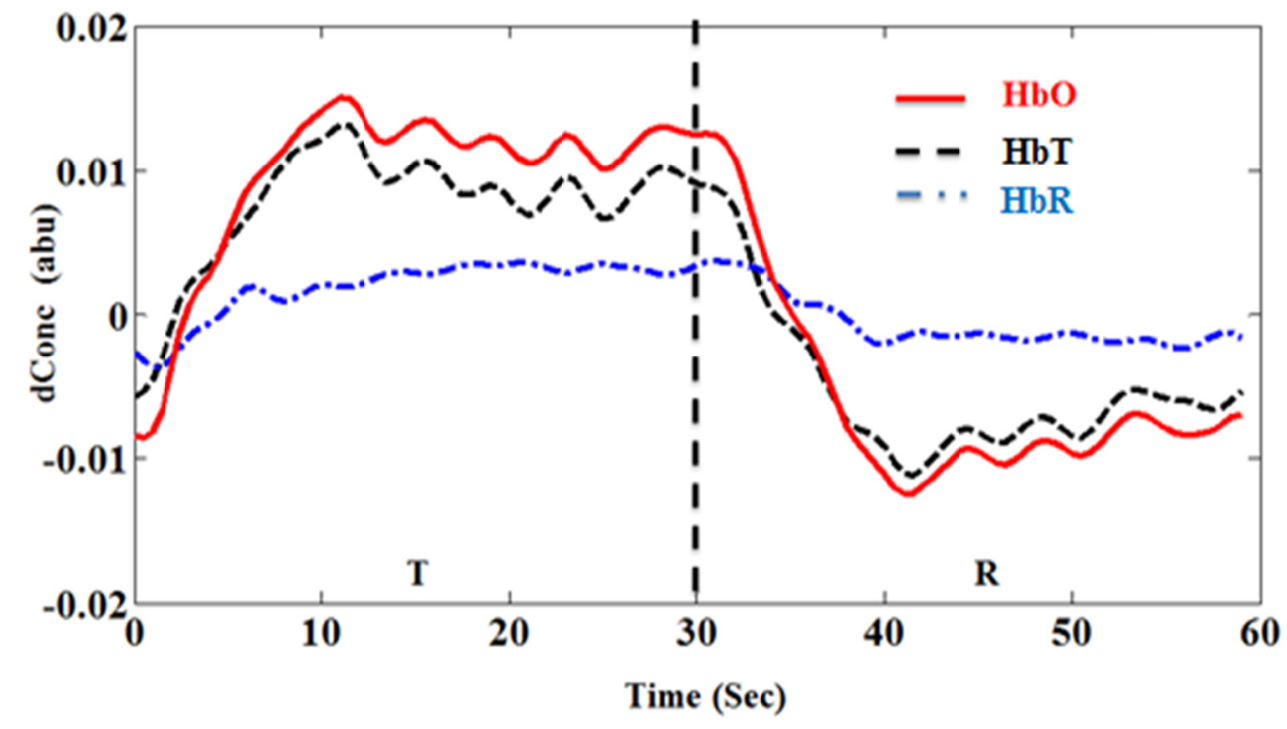

Figure 4.9: Change in concentration of $\mathrm{HbO}, \mathrm{HbR}$ and $\mathrm{HbT}$ averaged across the five blocks of task $(T)$ and rest $(R)$ period, obtained after processing change in optical density at $830 \mathrm{~nm}$ and $690 \mathrm{~nm}$, plotted against time.

\begin{tabular}{|l|l|l|}
\hline Physiological Signals & Response Timescale & Frequency Band \\
\hline Heart rate & $\sim 1 \mathrm{sec}$ period & $1,2,3 \mathrm{~Hz}$ \\
\hline \hline Respiration & $\sim 5 \mathrm{sec}$ period & $0.2-0.3 \mathrm{~Hz}$ \\
\hline Mayer waves & $\sim 10 \mathrm{sec}$ period & $0.08-0.12 \mathrm{~Hz}$ \\
\hline "Fast" signal & $\sim 0.050 \mathrm{sec}$ & $20+\mathrm{Hz}$ \\
\hline Hemodynamic response & $\sim 0.2-10 \mathrm{sec}$ & $0.1-5 \mathrm{~Hz}$ \\
\hline
\end{tabular}

Table 4.1: Different types of biological signals and their frequency range (modified and adapted from Boas \& Franceschini, 2004). 
The activation analysis described in this section elucidates the change in concentration of $\mathrm{HbO}, \mathrm{HbR}$ and $\mathrm{HbT}$ in the cortical regions of the brain under investigation without giving any information about the extent of synchronization between two cortical regions. Functional connectivity analysis is performed to investigate the extent of synchronization in the activity between two regions of the brain (as described in the following section).

\subsection{Functional connectivity analysis}

Functional connectivity as defined in section 2.3 .2 .2 , is fundamentally a statistical concept. Functional connectivity is defined as the (Friston 1994; Horwitz 2003; Lee et al. 2003) "temporal correlation between spatially remote neurophysiological events". During my doctoral work the functional connectivity analysis was performed using by computing the Pearson's correlation coefficient, as shown in Eqn. 4.4.

$$
\text { Correlation coefficient }=\frac{\sum_{i=1}^{n}\left(X_{i}-\bar{X}\right)\left(Y_{i}-\bar{Y}\right)}{\sqrt{\sum_{i=1}^{n}\left(X_{i}-\bar{X}\right)^{2}\left(Y_{i}-\bar{Y}\right)^{2}}}
$$

where in Eq. 4.4, $\bar{X}$ is the mean (across the time period of stimuli) of the averaged hemodynamic response ( $\mathrm{HbO}, \mathrm{HbR}$, or $\mathrm{HbT})$ corresponding to a given source-detector pair or channel in one region of brain under investigation; $\bar{Y}$ is mean (across the time period of stimuli) of the averaged hemodynamic response ( $\mathrm{HbO}, \mathrm{HbR}$, or $\mathrm{HbT}$ ) corresponding to a given source-detector pair or channel in another region of the brain under investigation; $X_{i}$ and $Y_{i}$ are the HRFs ( $\mathrm{HbO}, \mathrm{HbR}$, or $\mathrm{HbT}$ ) at each time sequence corresponding to two regions of the brain under investigation. Additionally, $n$ is the 
number of time sequence (limited by the NIRS instrument data acquisition speed) in each stimulus.

Functional connectivity measures simultaneous coupling between two time series. It does not give us information about whether two units are connected or not i.e. it is agnostic to the directional or causal relationship between two time series. Thus effective connectivity analysis was performed during my doctoral work to describe or make inferences about the direction of influence between regions (Friston 1994; Horwitz 2003). The effective connectivity analysis was performed using granger causality as described below in section 4.6. The experimental study for which the effective connectivity analysis was performed using granger causality is described in Chapter 6 .

\subsection{Effective connectivity analysis}

Granger causality (Goebel et al. 2003; Roebroeck et al. 2005) measure is derived from estimated parameters of an autoregressive model and summarizes the amount of variance in one region that is explained by the signal history of another region. Assuming that $U(n)$ and $V(n)$ are the measured time courses of two brain regions, where $n$ represents the time points in the time series, Granger causality quantifies the usefulness of unique information in one of the time series $(\mathrm{U}(n))$ in predicting values of the other time series $(V(n))$. Specifically, if incorporating past values of $U$ improves the prediction of the current value of $V$, we say that $U$ Granger causes $V$. Thus, the temporal precedence (or lag) is used to identify the direction of causality from information in the data. Thereby, the granger causality analysis provides the directional flow of information between the 
regions under investigation rather than just the strength of correlation or synchrony between the regions of brain as provided by functional connectivity.

To statistically explicate the granger causality analysis between two regions the two time series, $U(n)$ and $V(n)$ of equal length, corresponding to those two regions is used as an input to the granger causality algorithm. The other inputs which are needed for the algorithm are the significance level (alpha) and the maximum number of lags (maxLag) to be considered. The steps implemented in the granger causality algorithm are shown in flow chart given in Figure 4.10.

First the linear auto regression is performed on time series $U(n)$ to obtain residual of the time series $U(n)$. The residual is then used to determine the lag length of times series $U(n)$ to be included in the auto regression using Bayesian Information Criterion (Seth et al. 2005). The Bayesian Information Criterion (BIC) is used to find the proper model specification using the number of lags and it is calculated using Eq. 4.5.

$B I C=T * \log \frac{R S S}{T}+\max \operatorname{Lag} * \log (T)$

where in Eq. 4.5, $T$ is the length of the time series $\mathrm{U}(\mathrm{n}) ; R S S$ is the residual sum of square obtained after the auto regression of time series $U(n)$ and maxLag is the input maximum number of lags. The output of the auto regression of the time series $U(n)$ are residual sum of square $\left(R S S_{R}\right)$ and $U_{\text {lag. }}$. The auto regression of time series $U(n)$ is augmented by considering the lagged values of the time series $V(n)$, which upon linear regression gives the residual of time series $U(n)$ augmented with the lagged values of time series $V(n)$. The residual is further used to determine the lag length of times series $U(n)$ augmented 
with the lagged values of time series $V(n)$, to be included in the auto regression using Bayesian Information Criterion (Seth et al. 2005). The BIC is calculated using the formula shown in Eq. 4.5. The output of the auto regression of the time series U(n) augmented with the lagged values of time series $V(n)$ are residual sum of square $\left(R S S_{V}\right)$ and $V_{\text {lag. }}$ The residual sum of squares $R S S_{U}$ and $R S S_{V}$ and the lag lengths $U_{\text {lag }}$ and $V_{\text {lag }}$ determined using BIC are employed to test the hypothesis does granger $V(n)$ causes $U(n)$ using F- statistics. Thus, the final outputs of the granger causality analysis algorithm are values of F- statistics and the critical values from the F- distribution as the output. The value of F Value and critical Value is calculated using Eq. 4.6 and Eq. 4.7, respectively.

$F$ Value $=\frac{\frac{\left(R S S_{U}\left(U_{\text {lag }}\right)-R S S_{V}\left(V_{\text {lag }}\right)\right)}{V_{\text {lag }}}}{\frac{R S S_{V}\left(V_{\text {lag }}\right)}{\left(T-\left(U_{\text {lag }}+V_{\text {lag }}+1\right)\right)}}$

where in Eq. 4.6, $R S S_{U}$ is the residual sum of square of time series $\mathrm{U}(\mathrm{n})$, and $U_{\text {lag }}$ is the value of lag determined using the Bayesian Information Criterion; and $R_{S} S_{V}$ is the residual sum of square of the time series $V(n)$, and $\mathrm{V}_{\text {lag }}$ is the value of lag determined using the Bayesian Information Criterion.

Critical Value $=\operatorname{finv}\left(1-\right.$ alpha, $\left.V_{\text {lag }},\left(T-\left(U_{\text {lag }}+V_{\text {lag }}+1\right)\right)\right) \quad$ Eq. 4.7

where in Eq. 4.7, finv is the inverse of the F cumulative distribution function; alpha is the level of significance; $U_{\text {lag }}$ and $V_{\text {lag }}$ are the value of lag determined using the Bayesian Information Criterion. The $F$ value and the Critical Value from the F- distribution (obtained using the Eqs. 4.6 and 4.7, respectively) are compared for the inequality $F$ 
Value $>$ Critical Value; where in if F Value $>$ Critical Value the null hypothesis is rejected (i.e. $\mathrm{V}(\mathrm{n})$ does not granger cause $U(n))$.

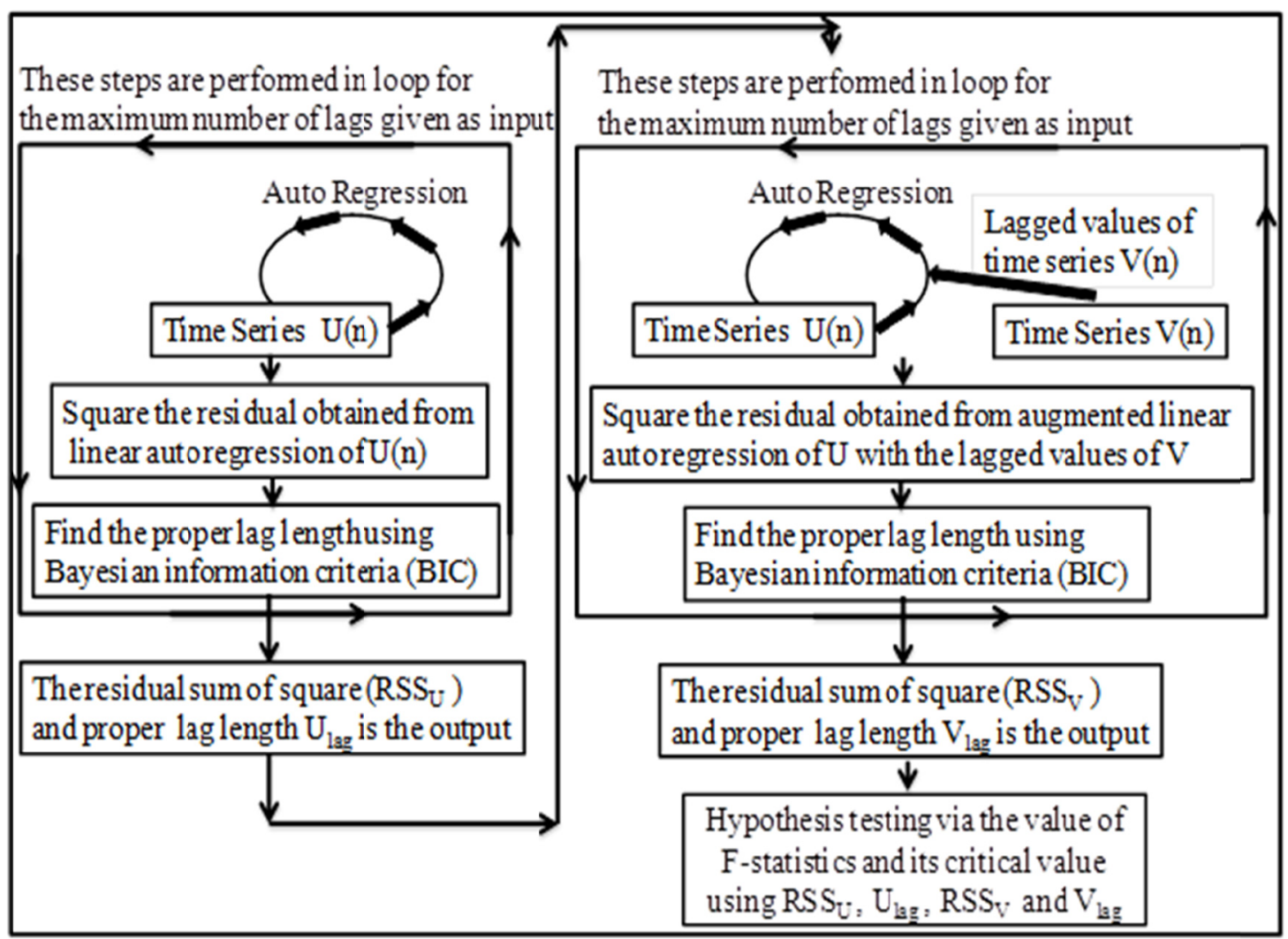

Figure 4.10: Flowchart depicting the implementation of granger causality algorithm for elucidating the effective connectivity across the cortical regions under investigation.

The lag length selection is chosen using Bayesian Information Criterion (Seth et al. 2005). The Bayesian Information Criterion is employed to find the proper model specification using the number of lags. The granger causality analysis algorithm gives the value of F-statistics (i.e. F value) and the critical value from the F- distribution as the output. The $F$ value gives the value of the probability distribution function of the relation granger $V(n)$ causes $U(n)$ for the lag specified in the algorithm. The critical value of the $\mathrm{F}$-distribution is the number obtained using the specified lags. The $F$ value and the 
Critical Value from the F- distribution obtained using the granger causality algorithm are compared for the inequality $F$ Value $>$ Critical Value; where in if $F$ Value $>$ Critical Value the null hypothesis is rejected (i.e. $V(n)$ does not granger cause $U(n)$ ).

The activation analysis (described in section 4.4), functional connectivity analysis (described in section 4.5) and effective connectivity analysis (described in section 4.6) provides information about change in cerebral hemodynamics, synchrony in the activity between different region and flow of information between different regions of brain, respectively but none of these methods provides information about the dominance feature of the brain. To elucidate the dominance feature of the brain, lateralization analysis is performed as described below.

\subsection{Lateralization analysis}

The lateralization analysis is performed using the $\mathrm{HbT}$ data as it contains information about the total change in concentration of hemoglobin in the region under investigation. To perform lateralization analysis using $\mathrm{HbT}$, NIRS laterality index, L, is calculated according to the formula shown in Eqn. 4.8.

$$
L(t)=\frac{\left(H b T_{\text {left }}(t)-H b T_{\text {right }}(t)\right)}{\left(H b T_{\text {left }}(t)+H b T_{\text {right }}(t)\right)}
$$

where in $\mathrm{Eq} 4.8, \mathrm{HbT}_{\text {left }}(\mathrm{t})$ is the total hemoglobin concentration of a given left channel of the region under investigation with respect to time and for each stimuli time period; $\mathrm{HbT}_{\text {right }}(\mathrm{t})$ is the total hemoglobin concentration of the corresponding right channel of the region under investigation with respect to time and for each stimuli time period. A positive laterality index indicates left-side dominance $(>0.25)$, and a negative laterality 
index indicates right-side dominance $(<-0.25)$ in the brain activation of the given channel pair (Gartus et al. 2009). A near-zero laterality index value indicates no dominance (between -0.25 to 0.25 ) between the channel pairs, also termed as bilateral activation.

\subsection{Statistical analysis}

Statistical analysis was performed to elucidate the differences in the hemodynamic response of the brain in response to various stimuli. Statistical tools are central to data processing of the optical data acquired from the brain. In the different studies described in chapters 5 to 12 , the various statistical tools employed include calculations of mean, standard deviation, paired t-test, Holm's Sequential Bonferroni Procedure, and/or confidence interval determination of data. Based on the experimental study performed, appropriate statistical tools as listed below were employed.

1) Mean: During optical imaging of brain the optical data is acquired from ' $p$ ' number of participant and also from a particular participant ' $b$ ' blocks of optical data are acquired. These optical data acquired either from ' $p$ ' number of participants or from ' $b$ ' blocks across a particular participant is averaged to obtain the average change across the entire participant and also across the blocks of optical data. It helps visualize the change in the trend of optical data acquired from all the subjects and across all the blocks of optical data. The mean is computed using Eq. 4.9.

Mean $=\left[\frac{X_{1}+X_{2}+X_{3}+\cdots+X_{b}}{b}\right]_{p}$ 
where $x_{1}, x_{2}, x_{3}, x_{n}$ corresponds to the optical data from the brain of either participant 1 , participant 2, participant 3, participant ' $p$ ' or from block 1, block 2, block 3, block ' $b$ ' of any of the participant. The Eq. 4.9, depicts that the optical data are first averaged across the blocks of each participants and then averaged across all the participants.

2) Standard deviation: As stated in the description of mean of the data during brain imaging the optical data are acquired from $p$ number of participant and also $b$ blocks of optical data are acquired from each participant. Each participant responds in differently in response to a particular stimulus. This difference in response induces variability in the optical data acquired from different participants. Hence standard deviation is computed to calculate the measure of variability in the data acquired from different participant's brain. The standard deviation is calculated using Eq. 4.10.

Standard deviation $=\sqrt{\frac{1}{p / b-1} \sum_{i=1}^{p / b}\left(x_{i}-\bar{x}\right)^{2}}$

Where $\mathrm{x}_{\mathrm{i}}$ corresponds to the data either from each individual participant or from each channel; $\bar{x}$ corresponds to the mean of the data either from all participants or from all channels and $p / b$ corresponds to either total number of participants in the study or the total number of blocks employed in the study.

3) Paired t-test: To compare two different stimuli student's t-test analysis was performed to compute the p-value as shown below:

Step1: The difference between change in $\mathrm{HbO}, \mathrm{HbR}$ and $\mathrm{HbT}$ from each pair of stimuli is calculated, as shown in Eq. 4.11 
$d_{t}=x_{t}-y_{t}$

where in Eq. $4.11, d_{t}$ is the difference between change in $\mathrm{HbO}, \mathrm{HbR}$ and $\mathrm{HbT}$ from a pair of two different stimuli $x_{t}$ and $y_{t}$ at time $t$ of the stimulus.

Step 2: The mean difference $d_{\text {mean }}$ is calculated

Step 3: The standard deviation of the differences is calculated, which is then used to calculate the standard error of the mean difference, as shown in Eq. 4.11.

$\left.S E\left(d_{\text {mean }}\right)=\frac{S_{d}}{\sqrt{n}}\right)$

where in Eq. 4.12, $S E\left(d_{\text {mean }}\right)$ is the standard deviation of the difference $S_{d}$ between the two pair of stimuli with $n$ number of time points.

Step 4: The t-statistics is calculated using the formula shown in Eq. 4.13

$T=\frac{d_{\text {mean }}}{S E\left(d_{\text {mean }}\right)}$

where in Eq. 4.13, T is the value of t-statistics calculated using the mean difference $d_{\text {mean }}$ and standard deviation $S E\left(d_{\text {mean }}\right)$ between the two stimuli pair. Under the null hypothesis, this statistics follows a t-distribution with $n-1$ degrees of freedom. A table of t-distribution is used to compare the value of $T$ to $t_{n-1}$ distribution. This gives the pvalue for the paired t-test. At $95 \%$ confidence interval if the $p$-value is less than 0.05 then it indicates significant difference while a p-value greater than 0.05 indicates no significant difference between two stimuli in terms of change in $\mathrm{HbO}, \mathrm{HbR}$ and $\mathrm{HbT}$. 
4) Holm's Sequential Bonferroni Procedure: If more than two stimuli are compared than holm's sequential bonferroni procedure is performed using the p-value computed via paired student's t-test between two stimuli. The t-tests are adjusted using Holm's sequential Bonferroni (http://mathworld.wolfram.com/BonferroniCorrection.html) procedure to control for the probability of a type I error. The steps to perform holm's sequential bonferroni procedure using the paired $\mathrm{p}$-values are given below:

Step 1: The p-value corresponding to change in $\mathrm{HbO}, \mathrm{HbR}$ each pair of stimuli is computed (Stimuli 1 vs. Stimuli 2, Stimuli 1 vs. Stimuli 3, and Stimuli 2 vs. Stimuli 3).

Step 2: The p-value of the given pair of data is sorted in ascending order.

Step 3: Each p-value is compared with the inequality shown in Eq. 4.14.

$p_{i} \leq \frac{\alpha}{1+k-i}$

Where in Eq. 4.14, $p_{i}$ corresponds to the p-value of the particular pair among the total numbers of pair; $\alpha$ is the statistical level of significance; $k$ is the total number of pairs in the comparison and $i$ corresponds to the particular pair among the total numbers of pair. If the inequality given by Eq. 4.14 is not met then there is no significant different between the given stimuli pair in terms of change in $\mathrm{HbO}, \mathrm{HbR}$ and $\mathrm{HbT}$ at the given statistical level of significance.

5) Confidence Interval: The optical data acquired from the brain are highly variable i.e. the optical data acquired from the brain varies across the participants and even between 
repetitions on each participant. Hence it is warranted to compute the confidence interval to determine the reliability of the estimate which is given by Eq. 4.15.

$C I=(X)_{\text {mean }} \pm t \times \frac{\operatorname{Std.Dev}(X)}{\sqrt{\text { Sample Size }}}$

Where $(x)_{\text {mean }}$ corresponds to the mean of the data either from each individual participant or from each channel computed using Eq. 4.9;Std.Dev $(x)$, is the standard deviation of the data either from each individual participant or from each channel computed using Eq.4.10; $t$ is the t-value of the data either from each individual participant or from each channel at a particular confidence interval and sample size is either the total number of participant or channels employed in the study.

Thus these statistical tools are indispensable for processing and analyzing the optical data acquired from the brain. These tools have been used appropriately in the various experimental studies described in chapters 5 to 12 . 
CHAPTER 5. Study 1a: Language Skills

During my doctoral research, NIRS was used to investigate the activation, connectivity and lateralization in the frontal region of the brain as described in this chapter. This work was published in BRAIN RESEARCH BULLETIN. (Ujwal Chaudhary, Michael Hall, Joe DeCerce, Gustavo Rey, Anuradha Godavarty, "Frontal activation and connectivity using near-infrared spectroscopy: Verbal fluency language study", Brain Research Bulletin, Volume 84, Issue 3, 28 February 2011, Pages 197-205).

\subsection{Introduction}

NIRS has been applied for various functional brain mapping studies as described in section 2.4. In the area of language development and brain activation/functionality using NIRS, researchers have performed studies using various experimental paradigms in normal adults and pediatric populations (Bortfeld et al, 2009; Herrmann et al, 2003; Hermann et al, 2006; Kuwabara et al, 2006; Sakatani et al, 1998). The studies were focused primarily in understanding the differences in the hemodynamic responses in different (typically frontal and temporal) regions of the brain in response to languagebased stimuli (Herrmann et al, 2003; Hermann et al, 2006; Jayakar et al, 2005; Kubota et al, 2005; Kuwabara et al, 2006; Quaresima et al, 2002; Sakatani et al, 1998). In addition, a few researchers have analyzed the cortical lateralization and dominance features of the brain in different populations, based on their application focus (e.g. schizophrenia, epilepsy) (Gallagher et al, 2008; Hermann et al, 2003; Kennan et al, 2002; Kubota et al, 2005; Noguchi et al, 2002; Watanabe et al, 2000; Watson et al, 2004). All the language studies carried out so far using NIRS (Gallagher et al, 2008; Herrmann et al, 2003, 
Jayakar et al, 2005; Kennan et al, 2002; Kubota et al, 2005; Kuwabara et al, 2006; Noguchi et al, 2002; Qaresima et al, 2002; Sakatani et al, 1998; Watanabe et al, 1998; Watson et al, 2004) have focused on the brain activity differences and/or cortical dominance. However, the fundamental aspect of the brain connectivity across spatial regions of the frontal cortex during a language task has not been performed to date.

In the current study, NIRS is used to understand the brain activation, functional connectivity, as well as the cortical lateralization in response to verbal fluency task. The study was performed using a multi-channel NIRS instrument to non-invasively map the frontal cortex of 15 normal adults in response to three stimuli - a verbal fluency word generation task, random jaw movement task, and rest conditions. The hemodynamic responses in terms of $\mathrm{HbO}, \mathrm{HbR}$, and $\mathrm{HbT}$ were used to statistically evaluate the differences in the brain activation amongst the three stimuli. Additionally, the functional connectivity of the left and right cortex was evaluated along with the cortical lateralization for left or right dominance. The brain activation, connectivity, and lateralization results were compared for any correlations amongst each other.

\subsection{Materials and methods}

\subsubsection{Instrumentation}

In the current study, the frontal cortex of the brain was imaged using a frequency-domain based optical imaging system, Imagent ( ISS, Inc., Champaign, IL) described in section 3.1. During this study 5 laser diodes sources (at each wavelength, 690 and $830 \mathrm{~nm}$ ) and 4 detectors was used. The source-detector layout (based on the international10-10 electrode placement system) on the pre- and anterior frontal cortex is schematically shown in 
Figure 5.1. A custom-built "brain cap" was used to hold the source and detector fibers in place during the imaging studies. Optical signals were acquired in real-time (at $\sim 1.3 \mathrm{~Hz}$ ) from each source -detector pair or channel (Ch.), in response to the stimuli presented (described in Section 5.2.3) using the software package (BOXY) of the instrument.

\subsubsection{Participants}

Fifteen normal and right handed adults were enrolled for the current study that was performed at The Brain Institute of Miami Children's Hospital (Miami, FL). This study was Florida International University's (FIU) Institutional Review Board (IRB) and WIRB approved, and written consent(s) were obtained from the participants.

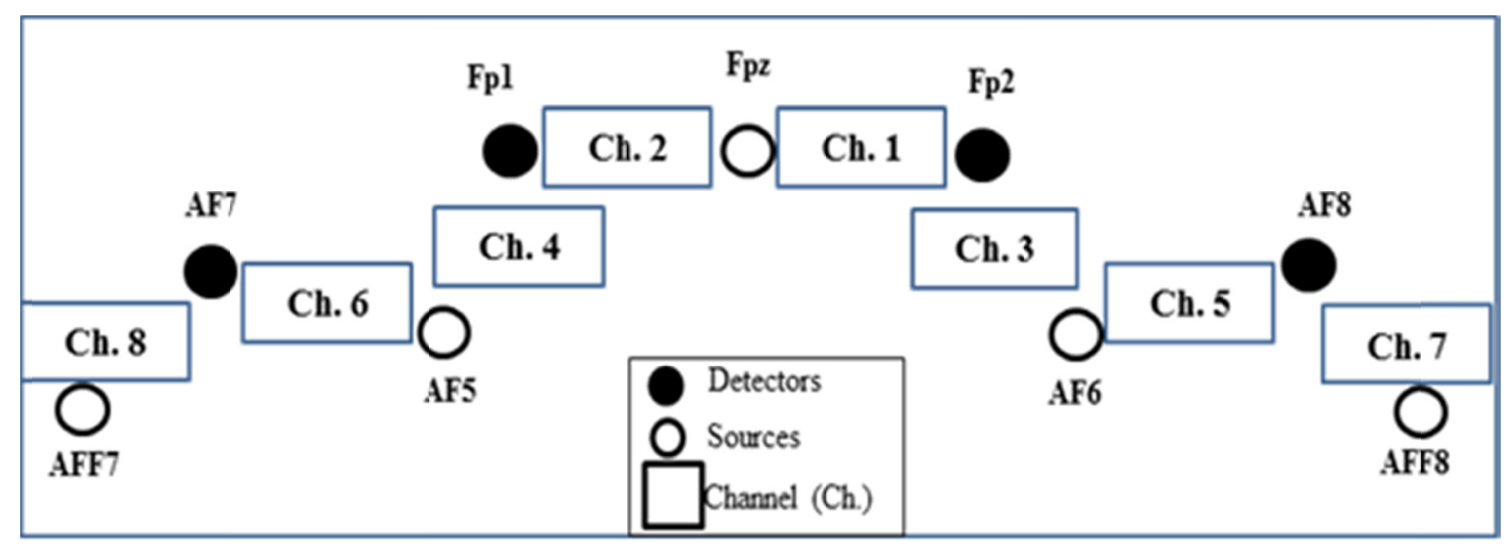

Figure 5.1: Source-detector layout corresponding to the prefrontal $(F p)$ and the anterior frontal (AF) region of standard 10-10 (Hoshi et al, 1994) electrode placement system. The solid circles and hollow circles used in the figure represent detectors and sources respectively. The hollow squares represent the path between the sources and detectors (i.e. channels). All the even number channels correspond to the left frontal cortex, and the odd number channels correspond to the right frontal cortex.

\subsubsection{Stimulus and Procedure}

The current NIRS study is focused on understanding how the brain responds to language cognitive tasks in the pre-frontal and anterior frontal cortex of the brain. Participants were seated in a comfortable chair (upright) in a quiet room and the custom-built optical 
cap was placed on their head (with minimal hair interference). The participants were asked to respond to the language-based verbal fluency task(s) presented to them. Prior to the block-designed tasks, there was an initial $30-\mathrm{sec}$ rest period. This was followed by the block-designed task that consisted of a $30-\mathrm{sec}$ of word generation (W), 30-sec of random Jaw movement $(\mathrm{J})$ and 30-sec of Rest(R). During the rest period, the participants were asked to minimize their thoughts and look at the sky (through the window). During the word generation task the participants were asked to say as many possible words (without repeating) beginning with a randomly presented alphabet. During the jaw movement task, the participants were asked to randomly move their jaw without actually speaking. The physiology behind the used block design is that the, $\mathrm{W}$ task consists both of cognitive thinking and jaw movement due to the requirement of task, as explained above, which necessitate both thinking and speaking. The process of speaking cannot be separated from jaw movement and hence $\mathrm{J}$ task (which entails pure jaw movement) was used without any cognitive thinking, thus providing the contrast between the two tightly coupled $\mathrm{W}$ and $\mathrm{J}$ tasks. The hypothesis of the study is that the hemodynamic response during a language-based cognitive task will be different from baseline rest condition or under random jaw movement, with no cognitive (verbal) response to presented stimuli. The W-J-R block was repeated 5 times on each participant. The entire study on each participant was repeated a second time after a 30 minute span between repetitions. During these NIRS studies, the participants were asked to keep their head still with minimal movement. 


\subsection{Optical data analysis}

In the current studies, only the average of the modulated light signal (i.e. DC signal) was employed in post-processing real-time one dimensional (1-D) hemodynamic responses across each source-detector pair (or channel). The optical data was post processed to obtain averaged HRF data as described in section 4.1. The $\mathrm{HbO}, \mathrm{HbR}$, and $\mathrm{HbT}$ data was further averaged over all the 15 participants and 2 repetitions in each participant (i.e. 15 participants $\times 2$ repetitions $=30$ trials). The averaged $\mathrm{HRF}$ data $(\mathrm{HbO}, \mathrm{HbR}$ and $\mathrm{HbT})$ was across the 90-sec block, consisting of $\mathrm{W}, \mathrm{J}$ and $\mathrm{R}$ task (30-sec each). The 0 - $30 \mathrm{sec}$ period corresponded to $\mathrm{W}$ task, $30-60 \mathrm{sec}$ to the J task, and $60-90 \mathrm{sec}$ to the $\mathrm{R}$ task. Prior to averaging the hemodynamic responses ( $\mathrm{HbO}, \mathrm{HbR}$, and $\mathrm{HbT}$ ) across all trials, the mean and standard deviation across trials was evaluated in order to assess the consistency in the responses across participants and repetitions. Three different studies were performed on the processed hemodynamic response data, which include: (i) activation, (ii) connectivity, and (iii) cortical lateralization study.

\subsubsection{Study 1-Activation}

Brain activation study (as described in section 4.4) was performed to elucidate the differences in the hemodynamic response ( $\mathrm{HbO}, \mathrm{HbR}$ and $\mathrm{HbT}$ ) as a function of time, in the frontal cortex and in response to the three presented stimuli (Word, Jaw and Rest).

\subsubsection{Study 2-Connectivity}

In the present study, functional brain connectivity was performed to characterize the ways in which the left and right frontal cortex was functionally integrated or works in synchrony in response to language-based cognitive stimuli. This study is carried out by 
computing zero lagged correlation (as described in section 4.5) to elucidate the correlation and synchrony in the hemodynamic response and hence the connectivity in the neuronal circuit between the left and right frontal cortex. Correlation calculations were performed between left vs. right frontal cortex for each stimulus (Word, Jaw and Rest) using the HRFs (HbO, HbR and HbT) data obtained from the following channels combinations: Ch.2 vs. Ch.1, Ch.4 vs. Ch.3, Ch.6 vs. Ch.5, and Ch.8 vs. Ch.7. The results of the connectivity study are depicted as histogram plots.

\subsubsection{Study 3- Cortical Lateralization}

Cortical lateralization study is performed to understand the hemispherical dominance of the language in the frontal cortex and its variation across the stimuli. The laterality index was computed (as described in section 4.7) between left vs. right frontal cortex for each stimuli (Word, Jaw and Rest) using the HbT data obtained from channels combination (Ch.2 vs. Ch.1, Ch.4 vs. Ch.3, Ch.6 vs.Ch.5, and Ch.8 vs. Ch.7) (Watanabe et al, 1998; Watson et al, 2004).

\subsection{Results}

\subsubsection{Study1: Activation}

The brain activation in response to verbal fluency $\operatorname{task}(\mathrm{s})$ is plotted (see Figure 5.2) in terms of the averaged (across 15 participants and 2 repetitions, i.e. 30 trials) relative $\mathrm{HbO}, \mathrm{HbR}$ and $\mathrm{HbT}$ changes across each stimulus (Word, Jaw and Rest) and each channel. It can be observed from the averaged $\mathrm{HRF}(\mathrm{HbO}, \mathrm{HbR}$ and $\mathrm{HbT}$ ) plots that the extent of activation in terms of $\mathrm{HbO}, \mathrm{HbR}$ and $\mathrm{HbT}$ was different across the three stimuli (Word, Jaw and Rest). The activation differences across stimuli were more obvious in 
terms of $\mathrm{HbO}$ and $\mathrm{HbT}$ than $\mathrm{HbR}$, as observed from literature as well (Kubota et al, 2005;

Sakatani et al, 1998).

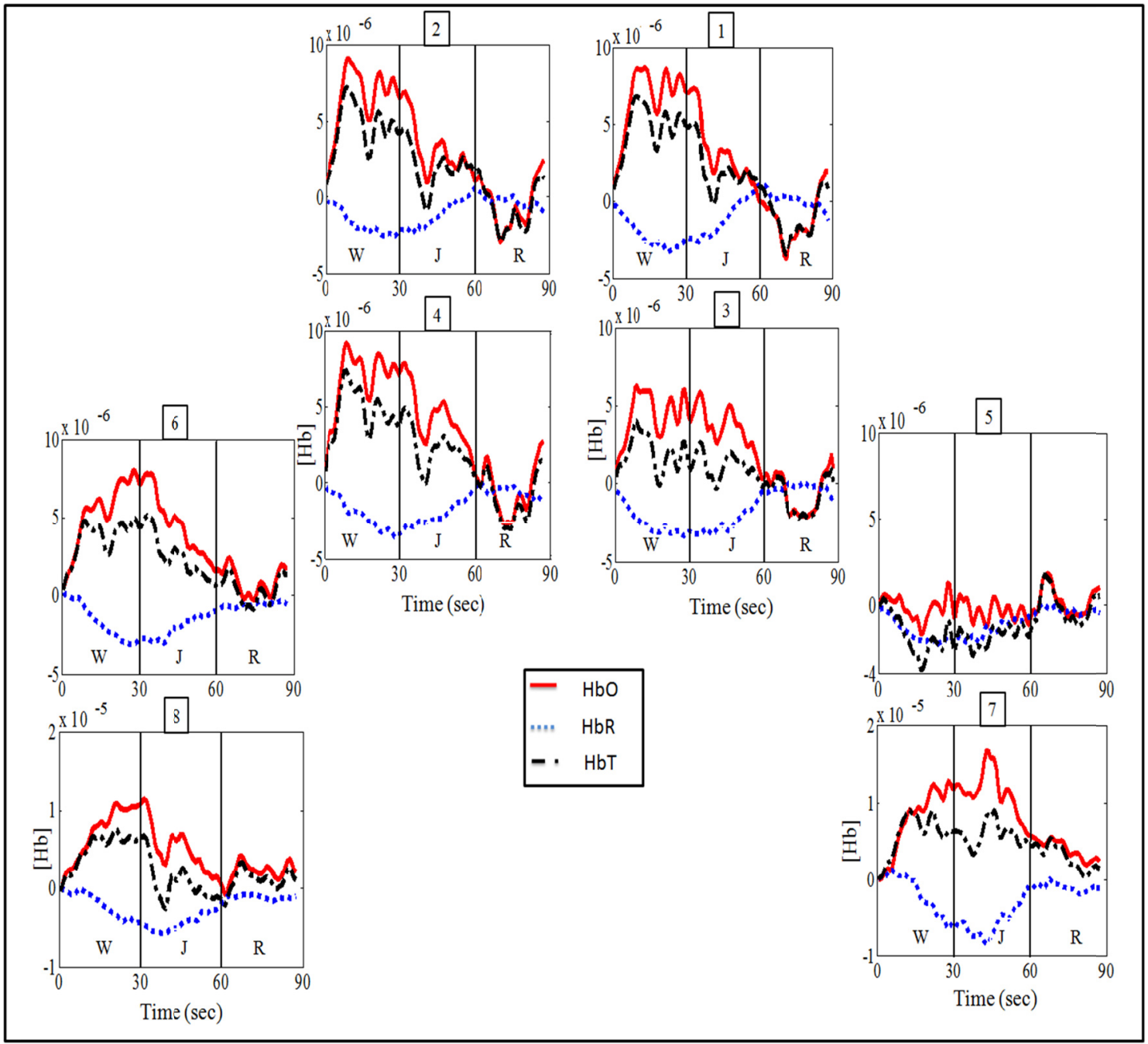

Figure 5.2: Averaged hemodynamic response function across all the participants (HRF) plotted against time (in seconds) for each channel (Ch. 1-8) in response to the languagebased stimuli. Each plot shows the HRF data for the block stimuli of Word (W), Jaw Movement (J), and Rest (R) performed by the participants. In each plot, the y-axis is the change in hemoglobin concentration, while $x$-axis is the time in seconds. In each plot, the red solid curve represents the change in HbO (oxy-hemoglobin), the blue dotted curve represents the change in HbR (deoxy-hemoglobin) and the black dash-dot curve represents the change in $\mathrm{HbT}$ (total hemoglobin). The hemodynamic changes from 0-30 sec corresponds to Word task, 30-60 sec to Jaw movement task, and 60-90 sec to Rest task. The numbers shown in square boxes on top of each plot corresponds to the number given to each source-detector combination or channel as shown in Figure 5.1. 
A marked increase in $\mathrm{HbO}$ and $\mathrm{HbT}$ was observed during the verbal fluency Word task in comparison to the baseline jaw movement and rest conditions. The rest condition has a non-zero signal initially (i.e. immediately after the Jaw movement condition) and tapers to a zero-signal (i.e. initial baseline at the beginning of the block) by the end of the 30 -sec rest period. The initial non-zero condition is because the effect of any stimuli continues in terms of the changes in the cerebral blood volume for a few seconds after the stimulus ends. Hence, the relative change in $\mathrm{HbO}, \mathrm{HbR}$, and $\mathrm{HbT}$ in response to the rest condition begins with a non-zero signal and eventually tapers to a zero-signal as seen from Figure 5.2, validating the effectiveness of the data. In addition, the HRFs ( $\mathrm{HbO}, \mathrm{HbR}$, and $\mathrm{HbT}$ ) were observed to be consistent across the 30 trials, as observed from the statistical analysis of the data in terms of mean and standard deviation. This demonstrates that the observed average change in brain activation in response to the three stimuli (W, J, and R) is consistent across all the participants.

Statistical analysis was performed to determine if differences in activation (in terms of $\mathrm{HbO}, \mathrm{HbR}$, and $\mathrm{HbT}$ ) between two stimuli (Word vs. Jaw, Word vs. Rest, and Jaw vs. Rest) and across each channel were significant. These results are shown in Figure 5.3, in terms of the evaluated p-value across the stimuli pair and for each channel. From these plots, it is obvious that the brain activation in terms of $\mathrm{HbO}, \mathrm{HbR}$ and $\mathrm{HbT}$ was significantly different for most of the channels when the stimuli compared were Word $v s$. Rest and Jaw vs. Rest. 


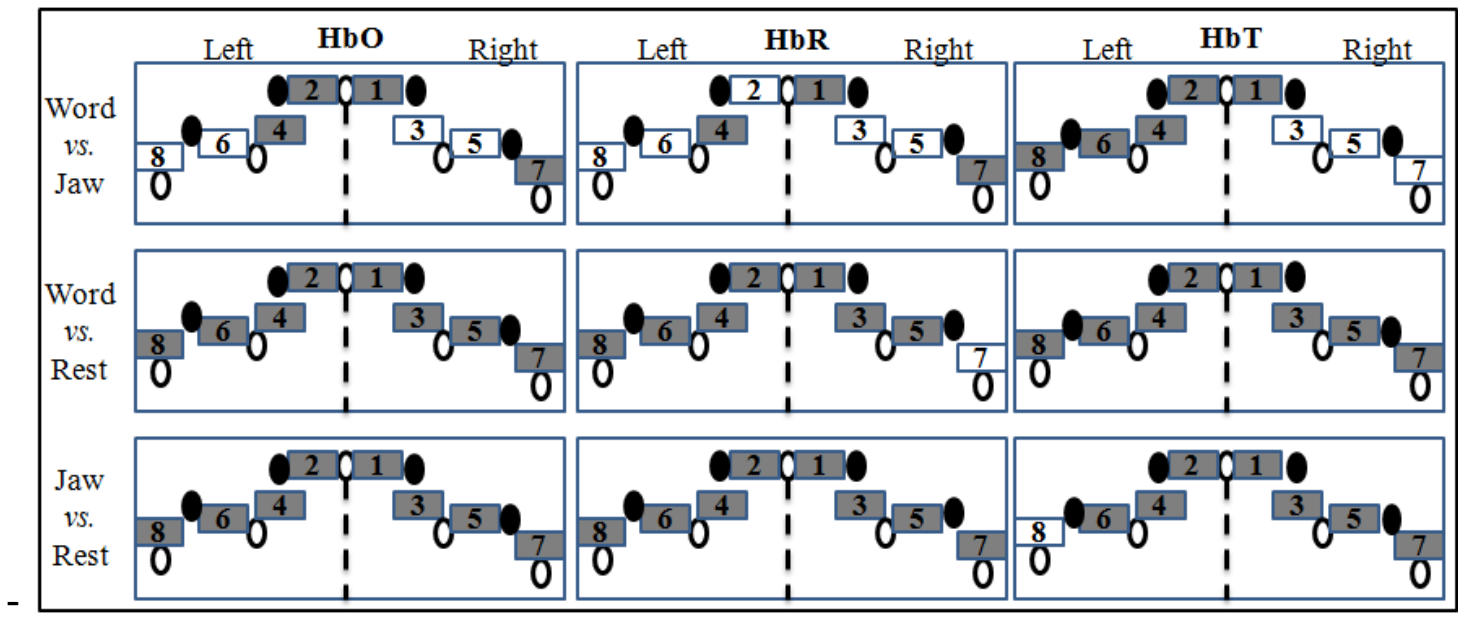

Figure 5.3: Pictorial representation of the differences in $H R F$ ( $H b O, H b R$, and $H b T)$ across the three stimuli $(W, J$, and $R$ ) in each channel, obtained from a three-paired $t$ test. The grey shaded channels represent significant differences between the two stimuli ( $W$ vs. $J, W$ vs. $R$, and $J$ vs. $R$ ) of interest for a given HRF data. The non-shaded white channels represent insignificant differences between the two stimuli of interest for a given HRF data. The solid black circles correspond to detectors and the hollow circles correspond to sources placed on the pre and anterior frontal region of brain as shown in Figure 5.1.

\subsubsection{Study 2: Connectivity}

The results from the connectivity study are shown as histogram plots (Figure 5.4) of the correlation coefficient obtained from the zero order correlation analysis, for each left vs. right paired channel, stimuli, and $\mathrm{HRF}(\mathrm{HbO}, \mathrm{HbR}, \mathrm{HbT})$.

The result for the inter stimuli zero order correlation analysis is shown using the source detector layout and a color code in Figure 5.5. The color codes correspond to the different range of the correlation value. Using the color code shown in Figure 5.5 correlation map was constructed for the inter stimuli (W vs. J, W vs. R and J vs. R) zero order correlation. 
Along with the zero order correlation analysis for each left vs. right paired channel for each stimulus, inter stimuli i.e. $\mathrm{W}$ vs. $\mathrm{J}, \mathrm{W}$ vs. $\mathrm{R}$ and $\mathrm{J}$ vs. $\mathrm{R}$, zero order correlation analysis was performed across each channel to ascertain; do the correlation varied w.r.t. the stimuli pair (W vs. J, W vs. R and J vs. R) or does it remains the same irrespective of the stimuli pair (W vs. J, W vs. R and J vs. R).

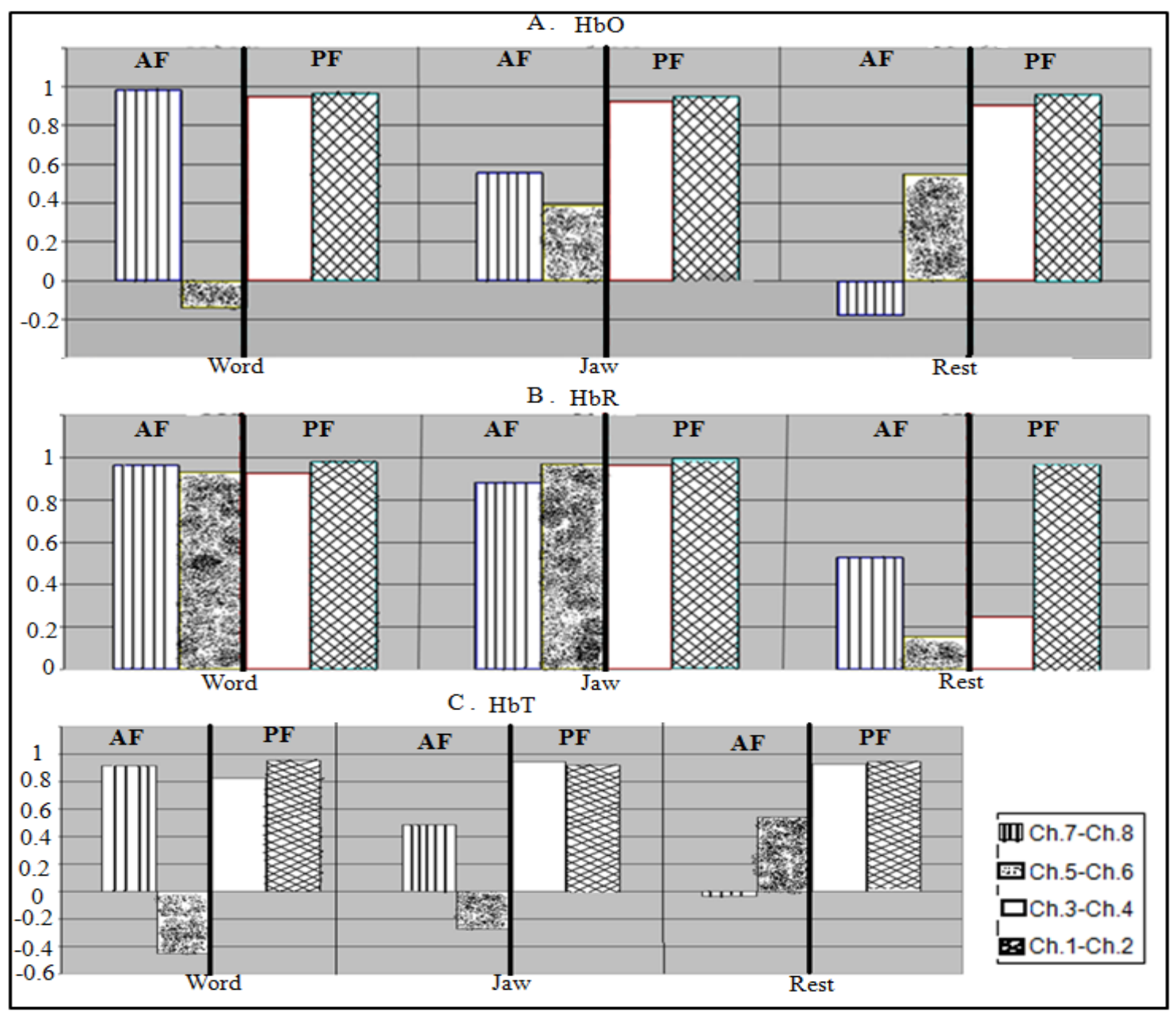

Figure 5.4: Bar plot of the correlation coefficient obtained from the zero order correlation analyses study in response to language-based stimuli on the frontal cortex. The plot depicts the correlation coefficient values obtained from (A) $\mathrm{HbO}$, (B) $\mathrm{HbR}$, and (C) HbT data, across the three stimuli (Word, Jaw, and Rest) and left vs. right channel pairs (4 of them) in the pre-frontal (PF) and anterior frontal (AF) cortex. 


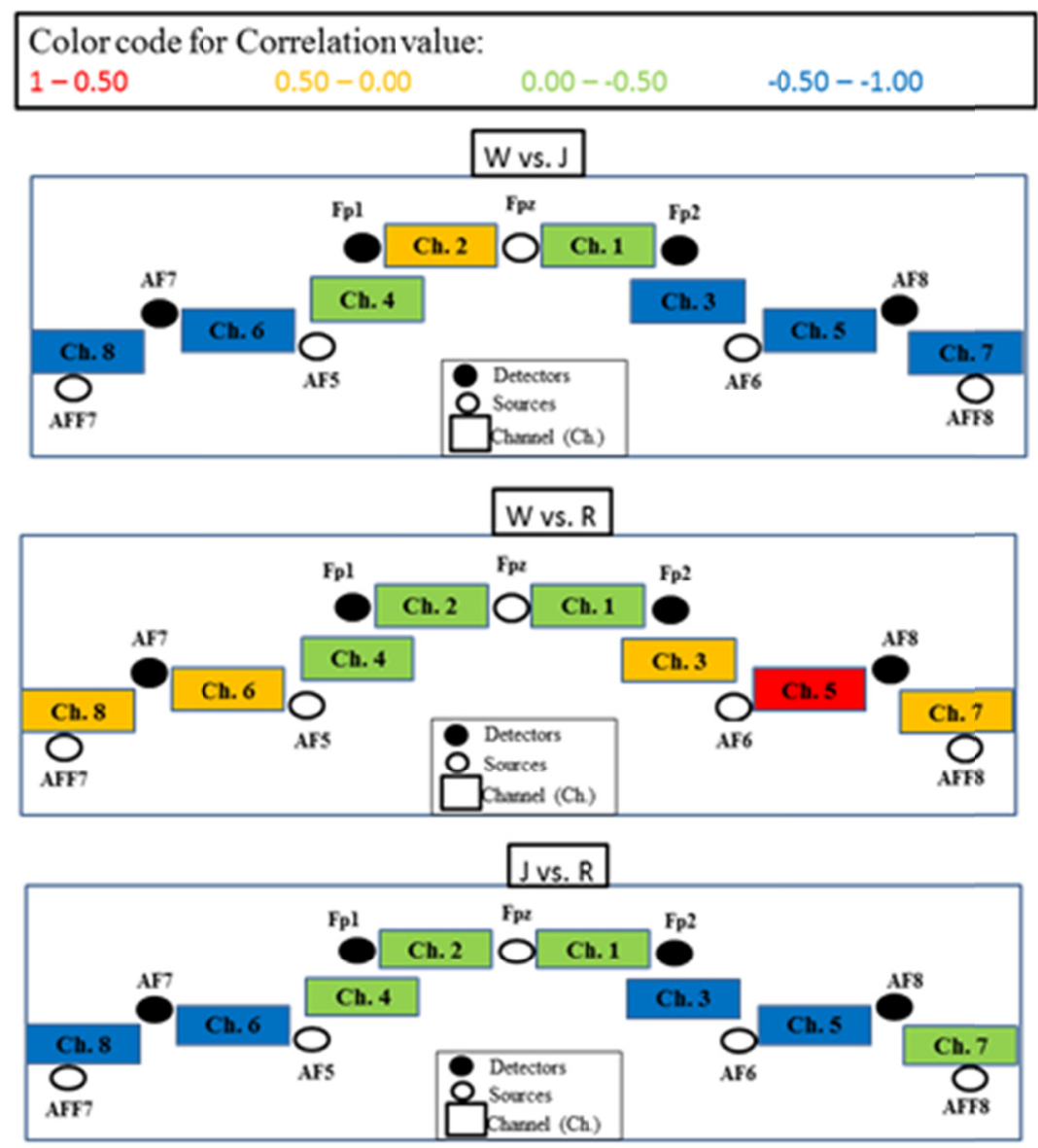

Figure 5.5: Inter stimuli ( $W$ vs. $J, W$ vs. $R$ and $J$ vs. $R$ ) zero order correlation analysis using $H b T$ data for all channels in the result using color code for the range of correlation values. As shown in the color code box correlation values in the range of 1.00 to 0.50 has been assigned red, 0.50 to 0.00 orange, 0.00 to -0.50 green and -0.50 to -1.00 blue. Using the source detector layout described in the Figure 1 each channel has been color coded, as described above, based on the correlation value obtained using HbT data for each stimuli pair i.e. $W$ vs. $J, W v s . R$ and $J$ vs. $R$.

From Figure 5.4, it is apparent that there is significant positive correlation or synchrony (>90\%) between the left and right pre-frontal cortex (Ch.1 vs. 2 and Ch.3 vs. 4) for all stimuli (W, J, R), and for all HRFs ( $\mathrm{HbO}, \mathrm{HbR}$, and $\mathrm{HbT})$. As we move away from the pre-frontal center region, the correlation between the left vs. right channels become less positively correlated, and in some cases, a negative correlation is observed. In other 
words, the left and right cortex's response to all stimuli begin to differ in the anterior frontal regions (Ch.6 vs. 5 and Ch.7 vs. 8), more preferably in the oxygenated blood flow (i.e. $\mathrm{HbO}$ ) than de-oxy blood flow (i.e. HbR).

From Figure 5.5, it is apparent that the correlation varied across the stimuli because if the HRF data ( $\mathrm{HbO}, \mathrm{HbR}$ and $\mathrm{HbT}$ ) only represented the systemic physiology, then the correlation across the stimuli would have been the same. However, from the results (Figure 5), it can be observed that the correlation varied across the stimuli, validating that the correlations represent the brain activity over systemic physiology.

\subsubsection{Study 3: Lateralization}

The lateralization index helps determine the dominant channel in a channel pair (here left $v s$. right) in response to a given stimuli. The schematic in Figure 5.6 shows the dominant channels (when comparing the left vs. right symmetric channels) in the frontal cortex in response to each of the stimuli, based on the overall HRF (i.e. HbT).

From Figure 5.6, it is observed that during the Word generation task neither the left nor right prefrontal cortex (Ch.1 and 2) was dominant, i.e. there was a bilateral activation. Upon moving to the anterior frontal cortex, a distinct left cortical dominance (Ch.4 and 6) is observed.

During the Jaw movement task, bilateral activation was observed in the pre-frontal cortex and random dominance of the channels were observed in the anterior frontal cortex (i.e., dominance of a single channel on the left (Ch.6) and on the right (Ch.7)). This random 
dominance may be due to the dominance in the muscle movement of one side of skull or the jaw movement pattern of the participant while performing the task. During the Rest task, there was bilateral dominance in the entire frontal cortex, except in the one channel of the anterior frontal region (Ch.7). The observance of bilateral dominance during Rest task is similar to the finding from previous $f$ MRI study, performed by Salvador et al. (Salvador et al, 2005) on 5 healthy subjects during resting state, where significant coherence and hence bilateral activation (no dominance) was observed in superior frontal gyrus dorsolateral and medial.

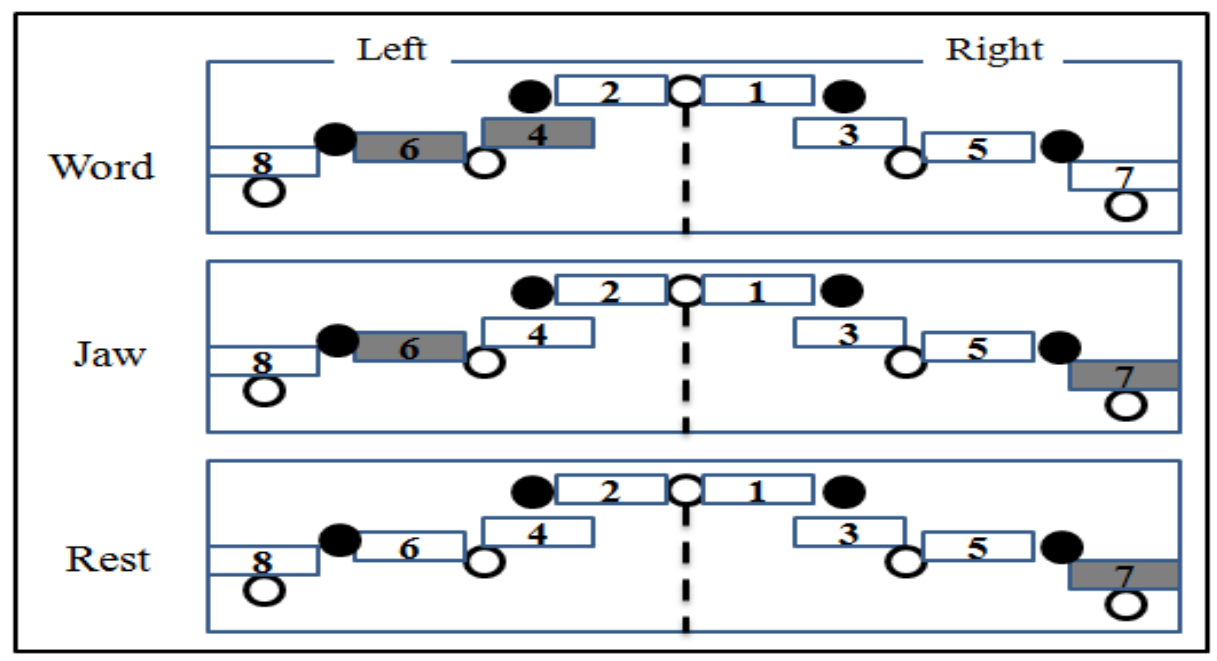

Figure 5.6: Cortical dominance representations using source detector layout as described in Figure 5.1 across all left vs. right channel pairs (4 of them) for each of the three stimuli (Word, Jaw, Rest) in the frontal cortex (averaged across all participants). The dominant channel pair (across the left and right cortex) is shaded grey.

\subsection{Discussion}

The brain activation in terms of $\mathrm{HbO}, \mathrm{HbR}$, and $\mathrm{HbT}$ were significantly different in only selective channels when the stimuli compared were Word vs. Jaw movement. The verbal fluency Word task tends to significantly differ from the Jaw movement task in the prefrontal (Ch.1, 2 and 4) and right anterior frontal cortex (Ch.7) as observed from $\mathrm{HbO}$ and 
HbR responses. The physical Jaw movement typically does not affect the pre-frontal cortex, causing a significant difference in the hemodynamic response from language cognition (i.e. verbal fluency Word task) in comparison the Jaw movement task. On the contrary, there is insignificant brain activation in the anterior frontal cortex (except in Ch.7) during Word task that it is masked by the Jaw movement task. This probably causes the no difference in $\mathrm{HbO}$ and $\mathrm{HbR}$ responses between Word vs. Jaw task, as observed in the previous study by Jayakar et al. (Jayakar et al, 2005). NIRS signal of muscular origin is present across the channels in the frontal cortex, since the muscles tend to move during Jaw movement (Jayakar et al, 2005). Hence the NIRS signal of cerebral origin cannot be distinguished from those of muscular origin during the Word generation and Jaw movement task, especially in the anterior frontal. When the $\mathrm{HbO}$ and $\mathrm{HbR}$ responses are combined into the overall hemodynamic $(\mathrm{HbT})$ response, it is clear that the left frontal cortex (pre- and anterior) significantly differs in the brain activation when comparing the Word vs. Jaw movement task.

The current NIRS brain activation observations are similar to past studies, demonstrating predominant left cortex activation over the right cortex during verbal fluency tasks (Sakatani et al, 1998; Watanabe et al, 2000). In addition, the activation of the anterior and pre-frontal cortex observed during the Word generation task is similar to past NIRS studies (Elfgren et al, 1998; Hermann et al, 2003; Hermann et al, 2006; Kubota et al, 2005; Matsuo et al, 2000; Sakatani et al, 1998), in terms of the increase in $\mathrm{HbO}$ and $\mathrm{HbT}$, and decrease in HbR concentrations. 
The connectivity studies demonstrated that there is symmetry in the hemodynamic response in the pre-frontal cortex independent of the stimuli, unlike in the anterior frontal cortex. A past $f$ MRI study also observed that the left and right frontal cortices were not in sync across the stimuli during brain activation (Binder et al, 1997). The result obtained from the connectivity analysis shows that the synchrony in the activity of the left and the right cortex differs with the cortical location (pre- or anterior frontal) and also with the kind of stimuli (Word, Jaw, or Rest) presented.

In general, the verbal fluency task consists of both executive (thinking) and language component. The possible reason for the difference in the laterality in the prefrontal and anterior frontal region is that the prefrontal region might be principally associated with the executive function of the verbal fluency task than language specific activation (Hermann et al, 2003). This is supported from past neuroimaging studies, such as PET or $f$ MRI, which has described a bilateral prefrontal activation for executive functions (Baker et al, 1996; D’Esposito et al, 1995). Additionally, in a recent NIRS study bilateral prefrontal activation was observed during the execution of the Wisconsin Card Sorting Test - a widely used test of executive function (Fallgatter et al, 1998). This further supports the current NIRS studies demonstrating statistically significant bi-lateral activation in the left and right prefrontal cortex during the verbal fluency task.

An earlier fMRI study performed by Binder et al. (Binder et al, 1997) demonstrated activation of the inferior, middle, and superior frontal gyri bilaterally during the language task along with Broca's area. The study also demonstrated that the frontal areas involved in language extend well beyond the traditional Broca's area to include much of the lateral 
and medial prefrontal cortex. This thereby indicates the existence of language cortex in several prefrontal regions outside Broca's area (Binder et al, 1997). Also, the left cortical hemispheric dominance observed during the Word generation task for right handed individuals in the current study is similar to the finding from previous NIRS (Noguchi et al, 2002) and fMRI (Binder et al, 1997; Posner et al, 1990; Rueckert et al, 1994).

In summary, significant differences in brain activation during verbal fluency (Word task) compared to baseline condition (Jaw and/or Rest) was observed. Lateralization results of left cortical dominance of language in the anterior frontal region and bilateral activation in the prefrontal region supports the observations in our functional brain connectivity study. The bilateral activation in the pre-frontal cortex (for any given stimuli) from the lateralization studies (Figure 5.6) appear as strong positive correlations in the same region from the functional connectivity analysis (see Figure 5.4). Similarly, the left/right dominance in different channels of the anterior frontal $(\mathrm{Ch} .4,6,7)$ for a given stimuli, as observed from the lateralization studies, appear as negative or weaker positive correlations in the $\mathrm{HbT}$ functional connectivity analysis of the anterior frontal cortex (see Figure 5.4). Hence bi-lateral activation mimics the significant positive correlation found in pre-frontal cortex (for any stimuli) and left anterior frontal cortical language lateralization mimics the negative and zero correlation found in the anterior frontal cortex (for any stimuli). Also differences in the connectivity in the anterior-frontal cortex across all the stimuli concur with the differences in the left/right dominance from the lateralization study. 
Additionally, the left cortical activation during verbal fluency is observed as left hemispherical dominance from the cortical lateralization study. Also, the asymmetry in the functional connectivity of the left and right anterior frontal cortex correlates to the left cortical dominance and significant activation in the same anterior frontal region. Thus, the results obtained from brain activation, connectivity, and cortical lateralization synchronize in concluding that the left frontal cortex responds significantly in verbal fluency, with preferred dominance and connectivity differences in the left anterior frontal.

\subsection{Conclusion}

Verbal fluency task was performed using NIRS technique to elucidate the brain activation, connectivity and lateralization of the language in the frontal cortex of the normal adult brain. In this study, the change in $\mathrm{HRF}(\mathrm{HbO}, \mathrm{HbR}$ and $\mathrm{HbT}$ ) during the activation supports the hypothesis (Hoshi et al, 1994; Kleinschmidt et al, 1996) that the blood oxygenation of the underlying region of interest depends upon the task requirements. Significant increase in brain activation was observed during the Word generation task in the prefrontal region of the brain as compared to baseline Jaw movement and Rest condition. The activation in the anterior frontal region during the word generation task was significantly different from the Rest condition but not from Jaw movement task. Connectivity study showed greater synchrony in the activity of the brain in the prefrontal region compared to the anterior frontal region. Also the synchrony in the activity changes with the tasks being performed by the participants. From the lateralization analysis it was observed that there was left cortical lateralization of 
language in the anterior frontal region and no cortical lateralization in the prefrontal region i.e. a bilateral activation was observed.

The primary future focus of the current study is towards epilepsy research. Surgical resection of epileptogenic tissue successfully alleviates seizures in the majority of patients with medically resistant partial epilepsy. Pre-surgical evaluation is carried out to map critical regions of the brain controlling motor and language functions so that these regions can be preserved during surgery. Eloquent cortical regions can be documented noninvasively by imaging techniques such as PET scans or $f$ MRI, but they are limited by inadequate temporal resolution. Furthermore, since these techniques require an awake and cooperative patient, they cannot be readily applied to the pediatric population. Mapping is therefore generally performed by electrically stimulating the cortex via subdurally implanted electrodes. Although highly effective, this technique incurs a potential albeit small risk for cortical tissue injury. The use of NIRS towards mapping the language and also motor functions in pediatric epilepsy patients, will prove more effective due to least impact from participant's movement during the study as well as maximum comfort in awake children. In addition to epileptic populations, NIRS approach involving activation and connectivity analyses has the potential to be applied to the schizophrenic and aphasic populations, where abnormality in the language processing is found. 
CHAPTER 6. Study 1b: NIRS and Language Skills in Fronto-Temporal Cortex

The study described in chapter 5 demonstrated the role of frontal cortical region of the brain in processing the language stimuli. Researchers have shown that along with the frontal region of brain several other regions situated in the fronto-temporal region also plays a role in processing the language stimuli. Hence in this chapter the study performed to investigate the activation, functional connectivity, effective connectivity and lateralization in the fronto-temporal region of brain in response to language stimuli has been described. This work has been submitted for publication in JOURNAL OF

\section{NEUROPHYSIOLOGY.}

\subsection{Introduction}

In the area of understanding language in the brain, several researchers have applied NIRS to investigate either activation (Kuwabara et al. 2006; Sato et al. 1999; Quaresima et al. 2002; Herrmann et al. 2003; Kubota et al. 2005; Kuwabara et al. 2006; Herrmann et al. 2006; Bortfeld et al. 2009), functional connectivity (Schecklmann et al. 2008) and/or lateralization (Watanabe et al. 1998; Kennan et al. 2002; Noguchi et al. 2002; Watson et al. 2004; Gallagher et al. 2008) (in isolation). However, the effective connectivity analysis of language regions using NIRS has not been attempted to date. Hence, in the current study NIRS has been used to investigate the activation, functional connectivity, effective connectivity and lateralization across the fronto-temporal cortical regions of the brain in response to language-based paradigms (i.e. word expression and word reception). The study was performed using a multi-channel NIRS instrument to non-invasively map the Broca's and Wernicke's region of 15 normal right-handed adults in response to these 
language paradigms. The hemodynamic response was used to statistically evaluate the functional connectivity, effective connectivity and lateralization across the frontotemporal cortical regions. The study demonstrates the feasibility of NIRS to investigate both the functional and effective connectivity in the language region of healthy adult population. As NIRS emerges as a non-invasive functional neuro-imaging technique, it circumvents several practical limitations of $f$ MRI (such as comfortable, portable, costeffective, robust to motion), which is well suited for clinical neuro-investigations in awake infants and children. In parallel, language is of importance for development and/or for some kinds of disease such as epilepsy, schizophrenia and aphasia. Therefore, if NIRS can be used to investigate the functional and effective connectivity across the language areas, complementary to $\mathrm{AMRI}$, NIRS could become a promising imaging modality for investigating changes of language-related brain interactions during development and/or impairment of patients.

\subsection{Materials and methods}

\subsubsection{Instrumentation}

In the current study, anterior left (AL) (which contains the Broca's region), posterior left $(\mathrm{PL})$ (which contains the Wernicke's region), anterior right (AR) and posterior right (PR) regions were non-invasively imaged using imaged using a frequency-domain based optical imaging system, Imagent (ISS, Inc., Champaign, IL) described in section 3.1. During this study 14 laser diodes sources (at each wavelength, 690 and $830 \mathrm{~nm}$ ) and 4 detectors were used. The source-detector layout (based on the 10-10 electrode placement) on the fronto-temporal regions is schematically shown in Figure 6.1. A 
custom-built "brain cap" was used to hold the source ( $400 \mathrm{~nm}$ core diameter) and detector fibers $(2.5 \mathrm{~mm}$ bundle diameter) in place during the NIRS studies where in the separation between the source and detector was $3 \mathrm{~cm}$. Optical signals were acquired in real-time (at $\sim 1.3 \mathrm{~Hz}$ ) from each source -detector pair or channel (Ch.), in response to the stimuli presented (as described in Section "Stimuli") using the software package (BOXY) of the instrument (Imagent).

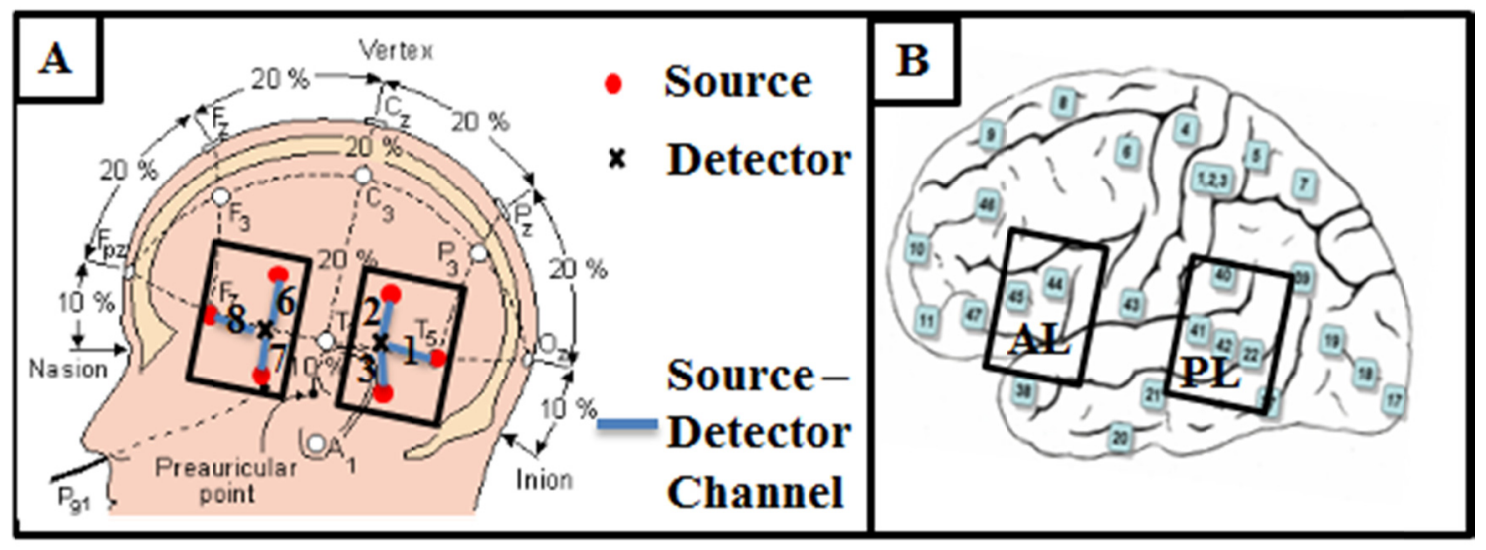

Figure 6.1: A. Arrangement of the source-detector layout employed during the study. The layout follows the standard International 10-10 (Jurcak et al. 2007) electrode placement system. The red circles and black x-mark represent sources and detectors, respectively. The line represents the path between the sources and detectors (i.e. channels). B. The Brodmann's layout of the different regions in the brain. The rectangular highlighted area represents the area investigated during the study. The figure represents the Brodmann area corresponding to the 10-10 electrode placement system. The channels 6, 7 and 8 were placed in such a way that it encompasses the Brodmann area 44 and 45 which contains the Broca's region (called Anterior left ( $A L)$ in the figure), while the channel 1, 2, and 3 were placed in such a way that it encompasses the Brodmann area 39 and 40 which contains the Wernicke's region (called Posterior left (PL) in the figure). The channels were correspondingly placed on the right side to form the right homologue (i.e. Anterior Right (AR) and Posterior Right (PR)) of the AL and PL, respectively. 


\subsubsection{Participants}

Fifteen healthy right-handed adults (between 18-30 years of age) were enrolled for the current study. All the participants were fluent English speakers, although their first language was not English. The handedness of the participant was based on the predominant use of a particular hand by the participant. The study was performed at the Brain Institute of Miami Children's Hospital (Miami, FL). This study was approved by the Florida International University's (FIU) Institutional Review Board (IRB), and written consent(s) were obtained from each participant before the study.

\subsubsection{Experimental design and protocol}

The current NIRS study is focused in understanding how the information flows across the Broca's and Wernicke's regions and their right homologue, as these underlying cortical regions responds to a language-based paradigm. During the study the participants were seated in a comfortable chair (upright) in a quiet room and the custom-built optical cap was placed on their head (with minimal hair interference). Two separate paradigms were presented during the study: (i) word expression paradigm and (ii) word reception paradigm. Both the paradigms were a block-based experimental design, with a total of 5 blocks in each paradigm, and each paradigm repeated twice (i.e. two replicates) by each participant.

\section{Word Expression Paradigm}

The word expression paradigm consists of 5 blocks of three 30-second stimuli, namely Word generation (W), Jaw movement (J), and Rest (R). Prior to the block-designed paradigm, an initial 30 -sec rest period was employed to bring the brain activity of the 
participants to baseline. During the word generation (W) stimuli the participants were asked to say as many possible words (without repetition) beginning with a randomly presented alphabet (in English). During the jaw movement (J) stimuli, the participants were asked to randomly move their jaw without actually speaking. During the rest (R), the participants were asked to minimize their thoughts and look at a white wall (Murias et al. 2007; Lu et al. 2010; Zhang et al. 2010; Chaudhary et al. 2011).

\section{Word Reception Paradigm}

The word reception task consists of 5 blocks of three 30-second stimuli, namely Forward audio, Reverse audio, and Rest stimuli. Prior to the block-designed paradigm, an initial 30 -sec rest period was employed to bring the brain activity of the participants to baseline. During the forward audio (FA) stimulus, the participants listened to a passage (rated at a $6^{\text {th }}$ grade reading level, http://www.fcat.fldoe.org). During the reverse audio (RA) stimulus, the participants listened to the same passage but in reverse order (i.e. from the last word to the first). During the rest (R), the participants were asked to minimize their thoughts and look at a white wall (Chaudhary et al. 2011; Lu et al. 2010; Murias et al. 2007; Zhang et al. 2010).

\subsection{NIRS signal analysis}

In the current studies, the average of the modulated light signal (i.e. DC signal) was employed in post-processing real-time one dimensional (1-D) hemodynamic responses across each source-detector pair (or channel). The optical data was post processed to obtain averaged hemodynamic response function (HRF) data as described in section 4.4. From 30 trials (i.e. 15 participants $\times 2$ repetitions), only 19 trials were used during the 
grand average in each task, while the 11 other trials were discarded. The trials with poor signal to noise ratio (SNR) were discarded based on the PMT's noise threshold level as determined by the Boxy software's interface. Prior to averaging the hemodynamic responses $(\mathrm{HbO}, \mathrm{HbR}$, and $\mathrm{HbT})$ across all trials, the mean and standard deviation across trials were evaluated in order to assess the consistency in the responses across participants and repetitions. Also p-value analysis was performed to ascertain the difference between the grand average of the repetitions across the subjects with each subject's hemodynamic response data. The result of the p-value analysis indicated that there was no significant difference between the grand average of the data and each subject's data. Further, the grand averaged channel-wise data was averaged to create 4 distinct regions anterior left (AL) (which contains the Broca's region), posterior left (PL) (which contains the Wernicke's region), anterior right (AR) and posterior right (PR). Each region was represented by the averaged individual channels corresponding to that region (as shown in Figure 6.1). The averaging is based on the reasoning that the source detector layout corresponding to anterior left encompasses the regions containing the Brodmann area BA 44 and BA 45, which represents the traditional Broca's area. While the source detector layout corresponding to posterior left encompasses the region containing Brodmann area BA 39 and BA 40, which represent the traditional Wernicke's region. Channels 4 and 5 were excluded from the regional analysis to circumvent the volume conduction effect. The volume conduction effect is potentially induced when a single source is shared by two different detectors (Rykhlevskaia et al. 2006). Paired t-test was performed between the averaged hemodynamic data of a particular region with respect to every channel data of that particular region, first at 5\% level of significance 
and then at $1 \%$ level of significance. The $p$-value of the paired t-test performed at $1 \%$ level of significance using averaged hemodynamic data for word expression and word reception paradigm is shown in Table 6.1 and Table 6.2, respectively.

\begin{tabular}{|l|c|c|c|c|c|c|c|c|c|}
\hline & \multicolumn{3}{|c|}{ HbO } & \multicolumn{3}{c|}{ HbR } & \multicolumn{3}{c|}{ HbT } \\
\cline { 2 - 10 } & Word & Jaw & Rest & Word & Jaw & Rest & Word & Jaw & Rest \\
\hline L1 vs. PL & 0.70 & 0.09 & 0.16 & 0.22 & 0.51 & 0.22 & 0.95 & 0.06 & 0.10 \\
\hline L2 vs. PL & 0.22 & 0.27 & 0.73 & 0.86 & 0.30 & 0.18 & 0.51 & 0.22 & 0.50 \\
\hline L3 vs. PL & 0.25 & 0.79 & 0.09 & 0.07 & 0.76 & 0.32 & 0.76 & 0.17 & 0.34 \\
\hline L6 vs. AL & 0.20 & 0.37 & 0.92 & 0.45 & 0.15 & 0.33 & 0.23 & 0.29 & 0.59 \\
\hline L7 vs. AL & 0.84 & 0.79 & 0.13 & 0.11 & 0.21 & 0.49 & 0.02 & 0.13 & 0.39 \\
\hline L8 vs. AL & 0.44 & 0.33 & 0.82 & 0.07 & 0.51 & 0.88 & 0.04 & 0.89 & 0.61 \\
\hline R1 vs. PL & 0.21 & 0.64 & 0.46 & 0.05 & 0.66 & 0.78 & 0.60 & 0.88 & 0.30 \\
\hline R2 vs. PL & 0.36 & 0.72 & 0.09 & 0.10 & 0.72 & 0.42 & 0.82 & 0.65 & 0.28 \\
\hline R3 vs. PL & 0.46 & 0.32 & 0.16 & 0.41 & 0.52 & 0.68 & 0.19 & 0.43 & 0.30 \\
\hline R6 vs. AL & 0.55 & 0.70 & 0.30 & 0.06 & 0.85 & 0.18 & 0.71 & 0.35 & 0.01 \\
\hline R7 vs. AL & 0.58 & 0.08 & 0.38 & 0.75 & 0.46 & 0.39 & 0.85 & 0.01 & 0.12 \\
\hline R8 vs. AL & 0.61 & 0.86 & 0.11 & 0.18 & 0.53 & 0.14 & 0.18 & 0.50 & 0.45 \\
\hline
\end{tabular}

Table 6.1: The p-values of the paired t-test performed using the average across all participants $\mathrm{HbO}, \mathrm{HbR}$ and $\mathrm{HbT}$ data of a particular region with the every single channel data of that particular region at $1 \%$ level of significance for word expression paradigm. A p-value $\geq 0.01$ indicates no significant difference between the regional data and each individual channel data.

It can be observed from these tables that no significant difference was observed between the averaged channel data (that forms a region) and individual channels within particular 
region, across any of the stimuli/paradigm. The t-test demonstrated that the regional data truly represents the average of channel data in that particular region. Henceforth, the averaged regional wise $\mathrm{HbO}, \mathrm{HbR}$ and $\mathrm{HbT}$ was used to perform 1) activation and lateralization, 2) functional connectivity and 3) effective connectivity across AL, PL, AR and PR regions for a) Word Expression paradigm and b) Word Reception paradigm.

\subsubsection{Activation and Lateralization Study}

Brain activation study, as described in section 4.4, was performed to explicate the differences in the hemodynamic response ( $\mathrm{HbO}, \mathrm{HbR}$ and $\mathrm{HbT})$ as a function of time, across the AL, PL, AR and PR and in response to the a) Word Expression paradigm and b) Word Reception paradigm. The entire window of $30 \mathrm{sec}$ for each stimuli (W, J, or R and FA, RA or R of the word expression and word reception paradigm respectively) was used to plot the averaged 1-D HRF plots (HbO - oxy-hemoglobin, HbR - deoxyhemoglobin and $\mathrm{HbT}$ - total hemoglobin) with respect to time for AL, PL, AR and PR region. Using the averaged hemodynamic data, three paired sample t-tests $(\mathrm{p}=0.05)$, as described in section 4.5, was performed for AL, PL, AR and PR region across two chosen 30-sec stimuli (i.e. $\mathrm{W}$ vs. J, W vs. R, and J vs. R for word expression paradigm and FA vs. RA, FA vs. R and RA vs. R for word reception paradigm). The three t-tests were adjusted using Holm's sequential Bonferroni procedure to control for the probability of a type I error. Alternatively, ANOVA analysis was also performed as described in Michael Hall's Master Thesis (http://digitalcommons.fiu.edu/etd/560/) where in the result of Holm's sequential Bonferroni procedure and ANOVA analysis was found to be same. Hence in this study Holm's sequential Bonferroni procedure was used. 
Cortical lateralization study, as described in section 4.7, was performed to determine the left vs. right hemispherical dominance in the anterior and posterior fronto-temporal regions, in response to each stimulus of the two paradigms.

\begin{tabular}{|l|c|c|c|c|c|c|c|c|c|}
\hline & \multicolumn{3}{|c|}{ HbO } & \multicolumn{3}{c|}{ HbR } & \multicolumn{3}{c|}{ HbT } \\
\cline { 2 - 10 } & FA & RA & Rest & FA & RA & Rest & FA & RA & Rest \\
\hline L1 vs. PL & 0.78 & 0.10 & 0.53 & 0.12 & 0.28 & 0.07 & 0.41 & 0.43 & 0.79 \\
\hline L2 vs. PL & 0.49 & 0.61 & 0.25 & 0.24 & 0.12 & 0.97 & 0.28 & 0.13 & 0.10 \\
\hline L3 vs. PL & 0.21 & 0.08 & 0.13 & 0.89 & 0.27 & 0.42 & 0.37 & 0.67 & 0.40 \\
\hline L6 vs. AL & 0.08 & 0.20 & 0.40 & 0.22 & 0.11 & 0.47 & 0.12 & 0.48 & 0.14 \\
\hline L7 vs. AL & 0.60 & 0.83 & 0.16 & 0.53 & 0.18 & 0.90 & 0.81 & 0.21 & 0.84 \\
\hline L8 vs. AL & 0.28 & 0.05 & 0.12 & 0.15 & 0.68 & 0.17 & 0.41 & 0.63 & 0.60 \\
\hline R1 vs. PL & 0.70 & 0.29 & 0.95 & 0.33 & 0.47 & 0.18 & 0.06 & 0.92 & 0.11 \\
\hline R2 vs. PL & 0.94 & 0.98 & 0.35 & 0.76 & 0.11 & 0.42 & 0.09 & 0.69 & 0.14 \\
\hline R3 vs. PL & 0.52 & 0.65 & 0.32 & 0.07 & 0.81 & 0.39 & 0.70 & 0.76 & 0.62 \\
\hline R6 vs. AL & 0.23 & 0.06 & 0.57 & 0.91 & 0.57 & 0.72 & 0.07 & 0.20 & 0.32 \\
\hline R7 vs. AL & 0.81 & 0.75 & 0.26 & 0.45 & 0.55 & 0.08 & 0.19 & 0.74 & 0.18 \\
\hline R8 vs. AL & 0.39 & 0.18 & 0.94 & 0.16 & 0.06 & 0.28 & 0.93 & 0.12 & 0.94 \\
\hline
\end{tabular}

Table 6.2: The p-value of the paired t-test performed using the average across all participants $\mathrm{HbO}, \mathrm{HbR}$ and $\mathrm{HbT}$ data of a particular region with the every single channel data of that particular region at $1 \%$ level of significance for word reception paradigm. A p-value $\geq 0.01$ indicates no significant difference between the regional data and each individual channel data. 


\subsubsection{Functional Connectivity Study}

In the present study, functional connectivity was performed to characterize the ways in which the left and right anterior and posterior regions i.e., AL, PL, AR and PR are functionally integrated or work in synchrony in response to language-based stimuli. This study was carried out by computing zero-lagged correlation, as described in section 4.5 , to elucidate the correlation and synchrony in the averaged hemodynamic data (i.e. $\mathrm{HbO}$, $\mathrm{HbR}$ and $\mathrm{HbT}$ ) and hence the connectivity in the neuronal circuit across AL, PL, AR and PR.

\subsubsection{Effective Connectivity Study}

Effective connectivity study was performed using granger causality, as described in section 4.7, to investigate the directional flow of information across the AL, PL, AR and PR as the language based stimuli was processed by these regions.

\subsection{Results}

The word expression and the word reception were two separate paradigms performed by the participants hence the activation and lateralization, functional connectivity and effective connectivity results will be presented separately for word expression and word reception paradigm.

\subsubsection{Study A: Word Expression Paradigm}

\subsubsection{Activation and Lateralization Study}

The hemodynamic response plots in terms of $\mathrm{HbO}, \mathrm{HbR}$ and $\mathrm{HbT}$ are provided in Figure 6.2 for each of the four fronto-temporal regions. 
The results of three-paired t-test statistical analysis at $p<0.05$ to determine the differences in activation (in terms of $\mathrm{HbO}, \mathrm{HbR}$, and $\mathrm{HbT}$ ) between two stimuli (Word vs. Jaw, Word vs. Rest, and Jaw vs. Rest) and across each AL, PL, AR and PR region is shown in Figure 6.3. The result of the three-paired t-test statistical analysis at $\mathrm{p}<0.05$ is also included in the Appendix A.1.

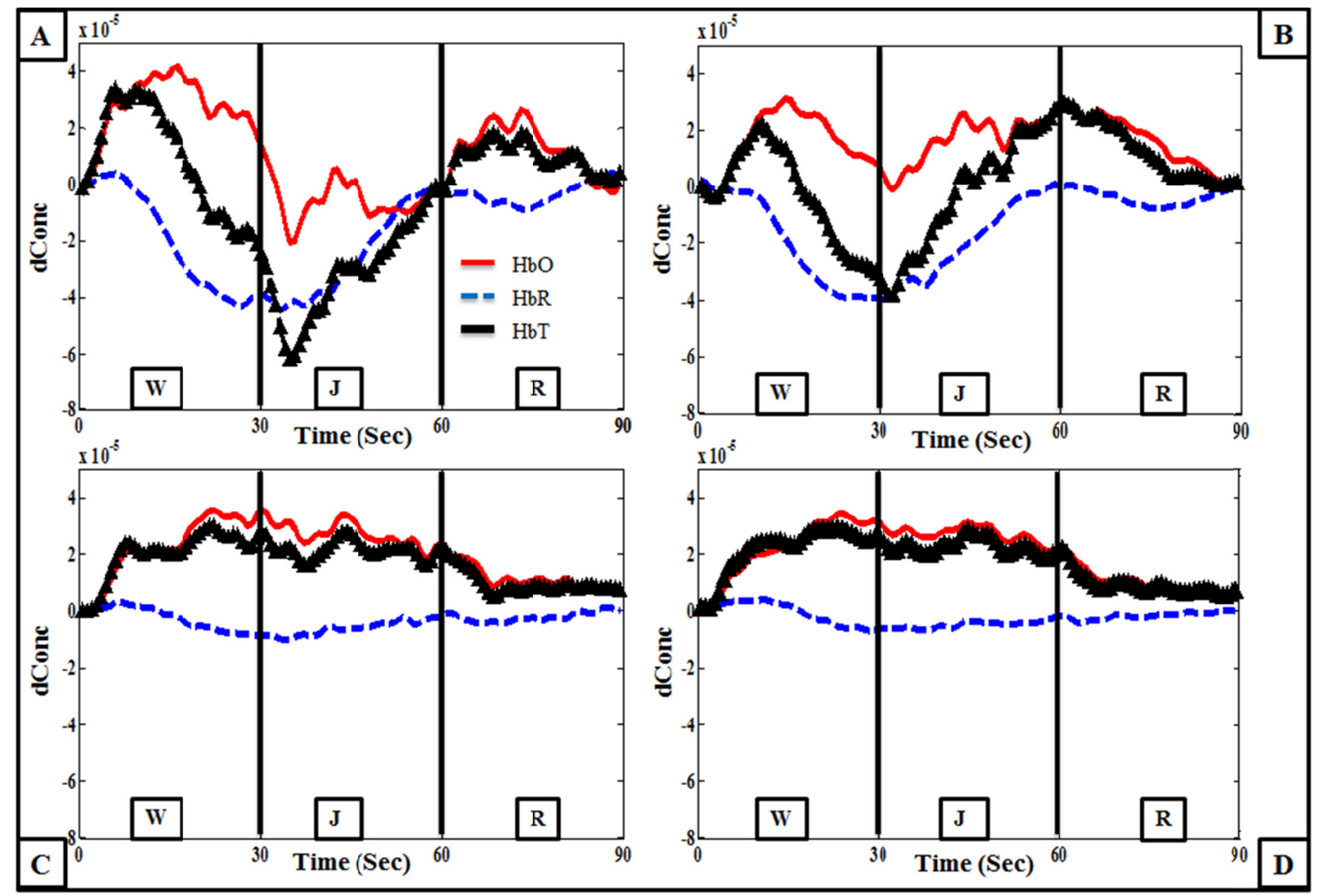

Figure 6.2: Hemodynamic change plotted against time (in seconds) for A. Anterior left $(A L)$ region, $B$. Anterior right (AR) region, C. Posterior left (PL) region and D. Posterior right (PR) region in response to the word expression based stimuli. Each plot shows the averaged hemodynamic data across all participants and for each stimuli including Word Generation (W) (0-30 sec), Jaw Movement (J) (31-60 sec), and Rest (R) (61-90 sec).

These results shown in Figure 6.3 are in terms of the evaluated p-value across the stimuli pair and for each AL, PL, AR and PR region. From these plots, it is can be seen that the brain activation in terms of $\mathrm{HbO}$ was significantly different in $\mathrm{AL}, \mathrm{PL}$ and $\mathrm{PR}$ region for 
the Word vs. Rest, Jaw vs. Rest and Jaw vs. Rest stimuli comparison. There was no significant difference in terms of HbR across any of the stimuli comparison and region except the Word vs. Rest and Jaw vs. Rest comparison in AL and AR region. In terms of $\mathrm{HbT}$ for the stimuli comparison Word vs. Jaw significant difference can only be found in the AL region, while there was significant difference for stimuli comparison Word vs. Rest and Jaw vs. Rest in all the regions.

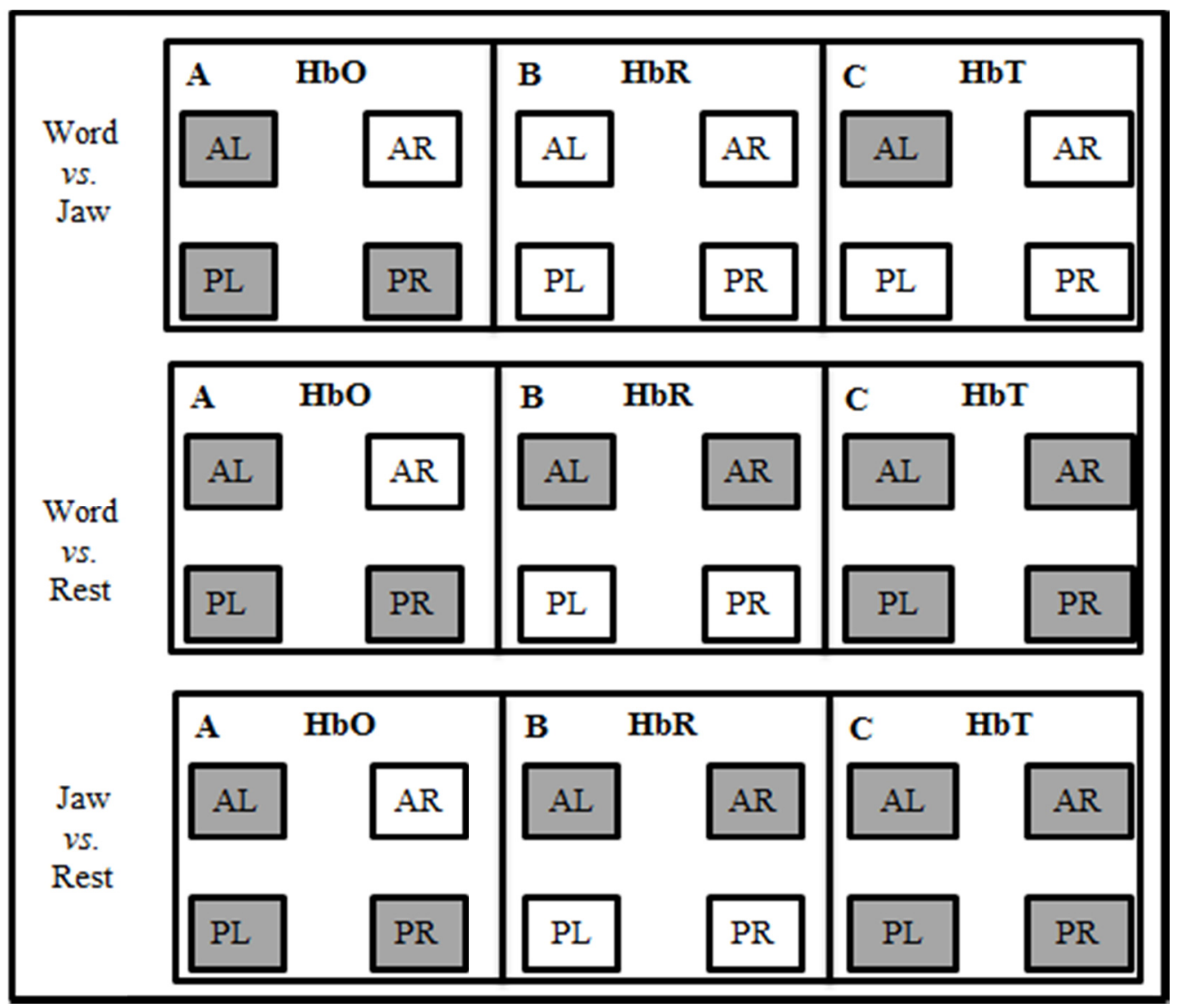

Figure 6.3: Pictorial representation of the differences in data ( $H b O, H b R$, and $H b T)$ across the three stimuli ( $W, J$, and $R$ ) in each region, obtained from a three-paired t-test. The grey shaded channels represent significant differences between the two stimuli ( $W v s$. $J, W$ vs. $R$, and $J$ vs. R) of interest for a given averaged (across all participants) hemodynamic data. The non-shaded white channels represent insignificant differences in the averaged hemodynamic data between the two stimuli of interest. 
The results from the lateralization study are shown in Figure 6.4, and it can be seen from Figure 6.4 that $\mathrm{AL}$ region is dominant during the Word Generation task. The AR region is dominant during the Jaw Movement task, and there is no dominance (or bilateral dominance) was observed during Rest.

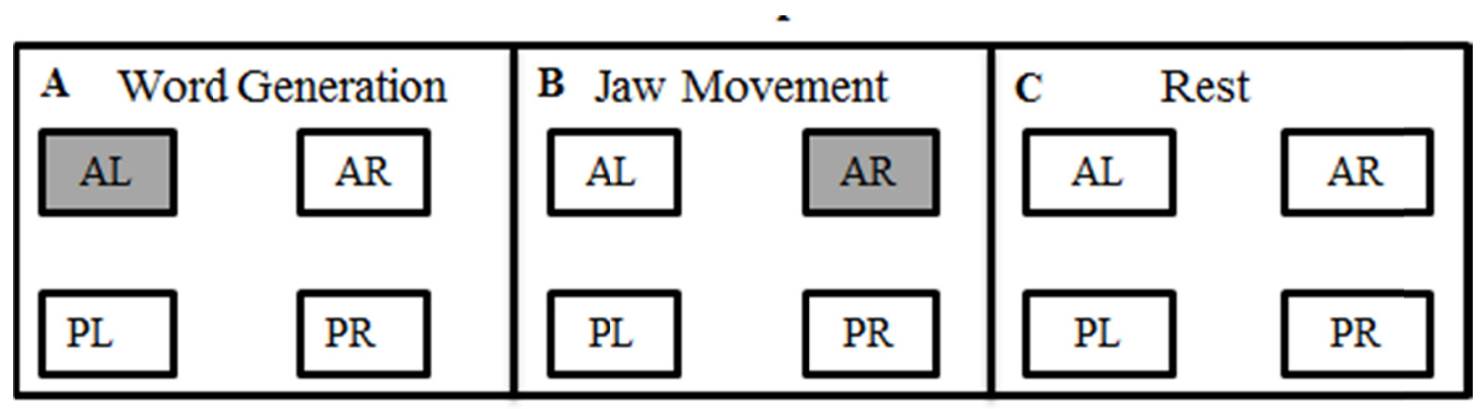

Figure 6.4: Lateralization diagram showing dominance (marked by grey) between left and right fronto-temporal regions during the three stimuli of the Word Expression Paradigm (obtained from the averaged HbT data across all participants). The four regions are - Left Anterior (LA), Right Anterior (RA), Left Posterior (LP), Right Posterior $(R P)$.

\subsubsection{Functional Connectivity Study}

The result from the functional connectivity as shown in Figure 6.5 is shown using four distinct weightage of solid line based on four different ranges of positive correlation value ( 0 to $0.25,0.25$ to $0.50,0.50$ to 0.75 and 0.75 to 0.1 ), and negative correlation value ( 0 to $-0.25,-0.25$ to $-0.50,-0.50$ to -0.75 and -0.75 to -0.1$)$.

The correlation coefficients obtained between two different regions for Word, Jaw and Rest using $\mathrm{HbO}, \mathrm{HbR}$ and $\mathrm{HbT}$ data is also shown in Figure 6.5, using a numerical value, along with the line representing the connectivity between two regions. 


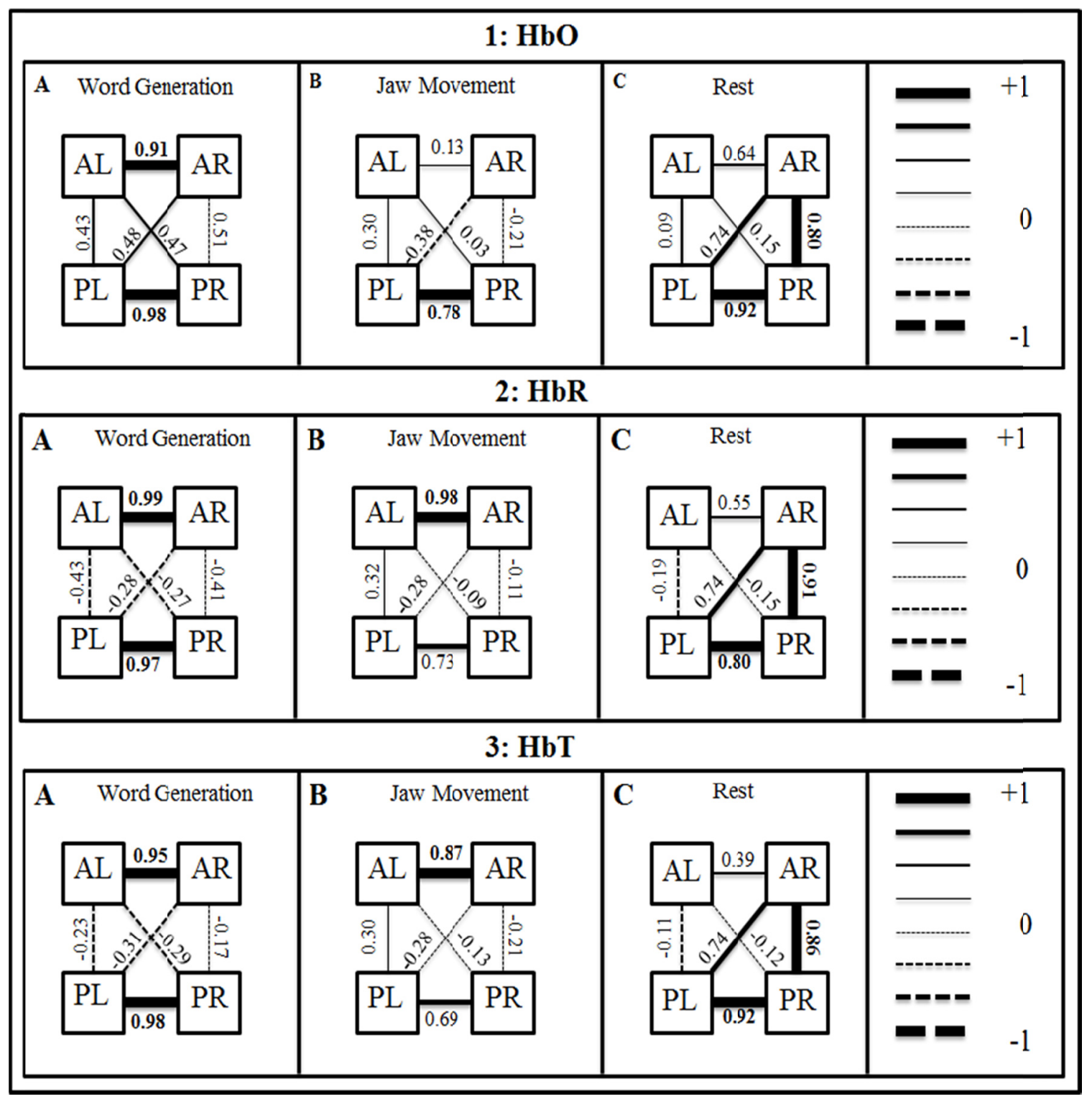

Figure 6.5: Pictorial representation of the result of functional connectivity analysis using zero order Pearson's correlation during word expression paradigm using average hemodynamic data across all participants 1: HbO, 2: HbR and 3: HbT data for (A) Word Generation, (B) Jaw Movement, and (C) Rest. The four regions under investigation are represented by four squares, where in the Anterior Left $(A L)$ region contains the Broca's region; Anterior Right (AR) region contains the right homologue of AL region; the Posterior Left (PL) region contains the Wernicke's region; and the Posterior Right (PR) region contains the right homologue of $P L$ region. 
From the Figure 6.5-1 (which corresponds to functional connectivity due to change in $\mathrm{HbO}$ ), Figure 6.5-2 (which corresponds to functional connectivity due to change in $\mathrm{HbR}$ ) and Figure 6.5-3 (which corresponds to functional connectivity due to change in $\mathrm{HbT}$ ) it can be seen that during the Word task there is significant positive correlation between AL vs. AR and PL vs. PR.

During Jaw Movement task there is significant positive correlation only between PL vs. $\mathrm{PR}$ in terms of change of $\mathrm{HbO}$ and $\mathrm{HbT}$ and between $\mathrm{AL}$ vs. AR in terms of change in HbR. During Rest there is significant positive correlation between PL vs. PR and AR vs. PR.

\subsubsection{Effective Connectivity Study}

The results from the effective connectivity are shown in Figure 6.6, where a thick arrow depicts strong output/input flow of information (i.e signal), and thin arrow depicts significantly weak output/input flow of information (i.e. signal). The strong or weak output/input flow of information was deduced based on the inequality $\mathrm{F}$ Value $>$ Critical Value (as described in Sub -Section "Effective Connective Study" of Section "Optical Data Analysis).

From Figure 6.6-1 (which corresponds to the effective connectivity due to changes in $\mathrm{HbO}$ ), it can be seen that during the Word task the AL region sends strong forward output flow of information to PL, AR and PR region; and the PL region sends strong forward output flow of information to AL and PR region. The AR region sends strong forward output flow of information to AL and PR region; and the PR region sends forward output flow of information only to AR region. During Jaw Movement task it can be seen that 
only AR sends strong forward output flow of information to PR, and the PR region sends strong forward output flow of information to AR and PL region. During Rest it can be seen that there is a strong forward output flow of information and backward input flow of information amongst $\mathrm{AL}, \mathrm{AR}, \mathrm{PL}$ and $\mathrm{PR}$.

From Figure 6.6-2 (which corresponds to the effective connectivity due to changes in $\mathrm{HbR})$, it can be seen that during the Word task there is a strong forward output flow of information and backward input flow of information amongst AL, AR, PL and PR. During Jaw Movement task it can be seen that there is a strong forward output flow of information from AL to PR, PL to AL and AR and PR to PL. During Rest it can be seen that there is a strong forward output flow of information and backward input flow of information amongst AL, AR, PL and PR except from AL to PR and PL to AR.

From Figure 6.6-3 (which corresponds to the effective connectivity due to changes in $\mathrm{HbT}$ ), it can be seen that during the Word task AL region sends strong forward output flow of information (i.e signal) to PL, AR and PR region; and the PL region sends strong forward output flow of information (i.e signal) to $\mathrm{AL}, \mathrm{AR}$ and $\mathrm{PR}$ region. The $\mathrm{AR}$ and PR sends strong forward output flow of information (i.e. signal) only to each other. During Jaw Movement, only AL sends strong output flow of information (i.e. signal) to PL, AR and AR; and AR to AL. During Rest it can be seen that there is a strong forward output flow of information (i.e. signal) and backward input flow of information (i.e. signal) amongst $\mathrm{AL}, \mathrm{AR}, \mathrm{PL}$ and $\mathrm{PR}$ except from $\mathrm{AL}$ to $\mathrm{PR}$ and $\mathrm{PL}$ to $\mathrm{AR}$. In short, the effective connectivity patterns in terms of $\mathrm{HbO}, \mathrm{HbR}$, and $\mathrm{HbT}$ differ across these front- 
temporal regions w.r.t the nature of the stimuli or Rest during the word expression paradigm.

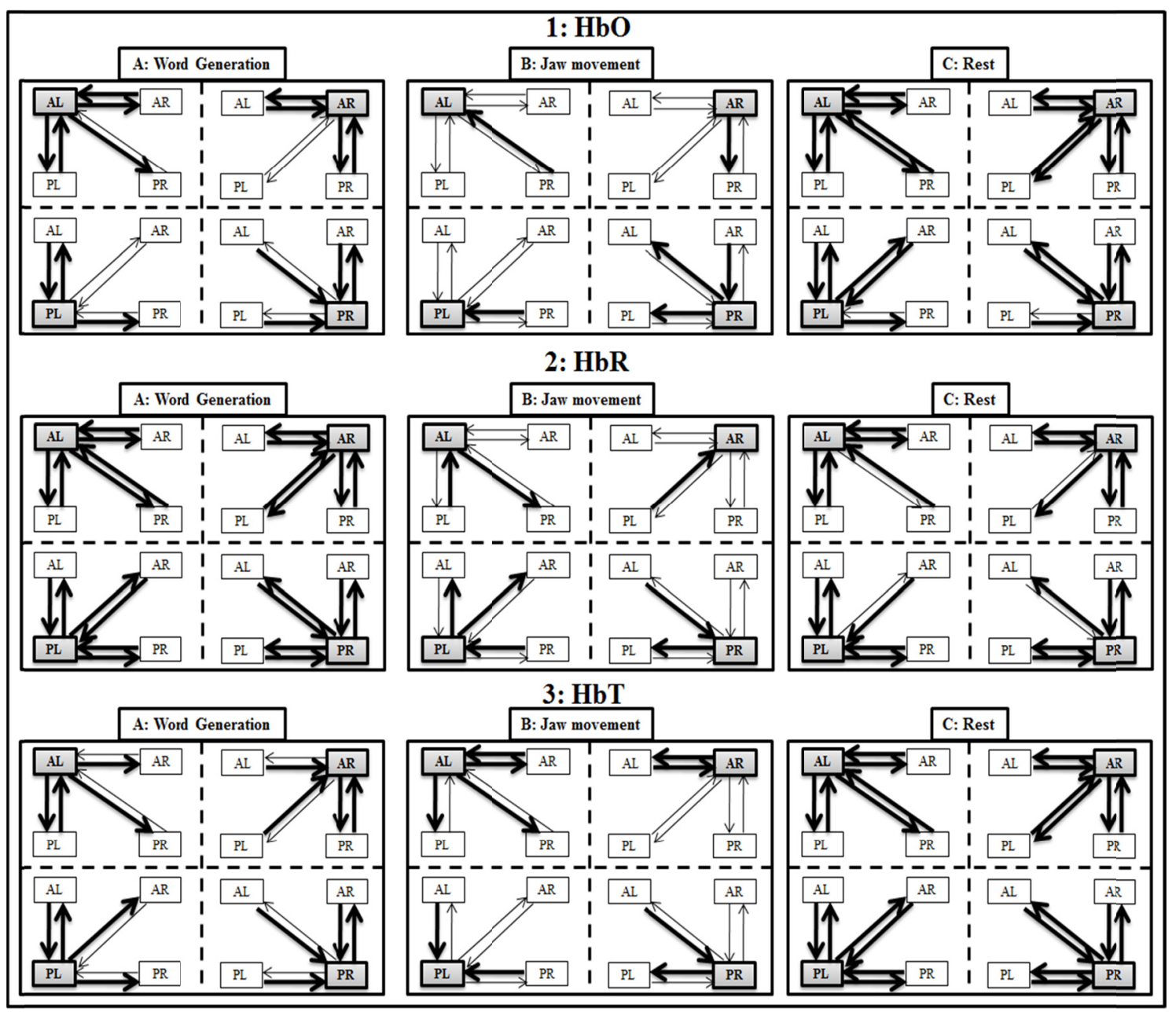

Figure 6.6: Pictorial representation of the causal and directional flow of information obtained from effective connectivity analysis (or granger causality analysis) during word expression paradigm using average hemodynamic data across all participants $1: H b O, 2$ : HbR and 3: HbT data, for (A) Word Generation, (B) Jaw Movement, and (C) Rest. The directional flow of information across the four regions (AL, PL, AR, and PR) is shown using thickening and thinning of arrows, where in the thick arrow shows strong flow of information and thin arrows shows weak flow of information. 


\subsubsection{Study B: Word Reception Paradigm}

\subsubsection{Activation and Lateralization Study}

The hemodynamic response plots in terms of $\mathrm{HbO}, \mathrm{HbR}$ and $\mathrm{HbT}$ are provided in Figure 6.7 for each of the four fronto-temporal regions.

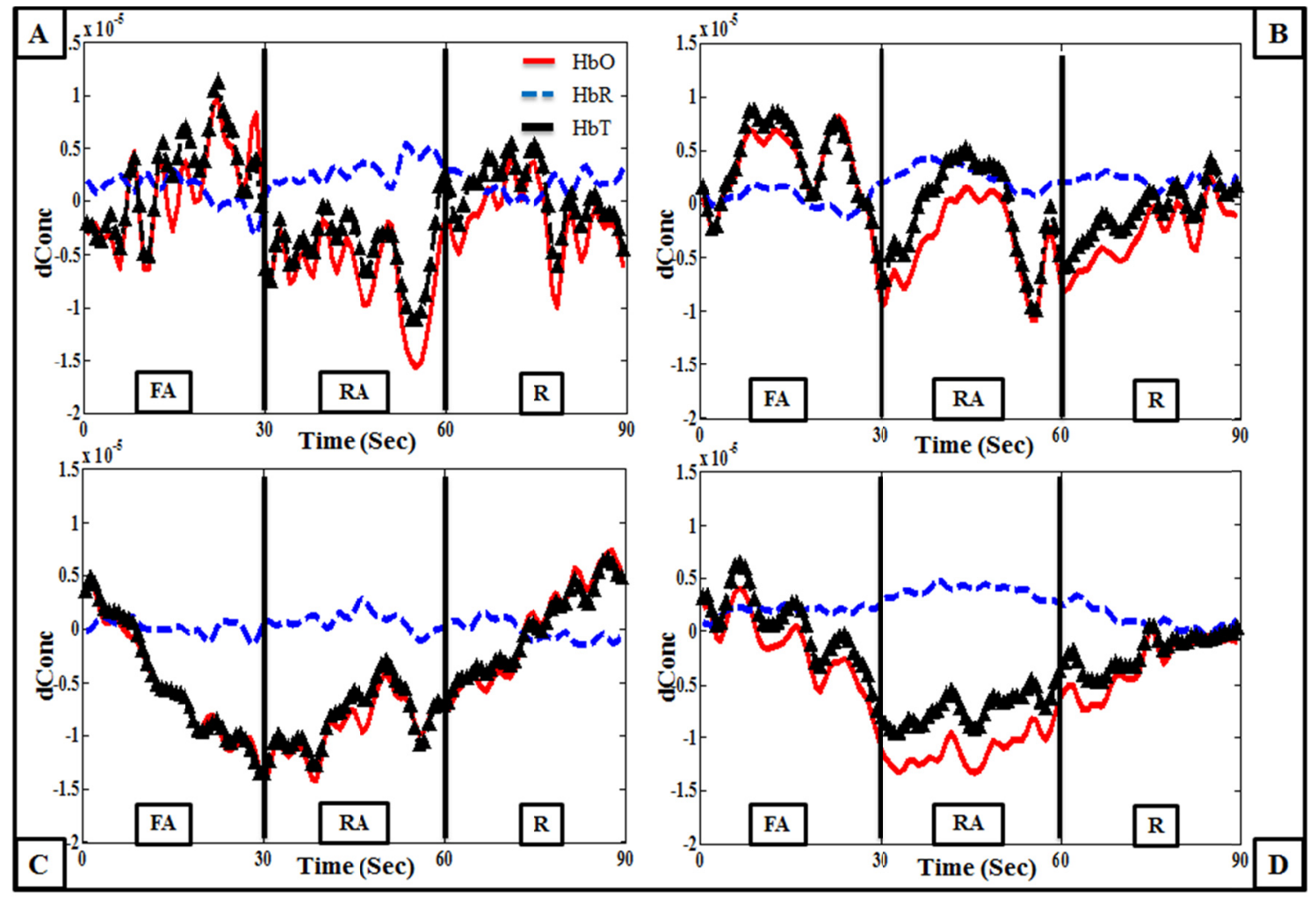

Figure 6.7: Averaged hemodynamic changes (across all participants) plotted against time (in seconds) for A. Anterior left (AL) region, B. Anterior right (AR) region, $C$. Posterior left (PL) region and D. Posterior right (PR) region in response to the word reception based stimuli. Each plot shows the averaged hemodynamic data for the block stimuli of Forward Audio task (FA), Reverse Audio task (RA), and Rest (R) performed by the participants.

The results of three-paired t-test statistical analysis at $p<0.05$ to determine the differences in activation (in terms of $\mathrm{HbO}, \mathrm{HbR}$, and $\mathrm{HbT}$ ) between two stimuli (Forward Audio vs. 
Reverse Audio, Forward Audio vs. Rest, and Reverse Audio vs. Rest) and across each AL, PL, AR and PR region is shown in Figure 6.8. The result of the three-paired t-test statistical analysis at $\mathrm{p}<0.05$ is also included in the Appendix A.2.

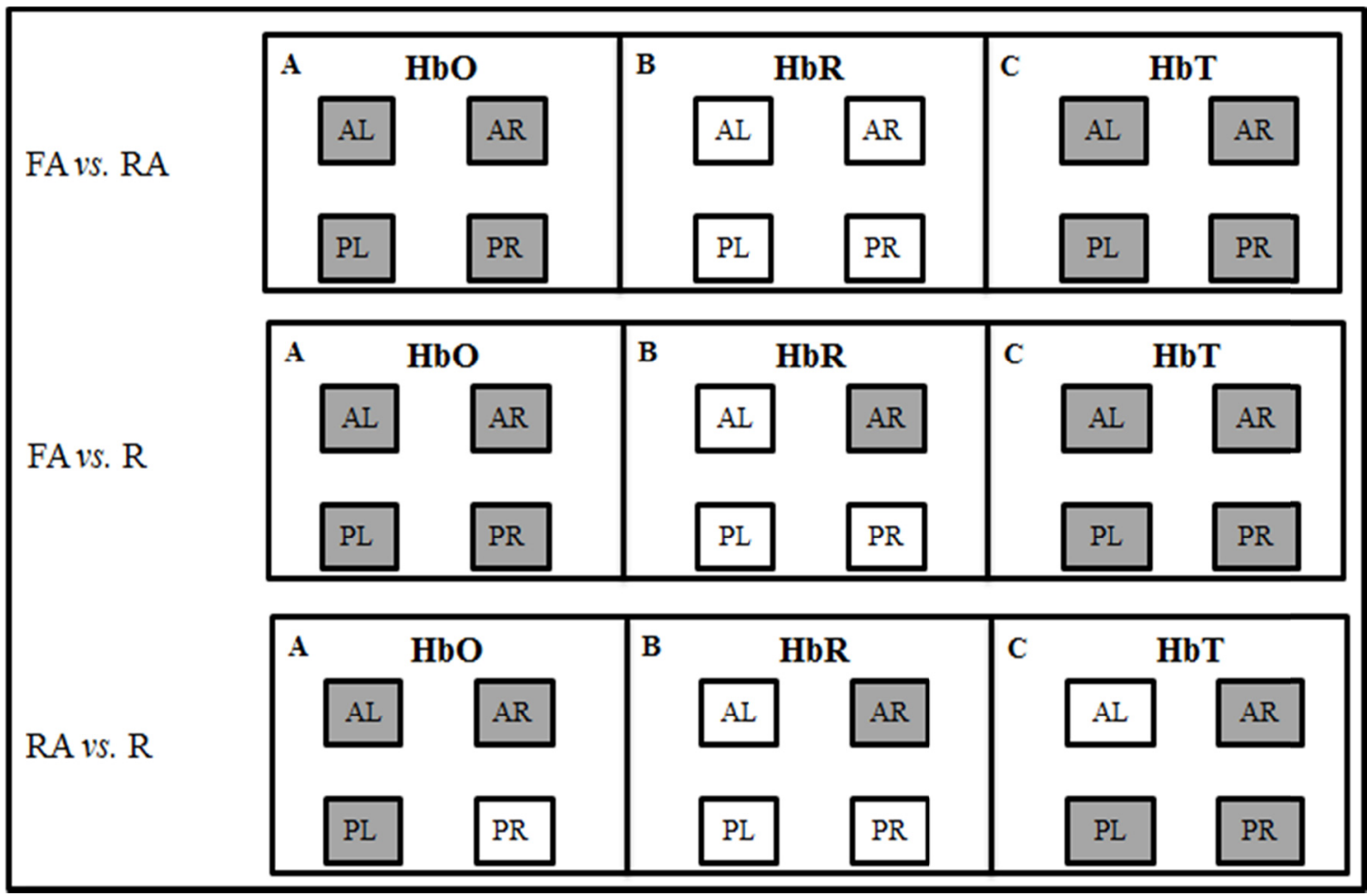

Figure 6.8: Pictorial representation of the differences in hemodynamic data ( $\mathrm{HbO}, \mathrm{HbR}$, and $H b T)$ across the three stimuli ( $F A, R A$, and $R$ ) in each region, obtained from a threepaired t-test. The grey shaded channels represent significant differences between the two stimuli ( $F A$ vs. RA, FA vs. $R$, and $R A$ vs. $R$ ) of interest for a given averaged hemodynamic data (across all participants). The non-shaded white channels represent insignificant differences between the two stimuli of interest for a given averaged hemodynamic data.

These results shown in Figure 6.8 are in terms of the evaluated p-value across the stimuli pair and for each AL, PL, AR and PR region. From these plots, it is can be seen that the brain activation in terms of $\mathrm{HbO}$ and $\mathrm{HbT}$ was significantly different across all the region for the Forward Audio vs. Reverse Audio, Forward Audio vs. Rest, and Reverse Audio 
vs. Rest stimuli comparison. There was no significant difference in terms of HbR across any of the stimuli comparison and region except the Forward Audio vs. Rest, and Reverse Audio vs. Rest comparison in AR region.

The results from the lateralization study are shown in Figure 6.9, and it can be seen from Figure 6.9 that PL region is dominant during the Forward Audio task. No dominance (or bilateral dominance) was observed during both the Reverse Audio task and Rest.

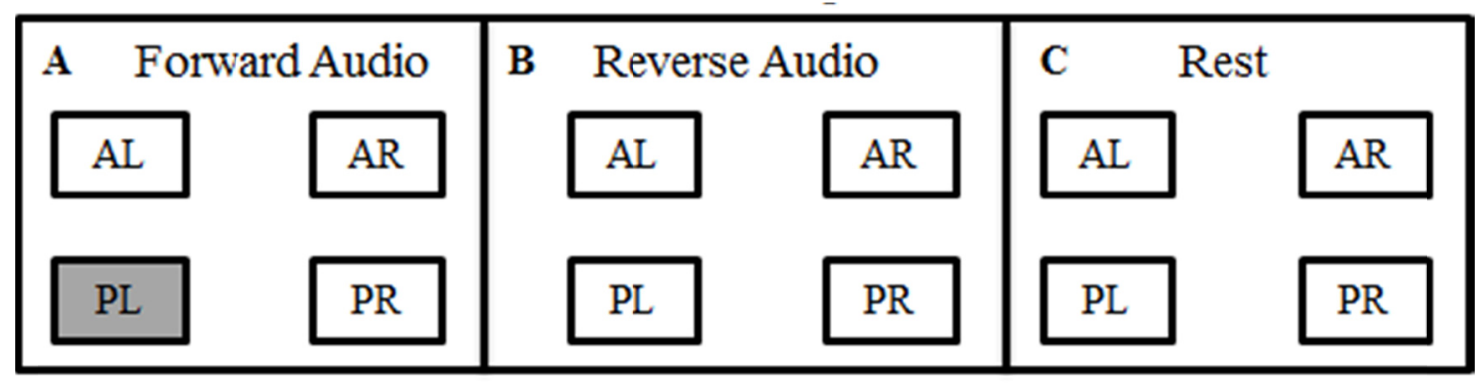

Figure 6.9: Lateralization diagram showing dominance (marked by grey) between left and right fronto-temporal regions during the three stimuli of the word expression paradigm obtained from averaged $H b T$ data (across all participants). The four regions are - Left Anterior (LA), Right Anterior (RA), Left Posterior (LP), Right Posterior (RP).

\subsubsection{Functional connectivity}

The result from the functional connectivity as shown in Figure 6.10 is shown using four distinct weightage of solid line based on four different range $(0-0.25,0.25-0.50,0.50$ -0.75 and $0.75-0.1$ ) of positive correlation value and four distinct weightage of dashed line based on four different range $(0--0.25,-0.25--0.50,-0.50--0.75$ and $-0.75--0.1)$ of negative correlation value. The correlation coefficient obtained between two different regions for Forward Audio, Reverse Audio and Rest using $\mathrm{HbO}, \mathrm{HbR}$ and $\mathrm{HbT}$ data is 
also shown in Figure 6.10, using a numerical value, along with the line representing the connectivity between two regions.

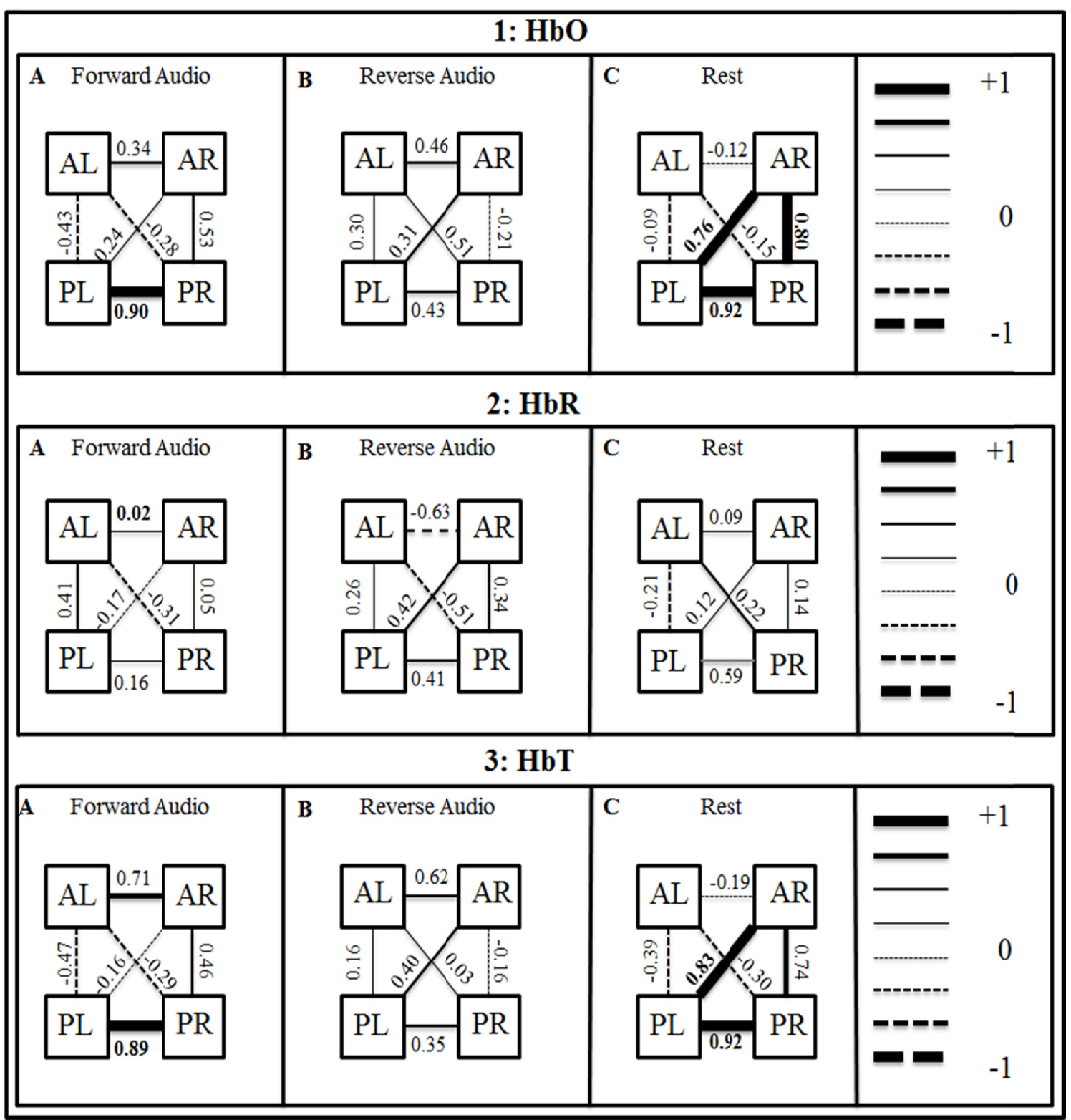

Figure 6.10: Pictorial representation of the result of functional connectivity analysis using zero order Pearson's correlation during word reception paradigm using average hemodynamic data across all participants 1: HbO, 2: HbR and 3: HbT data, for (A) Forward Audio, (B) Reverse Audio, and (C) Rest stimuli. 
From the Figure 6.10-1 (which corresponds to functional connectivity due to change in $\mathrm{HbO}$ ) and Figure 6.10-3 (which corresponds to functional connectivity due to change in $\mathrm{HbT}$ ) it can be seen that during the Forward Audio task there is significant positive correlation between PL vs. PR. During Reverse Audio task there is no significant positive correlation between any of the region. During Rest there is significant positive correlation between PL vs. PR, AR vs. PR and PL vs. AR. From the Figure 6.10-2 (which corresponds to functional connectivity due to change in $\mathrm{HbR}$ ) it can be seen that there is no significant correlation between any of the regions across Forward Audio task, Reverse Audio task and Rest.

\subsubsection{Effective Connectivity Study}

The results from the effective connectivity are shown in Figure 6.11, where a thick arrow depicts strong output/input flow of information (i.e. signal), and thin arrow depicts significantly weak output/input flow of information (i.e. signal). The strong or weak output/input of the signal was deduced based on the inequality $\mathrm{F}$ Value $>$ Critical Value (as described in Sub -Section "Effective Connective Study" of Section "Optical Data Analysis).

From the Figure 6.11-1 (which corresponds to the effective connectivity due to change in $\mathrm{HbO}$ ), it can be seen that during the Forward Audio task the AL region sends strong forward output flow of information to PL and AR region; and the PL region send strong forward output flow of information to AL and PR region. The AR and PR do not send any strong forward output flow of information. During Reverse Audio task it can be seen 
that only AL sends strong forward output flow of information to AR and the AR region sends strong forward output signal to PR region. During Rest it can be seen that there is a strong forward output flow of information and backward input flow of information amongst AL, AR and PR except PL.

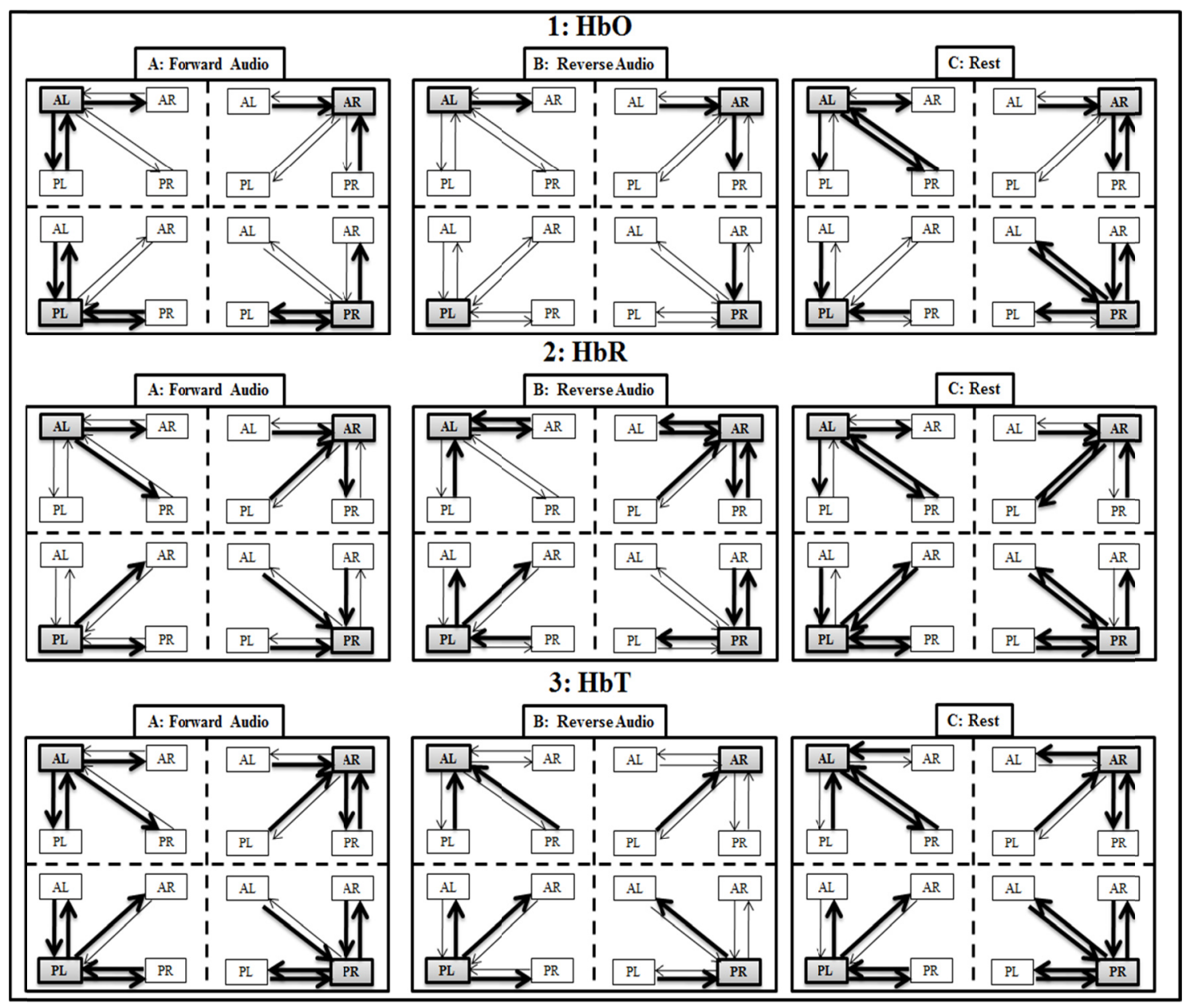

Figure 6.11: Pictorial representation of the causal and directional flow of information obtained from effective connectivity analysis (or granger causality analysis) during word reception paradigm using average hemodynamic data across all participants 1: $\mathrm{HbO}, 2$ : HbR and 3: HbT data, for (A) Forward Audio, (B) Reverse Audio, and (C) Rest. The directional flow of information between the regions is shown using thickening and thinning of arrows, where in the thick arrow shows strong flow of information and thin arrows shows weak flow of information. 
From the Figure 6.11-2 (which correspond to the effective connectivity due to change in $\mathrm{HbR}$ ), it can be seen that during the Forward Audio task AL sends strong forward output flow of information to AR and PR; and PL sends strong forward output flow of information to AR and PR. During Reverse Audio task it can be seen that there is a strong forward output flow of information from $\mathrm{AL}$ to $\mathrm{AR}, \mathrm{PL}$ to $\mathrm{AL}$ and $\mathrm{AR}, \mathrm{AR}$ to $\mathrm{AL}$ and $\mathrm{PR}$ and PR to PL and AR. During Rest it can be seen that there is a strong forward output flow of information and backward input flow of information amongst AL, AR, PL and PR except from PL to AL and AR to AL.

From the Figure 6.11-3 (which corresponds to the effective connectivity due to change in $\mathrm{HbT}$ ), it can be seen that during the Forward Audio task AL region sends strong forward output flow of information to PL, AR and PR region; and the PL region send strong forward output flow of information to $\mathrm{AL}, \mathrm{AR}$ and $\mathrm{PR}$ region. $\mathrm{AR}$ and $\mathrm{PR}$ sends strong forward output flow of information only to each other. During Reverse Audio only PL sends strong output flow of information to AL, AR and AR. During Rest it can be seen that there is a strong forward output flow of information and backward input flow of information amongst AL, AR, PL and PR except from AL to PL and AL to AR. In short, the effective connectivity patterns in terms of $\mathrm{HbO}, \mathrm{HbR}$, and $\mathrm{HbT}$ differ across these front-temporal regions w.r.t the nature of the stimuli or Rest during the word reception paradigm.

\subsection{Discussion}

The results of activation in the region under investigation during word generation w.r.t. jaw movement and rest (as shown in Figure 6.2) and forward audio w.r.t reverse audio 
and rest (as shown in Figure 6.7) demonstrate differences in activation, as observed from the hemodynamic plots as well as statistical analysis. These differences in activation during word generation w.r.t. jaw movement and rest and forward audio w.r.t reverse audio and rest concur with past research on fMRI (Gitelman et al. 2005; Papathanassiou et al. 2000; Wilke et al. 2009; Wonga et al. 2011; Yetkin et al. 1995) and NIRS towards language studies (Bortfeld et al. 2009; Herrmann et al. 2003; Herrmann et al. 2006; Kubota et al. 2005; Kuwabara et al. 2006; Quaresima et al. 2002; Sato et al. 1999). In addition, the lateralization studies (Figure 5.10 and Figure 5.15) demonstrate that there is not only differences in activation in the fronto-temporal regions in response to word generation and word reception stimulus, but also the fact that the AL region is dominant during word generation and PL region is dominant during Word reception. These results also concur with past work by other research groups employing NIRS (Watanabe et al. 1998) or fMRI (Cuenod et al. 1995; Kennan et al. 2002; Wilke et al. 2006) towards language studies (Geschwind \& Levistky 1968). Having demonstrated that the current results concur with the past work, the studies were extended to functional and effective connectivity analysis of these average hemodynamic responses in the four frontotemporal regions.

Functional and effective connectivity can be estimated either by model based methods or data driven methods (Friston 1994; Horwitz 2003; Lee et al. 2003). Model based methods attempt to characterize the relationships between time-series extracted from two or more regions. A model based method requires prior knowledge of the existence of the relationship between the regions of interest. The model based methods for functional 
connectivity estimation are coherence (Sun et al. 2004; Salvador et al. 2005; Murias et al. 2007), partial coherence (Murias et al. 2007; Salvador et al. 2005; Sun et al. 2004) or mutual information (Jeong et al. 2001; Na et al. 2002; Salvador et al. 2005) estimation. The model based method for effective connectivity estimation includes SEM (Structural equation modeling) (Gonzalez-Lima \& McIntosh 1994), MAR (multivariate autoregressive) modeling (Harrison et al. 2003) and DFA (dynamic factor analysis) (Molenaar et al. 1985; Wood \& Brown, 1994). On the other hand, data driven methods attempt to map connectivity in the whole brain without the prior knowledge of the region of interest. The data driven models can be modified to attempt describe connectivity between two regions. In most cases, there is little or no a priori knowledge of the existing functional and effective connectivity between the regions of interest. This lack of a priori knowledge reduces the competence of the application of model based methods to assess functional and effective connectivity between regions of interest. Hence, in this study, data driven approach was used to investigate the functional and effective connectivity across the regions under investigations. Data driven methods for functional connectivity estimation are PCA (principal component analysis) (Andersen et al. 1999; Bullmore et al. 1996; Friston et al. 1993; Mashal et al. 2005), ICA (independent component analysis) (Hyvarinen et al. 2010; Stone, 2002) calculation. In this study, a degenerate case of spatial PCA (principal component analysis) i.e. zero-order correlation (Biswal et al. 1995; Anand et al. 2005) across two chosen regions (amongst AL, PL, AR and PR) was employed to compute the functional connectivity. The data driven methods for effective connectivity estimation includes PPI (Psycho-physiological interactions) map (Friston et al. 1997) and Granger causal mapping (Goebel et al. 2003; Roebroeck et al. 2005). The 
granger causality analysis is simple to apply and is one of the most commonly used methods to explicate the effective connectivity (Roebroeck et al. 2005). Hence the granger causality was used to estimate the effective connectivity across AL, PL, AR and PR regions because of the lack of a priori knowledge between the regions under investigation.

\subsubsection{Study A: Work Expression Paradigm}

\subsubsection{Functional connectivity}

The results of strong positive functional connectivity (during Word Generation stimuli; as shown in Figure 6.5A) in the activity of the left vs. right anterior (AL vs. AR) and posterior ( $\mathrm{PL}$ vs. $\mathrm{PR}$ ) regions, in terms of change in $\mathrm{HbO}, \mathrm{HbR}$ and $\mathrm{HbT}$, indicates the involvement of all these fronto-temporal regions of the brain in processing the Word Generation stimuli. During the Jaw Movement stimuli (as shown in Figure 6.5B) strong positive functional connectivity is observed in $\mathrm{AL}$ and $\mathrm{AR}$ regions in terms of change in $\mathrm{HbO}, \mathrm{HbR}$ and $\mathrm{HbT}$. The $\mathrm{AL}$ and $\mathrm{AR}$ are those regions whose hemodynamic response function is expected to be affected by the Jaw Movement (based on the muscle movement), in comparison to the PL and PR regions (where weak functional connectivity was observed during Jaw Movement). The results of strong positive functional connectivity, in terms of change in $\mathrm{HbO}, \mathrm{HbR}$ and $\mathrm{HbT}$, during the Rest (as shown in Figure 6.5C), in the activity of AR vs. PR and PL vs. PR indicates the synchrony in the activity of right regions and posterior regions of the brain, even during the resting state. In summary there are differences in the functional connectivity patterns in the left vs. 
right anterior as well as posterior regions during Word Generation stimuli w.r.t the baseline stimuli (i.e. Jaw Movement and Rest).

\subsubsection{Effective connectivity}

The two language regions of the brain namely the left inferior frontal region (containing Broca's area) and the left posterior temporal region (containing Wernicke's area) are connected via the fibers of the arcuate fasciculus/superior longitudinal fasciculus (Brauer et al. 2011). Hence these two regions of brain should exert significant influence on each other as evident by the bidirectional flow of information between the AL and PL regions during the Word Generation stimuli. The result (as shown in Figure 6.6A) indicates the origination of the signal in the left anterior (AL) and left posterior (PL) regions of the brain, and its propagation to the other regions of brain under investigation. The unidirectional flow of information from AL region (which encompasses the Broca's region) and PL region (which encompasses the Wernicke's region) to the right region suggests that during the Word Generation stimuli the signal first originates in the left region of the brain, which then propagates to the right. These finding corroborates the findings from previous fMRI effective connectivity study on healthy right handed adults (Price et al., 1994; Wilke et al. 2005, 2006; Karunanayaka et al. 2007; Wilke et al. 2009), where the signal was found to originate in the left regions of the brain i.e. the Broca's and Wernicke's, which then propagates towards the right regions. The right regions of the brain under investigation, AR and PR, also seems to plays a significant role in processing the Word Generation stimuli, as there is bidirectional flow of information between AR and PR regions and additionally, they also receive input from AL and PL regions. The 
bidirectional flow of information between the AR and PR region, during the Word Generation stimuli, suggests that these two regions influence the flow of signal between each other. The significant influence of AR and PR region upon each other, during Word Generation stimuli indicates the involvement of right regions in the processing of language by the brain. This finding seems to corroborate the findings from a previous fMRI study on healthy right handed adults (Van Ettinger-Veenstra et al. 2010), which indicated the involvement of right region of brain during Word Generation stimuli.

During the Jaw Movement stimuli (as shown in Figure 6.6B) bidirectional flow of information is observed in the regions of the brain whose hemodynamic response function is prone to be affected by the Jaw Movement (i.e. between AL and AR). While no flow of information is observed between the regions of brain which are less prone to the effect of Jaw Movement (i.e. PL and PR). The result of effective connectivity during Rest indicates bidirectional flow of information across all the brain regions under investigation (as shown in Figure 6.6C) or, there is no specific origination of signal across all the regions under investigation. This is in contrast to the origination of signal in the left regions and its propagation to the right, during Word Generation. These differences in the signal origination pattern between Word Generation and Rest further corroborates that the Broca's and Wernicke's region play a significant role during language-based stimuli and not during Rest. 


\subsubsection{Study 2: Word Reception Paradigm}

\subsubsection{Functional connectivity}

The results of strong positive functional connectivity between PL and PR regions during the Forward Audio stimuli indicate synchrony in the activity between these two regions (as shown in Figure 6.10A). The results of weak functional connectivity across all fronto-temporal regions during the Reverse Audio stimuli indicate no synchrony in the activity across the fronto-temporal regions of the brain (as shown in Figure 6.10B). The results of strong positive functional connectivity during the Rest across the AR, PR and PL regions indicate the synchrony in the activity of right fronto-temporal and posterior regions of the brain (as shown in Figure 6.10C).

\subsubsection{Effective connectivity}

Sentence processing involves whole networks of brain areas (Vigneau et al. 2006; Hickok and Poeppel 2007; Doehrmann and Naumer 2008). Previous fMRI study of sentence processing (Vigneau et al. 2006) indicates that the left inferior frontal gyrus (i.e. AL region in this study) and the posterior temporal cortex (i.e. PL region in study) are the regions that support sentence processing. These findings from the present study concur with the previous finding, where flow of information was found across AL, AR, PL and PR regions. The results of effective connectivity analysis for Forward Audio stimuli indicate the bidirectional flow of information between AL vs. PL and PL vs. PR. It also shows unidirectional flow of information from AL (which encompasses the Broca's region) both to AR and PR (as shown in Figure 6.11A). This indicates the origination of the signal in the left anterior (which encompasses the Broca's region) and posterior 
regions (which encompasses the Wernicke's region) of the brain from where it propagates to the other regions of brain under investigation. It also indicates the involvement of right anterior and posterior regions of the brain in processing the Forward Audio stimuli. The results of bidirectional flow of information during Forward Audio stimuli, in PL vs. PR and PR vs. AR provides evidence to support the recent ongoing controversy of right regions' involvement during language comprehension (Van EttingerVeenstra et al. 2010).

The results of effective connectivity analysis for Reverse Audio stimuli indicates the origination of signal only in the left posterior (PL) region (which encompasses the Wernicke's region) of the brain, that is in cases when the language regions of the brain have to process the speech that is different from the normal speech syntax (as shown in Figure 6.11B). The results indicate that when a non-comprehensible audio stimuli, i.e. one with a gibberish sentence structure, is presented to the brain the signal originates in the left posterior (PL) region (which encompasses the Wernicke's region) of the brain to process the gibberish sentence structure. This result corroborates with the finding from previous fMRI studies (Price et al., 1994; Wilke et al. 2005, 2006; Karunanayaka et al. 2007; Wilke et al. 2009), which suggested the primary role of Wernicke's region when a gibberish language reception stimulus was presented to the healthy adult participant(s).

The results of functional and effective connectivity analysis during Rest (as shown in Figures $6.10 \mathrm{C}$ and $6.11 \mathrm{C}$, respectively) demonstrate that although there is a strong correlation across the right (AR vs. PR) and posterior regions (PL vs. PR and PL vs. AR), there is no specific origination of signal across all the regions under investigation. This is 
in contrast to the origination of signal in the left regions and its propagation to the right during word reception of comprehensible text (i.e. Forward Audio). This is also in contrast to the signal origination only in the Wernicke's region (i.e. PL) during word reception of non-comprehensible text (i.e. Reverse Audio). These differences in the signal origination pattern between word reception (both comprehensible and noncomprehensible) and Rest further corroborates that the Broca's and Wernicke's region play a significant role during Forward Audio and only Wernicke's region during Reverse Audio, unlike during Rest.

\subsection{Conclusion}

In summary, the findings from the present study indicate activation in the AL and PL region in response to Word Generation, Forward Audio and Reverse Audio task. The activation in response to these tasks was also observed in the right hemisphere homologues of AL and PL i.e. AR and PR. These findings are consistent with the finding from the previous research where the inferior frontal region, consisting of Broca's area, (AL in this study) has been implicated in phonological processing, semantic decision tasks, and sentence- and discourse-level processing. The superior temporal region, consisting of Wernicke's area (PL in this study) has shown activation during auditory processing of speech sounds, during semantic processing, and during syntactic processing (mostly in anterior regions). It has also been reported that the Wernicke's area is an epicenter for word comprehension. For the activation in the AR and PR region multiple studies have noted right hemisphere activations for language tasks (Mazoyer et al. 1993). The right hemisphere has been hypothesized to be particularly activated in response to 
interpreting sentence content, placing information in context, detecting semantic anomalies, interpreting prosody, and understanding metaphor (Cuenod et al., 1995). The right hemisphere may also be involved when the task requires the integration of information over time (Cuenod et al., 1995). However, as in the current study, right hemisphere activation has also been seen for language tasks requiring Word Generation, processing Forward Audio stimuli and Reverse Audio stimuli. Although it is possible that right hemisphere regions were activated simply as a by-product of their connections with homologous regions on the left, it is also possible that these regions are playing a specific role in language, which has yet to be fully determined. It is remarkable that the functional and effective connectivity indicates the involvement of vast network in the brain while processing the language based stimuli be it Word Generation, Forward Audio stimuli or Reverse Audio stimuli, thereby underlining the extensive network underlying language system (Price et al., 1994; Vigneau et al., 2006; Karunanayaka et al. 2007). The lateralization result indicates that although all the regions under investigation showed activation in response to Word Generation, Forward Audio stimuli or Reverse Audio stimuli and also the existence of vast language network in the brain it is the $\mathrm{AL}$ (comprising inferior region which consist of Broca's area) and PL regions (comprising of posterior temporal region which consists of Wernicke's area) which are dominant during Word Generation and Forward Audio stimuli, respectively.

The present work forms the foundation for the application of NIRS towards uncovering the vast language network present in the brain via investigation of activation, functional connectivity, effective connectivity and lateralization across different fronto-temporal 
regions of the brain in response to language stimuli. In our previous studies, NIRS was also used to investigate the brain activation, functional connectivity, as well as the cortical lateralization in the frontal cortex of healthy adults in response to verbal fluency paradigm (Chaudhary et al. 2011). The future focus is to perform high density NIRS to investigate the cognitive, language, and auditory regions of the brain simultaneously in response to language stimuli to further explicate the involvement of different regions of brain. These studies will potentially lay the foundation in applying NIRS towards investigation of language functions in various neurological disorders (e.g. epileptic, schizophrenic and aphasic populations), where abnormality in the language processing is found. 
CHAPTER 7. Study 2a: NIRS and Joint Attention of Frontal Cortex in Healthy Adults

In this chapter the NIRS study performed to elucidate the intensity and phase dependent connectivity in the frontal region of brain in response to joint attention skills has been described. The work was published in the JOURNAL OF NEAR INFRARED SPECTROSCOPY. (Ujwal Chaudhary, Banghe Zhu, and Anuradha Godavarty," Frontal Cortical Connectivity and Lateralization of Joint Attention Experience Using NearInfrared Spectroscopy", J. Near Infrared Spectrosc. 19, 105-116 (2011)).

\subsection{Introduction}

Brain-behavior studies of joint attention may be critical to understanding autism because the early social communication disturbance of autism is exemplified by a developmental failure in this domain (Dawson et al, 2002; Mundy et al, 2000; Mundy et al, 2005; Mundy et al 1989). Neuro-imaging techniques are essential in examining the underlying neural processes and connectivity associated with autism. Current imaging techniques (EEG, PET, $f \mathrm{MRI})$ are limited either by temporal/spatial resolution, costs, or complexities in imaging awake children as described in section 2.1.4. The limitations of EEG, PET, or $f$ MRI towards autism research may be overcome by the near infrared spectroscopy (NIRS) technology as described in section 2.1.4.

Various research groups have demonstrated the ability of NIRS to be successfully implemented towards functional brain mapping in awake adults, infants and children as well in response to an active stimulus (Strangman et al, 2002; Tsujimoto et al, 2004). However, application of NIRS towards autism research has been very limited, and in particular to 
joint attention studies there have been none to date. Recently, in our Optical Imaging Laboratory, NIRS was used to understand the neuronal activity in the frontal cortex in response to joint attention skills in normal adults (Zhu et al, 2009). However, to date, NIRS has not been applied to the study of the neural correlates of social impairments (specifically joint attention) in children with autism, which is the focus of our ongoing research.

In a recent study in our Optical Imaging Laboratory, NIRS was used for the first time to investigate the hemodynamic responses to joint attention experience in normal adults (Zhu et al, 2009). The hemodynamic responses to joint-attention experience using NIRS were similar to that obtained using fMRI in the frontal cortex (Williams et al, 2005), demonstrating the feasibility of implementing NIRS for the first time towards sociocommunication. The study was performed using a frequency based NIRS system, described in section 3.1. The intensity and phase shift component of the acquired optical data provide different information regarding the propagation of NIR (Near Infrared) light in the superficial cortical region. The intensity data provides information about the decay in the input signal as it propagates through the absorbing media in the superficial cortex. On the other hand the phase shift data elucidates the time of flight (or pathlength, or in other words the scattering properties) of the NIR light (Gratton et al, 2010; Rykhlevskaia et al, 2006). The combined intensity and phase shift can potentially provide new insight in the stimulus processing by the superficial cortical region as the light propagates through it. The current study is focused on the functional connectivity (or correlation) and cortical dominance analyses along with the hemodynamic response studies (from the past). These studies may form a foundation in better understanding the underlying 
neuronal processes and pathways during frontal brain activation in response to sociocommunicative tasks (i.e. joint attention experience).

\subsection{Materials and methods}

\subsubsection{Instrumentation}

In the current study, anterior left (AL) (which contains the Broca's region), posterior left (PL) (which contains the Wernicke's region), anterior right (AR) and posterior right (PR) regions were non-invasively imaged using imaged using a frequency-domain based optical imaging system, Imagent ( ISS, Inc., Champaign, IL) described in section 3.1. A custom-built optical probe-based cap was developed, and the (source/detector) fibers were positioned using the 10-20 electrode placement system. The optical fibers were placed in the frontal cortex at the Fp1, Fp2, Fpz, AFz, AF3 and AF4 (Fp stands for prefrontal and AF stands for anterior frontal) location of the 10-20 electrode placement system, as shown in Figure 7.1.

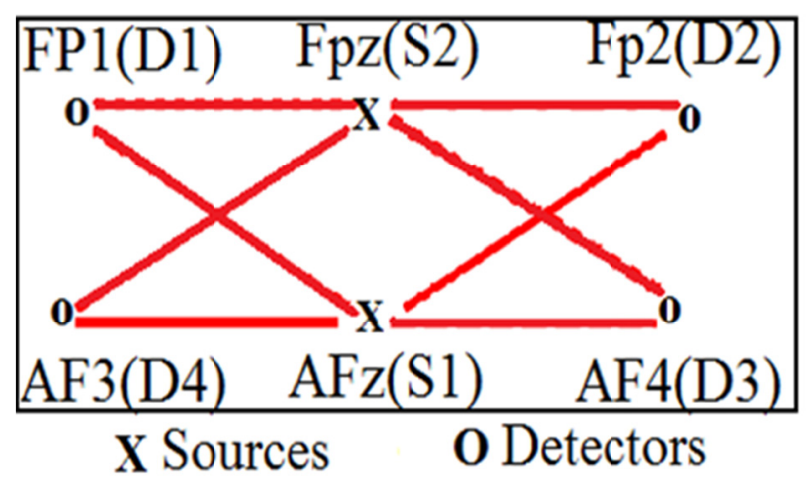

Figure 7.1: Source-detector layout corresponding to the prefrontal and the anterior frontal brain region of the standard 10-20 electrode placement system. 


\subsubsection{Subjects}

Eleven healthy right handed adults were enrolled for the current study that was performed at The Brain Institute of Miami Children's Hospital (Miami, FL). This study was approved by the Florida International University's (FIU) Institutional Review Board (IRB), and written consent(s) were obtained from the subjects.

\subsubsection{Experimental design and task}

Video clips (Williams et al, 2005) developed by other researchers that engendered an experience of joint attention contrasted with a very similar but non-joint attention condition, were used during our functional optical imaging studies. Joint attention refers to the capacity of subject to coordinate attention with a social partner in relation to some object or event (here the moving dot). In the case of non-joint attention, the subject(s) gazes the object (moving dot) without coordinating with the social partner.

A block experimental design, similar to that performed in the past fMRI studies (Williams et al, 2005), was chosen in stimulating the subjects with the following sequence: joint-attention clip (J), followed by a non-joint attention clip (NJ), followed by a baseline rest (R) (where the subject viewed a blank screen). The sequence of tasks (JNJ-R, $30 \mathrm{sec}$ each task) was repeated five times during an experiment and the entire experiment was repeated 3 times for each subject. In all these studies, the subjects were asked to keep their head still and only to shift gaze by eye movements in order to follow red dot in the presented video clips. 


\subsection{Optical data analysis}

The NIRS imaging technique with its ms-scale temporal resolution and sub-cm spatial resolution can allow functional connectivity analyses from a dynamic and static perspective (Gratton et al, 2010; Rykhlevskaia, 2006; Gratton et al, 2001; Gratton, 2003). In the current study, functional brain connectivity was carried out by computing zero lagged correlation as described in section 4.5, elucidate the correlation and synchrony in the hemodynamic response and hence the connectivity in the neuronal circuit between the left and right pre and anterior frontal cortex.

Optical measurements were obtained from eight source-detector pairs (or channels) in real-time at an update rate of $1.3 \mathrm{~Hz}$ in response to the different stimuli, for the entire experimental period and in each subject. The acquired optical data consisted of DC, AC and relative phase shift component. In the current studies, the temporal aspect of the time-dependent measurements (i.e. relative phase shift in frequency-domain) and intensity (i.e. DC, or average of AC) was employed in order to assess the connectivity in the frontal cortex. The intensity data in terms of AC and DC showed similar activation (in terms of $\mathrm{HbO}$ and $\mathrm{HbR}$ ), and hence only DC data was used in the current studies.

Functional brain connectivity study was performed to characterize the ways in which the left and right frontal cortex was functionally integrated or works in synchrony in response to social communication based stimuli. This study was carried out using both intensity (DC) and relative phase shift components of the optical data by computing zero lagged correlations, in order to elucidate the correlation (or synchrony) in the hemodynamic response and relative phase shift, respectively. This will in turn help 
understand the functional connectivity in the neuronal circuit between the left and right frontal cortex. Herein, two different studies were performed using intensity and relative phase shift data, namely: (i) Connectivity and cortical lateralization using intensity data, and 2) Connectivity using time dependent phase shift data.

\subsubsection{Study 1: Connectivity and cortical lateralization using intensity data}

In the present work intensity (DC) data was used to study the functional connectivity between the left and right frontal cortex (pre- and anterior, separately). The average of the modulated light signal (i.e. DC signal) was post-processed (as described in the earlier work8) to obtain the hemodynamic response function ( $\mathrm{HRF}$ ) of $\mathrm{HbO}, \mathrm{HbR}$, and $\mathrm{HbT}$ (HbO - oxy-hemoglobin, HbR - deoxy-hemoglobin and $\mathrm{HbT}$ - total hemoglobin) (averaged over the 5 blocks in each subject). The $\mathrm{HbO}, \mathrm{HbR}$, and $\mathrm{HbT}$ data was further averaged over all the 11 subjects and 3 repetitions in each subject (i.e. 11 subjects $\times 3$ repetitions $=33$ trials $).$ The averaged HRF data $(\mathrm{HbO}-$ oxy-hemoglobin, $\mathrm{HbR}-$ deoxyhemoglobin and $\mathrm{HbT}$ - total hemoglobin) was across the 90 -sec block, consisting of J, NJ and $\mathrm{R}$ task (30-sec each). The $0-30 \mathrm{sec}$ period corresponded to J task, $30-60 \mathrm{sec}$ to the NJ task, and $60-90$ sec to the R task. The averaged HRF data was further averaged across all the trials (11 subject $\times 3$ Repetitions) to obtain a mean HRF of all subjects and also standard deviation was computed using the averaged HRF data across all the trials. The consistency (similarity in change in HRF for each stimulus) of the HRF obtained from each subject and repetition across the three stimuli was performed to ascertain the uniformity in the nature of the response during stimulus and rest conditions. Statistical analysis (using the mean and standard deviation of HRF data) over all subjects has shown 
consistency in the nature of the response during stimulus and rest conditions (Zhu et al, 2009).

. The entire window of $30 \mathrm{sec}$ for each stimuli (J, NJ, or R) was used to plot the averaged 1-D HRF plots (HbO - oxy-hemoglobin, HbR - deoxy-hemoglobin and HbT total hemoglobin) between the left and right pre- and anterior frontal cortex with respect to time. The 1-D HRF plot demonstrated similar contrast in its trend across the three stimuli, independent of the time window chosen in each stimulus (i.e. 10-sec, 15-sec, or 20-sec windows). This demonstrates that the HRF differences across the stimuli do not vary with the time window of each block, but truly varies with the nature of the stimuli.

The averaged HRF data was used to compute zero order correlations between the left and right pre- and anterior frontal cortex (channels Fp1-Fpz vs. Fpz-Fp2, AF3-Fpz vs. AF4 -Fp4, Fp1-AFz vs. AFz -Fp2 and AF3-AFz vs. AFz-AF4 of Figure 7.1), as described in section 4.5 .

In addition to the correlation studies across left and right frontal cortex, zero-order correlation studies were also performed across the stimuli or inter stimuli (i.e. W vs. J, W vs. R, and J vs. R) using HbT (total hemoglobin) data. These studies were carried out in an attempt to determine if the differences in the connectivity were from variation in the stimuli presented or from systemic physiology. If the correlation was predominantly from systemic physiology, the connectivity would remain the same and independent of the stimuli presented. In other words, a strong correlation would be observed from the inter stimuli correlation analyses if the correlation was predominantly from systemic physiology and independent of the presented stimuli. The correlation coefficients 
obtained from the functional connectivity study is tabulated as well as presented as histogram plots for better visual representation. In the histogram plots, the y-axis is the correlation coefficient which varies from -1 to 1 , where -1 depicts negative correlation, 0 depicts no correlation, and 1 depicts positive correlation between the two channels that are compared.

Additionally, cortical lateralization study was performed to understand the hemispherical dominance of the social communication in the frontal cortex and its variation across the stimuli. The laterality index was computed between left $v s$. right frontal cortex for each stimulus ( $\mathrm{J}, \mathrm{NJ}$ and $\mathrm{R}$ ) using the $\mathrm{HbT}$ data obtained from the following channels: Fp1-Fpz vs. Fp2-Fpz, AF3-Fpz vs. AF4-Fpz, Fp1-AFz vs. Fp2-AFz and AF3-AFz vs. AF4-AFz (see Figure 7.1). The NIRS laterality index, L, for total hemoglobin $(\mathrm{HbT})$ was calculated as described in section 4.7.

\subsubsection{Study 2: Connectivity using time dependent phase data}

The relative phase shift component of the optical data was post-processed differently compared to the intensity data. Initially, the relative phase shift data was filtered using a band pass filter, with a band of $0.3 \mathrm{~Hz}$ and $0.0016 \mathrm{~Hz}$ (similar to that filtered in the DC data $^{8}$ ). The filtered data was further processed by subtracting each data point of the 30sec stimuli with respect to its initial onset point (i.e. at $\mathrm{t}=0$ sec for each 30 -sec stimuli). The subtracted data was then averaged across all the 5 blocks of the J-NJ-R task. This resulted in a single 90 -sec block of J-NJ-R task, thus providing an average relative phase shift over a 90 -sec time period (i.e. one J-NJ-R block). Mean and standard deviation of the average subtracted data across the 11 subjects and 3 repetitions on each subject (i.e. 
33 trials) was further estimated to check the consistency in the relative phase shift data across the trials. Since the standard deviation was small compared to the mean of the processed optical data, the relative phase shift data was considered consistent across all the trials. The mean (or average) relative phase shift data across the 33 trials was thus used to study the correlation between the left and right frontal cortical regions of the brain (channels Fp1-Fpz vs. Fpz-Fp2, AF3-Fpz vs. AF4 -Fp4, Fp1-AFz vs. AFz -Fp2 and AF3-AFz vs. AFz-AF4 in Figure 7.1). The correlation analysis was performed by computing the Pearson's correlation coefficient, as described in section 4.5.

\subsection{Result and discussion}

7.4.1 Study 1: Connectivity and cortical lateralization using intensity data

The relative change in $\mathrm{HbO}, \mathrm{HbR}$ and $\mathrm{HbT}$ in response to joint attention task(s) is plotted (Figures 7.2, 7.3 and 7.4, respectively) in terms of the averaged (across 33 trials) changes for each stimulus (J, NJ and R) and each channel, in order to assess the change in HRF during the J, NJ and R tasks between left and right frontal cortex (in both the preand anterior regions). It is apparent from Figure 7.2 that there is a significant correlation between the left and right frontal cortex (in both the pre- and anterior regions) for each stimuli ( $\mathrm{J}, \mathrm{NJ}$ and $\mathrm{R}$ ) in terms of change in $\mathrm{HbO}$ and $\mathrm{HbT}$ (Figures 7.2 and 7.4), and no significant correlation in terms of HbR (Figure 7.3). The extents of correlation in the change of $\mathrm{HbO}, \mathrm{HbR}$ and $\mathrm{HbT}$ for each stimulus are quantified via functional connectivity analysis described in Section 7.3.1. The correlation coefficients obtained from the functional connectivity study are shown in the Table 7.1 and also as histograms in Figure 7.5. It can be observed from Figure 7.5 that the extent of correlation is similar 
for each stimulus ( $\mathrm{J}, \mathrm{NJ}$ and $\mathrm{R}$ ) in terms of change in $\mathrm{HbO}$ and $\mathrm{HbT}$ (Figure 7.5A and $7.5 \mathrm{C})$.
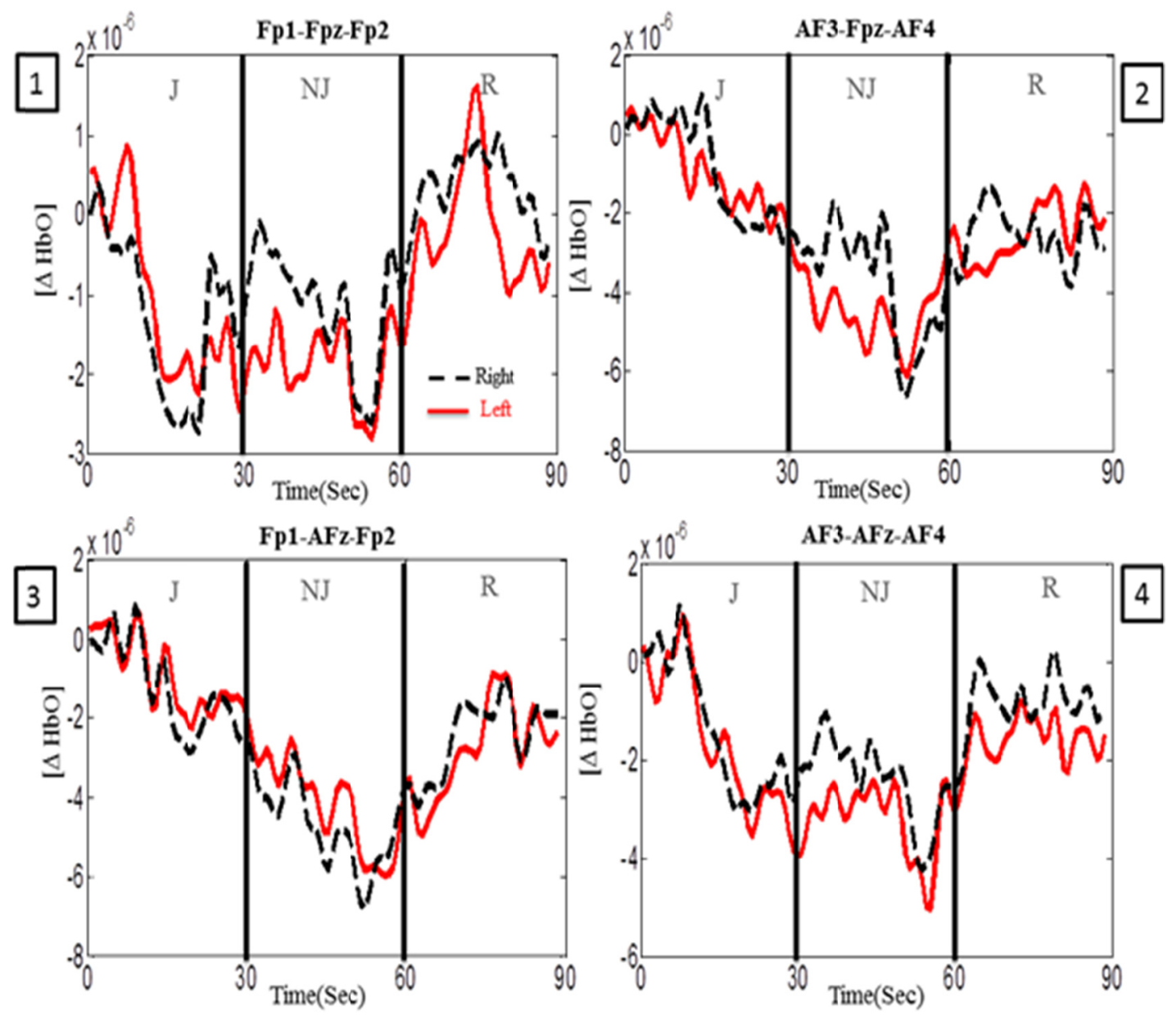

Figure 7.2: Hemodynamic response function (HRF) (averaged across all participants) in terms of $\mathrm{HbO}$ (Oxy-Hemoglobin) with respect to time, plotted for each 30-sec stimuli (J, $N J$, and $R$ ) and for each symmetric channel pair (left and right) on the same plot.

The results for the inter stimuli zero-order correlation analysis is shown in the Table 7.2. From Table 7.2, it is apparent that there was no significant correlation across the stimuli. If the HRF data only represented the systemic physiology, a strong correlation is expected across the stimuli. However, from the results shown in Table 7.2, it was 
observed that there was no significant correlation across the stimuli. This further validates that the connectivity results do represent the brain activity and not just the underlying systemic physiology (or noise).
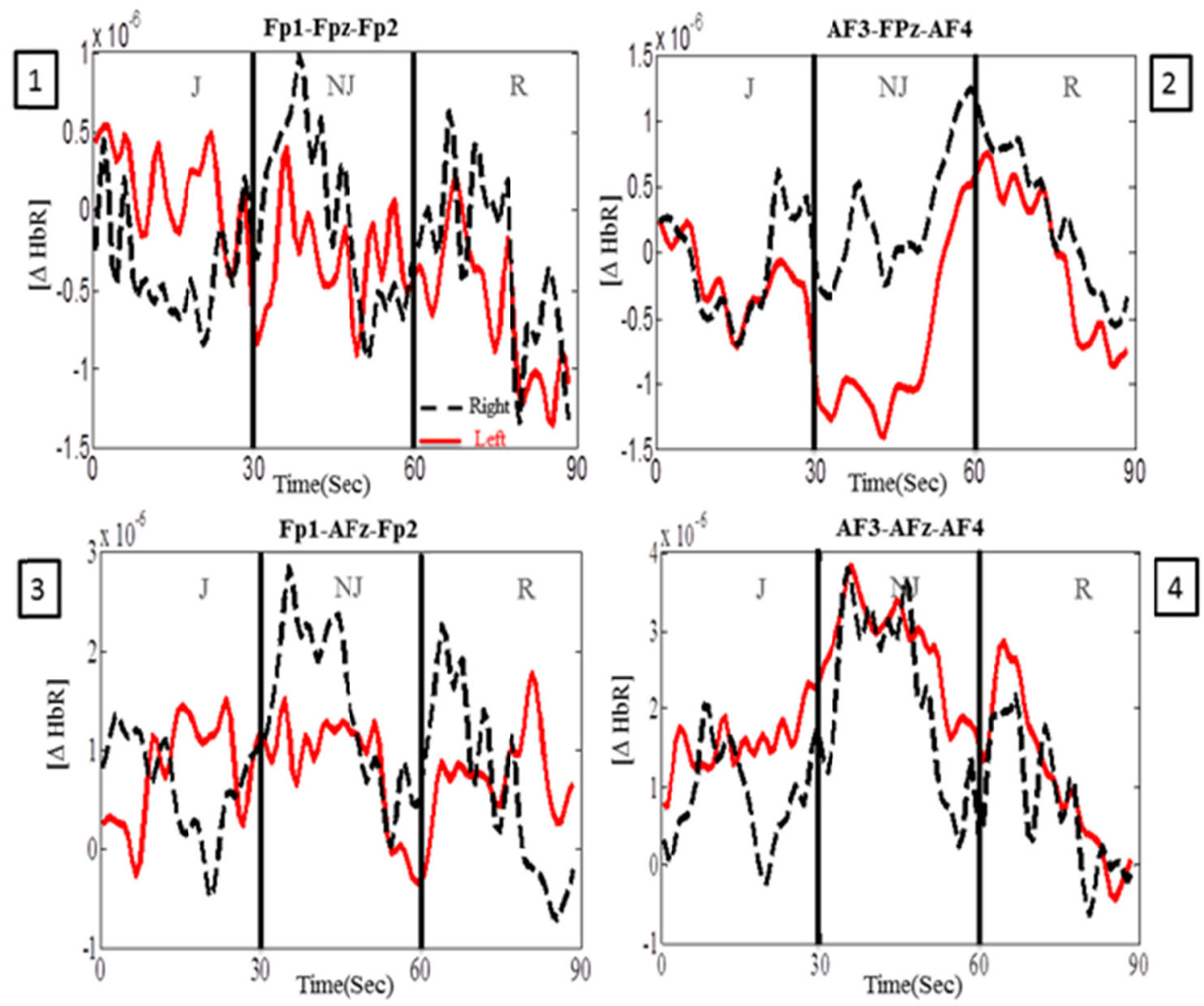

Figure 7.3: Hemodynamic response function (HRF) (averaged across all participants) in terms of HbR (deoxy-Hemoglobin) with respect to time, plotted for each 30-sec stimuli (J, $N J$, and $R$ ) and for each symmetric channel pair (left and right) on the same plot.

The functional connectivity analysis provides information related to the correlation in the activity of the left and right frontal cortex. However, it does not help determine the dominance of the frontal cortex in response to each stimuli. The dominance of the frontal cortex was determined for each stimulus by performing lateralization studies described in 
Section 7.3.1. The results of the lateralization study is depicted as histograms in Figure 7.6. It can be observed from Figure 7.6 that the cortical dominance differs for each stimuli (J, NJ and R) and across symmetric (left vs. right) channels.
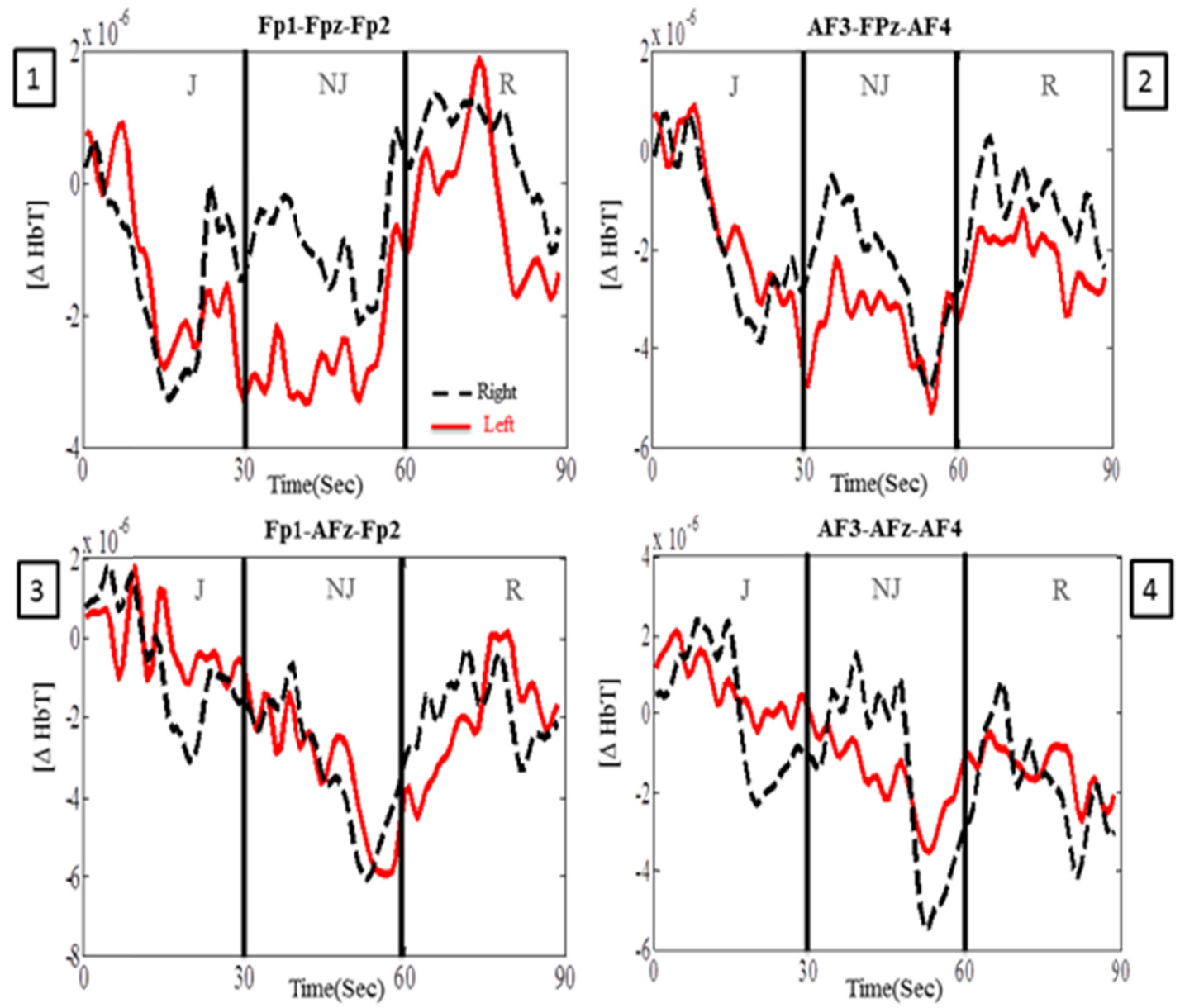

Figure 7.4: Hemodynamic response function (HRF) (averaged across all participants) in terms of HbR (Deoxy-Hemoglobin) with respect to time, plotted for each 30-sec stimuli $(J, N J$, and $R$ ) and for each symmetric channel pair (left and right) on the same plot.

Case 1: Joint attention $(\mathrm{J})$ related connectivity and lateralization

The hemodynamic responses in terms of change in $\mathrm{HbO}$ and $\mathrm{HbT}$ with respect to time (shown in Figures 7.2 and 7.4, respectively) and the corresponding correlation coefficients for $\mathrm{HbO}$ and $\mathrm{HbT}$ (shown as histograms in Figures $7.5 \mathrm{~A}$ and $7.5 \mathrm{C}$, 
respectively and in Table 7.1) demonstrates a high level of correlation between the left and right frontal cortex (in both the pre- and anterior regions) during joint attention $(\mathrm{J})$ stimulus. The hemodynamic response in terms of change in HbR (Figure 7.3) and the corresponding correlation coefficient for $\mathrm{HbR}$ (see Figure 7.5B and Table 7.1) demonstrates no significant correlation between the left and right frontal cortex (in both the pre- and anterior regions). Lateralization analysis (using $\mathrm{HbT}$ data) demonstrated a bilateral activation in both the pre- and anterior frontal cortex (as shown in Figure 7.6A).

\begin{tabular}{|l|c|c|c|c|c|c|c|c|c|}
\hline \multirow{2}{*}{$\begin{array}{l}\text { Source-Detector } \\
\text { Pair/ Stimuli }\end{array}$} & \multicolumn{3}{|c|}{ HbO } & \multicolumn{3}{c|}{ HbR } & \multicolumn{3}{c|}{ HbT } \\
\cline { 2 - 11 } & $\mathrm{J}$ & $\mathrm{NJ}$ & $\mathrm{R}$ & $\mathrm{J}$ & $\mathrm{NJ}$ & $\mathrm{R}$ & $\mathrm{J}$ & $\mathrm{NJ}$ & $\mathrm{R}$ \\
\hline Fp1 - Fpz - Fp2 & 0.81 & 0.77 & 0.74 & 0.55 & 0.92 & 0.93 & 0.72 & 0.64 & 0.75 \\
\hline AF3 - Fpz - AF4 & 0.92 & 0.74 & 0.72 & 0.18 & 0.34 & 0.70 & 0.88 & 0.74 & 0.80 \\
\hline Fp1 - AFz - Fp2 & 0.91 & 0.86 & 0.86 & -0.70 & 0.64 & -0.05 & 0.60 & 0.82 & 0.36 \\
\hline AF3 - AFz - AF4 & 0.84 & 0.50 & -0.1 & 0.20 & 0.89 & 0.83 & 0.74 & 0.73 & 0.62 \\
\hline
\end{tabular}

Table 7.1: Zero-order correlation coefficient for changes in HbO (Oxy-Hemoglobin), HbR (Deoxy-Hemoglobin), and HbT (Total Hemoglobin) data (averaged across all participants), for each symmetric channel pair (left and right), across the three stimuli Joint (J), Non joint (NJ) and Rest (R). The correlation coefficients range from -1 to +1 , with -1 is strong negative correlation, +1 is strong positive correlation, and zero is no correlation.

Case 2: Non joint attention (NJ) related connectivity and lateralization

The hemodynamic responses in terms of change in $\mathrm{HbO}$ and $\mathrm{HbT}$ with respect to time (shown in Figures 7.2 and 7.4, respectively) and the corresponding correlation coefficients for $\mathrm{HbO}$ and $\mathrm{HbT}$ (shown as histograms in Figures $7.5 \mathrm{~A}$ and $7.5 \mathrm{C}$, respectively and in Table 7.1, demonstrate a high level of correlation between the left and right frontal cortex (in both the pre- and anterior regions) during non-joint attention (NJ) stimulus. The hemodynamic response in terms of change in HbR with respect to time 
(Figure 7.3) and the corresponding correlation coefficient for $\mathrm{HbR}$ (see Figure 7.5B and Table 7.1) demonstrates significant correlation between all the channel pairs, except AF3-Fpz-AF4. The lateralization analysis (using HbT data) demonstrated right prefrontal dominance and bilateral activation in the anterior frontal cortex (see Figure 7.6B).

\begin{tabular}{|l|l|c|c|c|}
\hline \multicolumn{2}{|l|}{ Source Detector Pair/ Stimuli pair } & J vs. NJ & J vs. R & NJ vs. R \\
\hline \multirow{2}{*}{$\begin{array}{l}\text { Left } \\
\text { Frontal }\end{array}$} & Fp1 $-\mathrm{Fpz}$ & -0.30 & -0.01 & -0.36 \\
\cline { 2 - 5 } & $\mathrm{Fp} 1-\mathrm{AFz}$ & 0.53 & 0.64 & 0.55 \\
\cline { 2 - 5 } & $\mathrm{AF} 3-\mathrm{Fpz}$ & 0.63 & 0.02 & 0.47 \\
\cline { 2 - 5 } & $\mathrm{AF} 3-\mathrm{AFz}$ & 0.52 & -0.53 & -0.44 \\
\hline \multirow{2}{*}{$\begin{array}{l}\text { Right } \\
\text { Contal }\end{array}$} & $\mathrm{Fp} 2-\mathrm{Fpz}$ & 0.21 & -0.37 & -0.11 \\
\cline { 2 - 5 } & $\mathrm{Fp} 2-\mathrm{AFz}$ & 0.69 & 0.41 & 0.65 \\
\cline { 2 - 5 } & $\mathrm{AF} 4-\mathrm{Fpz}$ & 0.31 & 0.22 & 0.68 \\
\cline { 2 - 5 } & $\mathrm{AF} 4-\mathrm{AFz}$ & 0.45 & -0.17 & 0.32 \\
\hline
\end{tabular}

Table 7.2: Correlation coefficients for Inter stimuli (Joint (J) vs. Non Joint, Joint (J) vs. Rest $(R)$ and Non Joint (NJ) vs. Rest (R)) zero order correlation analysis using HbT (Total Hemoglobin) data (averaged across all participants) for all channels. The correlation coefficients range from -1 to +1 , with -1 is strong negative correlation, +1 is strong positive correlation, and zero is no correlation.

Case 3: Rest $(\mathrm{R})$ related connectivity and lateralization

The hemodynamic responses in terms of change in $\mathrm{HbO}, \mathrm{HbR}$, and $\mathrm{HbT}$ with respect to time (shown in Figure 7.2) demonstrate differences in the level of correlation between the left and right channels during rest (R) stimulus. The quantitative analysis of correlation coefficients of changes in $\mathrm{HbO}, \mathrm{HbR}$, and $\mathrm{HbT}$ (see Figure 7.5 and Table 7.1) confirms that there is significant correlation between left and right pre-frontal cortex. However, the correlation tends to weaken in either of the anterior frontal channels, more predominantly in $\mathrm{HbO}$ and $\mathrm{HbR}$ (as shown in Figure 7.5 and Table 7.1). The 
lateralization analysis (using $\mathrm{HbT}$ data) demonstrated right pre-frontal dominance and bilateral activation in the anterior frontal cortex (see Figure 7.6C).

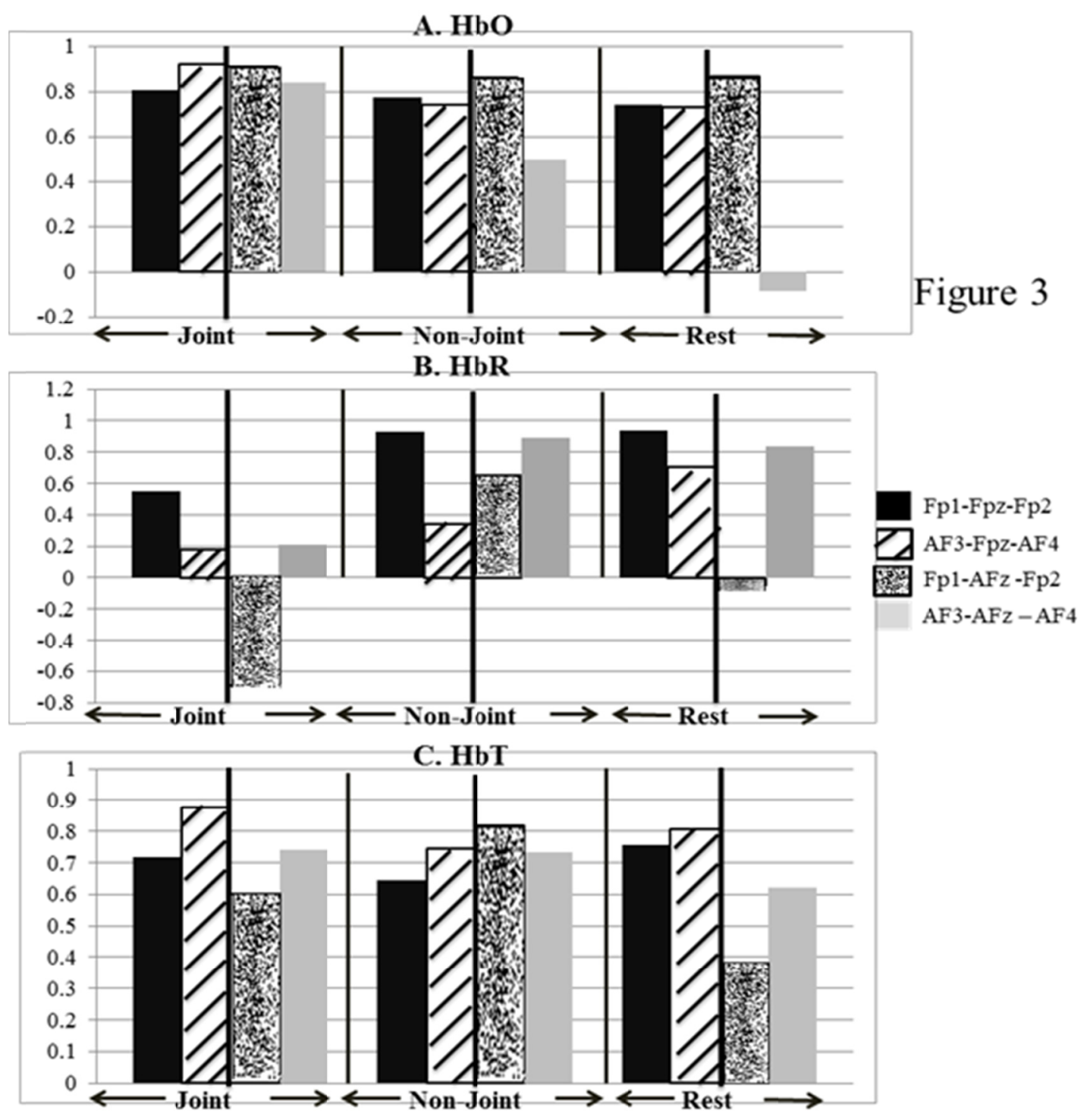

Figure 7.5: Zero-order correlation bar plots for (A) HbO (Oxy-Hemoglobin), (B) HbR (Deoxy-Hemoglobin), and (C) HbT (Total Hemoglobin) data (averaged across all participants), for each symmetric channel pair (left and right) and across the three stimuli ( $J, N J$ and $R)$. 

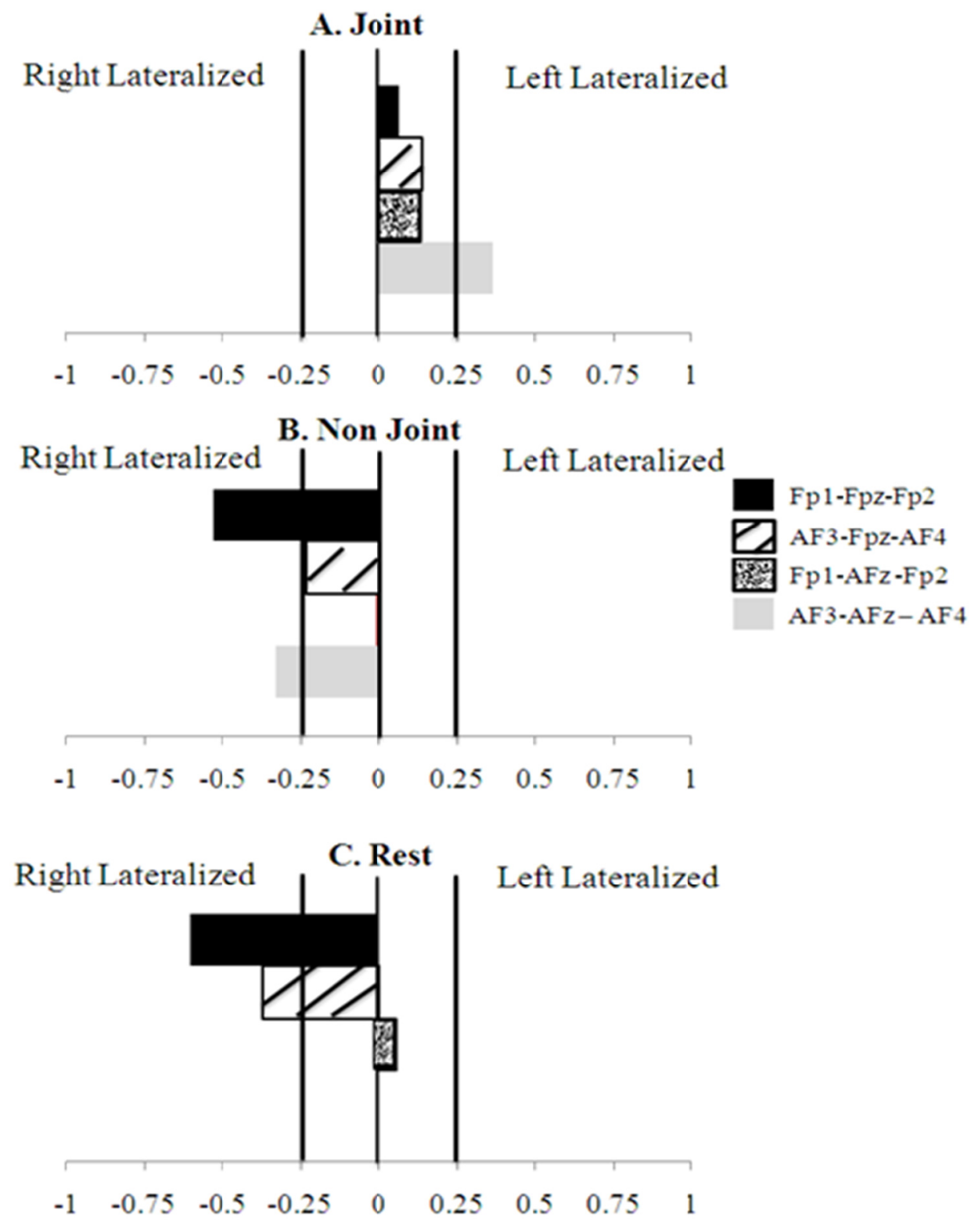

Figure 7.6: Cortical lateralization bar plot for each symmetric channel pair (left and right) and across the three stimuli ( $J, N J$ and $R)$. Laterality index value $(L)>0.25$ indicates left cortical lateralization, $L<-0.25$ indicates right cortical lateralization and $-0.25<L<0.25$ indicates no dominance or bilateral activation.

A comparison of the results from the connectivity and cortical lateralization studies was carried out to assess the significance of the findings. In the pre-frontal cortex, 
bilateral activation was observed during J-task; whereas right dominance was observed during NJ and R-tasks (see Figure 7.6). On the other hand, strong correlations (in terms of $\mathrm{HbO}$ and $\mathrm{HbT}$ ) were observed between left and right pre-frontal cortex, irrespective of the stimuli (see Table 7.1 and Figures $7.5 \mathrm{~A}, 7.5 \mathrm{C}$ ). In terms of $\mathrm{HbR}$, the connectivity varied irrespective of the stimuli (J, NJ and R) (see Table 7.1 and Figure 7.5B).

In the anterior frontal cortex, bilateral activation was observed for all the three stimuli (see Figure 7.6). Also, strong connectivity (in terms of $\mathrm{HbO}, \mathrm{HbR}$, and $\mathrm{HbT}$ ) was observed in the anterior frontal cortex irrespective of the stimuli (except for one channel during R-task in terms of $\mathrm{HbO}$ ) (see Table 7.1 and Figure 7.5).

In summary, the connectivity (in terms of $\mathrm{HbO}$ or $\mathrm{HbT}$ ) demonstrated symmetry in the functionality of the left and right frontal cortex, irrespective of the stimuli. This in turn demonstrated similarity in the structure of the left and right frontal cortex. This is possibly due to similarity in the density of the underlying cortical region through which the optical signals propagates. These findings corroborate with similar findings from a previous study of parcellation of the human frontal cortex using MRI, (Wible et al, 1997) which has demonstarted similarity in the volume of left and right pre frontal cortex grey matter and hence density. The value of left and right frontal cortex grey matter reported in the literature (Wible et al, 1997) was 8.28 (1.7) and 8.92 (1.1) respectively. Another study (Rant et al, 2009) reported the density of left and right frontal cortex as a whole (231 \pm 21 and $231 \pm 22$, respectively) and also the density of left and right frontal cortex

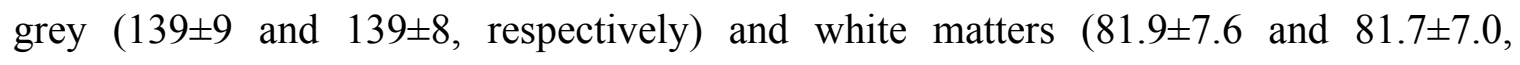
respectively) which were similar. This similarity in volume and hence density posssibly 
causes similar light intensity changes as it propogates through the left and right frontal cortex.

While connectivity describes the synchrony in the flow pattern of the optical signals (either in terms of intensity, HRF or relative phase shift data), the differences in the cerebral blood flow volumes between the left and right is better understood from the cortical lateralization using $\mathrm{HbT}$ data. In other words, the cortical lateralization study expands the findings from the brain connectivity study, by determining the cortical dominance for a given stimuli. In the current study, although there is symmetry in the flow patterns of the optical signals (or cerebral blood flow) in terms of $\mathrm{HbO}$ and $\mathrm{HbT}$ in the frontal cortex (i.e. synchronous flow of signals was observed), a right pre-frontal cortical dominance was observed from lateralization study during NJ and R tasks. Our past studies (Zhu et al, 2009) demonstrated no significant differences in brain activation across two stimuli (J vs. R, J vs. NJ and NJ vs. R), when the HRF data was in terms of HbR. However, significant differences in the level of activation across two stimuli were observed in terms of $\mathrm{HbO}$ (and $\mathrm{HbT}$ ). Hence, the connectivity in terms of $\mathrm{HbR}$ is not compared with the lateralization data (in terms of $\mathrm{HbT}$ ).

\subsubsection{Study 2: Connectivity using time dependent phase data}

The correlation analysis carried out between the left and right frontal cortex in terms of the relative phase shift data, elucidates the extent of synchrony in relative phase shift, with $+/-1$ representing strong positive/negative correlation (or synchrony) and 0 representing no correlation. The average relative phase shift (across 33 trials) in response to each stimulus (J, NJ, and R) and at each channel is plotted for the $830 \mathrm{~nm}$ and $690 \mathrm{~nm}$ 
wavelengths in Figures 7.7 and 7.8, respectively; and the correlation coefficient due to the relative phase shift at $830 \mathrm{~nm}$ and $690 \mathrm{~nm}$ between left and right frontal cortical region is shown in Table 7.3.

\begin{tabular}{|l|c|c|c|c|c|c|}
\hline $\begin{array}{l}\text { Source-Detector } \\
\text { Pair/Stimuli }\end{array}$ & \multicolumn{2}{|c|}{ Joint Attention } & \multicolumn{2}{|c|}{$\begin{array}{l}\text { Non joint } \\
\text { Attention }\end{array}$} & \multicolumn{2}{c|}{ Rest } \\
\cline { 2 - 7 } (Left $v s$. Right) & $830 \mathrm{~nm}$ & $690 \mathrm{~nm}$ & $830 \mathrm{~nm}$ & $690 \mathrm{~nm}$ & $830 \mathrm{~nm}$ & $690 \mathrm{~nm}$ \\
\hline Fp1 - Fpz - Fp2 & 0.99 & 0.98 & 0.99 & 0.98 & 0.97 & 0.98 \\
\hline AF3 - Fpz - AF4 & 0.13 & -0.12 & -0.04 & 0.01 & -0.13 & 0.05 \\
\hline Fp1 - AFz - Fp2 & 0.07 & 0.03 & 0.17 & 0.10 & 0.11 & 0.50 \\
\hline AF3 - AFz - AF4 & 0.07 & 0.06 & 0.04 & -0.13 & -0.11 & 0.05 \\
\hline
\end{tabular}

Table 7.3: Correlation coefficients for relative phase shift at $830 \mathrm{~nm}$ and relative phase shift at $690 \mathrm{~nm}$ (averaged across all participants), across the three stimuli Joint (J), Non joint (NJ) and Rest (R) and for each symmetric channel pair. The correlation coefficients range from -1 to +1 , with -1 is strong negative correlation, +1 is strong positive correlation, and zero is no correlation.

Based on the plot depicted in the Figures 7.7 and 7.8 and the correlation coefficient values shown in Table 7.3 it can be observed that there is a significant similarity in the relative phase shift between the left and right prefrontal region (Fp1-Fpz-Fp2) of the brain at both the wavelengths (690 and $830 \mathrm{~nm}$ ) and for each stimulus (J, NJ and R). It is also apparent from Figures 7.7 and 7.8 and the values in Table 7.3 that the relative phase shift is significantly varying in the left over the right frontal cortical regions, except for Fp1-Fpz-Fp2 (pre-frontal). These differences in the relative phase shifts depict the possibility of a difference in the optical signal pathways between the left and right frontal cortex (preferably in the anterior frontal). 

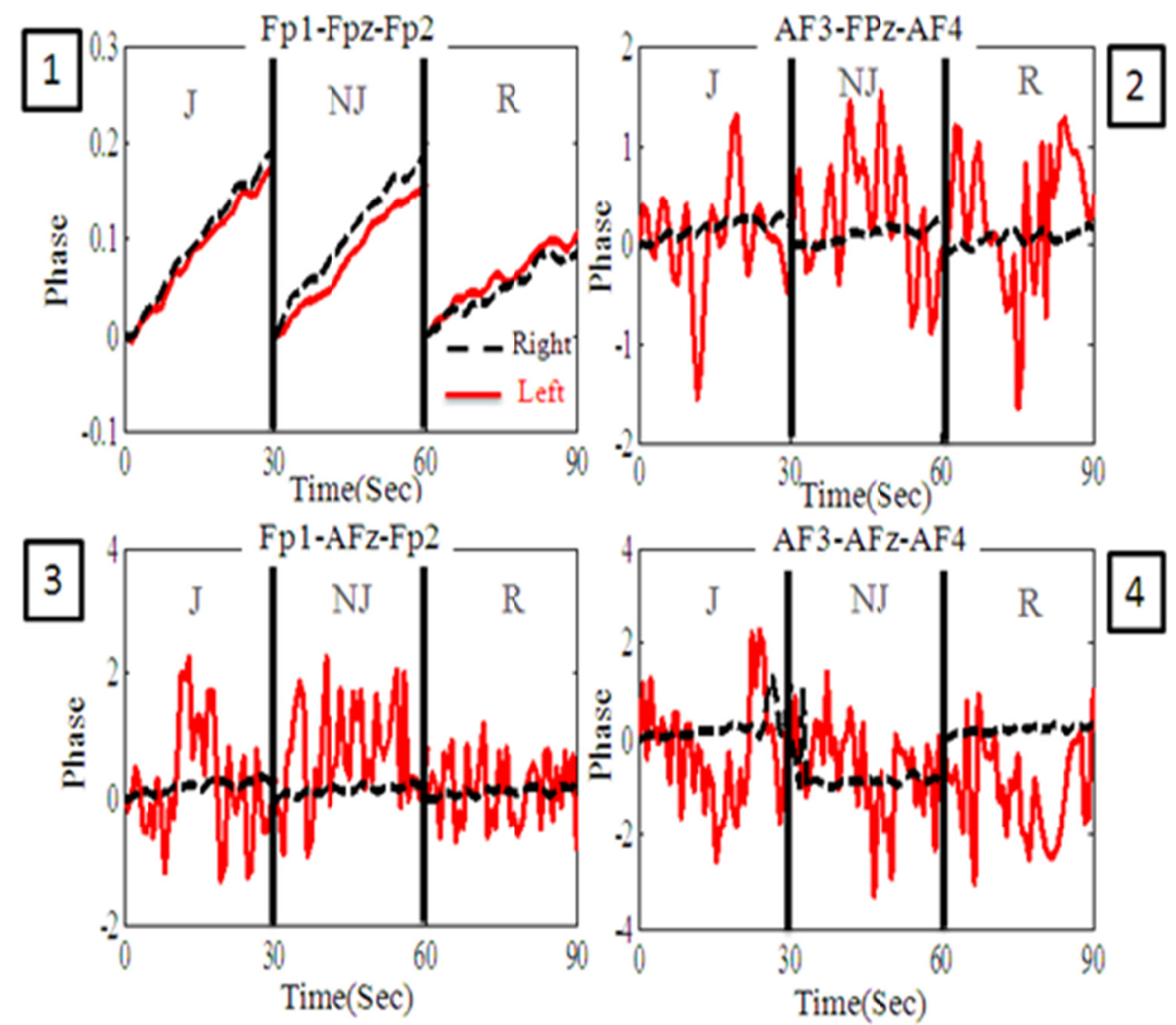

Figure 7.7: Relative phase shift (at $830 \mathrm{~nm}$ ) (averaged across all participants) across the three stimuli (J, NJ and R) for each symmetric channel pair (left and right).

It can also be observed from Figures 7.7 and 7.8 that the relative phase shift of the left/right pre-frontal channel (Fp1-Fpz-Fp2) was smaller for the R stimulus in comparison to the $\mathrm{J}$ and $\mathrm{NJ}$ stimulus, at both the wavelengths. 

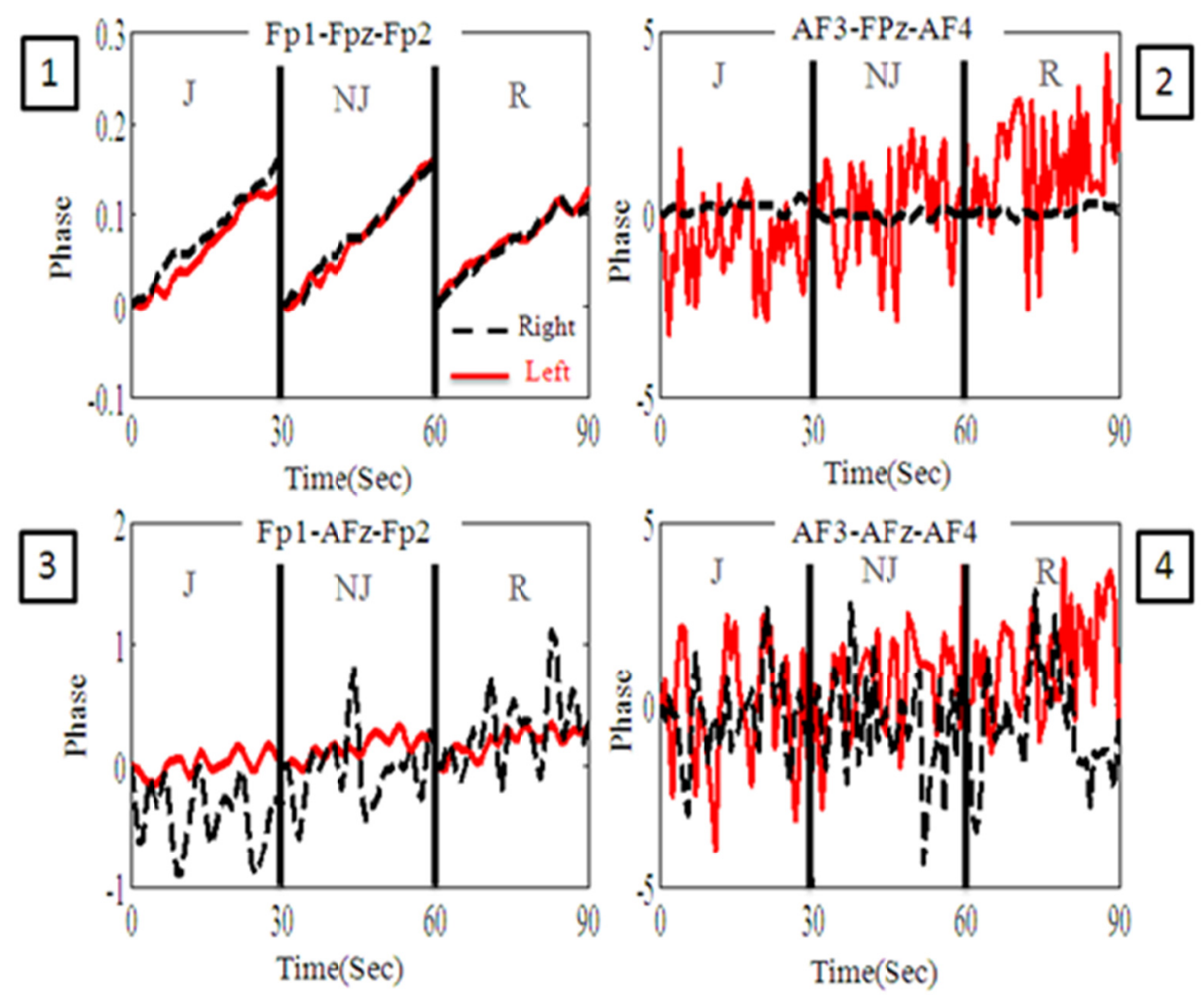

Figure 7.8: Relative phase shift (at $690 \mathrm{~nm}$ ) (averaged across all participants) across the three stimuli (J, NJ and R) for each symmetric channel pair (left and right).

This demonstrates that the pathways the signal travels in the underlying cortical region differ during a socio-communicative task in comparison to the rest conditions. The correlation coefficient obtained from functional connectivity analysis results are shown as histogram plots (Figure 7.9), for each left vs. right channel, stimulus, and wavelength (i.e. $830 \mathrm{~nm}$ in Figure 7.9A and $690 \mathrm{~nm}$ in Figure 7.9B). Significant correlation between the left and right pre-frontal cortex (Fp1-Fpz-Fp2) for the three stimuli (J, NJ and R) and at both the wavelengths ( 830 and $690 \mathrm{~nm}$ ) can be clearly visualized from the histograms 
(Figure 7.9). In all the remaining left vs. right channels, no correlation was observed (as demonstrated from the relative phase shift at $830 \mathrm{~nm}$ and $690 \mathrm{~nm}$ with respect to time).

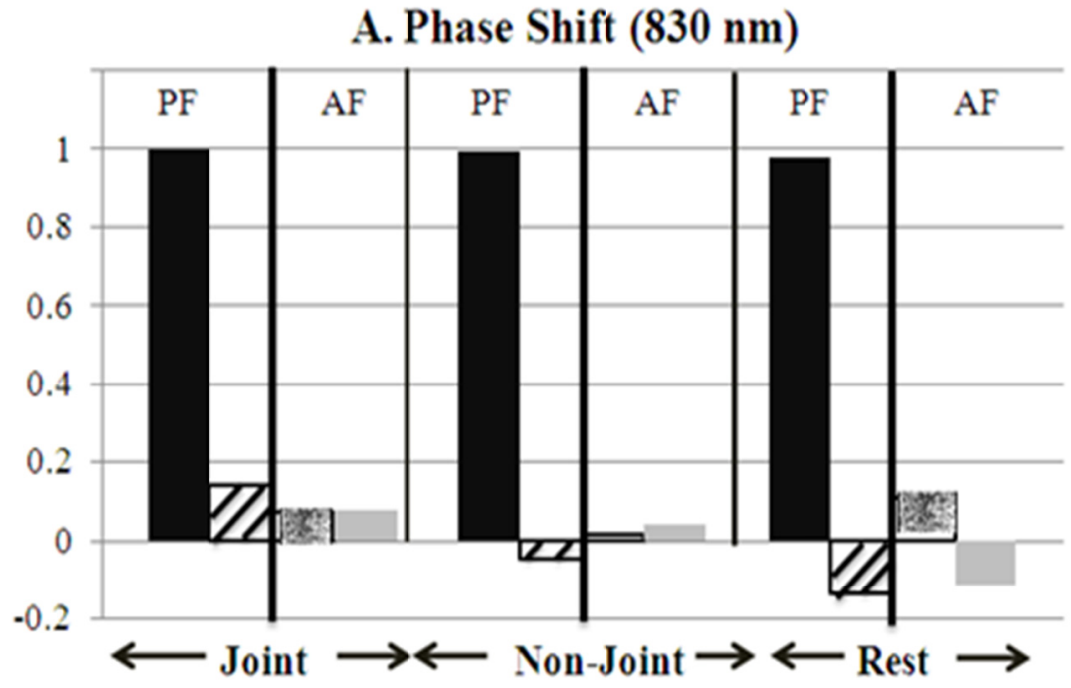

B. Phase Shift $(690 \mathrm{~nm})$

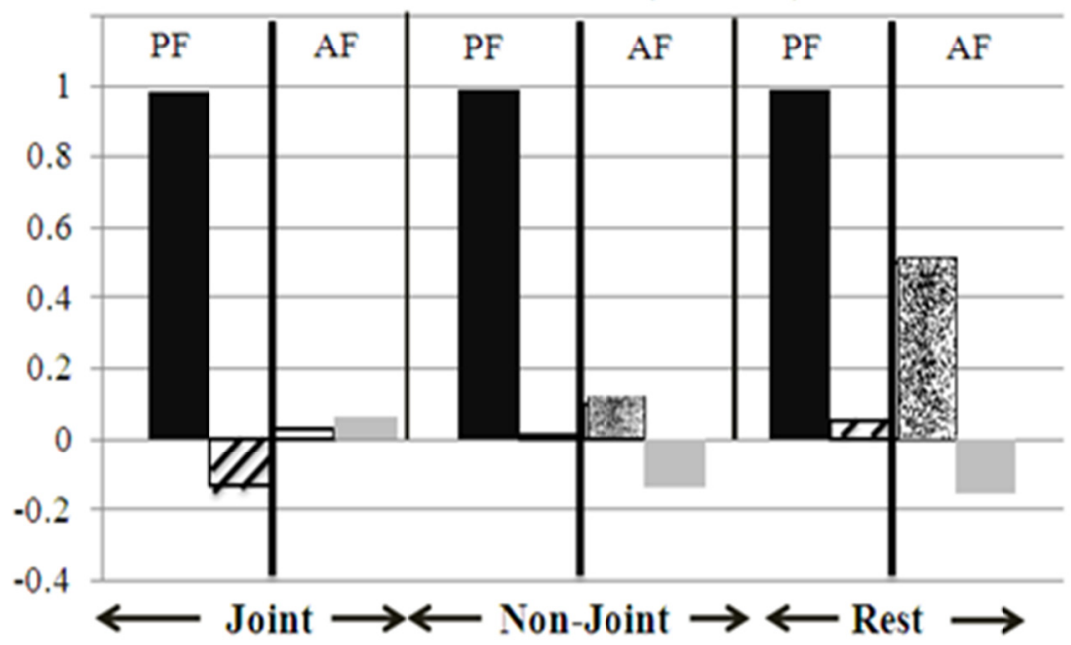

Figure 7.9: Zero-order correlation bar plots for (A) Relative phase shift at $830 \mathrm{~nm}$ and (B) Relative phase shift at $690 \mathrm{~nm}$, (averaged across all participants) across the three stimuli $(J, N J$ and $R)$ and for each symmetric channel pair, where PF is pre-frontal and $A F$ is anterior frontal cortex.

The relative phase shift based optical data is related to the temporal information, i.e. the time delay of the signal as it travels through the underlying cortical region(s). The 
results from the present study demonstrate that there is similarity in the time of flight of the optical signal and possibly similarity in the pathlength travelled in left and right prefrontal cortex while integrating the social cues presented to the subjects. The result of no connectivity in the anterior frontal cortex demonstartes dissimilarity in time of flight of optical signal and possibly difference in the pathlength travelled during information integration in anterior frontal cortex.

The result of bilateral pre-frontal activation during the $\mathrm{J}$ stimuli obtained from the cortical lateralization analysis of intensity (DC) data complements the result of similar relative phase shift between the left and right pre-frontal cortex. While strong connectivity (using phase data) was observed in the pre-frontal cortex, irrespective of the stimulus (see Figure 7.9) (similar to connectivity in terms of $\mathrm{HbO}$ and $\mathrm{HbT}$ ), the dominance changed with respect to the stimulus (see Figure 7.6). When the stimulus was either NJ or R, there was correlation in the pathlengths of the optical signals between the left and right pre-frontal cortex, although the cortical dominance shifted to the right. This explicates that although the pathways may be similar in pre-frontal, the extent of activation changes with stimuli. In anterior frontal cortex, there were weak correlations (using phase data) between the left and right regions for all the stimuli (see Figure 7.9). Also, from cortical lateralization study, no significant dominance was observed (i.e. bilateral activation) (see Figure 7.6). This states that although extent of activation is similar between left and right anterior frontal for any given stimuli, the pathways are different as observed from phase data. In other words, it is apparent that eventhough the density of the underlying left vs. right cortical region through which the light propagates 
is similar, this similarity does not imply that the time delay in the light propogation through that cortical region will be same or vice-versa. This is because the time delay of the signal depends on the path length travelled and not on the density of the underlying cortical region.

Hence, it can be stated that the information obtained from cortical laterlization study (using intensity data) and connectivity study (using relative phase shift data) has the potential to elucidate the density of the underlying cortical region and also the time of flight and eventually the length of pathways travelled during the stimulus processing by the underlying cortical regions.

\subsection{Conclusion}

Socio communicative study was performed using NIRS technique to elucidate the brain connectivity and lateralization in the fortal cortex of a normal adult. The primary focus of the current study is to investigate functional connectivity and cortical dominance in the frontal brain based on the intensity and relative phase shift information obtained from the frequency-domain optical measurements. A socio-communicative based stimuli was presented as a block design (J-NJ-R) to the subjects and time-dependent NIRS signals (in terms of intensity and phase shift data) were recorded in real-time. The study demonstrated strong functional connectivity (in terms of $\mathrm{HbO}, \mathrm{HbT}$ and relative phase shift data) in the pre-frontal cortex, irrespective of the stimuli (J, NJ, or R). However, in the anterior frontal cortex, although the connectivity (based on $\mathrm{HbO}$ and $\mathrm{HbT}$ ) demonstrated a strong correlation between the left and right regions, the connectivity (based on relative phase shift data) demonstrated a weaker correlation. This analyses 
depicts that the density and pathlengths of the optical signals in the pre-frontal cortex are similar. However, the density or symmetry of the left and right anterior frontal cortex differs from the pathlengths of the optical signals. Future studies involving other cortical regions of the brain during socio-communicative tasks can better elucidate the underlying phenomenon of functional connectivity.

The cortical lateralization study on the other hand demonstrated a right pre-frontal dominance during $\mathrm{NJ}$ and $\mathrm{R}$ stimuli, and not so in the $\mathrm{J}$ stimulus. The lateralization study complements the connectivity study by stating that, although both the density and pathlengths of the left and right pre-frontal cortex are similar, the dominance can vary with respect to stimuli.

Currently, the functionality of the brain can be obtained using other imaging modalities (e.g. EEG, MRI etc.). However, the images do not provide any information about the neuronal pathlength or the time delay of the signal propogation across two different regions of the brain. NIRS with its fast temporal resolution and also the ease with which it can be applied to the pediatric population, has a potential to elucidate the connectivity pattern of the developing brain. This is made possible using time-dependent optical measurements, thus providing additional analyses of the brain's activity in response to the presented stimulus. Additionally, the ability to apply NIRS technique to awake pediatric populations towards real-time imaging has greater benefits over the currently applied imaging techniques in children.

The next step to the current research is to carry out NIRS-based imaging studies in autistic and typically developing children, in response to the socio-communicative tasks 
(e.g. joint attention experience). Understanding the differences in the hemodynamic responses across the subject groups along with the differences in the functional connectivity and cortical lateralization will have potential to differentiate the two groups of children. This in turn may have a significant impact in early stage diagnostic intervention of autism from NIRS studies in much younger children $(<3$ years of age $)$ versus psychological assessments on children, which is feasible only for children above 3 years of age. 
CHAPTER 8. Study 2b: NIRS and Joint Attention of Frontal Cortex in Children

In this chapter the application of NIRS to investigate the difference in activation between typically developing children and children with autism in response to joint attention skills has been described. The joint attention study performed on healthy adults, described in chapter 7, demonstrated the feasibility of NIRS to investigate the change in frontal region in response to joint attention skills. Hence the joint attention skill was used to investigate the response of frontal cortex of typically developing children and children with autism as described in this chapter. This work was published in the conference proceedings of SPIE 2011. (Ujwal Chaudhary, Michael Hall, Anibal Gutierrez, Daniel Messinger, Gustavo Rey and Anuradha Godavarty," Activation in Frontal Cortex of Typically Developing and Children with Autism in Response to Joint Attention Skill using Near Infrared Spectroscopy”, SPIE BiOS, 788348-788348-6).

\subsection{Introduction}

Autism is a neuro-developmental disorder that is characterized by impaired social interactions, communication deficits and a restricted, repetitive pattern of interests and activities. Autism is classified as a part of Autism Spectrum Disorders (ASD), also known as Pervasive Developmental Disorders, which are a broad spectrum of developmental disorders affecting children and adults (American Psychiatric Association, 2000; National Institute of Mental Health, 2004; World Health Organization, 1993). One of the primary characteristics of the Children with autism is that they display robust levels of impairments in joint attention development from as early as 12 to 18 months of 
age (Osterling et al., 2002; Swettenham et al., 1998). Joint Attention is the process of sharing one's experience of observing an object or event, by following gaze or pointing gestures. It is critical for social development, language acquisition, and cognitive development (Mundy \& Crowson, 1997). Thus brain-behavior studies of joint attention may be critical to understanding autism (Dawson et al., 2002; Mundy et al., 2000) as the early social communication disturbance of autism involves deficits in this domain (Mundy \& Sigman, 1989).

An earlier fMRI study (Williams et al., 2005) of joint attention experience demonstrated an increased activation in the right ventral medial frontal cortex and the left anterior frontal cortex during a responding to joint attention (RJA) based stimuli. The activation was also observed in other areas such as the cingulate cortex, bilateral caudate nuclei, and right anterior frontal lobe (Williams et al., 2005). In particular, the differences amongst the two RJA stimuli (i.e. joint and non-joint attention) and baseline rest were distinctly observed in the frontal cortical brain regions. Hence, based on the paradigm used in the earlier fMRI study, NIRS was employed for the first time in order to study the hemodynamic responses to joint attention experience (using responding to joint attention, or RJA-based stimuli) in normal adults (Zhu et al., 2009). The results obtained from this NIRS study concurred with the results from an earlier $f$ MRI study. Differences in, hemodynamic response in terms of $\mathrm{HbO}$ (oxy hemoglobin) was different between Joint attention and Non-Joint attention (in the entire frontal cortex), and Joint attention and Rest (preferably on the right frontal cortex) in normal adults. Having demonstrated the feasibility of studying joint attention based behavioral paradigm using NIRS, the 
objective of the present study is to extend the application of NIRS to pediatric populations in the area of autism. NIRS-based imaging studies in TD and children with autism were performed in response to the socio-communicative (joint attention) stimuli, in order to understand the differences in the frontal cortical brain activation between the two subject groups.

\subsection{Materials and methods}

\subsubsection{Instrumentation}

The joint attention study was performed using a frequency-domain based optical imaging system, Imagent (ISS, Inc., Champaign, IL) described in section 3.1. The optical probes were placed on the frontal cortex, using a custom-built optical probe-based cap, in accordance with the 10-20 electrode placement system at the Fp1, Fp2, Fpz, AFz, AF3 and AF4 (Fp stands for prefrontal and AF stands for anterior frontal) locations, as shown in Figure 8.1. A picture depicting the placement of optical cap on a pediatric subject is shown in Figure 8.2 .

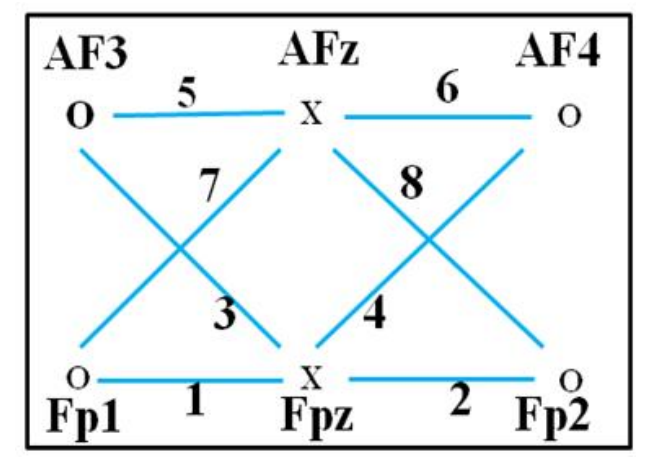

Figure 8.1: Source-detector layout on the prefrontal and the anterior frontal brain region of the brain, in accordance to the standard 10-20 electrode placement system, during the NIRS joint attention studies. 


\subsubsection{Participants}

Four TD and one child diagnosed with autism in the age range of 4-8 years were enrolled for the current study. The child with autism was a 5 year-old boy with a clinical diagnosis of autism confirmed by a research reliable rater on the Autism Diagnostic Observation Schedule (Lord et al., 2000).

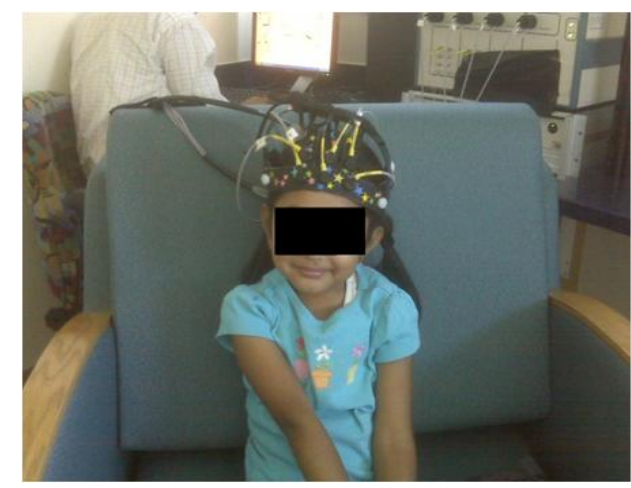

Figure 8.2: Participant with the optical cap during the brain imaging study. The source and detector fibers were placed in the frontal cortex.

\subsubsection{Experimental design and protocol}

The subjects were shown an animated video clips, which engendered an experience of joint attention contrasted with a very similar but non-joint attention condition in the subjects. The animated video clips were developed in the FIU-OIL (Florida International University Optical Imaging Laboratory) by modifying and adapting the original video clips developed by Williams et al., 2005. The original video clip was aesthetically unappealing and it was very difficult to engage the visual attention of the children during the study. Hence, in the newly developed video clips the presenter and object of the existing video was replaced with animated cartoon characters such that it is more appealing to children. The Joint attention stimuli presented in the video clip refers to the 
capacity of subject to coordinate attention with a social partner (here the cartoon of lady in the center of the screen) in relation to some object or event (here the moving cartoon character appearing on the corner of the screen). In the case of non-joint attention, the subject(s) gazes the object (moving cartoon characters) that are incongruent from the social partner's gaze (here the cartoon of lady in the center of the screen). A snap shot of the animated video presented to the subject for joint and non-joint attention based stimuli is shown in Figures 8.3A and 8.3B, respectively.

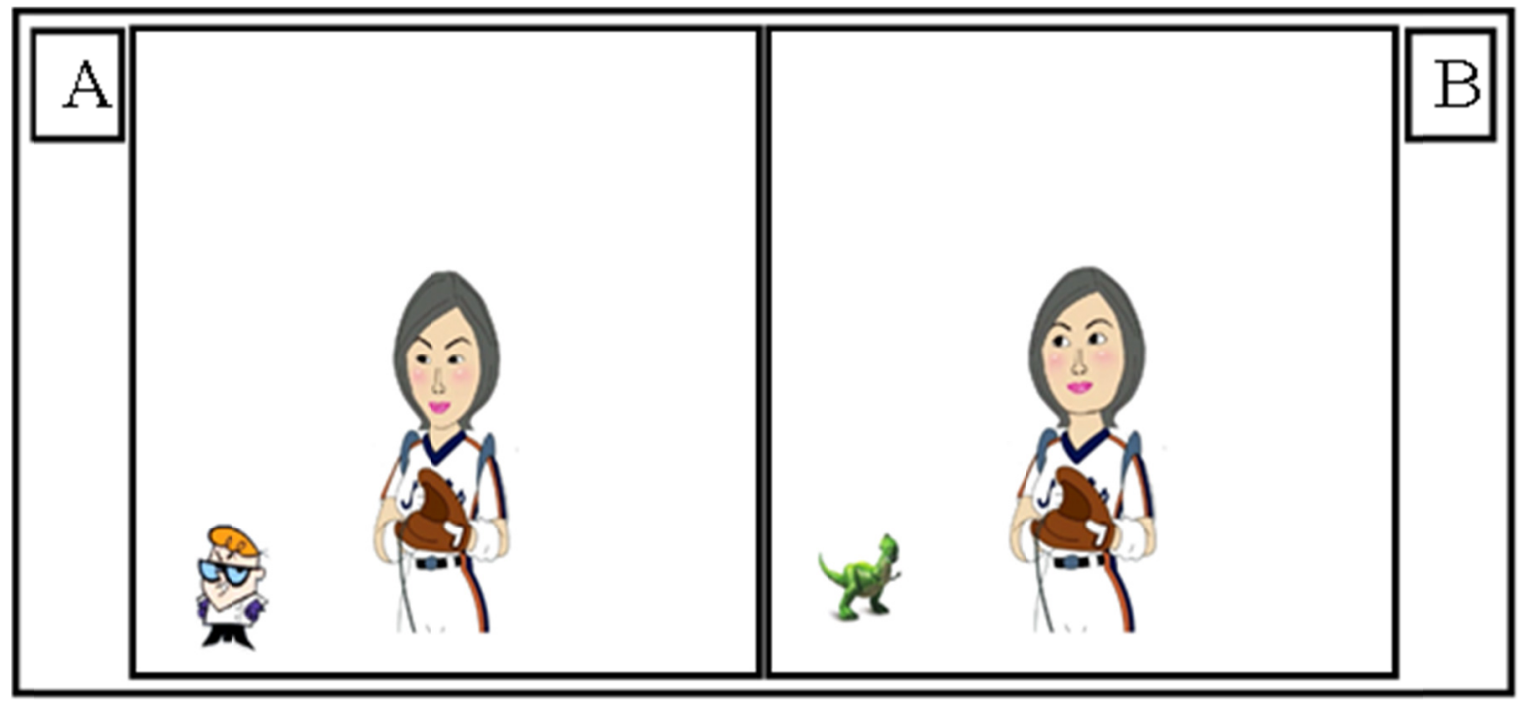

Figure 8.3: (A) Joint (J) attention stimulus, movement of the gaze of the presenter (lady in the center of cartoon) is congruent with the presentation of the moving object (the cartoon near the edge of square, here it is Johnny bravo). (B) Non Joint attention (NJ) stimulus, movement of the gaze of the presenter (lady in the center of cartoon) is incongruent with the presentation of the moving object (the cartoon near the edge of square, here it is pink cartoon character).

A block experimental design, similar to that performed in the past fMRI studies (Williams et al., 2005), was chosen in stimulating the subjects. The sequence of the videos during a single block of the experiment involved: 30 -sec joint-attention clip (J), followed by a 30 -sec non-joint attention clip (NJ), and followed by a 30 -sec baseline rest 
(R) (where the subject viewed a blank screen). The sequence of tasks (J-NJ-R, $30 \mathrm{sec}$ each task) was repeated five times during an experiment and the entire experiment was performed twice on each TD subject and only once on each ASD subject. In all these studies, the subjects were asked to keep their head still and only to shift gaze by eye movements in order to follow moving cartoon characters in the presented video clips.

\subsection{NIRS signal analysis}

Frequency-domain optical measurements were obtained in real-time (every $0.7 \mathrm{sec}$ ) in response to the different stimulus for the entire experimental period, for each subject. The acquired optical data consisted of DC, AC and phase shift information of the attenuated diffuse NIR light emerging from the frontal cortex of the brain. In the current studies, only the average of the modulated light signal (i.e. DC signal) was employed in postprocessing real-time hemodynamic responses across each source-detector pair (or channel). The optical data was post processed to obtain averaged HRF data as described in section 4.4. The 1-D HRF data was averaged across the five experimental blocks to obtain single $90 \mathrm{sec}$ block consisting of J, NJ and R task (30-sec each). The 0 - $30 \mathrm{sec}$ period corresponded to J task, $30-60 \mathrm{sec}$ to the NJ task, and $60-90 \mathrm{sec}$ to the R task. The averaged 1-D HRF data was then interpolated using the data obtained from all the source-detector pair to obtain 2-D activation plots.

A brain activation study was performed to explicate the differences in the hemodynamic response $(\mathrm{HbO}, \mathrm{HbR}$ and $\mathrm{HbT})$ as a function of time, in the frontal cortex and in response to the three presented stimuli (J, NJ and R). A 2-D activation plot was generated using a window of 10, 15, 20 and $30 \mathrm{sec}$ for each stimulus (J, NJ and R) from the calculated 
averaged hemodynamic response function (HRF). The 2-D response was found to be similar irrespective of the window size, thereby validating the consistency of data. Henceforth, a window of $30 \mathrm{sec}$ was used to construct 2-D activation plot.

\subsection{Results}

Activation plots depicting change in hemodynamic response during $\mathrm{J}$, NJ and $\mathrm{R}$ in TD and ASD children are shown in Figures $8.4 \mathrm{~A}$ and B, respectively. It is evident from Figure 8.4A (activation plot for TD children) that the activation in the pre and anterior frontal brain region of TD's differs across the stimuli i.e., the pattern and extent of activation is different between the stimuli ( $\mathrm{J}$ vs. NJ, J vs. R and NJ vs. R). The activation plot for the child with autism (Figure 8.4B) shows no difference in activation across the stimuli. The observation of difference between the stimuli (J vs. NJ, J vs. R and NJ vs. R) in TD children and of no difference between the stimuli (J vs. NJ, J vs. R and NJ vs. R) in child with autism indicates the difference in the response of pre and anterior frontal cortex to joint attention based stimuli across the two subject groups (i.e. TD and child with autism). Even in the absence of an RJA-stimulus (i.e. joint or non-joint attention) to the brain, i.e. under rest conditions the HRF (in terms of $\mathrm{HbO}, \mathrm{HbR}$ and $\mathrm{HbT}$ ) differ across TD and ASD children. These preliminary results are significant in the area of autism research, where NIRS has potential in early stage intervention of autism in children at a much younger age. 


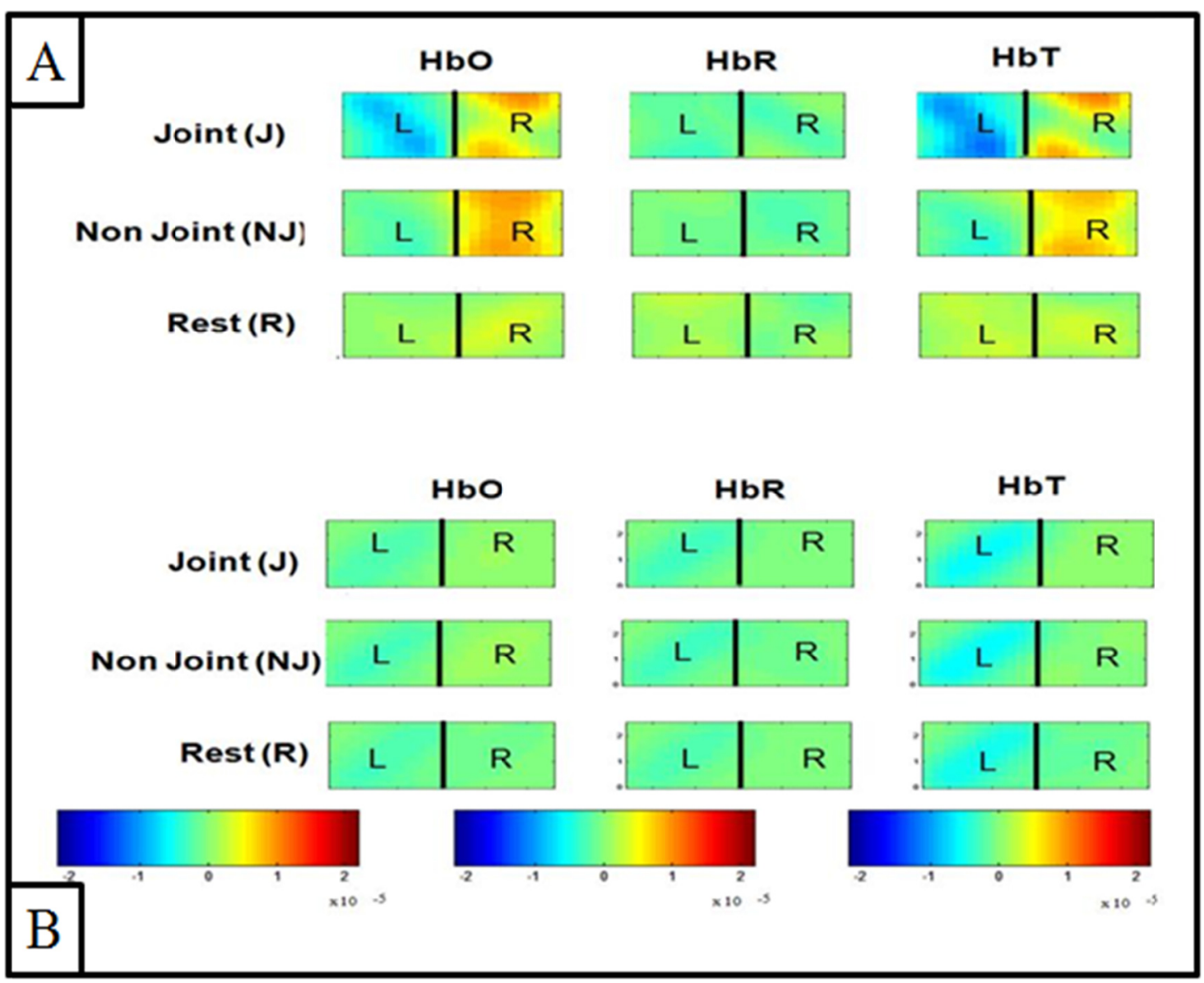

Figure 8.4: (A) Activation plots depicting changes in hemodynamic response (in terms of $\mathrm{HbO}, \mathrm{HbR}$, and $\mathrm{HbT}(\mathrm{HbO}$ - oxy-hemoglobin, HbR - deoxy-hemoglobin and HbT - total hemoglobin)) during $J, N J$ and $R$ in a Typically Developing (TD) Children. In the Figure, $L$ corresponds to left frontal cortex and $R$ to correspond to the right frontal cortex. (B) Activation plots depicting changes in hemodynamic response (in terms of $\mathrm{HbO}, \mathrm{HbR}$, and $H b T$ (HbO - oxy-hemoglobin, HbR - deoxy-hemoglobin and HbT - total hemoglobin)) during $J, N J$ and $R$ in a Child with autism. In the Figure, L corresponds to left frontal cortex and $R$ to correspond to the right frontal cortex.

\subsection{Conclusion}

In conclusion, NIRS is employed for the first time to study the joint attention based stimuli's response in TD and ASD children between 4-8 years of age. Preliminary frontal cortical imaging study results indicate difference in activation between $\mathrm{J}$ vs. NJ, J vs. R 
and NJ vs. R stimuli in TD and ASD children. The activation also differs during the stimuli i.e., J, NJ and R between TD and ASD children. Thus the result has a potential to differentiate the two groups of children. These studies when performed extensively on many subjects can potentially form a foundation in understanding the underlying differences in the neuronal networks in TD and ASD children. 
CHAPTER 9. Study 3a: Investigation of Planning and Execution in Individuals with

Cerebral Palsy

In this chapter the application of NIRS to investigate the difference in relative change in total hemoglobin and cortical lateralization between controls and individuals with cerebral palsy has been described. This work was published in the conference proceedings of OSA 2012. (Ujwal Chaudhary, Michael Hall, Jean Gonzalez, Leonard Elbaum, Martha Bloyer and Anuradha Godavarty, "Cognitive Response to Motor Tasks Using NIRS: Pilot Studies of Adults With And Without Spastic Cerebral Palsy", optical society of America, 2012).

\subsection{Introduction}

The use of NIRS in field of CP provides researchers with a functional neuroimaging technique which can elucidate the change in cerebral hemodynamics in the brain of individuals with $\mathrm{CP}$ as they attend to stimuli, something which is not possible with the current functional neuroimaging technique in use. Hence in this study NIRS is used for the first time, to the best of our knowledge, to investigate the planning and execution of motor skill tasks in the prefrontal region of the brain of individuals with CP. The prefrontal region of the brain plays a prominent role in memory, motor set and preparation for movement (Duncan \& Owen, 2000). Researchers have shown that the initiation of motor action in response to sensory stimuli takes place in prefrontal cortex, where the sensory stimuli are integrated to produce a perceivable change in the environment (Faw, 2003). Thus the prefrontal region plays a dominant role in the planning and execution of motor skill tasks (Duncan \& Owen, 2000; Faw, 2003). 
Therefore, in the present pilot study NIRS was used to investigate the prefrontal cortex of individuals with $\mathrm{CP}$ and controls to elucidate differences (if any) in the brain's response to planning and execution of simple motor tasks (e.g. ball throw) in individuals with $\mathrm{CP}$ with respect to controls.

\subsection{Materials and methods}

\subsubsection{Instrumentation}

An in-house developed continuous-wave (CW) NIR spectroscopy (NIRS) system as described in section 3.2 was used for the study. During this study the output optical power of the laser diodes was $5 \mathrm{~mW}$ and the optical cap with the source detector layout were placed on the prefrontal region of the brain in accordance with the international 1020 electrode placement system, as shown in Figure 9.1. Two different source-detector pairs or channels corresponding to left and right prefrontal regions, i.e. Fp1-Fpz and Fp2Fpz, (according to international 10-20 electrode placement system) respectively were used for this study, as the prefrontal region plays a dominant role in planning and execution of motor tasks (Duncan \& Owen, 2000; Faw, 2003 ).

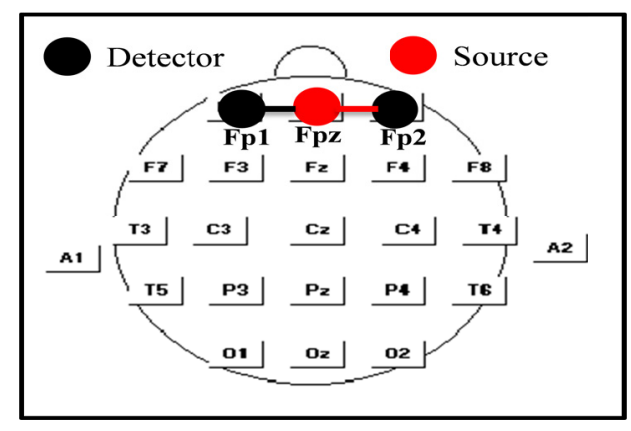

Figure 9.1: Source detector placement is same as the 10-20 system of electrode placement for the pre frontal region of the brain. Each source detector pair is called channel. Fp1 and Fp2 represent the detectors placed on the left and right prefrontal, respectively. Fpz represent the source placed on the mid pre frontal. 


\subsubsection{Participants}

One left handed and one right handed adult male 26 and 28 years old respectively with a primary diagnosis of spastic, quadriplegic cerebral palsy (CP) and seven controls (4 right handed and 3 left handed) in the age range of 21-30 year old were recruited for the study. The individuals with CP were recruited through the physical therapy department in FIU. The participants conveyed their handedness based on the dominant use of their particular hand. This study was FIU-IRB approved and written consent(s) were obtained from the participants.

\subsubsection{Stimulus and Procedure}

The focus of the current study is to investigate how the planning and execution of motor skill tasks manifests itself in the prefrontal region of the brain of individuals with CP. During the study the individuals with CP remained on their wheel chair which was wheeled into a quiet imaging room. The custom-built optical cap was placed on the prefrontal region of their head and the tasks to be performed during the study were explained to them by their physical therapist. While, the control participants were seated comfortably in an upright chair in the same quiet room imaging and the custom-built optical cap was also placed on the prefrontal region of their head. The study begins with a $30-\mathrm{sec}$ rest period, followed by $30 \mathrm{sec}$ of ball throw task (T) and $30 \mathrm{sec}$ of $\operatorname{Rest}(\mathrm{R})$. The T-R block was repeated for 5 times by each participant. During the rest the participants were asked to close their eyes and try not to think anything. During the ball throw task period the participants were asked to throw balls in a container placed at a distance of four feet from their palm. The balls used for this study were soft and spongy, with 
grooves on their surface and of the size of golf ball $(4 \mathrm{~cm}$ in diameter). The task of throwing the ball in the container involved picking up the ball from the box containing the ball, aiming the ball at the container placed at a distance of four feet and finally releasing the ball at such a trajectory that it lands in the container. The task may seem to be easy for controls but for individuals with $\mathrm{CP}$ who have impairment in muscular mobility the task of picking up and throwing the ball in the container is full of challenges. During the study it was observed that individuals with CP had to put lot of strain on their fingers to grab a ball from the box containing the balls, and above all they were unable to throw the ball in the container placed at a distance of four feet. Therefore for individuals with $\mathrm{CP}$ the container was placed right below their hand and they were asked to pick up the ball from the box containing the balls and drop it in the container, even this task was very challenging for them. On the other hand, the ball throw task was performed by seven controls by maintaining a distance of four feet between the palm and the container, in which the participant were asked to throw the balls. The participants performed the task with their dominant hand.

\subsection{NIRS signal analysis}

The in-house developed CW NIRS system, described in section 3.2, acquires the changes in the detected optical signals in response to the stimuli and as a function of time. The NIRS system used during the study employed $785 \mathrm{~nm}$ (close to isosbestic point (Chance

et al., 1988) wavelength of NIR light. Since the absorption spectra of $\mathrm{HbO}$ and $\mathrm{HbR}$ intersect at isosbestic point the change in optical density calculated using wavelength of light close to isosbestic point is equal to the relative change in total hemoglobin 
concentration (HbT) (Chance et al., 1988). Thus the acquired optical signal was processed as descried in section 4.4 to calculate the change in optical density which is equal to the relative change in $\mathrm{HbT}$, for the reason described above. The averaged relative change in $\mathrm{HbT}$ obtained from the controls were further averaged across the right handed controls and left handed controls to obtain the grand average of relative change in $\mathrm{HbT}$, separately for right handed and left handed controls. To demonstrate the consistency in the relative change in $\mathrm{HbT}$ across the participants, mean and standard deviation of the relative change in $\mathrm{HbT}$ for each participant was calculated before averaging. The calculated mean of relative change in $\mathrm{HbT}$ across each participant was greater than the standard deviation in relative change in $\mathrm{HbT}$ across each participant demonstrating the consistency of the change in $\mathrm{HbT}$ acquired from the participants. While the relative change in $\mathrm{HbT}$ obtained from the individuals with $\mathrm{CP}$ was not averaged across the participants to obtain grand average as the study was performed on one right handed and one left handed individuals with CP. Herein, two different studies were performed using intensity data, namely: 1) Relative change in total hemoglobin ( $\mathrm{HbT}$ ), and 2) Cortical lateralization.

\subsubsection{Relative change in total hemoglobin}

The relative change in $\mathrm{HbT}$ for right handed and left handed controls as well as right handed and left handed individuals with CP was calculated as described in section 9.3. The relative change in average $\mathrm{HbT}$ for all the participant types was also quantified by computing the total change in $\mathrm{HbT}$ for the central $20 \mathrm{sec}$ period of the ball throw task and rest period. As mentioned in the section 9.3, the duration of each stimulus was $30 \mathrm{sec}$ but 
only the central $20 \mathrm{sec}$ period, of each stimulus from all the participants' type, was used to calculate the total change in $\mathrm{HbT}$ to negate the confounding effect of one stimulus over the other.

\subsubsection{Cortical lateralization}

Cortical lateralization study was performed to understand the hemispherical dominance of the planning and execution task in the frontal cortex and its variation across the stimuli for each participant type. The laterality index was computed for left vs. right frontal cortex for each stimulus ( $\mathrm{T}$ and $\mathrm{R}$ ) using the relative change in $\mathrm{HbT}$ obtained from the channels: Fp1-Fpz vs. Fp2-Fpz (see Figure 9.1), as described in section 4.7.

\subsection{Result and discussion}

\subsubsection{Relative change in total hemoglobin}

The averaged relative change in total hemoglobin across the T-R block obtained from the prefrontal cortex (Fp1-Fpz-Fp2) of left and right handed individuals with $\mathrm{CP}$ and controls, as a function of time, is shown in Figure 9.2. The total relative change in HbT obtained from right handed controls (as shown in Figure 9.2A), left handed controls (as shown in Figure 9.2B), right handed individuals with CP (as shown in Figure 9.2C) and left handed individuals with CP (as shown in Figure 9.2D) is represented by horizontal dotted black line for Fp1-Fpz channel and by horizontal solid red line for Fp2-Fpz channel in Figure 9.2.

It can be observed from Figure 9.2A and 9.2B which correspond to relative change in $\mathrm{HbT}$ in right handed and left handed controls, respectively, that there is a difference in HbT between ball throw task and rest across both the channels (Fp1-Fpz and Fp2-Fpz). 
The quantified change in $\mathrm{HbT}$ across Fp1-Fpz channel during the ball throw task and rest performed by right handed controls, as shown in Figure 9.2A by black dotted horizontal lines, was $0.045(\mathrm{abu})$ and $-0.015(\mathrm{abu})$, respectively. The quantified change in $\mathrm{HbT}$ across Fp2-Fpz channel during the ball throw task and rest performed by right handed controls, as shown in Figure 9.2A by red solid horizontal lines, was 0.025 (abu) and $0.038(\mathrm{abu})$, respectively.

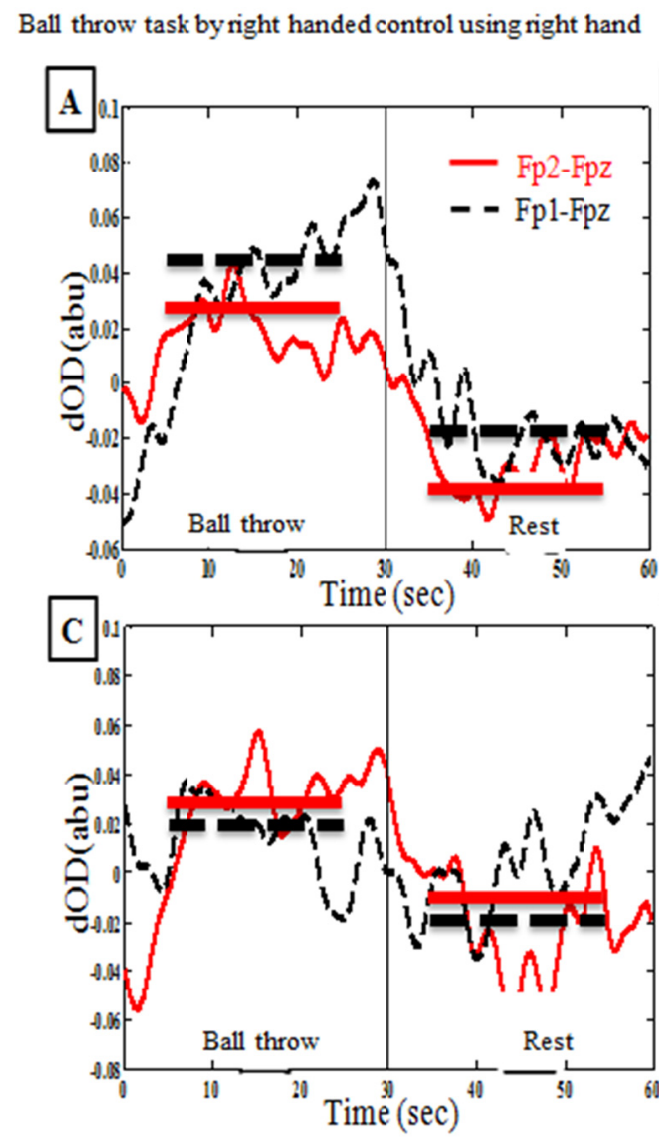

Ball throw task byleft handed control using left hand

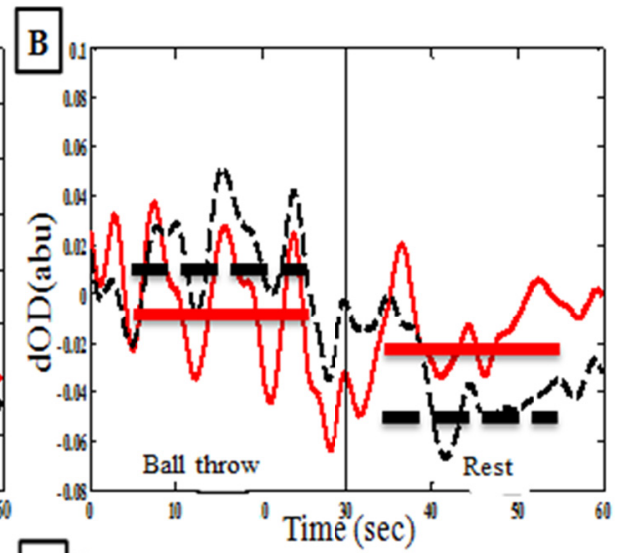

Ball throw task by right handed adult with $\mathrm{CP}$ using right hand Ball throw task by right handed adult with $\mathrm{CP}$ using right hand

Figure 9.2: Average change in total hemoglobin across all participants (HbT) as a function of time across the source detector combination Fp1-Fpz (plot in dashed black) and Fp2-Fpz (plot in solid red). The quantified change in HbT calculated by summing the change in HbT for the central $20 \mathrm{sec}$ period of the ball throw task and rest period is shown as thick dotted black line and thick solid red line across the source detector combination Fp1-Fpz and Fp2-Fpz, respectively. The first $30 \mathrm{sec}$ represent the change in $H b T$ during ball throw task (BT) and the last 30 sec represent Rest period (R). 
Similarly, the quantified change in HbT across Fp1-Fpz channel during the task and rest performed by left handed controls, as shown in Figure 9.2B by black dotted horizontal lines, was $0.018(\mathrm{abu})$ and $-0.05(\mathrm{abu})$, respectively. The quantified change in $\mathrm{HbT}$ across Fp2-Fpz channel during the task and rest performed by left handed controls, as shown in Figure 9.2B by red solid horizontal lines, was -0.05 (abu) and $-0.022(\mathrm{abu})$, respectively. Thus the relative change in total hemoglobin depicts greater relative change in $\mathrm{HbT}$ during the task than during the rest period both in right handed and left handed controls.

It can be observed from Figure 9.2C and 9.2D, which correspond to the change in $\mathrm{HbT}$ in right handed and left handed adult with $\mathrm{CP}$, respectively, that there is a difference in $\mathrm{HbT}$ between task and rest across both the channels (Fp1-Fpz and Fp2-Fpz). The quantified change in $\mathrm{HbT}$ across Fp1-Fpz channel during the task and rest performed by right handed adult with $\mathrm{CP}$, as shown in Figure $9.2 \mathrm{C}$ by black dotted horizontal lines, was $0.02(\mathrm{abu})$ and $-0.02(\mathrm{abu})$, respectively. The quantified change in HbT across Fp2-Fpz channel during the task and rest performed by right handed adult with $\mathrm{CP}$, as shown in Figure $9.2 \mathrm{C}$ by red solid horizontal lines, was 0.03 (abu) and -0.01 (abu), respectively. Similarly, the quantified change in $\mathrm{HbT}$ across Fp1-Fpz channel during the task and rest performed by left handed adult with $\mathrm{CP}$, as shown in Figure 9.2D by black dotted horizontal lines, was $0.02(\mathrm{abu})$ and $-0.018(\mathrm{abu})$, respectively. The quantified change in $\mathrm{HbT}$ across Fp2-Fpz channel during the task and rest performed by left handed adult with $\mathrm{CP}$, as shown in Figure 9.2D by red solid horizontal lines, was 0.08 (abu) and -0.001 (abu), respectively. Thus the relative change in total hemoglobin depicts greater change 
in $\mathrm{HbT}$ during the task than during the rest period both in right handed and left handed controls.

Thus the relative change in $\mathrm{HbT}$ across the Fp1-Fpz and Fp2-Fpz channels for all the participant types indicates that the change in $\mathrm{HbT}$ during the task is greater than the change in $\mathrm{HbT}$ during the rest (from the values plotted in the Figure 9.2). The relatively greater change in $\mathrm{HbT}$ during the task as compared to rest indicates the increase in the blood flow to the prefrontal region of the brain to support the increased metabolic activity due to the activation of the prefrontal region of the brain during the planning and execution of motor skill task.

\subsubsection{Cortical lateralization}

The result of the cortical lateralization study for all the participant type is shown in Table 9.1 and Figure 9.3, which contains the laterality index for each participant during the task and rest period. The laterality index value for right handed control (shown in Table 9.1A and Figure 9.3A) and for left handed control (shown in Table 9.1B and Figure 9.3B), indicates that there is a bilateral dominance during the task and contralateral dominance during the rest in the prefrontal cortex of controls (left/right handed). The laterality index value for right handed individuals with CP (shown in Table 9.1C and Figure 9.3C) and for left handed individuals with CP (shown in Table 9.1D and Figure 9.3D), indicates that there is an ipsilateral dominance during the task and contralateral dominance during the rest in the prefrontal cortex of individuals with $\mathrm{CP}$ (left/right handed). 


\begin{tabular}{|c|c|c|c|}
\hline \multirow{2}{*}{ Row No. } & \multirow{2}{*}{ Participant Type } & \multicolumn{2}{|c|}{ Dominance } \\
\cline { 3 - 4 } & Right handed control & 0.02 (Bilateral) & $\begin{array}{c}0.38 \\
\text { (Contralateral) }\end{array}$ \\
\hline (A) & Rest \\
\hline (B) & Left handed control & -0.1 (Bilateral) & $\begin{array}{c}-0.29 \\
\text { (Contralateral) }\end{array}$ \\
\hline (C) & $\begin{array}{c}\text { Right handed adult with } \\
\text { cerebral palsy (CP) }\end{array}$ & -0.75 (Ipsilateral) & $\begin{array}{c}0.29 \\
\text { (Contralateral) }\end{array}$ \\
\hline (D) & $\begin{array}{c}\text { Left handed adult with } \\
\text { cerebral palsy (CP) }\end{array}$ & 0.26 (Ipsilateral) & $\begin{array}{c}-0.35 \\
\text { (Contralateral) }\end{array}$ \\
\hline
\end{tabular}

Table 9.1: Pre frontal cortical lateralization or dominance in response to planning and execution of motor skill task and rest task in controls and adult (s) with cerebral palsy.

The result of change in $\mathrm{HbT}$ and cortical lateralization shows that although there was difference in the relative change in $\mathrm{HbT}$ between task and rest period across all the participant type the cortical lateralization differs between controls and individuals with $\mathrm{CP}$ during the task period. The result of cortical lateralization during the task shows contralateral dominance in controls and ipsilateral dominance in individuals with $\mathrm{CP}$. The present study was performed on small number of participants because of which no statistically significant conclusion can be made but the preliminary results suggests difference in the response of underlying prefrontal cortical region of controls and individuals with CP. The preliminary results also raises the possibility of use of NIRS as a reliable tool to investigate the planning and execution of motor skills task in individuals with $\mathrm{CP}$ and upon further extensive studies NIRS has the potential to elucidate the neural basis of cognition in individuals with $\mathrm{CP}$. 


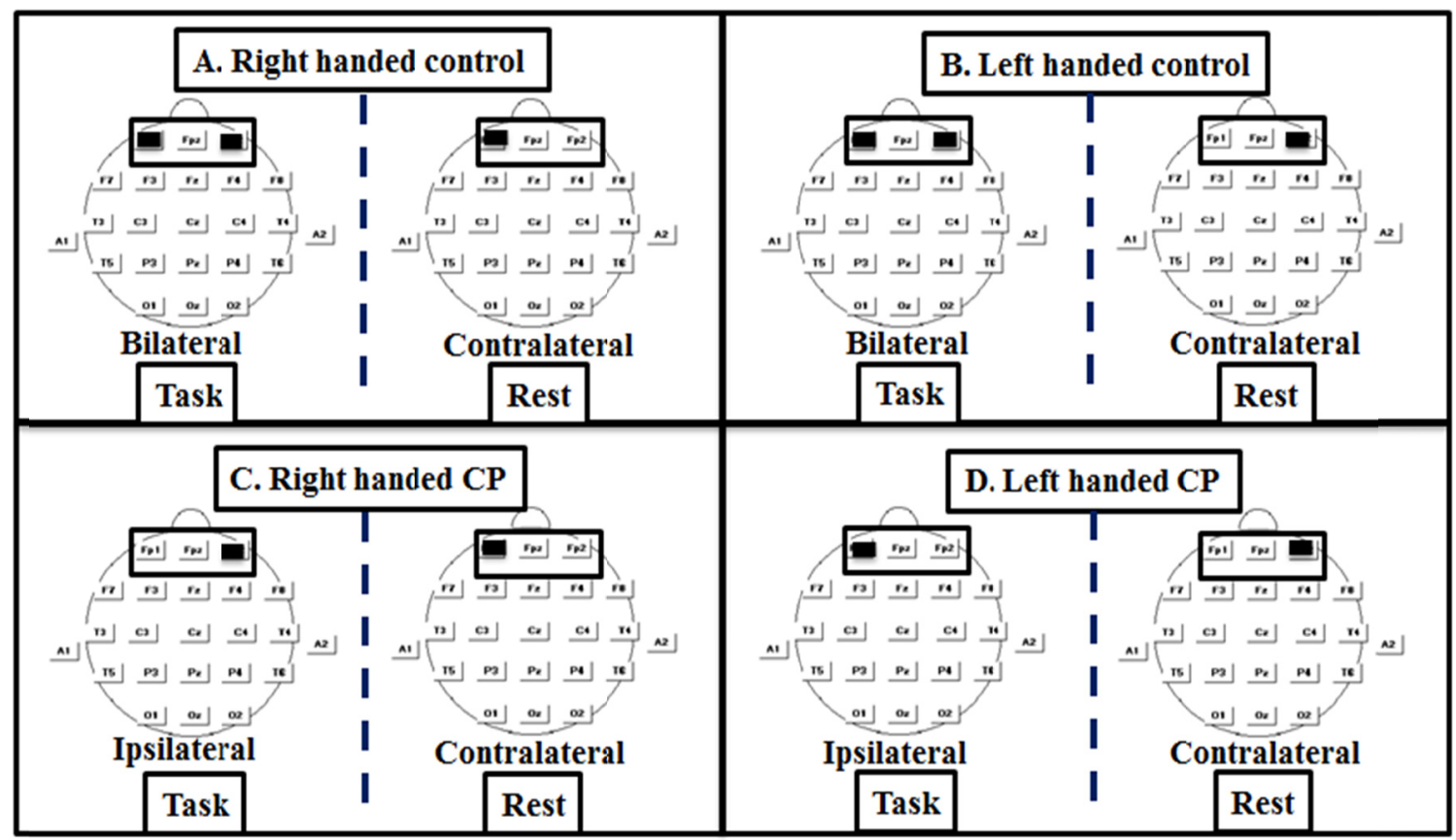

Figure 9.3: Cortical lateralization/dominance map during task and rest for all the participant type. In the map the highlighted rectangular box represent the prefrontal region under investigation and the thick line denotes the dominant side. A-Dominance map for the right handed control. B - Dominance map of the left handed control. $C$ Dominance map of the right handed individuals with CP. D-Dominance map of the left handed individuals with $C P$.

\subsection{Conclusion}

Motor skill task was performed by controls and individuals with $\mathrm{CP}$ and the optical signal was measured using a continuous wave based NIRS system. The preliminary results show difference, in terms of change in $\mathrm{HbT}$, between ball throw task and rest period both in controls and in individuals with $\mathrm{CP}$. Although there was difference in the relative change in $\mathrm{HbT}$ between task and rest period across all the participant type, the cortical lateralization differed between the controls and individuals with $\mathrm{CP}$ during the task period. The cortical lateralization showed bilateral dominance in controls and 
contralateral dominance in individuals with $\mathrm{CP}$ during the task period, in the prefrontal region of brain.

The main aim of the present work was to demonstrate the feasibility of NIRS towards investigation of the difference if any in planning and execution of simple motor tasks in controls vs. $\mathrm{CP}$, such that it can be developed as a reliable tool to aid in the rehabilitation of individuals with CP. During the study it was observed that the individuals with CP had problem in performing the ball throw task because of the limited mobility of the upper extremity of individuals with $\mathrm{CP}$. Hence it was decided to change the ball throw task to a simpler planning and execution of motor skill stimuli, known as target touching task which will be described in chapter 10. Extensive studies were performed on controls in response to different variant of target touching tasks along with ball throw tasks to investigate the response of frontal cortex before applying the target touching tasks on individuals with $\mathrm{CP}$. 
CHAPTER 10. Study 3b: Motor Skill Investigation of Planning and Execution in

\author{
Healthy Adults
}

The preliminary ball throw task study described in chapter 9 demonstrated the role of pre frontal cortex in planning and execution of motor skill stimuli. Hence in this chapter the extensive studies performed to statistically validate the role of pre and anterior frontal cortex in response to different planning and execution of motor skills stimuli have been described.

\title{
10.1 Introduction
}

Dorsolateral cortex of the frontal lobe plays a prominent role in memory, motor set and the preparation for movement (Duncan \& Owen, 2000). The frontal cortex of the brain is the integration center of the brain where the integration of sensory and perceptual information takes place (Faw, 2003). The motor actions which arise due to the sensory stimuli are organized in the frontal cortex which produces a perceivable change in the environment (Duncan \& Owen, 2000). The global scheme of action associated with the motor actions is represented and monitored during execution in prefrontal region. Thus the prefrontal region is responsible for planning and execution of motor skills stimuli. The prefrontal region is also responsible for the action of skeletal muscle in response to sensory stimuli (Duncan \& Owen, 2000; Faw, 2003).

In the current study NIRS was used to investigate the response of pre and anterior frontal cortical region of healthy adult human participants. The participants performed three different (novel) planning and execution of motor skills stimuli with varying level of 
complexity and the response of the pre and anterior frontal region of brain was evaluated to determine the tasks which elicits maximum response. The goal of the current study is design paradigms pertinent to the everyday task which can be used to stimulate the frontal cortex of individuals with cerebral palsy to help them in their rehabilitation.

\subsection{Materials and methods}

\subsubsection{Instrumentation}

An in-house developed continuous-wave (CW) NIR spectroscopy (NIRS) system as described in section 3.2 was used for the study. During this study the output optical power of the laser diodes was $5 \mathrm{~mW}$ and the optical cap with the source detector layout were placed on the frontal region of the brain in accordance with the international 10-10 electrode placement system, as shown in Figure 10.1. Four different source-detector pairs or channels namely Fp1-Fpz (Ch.1), Fp2-Fpz (Ch.2), AF3-AFz (Ch.3) and AF4AFz (Ch.4) were used for the study as shown in Figure 10.1.

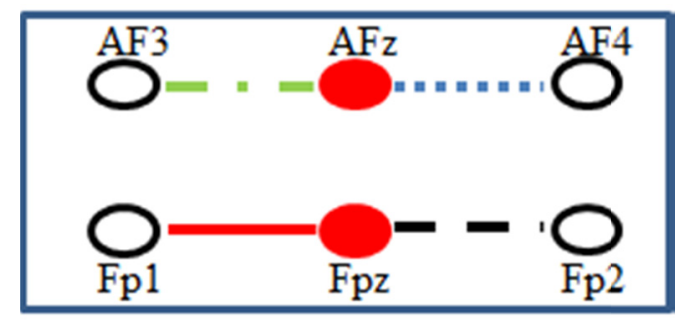

Figure 10.1: Source detector layout corresponding to the 10-10 electrode placement system showing the placement of source (solid circle) and detector (hollow circle) on the pre and anterior frontal region of brain. Each source detector pair is called channel. Fpl and Fp2 represent the detector placed on the left and right prefrontal, respectively. Fpz represent the source placed on the mid pre frontal. AF3 and AF4 represent the detector placed on the left and right anterior frontal, respectively. AFz represent the source placed on the mid anterior frontal. 


\subsubsection{Participants}

Seventeen healthy right-handed adults between 21-30 years of age were enrolled for the current study. The participants conveyed their handedness based on the dominant use of their particular hand. This study was FIU-IRB approved and written consent(s) were obtained from the participants.

\subsubsection{Experimental design and protocol}

The goal of the current NIRS is to elucidate the response of the pre and anterior frontal region of brain in response to different planning and execution of motor skill tasks. Different innovative paradigms were designed to investigate the response of pre and anterior region of brain in response to the variation in complexity and kind of the task. The study took place in a quiet imaging room with dim ambient light. During the study custom-built optical cap was placed on the frontal region of their head (with minimal hair interference) and the participants were asked to seat comfortably on an upright chair. The participants attended to three different block designed paradigm as described below in section 2.3.1, 2.3.2 and 2.3.3, with a break of 15 minutes between each paradigm.

\subsubsection{Paradigm 1: Dot placement - Coarser motor control}

The dot placement tasks were used to investigate the frontal region of brain of controls with the aim to design a paradigm to address the problem of motor movement in individuals with CP. During this paradigm a table was placed in front of the participants with an A4 sheet firmly secured on the top of table within the reaching distance of participants' hand. The A4 sheet consists of circular ring of 0.6 " diameter drawn on its four corners. The paradigm used during the study consists of 3 blocks of four 30 -second 
stimuli, namely the dot placement (DP-C), rest (R1), dot placement with balance (DP$\mathrm{CB}$ ) and rest (R2) in a sequential order. Prior to the block-designed paradigm, an initial 30 -sec rest period was employed to determine the baseline of the brain activity of the participants. During the dot placement stimuli (DP-C) the participants were asked to hold a pen in writing style and place dots in the center of four circles as fast and as precisely as they can in such a way that their hand movement pattern resembles a "Z". During Rest (R1) the participants were asked to close their eyes and try not to think anything. During the dot placement with balance (DP-CB) the participants were asked to hold a pen in writing style and a $100 \mathrm{ml}$ beaker filled with $50 \mathrm{ml}$ of water was placed on their hand. Then the participants were asked to place dots in the center of four circles as fast and as precisely as they can, while balancing the beaker without spilling water, in such a way that their hand movement pattern resembles a " $Z$ ". The rationale behind adding a beaker with water during DP-CB was to increase the complexity of the task thereby increasing the cognitive load on the frontal region of brain. The rest period R2 was similar to similar to rest period R1, the only difference was that the R1 followed DP-C and R2 followed DP-CB. The participants performed the task with both of their hand, first with dominant hand and then with non-dominant hand.

\subsubsection{Paradigm 2: Dot placement - Finer motor control}

The individuals with $\mathrm{CP}$ often have problem with finer motor movement hence the tasks described in paradigm 1 was modified to address the question of finer motor movement. The paradigm 2 was similar to paradigm 1 except the size of the circle drawn on the A4 sheet. For paradigm 2 the circles drawn on the four corner of A4 sheet were 0.3 " in 
diameter, decreasing the size of circle and thus increase the complexity of the task. The participants performed the 3 blocks of four 30 -second stimuli, namely the dot placement (DP-F), rest (R1), dot placement with balance (DP-FB) and rest (R2) in a sequential order. The rationale of this paradigm was that the decrease in the diameter of the circle in which they were asked to place a dot would force the participants to adjust their motor skill associated with movement to perform finer movement. Thus the goal of performing two different variant of same task was to elucidate the response of pre and anterior frontal cortex of brain as the complexity of task varies.

\subsubsection{Paradigm 3: Ball throws}

This paradigm was designed to investigate the grabbing and throwing aspect of motor movement with the future aim to apply it on individuals with CP. During this paradigm the participants were asked to throw balls in a cubic container of dimension $20 \times 20 \times 20$ $\mathrm{cm}^{3}$ placed on an elevated platform, two feet in height, at a distance of four feet from the participant. The paradigm used during the study again consists of 3 blocks of four 30second stimuli, namely the ball throw with dominant hand (BTD), rest (R1), ball throw with non-dominant hand (BTND) and rest (R2) in a sequential order. Prior to the blockdesigned paradigm, an initial 30-sec rest period was employed to determine the baseline of the brain activity of the participants. During the ball throw task either with dominant hand (BTD) or non-dominant hand (BTND) the participants were asked to throw balls in a container placed at a distance of four feet from their palm. The balls used for this study were soft and spongy, with grooves on their surface and of the size of golf ball $(4 \mathrm{~cm}$ in diameter). The task of throwing the ball in the container involved picking up the ball 
from the box containing the ball, aiming the ball at the container placed at a distance of four feet and finally releasing the ball at such a trajectory that it lands in the container. During the rest R1 and R2 the participants were asked to close their eyes and try not to think anything.

The tasks employed in paradigm 1 and 2 are completely different from the task employed in paradigm 3. The rationale behind such a task was to investigate how does the response in pre- and anterior frontal cortex changes as the task becomes completely different. Thus during this experimental study three different novel and innovative paradigm were designed and used to investigate and elucidate the role of pre and anterior frontal cortex of brain; as (1) the complexity of task changes, (2) the tasks demands comparatively finer motor control, and (3) the tasks changes to completely different everyday task.

\subsection{NIRS signal analysis}

The acquired optical signal was post processed using custom Matlab based code to determine the change in total hemoglobin concentration $(\mathrm{HbT})$. The acquired optical signal was first filtered using a band pass filter $(0.016 \mathrm{~Hz}-0.3 \mathrm{~Hz})$ to remove the systemic physiological noise. The filtered optical signal was then normalized and post processed to calculate the change in $\mathrm{HbT}$ using steps described in details in section 4.4. The averaged change in $\mathrm{HbT}$ obtained from each participant then was averaged across all the participants to obtain the grand averaged change in $\mathrm{HbT}$. Prior to averaging the change in $\mathrm{HbT}$ across all participants, the mean and standard deviation across participants was evaluated in order to assess the consistency in the responses across participants. Three different kind of analyses were performed on the processed $\mathrm{HbT}$ data obtained 
from paradigm 1, 2 and 3 which include: (i) change in total hemoglobin, (ii) connectivity, and (iii) cortical lateralization study.

\subsubsection{Relative change in total hemoglobin}

The relative change in total hemoglobin study was performed to elucidate the differences in $\mathrm{HbT}$ as a function of time, in the frontal cortex in response to the stimuli presented during the three different paradigms. The entire window of $30 \mathrm{sec}$ for each stimulus was used to plot the averaged change in $\mathrm{HbT}$ plot with respect to time for each sourcedetector pair or channel (Ch.), separately for each paradigm. Preliminary studies were carried out by using a smaller window $(10 \mathrm{sec}, 15 \mathrm{sec}$, and $20 \mathrm{sec})$ of the entire $30 \mathrm{sec}$ window of the stimulus in order to observe if the contrast in the stimuli was any different. Using the averaged $\mathrm{HbT}$ data, paired $\mathrm{t}$-tests $(\mathrm{p}=0.05)$ was performed for each individual channel (Ch.) across two chosen 30-s stimuli, separately for each paradigm. The relative change in averaged $\mathrm{HbT}$ was also quantified by computing the total change in $\mathrm{HbT}$ for the central $20 \mathrm{sec}$ period of each $30 \mathrm{sec}$ stimulus, separately for each paradigm. As mentioned in the section 10.2.3, the duration of each stimulus was $30 \mathrm{sec}$ but only the central $20 \mathrm{sec}$ period was used to calculate the total change in $\mathrm{HbT}$ to negate the confounding effect of one stimulus over the other.

\subsubsection{Functional connectivity analysis}

In the present study, functional brain connectivity was performed to characterize the ways in which the left and right frontal cortex was functionally integrated or works in synchrony in response to planning and execution of motor skill stimuli. This study is carried out by computing zero lagged correlation (as described in section 4.5) to elucidate 
the correlation and synchrony in the hemodynamic response and hence the connectivity in the neuronal circuit between the left and right frontal cortex. Correlation calculations were performed between left vs. right frontal cortex for each stimulus using the HbT data obtained from the following channels combinations: Ch.2 vs. Ch.1 and Ch.4 vs. Ch.3, separately for each paradigm.

\subsubsection{Lateralization analysis}

Cortical lateralization study is performed to understand the hemispherical dominance of the language in the frontal cortex and its variation across the stimuli. The laterality index (as described in section 4.7) was computed for left vs. right frontal cortex for each stimulus using the change in HbT obtained from channel combination (Ch.2 vs. Ch.1 and Ch.4 vs. Ch.3).

\subsection{Results and discussion}

The three different kind of data analyses described in section 3 namely change in $\mathrm{HbT}$, functional connectivity, and lateralization were performed separately for each of the three different paradigms described in section 10.2.3. In this section the results obtained from the different analysis will be presented separately for each paradigm.

\subsubsection{Paradigm1: Dot placement - coarser motor control}

\subsubsection{Relative change in $\mathrm{HbT}$ and lateralization results}

The averaged relative change in $\mathrm{HbT}$ across all the participants in response to dot placement-Coarser motor control tasks performed via both dominant and non-dominant hand is shown in Figure 10.2 across each stimulus (DP-C, R1, DP-CB and R2) and each 
channel. The Figure 10.2A and 10.2B corresponds to the averaged relative change in $\mathrm{HbT}$ for the tasks performed by participants using their dominant and non-dominant hand, respectively. It can be observed from the averaged relative change in $\mathrm{HbT}$ plots that the increase in $\mathrm{HbT}$ during DP-C and DP-CB was different from the decrease in $\mathrm{HbT}$ during R1 and R2, both for the task performed using dominant and non-dominant hand. The relative increase in $\mathrm{HbT}$ during task DP-C and DP-CB supports the hypothesis that when the region of the brain becomes activated in response to a task the cerebral blood flow to the activated region increases (Hoshi et al., 1994). The increase in blood flow in the activated region is to support the increased metabolic activity in the activated region (Kleinschmidt et al., 1996). The results of paired t-test between DP-C vs. R1 and DP-CB vs. R2 indicated significant difference between DP-C and R1 as well as DP-CB and R2 at a significance level of 0.05 , both for the task performed using dominant and nondominant hand. The rest conditions (R1 and R2) have a non-zero signal initially which tapers to a zero-signal (i.e. initial baseline at the beginning of the block) by the end of the rest period. The initial non-zero condition is because the effect of any stimuli continues in terms of the changes in the cerebral blood volume for a few seconds after the stimulus ends. Hence, the relative change in $\mathrm{HbT}$ in response to the rest condition begins with a non-zero signal and eventually tapers to a zero-signal as seen from Figure 10.2, validating the effectiveness of the data. The decrease in metabolic activity during the rest period indicates that the participants were indeed able to decrease their thought process which elicited decrease in the relative change in $\mathrm{HbT}$. 
In addition, the relative change in $\mathrm{HbT}$ was observed to be consistent across the 17 participants, as observed from the statistical analysis of the data in terms of mean and standard deviation. This demonstrates that the observed average relative change in $\mathrm{HbT}$ in response to the stimuli (DP-C, R1, DP-CB and R2) was consistent across all the participants. The averaged relative change in $\mathrm{HbT}$ across all the participants was also plotted along with the change in $\mathrm{HbT}$ obtained from each participant to demonstrate the similarity in the response obtained from all the participants, described in details in Appendix A.3 and A.4.
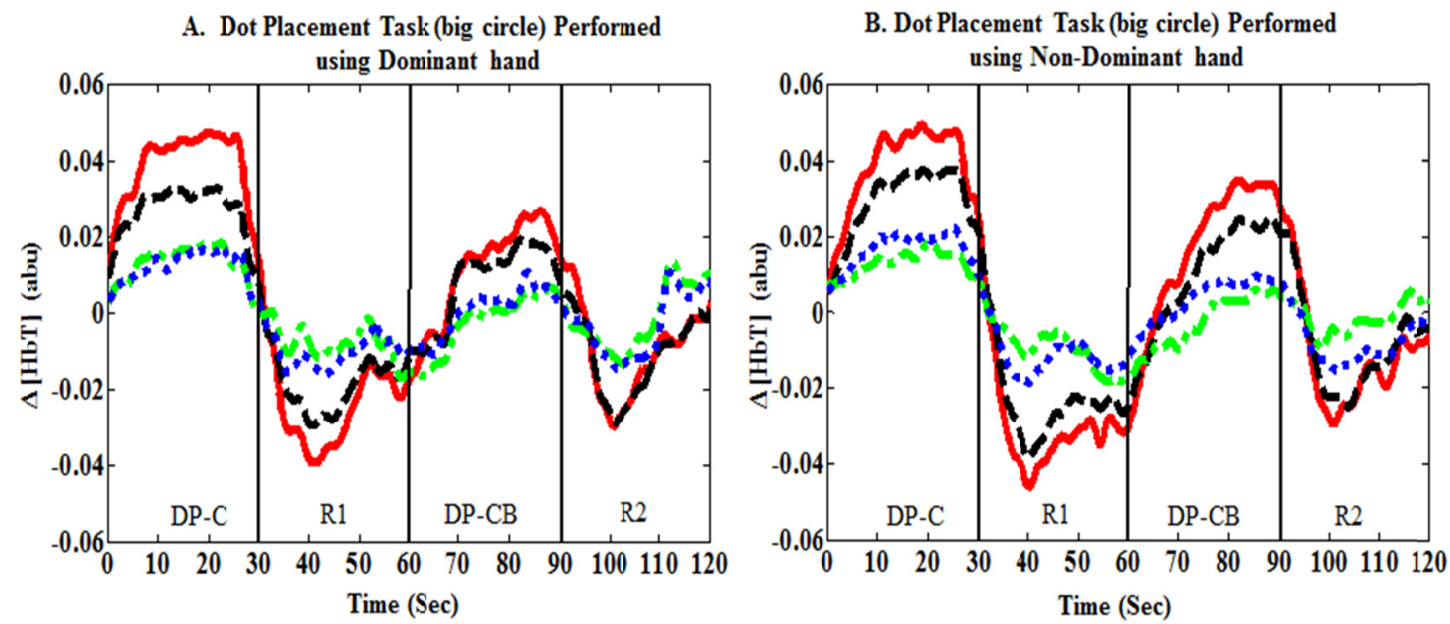

Figure 10.2: Change in total hemoglobin (HbT) (averaged across all participants) as a function of time for the source detector combination Fp1-Fpz (plot in solid red), Fp2-Fpz (plot in dashed black), AF3-AFz (plot in dash-dot green) and AF4-AFz (plot in dotted blue) during dot placement coarser motor control paradigm. In the plot, the y-axis is the relative change in $H b T$, while $x$-axis is the time in seconds. The relative change in $H b T$ from 0-30 sec corresponds to dot placement task (DP-C), 30-60 sec to rest period (R1), 60-90 sec to dot placement with balance task (DP-CB) and 90-120 sec to second rest period (R2). A-Depicts the relative change in $H b T$ when the participants performed the tasks via their dominant hand. B - Depicts the relative change in HbT when the participants performed the tasks via their non-dominant hand. 
The quantified change in $\mathrm{HbT}$ both for the task performed using dominant and nondominant hand is plotted as histogram for each channel and tasks as shown in Figure 10.3 along with the values of the relative change in $\mathrm{HbT}$. It can be observed from Figure 3, that the extent of change in $\mathrm{HbT}$ both for DP-C and DP-CB was greater in prefrontal channels (Ch. 1 and Ch. 2) than the anterior frontal channels (Ch. 3 and C. 4), both for the task performed using dominant and non-dominant hand. Within the prefrontal channels, the change in HbT was greater in the left channels than the right channels both for DP-C and DP-CB and within the tasks DP-C and DP-CB the change in HbT was greater during DP-C than during DP-CB, both for the task performed using dominant and non-dominant hand. Finally within the task performed via either dominant or nondominant hand the relative change in $\mathrm{HbT}$ during the task performed via non-dominant hand was greater than during the task performed via dominant hand.

The results from lateralization analysis are shown in Figure 10.3 by using lines of different style and thickness connecting a particular source and detector pair. A thin, thick dash and thick dash-dot horizontal line drawn between a particular source and detector pair indicates no dominance, left dominance and right dominance respectively. The left pre frontal cortex was found to be dominant over the right pre frontal when the participants performed the task DP-C using their dominant hand, shown by thick dash line connecting the pre frontal source and detector pair in Figure 10.3A. While no dominance was found in terms of change in $\mathrm{HbT}$ between left vs. right pre and anterior frontal cortex for task DP-CB performed using dominant hand, task DP-C and DP-CB performed using non-dominant hand, shown by thin line connecting the source and detector pair in Figure 10.3B, 10.3C and 10.3D respectively. 
DP-C + Rl

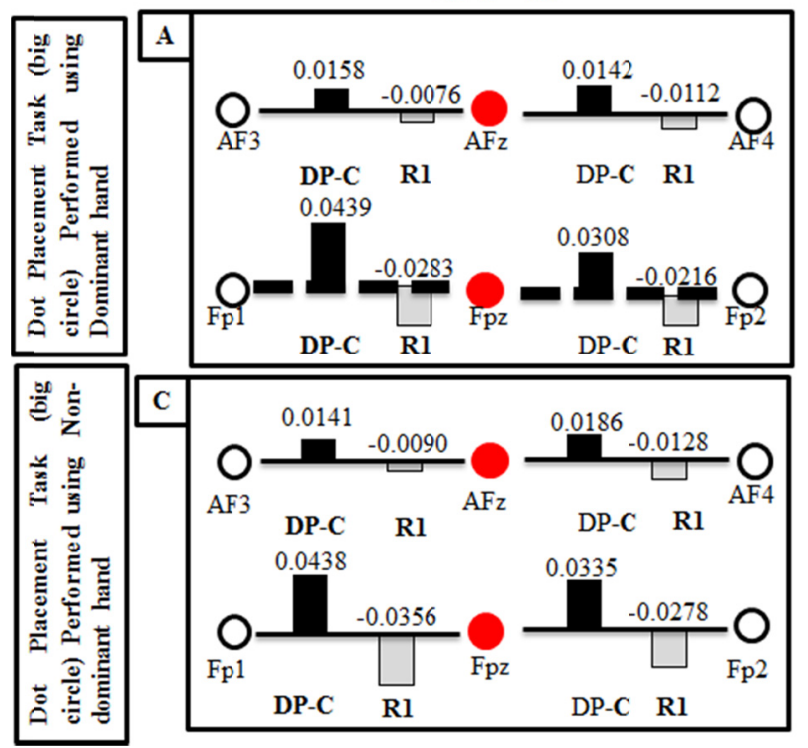

$\mathrm{DP}-\mathrm{CB}+\mathrm{R} 2$

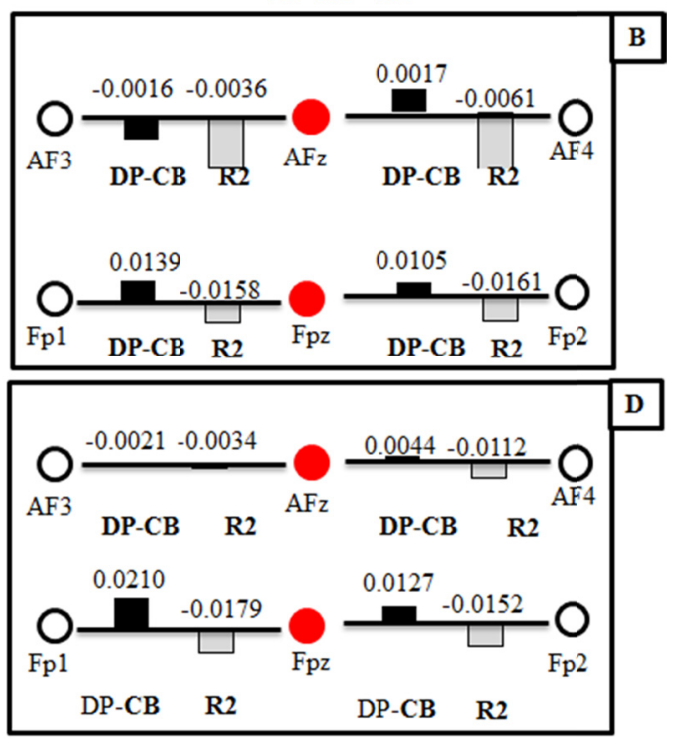

Figure 10.3: Bar plot depicting the quantified relative change in HbT across different source-detector pairs for the dot placement coarser motor control paradigm performed by the participants via their dominant and non-dominant hand. In each plot the relative change in HbT across a particular source-detector pair is shown by a bar plot as well as a quantitative value calculated by averaging the central $20 \mathrm{sec}$ of the relative change in $H b T$ during a particular stimulus, where (A) includes task (Dot placement coarser motor control: DP-C) and rest (RI) using dominant hand, (B) includes task (Dot placement coarser motor control with beaker: $D P-C B$ ) and rest (R2) using dominant hand, $(C)$ includes task (DP-C) and rest (R1) using non-dominant hand, and (D) includes task (DP$C B)$ and rest (R2) using non-dominant hand. In each plot the bar in black and grey represents the quantitative relative change in $H b T$ during task (DP-C or DP-CB) and rest period ( $R 1$ and $R 2$ ) respectively. The results from lateralization analysis are also shown in Figure 10.3 by using lines of different style and thickness connecting a particular source and detector pair. In Figure 10.3 the thick dash line drawn to represent the prefrontal channels, denotes left dominance.

Combining the result of relative change in HbT during task DP-C and DP-CB it can be observed that if the task is relatively simple then the contralateral pre frontal region of the brain plays a dominant role in processing the stimulus as evident by the left prefrontal cortical lateralization during the task DP-C performed by dominant (right) hand. The result of no cortical dominance or bilateral activation during task DP-C performed via non-dominant (left) hand as well as task DP-CB performed via either non-dominant (left) 
hand or dominant (right) hand indicates the involvement of both left and right region to process the relatively complex task. This suggests that as the task becomes more complex both the hemisphere i.e. left and right pre frontal region of the brain seems to plays dominant role in processing the stimulus.

\subsubsection{Functional connectivity results}

The results from the functional connectivity analysis are shown as a table of correlation coefficient obtained from zero-order correlation analysis in Table 10.1. It is apparent from Table 10.1 that there was significant synchrony/correlation in terms of change in $\mathrm{HbT}$ between left vs. right pre and anterior frontal channels across any tasks, both for the task performed using dominant and non-dominant hand. Significant correlation between left vs. right pre and anterior frontal region was also observed during rest period as shown in Table 10.1.

\begin{tabular}{|c|c|c|c|c|c|}
\hline Task Handedness & Channel/Stimuli & DP-C & R1 & DP-CB & $\mathrm{R} 2$ \\
\hline \multirow[t]{2}{*}{ Dominant Hand } & Fp1-Fpz vs. Fp2-Fpz & .91 & .99 & .98 & .97 \\
\hline & $\mathrm{AF} 3-\mathrm{AFz}$ vs. $\mathrm{AF} 4-\mathrm{AFz}$ & .90 & .97 & .95 & .98 \\
\hline \multirow{2}{*}{$\begin{array}{c}\text { Non-dominant } \\
\text { Hand }\end{array}$} & Fp1-Fpz vs. Fp2-Fpz & .98 & .99 & .99 & .98 \\
\hline & $\mathrm{AF} 3-\mathrm{AFz}$ vs. $\mathrm{AF} 4-\mathrm{AFz}$ & .90 & .82 & .96 & .82 \\
\hline
\end{tabular}

Table 10.1: Correlation value between left vs. right pre and anterior frontal channel, when the participants performed the dot placement coarser motor control paradigm using their dominant and non-dominant hand. 


\subsubsection{Dot placement - coarser motor control}

\subsubsection{Change in $\mathrm{HbT}$ and lateralization results}

The averaged change in $\mathrm{HbT}$ across all the participants in response to dot placementFiner motor control tasks performed via both dominant and non-dominant hand is shown in Figure 10.4 across each stimulus (Dot placement finer motor control:DP-F, R1, Dot placement finer motor control with beaker:DP-FB and R2) and each channel. The Figure 10.4A and $10.4 \mathrm{~B}$ corresponds to the averaged change in $\mathrm{HbT}$ for the tasks performed by participants using their dominant and non-dominant hand, respectively. It can be observed from the averaged change in $\mathrm{HbT}$ plots that the increase in $\mathrm{HbT}$ during $\mathrm{DP}-\mathrm{F}$ and DP-FB was different from the decrease in $\mathrm{HbT}$ during R1 and R2, both for the task performed using dominant and non-dominant hand. The results of paired t-test between DP-F vs. R1 and DP-FB vs. R2 indicated significant difference between DP-F and R1 as well as DP-FB and R2 at a significance level of 0.05 , both for the task performed using dominant and non-dominant hand. The change in $\mathrm{HbT}$ observed during rest conditions $\mathrm{R} 1$ and R2 of dot placement with finer motor control was similar to change in $\mathrm{HbT}$ observed during rest conditions R1 and R2 of dot placement with coarse motor control as described in section 10.4.1.1. In addition, the change in $\mathrm{HbT}$ was observed to be consistent across the 17 participants, as observed from the statistical analysis of the data in terms of mean and standard deviation. This demonstrates that the observed average change in $\mathrm{HbT}$ in response to the stimuli (DP-F, R1, DP-FB and R2) was consistent across all the participants. The averaged change in $\mathrm{HbT}$ across all the participants was also plotted along with the change in $\mathrm{HbT}$ obtained from each participant to demonstrate 
the similarity in the response obtained from all the participants, described in details in Appendix A.5 and A.6.
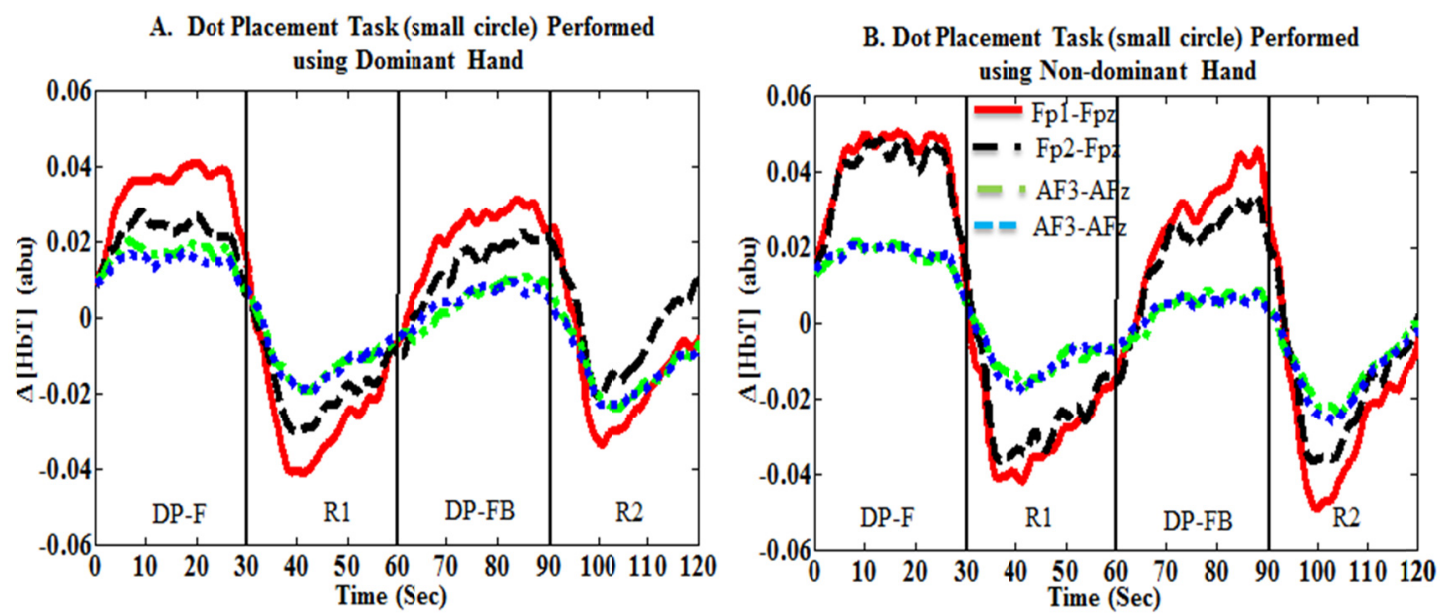

Figure 10.4: Change in total hemoglobin (HbT) (averaged across all participants) as a function of time across the source detector combination Fp1-Fpz (plot in solid red), Fp2Fpz (plot in dashed black), AF3-AFz (plot in dash-dot green) and AF4-AFz (plot in dotted blue) during dot placement finer motor control paradigm. In the plot, the y-axis is the change in HbT, while $x$-axis is the time in seconds. The change in HbT from 0-30 sec corresponds to dot placement task (DP-F), 30-60 sec to rest period (R1), 60-90 sec to dot placement with balance task (DP-FB) and 90-120 sec to second rest period (R2), when using the (A) dominant hand, and (B) non-dominant hand.

The quantified relative change in $\mathrm{HbT}$ both for the task performed using dominant and non-dominant hand is plotted as a bar plot for each channel and tasks as shown in Figure 10.5 along with the values of the relative change in $\mathrm{HbT}$. The pattern of change in $\mathrm{HbT}$ during the dot placement with finer motor control was similar to the change in $\mathrm{HbT}$ during the dot placement with coarser motor control as described in section 10.4.1.1. 
DP-F + Rl

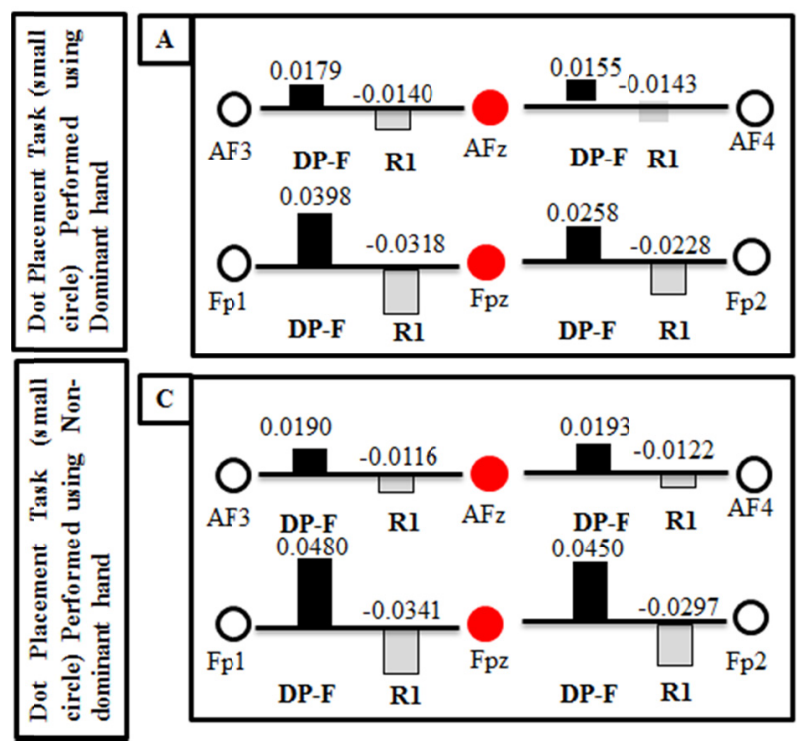

DP-FB + R2
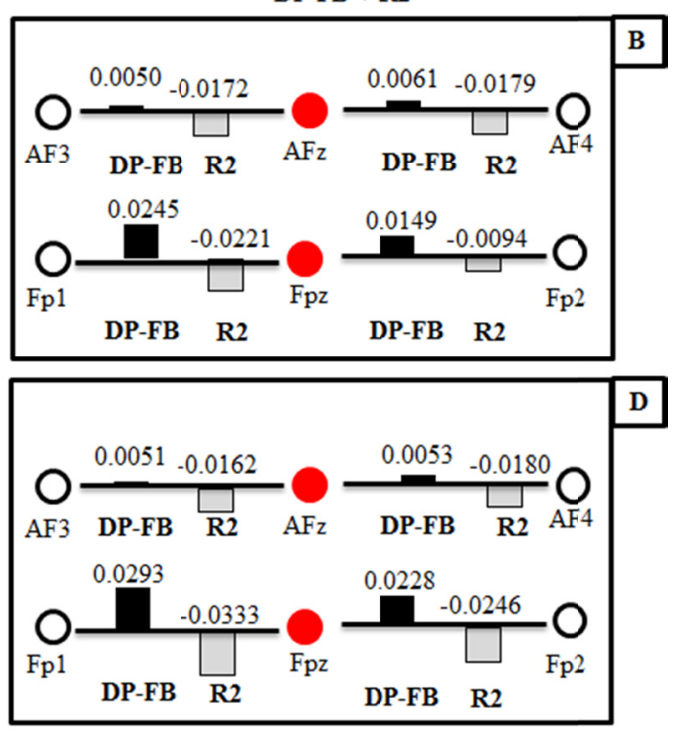

Figure 10.5: Bar plot depicting the quantified relative change in HbT across different source-detector pairs for the dot placement finer motor control paradigm performed by the participants via their dominant and non-dominant hand. In each plot the relative change in HbT across a particular source-detector pair is shown by a bar plot as well as a quantitative value calculated by averaging the central $20 \mathrm{sec}$ of the relative change in $H b T$ during a particular stimulus, where (A) includes task (Dot placement finer motor control: DP-F) and rest (RI) using dominant hand, (B) includes task (Dot placement coarser finer control with beaker: DP-FB) and rest (R2) using dominant hand, (C) includes task (DP-F) and rest (RI) using non-dominant hand, and (D) includes task (DP$F B)$ and rest (R2) using non-dominant hand. In each plot the bar in black and grey represents the quantitative relative change in $H b T$ during task (DP-F or DP-FB) and rest period ( $R 1$ and $R 2$ ) respectively. The results from lateralization analysis are also shown in Figure 10.5 by using lines of different style and thickness connecting a particular source and detector pair. In Figure 10.5 the thin line drawn to represent the channels denotes bilateral dominance.

The results from lateralization analysis are shown in Figure 10.5 by using lines of different style and thickness connecting a particular source and detector pair described in section 10.4.1.3. No dominance was found in terms of change in $\mathrm{HbT}$ between left vs. right pre and anterior frontal cortex for any of the task performed using both dominant and non-dominant hand, shown by thin line connecting the source and detector pair in Figure 10.5 . 


\subsubsection{Functional connectivity results}

The results from the functional connectivity analysis are shown as a table of correlation coefficient obtained from zero-order correlation analysis in Table 10.2. It is apparent from Table 10.2 that there was significant synchrony/correlation in terms of change in $\mathrm{HbT}$ between left vs. right pre and anterior frontal channels across any tasks, both for the task performed using dominant and non-dominant hand. Significant correlation between left vs. right pre and anterior frontal region was also observed during rest period as shown in Table 10.2.

\begin{tabular}{|c|c|c|c|c|c|}
\hline Task Handedness & Channel/Stimuli & DP-F & R1 & DP-FB & R2 \\
\hline \multirow{2}{*}{ Right Hand } & Fp1-Fpz vs. Fp2-Fpz & .72 & .98 & .96 & .91 \\
\cline { 2 - 6 } & AF3- AFz vs. AF4-AFz & .93 & .99 & .91 & .99 \\
\hline \multirow{2}{*}{ Left Hand } & Fp1-Fpz vs. Fp2-Fpz & .98 & .99 & .99 & .99 \\
\cline { 2 - 6 } & AF3- AFz vs. AF4-AFz & .94 & .98 & 96 & .98 \\
\hline
\end{tabular}

Table 10.2: Correlation value between left vs. right pre and anterior frontal channel, when the participants performed the dot placement finer motor control paradigm using their dominant and non-dominant hand.

The dot placement finer motor control tasks is relatively more complex than the dot placement coarser motor control task as the participants performed the task of dot placements DP-F and dot placements with balance DP-FB with their left (non-dominant) hand. The change in $\mathrm{HbT}$ during the dot placement finer motor control tasks is similar to the relative change in $\mathrm{HbT}$ during the dot placement coarser motor control except two major differences. First there was no cortical lateralization across any regions for any of 
the tasks performed via either non-dominant (left) or dominant (right) hand which again suggests the involvement of both the hemisphere of the pre and anterior frontal region to process the complex stimuli. Second the increase in relative change in $\mathrm{HbT}$ was greatest when the participants performed the task DP-F with their left (non-dominant) hand, which suggests an increase in blood flow to support the increase in cognitive load to process the complex stimuli. The result of increases in cerebral blood flow to support the increased cognitive load to process the complex stimuli is similar to the result reported by fMRI (Binder et al., 1997), PET (Baker et al., 1996) and NIRS (Esposito et al., 1995; Fallgatter et al., 1997) studies.

The dot placement with balance task of both the coarser (DP-CB) and finer motor control (DP-FB) was more complex than the just dot placement task DP-C and DP-F, still the increase in relative change in $\mathrm{HbT}$ during DP-C and DP-F was greater than during DP$\mathrm{CB}$ and DP-FB. This suggests increase in metabolic activity in the pre and anterior frontal cortical region of the brain to process the more complex DP-CB and DP-FB task than the task DP-C and DP-F. The comparison of the change in HbT between the dot placement tasks of paradigm 1 and paradigm 2 indicates greater increase in the change in $\mathrm{HbT}$ during finer motor control than coarser motor control across any of the tasks and channels. Thus this suggests that the cerebral blood flow to the pre and anterior frontal region of the brain surges to support the increased metabolic activity associated with the increase in cognitive load to process the complex and difficult finer motor control task, supported by findings from other neuroimaging studies (Duncan \& Owen, 2000; Faw, 2003). 


\subsubsection{Ball throw}

\subsubsection{Change in $\mathrm{HbT}$ and lateralization results}

The averaged change in $\mathrm{HbT}$ across all the participants in response to ball throws task is shown in Figure 10.6 across each stimulus ball throw with dominant hand (BTD), rest (R1), ball throw with non-dominant hand (BTND) and rest (R2) and each channel.

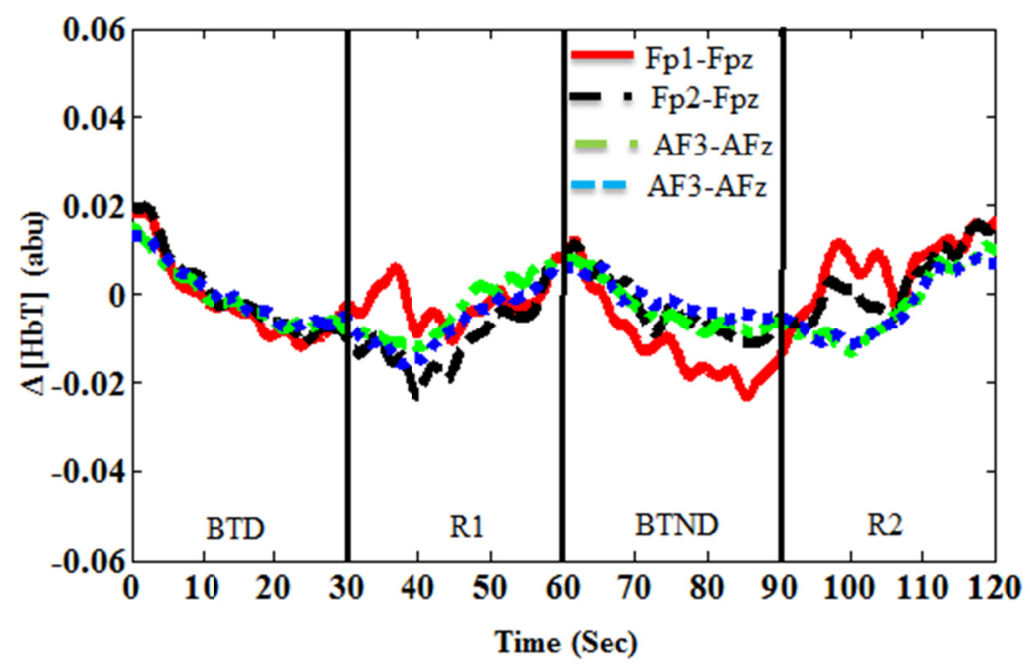

Figure 10.6: Change in total hemoglobin (HbT) (averaged across all participants) as a function of time across the source detector combination Fp1-Fpz (plot in solid red), Fp2Fpz (plot in dashed black), AF3-AFz (plot in dash-dot green) and AF4-AFz (plot in dotted blue) during ball throws paradigm. In the plot, the y-axis is the relative change in $H b T$, while $x$-axis is the time in seconds. The relative change in $H b T$ from 0-30 sec corresponds to ball throws using dominant hand task (BTD), 30-60 sec to rest period (R1), 60-90 sec to ball throws using non-dominant hand task (BTND) and 90-120 sec to second rest period ( $R 2)$.

The results of paired t-test between BTD vs. R1 and BTND vs. R2 indicated no significant difference between BTD and R1 as well as BTND and R2 at a significance level of 0.05 . The rest conditions (R1 and R2) have a non-zero signal initially which tapers to a zero-signal (i.e. initial baseline at the beginning of the block) by the end of the rest period for the reason described in section 10.4.1.1. 
Thus, the change in $\mathrm{HbT}$ in response to the rest condition begins with a non-zero signal and eventually tapers to a zero-signal as seen from Figure 10.6 again validating the effectiveness of the data.

The quantified change in $\mathrm{HbT}$ for the ball throws task is plotted as a bar plot for each channel and tasks as shown in Figure 10.7 along with the values of the relative change in $\mathrm{HbT}$. It can be observed from Figure 10.7, that there was no difference in extent of relative change in $\mathrm{HbT}$ for both $\mathrm{T} 1$ and $\mathrm{T} 2$ across any of the channels.

The results from lateralization analysis are shown in Figure 10.7 by using lines of different style and thickness connecting a particular source and detector pair described in section 10.4.1.3. No dominance (in other words, bilateral dominance) was found in terms of change in $\mathrm{HbT}$ between left vs. right pre and anterior frontal cortex for any of the task performed, shown by thin line connecting the source and detector pair in Figure 10.7.
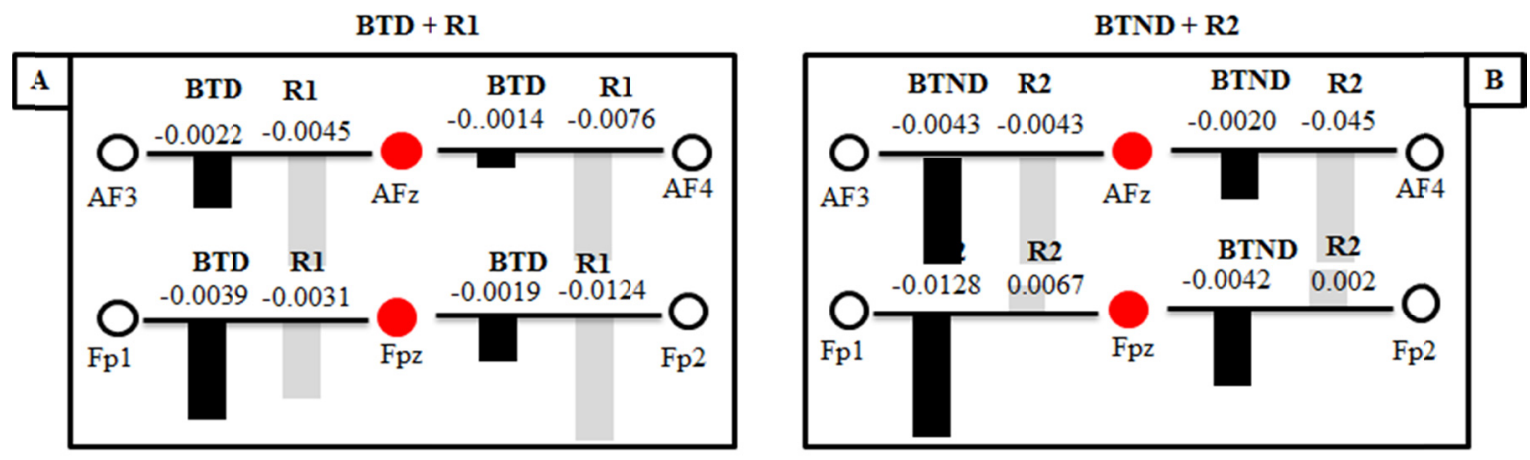

Figure 10.7: Bar plots depicting the quantified change in HbT across different sourcedetector pairs for the ball throws paradigm. In each plot the relative change in HbT across a particular source-detector pair is shown by a bar plot as well as a quantitative value calculated by averaging the central $20 \mathrm{sec}$ of the relative change in HbT during a particular stimulus where (A) includes task (Ball throw with dominant hand: BTD) and rest (RI) and (B) includes task (Ball throw with non-dominant hand: BTND) and rest period (R2). The results from lateralization analysis are also shown in Figure 10.7 by using lines of different style and thickness connecting a particular source and detector pair. In Figure 10.7 the thin line drawn to connect channels denotes bilateral activation or no dominance across any channels. 


\subsubsection{Functional connectivity results}

The results from the functional connectivity analysis are shown as a table of correlation coefficient obtained from zero-order correlation analysis in Table 10.3. It is apparent from Table 10.3 that there was significant synchrony/correlation in terms of change in $\mathrm{HbT}$ between left vs. right pre and anterior frontal channels both for task BTD and BTND. Significant correlation between left vs. right pre and anterior frontal region was also observed during rest period as shown in Table 10.2.

\begin{tabular}{|c|c|c|c|c|}
\hline Channel/Stimuli & BTD & R1 & BTND & R2 \\
\hline Fp1-Fpz vs. Fp2-Fpz & .97 & .85 & .99 & .98 \\
\hline AF3-AFz vs. AF4-AFz & .93 & .84 & .95 & .98 \\
\hline
\end{tabular}

Table 10.3: Correlation value between left vs. right pre and anterior frontal channel, when the participants performed the ball throws paradigm.

The ball throws task elicits a completely different kind of response in the pre and anterior frontal region of the brain as compared to the response of the pre and anterior frontal region of the brain to dot placement coarser and finer motor control task. Instead of an increase in relative change in $\mathrm{HbT}$ as observed in response to dot placement coarser and finer motor control task a decrease in $\mathrm{HbT}$ was observed both in pre and anterior frontal region of the brain in response to both ball throw tasks BTD and BTND. This decrease in change in $\mathrm{HbT}$ was not significantly different from the decrease in change in $\mathrm{HbT}$ during rest period. The lack in significant change in cerebral blood flow to the pre and anterior 
frontal region of the brain during ball throw task may be due to the simplicity of the task. The results from different neuroimaging studies have shown that if the human participants become proficient in certain tasks then the neural response associated with that task decreases (Kubota et al., 1998). Since the human performs tasks similar to ball throws tasks knowingly or unknowingly in everyday life there may have been an established neural basis associated with it which explains the response of significantly no change in cerebral blood flow.

Thus the pre and anterior frontal cortex of the brain plays a significant role in the planning and execution of motor skills and the extent of change in HbT differs based on the novelty, complexity and the type of task. Three different paradigms used to investigate the response of pre and anterior frontal region of brain elicits different response in pre and anterior region of brain based on the novelty and complexity of the task. The result of increase in change in $\mathrm{HbT}$ during both dot placement coarser and finer motor control tasks shows greater increase in relative change in $\mathrm{HbT}$ in pre frontal than the anterior frontal region. It was also observed that as the complexity of tasks increases both the left and right pre frontal cortical region of brain plays dominant role in processing the stimuli. Thus the pre and anterior frontal region of the brain which controls the planning and execution of motor skills task respond differently for each stimulus, based on the novelty and complexity of the task.

\subsection{Conclusion}

Near infrared spectroscopy was used to investigate the relative change in $\mathrm{HbT}$, functional connectivity and lateralization in the pre and anterior frontal region of brain in response 
to three different planning and execution of motor skills paradigm. First paradigm called as dot placement coarser motor control consists of four different stimuli namely DP-C, R1, DP-CB and R2. During task DP-C participants placed dots in the center of four circles printed on the four corner of A4 sheet in a sequential order called task and during task DP-CB participants performed the task similar to task DP-C but while balancing a beaker on the hand. R1 and R2 were the two rest periods at the end of each task DP-C and DP-CB, respectively. Second paradigm called as dot placement finer motor control also consisted of four different stimuli namely DP-F, R1, DP-FB and R2. The second paradigm was similar to first paradigm except the size of circle of in which the participants placed the dot. The size of the circle used in dot placement finer motor control task was smaller than the size of the circle of used in dot placement coarser motor control task. The rationale behind such a paradigm was to investigate the relative change in $\mathrm{HbT}$ in pre and anterior frontal region in response to similar task of different complexity level. Third paradigm called as ball throws consisted of throwing balls in a container placed at a distance of 4 feet from the participants.

The result of change in $\mathrm{HbT}$ shows that there was an increase in change in $\mathrm{HbT}$ in the pre and anterior frontal region of brain during tasks DP-C, DP-CB, DP-F and DP-FB of dot placement with coarser and finer motor control paradigms. The increase in change in $\mathrm{HbT}$ in the pre frontal region was greater than the increase in $\mathrm{HbT}$ in the anterior frontal region for both the dot placement with coarser and finer motor control paradigms. The increase in the change in $\mathrm{HbT}$ during dot placement finer motor control was greater than the dot placement coarser motor across any of the tasks and channels, suggesting increase in the cerebral blood flow to the pre and anterior frontal region. This greater increase in 
cerebral blood flow during dot placement finer motor control was to support the increased metabolic activity associated with the increase in cognitive load to process the complex and difficult finer motor control task. The result of connectivity analysis showed significant synchrony in the relative change in $\mathrm{HbT}$ between left vs. right pre and anterior frontal region during any task of both the dot placement paradigms. The result of lateralization analysis suggests that as the task become increasingly complex both the left and right frontal (pre and anterior) region of the brain plays dominant role in processing the complex stimuli. The result of change in $\mathrm{HbT}$ during the ball throws paradigm was completely different from both the dot placement paradigms where a decrease in $\mathrm{HbT}$ was observed instead of the increase observed during the dot placement paradigm. The decrease in $\mathrm{HbT}$ during the ball throw task was not significantly different from the rest period suggesting the failure of the ball throw tasks to elicit any significant change in blood flow in the pre and anterior frontal region of the brain.

Thus the significance of this work is that it will help in determining the tasks to be included in the physical therapy regime of individuals with $\mathrm{CP}$, which can maximally activate the frontal cortical regions of individuals with $\mathrm{CP}$. 
CHAPTER 11. Study 3c: Simultaneous NIRS and Kinematics Study of Planning and Execution of Motor Skills (Single Wavelength Study)

The studies described in chapters 9 and 10 demonstrated the response of the frontal cortex of controls in response to planning and execution of motor skills. The goals of these studies are to design experimental paradigms to investigate the response of planning and execution of motor skill in individuals with cerebral palsy to help in their rehabilitation. Thus it is imperative to investigate both the response of the brain as well as the movement of the arm of the participants as they are performing the task. Hence a study was designed to simultaneously investigate the response of the brain and the movement of the arm as the participants performs the planning and execution of motor skills. This work was published in the conference proceeding of SPIE 2013. (Ujwal Chaudhary, Bryant Thompson, Jean Gonzalez, Young-Jin Jung, Jennifer Davis, Patricia Gonzalez, Kyle Rice, Martha Bloyer, Leonard Elbaum, Anuradha Godavarty, "Simultaneous NIRS and kinematics study of planning and execution of motor skill task: towards cerebral palsy rehabilitation", SPIE Photonics West Bios, San Francisco, CA, Feb. 2-7, 2013).

\subsection{Introduction}

Individuals with cerebral palsy often have motor impairment and research have shown that individuals with $\mathrm{CP}$ who participate in physical therapy programs generally demonstrate some degree of decreased motor impairment and functional improvement. Therefore, activities to include in physical therapy programs for any given person with $\mathrm{CP}$, (or for adults with acquired brain lesions, might best be chosen based on the 
magnitude, duration, and location of CNS activation they engender, with the assumption that these activations will facilitate plastic changes within the CNS that will ultimately yield the best possible functional outcomes. Unfortunately, the current understanding of the relationships between neural plasticity, neural activation, and specific motor activities are quite limited. In order to better understand these relationships, many researchers have implemented neural imaging before, after, and/or in synchrony with motor activities in non-disabled individuals as well as in individuals with CP. NIRS neuroimaging technique has several advantages as described in section 2.4 which makes it a reliable tool to investigate the response of brain during a motor activity performed by participants. Therefore, in the present study NIRS neuroimaging technique in conjunction with motion capture system was used to simultaneously investigate the correlation between the brain's planning and execution activity during and with arm movement in healthy individuals. The significance of this work is that is lays the foundation for the use of simultaneous NIRS and motion capture study towards investigation of the difference if any in planning and execution of simple motor tasks in controls, such that it can be developed as a reliable tool to aid in the rehabilitation of individuals with $\mathrm{CP}$.

\subsection{Materials and methods}

\subsubsection{Instrumentation}

\subsubsection{NIRS system}

An in-house developed continuous-wave (CW) NIR spectroscopy (NIRS) system as described in section 3.2 was used for the study. During this study the output optical 
power of the laser diodes was $5 \mathrm{~mW}$ and the optical cap with the source detector layout were placed on the prefrontal region of the brain in accordance with the international 1020 electrode placement system, as shown in Figure 11.1. Two different source-detector pairs or channels corresponding to left and right prefrontal regions, i.e. Fp1-Fpz and Fp2Fpz, (according to international 10-20 electrode placement system) respectively were used for this study, as the prefrontal region plays a dominant role in planning and execution of motor tasks (Duncan \& Owen, 2000; Faw, 2003).

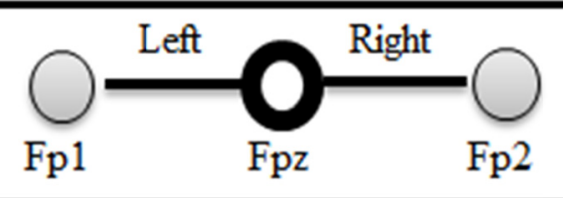

Figure 11.1: Source detector layout corresponding to the 10-20 electrode placement system showing the placement of source (hollow circle) and detector (solid circle) on the pre and anterior frontal region of brain. Each source detector pair is called channel. Fp1 and Fp2 represent the detector placed on the left and right prefrontal, respectively. Fpz represent the source placed on the mid pre frontal.

\subsubsection{Kinematics system}

The motion capture system from Qualisys, which comes with an integrated hardware and software system (Qualisys, Ojus Camera and QTM Software, Release 2.75, Qualisys Medical AB, Gothenburg, Sweden), was used to record the upper extremity kinematics. During the study four oqus series of camera from Qualisys was used which is capable of recording high-speed and high-resolution video in real time. The oqus series of camera were operated at $120 \mathrm{~Hz}$ to record upper extremity kinematics. 


\subsubsection{Participants}

Ten healthy right-handed adults between 18-25 years of age were enrolled for the current study. The study was performed at the Optical Imaging Laboratory of Florida International University (Miami FL). The participants conveyed their handedness based on the dominant use of their particular hand. This study was FIU-IRB approved and written consent(s) were obtained from the participants.

\subsubsection{Experimental design and protocol}

During the study the retro-reflective markers were placed on both the arms and index finger of the participants and the custom-built optical cap was placed on the frontal region of their head (with minimal hair interference). The complete experimental set up during the study is shown in Figure 11.2 and the visual 3D representation of the placement of the retro-reflective marker on both the arm of the participants is shown in Figure 11.3. The participants were seated in a comfortable upright chair (in a quiet room) in front a table with an A4 sheet fastened to the table within reaching distance of the subjects' hand. The A4 sheet consists of circular ring (1" in diameter) drawn on its four corner with the retro-reflective marker placed in the center of each circle. The paradigm used during the study consists of 5 blocks of two 30-second stimuli, namely the target touching (TT) and rest (R). Prior to the block-designed paradigm, an initial 30-sec rest period was employed to bring the brain activity of the human participants to the baseline. During the target touching (TT) stimuli the participants were asked to touch the center of four circles as fast and as precisely as they can in such a way that their touching pattern 
resembles a "Z" as shown in Figure 11.4. During rest $(\mathrm{R})$ the participants were asked to close their eyes and try not to think anything.

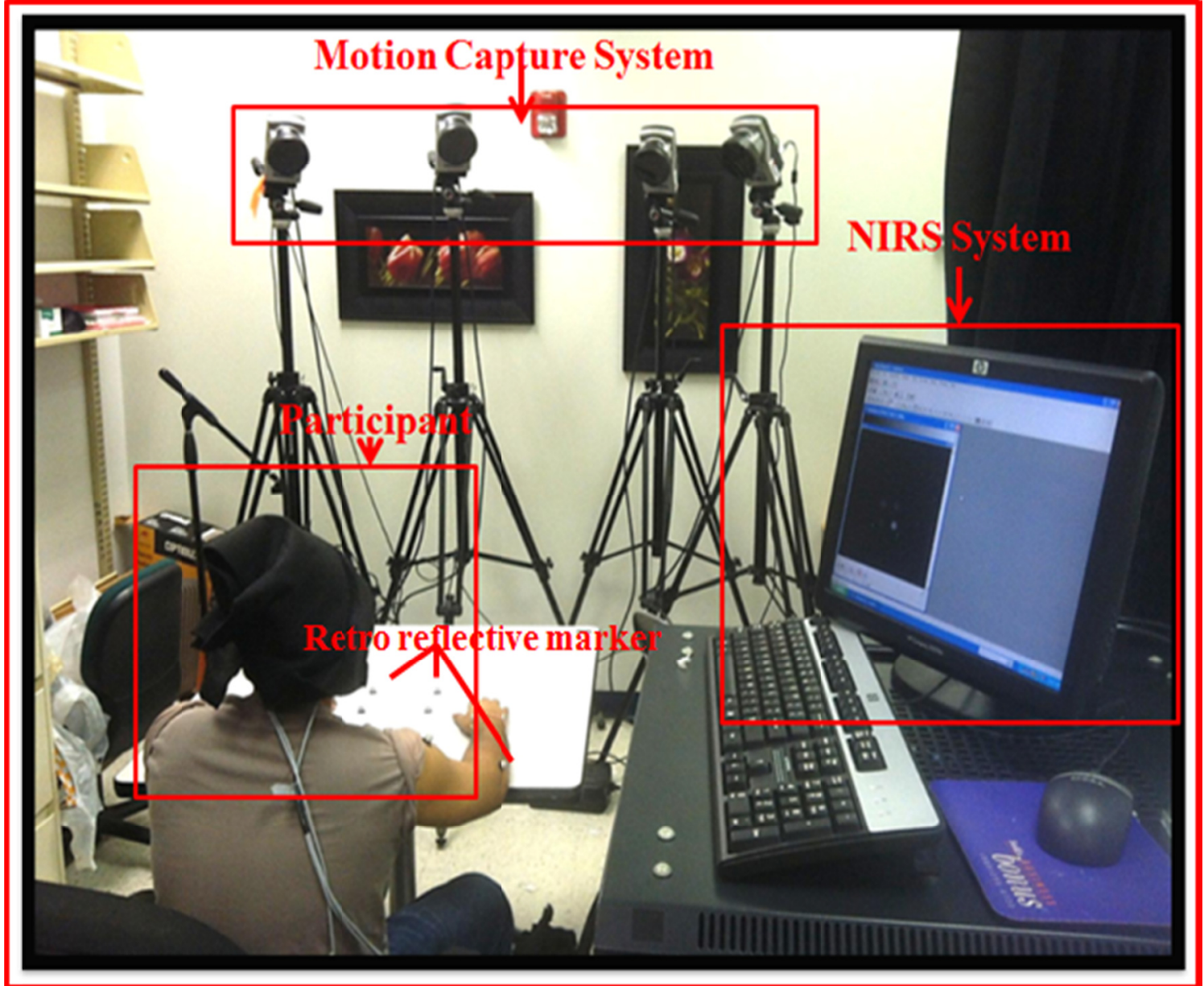

Figure 11.2: The complete experimental setup of the simultaneous kinematics and NIRS system. The set-up shows the participant sitting in front of motor capture system with the optical probes on his head connected to NIRS system. 


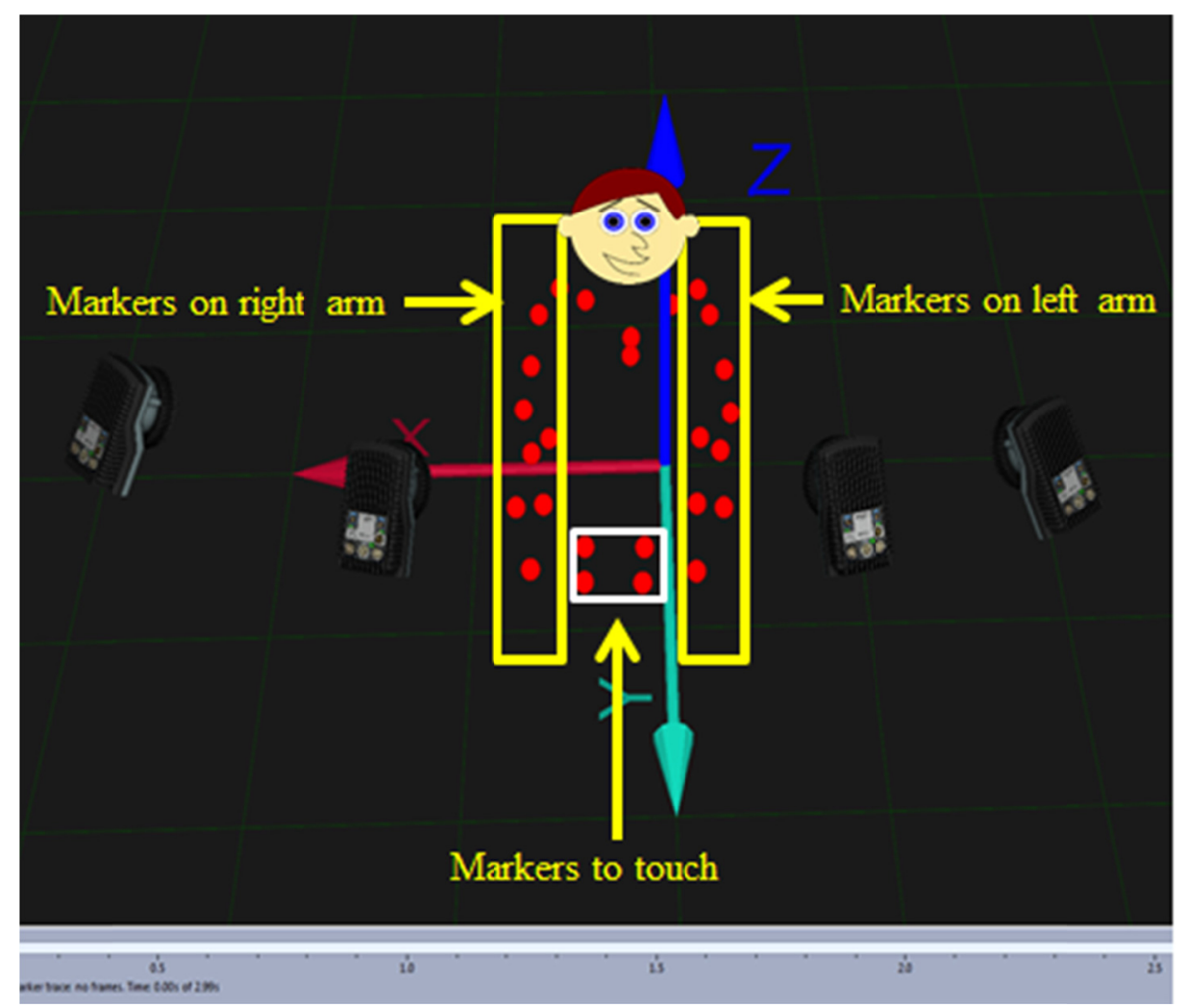

Figure 11.3: Visual 3-D representation of retroreflective markers placed on the arms of the participants (shown by yellow rectangle) and on the A4 sheet (shown by white square).

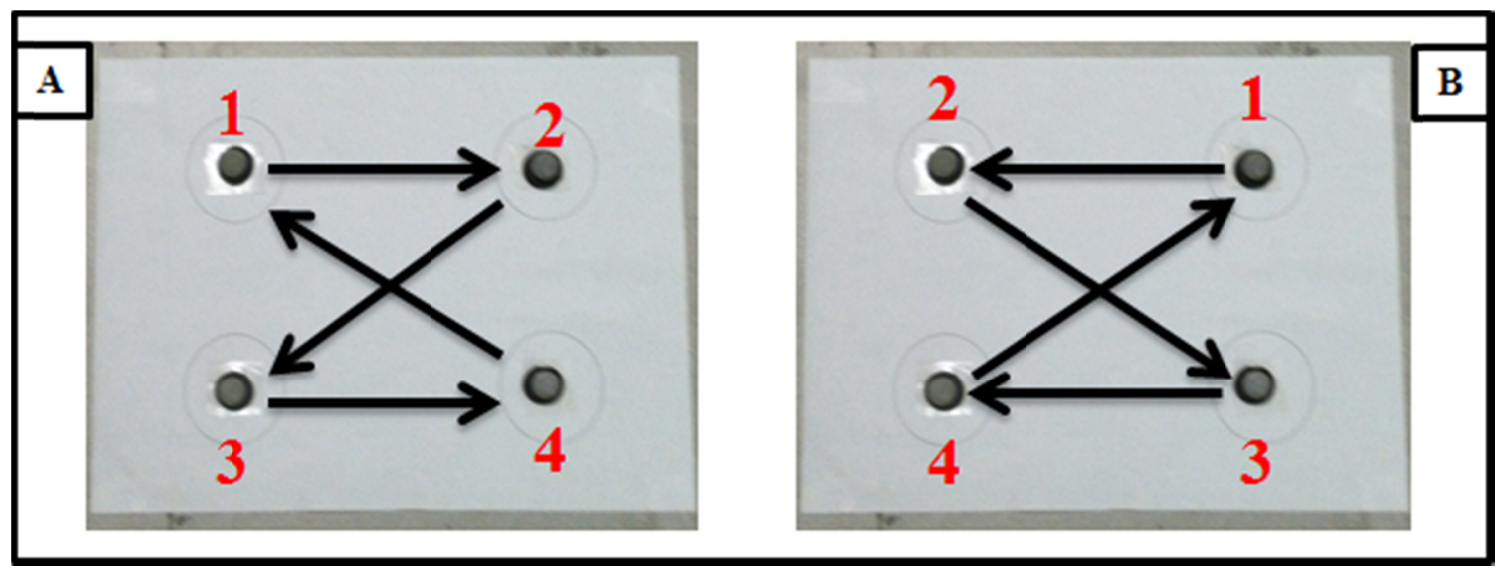

Figure 11.4: The A4 sheet showing circle drawn on its four corners with retro-reflective marker placed in the center of each circle. (A)- shows the target touching pattern performed by participants using their dominant hand, and (B)- shows the target touching pattern performed by participants using their non-dominant hand. 
The participants performed the task with both of their hand, first with dominant hand and then with non-dominant hand. The motion of the arm of the participants during the study was visualized in real-time in 3-D using the QTM (Qualyis Track Manager) software of the Qualysis motion capture system.

\subsection{Data analysis}

\subsubsection{NIRS signal analysis}

The in-house developed CW NIRS system used for this study employed $785 \mathrm{~nm}$ (close to isosbestic point (Chance et al., 1988) wavelength of NIR light, which provides information about the change in total hemoglobin concentration ( $\mathrm{HbT})$. The acquired optical signal was processed as descried in section 4.4 to calculate the change in HbT. The averaged change in $\mathrm{HbT}$ from each participant was further averaged across all participants in order to obtain grand averaged change in $\mathrm{HbT}$ for each stimulus. The details of the post-processing steps employed are described elsewhere. Prior to averaging the change in optical density across all the participants the mean and standard deviation of the change in optical density across each participant was computed to ascertain the consistency of the data. The grand averaged change in HbT was further used to elucidate 1) change in total hemoglobin $(\mathrm{HbT}), 2)$ functional connectivity analysis, and 3) lateralization analysis.

\subsubsection{Change in total hemoglobin}

The change in total hemoglobin study was performed to elucidate the differences in $\mathrm{HbT}$ as a function of time, in the frontal cortex in response to the planning and execution of motor skills based stimuli. The entire window of $30 \mathrm{sec}$ for each stimulus was used to 
plot the averaged relative change in HbT plot with respect to time for Fp1-Fpz and Fp2Fpz source-detector pair or channel (Ch.). Preliminary studies were carried out by using a smaller window $(10 \mathrm{sec}, 15 \mathrm{sec}$, and $20 \mathrm{sec})$ of the entire $30 \mathrm{sec}$ window of the stimulus in order to observe if the contrast in the stimuli was any different. Using the averaged HbT data, paired t-tests $(\mathrm{p}=0.05)$ was performed for each individual channel $(\mathrm{Ch}$. between task (TT) and rest (R) period. The change in $\mathrm{HbT}$ obtained from each participant during the five blocks of the stimuli was also plotted for each source-detector pair, both for the task performed using non-dominant and dominant hand. The change in $\mathrm{HbT}$ across a particular block of task stimuli was quantified by computing the total change in $\mathrm{HbT}$ for the central $20 \mathrm{sec}$ period of each $30 \mathrm{sec}$ and also by changing the window size to $15 \mathrm{sec}$ and $25 \mathrm{sec}$ stimulus. It was observed that changing the window size did not change the result hence in this study the results using the $20 \mathrm{sec}$ window have been presented.

\subsubsection{Functional Connectivity Analysis}

In the present study, functional brain connectivity was performed to characterize the ways in which the left and right frontal cortex was functionally integrated or works in synchrony in response to planning and execution of motor skill stimuli. This study was carried out by computing zero lagged correlation (as described in section 4.5) to elucidate

the correlation and synchrony in the hemodynamic response and hence the connectivity in the neuronal circuit between the left and right frontal cortex. 


\subsubsection{Cortical lateralization}

Cortical lateralization study was performed to understand the hemispherical dominance of the planning and execution task in the frontal cortex and its variation across the stimuli for each participant type. The laterality index was computed for left vs. right frontal cortex for each stimulus ( $\mathrm{T}$ and $\mathrm{R}$ ) using the relative change in $\mathrm{HbT}$ obtained from the channels: Fp1-Fpz vs. Fp2-Fpz (see Figure 9.1), as described in section 4.7.

\subsubsection{Kinematics data analysis}

The motion data acquired were exported to Visual 3D (v4.90, C-Motion, Inc., Germantown, Maryland) to calculate position of the index fingertip during the target touching task. Kinematic data were filtered with a $4^{\text {th }}$ order single low-pass Butterworth filter with a cut-off frequency of $12 \mathrm{~Hz}$. The $\mathrm{x}$ and $\mathrm{y}$ coordinates were extracted from all the participants except the participant number 5 and 6 because of the poor resolution of the recorded motion data. The $\mathrm{x}$ and $\mathrm{y}$ coordinates were used to calculate the change in relative distance of each touch from the origin (the left most bottom corner) of the A4 sheet, using the distance formula. Hence forth the total number of touches performed by each participant across the five blocks of target touching stimulus was calculated and averaged to obtain the average number of touches performed by each participant.

\subsection{Results}

\subsubsection{NIRS data results}

\subsubsection{Change in $\mathrm{HbT}$}

The averaged change in $\mathrm{HbT}$ across all the participants in response to planning and execution of motor skill task performed via both dominant and non-dominant hand is 
shown in Figure 11.5. The Figure 11.5A and 11.5B corresponds to the averaged change in $\mathrm{HbT}$ for the tasks performed by participants using their non-dominant and dominant hand, respectively. It can be observed from the averaged change in $\mathrm{HbT}$ increases during task (TT) which gradually decrease during the rest period, both for the task performed using non-dominant and dominant hand. The results of paired t-test between task (TT) and rest $(\mathrm{R})$ indicated significant difference between task (TT) and rest (R) at a significance level of 0.05 , both for the task performed using non-dominant and dominant hand. The rest period $(\mathrm{R})$ has a non-zero signal initially which tapers to a zero-signal (i.e. initial baseline at the beginning of the block) by the end of the rest period. The initial non-zero condition is because the effect of any stimuli continues in terms of the changes in the cerebral blood volume for a few seconds after the stimulus ends. Hence, the change in $\mathrm{HbT}$ in response to the rest condition begins with a non-zero signal and eventually tapers to a zero-signal as seen from Figure 11.5, validating the effectiveness of the data.

The block wise change in $\mathrm{HbT}$ across the five blocks of planning and execution of motor skill was plotted for each participants and the change in $\mathrm{HbT}$ was also quantified and plotted as bar plot for each participants. Here in the block wise and the quantified change in $\mathrm{HbT}$ across the five blocks of planning and execution of motor skill is shown from a randomly chosen participant in Figure 11.6 and Figure 11.7, respectively. 


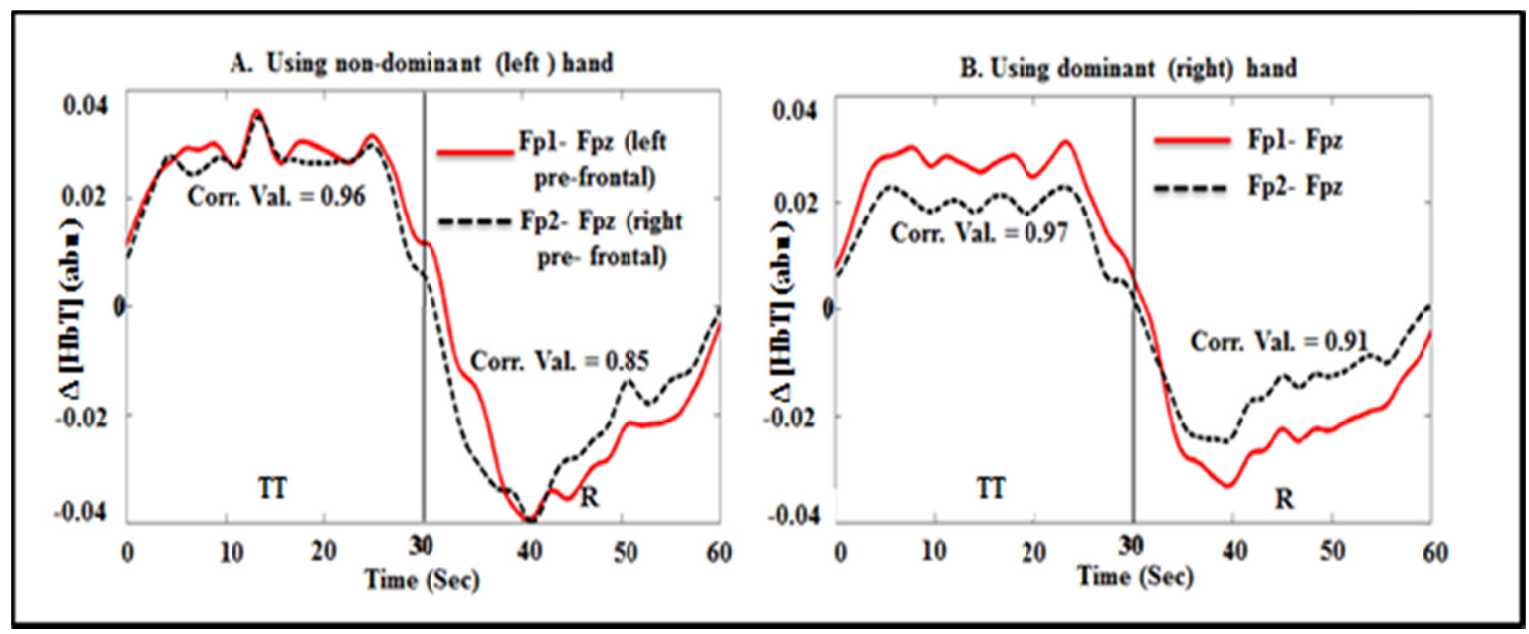

Figure 11.5: Change in total hemoglobin (HbT), averaged across all participants, as a function of time across the source detector combination Fpl-Fpz (plot in solid red line) correspond to left pre-frontal channel and Fp2-Fpz (Plot in dashed black line) correspond to right pre-frontal channel. The numerical value shown in each 30 second period (with the label corr. val.) is the correlation coefficient between left and right pre frontal region of the brain.
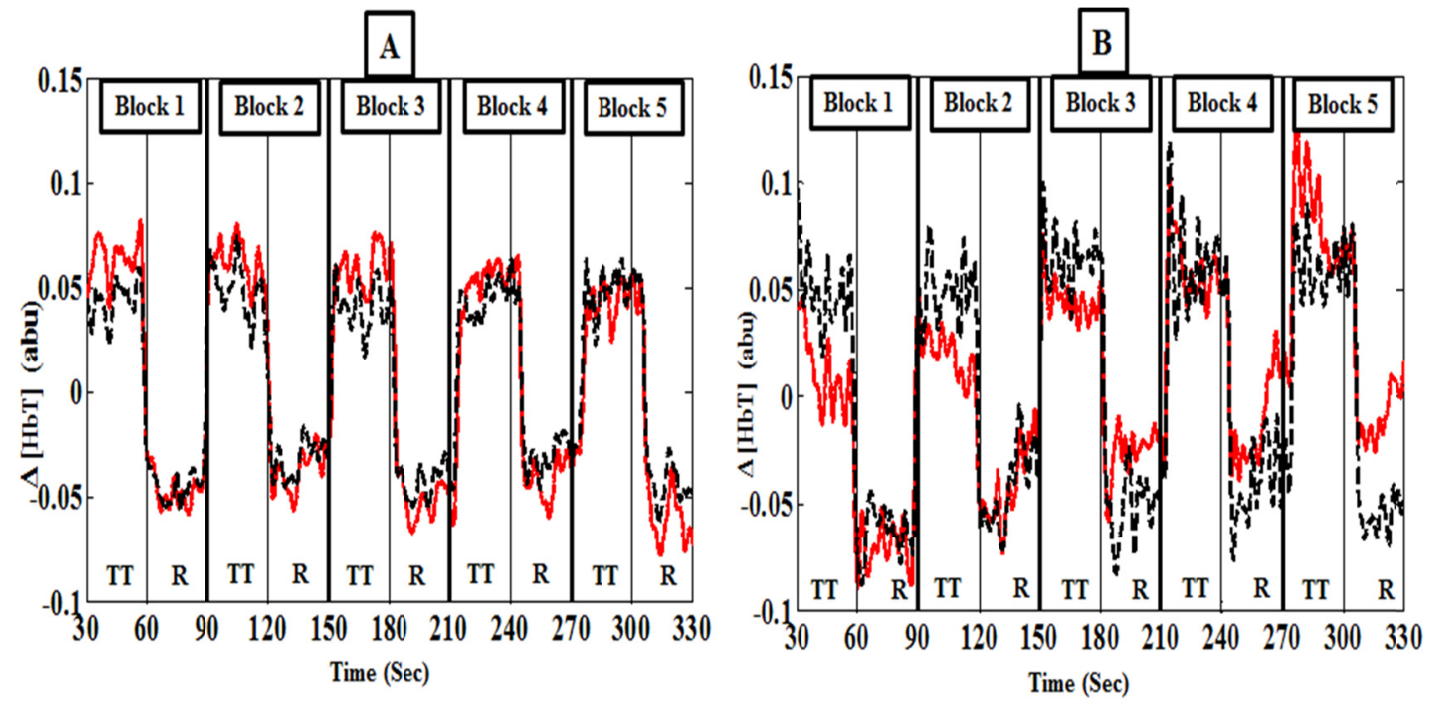

Figure 11.6: Block wise change in total hemoglobin (HbT) as a function of time across the source detector combination Fp1-Fpz (plot in solid red), Fp2-Fpz (plot in dashed black). In the plot, the $y$-axis is the change in HbT, while x-axis is the time in seconds. In each block $T$ denotes the target touching task period and $R$ the rest period. A-Random block wise plot from the group of participants among whom the change in HbT decreases as block progresses. B - Random block wise plot from the group of participants among whom the change in HbT increases as block progresses. 


\subsubsection{Functional Connectivity}

The result of the functional connectivity analysis is shown by the correlation values in Figure 11.5. The functional connectivity analysis result shows significant correlation in the change in $\mathrm{HbT}$ between the left and right pre-frontal channel irrespective of the stimuli and hand used to perform the task.

\subsubsection{Lateralization}

The result of the lateralization analysis is shown in Table 11.1. The lateralization analysis result showed bilateral activation between the left and right pre-frontal channel during the task and rest whether the participants performed the task with their dominant (right) hand or non-dominant (left) hand. The observance of bilateral dominance during Rest task is similar to the finding from previous fMRI study, performed by Salvador et al. (Salvador et al, 2005) on 5 healthy subjects during resting state, where significant coherence and hence bilateral activation (no dominance) was observed in superior frontal gyrus dorsolateral and medial.

Thus over all, the averaged change in $\mathrm{HbT}$ across all the participants shows that the change in $\mathrm{HbT}$ increases rapidly at the onset of the task period and remains at an elevated level until the end of task period. The change in $\mathrm{HbT}$ then starts to decrease gradually at the onset of the rest and reaches the baseline by the end of the rest. The significant increase in $\mathrm{HbT}$ during target touching demonstrates the increase in blood flow to the prefrontal region of the brain to support cognitive load during planning and execution of motor skill task. The left and right prefrontal region of brain worked in synchrony to process the planning and execution of motor skill stimuli and both the regions were non- 
dominant over each other. This is supported from previous neuroimaging studies, such as PET or fMRI, which has described the role of prefrontal region in planning and execution of motor skill stimuli as well as bilateral prefrontal activation in response to such stimuli (Duncan \& Owen 2000).
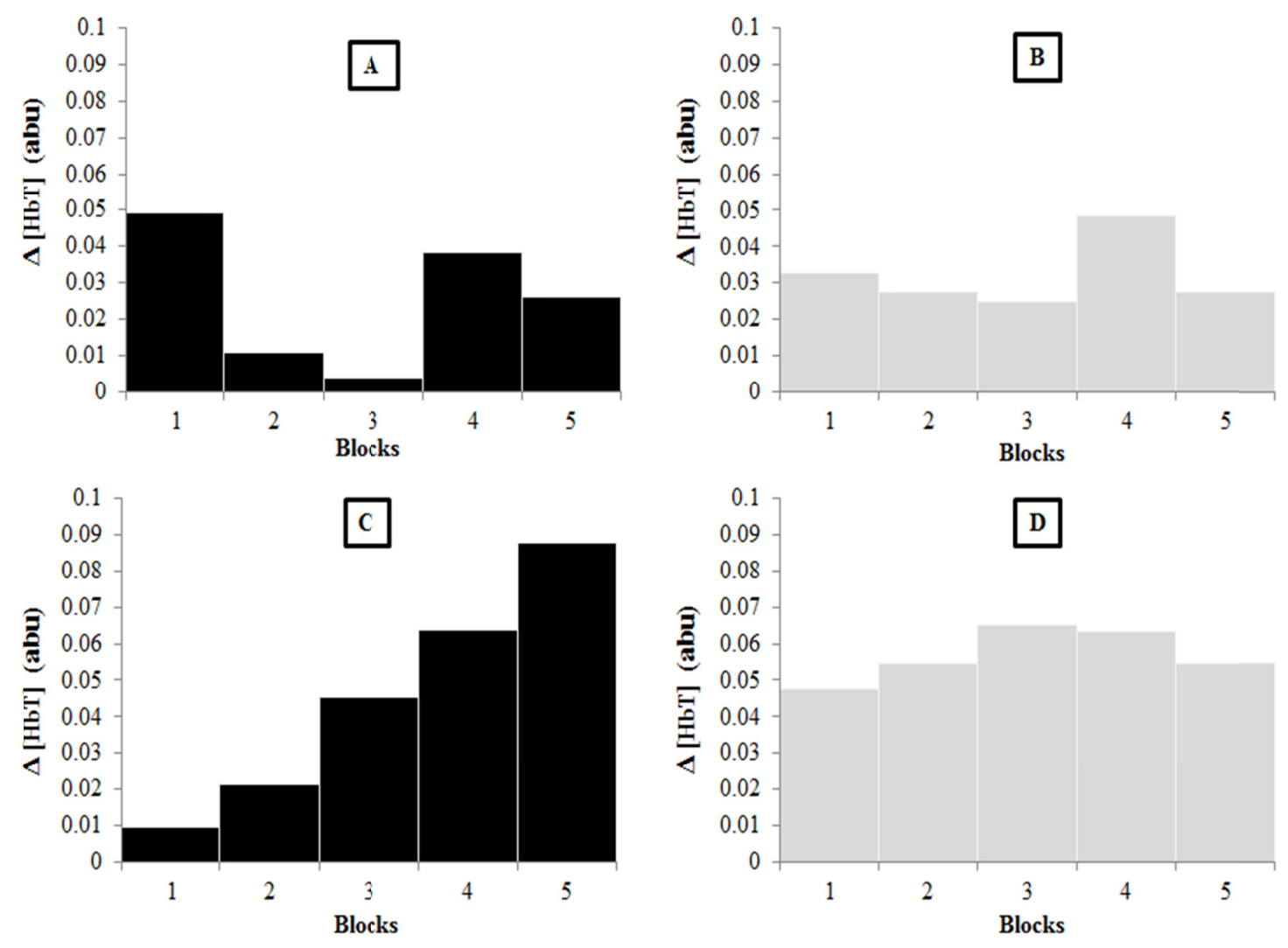

Figure 11.7: Quantified bar plot of the block wise change in HbT plotted in Figure 6. A and $B-$ Depict the quantitative relative change in $H b T$ across the left and right pre frontal region of brain, respectively, for plot 11.6A. C and D-Depict the quantitative relative change in $\mathrm{HbT}$ across the left and right pre frontal region of brain, respectively, for plot $11.6 B$. 


\begin{tabular}{|c|c|c|c|}
\hline \multirow{2}{*}{ Row No. } & \multirow{2}{*}{ Task Handedness } & \multicolumn{2}{|c|}{ Dominance } \\
\cline { 3 - 4 } & & Target Touching & Rest \\
\hline (A) & Dominant hand & 0.18 (Bilateral) & 0.10 (Bilateral) \\
\hline (B) & Non-dominant hand & 0.05 (Bilateral) & 0.018 (Bilateral) \\
\hline
\end{tabular}

Table 11.1: Pre frontal cortical lateralization or dominance (averaged across all participants) in response to planning and execution of motor skill task and rest task in healthy adults.

\subsubsection{Kinematics data results}

The result from the motion capture system showing the total number of touches performed by each participant using their dominant and non-dominant hand is shown by the bar plot in Figure 11.8. It can be seen from Figure 11.8 that different participants performed different number of touches using their left and right hand. The number of touches performed by the right handed participants using their right (or dominant) hand was greater than the number of touches performed using their left (or non-dominant) hand except participant 8 and 10, where the trend was vice-versa.. To correlate the change in $\mathrm{HbT}$ due to the number of touches performed by each participant the number of touches performed by each participant during each block of the task was also plotted. The participant whose block wise data was plotted and quantified, his/her kinematics data was also plotted block wise as shown in Figure 11.9. 


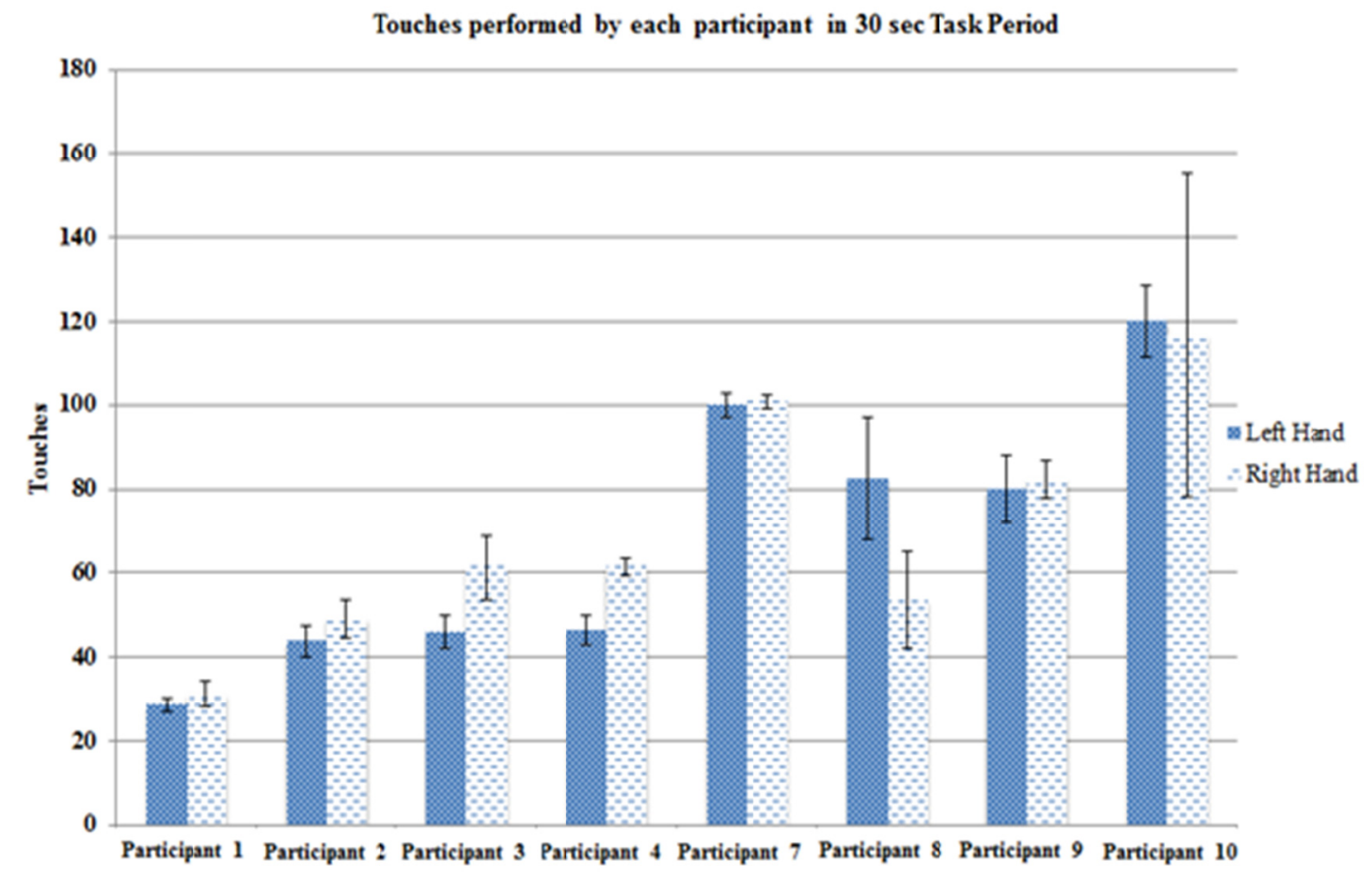

Figure 11.8: Bar plot showing the average number of touches, along with the standard deviation, performed by each participant using their left and right hand.

A.

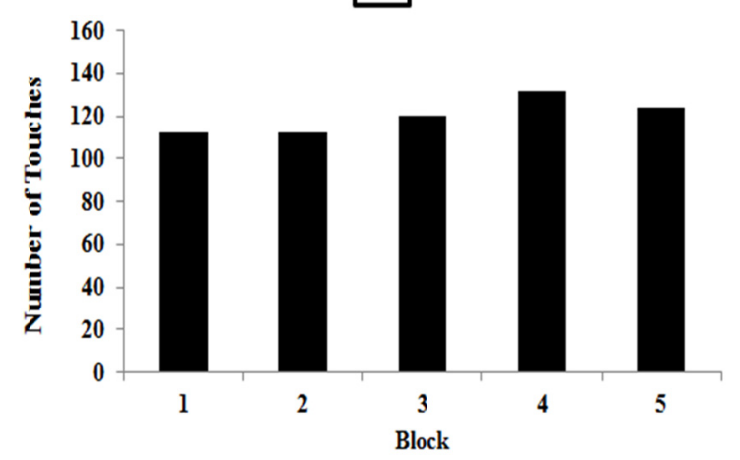

B

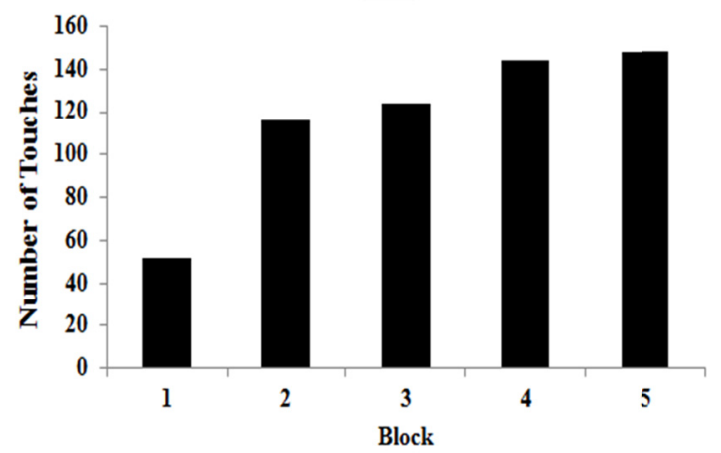

Figure 11.9: Bar plot showing the number of touches performed by the randomly chosen participant, for whom the change in HbT was plotted in Figure 11.6, block wise. ADepicts the number of touches performed by the participant for whom the change in HbT was plotted in Figure 11.6A. B-Depicts the number of touches performed by the participant for whom the change in HbT was plotted in Figure 11.6B. 
The block wise analysis of number of touches performed by each participant during each block of the target touching task revealed two different kind of touch pattern. It was observed that $50 \%$ of the participants on the average performed similar number of touches across the five blocks of tasks with standard deviation very less than the average number of touches (both with their dominant and non-dominant hand). The other $50 \%$ of the participants performed increasingly greater number of touches as the block progresses and the standard deviation of the number of touches performed across the block was comparable to the average number of touches performed (both with their dominant and non-dominant hand). Similarly, the block wise analysis of relative change in $\mathrm{HbT}$ in the prefrontal region also revealed two different kind of pattern of relative change in $\mathrm{HbT}$. It was observed that in $50 \%$ of the participants the extent of increase in HbT gradually dropped while in another $50 \%$ it either remained same or increased as the block progresses (both for the task performed with their dominant and non-dominant hand).

The comparison of block wise number of touches performed by participants and the change in $\mathrm{HbT}$ in the prefrontal region of the participants showed that when the participants performed similar number of touches across the block, the change in $\mathrm{HbT}$ decreases as the block progresses. While the change in $\mathrm{HbT}$ remained same or increased in the prefrontal region of participants who performed increasingly greater number of touches as the block progressed. Thus the combined analysis of relative change in $\mathrm{HbT}$ in the prefrontal region of the brain of the participant as they performed the target touching tasks suggests two different phenomena. Firstly, if the prefrontal region of the brain processes similar stimulus, without any change in the intensity of the task 
performed, as time passes the change in $\mathrm{HbT}$ in the prefrontal region decreases. This decrease in the change in $\mathrm{HbT}$ may be due to decrease in the cognitive load required to perform the task as the participants performs the same task without any change in the rigor or intensity of the task. Secondly, if the prefrontal region of the brain processes stimuli which gradually increases in intensity as the time passes then the change in $\mathrm{HbT}$ also increases gradually. This gradual increase in change in $\mathrm{HbT}$ may be due to increase in cognitive load associated with the increase in intensity of task performed by the participants. Thus this preliminary result gives an insight into the processing of planning and execution of motor skills stimuli by the prefrontal region of brain wherein if the intensity of task performed remained the same the hemodynamic activity evoked in response to that task decreases. However, if the intensity of task increases then the hemodynamic activity associated with the task also increases.

\subsection{Conclusion}

Motor skill task was performed by right handed healthy adults, and the NIRS and motion capture system were simultaneously used in order to correlate the brain's planning and execution activity during and with arm movement. The preliminary results show differences, in terms of change in $\mathrm{HbT}$, between target touching and rest in healthy adults. The result also demonstrate the plastic and learning behavior of brain wherein if the number of touches performed across the block remained the same then the change in $\mathrm{HbT}$ decreases in the prefrontal region of brain. On the contrary, if the number of the touches performed by the participant increases gradually then the change in $\mathrm{HbT}$ either remains same or increase gradually in the prefrontal region of brain. 
The main aim of the present work is to demonstrate the feasibility and importance of simultaneous NIRS and motion capture study towards investigation of the differences if any in planning and execution of simple motor tasks in controls, such that it can be developed as a reliable tool to aid in the rehabilitation of individuals with $\mathrm{CP}$. The present ongoing work is to apply simultaneous NIRS (dual wavelength based system) and motion capture system to elucidate the correlation (if any) between brain's planning and execution activity during and with arm movement in subjects with cerebral palsy. The combined NIRS and kinematics study can be synchronized for a better understanding and intervention options offered to $\mathrm{CP}$ subjects, by physical therapists. The significance of our result is that in the long term it has the potential to help in designing treatment strategies to augment the rehabilitation of individuals with $\mathrm{CP}$. 
CHAPTER 12. Study 3d: Hemodynamic Response Measurement of Simultaneous NIRS and Kinematics Study of Planning and Execution of Motor Skills

The simultaneous NIRS and kinematics study described in chapter 11 was performed using a single wavelength based NIRS system which only elucidates the change in $\mathrm{HbT}$ (total hemoglobin). Moreover the change in $\mathrm{HbT}$ in the pre frontal region of brain was investigated in response to target touching task performed using index finger. In this chapter the hemodynamic response function or the change in $\mathrm{HbO}, \mathrm{HbR}$ and $\mathrm{HbT}$ both in the pre and anterior frontal region of brain was investigated in response to two different variant of target touching task i.e. with index finger and with pen.

\subsection{Introduction}

Individuals with cerebral palsy often has problem with planning and execution of motor skill stimuli. The current state of art in the field of physical therapy of individuals with $\mathrm{CP}$ is that they are trained in certain motor task to help them in their everyday activity. If the activation in the brain of individuals with $\mathrm{CP}$ can be monitored during their training routine then an effective and customized training routine can be designed for them based on their brain activation pattern. The frontal region of the brain plays a dominant role in planning and execution of motor skill stimuli which can be easily investigated using NIRS. The investigation of frontal region of brain along with the monitoring of the kinematics of arm will help in better understanding the effect of training on the brain's response in conjunction with the measurement of the kinematics of muscle movement. The combined NIRS and kinematics study can be synchronized for a better understanding and intervention options offered to $\mathrm{CP}$ subjects, by physical therapists. Thus in the 
current study hemodynamic response function of the frontal region of brain was investigated along with the motion of the hand in response to planning and execution of motor skill stimuli. The significance of our result is that in the long term it has the potential to help in designing treatment strategies to augment the rehabilitation of individuals with $\mathrm{CP}$, using both kinematics of the arm movement and the brain activation, determined using NIRS, associated with arm movement.

\subsection{Materials and methods}

\subsubsection{NIRS Instrument}

In the current study, the frontal cortex of the brain was imaged using an in-house developed continuous wave based NIRS system with dual wavelength light sources, described in section 3.3. During this study 2 laser diodes sources (at each wavelength, 690 and $830 \mathrm{~nm}$ ) and 4 detector fiber bundles were used. The source-detector layout (based on the 10-20 electrode placement) on the pre- and anterior frontal cortex is schematically shown in Figure 12.1. During this study the output optical power of the laser diodes was $3.5 \mathrm{~mW}$ and a custom-built "optical cap" was used to hold the source and detector fibers in place during the imaging studies. Optical signals were acquired in real-time (at $2 \mathrm{~Hz}$ ) from each source -detector pair or channel (Ch.), in response to the stimuli presented (described in Section 12.2.3) using the WinView software package of the instrument. The sources and detectors were placed on the frontal region of the brain as it plays a dominant role in planning and execution of motor tasks (Duncan \& Owen, 2000; Faw, 2003). 


\subsubsection{Participants}

Thirteen healthy right-handed adults between 21-30 years of age were enrolled for the current study. The study was performed at the Optical Imaging Laboratory of Florida International University (Miami FL). The participants conveyed their handedness based on the dominant use of their particular hand. This study was FIU-IRB approved and written consent(s) were obtained from the participants.

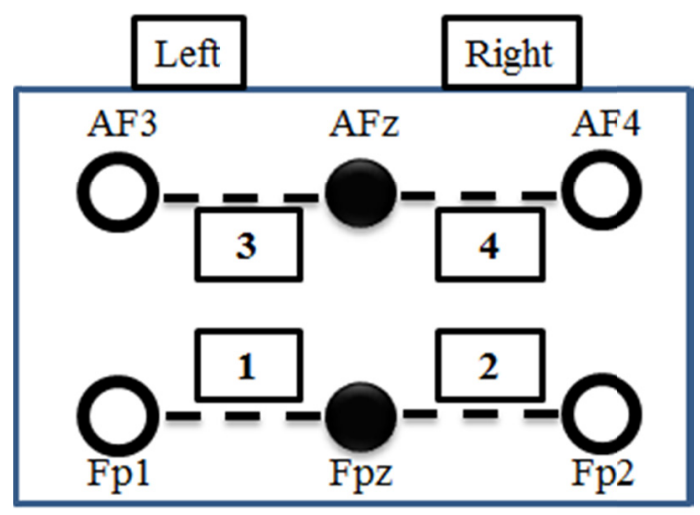

Figure 12.1: Source-detector layout corresponding to the prefrontal and the anterior frontal region of standard 10-20 electrode placement system. The solid circles and hollow circles used in the figure represent sources and detectors respectively. The dashed line represents the path between the sources and detectors (i.e. channels). All the odd number channels correspond to the left frontal cortex, and the even number channels correspond to the right frontal cortex.

\subsubsection{Experimental Design and Stimulus}

The current NIRS study is focused on understanding how the hemodynamics in the frontal region of the brain changes in response to planning and execution of motor skill stimuli. During the study the custom-built optical cap was placed on their forehead with minimal hair interference. The participants attended to two different block designed paradigm (as described below), with a break of 15 minutes between each paradigm. 


\subsubsection{Paradigm 1: Target touching with finger}

During this paradigm a table was placed in front of the participants with an A4 sheet firmly secured on it within the reaching distance of participants' hand. The A4 sheet consists of circular ring of 1 inch diameter drawn on its four corners. The paradigm used during the study consists of 5 blocks of two 30-second stimuli, namely the target touching (TT) and rest (R). Prior to the block-designed paradigm, an initial 30-sec rest period was employed to bring the brain activity of the human participants to the baseline. During the target touching (TT) stimuli the participants were asked to touch the center of four circles as fast and as precisely as they can in such a way that their touching pattern resembles a "Z" as shown in Figure 12.2.

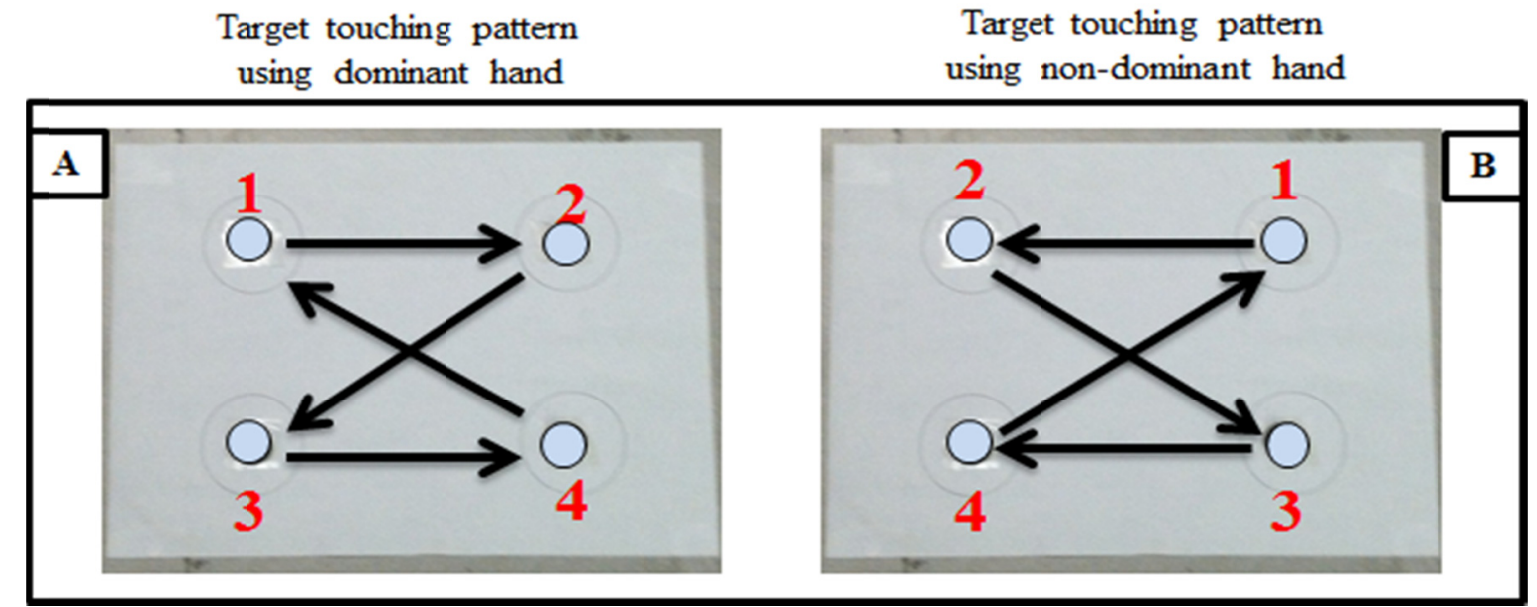

Figure 12.2: The A4 sheet showing circle drawn on its four corners, showing the target touching pattern performed by participants using their (A) dominant hand, and (B) nondominant hand. 
During rest (R) the participants were asked to close their eyes and try not to think anything. The participants performed the target touching task with the index finger of their hand, first with dominant hand and then with their non-dominant hand.

\subsubsection{Paradigm 2: Target touching with pen}

The paradigm 2 is similar to paradigm 1 except that the participants performed the target touching task with pen in their hand. The participants again performed the target touching task with pen in their hand, first with dominant hand and then with nondominant hand. The motion of the hand of the participants during the study was recorded using the Logitech webcam.

\subsection{Data analysis}

\subsubsection{Optical Data Analysis}

The in-house developed continuous wave based NIRS system with dual wavelength light sources acquires the attenuated light intensity of two different wavelength which is quantified to elucidate the hemodynamic responses ( $\mathrm{HbO}, \mathrm{HbR}$ and $\mathrm{HbT}$ ) across each

source-detector pair (or channel). The optical data was post processed to obtain averaged $\mathrm{HRF}$ data as described in section 4.4. The $\mathrm{HbO}, \mathrm{HbR}$, and $\mathrm{HbT}$ data was further averaged over all the 13 participants and 2 repetitions in each participant (i.e. 13 participants $\times 2$ repetitions $=26$ trials). The averaged $\mathrm{HRF}$ data $(\mathrm{HbO}, \mathrm{HbR}$ and $\mathrm{HbT})$ was across the 60sec block, consisting of $\mathrm{T}$ and $\mathrm{R}$ task ( 30 -sec each). The $0-30 \mathrm{sec}$ period corresponded to target touching task and $30-60 \mathrm{sec}$ to the rest period. Prior to averaging the hemodynamic responses $(\mathrm{HbO}, \mathrm{HbR}$, and $\mathrm{HbT})$ across all trials, the mean and standard deviation across trials was evaluated in order to assess the consistency in the responses 
across participants and repetitions. The data is shown in Appendix A.8. Three different studies were performed on the processed hemodynamic response data, which include: (i) activation, (ii) connectivity, and (iii) cortical lateralization study.

\subsubsection{Study 1-Activation}

Brain activation study (as described in section 4.4) was performed to elucidate the differences in the hemodynamic response ( $\mathrm{HbO}, \mathrm{HbR}$ and $\mathrm{HbT}$ ) as a function of time, in the frontal cortex and in response to the target touching task (T) and rest period (R) of paradigm 1 and 2.

\subsubsection{Study 2- Connectivity}

In the present study, functional brain connectivity was performed to characterize the ways in which the left and right frontal cortex was functionally integrated or works in synchrony in response to planning and execution of motor skills stimuli. This study is carried out by computing zero lagged correlation (as described in section 4.5) to elucidate the correlation and synchrony in the hemodynamic response and hence the connectivity in the neuronal circuit between the left and right frontal cortex. Correlation calculations were performed between left $v s$. right frontal cortex for each stimulus (target touching and rest) of paradigm 1 and 2 using the HRFs ( $\mathrm{HbO}, \mathrm{HbR}$ and $\mathrm{HbT}$ ) data obtained from the following channels combinations: Ch.2 vs. Ch.1 and Ch.4 vs. Ch.3. The results of the connectivity study are depicted as histogram plots.

\subsubsection{Study 3-Cortical Lateralization}

Cortical lateralization study is performed to understand the hemispherical dominance of the language in the frontal cortex and its variation across the stimuli. The laterality index 
was computed (as described in section 4.7) between left vs. right frontal cortex for each stimuli (target touching and rest) of paradigm1 and 2 using the $\mathrm{HbT}$ data obtained from channels combination (Ch.2 vs. Ch.1 and Ch.4 vs. Ch.3).

\subsubsection{Kinematics Data Analysis}

The motion of the hand recorded during the task was analyzed to determine the number of touches performed by each participant. The number of touches performed by each participant during each block of the target touching task was counted and averaged across the five blocks of target touching task to determine the average number of touches performed by each participant during paradigm 1 and 2 .

\subsection{Results and discussion}

\subsubsection{Paradigm 1: Target touching with finger}

\subsubsection{Study1: Activation}

The brain activation in response to target touching task performed with the dominant and non-dominant hand's index finger is plotted, as shown in Figure 12.3 and 12.4, in terms of the averaged relative $\mathrm{HbO}, \mathrm{HbR}$ and $\mathrm{HbT}$ changes across each stimulus (target touching and rest) and each channel.

It can be observed from the averaged $\mathrm{HRF}(\mathrm{HbO}, \mathrm{HbR}$ and $\mathrm{HbT}$ ) plots that the extent of activation in terms of $\mathrm{HbO}$ and $\mathrm{HbT}$ was different between the target touching and rest, for the task performed using both the dominant and non-dominant hand, only in the prefrontal region and also from the result of paired t-test at $\mathrm{p}$-value $<0.05$ (see Table 12.1A). There was no significant difference in activation in terms of $\mathrm{HbO}, \mathrm{HbR}$ and $\mathrm{HbT}$ between the target touching and rest in the anterior frontal region of brain from the result 
of paired t-test at p-value $<0.05$ (see Table 12.1A). The activation differences across stimuli in the prefrontal region were more obvious in terms of $\mathrm{HbO}$ and $\mathrm{HbT}$ than $\mathrm{HbR}$, as observed from literature as well (Kubota et al., 2005; Sakatani et al, 1998).

\section{A. Dominant Hand}
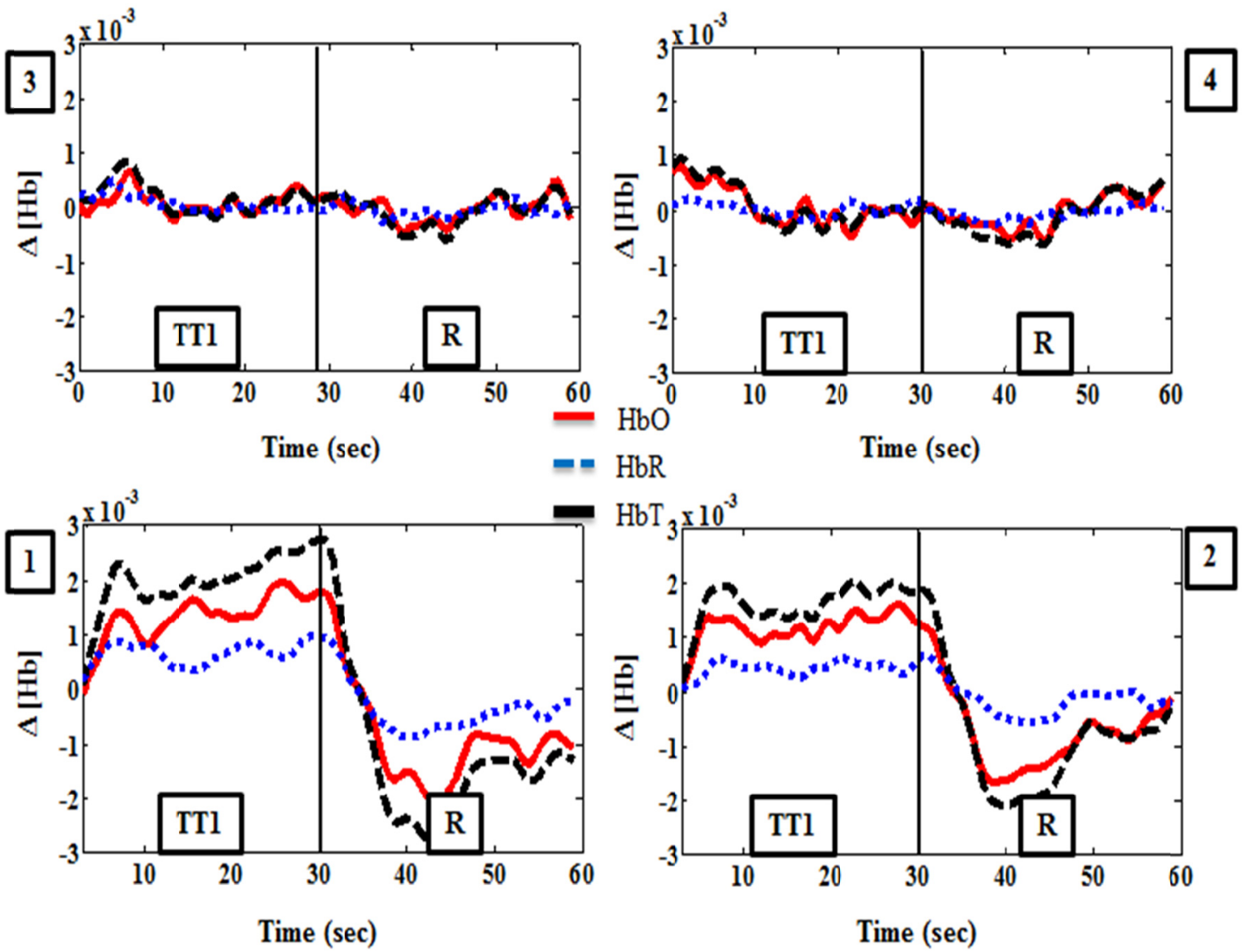

Figure 12.3: Hemodynamic response function $(H R F)$, averaged across all participants, plotted against time (in seconds) for each channel (Ch. 1-4) in response to the planning and execution of motor skill-based stimuli performed by participants using their dominant hand's index finger. Each plot shows the HRF data for the block stimuli of target touching task $(T)$, and Rest $(R)$ performed by the participants. The hemodynamic changes from 0-30 sec correspond to target touching task and 30-60 sec to Rest task. The numbers shown in square boxes on top of each plot corresponds to the number given to each source-detector combination or channel as shown in Figure 12.1. 


\section{B. Non-Dominant Hand}
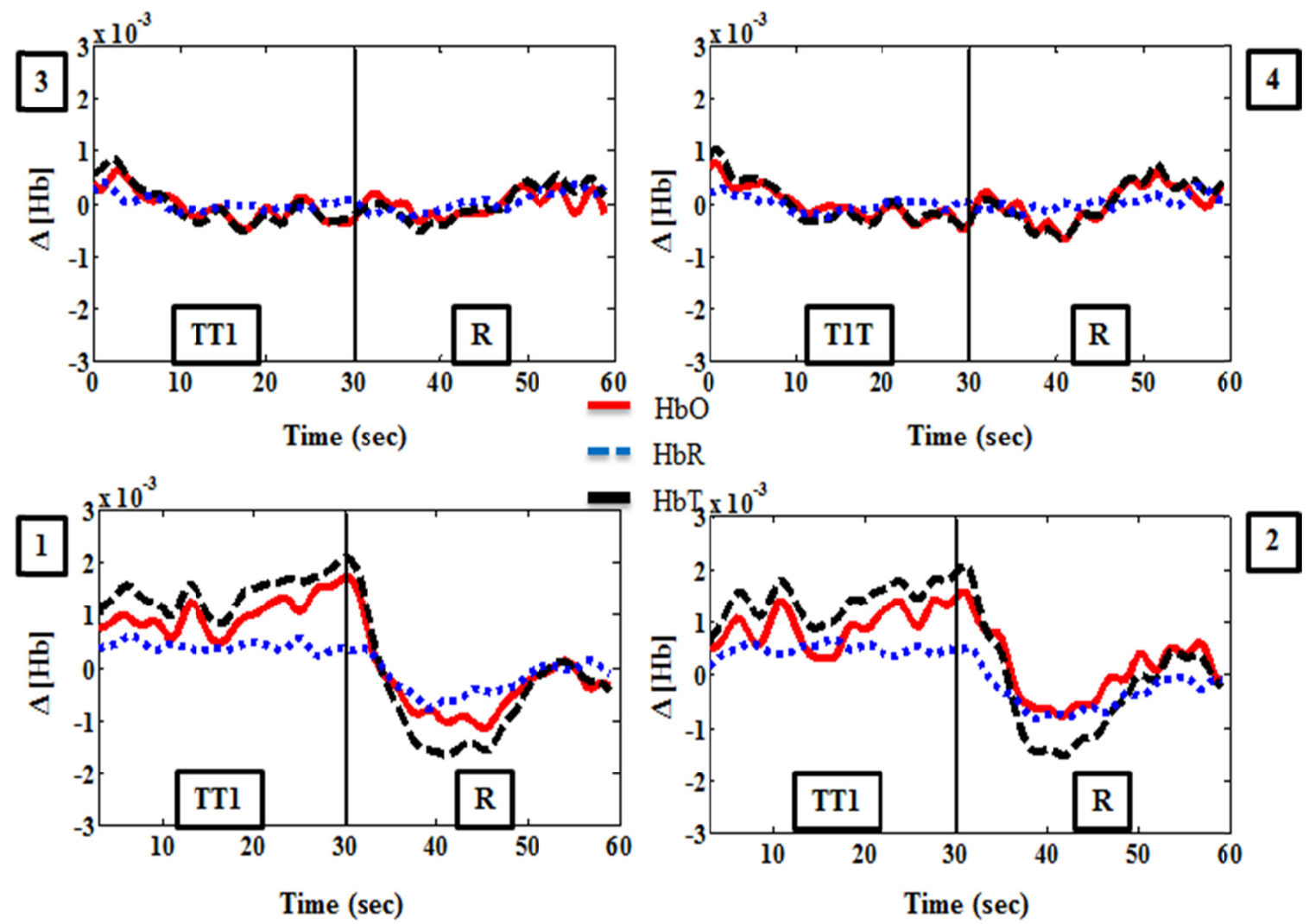

Figure 12.4: Hemodynamic response function (HRF), averaged across all participants, plotted against time (in seconds) for each channel (Ch. 1-4) in response to the planning and execution of motor skill -based stimuli performed by participants using their nondominant hand's index finger. The details of the figure are same as described in caption of Figure 12.3.

A marked increase in $\mathrm{HbO}$ and $\mathrm{HbT}$ was observed during the target touching task in comparison to the rest conditions. The rest condition has a non-zero signal and tapers to baseline by the end of the $30-\mathrm{sec}$ rest period. The initial non-zero condition is because the effect of any stimuli continues in terms of the changes in the cerebral blood volume for a few seconds after the stimulus ends. Hence, the change in $\mathrm{HbO}, \mathrm{HbR}$, and $\mathrm{HbT}$ in response to the rest condition begins with a non-zero signal and eventually tapers to a zero-signal as seen from Figure 12.3 and 12.4, validating the effectiveness of the data. In 
addition, the HRFs ( $\mathrm{HbO}, \mathrm{HbR}$, and $\mathrm{HbT}$ ) were observed to be consistent across the 26 trials, as observed from the statistical analysis of the data in terms of mean and standard deviation.

This demonstrates that the observed average change in brain activation in response to the target touching task and rest period is consistent across all the participants. Statistical analysis performed to determine the differences, if any, in activation (in terms of $\mathrm{HbO}$, $\mathrm{HbR}$, and $\mathrm{HbT}$ ) between target touching task and rest period demonstrated significant difference between target touching and rest period only in the prefrontal region, for both the task performed using dominant and non-dominant hand. The result from paired t-test at $\mathrm{p}$-value $<0.05$ is shown in Table $12.1 \mathrm{~B}$.

\section{A. Dominant Hand}

\begin{tabular}{|c|c|c|c|}
\hline & $\mathrm{HbO}$ & $\mathrm{HbR}$ & $\mathrm{HbT}$ \\
\cline { 2 - 4 } & TT1 vs. R & TT1 vs. R & TT1 vs. $\mathrm{R}$ \\
\hline $\mathrm{Fp} 1-\mathrm{Fpz}$ & $<0.001$ & 0.02 & $<0.001$ \\
\hline $\mathrm{Fp} 2-\mathrm{Fpz}$ & $<0.001$ & 0.04 & $<0.001$ \\
\hline $\mathrm{AF} 3-\mathrm{AFz}$ & 0.40 & 0.33 & 0.32 \\
\hline $\mathrm{AF} 4-\mathrm{AFz}$ & 0.39 & 0.39 & 0.43 \\
\hline
\end{tabular}

\begin{tabular}{|c|c|c|c|}
\hline & $\mathrm{HbO}$ & $\mathrm{HbR}$ & $\mathrm{HbT}$ \\
\cline { 2 - 4 } & TT1 vs. $\mathrm{R}$ & TT1 vs. $\mathrm{R}$ & TT1 vs. $\mathrm{R}$ \\
\hline Fp1-Fpz & $<0.001$ & 0.01 & $<0.001$ \\
\hline Fp2-Fpz & $<0.001$ & 0.02 & $<0.001$ \\
\hline $\mathrm{AF} 3-\mathrm{AFz}$ & 0.35 & 0.29 & 0.32 \\
\hline $\mathrm{AF} 4-\mathrm{AFz}$ & 0.34 & 0.31 & 0.33 \\
\hline
\end{tabular}

Table 12.1: The results of paired t-test statistical analysis at $p<0.05$ to determine the differences in activation (in terms of $\mathrm{HbO}, \mathrm{HbR}$, and $\mathrm{HbT}$ ) between target touching with finger (TT1) vs. Rest for the both the task performed using (A) dominant hand and (B) non-dominant hand.

\subsubsection{Study 2: Connectivity}

The results from the connectivity study for both the task performed using dominant and non-dominant hand's index finger are shown as bar plots (Figure 12.5 and 12.6) of the 
correlation coefficient obtained from the zero order correlation analysis, for each left vs. right paired channel, stimuli, and $\mathrm{HRF}(\mathrm{HbO}, \mathrm{HbR}, \mathrm{HbT})$.

\section{Dominant Hand}
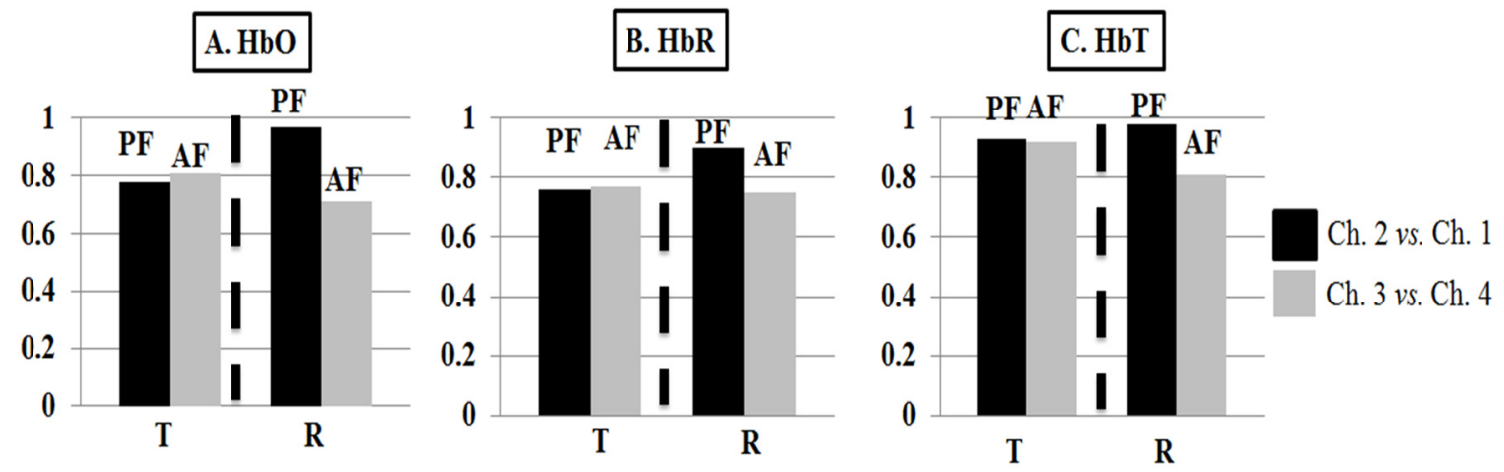

Figure 12.5: Bar plot of the correlation coefficient obtained from the zero order correlation analyses study in response to planning and execution of motor skill-based stimuli performed by participants using their dominant hand's index finger. The plot depicts the correlation coefficient values obtained from (A) $\mathrm{HbO}$, (B) $\mathrm{HbR}$, and (C) $\mathrm{HbT}$ data, across the two stimuli (target touching and Rest) and left vs. right channel pairs (2 of them) in the pre-frontal $(P F)$ and anterior frontal $(A F)$ cortex.

From Figure 12.5 and 12.6, it is apparent that there is significant positive correlation or synchrony $(>75 \%)$ between the left and right pre and anterior frontal cortex (Ch.1 vs. 2 and Ch. 3 vs. 4) for both the task (T and R) performed using dominant and non-dominant hand, and for all HRFs (HbO, HbR, and $\mathrm{HbT})$. 
Non-Dominant Hand
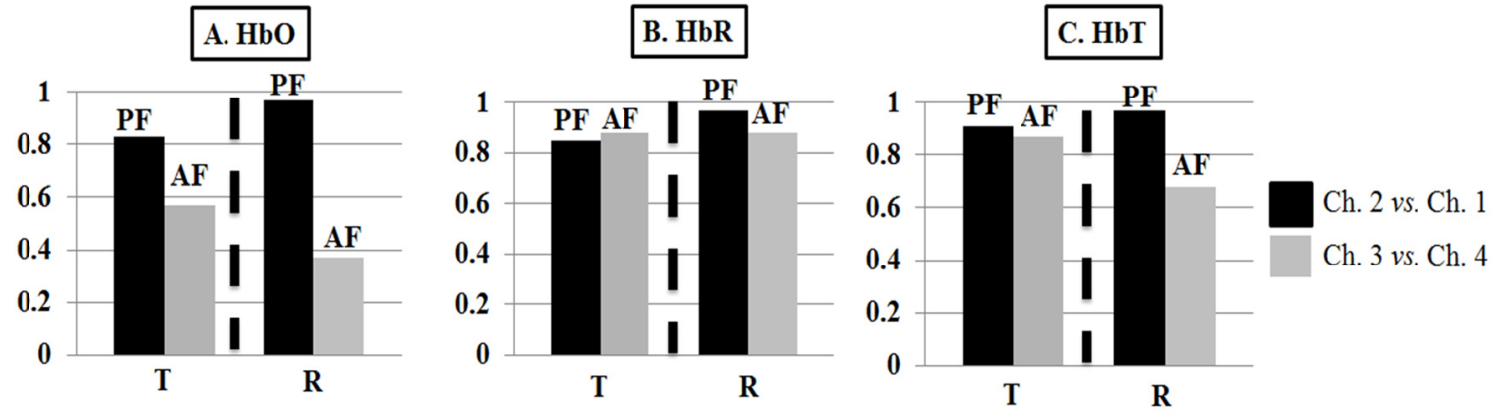

Figure 12.6: Bar plot of the correlation coefficient obtained from the zero order correlation analyses study in response to planning and execution of motor skill-based stimuli performed by participants using their dominant hand's index finger. The details of the figure are same as described in the caption of Figure 12.5.

\subsubsection{Study 3: Cortical Lateralization}

The lateralization index helps determine the dominant channel in a channel pair (here left $v s$. right) in response to a given stimuli. The schematic in Figure 12.7 shows the dominant channels (when comparing the left $v s$. right symmetric channels) in the frontal cortex in response to each of the stimuli, based on the overall HRF (i.e. HbT).

From Figure 12.7 A and B, it is observed that during the target touching task performed with index finger of dominant and non-dominant hand neither the left nor right prefrontal cortex (Ch.1 and 2) was dominant, i.e. there was a bilateral activation. Upon moving to the anterior frontal cortex, a distinct left cortical (Ch.3) and right cortical (Ch. 4) dominance is observed when the participants performed the task with dominant (right) hand and non-dominant (left) hand, respectively. During the Rest task, there was bilateral dominance in the entire frontal cortex. The observance of bilateral dominance during rest task is similar to the finding from previous $f$ MRI study, performed by Salvador et al. (Salvador et al, 2005) on 5 healthy subjects during resting state, where 
significant coherence and hence bilateral activation (no dominance) was observed in superior frontal gyrus dorsolateral and medial.

A. Dominant Hand

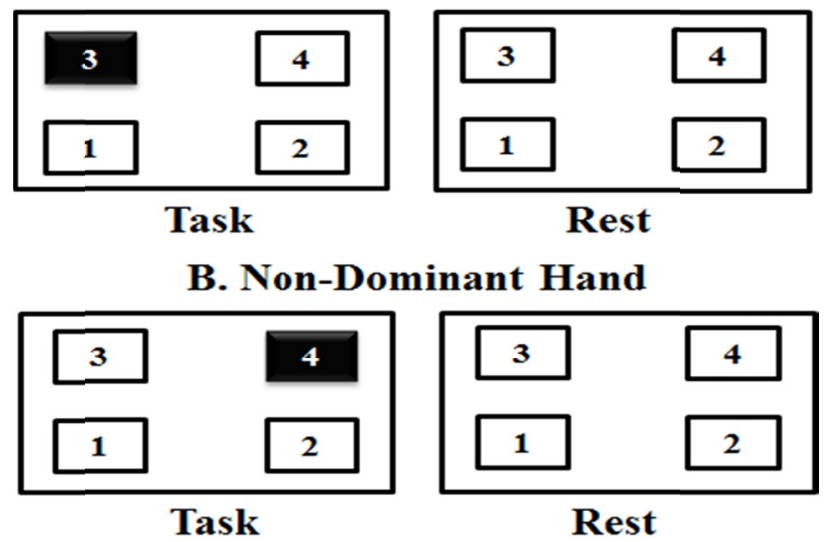

Figure 12.7: Cortical dominance representations using source detector layout as described in Figure 12.1 across all left vs. right channel pairs (2 of them) for each of the two stimuli (target touching and rest) in the frontal cortex. The figure depicts the lateralization when the participants perform the target touching task with (A) dominant hand, and (B) non-dominant hand. The dominant channel pair (across the left and right cortex) is highlighted in black.

\subsubsection{Kinematics}

The result from the kinematics analysis showing the total number of touches performed by each participant using their dominant and non-dominant hand during two different trials is shown by bar plots in Figure 12.8. It can be seen from Figure 12.8 that different participant performed different number of touches using their non-dominant and dominant hand. Participants seemed to perform greater number of touches using their dominant hand than their non-dominant hand except participant 4 and 5, where the number of touches performed using non-dominant hand was greater than the dominant 
hand despite being right handed. It can also be observed from Figure 12.8 A and B that the participants seemed to perform more number of touches during trial 1 than trial 2.

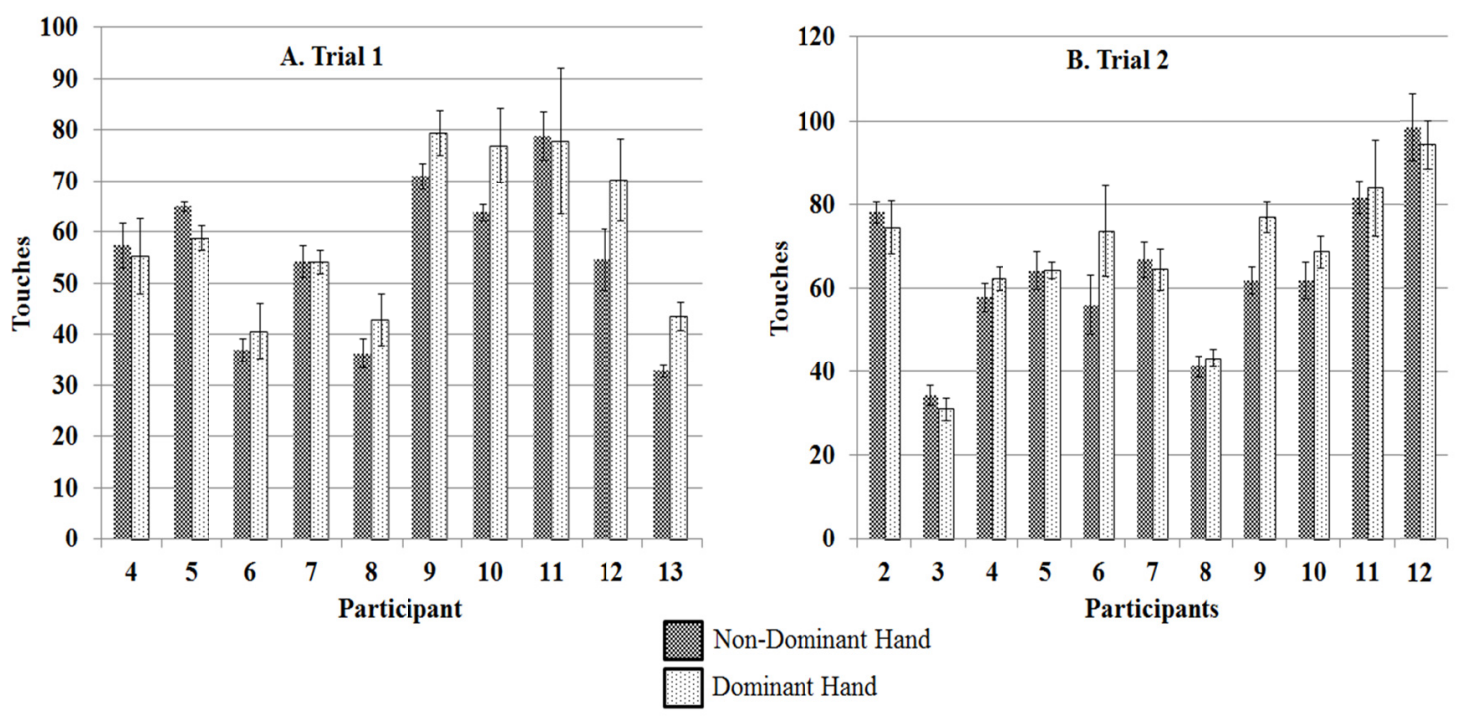

Figure 12.8: Bar plot showing the average number of touches (along with the standard deviation) during the target touching task performed by each participant using their dominant and non-dominant hand's index finger during (A) Trial 1 and (B) Trial 2.

Thus the activation, connectivity and lateralization in pre and anterior frontal cortex in response to target touching task performed by the participants using the index finger of their dominant and non-dominant hand demonstrate the dominant role played by prefrontal cortex during planning and execution of motor skill stimuli. The activation results indicate increase in the change in $\mathrm{HbO}$ and $\mathrm{HbT}$ only in the pre frontal cortex for the target touching task performed using both dominant and non-dominant hand's index finger which returned to baseline by the end of rest period. This shows that the prefrontal region of the brain is involved in planning and execution of motor skill stimuli as supported by the findings from other fMRI and PET studies (Baker et al., 1996; Binder et al., 1997). The left and right pre frontal region of brain seemed to work in synchrony as 
well as equally to process the planning and execution of motor skill stimuli evident from the connectivity and lateralization results.

\subsubsection{Paradigm 2: Target touching with pen}

\subsubsection{Study 1: Activation}

The brain activation in response to target touching task performed while holding pen with the dominant and non-dominant hand is plotted as shown in Figure 12.9 and 12.10, in terms of the averaged change in $\mathrm{HbO}, \mathrm{HbR}$ and $\mathrm{HbT}$ across each stimulus (target touching and rest) and each channel.

\section{A. Dominant Hand}
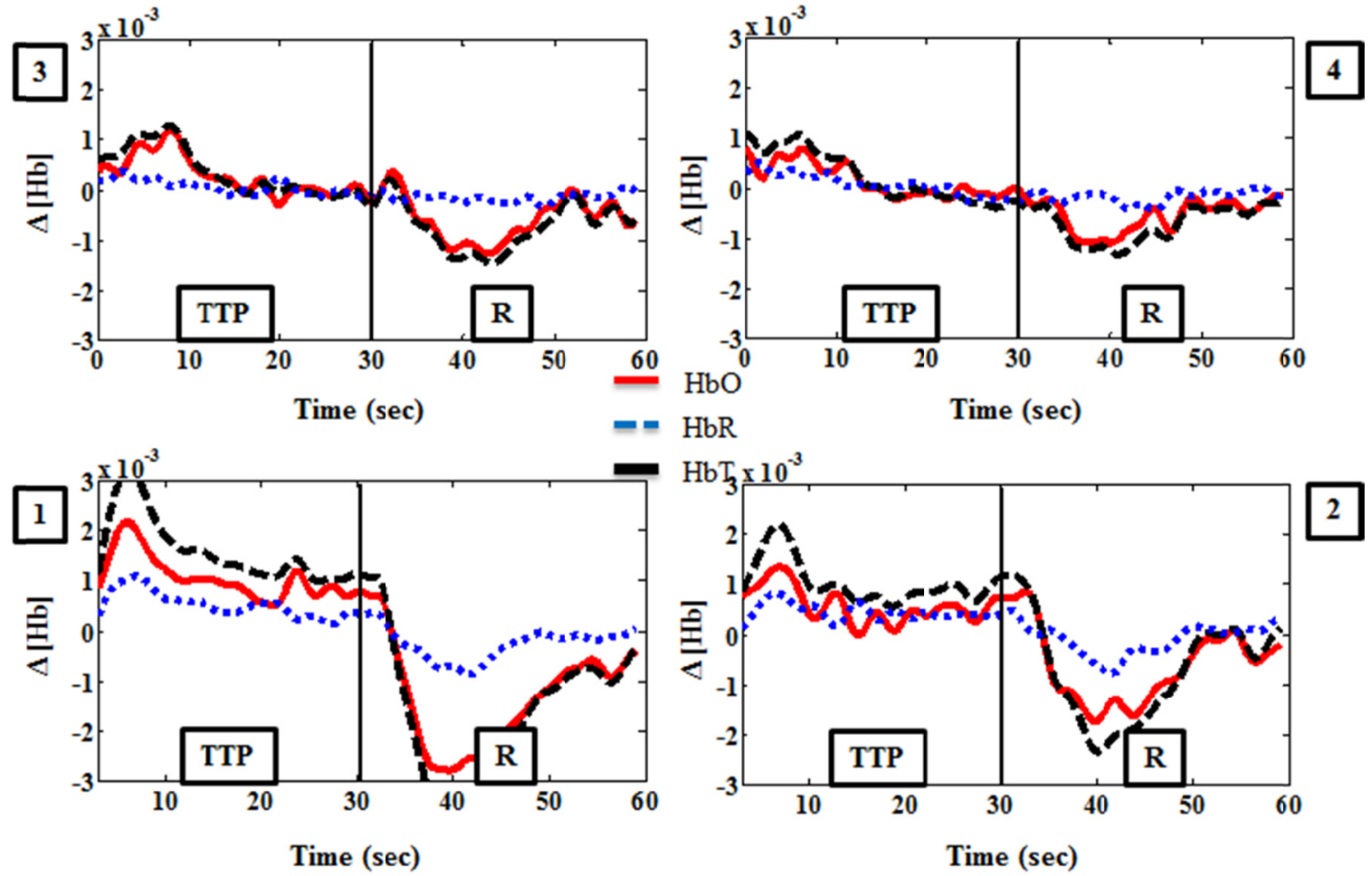

Figure 12.9: Hemodynamic response function (HRF), averaged across all participants, plotted against time (in seconds) for each channel (Ch. 1-4) in response to the planning and execution-based stimuli performed by participants holding pen with their dominant hand. The details of the figure are same as described in caption of Figure 12.3. 
The observed activation in terms of change in $\mathrm{HbO}, \mathrm{HbR}$ and $\mathrm{HbT}$ during the target touching task with pen and rest, for both the task performed using dominant and nondominant hand, was similar to as observed during the target touching task performed using the index finger. The result of paired t-test at $\mathrm{p}$-value $<0.05$ between touching task with pen and rest using dominant and non-dominant hand is shown in Table 12.2A and 12.2B, respectively.

\section{B. Non-Dominant Hand}
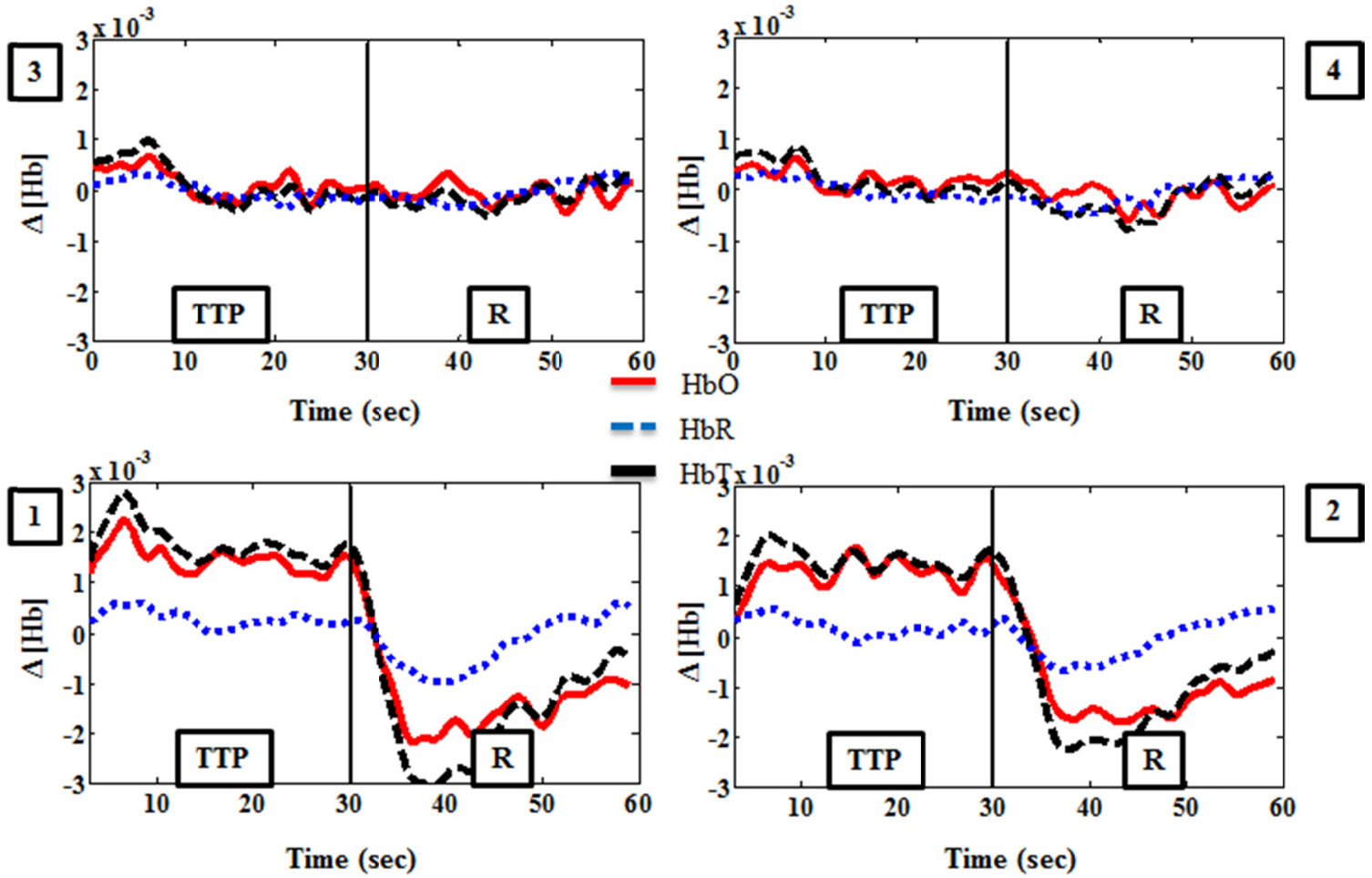

Figure 12.10: Hemodynamic response function (HRF), averaged across all participants, plotted against time (in seconds) for each channel (Ch. 1-4) in response to the planning and execution-based stimuli performed by participants holding pen with their nondominant hand. The details of the figure are same as described in caption of Figure 12.3. 
A. Dominant Hand

\begin{tabular}{|c|c|c|c|}
\hline & $\mathrm{HbO}$ & $\mathrm{HbR}$ & $\mathrm{HbT}$ \\
\cline { 2 - 4 } & TTP vs. $\mathrm{R}$ & TTP vs. $\mathrm{R}$ & TT1 vs. $\mathrm{R}$ \\
\hline $\mathrm{Fp1} 1-\mathrm{Fpz}$ & $<0.001$ & 0.04 & $<0.01$ \\
\hline $\mathrm{Fp} 2-\mathrm{Fpz}$ & $<0.001$ & 0.03 & 0.75 \\
\hline $\mathrm{AF} 3-\mathrm{AFz}$ & 0.32 & 0.29 & 0.30 \\
\hline $\mathrm{AF} 4-\mathrm{AFz}$ & 0.31 & 0.27 & 0.29 \\
\hline
\end{tabular}

\begin{tabular}{|c|c|c|c|}
\hline & $\mathrm{HbO}$ & $\mathrm{HbR}$ & $\mathrm{HbT}$ \\
\cline { 2 - 4 } & TTP vs. $\mathrm{R}$ & TTP vs. $\mathrm{R}$ & TTP vs. $\mathrm{R}$ \\
\hline Fp1-Fpz & $<0.001$ & 0.05 & $<0.001$ \\
\hline Fp2-Fpz & $<0.001$ & 0.04 & $<0.001$ \\
\hline AF3-AFz & 0.28 & 0.31 & 0.36 \\
\hline AF4-AFz & 0.29 & 0.32 & 0.33 \\
\hline
\end{tabular}

Table 12.2: The results of paired t-test statistical analysis at $p<0.05$ to determine the differences in activation (in terms of $\mathrm{HbO}, \mathrm{HbR}$, and $\mathrm{HbT}$ ) between target touching with pen (TTP) vs. Rest for the both the task performed using (A) dominant hand and (B) nondominant hand.

\subsubsection{Study 2: Connectivity}

The results from the connectivity study for both the target touching task performed while holding pen in dominant and non-dominant hand are shown as bar plots (Figure 12.11 and 12.12) of the correlation coefficient obtained from the zero order correlation analysis, for each left vs. right paired channel, stimuli, and $\mathrm{HRF}(\mathrm{HbO}, \mathrm{HbR}, \mathrm{HbT})$.

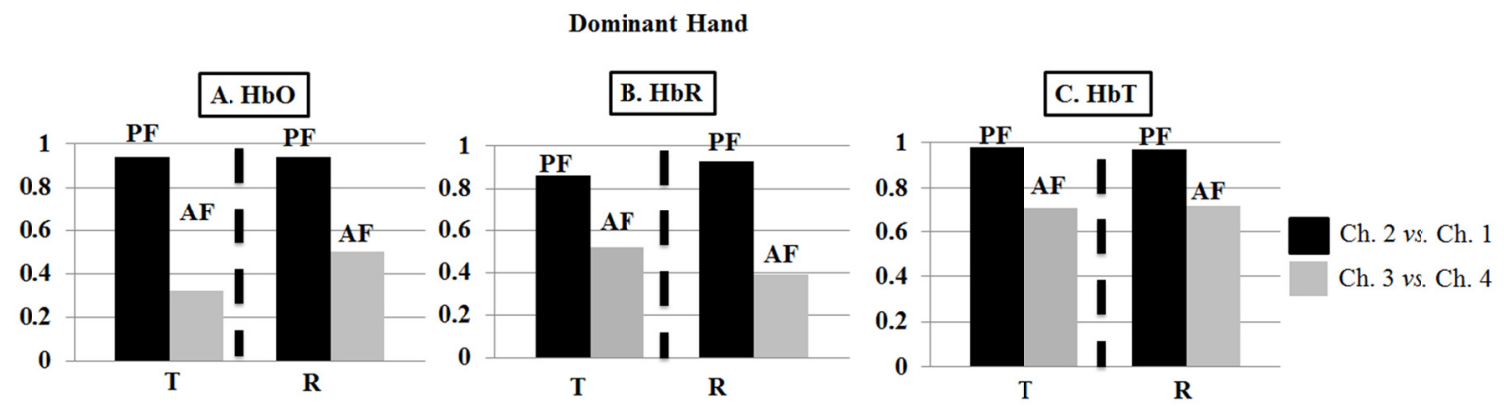

Figure 12.11: Bar plot of the correlation coefficient obtained from the zero order correlation analyses study in response to planning and execution of motor skill-based stimuli performed by participants holding pen with their dominant hand. The details of the figure are same as described in caption of Figure 12.5. 


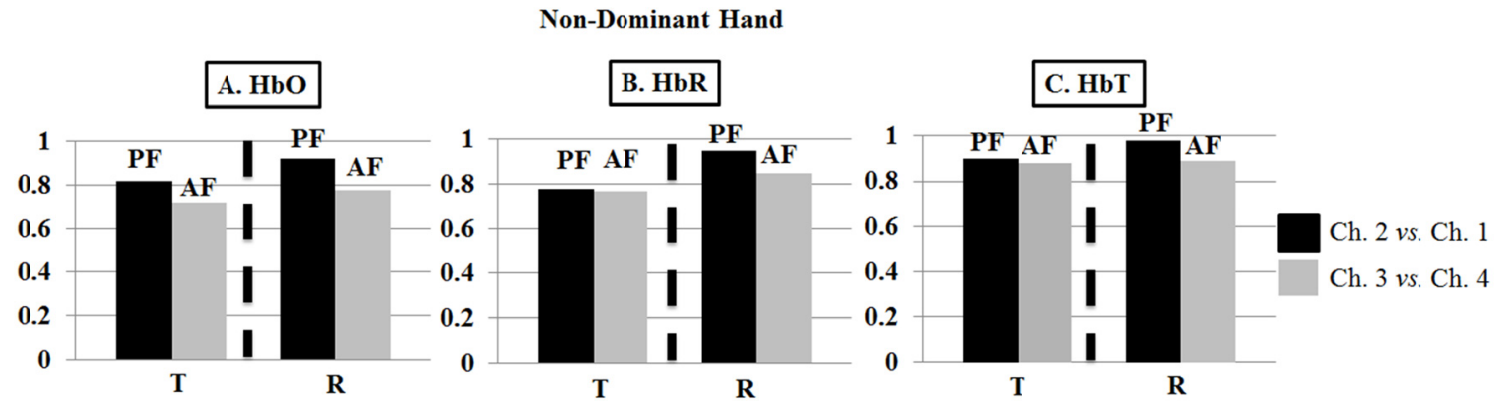

Figure 12.12: Bar plot of the correlation coefficient obtained from the zero order correlation analyses study in response to planning and execution of motor skill-based stimuli performed by participants holding pen with their non-dominant hand. The details of the figure are same as described in caption of Figure 12.5.

The synchrony in the activity in left $v s$. right pre and anterior frontal cortex during the target touching task with pen and rest, for both the task performed using dominant and non-dominant hand, was similar to the synchrony observed during the target touching task performed using the index finger.

\subsubsection{Study 3: Cortical Lateralization}

The cortical lateralization result during the target touching task performed with pen in hand was the same as the target touching task performed with finger, for both the task performed using dominant and non-dominant hand. Hence the Figure 12.7 also shows the cortical lateralization in the pre and anterior frontal region of brain during target touching task performed with pen and rest period, for both the task performed using dominant and non-dominant hand.

\subsubsection{Kinematics}

The result from the kinematics analysis showing the total number of touches performed by each participant while holding pen in their dominant and non-dominant hand during 
two different trials is shown by bar plots in Figure 12.13. It can be seen from Figure 12.13 that different participants performed different number of touches using their nondominant and dominant hand. Participants seemed to perform greater number of touches using their dominant hand than their non-dominant hand. It can also be observed from Figure 12.13 A and B that the participants seemed to perform similar number of touches during trial 1 than trial 2.

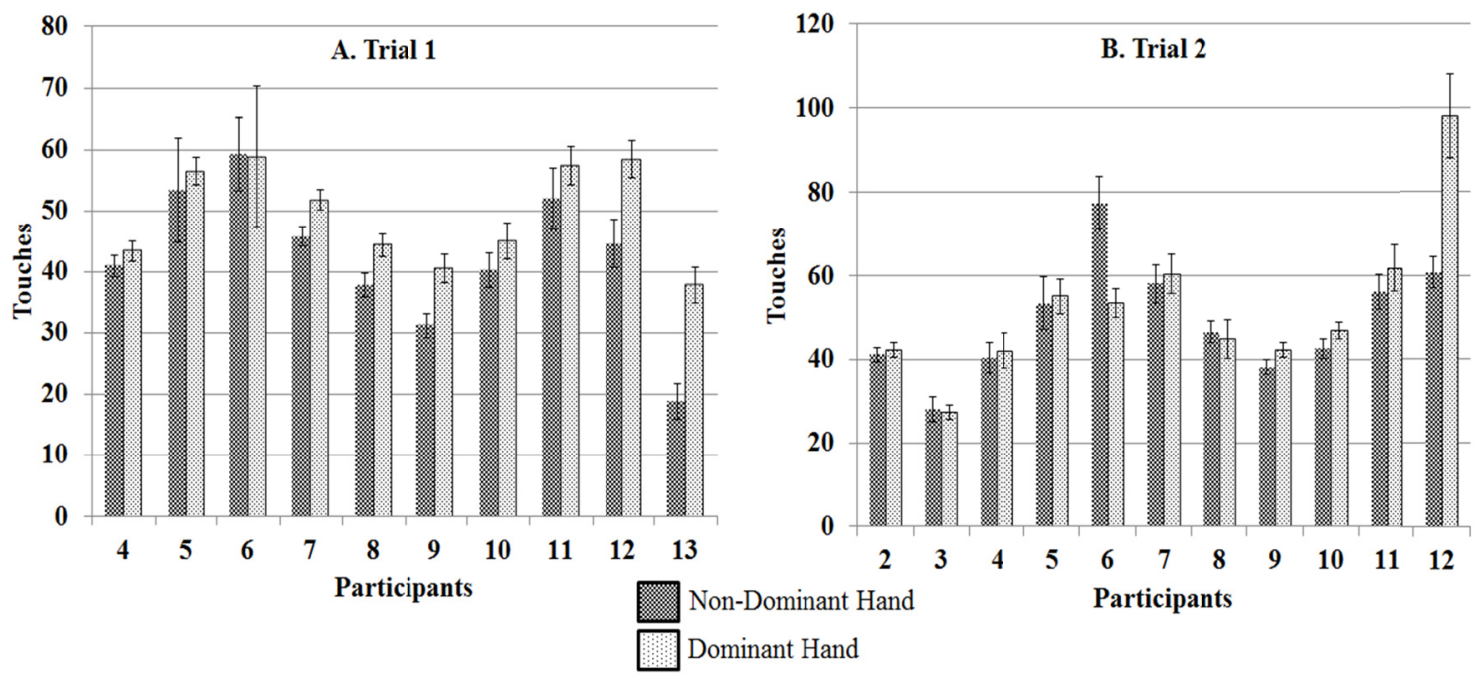

Figure 12.13: Bar plot showing the average number of touches along with the standard deviation during the target touching task performed by each participant while holding pen with their dominant and non-dominant hand during (A) Trial 1 and (B) Trial 2.

Comparing the activation (by computing the total change in $\mathrm{HbO}$ and $\mathrm{HbT}$ for the central $20 \mathrm{sec}$ period of $30 \mathrm{sec}$ target touching stimulus) in terms of change in $\mathrm{HbO}$ and $\mathrm{HbT}$, it was observed that there was no significant difference between the target touching task performed using the index finger and with pen (as shown in Table 12.3). There was also no significant difference in connectivity and lateralization in pre and anterior frontal cortex in response to target touching task performed by the participants using the index 
finger and with pen. This suggests that the prefrontal region of the healthy individuals processes the two different variant of the planning and execution of motor skill stimuli in similar fashion. The similarity in response may be due to failure of prefrontal region of brain to respond significantly different probably due to subtle differences between the two different target touching tasks.

\begin{tabular}{|c|c|c|c|c|}
\hline \multirow{2}{*}{} & \multicolumn{4}{|c|}{ A. HbO } \\
\cline { 2 - 5 } & Dominant Hand & Non-dominant hand & Dominant Hand & Non-dominant hand \\
\hline Trial 1 & 0.0011 & 0.0015 & 0.0013 & 0.0004 \\
\hline Trial 2 & 0.0005 & 0.0014 & 0.0012 & 0.0008 \\
\hline
\end{tabular}

\begin{tabular}{|c|c|c|c|c|}
\hline \multirow{2}{*}{} & \multicolumn{3}{|c|}{ A. HbR } \\
\cline { 2 - 5 } & Dominant Hand & Non-dominant hand & Dominant Hand & Non-dominant hand \\
\hline Trial 1 & 0.0006 & 0.0003 & 0.0006 & 0.0004 \\
\hline Trial 2 & 0.0005 & 0.00002 & 0.0005 & 0.0005 \\
\hline
\end{tabular}

\begin{tabular}{|c|c|c|c|c|}
\hline \multirow{2}{*}{} & \multicolumn{3}{|c|}{ C. HbR } \\
\cline { 2 - 5 } & Dominant Hand & Non-dominant hand & Dominant Hand & Non-dominant hand \\
\hline Trial 1 & 0.0018 & 0.0019 & 0.0019 & 0.0013 \\
\hline Trial 2 & 0.0011 & 0.0016 & 0.0016 & 0.0014 \\
\hline
\end{tabular}

Table 12.3: The results of quantitative change in (A) $\mathrm{HbO},(B) \mathrm{HbR}$ and $(C) \mathrm{HbT}$ during the target touching task performed by participants with finger and with pen using their dominant and non-dominant hand across trial 1 and trial 2.

\subsection{Conclusion}

The hemodynamic response function in the pre and anterior region of brain was investigated in response to two different planning and execution of motor skill stimuli 
using near infrared spectroscopy. The change in activation, functional connectivity and lateralization in the pre and anterior frontal region of brain in response to two different planning and execution of motor skills paradigms was demonstrated. The activation results indicate increase in the change in $\mathrm{HbO}$ and $\mathrm{HbT}$ only in the pre frontal cortex for the target touching performed using index finger and with pen, for both using both dominant and non-dominant hand, which returned to baseline by the end of rest period. There was no significant difference in activation between the target touching performed using index finger and with pen and rest period, for both using both dominant and nondominant hand. There was significant correlation in the activity of left $v s$. right pre and anterior frontal region of brain in response to target touching performed using index finger and with pen, for both using both dominant and non-dominant hand, as well as during the rest period. Bilateral activation (no dominance) and contralateral dominance was observed in the pre and anterior frontal region of brain, respectively in response to target touching performed using index finger and with pen, for both using both dominant and non-dominant hand.

The future work is to implement the developed NIRS instrument as well as the designed planning and execution of motor skill stimuli to investigate both the frontal cortex and motor cortex of individuals with $\mathrm{CP}$ along with kinematics study, with the future application towards their rehabilitation. 


\section{CHAPTER 13. Conclusion and Future Work}

During my doctoral work near infrared spectroscopic imaging of the brain was performed in response to language, joint attention and planning and execution of motor skill stimuli with the future application towards epilepsy, autism and cerebral palsy research, respectively. Near infrared spectroscopy (NIRS) of the brain in response to language and joint attention stimuli were performed using commercial NIRS system, Imagent, while NIRS of the brain in response to planning and execution of motor skill stimuli was performed using in-house developed NIRS system. The NIRS studies of planning and execution of motor skill stimuli was also performed in conjunction with kinematics study to simultaneously investigate the response of the frontal cortex and the motion of the hand. The significance of the language studies forms the foundation for the application of NIRS towards uncovering the vast language network present in the brain via investigation of activation, functional connectivity, effective connectivity and lateralization across frontal and fronto-temporal regions of the brain in response to language stimuli. These studies potentially lays the foundation in applying NIRS towards investigation of language functions in various neurological disorders (e.g. epileptic, schizophrenic and aphasic populations), where abnormality in the language processing is found. The joint attention studies have the potential to differentiate the two groups of children (typically developing and children with aurism) by understanding the differences in the hemodynamic responses across the subject groups along with the differences in the functional connectivity and cortical lateralization. This in turn may have a significant impact in early stage diagnostic intervention of autism from NIRS studies in much younger children $(<3$ years of age) versus psychological assessments on children, which 
is feasible only for children above 3 years of age. The NIRS studies of planning and execution of motor skills helped determine the feasibility as well as the type of planning and execution of motor skill stimuli to be used to investigate the frontal cortex of individuals with cerebral palsy. The current state of art in the field of physical therapy of individuals with cerebral palsy is that, they are trained in certain motor task to help them in their everyday activity. If the activation in the brain of individuals with $\mathrm{CP}$ can be monitored during their training routine then an effective and customized training routine can be designed for them based on their brain activation pattern. Hence a simultaneous NIRS of brain and kinematics were performed to investigate both the kinematics of the arm and the response of the frontal and motor region of the brain in response to the planning and execution of motor skill tasks. The significance of the simultaneous NIRS and kinematics studies is that it demonstrated the feasibility and importance of simultaneous NIRS and kinematics study towards investigation of the differences if any in planning and execution of simple motor tasks, such that it can be developed as a reliable tool to aid in the rehabilitation of individual with cerebral palsy.

\subsection{Ongoing and future work}

The work performed during my doctoral work addressed three major aspects of near infrared spectroscopy of brain. The first aspect was development of NIRS instrument to perform NIRS of brain. The second aspect was the development of techniques and software to process the neuro-optical data acquired from the different region of brain in response to different stimuli. The third aspect was to use the developed NIRS instrument and data processing technique to investigate the different regions of brain of healthy 
participants in response to three different stimuli namely, language, joint attention and planning and execution of motor skill stimuli. Thus the immediate future work to address each aspect of my doctoral work is divided in three major categories as described below:

\subsubsection{Instrumentation}

The NIRS instrument developed during my doctoral work has three pair of sources ( 3 of $830 \mathrm{~nm}$ laser source and 3 of $690 \mathrm{~nm}$ laser source) and eight detector fiber bundle. The immediate future work according to me on the instrumentation side is to increase the number of sources and detectors and design optical caps capable of holding the increased number of sources and detectors to enable NIRS of larger region of brain. The current inhouse developed NIRS system is designed in such a way that the number of sources and detectors can be easily increased. The number of sources can be increased to any number of sources required to cover larger of brain but the number of detector can only be increased up to 16 detector fiber bundles, limited by the size of the c-mount which couples with the face of ICCD camera. Thus upon increasing the number of sources and detectors the in-house developed NIRS system will be capable of performing high density real time dynamic imaging of larger brain area which would result in better brain activation localization.

\subsubsection{Data processing techniques}

The neuro-optical data acquired from the different regions of brain were processed offline using custom developed Matlab based software. There is a need to develop software to perform real time online processing of neuro-optical data to enable online monitoring of changes in the brain in response to the stimuli being processed by the underlying cortical 
region of the brain. The online monitoring will enable researchers to assess and make changes in the stimuli attended by the participants on the go during the study thereby making NIRS of the brain interactive with feedback. During my doctoral work I employed mathematical model based on granger causality approach to explicate the directional flow in the regions of brain in response to language stimuli. The neuro-optical which is acquired from different regions of brain is complex data, which contains different information pertaining to the activity in the brain. Hence there is also a need to develop other mathematical models to decipher the complex neuro-optical data. The different mathematical models which can be applied to decode the information contained in the neuro-optical data are dynamic causal model, entropy, psycho-physiological interaction, coherence, partial coherence and mutual information among several other methods.

\subsubsection{Participants recruitment}

The NIRS of brain studies which I performed during my doctoral work in response to language, joint attention and planning and execution of motor skill stimuli were mainly performed on healthy participants. The NIRS of brain studies were also performed very preliminarily on children with autism and individuals with cerebral palsy in response to joint attention and planning and execution of motor skills stimuli, respectively.

Thus the ongoing effort in the NIRS study of joint attention skill is to recruit more typically developing children and children with autism to statistically validate the result obtained from the preliminary study as described in chapter 8 . The next step to the current research will be to carry out NIRS-based imaging studies in typically developing 
and children with autism, in response to different socio-communicative tasks. Understanding the differences in the hemodynamic responses across the subject groups along with the differences in the functional connectivity and cortical lateralization will have potential to differentiate the two groups of children. This in turn may have a significant impact in early stage diagnostic intervention of autism from NIRS studies in much younger children $(<3$ years of age) versus psychological assessments on children, which is feasible only for children above 3 years of age.

The future work in the NIRS study of planning and execution of motor skill stimuli is to recruit more individuals with $\mathrm{CP}$ and controls to investigate the effect of training on the brain's response in conjunction with the measurement of the kinematics of muscle movement. The planning and execution of motor skill stimuli will also be used to investigate the motor regions of brain along with the frontal region of brain. The combined NIRS and kinematics study can be synchronized for a better understanding and intervention options offered to $\mathrm{CP}$ subjects, by physical therapists. The significance of this study is that in the long term it has the potential to help in designing treatment strategies to augment the rehabilitation of individuals with $\mathrm{CP}$, using both kinematics of the arm movement and the brain activation, determined using NIRS, associated with arm movement.

\subsection{Challenges and recommendation}

Several challenges were encountered and lessons were learned throughout the dissertation process. One lesson is the importance of maintaining a systematic experimental approach at every stage in the research. If a new instrument is developed it 
can be tempting to immediately try it to perform the studies on human participants without systematically testing every aspect of the instrument. However, any new instrument must be systematically tested in incremental stage in order to be confident that the data recorded truly represents the activity of the brain. During the modification and adaptation of optical mammography system to perform NIRS of brain, I gained experience in instrument development. The development of instrument can be a strenuous process as it is challenging to choose the right component to develop the instrument and then wait for weeks for the delivery of components. Instrument development was just one jigsaw of a big puzzle as near infrared spectroscopy of brain is riddled with several challenges like the recruitment and accurate placement of optical cap on the head of participants. The most forbidding challenge is to recruit neurologically challenged participants i.e. the children with autism and individuals with cerebral palsy. Even though it was not so difficult to recruit healthy participants it required careful maneuvering to place the optical cap on the head of the participants with minimal hair interference. The task of placing the optical cap on the head of the participants became even more challenging if the participants were either children with autism or individuals with cerebral palsy. The accurate placement of the optical cap on the head of participants requires patience, practice and knowledge of the region of brain. Thus it is important to understand the anatomy of brain and gain expertise in accurate and efficient placement of optical cap on participants head. The scientific challenges are sometimes surpassed by the challenge of collaborating with other institutions to recruit neurologically challenged participants. 
Several major lessons were learned during this process like although the participants are asked to remain still during the imaging procedure; there will inevitably be some movement which can impact the results. The study must be performed efficiently within a stipulated time frame to keep the participants motivated and not to waste their time. Another lesson is that the behavioral conduct of the researchers performing the experiment must be formal; they must exhibit friendly behavior and never coerce the participants if they do not want to continue with the study. It is also imperative to understand the condition of the participants and address any of their qualms regarding the procedure followed during the study. It is also important to maintain awareness of the challenges faced by the neurologically challenged participants and never laugh on and make fun of their condition. Written consent must be taken by the participants before the study, the study should be clearly described to them and their concerns and questions should be addressed properly. It must be kept in mind that the participant's information is confidential and it must be never disclosed to person not involved in the study. The identity of the subject should not be associated with the data collected, and the medical records must be kept locked to protect the privacy of the subject. The lessons learned during the dissertation process will be incorporated in the ongoing and future near infrared spectroscopy study on neurologically challenged participants using the developed NIRS system. These studies will help make NIRS a reliable tool to investigate the underlying neural basis of a neurological disorder in the clinical settings. 


\section{REFERENCES}

Accardo J., Kammann H., Hoon JR A. H. Neuroimaging in cerebral palsy. J. Pediatr. 145:S19-S27, 2009.

Adelson PD, Nemoto E, Scheuer M, Painter M, Morgan J, Yonas H. Noninvasive continuous monitoring of cerebral oxygenation perictally using near-infrared spectroscopy: a preliminary report. Epilepsia 40:1484-1489, 1999.

Aertsen AM, Gerstein GL, Habib MK, Palm G. Dynamics of neuronal firing correlation: modulation of "effective connectivity." J Neurophysiol 61:900-917, 1989.

Aertsen A., Preissl H. Dynamics of activity and connectivity in physiological neuronal networks. $V C H$ 281-302, 1991

Aertsen A, Erb M, Palm G. Dynamics of functional coupling in the cerebral cortex: an attempt at a model-based interpretation. Physica D 75:103-128, 1994.

Akin A, Bilensoy D. Cerebrovascular reactivity to hypercapnia in migraine patients measured with near-infrared spectroscopy. Brain Res. 1107:206-214, 2006.

Akiyama T, Ohira T, Kato T, Toda Y, Orii M, Hiraga K, Fukunaga A, Kobayashi M, Onozuka S, Kawase T. Motor-related intracortical steal phenomenon detected by multichannel functional near-infrared spectroscopy imaging. Cerebrovasc. Dis. 20:337346, 2005.

Alavi A, Reivich M, Greenberg J, Rosenquist A, Hand P, Rintelmann W, Christman D, Fowlder J, Goldman A, MacGregor R, Wolf A. Mapping of functional activity in brain with 18F-fluoro-deoxyglucose. Semin Nucl Med 11:24-31, 1981.

Amunts K, Schleicher A, Bu" lger U, Mo"hlberg H, Uylings HBM, Roland PE, Zilles K. Broca's region revisited: Cytoarchitecture and intersubject variability. J Comp Neurol 12:319-341, 1999.

Anand A, Li Y, Wang Y, Wu J, Gao S, Bukhari L, Mathews VP, Kalnin A, Lowe MJ. Activity and connectivity of brain mood regulating circuit in depression: a functional magnetic resonance study. Biol Psychiatry 57:1079-1088, 2005.

Anand A., Li Y.,Wang Y.,Wu J., Gao S., Bukhari L., Mathews V.P., Kalnin A., Lowe M.J. Activity and connectivity of brain mood regulating circuit in depression: a functional magnetic resonance study. Biol. Psychiatry 570:1079-1088, 2005. 
Andersen AH, Gash DM, Avison MJ. Principal component analysis of the dynamic response measured by fMRI: a generalized linear systems framework. Magn Reson Imaging 17:795-815, 1999.

Arridge SR, Cope M, Delpy DT. The theoretical basis for the determination of optical path-lengths in tissue: temporal and frequency analysis. Physics in medicine and biology, 37:1531-1560, (1992).

Baker SC, Rogers RD, Owen AM, Frith CD, Dolan RJ, Frackowiak RSJ, Robbins TW. Neural systems engaged by planning: a PET study of the Tower of London task. Neuropsychologia. 34:515-526, 1996.

Batista C.E., Chugani H.T., Juhász C., Behen M.E., Shankaran S. Transient hypermetabolism of the basal ganglia following perinatal hypoxia. Ped. neurol. 36: 330$3,2007$.

Bax M., Goldstein M., Rosenbaum P., Leviton A., Paneth N., Dan B. Jacobsson B., Damiano D. Executive Committee for the Definition of Cerebral Palsy, Proposed definition and classification of cerebral palsy. Dev. Med. Child Neurol. 47:571-576, 2005.

Benaron DA, Stevenson DK. Optical time-of-flight and absorbance imaging of biologic media. Science 259:1463- 1466, 1993.

Binder JR, Frost JA, Hammeke TA, Cox RW, Rao SW, Prieto T. Human brain language areas identified by functional magnetic resonance imaging. $J$ Neurosci. 17: 353-362, 1997.

Biswal B, Yetkin FZ, Haughton VM, Hyde JS. Functional connectivity in the motor cortex of resting human brain using echo-planar MRI. Magn Reson Med 34:537-541, 1995.

Biswal B., Yetkin F.Z., Haughton V.M., Hyde J.S. Functional connectivity in the motor cortex of resting human brain using echo-planar MRI. Magn. Reson. Med. 340:537-541, 1999.

Boas DA, Dale AM, Franceschini MA. Diffuse optical imaging of brain activation: approaches to optimizing image sensitivity, resolution, and accuracy. Neuroimage 23:S275-S288, 2004.

Boden S, Obrig H., Kohncke C., Benav H., Koch SP, Steinbrink J. The oxygenation response to functional stimulation: is there a physiological meaning to the lag between parameters? Neuroimage 36:100-107, 2007. 
Bogen JE, Bogen GM. Wernicke's region-Where is it?. Ann NY Acad Sci 280:834-843, 1976.

Bortfeld H, Fava E, Boas DA. Identifying Cortical Lateralization of Speech Processing in Infants Using Near-Infrared Spectroscopy. Dev Neuropsychol 34:52-65, 2009.

Bottini G, Corcoran R, Sterzi R, Paulesu E, Schenone P, Scarpa P, Frackowiak RSJ, Frith D. The role of the right hemisphere in the interpretation of figurative aspects of language. A positron emission tomography activation study. Brain 117:1241-53, 1994.

Brauer J, Anwander A, Friederici AD. Neuroanatomical Prerequisites for Language Functions in the Maturing Brain. Cereb Cortex 21:459-466, 2011.

Broca P. Remarques sur le sie 'ge de la faculte' du language articule', suivies d'une observation d'aphe'mie (perte de la parole). Bull Me'moires Soc Anat Paris 36:330-357, 1861.

Buchanan TW, Lutz K, Mirzazade S, Specht K, Shah NJ, Zilles K, Jancke L. Recognition of emotional prosody and verbal components of spoken language: an fMRI study. Cogn Brain Res 9:227-38, 2000.

Bullmore ET, Rabe-Hesketh S, Morris RG, Williams SC, Gregory JA, Gray JA, Brammer MJ. Functional magnetic resonance image analysis of a large-scale neurocognitive network. Neuroimage 4:16-33, 1996.

Burton H., Dixit S., Litkowski P., Wingert J.R. Functional connectivity for somatosensory and motor cortex in spastic diplegia. Somatosens. Mot. Res. 26:90-104, 2009.

Cannestra A.F., Wartenburger I., Obrig H., Villringer A., Toga A.W Functional assessment of Broca's area using near infrared spectroscopy in humans. NeuroReport 14:1961-1965, 2003.

Capillary-oxygenation-level-dependent near-infrared spectrometry in frontal lobe of humans. J. Cereb. Blood Flow Metab. 27:1082-1093, 2007.

Chance B., Leigh J. S., Miyake H., Smiths D. S., Nioka S., Greenfeld R., Finander M., Kaufmann K., Levy W., Young M., Cohen P., Yoshioka H., Boretsky R. Comparison of time-resolved and -unresolved measurements of deoxyhemoglobin in brain. Proc. Nati. Acad. Sci. 85:4971-4975, 1988. 
Chaudhary U, Hall M, DeCerce J, Rey G, Godavarty A. Frontal activation and connectivity using near-infrared spectroscopy: Verbal fluency language study. Brain Res Bull 84:197-205, 2011.

Chen WG, Li PC, Luo QM, Zeng SQ, Hu B. Hemodynamic assessment of ischemic stroke with near-infrared spectroscopy. Space Med. Med. Eng. 13:84-89, 2000.

Cheong WF, Prahl SA, Welch AJ. A review of the optical properties of biological tissues. IEEE J Quantum Electron. 26:2166-2185, 1990.

Chiang TC, Vaithianathan T, Leung T, Lavidor M, Walsh V, Delpy DT. Elevated haemoglobin levels in the motor cortex following $1 \mathrm{~Hz}$ transcranial magnetic stimulation: a preliminary study. Exp. Brain Res. 181:555-560, 2007.

Cohen J.D., Tong F. The face of controversy. NEUROSCIENCE 2930:2405-2407, 2001.

Colier WN, Quaresima V, Baratelli G, Cavallari P, van der Slujis M, Ferrari M. Detailed evidence of cerebral hemoglobin oxygenation changes in response to motor cortical activation revealed by continuous wave spectrophotometer with $10 \mathrm{~Hz}$ temporal resolution. SPIE 2979:390-396, 1997.

Colier WN, Quaresima V, Oeseburg B, Ferrari M. Human motor-cortex oxygenation changes induced by cyclic coupled movements of hand and foot. Exp. Brain Res 129:457-461, 1999.

Contini D, Torricelli A, Pifferi A, Spinelli L, Taroni P, Quaresima V, Ferrari M, Cubeddu R. Multichannel time-resolved tissue oximeter for functional imaging of the brain. IEEE Trans. Instrum. Meas. 55:85-90, 2006.

Cope M, Delpy DT, Reynolds EOR, Wray S, Wyatt J, Van der Zee P. Methods of quantitating cerebral near infrared spectroscopy data. Adv Exp Med Biol 222:183-189, 1987.

Cuccia DJ, Bevilacqua F, Durkin AJ, Tromberg BJ. Modulated imaging: quantitative analysis and tomography of turbid media in the spatial-frequency domain. Optics letters 30: 1354-6, 2005.

Cuenod CA, Bookheimer SY, Hertz-Pannier L, Zeffiro TA, Theodore WH, Le Bihan D. Functional MRI during word generation, using conventional equipment: a potential tool for language localization in the clinical environment. Neurology. 45:1821-1827, 1995. 
Culver JP, Schlaggar BL, Dehghani H, Zeff BW. Diffuse optical tomography for mapping human brain function. presented at the Human Brain Mapping Meeting 11-15 June, 206, Florence, Italy, paper 684 T-PM

Dawson G, Webb S, Schellenberg GD, Dager S, Friedman S, Aylward E, Richards T. Defining the broader phenotype of autism: Genetic, brain, and behavioral perspectives. Dev Psychopathol 14: 581-611, 2002.

Delpy DT, Cope M, Zee P van der, Arridge SR, Wray S, Wyatt JS, Estimation of optical path-length through tissue from direct time of flight measurement. Physics in medicine and biology 33: 1433-1442, 1988.

Delpy DT, Cope M. Quantification in tissue near-infrared spectroscopy. Philosophical Transactions of the Royal Society B: Biological Sciences 352:649-659, 1997.

De Rinaldis M., Losito L., Gennaro L., Trabacca A. Long-term oral baclofen treatment in a child with cerebral palsy: electroencephalographic changes and clinical adverse effects. J. of Child Neurol. 25:1272-4, 2010.

Doehrmann O, Naumer MJ. Semantics and the multisensory brain: how meaning modulates processes of audio-visual integration. Brain Res 1242:136-150, 2008.

Duncan J., Owen A. M. Common regions of the human frontal lobe recruited by diverse cognitive demands. Trends Neurosci. 23:475-483, 2000.

Durduran T, Yu G, Burnett MG, Detre JA, Greenberg JH, Wang JZ, Zhou C, Yodh A G. Diffuse optical measurement of blood flow, blood oxygenation, and metabolism in a human brain during sensorimotor cortex activation. Opt. Lett. 29:1766-1768, 2004.

Elfgren CI, Risberg J. Lateralized frontal blood flow increases during fluency tasks: influence of cognitive strategy. Neuropsychologia. 36:505-512, 1998.

Eschweiler GW, Wegerer C, Schlotter W, Spandl C, Stevens A, Bartels M, Buchkremer G. Left prefrontal activation predicts therapeutic effects of repetitive transcranial magnetic stimulation (rTMS) in major depression. Psychiatry Res. 99:161-172, 2000.

Esposito MD, Detre JA, Alsop DC, Shin RK, Atlas S, Grossman M. The neural basis of the central executive system of working memory. Nature 378:279-281,1995.

Everdell NL, Gibson AP, Tullis IDC, Vaithianathan T, Hebden JC, Delpy DT, A frequency multiplexed near-infrared topography system for imaging functional activation in the brain. Rev. Sci. Instrum. 76:093705, 2005. 
Fallgatter AJ, Roesler M, Sitzmann L, Heidrich A, Mueller TJ, Strik WK. Loss of functional hemispheric asymmetry in Alzheimer's dementia assessed with near-infrared spectroscopy. Brain Res. Cogn. Brain Res. 6:67-72, 1997.

Fallgatter AJ, Strik WK. Frontal brain activation during the Wisconsin Card Sorting Test assessed with two-channel near-infrared spectroscopy. Eur. Arch. Psychiatry Clin. Neurosci. 248: 245-249, 1998.

Fallgatter AJ, Strik WK. Reduced frontal functional asymmetry in schizophrenia during a cued continuous performance test assessed with near-infrared spectroscopy. Shizophr. Bull. 26:913-919, 2006.

Fariaa A. V., Zhanga J., Oishia K., Li X., Jiang H., Akhter K., Hermoye L., Lee S-K., Hoon, Stashinko E. A., Miller M. I., van Zijla P.C.M., Mori S. Atlas-Based Analysis of Neurodevelopment from Infancy to Adulthood Using Diffusion Tensor Imaging and Applications for Automated Abnormality Detection. Neuroimage 52: 415-428, 2011.

Faw B., Pre-frontal executive committee for perception, working memory, attention, long-term memory, motor control, and thinking: A tutorial review. Cons. and Cog. 12:83-139, 2003.

Fitzgerald K.C., Williams L.S., Garg B.P., Golomb M.R. Epilepsy in children with delayed presentation of perinatal stroke. J. of Child Neurol. 22: 1274-80, 2007

Fox SL, Blasi A, Elwell CE. Illuminating the developing brain: The past, present and future of functional near infrared spectroscopy. Neuroscience and Biobehavioral Reviews 34:269-284, 2010.

Franceschini MA, Toronov V, Filiaci ME, Gratton E, Fantini S. On-line optical imaging of the human brain with 160-ms temporal resolution. Optics Express 6:49-57, 2000.

Franceschini MA, Joseph DK, Huppert TJ, Diamond SG, Boas DA. Diffuse optical imaging of the whole head. J. Biomed. Opt. 11:054007, 2006.

Franceschini MA, Thaker S, Themelis G, Krishnamoorthy KK, Bortfed H, Diamond SG, Boas DA, Arvin K and Grant PE. Assessment of Infant Brain Development With Frequency-Domain Near-Infrared Spectroscopy. Pediactric Research 61:5, 2007.

Franceschini MA, Fantini S, Thompson JH, Culver JP, Boas DA. Hemodynamic evoked response of the sensorimotor cortex measured non-invasively with near infrared optical imaging. Psychophysiology 40:548-560, 2008. 
Friston KJ, Frith CD, Liddle PF, Frackowiak RS. Functional connectivity: The principal component analysis of large (PET) data sets. J Cereb Blood Flow Metab 13:5-14, 1993.

Friston KJ. Functional and effective connectivity in neuroimaging: a synthesis. Hum Brain Mapp 2:56-78, 1994.

Friston KJ, Buechel C, Fink GR, Morris J, Rolls E, Dolan RJ. Psychophysiological and modulatory interactions in neuroimaging. Neuroimage 6:218 - 229, 1997.

Frith CD, Friston K, Liddle PF, Frackowiak RS. A PET study of word finding. Neuropsychologia 29:1137-48, 1991.

Fujiwara N, Sakatani K, Katayama Y, Murata Y, Hoshino T, Fukaya C, Yamamoto T. Evoked-cerebral blood oxygenation changes in false-negative activations in BOLD contrast functional MRI of patients with brain tumors. Neuroimage 21:1464-1471, 2004.

Galaburda AM. Le re'gion de Broca: Observations anatomiques faites apre's la mort de son de'couvreur. Rev Neurol 136:609-616, 1980.

Gallagher A, Bastien D, Pelletier I, Vannasing P, Legatt AD, Moshe SL, Jehle R, Carmant L, Lepore F, Be'land R, Lassonde M. A noninvasive, presurgical expressive and receptive language investigation in a 9-year-old epileptic boy using near-infrared spectroscopy. Epilepsy \& Behavior 12:340-346, 2008.

Garfinkle J., Shevell M.I. Cerebral palsy, developmental delay, and epilepsy after neonatal seizures. Pedia. Neurol. 44: 88-96, 2001.

Garfinkle J., Shevell M.I. Prognostic factors and development of a scoring system for outcome of neonatal seizures in term infants. Eur. J. of Pedia. Neurol. 15:222-9, 2009.

Gartus A, Foki T, Geissler A, Beisteiner R. Improvement of Clinical Language Localization with an Overt Semantic and Syntactic Language Functional MR Imaging Paradigm. Am. J. Neuroradiol 30:1977-1985, 2009.

Gartus A., Foki T., Geissler A., Beisteiner R. Improvement of Clinical Language Localization with an Overt Semantic and Syntactic Language Functional MR Imaging Paradigm. American Journal of Neuroradiology 30:1977-1985, 2009.

Ge J, Zhu B, Regalado S, and Godavarty A. Three-dimensional fluorescence-enhanced optical tomography using a hand-held probe based imaging system. Med. Med. Phys. 35, 2008.

Gerstein G.L. Functional association of neurons: detection and interpretations. The Neurosciences: Second Study Program 648-661, 1970. 
Gerstein G.L., Aertsen A.M. Representation of cooperative firing activity among simultaneously recorded neurons. J. Neurophysiol 54:1513-1528, 1985.

Gerstein GL, Perkel DH. Simultaneously recorded trains of action potentials: analysis and functional interpretation. Science 164:828-830, 1969.

Geschwind N, Levitsky W. Human brain left-right asymmetries in temporal speech region. Science 161:186-187, 1968.

Geschwind N. The organization of language and the brain. Science 170:940-944, 1970.

Gibson A.P., Hebden J.C., Arridge S.R. Recent Advances in Diffuse Optical Imaging. Phys. Med. Biol. 50:R1-43, 2005.

Gitelman DR, Nobre AC, Sonty S, Parrish TB, Mesulam MM. Language network specializations: an analysis with parallel task designs and functional magnetic resonance imaging. Neuroimage 26:975-985, 2005.

Goebel R, Roebroeck A, Kim DS, Formisano E. Investigating directed cortical interactions in time-resolved fMRI data using vector autoregressive modeling and Granger causality mapping. Magn Reson Imaging 2:1251-1261, 2003.

Gonzalez-Lima F, McIntosh AR. Structural equation modeling and its application to network analysis in functional brain imaging. Hum Brain Mapp 2:2-22, 1994.

Gratton E, Fantini S, Franceschini MA, Gratton G, Fabiani M. Measurements of scattering and absorption changes in muscle and brain. Philos Trans $R$ Soc Lond B Biol Sci 352:727-735, 1997.

Gratton G., Fabiani M. Shedding light on brain function: the event-related optical signal. Trends Cogn Sci. 5:357-363, 2001.

Gratton G, and Fabiani M. Fast optical imaging of human brain function. Front. Hum. Neurosci. 4:52, 2010.

Gratton G., Fabiani M. The event related optical signal (EROS) in visual cortex: reliability, consistency, localization and resolution. Psychophysiology 40:561-571, 2003.

Griffiths P.D., Radon M.R., Crossman R., Zurakowski D., Connolly D.J. Anatomic localization of dyskinesia in children with "profound" perinatal hypoxic-ischemic injury. AJNR 3: 436-41, 2010. 
Hanlon EB, Itzkan I, Dasari RR, Feld MS, Ferrante RJ, McKee AC, Lathi D, Kowall NW. Near infrared fluorescence spectroscopy detects Alzheimer's disease in vitro. Photochem. Photobiol. 70:236-242, 1999.

Harada T., Miyai I., Suzuki M., Kubota K. Gait capacity affects cortical activation patterns related to speed control in the elderly. Exp. Brain Res. 193:445-454, 2009.

Harrison L, Penny WD, Friston K. Multivariate autoregressive modeling of fMRI time series. Neuroimage 19:1477-1491, 2003.

Hatakenaka M., Miyai I.,Mihara M., Sakoda S., Kubota K. Frontal regions involved in learning of motor skill—a functional NIRS study. Neuroimage 34:109-116, 2007.

Hebden JC, Arridge SR, Delpy DT. Optical imaging in medicine: I. Experimental techniques. Physics Med Biol 42:825-840, 1997.

Hebden JC. Advances in optical imaging of the newborn infant brain. Blackwell Publishing Inc, Psychophysiology. 40:501-510, 2003.

Hebden JC, Austin T. Jeremy C. Hebden, Topun Austin. Eur Radiol Pediactric 17:29262933, 2007.

Hebden JC, Gibson A, Yusof RM, Everdell N, Hillman EMC, Delpy DT, Arridge SR, Austin T, Meek JH and Wyatt JS. Three-dimensional optical tomography of the premature infant brain. PHYSICS IN MEDICINE AND BIOLOGY 47:4155-4166, 2002.

Heeger DJ, Boynton GM, Demb JB, Seidemann E, Newsome WT. Motion opponency in visual cortex. J. Neurosci. 19:7162-7174, 1999.

Heeger, DJ, Huk AC, Geisler WS, Albrecht DG. Spikes versus bold: what does neuroimaging tell us about neuronal activity? Nat. Neurosci. 3: 631-633, 2000.

Heekeren HR, Obrig H, Wenzel R, Eberle K, Ruben J, Villringer K, Kurth R, Villringer A. Cerebral haemoglobin oxygenation during sustained visual stimulation-A nearinfrared spectroscopy study. Philos. Trans. R. Soc. Lond 352:743- 750,1997.

Herrmann MJ, Ehlis AC, Fallgatter AJ. Frontal activation during a verbal-fluency task as measured by near-infrared spectroscopy. Brain Res Bull 61:51-56, 2003.

Herrmann MJ, Walter A, Ehlis AC, Fallgatter AJ. Cerebral oxygenation changes in the prefrontal cortex: effects of age and gender. Neurobiol Aging. 27:888-894, 2006.

Hess, A, Stiller D, Kaulisch T, Heil P, Scheich H. New insights into the hemodynamic blood oxygenation level-dependent response through combination of functional magnetic 
resonance imaging and optical recording in gerbil barrel cortex. J. Neurosci. 20:33283338,2000 .

Hickok G, Poeppel D. Opinion - the cortical organization of speech processing. Nat Rev Neurosci 8:393—402, 2007.

Hintz1 SR, Benaron DA, Siegel AM, Zourabian A, Stevenson DK, and Boas DA. Bedside functional imaging of the premature infant brain during passive motor activation. J. Perinat. Med. 29:335-343, 2001.

Hirth C, Obrig H, Villringer K, Thiel A, Bernarding J, Muhlnickel W, Flor H, Dirnagl W, Villringer A. Non-invasive functional mapping of the human motor cortex using nearinfrared spectroscopy. NeuroReport 7:1977-1981, 1996.

Hock C, Villringer K, Spahn FM, Hofmann M, Hofer SS, Heekeren H, Wenzel R, Dirnagl U, Villringer A. Near infrared spectroscopy in the diagnosis of Alzheimer's disease. Ann. N. Y. Acad. Sci.777:22-29, 1996.

Hoge RD, Franceschini MA, Covolan RJ, Huppert T, Mandeville JB, Boas DA. Simultaneous recording of task-induced changes in blood oxygenation, volume, and flow using diffuse optical imaging and arterial spin-labeling MRI. Neuroimage 25, 701-707, 2005.

Holper L, Biallas M, Wolf M. Task complexity relates to activation of cortical motor areas during uni- and bimanual performance: a functional NIRS study. Neuroimage 46:1105-1113, 2009.

Homan RW, Hermanb J, Purdy P. Cerebral location of international 10-20 system electrode placement. Electroencephalography and Clinical Neurophysiology 66:376-382, 1987.

Horridge K., Johnston J., Phatak V., Guadagno A. Magnetic resonance imaging of the brain in children and young people with cerebral palsy: who reports matters? Dev. Med. Child Neurol. 53: 375-7, 2011.

Horwitz B. The elusive concept of brain connectivity. NeuroImage 19:466-470, 2003.

Hoshi Y, Onoe H, Watanabe Y, Anderson J, Bergstra`m M, Lilja A, Långstro“m B, Tamura M. Non-synchronous behavior of neuronal activity, oxidative metabolism and blood supply during mental tasks in man. Neurosci Lett. 172:129-133, 1994. 
Huppert TJ, Hoge RD, Diamond SG, Franceschini MA, Boas DA. A temporal comparison of BOLD, ASL, and NIRS hemodynamic responses to motor stimuli in adult humans. Neuroimage 29:368-382, 2006.

Huppert TJ, Diamond SG, Franceschini MA, Boas DA. HomER: a review of time-series analysis methods for near-infrared spectroscopy of the brain. Appl Opt. 10:280-298, 2009

Hyvarinen A, Ramkumar P, Parkkonen L, Hari R. Independent component analysis of short-time Fourier transforms for spontaneous EEG/MEG analysis. Neuroimage 49:257$271,2010$.

Ide K., Horn A., Secher N.H. Cerebral metabolic response to sub maximal exercise. J. Appl. Physiol. 87:1604-1608, 1999.

Ikegami T., Taga G. Decrease in cortical activation during learning of a multijoint discrete motor task. Exp. Brain Res. 191:221-236, 2008.

Investigation of human brain hemodynamics by simultaneous near-infrared spectroscopy and functional magnetic resonance imaging. Med. Phys. 28:521-527, 2001.

Ishizu T., Noguchi A., Ito Y., Ayabe T., Kojima S. Motor activity and imagery modulate the body-selective region in the occipital-temporal area: a near-infrared spectroscopy study. Neurosci. Lett. 465:85-89, 2009.

Janis J. D., Robert L. R. Construction of Efficacious Gait and Upper Limb Functional Interventions Based on Brain Plasticity Evidence and Model-Based Measures For Stroke Patients. The Scientific World JOURNAL. 7: 2031-2045, 2007.

Jasdzewski G., Strangman G., Wagner J., Kwong K.K., Poldrack R.A., Boas D.A. Differences in the hemodynamic response to event-related motor and visual paradigms as measured by near-infrared spectroscopy. Neuroimage 20:479-488, 2003.

Jayakar A, Dunoyer C, Rey G, Yaylali I, Jayakar P. Near Infrared Spectroscopy to Define Cognitive Frontal Lobe Functions. Journal of Clinical Neurophysiology. 22: 415-417, 2005.

Jeong J, Gore JC, Peterson BS. Mutual information analysis of the EEG in patients with Alzheimer's disease. . Clin Neurophysiol 1120:827-835, 2001.

Jobsis FF. Noninvasive infrared monitoring of cerebral and myocardial sufficiency and circulatory parameters. Science 198:1264-1267, 1997. 
Joseph DK, Huppert TJ, Franceschini MA, and Boas DA. Diffuse optical tomography system to image brain activation with improved spatial resolution and validation with functional magnetic resonance imaging. Applied Optics 45:31, 2006

Jurcak V, Tsuzuki D, Dan I. 10/20, 10/10, and 10/5 systems revisited: Their validity as relative head-surface-based positioning systems. NeuroImage 34:1600-1611, 2007.

Karunanayaka PR, Holland SK, Schmithorst VJ, Solodkin A, Chen EE, Szaflarski JP, Plante E. Age-related connectivity changes in fMRI data from children listening to stories. NeuroImage 34:349-360, 2007.

Kashyap DR, Chu N, Apte A, Wang BP, Liu H. Development of broadband multichannel NIRS (near-infrared spectroscopy) imaging system for quantification of spatial distribution of hemoglobin derivatives. Proc. SPIE 6434, 6434IX (2007).

Kawaguchi F, Ichikawa N, Fujiwara N, Yamashita Y, Kawasaki S. Clinically Available Optical Topography System. Hitachi Review 50:18-22, 2001.

Kawakubo Y, Kuwabara H, Watanabe K, Minowa M, Someya T. Impaired Prefrontal Hemodynamic Maturation in Autism and Unaffected Siblings. PLoS ONE 4:6881, 2009.

Kek KJ, Samizo M, Miyakawa T, Kudo N, Yamamoto K. Imaging of regional differences of muscle oxygenation during exercise using spatially resolved NIRS. in IEEE Eng. Med. Biol. 27th Annu. Int. Conf., 1-4 September 2005, Shanghai, China, pp. 2622-2625.

Kennan RP, Kim D, Maki A, Koizumi H, Constable RT. Noninvasive assessment of language lateralization by transcranial near infrared optical topography and functional MRI. Hum Brain Mapp. 16:183-189, 2002.

Khan B., Tian F., Behbehani K., Romero M. I., Delgado M. R., Clegg N. J., Smith L., Reid D., Liu H., Alexandrakis G. Identification of abnormal motor cortex activation patterns in children with cerebral palsy by functional near-infrared spectroscopy. $J$. Biomed. Opt. 15:036008, 2010.

Kirby R.S., Wingate M.S., Van Naarden Braun K ., Doernberg N.S., Arneson C.L., Benedict R.E., Mulvihill B., Durkin M.S., Fitzgerald R.T., Maenner M.J., Patz J.A., Yeargin-Allsopp M. Prevalence and functioning of children with cerebral palsy in four areas of the United States in 2006: a report from the Autism and Developmental Disabilities Monitoring Network. Res. Dev. Disabil. 32:462-469, 2011.

Kleinschmidt A, Obrig H, Requardt M, Merboldt KD, Dirnagl U, Villringer A, Frahm J. Simultaneous recording of cerebral blood oxygenation changes during human brain 
activation by magnetic resonance imaging and near-infrared spectroscopy. J. Cereb. Blood Flow Metab. 16:817-826, 1996.

Kubota Y, Toichi TM, Shimizu M, Mason RA, Coconcea CM, Findling RL, Yamamoto $\mathrm{K}$, Calabrese JR. Prefrontal activation during verbal fluency tests in schizophrenia-a near-infrared spectroscopy (NIRS) study. Schizophrenia Research 77:65-73, 2005.

Kuboyama N, Nabetani T, Shibuya K, Machida K, Ogaki T. The effect of maximal finger tapping on cerebral activation. J. Physiol. Anthropol. Appl. Hum. Sci. 23:105-110, 2004.

Kuebler WM, Sckell A, Habler O, Kleen M, Kuhnle GEH, Welte M, Messmer K, Goetz AE. Noninvasive measurement of regional cerebral blood flow by near-infrared spectroscopy and indocyanine green. J. Cereb. Blood Flow Metab. 18:445-456, 1998

Kulak W., Sobaniec W., Kubas B. Spastic Cerebral Palsy: Clinical Magnetic Resonance Imaging Correlation of 129 Children. J. of Child Neurol. 22: 8-14, 2007.

Kułak W., Sobaniec W., Kubas B., Walecki J. Corpus callosum size in children with spastic cerebral palsy: relationship to clinical outcome. J. of child Neurol. 22: 371-4, 2007.

Kułak W., Sobaniec W., Gościk M., Oleński J., Okurowska-Zawada B. Clinical and neuroimaging profile of congenital brain malformations in children with spastic cerebral palsy. Ad. in Med. Sci. 53: 42-8, 2008.

Kuwabara H, Kasai K, Takizawa R, Kawakubo Y, Yamasue H, Rogers MA, Ishijima M, Watanabe K, Kato N. Decreased prefrontal activation during letter fluency task in adults with pervasive developmental disorders: A near-infrared spectroscopy study. Behavioural Brain Research 172:272-277, 2006.

Lee J.D., Park H-J., Park E.S., Kim D.G., Rha D-W., Kim E. Y., Kim D. I., Kim J-J., Yun M., Ryu Y. H., Lee J., Jeong J.M., Lee D.S., Lee M.C., Il Park C. Assessment of regional $\mathrm{GABA}(\mathrm{A})$ receptor binding using $18 \mathrm{~F}$-fluoroflumazenil positron emission tomography in spastic type cerebral palsy. NeuroImage. 34: 19-25, 2007.

Lee L, Harrison LM, Mechelli A. A report of the functional connectivity workshop, Dusseldorf 2002. NeuroImage 19:457-65, 2003.

Leff DR, Espina FO, Elwell CE, Athanasiou T, Delpy DT, Darzi AW, Yang GZ. Assessment of the cerebral cortex during motor task behaviours in adults: A systematic review of functional near infrared spectroscopy (fNIRS) studies. NeuroImage 54:29222936, 2011. 
Leon-Carrion J, Damas J, Izzetoglu K, Pourrezai K, Martin-Rodriguez JF, Barroso y Martin JM, Dominguez-Morales MR. Differential time course and intensity of PFC activation for men and women in response to emotional stimuli: A functional nearinfrared spectroscopy (fNIRS) study. Neurosci. Lett. 403:90-95, 2006.

Li J, Dietsche G, Iftime D, Skipetrov SE, Maret G, Elbert T, Rockstroh B, Gisler T. Noninvasive detection of functional brain activity with near-infrared diffusing-wave spectroscopy. J. Biomed. Opt. 10:440-4402, 2005.

Li CJ, Gong H, Gan Z, Zeng SQ, Luo QM. Verbal working memory load affects prefrontal cortices activation: Evidence from a functional NIRS study in humans. Proc. SPIE 5696. 33-40 (2005).

Liebert A, Kacprzak M, Maniewski R. Time-resolved reflectometry and spectroscopy for assessment of brain perfusion and oxygenation. presented at the Opt. Meth. Med. Diagn. Conf. 13-16 October 2005 Warsaw, pp. 113-121

Lloyd-Fox S, Blasi A, Elwell CE. Illuminating the developing brain: The past, present and future of functional near infrared spectroscopy. Neurosci Biobehav Rev 34:269-284, 2010.

Lord C, Risi S, Lambrecht L, Cook E H Jr, Leventhal BL, DiLavore PC. The autism diagnostic observation schedule-generic: A standard measure of social and communication deficits associated with the spectrum of autism. Journal of Autism and Developmental Disorders 30:205-223, 2000.

Lu CM, Zhanga YJ, Biswal BB, Zanga YF, Penga DL, Zhu CZ. Use of fNIRS to assess resting state functional connectivity. J Neurosci Methods 186:242-249, 2010.

Maki A, Yamashita Y, Ito Y, Watanabe E, Mayanagi Y, Koizumi H. Spatial and temporal analysis of human motor activity using noninvasive NIR topography. Med. Phys. 22:1997-2005, 1995.

Maki A, Yamashita Y, Watanabe E, Koizumi H. Visualizing human motor activity by using non-invasive optical topography. Front. Med. Biol. Eng. 7:285-297, 1996.

Mashal N, Faust M, Hendler T. The role of the right hemisphere in processing nonsalient metaphorical meanings: application of principal components analysis to fMRI data. Neuropsychologia 43:2084-100, 2005.

Matcher SJ, Cooper CE. Absolute quantification of deoxyhaemoglobin concentration in tissue near infrared spectroscopy. Physics in medicine and biology, 39:1295-1312, 1994. 
Matsuo K, Kato T, Fukuda M, Kato N. Alteration of hemoglobin oxygenation in the frontal region in elderly depressed patients as measured by near-infrared spectroscopy. $J$. Neuropsychiatry Clin.Neurosci. 12:465-471, 2000.

Mazoyer BM, Tzourio N, Frak V, Syrota A, Murayama N, Liverier O, Salamon G, Dehaene $\mathrm{S}$, Cohen L, Mehler J. The cortical representation of speech. J Cogn Neurosci 5:467-79, 1993.

McCormick P.W., Stewart M., Lewis G., Dujovny M., Ausman J.I. Intracerebral penetration of infrared light: technical note. J. Neurosurg. 76: 315-318, 1992.

Meek JH, Elwell CE, Khan MJ, Romaya J, Wyatt JS, Delpy DT, Zeki S. Regional changes in cerebral hemodynamics as a result of a visual stimulus measured by near infrared spectroscopy. Proc. R. Soc. Lond 261:351-356, 1995.

Mehagnoul-Schipper DJ, van der Kallen BF, Colier WN, van der Sluijs MC, van Erning LJ, Thijssen HO, Oeseburg B, Hoefnagels WH, Jansen RW. Simultaneous measurements of cerebral oxygenation changes during brain activation by near-infrared spectroscopy and functional magnetic resonance imaging in healthy young and elderly subjects. Hum. Brain Mapp. 16:14-23, 2002.

Mihara M., Miyai I., Hatakenaka M., Kubota K., Sakoda S. Sustained prefrontal activation during ataxic gait: a compensatory mechanism for ataxic stroke? Neuroimage 37: 1338-1345, 2007.

Miyai, I., Tanabe, H.C., Sase, I., Eda, H., Oda, I., Konishi, I., Tsunazawa, Y., Suzuki, T. Cortical mapping of gait in humans: a near-infrared spectroscopic topography study. Neuroimage 14, 1186-1192.

Miyai I., Yagura H., Oda I., Konishi I., Eda H., Suzuki T., Kubota K. Premotor cortex is involved in restoration of gait in stroke. Ann. Neurol. 52:188-194, 2002.

Miyai I., Yagura H., Hatakenaka M., Oda I., Konishi, I., Kubota K. Longitudinal optical imaging study for locomotor recovery after stroke. Stroke 34:2866-2870, 2003.

Molenaar PCM. A dynamic factor model for the analysis of multivariate time-series. Psychometrika 50:181-202, 1985.

Montcel B, Chabrier R, Poulet P. Detection of cortical activation with time-resolved diffuse optical methods. Appl. Opt. 44:1942-1947, 2005.

Muhlemann T, Haensse D, Wolf M. Ein drahtloser sensor fur die bildgebende in-vivo nahinfrarotspektroskopie. presented at the 3-Landertreffen der Deutschen, 
Osterreichischen and Scheweizerishen Gesellschaft fiir Biomedizinische Technik, Swiss Society of Biomedical Engineering, 6-9 September 2006, Zurich, p. P60.

Mundy P, Card J, Fox N. Fourteen month cortical activity and different infant joint attention skills. Developmental Psychobiology 36:325-338, 2000;

Mundy P, Crowson M. Joint Attention and Early Social Communication; Implications for Research on Intervention with Autism. Journal of Autism and Developmental Disorders 27:6, 2007.

Mundy P, Sigman M. The theoretical implications of joint-attention deficits in autism. Development and Psychopathology 1:173-183, 1989.

Mundy P., Burnette C., Joint attention and neurodevelopment. Handbook of Autism and Pervasive Developmental Disorders 3:650-681, 2005.

Mundy P., Sigman M., Ungerer J. A. Specifying the nature of the social impairment in autism. Autism: Nature, diagnosis, and treatment, Geraldine Dawson: Guilford Press 28:3-21 (1989).

Murata Y, Sakatani K, Katayama Y, Fukaya C. Increase in focal concentration of deoxyhaemoglobin during neuronal activity in cerebral ischaemic patients. J. Neurol. Neurosurg. Psychiatry 73:182-184, 2002.

Murias M, Webb SJ, Greenson J, Dawson G. Resting state cortical connectivity reflected in EEG coherence in individuals with autism. Biol Psychiatry 620:270-273, 2007.

Na S.H., Jin S.H., Kim S.Y., Ham B.J. EEG in schizophrenic patients: mutual information analysis. Clin. Neurophysiol. 1130:1954-1960, 2002.

Nambu I, Osu R, Sato MA, Ando S, Kawato M, Naito E. Single-trial reconstruction of finger-pinch forces from human motor-cortical activation measured by near-infrared spectroscopy (NIRS). Neuroimage 47:628-637, 2009.

Nemoto EM, Yonas H, Kassam A. Clinical experience with cerebral oximetry in stroke and cardiac arrest. Crit. Care Med. 28:1052-1054, 2000.

Nevalainen P., Pihko E., Maenpaa H., Valanne L., Nummenmaa L., Lauronen L. Bilateral alterations in somatosensory cortical processing in hemiplegic cerebral palsy. Dev. Med. Child. Neurol. 54:361-367, 2012.

Nielsen H.B., Boushel R., Madsen P., Secher N.H. Cerebral desaturation during exercise reversed by O2 supplementation. Am. J. Physiol. 277:1045-1052, 1999. 
Nissila I, Noponen T, Kotilathi K, Katila T, Liainen L, Tarvainen T, Scheweiger M, Arridge S. Instrumentation and calibration methods for the multichannel measurement of phase and amplitude in optical tomography. Rev. Sci. Instrum. 76:044302, 2005.

Nioka S, Luo Q, Chance B. Human brain functional imaging with reflectance CWS. Adv Exp Med Biol 428:237-242, 1997.

Noguchi Y, Takeuchi T, Sakai KL. Lateralized activation in the inferior frontal cortex during syntactic processing: event-related optical topography study. Hum Brain Mapp. 17:89-99, 2002.

Ntziachristos V, Ma XH, Yodh AG, Chance B. Multichannel photon counting instrument for spatially resolved near infrared spectroscopy. Review of Scientific Instruments 70:193-201, 1999.

Obrig H, Hirth C, Junge-Hulsing JG, Doge C, Wolf T, Dirnagl U, Villringer A. Cerebral oxygenation changes in response to motor stimulation. J. Appl. Physiol 81:1174-1183, 1996.

Obrig H, Hirth C, Junge-Hulsing JG, Doge C, Wenzel R, Wolf T, Dirnagl U, Villringer A. Length of resting period between stimulation cycles modulates hemodynamic response to a motor stimulus. Adv. Exp. Med. Biol. 411:471-480, 1997.

Obrig H, Wenzel R, Kohl M, Horst S, Wobst P, Steinbrink J, Thomas F, Villringer A. Near-infrared spectroscopy: does it function in functional activation studies of the adult brain? Int. J. Psychophysiol. 35:125-142, 2000.

Obrig H, Wolf T, Doge C, Hulsing JJ, Dirnagl U, Villringer A. Cerebral oxygenation changes during motor and somatosensory stimulation in humans, as measured by nearinfrared spectroscopy. Adv. Exp. Med. Biol. 388:219-224, 1996.

Okada F, Takahashi N, Tokumitsu Y. Dominance of the non dominant hemisphere in depression. J. Affect Disord.. 37:13-21, 1996.

Okada F, Tokumitsu Y, Hoshi Y, Tamura M. Impaired interhemispheric integration in brain oxygenation and hemodynamics in schizophrenia. Eur. Arch. Psychiatry Clin. Neurosci. 244:17-25 2004

Okamoto M., Dan H., Shimizu K., Takeo K., Amita T., Oda I., Konishi I., Sakamoto K., Isobe S., Suzuki T., Kohyama K., Dan, I. Multimodal assessment of cortical activation during apple peeling by NIRS and fMRI. Neuroimage 21:1275-1288, 2004. 
Osterling JA, Dawson G, Munson JA. Early recognition of 1-year-old infants with autism spectrum disorder versus mental retardation. Dev Psychopathol 14:239-251, 2002.

Palmer KF, Williams D. Optical properties of water in the near infrared. Journal of the Optical Society of America 64:1107-1110, 1974.

Papathanassiou D, Etard O, Mellet E, Zago L, Mazoyer B, Tzourio-Mazoyer N. A common langage network for comprehension and production: a contribution to the definition of language epicenters with PET. Neuroimage 11:347-357, 2000.

Patel J, Marks K, Roberts I, Azzopardi D, Edwards AD. Measurement of cerebral blood flow in newborn infants using near infrared spectroscopy with indocyanine green. Pediatr. Res. 43:34-39, 1998.

Pires G., Nunes U., Castelo-Branco M. Statistical spatial filtering for a P300-based BCI: tests in able-bodied, and patients with cerebral palsy and amyotrophic lateral sclerosis. $J$. of Neurosci. Meth. 195: 270-81, 2011.

Plichta MM, Herrmann MJ, Ehlis AC, Baehne CG, Richter MM, Fallgatter AJ. Eventrelated visual versus blocked motor task: detection of specific cortical activation patterns with functional near-infrared spectroscopy. Neuropsychobiology 53:77-82, 2006.

Pogue BW, Patterson MS. Frequency-domain opticalabsorption spectroscopy of finite tissue volumes using diffusion- theory. Phys Med Biol 39:1157-1180, 1994.

Posner MI, Petersen SE, Fox PT, Raichle ME. Localization of cognitive operations in the human brain. Science 240:1627-1631, 1990.

Price CJ, Wise RJ, Watson JD, Patterson K, Howard D, Frackowiak RS. Brain activity during reading. The effects of exposure duration and task. Brain 117:1255-69, 1994.

Quaresima V, Ferrari M, Sluijs MCP van der, Menssen J, Colier WNJM. The Lateral frontal cortex oxygenation changes during translation and language switching revealed by non-invasive near infrared multi point measurements. Brain Res Bull 59:35-43, 2002.

Ranta E.M., Crocetti D., Clauss A. J., Kraut A. M., Mostofsky H. S., Kaufmann E. W. Manual MRI parcellation of the frontal lobe. Psychiatry Research: Neuroimaging 172:147-154, 2009.

Rasmussen P, Dawson EA, Nybo L, van Lieshout JJ, Secher NH, Gjedde A. Capillaryoxygenation-level-dependent near-infrared spectrometry in frontal lobe of humans. J. Cereb. Blood Flow Metab. 27:1082-1093, 2007 
Rha D., Chang W.H., Kim J., Sim E.G., Park E.S. Comparing quantitative tractography metrics of motor and sensory pathways in children with periventricular leukomalacia and different levels of gross motor function. Neuroradio. 54: 615-621, 2011.

Robinson MN, Peake LJ, Ditchfield MR., Reid SM, Lanigan A, Reddihough DS. Magnetic resonance imaging findings in a population-based cohort of children with cerebral palsy. Dev. Med. Child. Neurol. 51(1): 39-45, 2009.

Roebroec A, Formisano E, Goebel R. Mapping directed influence over the brain using Granger causality and fMRI. Neuroimage 25:230-42, 2005.

Rogersa BP, Morgana VL, Newton AT, Gore JC. Assessing functional connectivity in the human brain by Fmri. Magnetic Resonance Imaging 25:1347-1357, 2005.

Ruben J, Wenzel R, Obrig H, Villringer K, Bernarding J, Hirth C, Heekeren H, Dirnagl $\mathrm{U}$, Villringer A. Haemoglobin oxygenation changes during visual stimulation in the occipital cortex. Adv. Exp. Med. Biol. 428:181-187, 1997.

Rueckert L., Appollonio I., Grafman J., Jezzard P., Johnson R., Jr. Le Bihan, Turner D., and Turner R. Magnetic resonance imaging functional activation of left frontal cortex during covert word production. J. Neuroimag. 4:67-70, 1994.

Rupp T., Perrey S. Prefrontal cortex oxygenation and neuromuscular responses to exhaustive exercise. Eur. J. Appl. Physiol. 102:153-163, 2008.

Rykhlevskaia E, Fabiani M, Gratton G. Lagged covariance structure models for studying functional connectivity in the brain. NeuroImage 30:1203 - 1218, 2006.

Saitou H, Yanagi H, Hara S, Tsuchiya S, Tomura S. Cerebral blood volume and oxygenation among poststroke hemiplegic patients: effects of 13 rehabilitation tasks measured by near-infrared spectroscopy. Arch. Phys. Med. Rehabil. 81:1348-1356, 2000.

Sakatani K, Chen S, Lichty W, Zuo H, Wang YP. Cerebral blood oxygenation changes induced by auditory stimulation in newborn infants measured by near infrared spectroscopy. Early Hum. Dev 55:229-236, 2006.

Sakatani K, Murata Y, Fukaya C, Yamamoto T, Katayama Y. BOLD functional MRI may overlook activation areas in the damaged brain. Acta Neurochir. Suppl. 87:59-62, 2003.

Sakatani K., Xie Y., Lichty W., Li S., Zuo H. Language-Activated Cerebral Blood Oxygenation and Hemodynamic Changes of the Left Prefrontal Cortex in Poststroke Aphasic Patients : A Near-Infrared Spectroscopy Study. Stroke 29:1299-1304, 1998. 
Salvador R, Suckling J, Schwarzbauer C, Bullmore E. Undirected graphs of frequencydependent functional connectivity in whole brain networks. Philos Trans R Soc Lond B Biol Sci 3600:937-946, 2005.

Sato H, Kiguchi M, Maki A, Fuchino Y, Obata A, Yoro T, Koizumi H. Within subject reproducibility of near-infrared spectroscopy signals in sensorimotor activation after 6 months. J. Biomed. Opt. 11:014021, 2006.

Sato H, Takeuchi T, Sakai KL. Temporal cortex activation during speech recognition: an optical topography study. Cognition 73:55-66, 1999.

Sato T, Ito M, Suto T, Kameyama M, Suda M, Yamagishi Y, Ohshima A, Uehara T, Fukuda M, Mikuni M. Time courses of brain activation and their implications for function: a multichannel near-infrared spectroscopy study during finger tapping. Neurosci. Res. 58:297-304, 2007.

Schmidt FE, Fry ME, Hillman EM, Hebden JC, Delpy DT. A 32-channel time-resolved instrument for medical optical tomography. Rev. Sci. Instrum. 71:256-265, 2000.

Schmitz CH, Locker M, Lasker JM, Hielscher AH, Barbour RL. Instrumentation for fast functional optical tomography. Rev. Sci. Instrum. 73:429-439, 2002.

Schnitzler A, Gross J. Functional connectivity analysis in magnetoencephalography. Int Rev Neurobiol 68:173-195, 2005.

Schwartz TH. The application of optical recording of intrinsic signals to simultaneously acquire functional, pathological and localizing information and its potential role in neurosurgery. Stereotact Funct Neurosurg 83:36-44, 2005.

Schwilling E., Krägeloh-Mann I., Konietzko A., Winkler and S., Lidzba K. Testing the language of German cerebral palsy patients with right hemispheric language organization after early left hemispheric damage. Cli. Ling. \& phon. 26: 135-47, 2012.

Selb J, Stott JJ, Franceschini MA, Sorensen AG, Boas DA. Improved sensitivity to cerebral hemodynamics during brain activation with a time-gated optical system: analytical model and experimental validation. J. Biomed. Opt. 10:110-113, 2005.

Selb J, Joseph DK, Boas DA. Time-gated optical system for depth-resolved functional brain imaging. J. Biomed. Opt. 11:044008, 2006.

Seth AK. Causal connectivity of evolved neuronal networks during behavior. Network 16:35-54, 2005. 
Shibusawa M., Takeda T., Nakajima K., Ishigami K., Sakatani K. Functional nearinfrared spectroscopy study on primary motor and sensory cortex response to clenching. Neurosci. Lett. 449:98-102, 2009.

Siegel AM, Marota JJA, Boas DA. Design and evaluation of a continuous-wave diffuse optical tomography system. Optics Express 4:287-298, 1999.

Skrandies W, Jedynak A. Associative learning in humans - conditioning of sensoryevoked brain activity. Behavio. Bra. Res. 107(1): 1-8, 2000.

Sokol DK, Markand ON, Daly EC, Luerssen TG, Malkoff MD. Near infrared spectroscopy (NIRS) distinguishes seizure types. Seizure 9:323-327, 2000.

Steinhoff BJ, Herrendorf G, Kurth C. Ictal near infrared spectroscopy in temporal lobe epilepsy: a pilot study. Seizure 5:97-101, 2005.

Stone JV. Independent component analysis: an introduction. Trends Cogn. Sci 6:59-64, 2002.

Strangman G, Boas DA, Sutton JP. Non-invasive neuroimaging using near-infrared light. Biol Psychiatry. 52:679-693, 2002.

Strangman G, Culver JP, Thompson JH, Boas DA. A quantitative comparison of simultaneous BOLD fMRI and NIRS recordings during functional brain activation. Neuroimage 17: 719-731, 2002b.

Subudhi A.W., Miramon B.R., Granger M.E., Roach R.C. Frontal and motor cortex oxygenation during maximal exercise in normoxia and hypoxia. J. Appl. Physiol. 106:1153-1158, 2009.

Sun F.T., Miller L.M., D'Esposito M. Measuring interregional functional connectivity using coherence and partial coherence analyses of fMRI data. NeuroImage 210:647-658, 2004.

Sutcliffe T.L., Gaetz W.C., Logan W.J., Cheyne D.O., Fehlings D.L. Cortical reorganization after modified constraint-induced movement therapy in pediatric hemiplegic cerebral palsy. J. of child neurol. 22: 1281-7, 2007.

Sutcliffe T.L., Logan W.J., Fehlings D.L. Pediatric constraint-induced movement therapy is associated with increased contralateral cortical activity on functional magnetic resonance imaging. J. of child neurol. 24:1230-5, 2009.

Swanson LW. Brain Architecture. Oxford University Press, 2003 
Swettenham J, Baron-Cohen S, Charman T, Cox A, Baird G, Drew A, Rees L, Wheelwright $\mathrm{S}$. The frequency and distribution of spontaneous attention shifts between social and nonsocial stimuli in autistic, typically developing, and nonautistic developmentally delayed infants. J Child Psychol Psychiatry Allied Discip 39:747-753, 1998.

Sööt A., Tomberg T., Kool P., Rein R., Talvik T. Magnetic resonance imaging in children with bilateral spastic forms of cerebral palsy. Ped. Neurol. 38: 321-8, 2008.

Taga G, Asakawa K, Maki A, Konishi Y, Koizumi H. Brain imaging in awake infants by near-infrared optical topography. PNAS 100:10722-10727, 2003

Takeda K., Gomi Y., Imai I., Shimoda N., Hiwatari M., Kato H. Shift of motor activation areas during recovery from hemiparesis after cerebral infarction: a longitudinal study with near-infrared spectroscopy. Neurosci. Res. 59:136-144, 2007.

Teflioudi EP, Zafeiriou DI, Vargiami E, Kontopoulos E, Tsikoulas I. Somatosensory evoked potentials in children with bilateral spastic cerebral palsy. Pediatric neurology. 44(3): 177-182, 2011.

Thiel A, Herholz K, vonStockhausen HM, vanLeyenPilgram K, Pietrzyk U, Kessler J, Wienhard K, Klug N, Heiss WD. Localization of language-related cortex with O-15labeled water PET in patients with gliomas. Neuroimage 7:284-295, 1998.

Thomas R., Stephane P. Prefrontal cortex oxygenation and neuromuscular responses to exhaustive exercise. Eur. J. Appl. Physiol. 102:153-163, 2008.

Tian F., Delgado M. R., Dhamne S. C., Alexandrakis G., Romero M. I., Smith L., Khan B., Reid D., Clegg N. J., Liu H. Quantification of functional near infrared spectroscopy to assess cortical reorganization in children with cerebral palsy. Opt. Exp. 18:25973-25986, 2010.

Ting Li, Hui Gong, Qingming Luo. Visualization of light propagation in visible Chinese human head for functional near-infrared spectroscopy J. Biomed. Opt. 16, 2011.

Toga AW, Mazziotta JC, Brain Mapping: The Methods. Academic Press 2002.

Toronov V, Franceschini MA, Filiaci M., Fantini S, Wolf M, Michalos A, Gratton E. Near-infrared study of fluctuations in cerebral hemodynamics during rest and motor stimulation: temporal analysis and spatial mapping. Med. Phys. 27:801-815, 2000. 
Toronov V., Walker S., Gupta R., Choi J.H., Gratton E., Hueber D., Webb A. The roles of changes in deoxyhemoglobin concentration and regional cerebral blood volume in the fMRI BOLD signal. Neuroimage 19:1521-1531, 2003.

Toronov V., Webb A., Choi J.H., Wolf M., Michalos A., Gratton E., Hueber D. Investigation of human brain hemodynamics by simultaneous near-infrared spectroscopy and functional magnetic resonance imaging. Med. Phys. 28:521-527, 2001.

Tsujimoto S., Yamamoto T., Kawaguchi H., Koizumi H., Sawaguchi T. Prefrontal cortical activation associated with working memory in adults and preschool children: an event-related optical topography study. Cerebral Cortex 14:703-712, 2004.

Ueda Y, Yamanaka T, Yamashita D, Suzuki T, Ohmae E, Oda M, Yamashita Y. Reflectance diffuse optical tomography: Its application to human brain mapping. Jpn. J. Appl. Phys. 44:L1203-L1206, 2005.

Van Ettinger-Veenstra HM, Ragnehed M, Hällgren M, Karlsson T, Landtblom AM, Lundberg P, Engström M. Right-hemispheric brain activation correlates to language performance. NeuroImage 49:3481-3488, 2010.

Vernieri F, Rosato N, Pauri F, Tibuzzi F, Passarelli F, Rossini PM. Near infrared spectroscopy and transcranial Doppler in monohemispheric stroke. Eur. Neurol. 41:159$162,1999$.

Vigneau M, Beaucousin V, Herve' PY, Duffau H, Crivello F, Houde' O, Mazoyer B, Tzourio-Mazoyer N. Meta-analyzing left hemisphere language areas: phonology, semantics, and sentence processing. Neuroimage 30:1414-1432, 2006.

Watanabe E, Maki A, Kawaguchi F, Takashiro K, Yamashita Y, Koizumi H, Mayanagi Y. Non-invasive assessment of language dominance with near-infrared spectroscopy mapping. Neurosci Lett. 256:49-52, 1998.

Watanabe E, Maki A, Kawaguchi F, Yamashita Y, Koizumi H, Mayanagi Y. Noninvasive cerebral blood volume measurement during seizures using multichannel near infrared spectroscopic topography. J.Biomed. Opt. 5:287-290, 2000.

Watson NF, Dodrill C, Farrell D, Holmes MD, Miller JW. Determination of language dominance with near-infrared spectroscopy: comparison with the intracarotid amobarbital procedure. Seizure 13:399-402, 2004

White BR, Snyder AZ, Cohe AL, Petersen SE, Raichle ME, Schlaggar BL, Culver JP. Resting-state functional connectivity in the human brain revealed with diffuse optical tomography. NeuroImage 47:148-156, 2009. 
Wible G.C., Shenton E.M., Fischer A.I., Allard E.J., Kikinis R., Jolesz A.F., Iosifescu V.D., McCarley W.R. Parcellation of the human prefrontal cortex using MRI. Psychiatry Research: Neuroimaging Section 76:29-40, 1997.

Wilke M, Lidzba K, Krägeloh-Mann I. Combined functional and causal connectivity analyses of language networks in children: A feasibility study. Brain Lang 108:22-29, 2009.

Wilke M, Lidzba K, Staudt M, Buchenau K, Grodd W, Krägeloh-Mann I. An fMRI task battery for assessing hemispheric language dominance in children. NeuroImage 32:400410, 2006.

Wilke M, Lidzba K, Staudt M, Buchenau K, Grodd W, Krägeloh-Mann I. Comprehensive language mapping in children, using functional magnetic resonance imaging: What's missing counts. Neuroreport 16:915-919, 2005.

Williams JHG, Waiter GD, Perra O, Perrett DI, Whiten A. An fMRI study of joint attention experience. J. Neuroimage 25:133-140, 2005.

Wingert J. R., Sinclair R.J., Dixit S., Damiano D. L., Burton H. Somatosensory-evoked Cortical Activity in Spastic Diplegic Cerebral Palsy. Hum. Brain Mappi. 11:1772-1785, 2010.

Wong V.C.N., Sun J-G., Yeung D.W.C. Pilot Study of Positron Emission Tomography (PET) Brain Glucose Metabolism to Assess the Efficacy of Tongue and Body Acupuncture in Cerebral Palsy. J. of Child Neurol. 21: 455-462, 2006.

Wonga D, Dzemidzic M, Talavage TM, Romito LM, Byrd KE. Motor control of jaw movements: An fMRI study of parafunctional clench and grind behavior. Brain Res 383: 206-217, 2011.

Wood P, Brown D. The study of intraindividual differences by means of dynamic factor models: rationale, implementation, and interpretation. Psych Bull 116:166-186, 1994.

Wray S, Cope M, Delpy DT, Wyatt JS, Reynolds EOR. Characterization of the near infrared absorption spectra of cytochrome aa3 and haemoglobin for the non-invasive monitoring of cerebral oxygenation. Biochimica et biophysica acta 933:184-192, 1988.

Yanagida, T., Kubota, K., 2001. Cortical mapping of gait in humans: a near-infrared spectroscopic topography study. Neuroimage 14, 1186-1192. 
Yetkin FZ, Hammeke TA, Swanson SJ, Morris GL, Mueller WM, McAuliffe TL, Haughton VM. A Comparison of Functional MR Activation Patterns during Silent and Audible Language Tasks. AJNR Am J Neuroradiol 16:1087-1092, 1995.

Yodh AG, Boas DA. Functional imaging with diffusing light. Biomedical Photonics Handbook Tuan Vo- Dinh, 2003.

Yoshida S., Hayakawa K., Oishi K., Mori S., Kanda T., Yamori Y., Yoshida N., Hirota H., Iwami M., Okano S. Athetotic and Spastic Cerebral Palsy: Anatomic Characterization Based on Diffusion-Tensor Imaging. Radiology 260: 511-520, 2011.

Zhang H, Zhang YJ, Lu CM, Ma SY, Zang YF, Zhu CZ. Functional connectivity as revealed by independent component analysis of resting-state fNIRS measurements. NeuroImage 51:1150-1161, 2002.

Zhang H, Zhang YJ, Lu CM, Ma SY, Zang YF, Zhu CZ. Functional connectivity as revealed by independent component analysis of resting-state fNIRS measurements. NeuroImage 51:1150-1161, 2010.

Zhu B, Yadav N, Rey G, Godavarty A. Diffuse optical imaging of brain activation to joint attention experience. Behavioural Brain Research 202:32-39, 2009. 


\section{APPENDICES}

Appendix A.1

\begin{tabular}{|c|c|c|c|c|c|c|c|c|c|}
\hline \multirow{2}{*}{} & \multicolumn{3}{|c|}{ HbO } & \multicolumn{3}{c|}{ HbR } & \multicolumn{3}{c|}{ HbT } \\
\cline { 2 - 10 } & W vs. J & W vs. R & J vs. R & W vs. J & W vs. R & Jvs. R & W vs. J & W vs. R & J vs. R \\
\hline AL & $<0.01$ & $<0.01$ & $<0.01$ & 0.13 & $<0.01$ & $<0.01$ & $<0.01$ & $<0.01$ & $<0.01$ \\
\hline AR & 0.40 & 0.94 & 0.79 & 0.08 & $<0.01$ & $<0.01$ & 0.75 & $<0.01$ & $<0.01$ \\
\hline PL & $<0.01$ & $<0.01$ & $<0.01$ & 0.06 & 0.33 & 0.09 & 0.32 & $<0.01$ & $<0.01$ \\
\hline PR & $<0.01$ & $<0.01$ & $<0.01$ & 0.09 & 0.12 & 0.11 & 0.43 & $<0.01$ & $<0.01$ \\
\hline
\end{tabular}

Table A.1: The results of three-paired t-test statistical analysis at $p<0.05$ to determine the differences in activation (in terms of $\mathrm{HbO}, \mathrm{HbR}$, and $\mathrm{HbT}$ ) between two stimuli (Word vs. Jaw, Word vs. Rest, and Jaw vs. Rest) and across each AL, PL, AR and PR region.

Appendix A.2

\begin{tabular}{|c|c|c|c|c|c|c|c|c|c|}
\hline \multirow{2}{*}{} & \multicolumn{3}{|c|}{ HbO } & \multicolumn{3}{c|}{ HbR } & \multicolumn{3}{c|}{ HbT } \\
\cline { 2 - 10 } & FA vs. RA & FA vs. R & RA vs. R & FAvs. RA & FAvs. R & RA vs. R & FAvs. RA & FAvs. R & RA vs. R \\
\hline AL & $<0.01$ & $<0.01$ & $<0.01$ & $<0.01$ & 0.04 & $<0.01$ & $<0.01$ & $<0.01$ & 0.12 \\
\hline AR & $<0.01$ & $<0.01$ & 0.45 & $<0.01$ & $<0.01$ & $<0.01$ & $<0.01$ & $<0.01$ & $<0.01$ \\
\hline PL & $<0.01$ & $<0.01$ & $<0.01$ & 0.06 & $<0.01$ & $<0.01$ & $<0.01$ & $<0.01$ & $<0.01$ \\
\hline PR & $<0.01$ & 0.06 & $<0.01$ & $<0.01$ & $<0.01$ & $<0.01$ & $<0.01$ & $<0.01$ & $<0.01$ \\
\hline
\end{tabular}

Table A.2: The results of three-paired t-test statistical analysis at $p<0.05$ to determine the differences in activation (in terms of $\mathrm{HbO}, \mathrm{HbR}$, and $\mathrm{HbT}$ ) between two stimuli (Forward Audio vs. Reverse Audio, Forward Audio vs. Rest, and Reverse Audio vs. Rest) and across each AL, PL, AR and PR region. 


\section{Appendix A.3}

The plot shown below demonstrates the consistency of the data acquired from all the participants during dot placement coarser motor control paradigm performed by participants using their dominant hand.

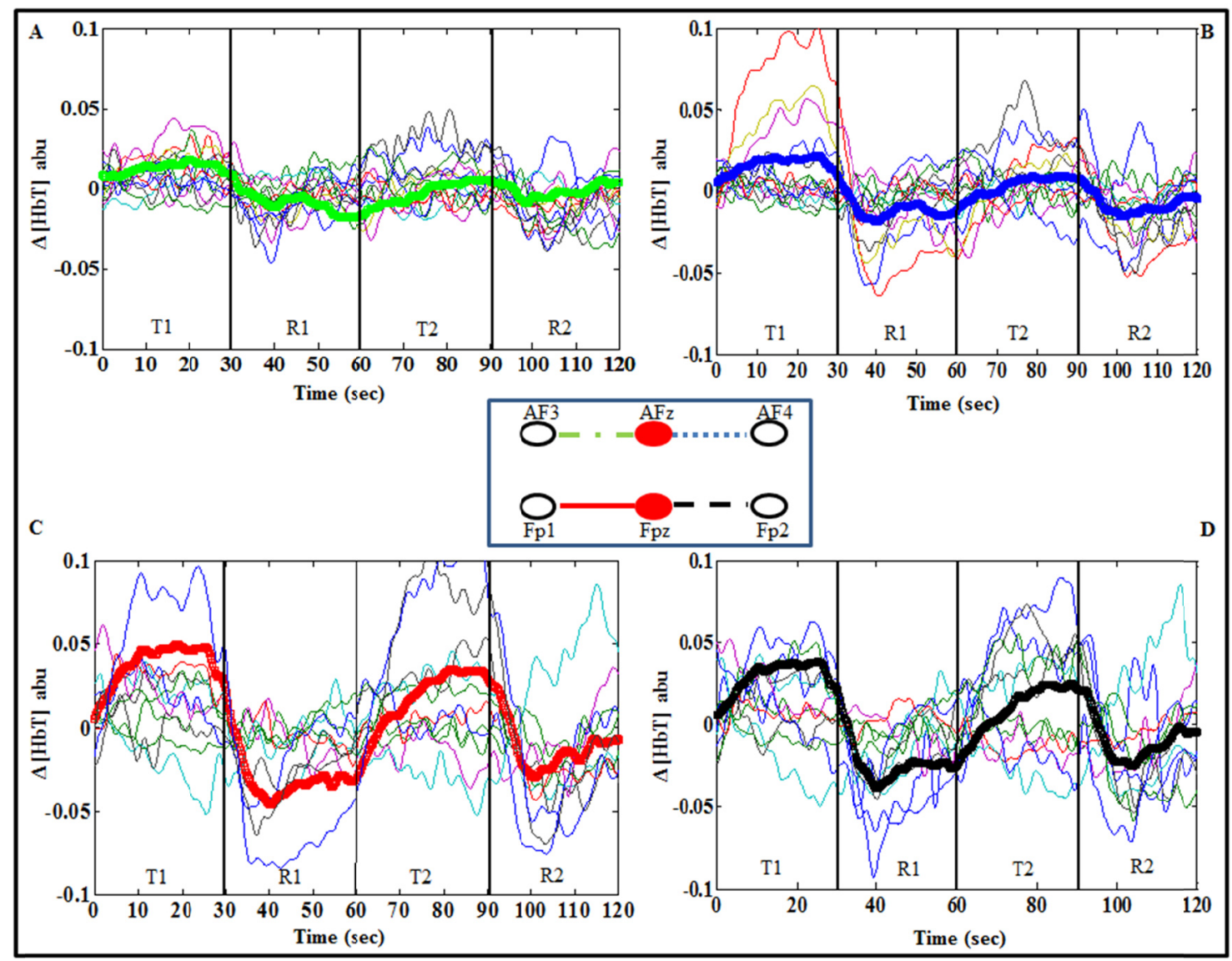

Figure A.3: Plot depicting the change in HbT across all the participants as well as the average of all the participants in the same plot corresponding to the dot placement coarser motor control paradigm performed by participants using their dominant hand. In each plot the change in HbT plotted is using thin and thick curve which corresponds to the relative change in $H b T$ obtained from individual participants and averaged relative change in HbT across all the participants respectively. The HbT changes are shown for (A) AF3-AFz channel, (B) AF4-AFz channel, (C) Fp1- Fpz channel, and (D) Fp2- Fpz channel. The change in HbT from 0-30 sec corresponds to dot placement task (T1), 30-60 sec to rest period (RI), 60-90 sec to dot placement with balance task (T2) and 90-120 sec to second rest period ( $R 2)$. 
The plot shown below demonstrates the consistency of the data acquired from all the participants during dot placement coarser motor control paradigm performed by participants using their non-dominant hand.

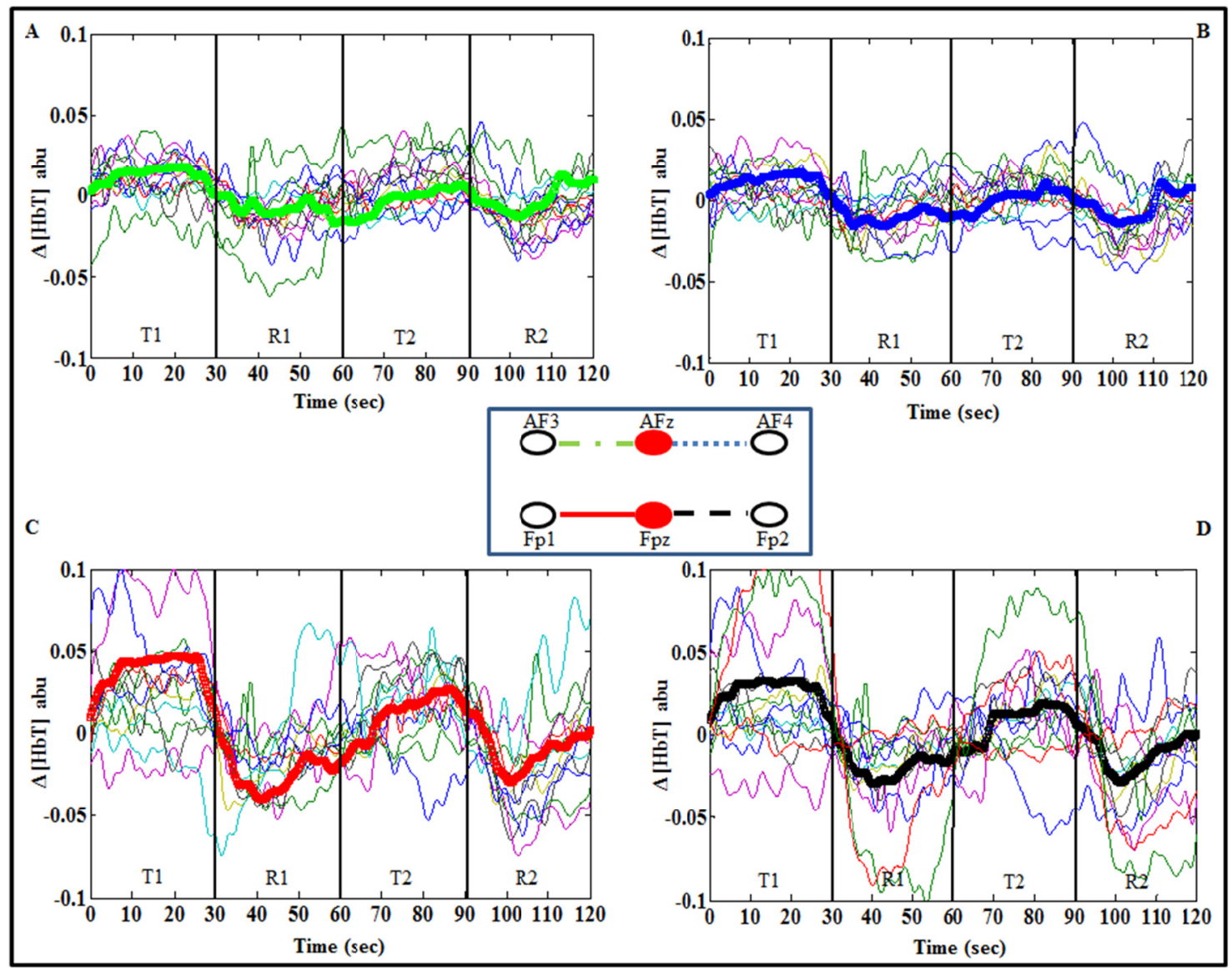

Figure A.4: Plot depicting the change in HbT across all the participants as well as the average of all the participants in the same plot corresponding to the dot placement coarser motor control paradigm performed by participants using their non-dominant hand. The details of the figure are same as described in caption of Figure A.3. 


\section{Appendix A.5}

The plot shown below demonstrates the consistency of the data acquired from all the participants during dot placement finer motor control paradigm performed by participants using their dominant hand.

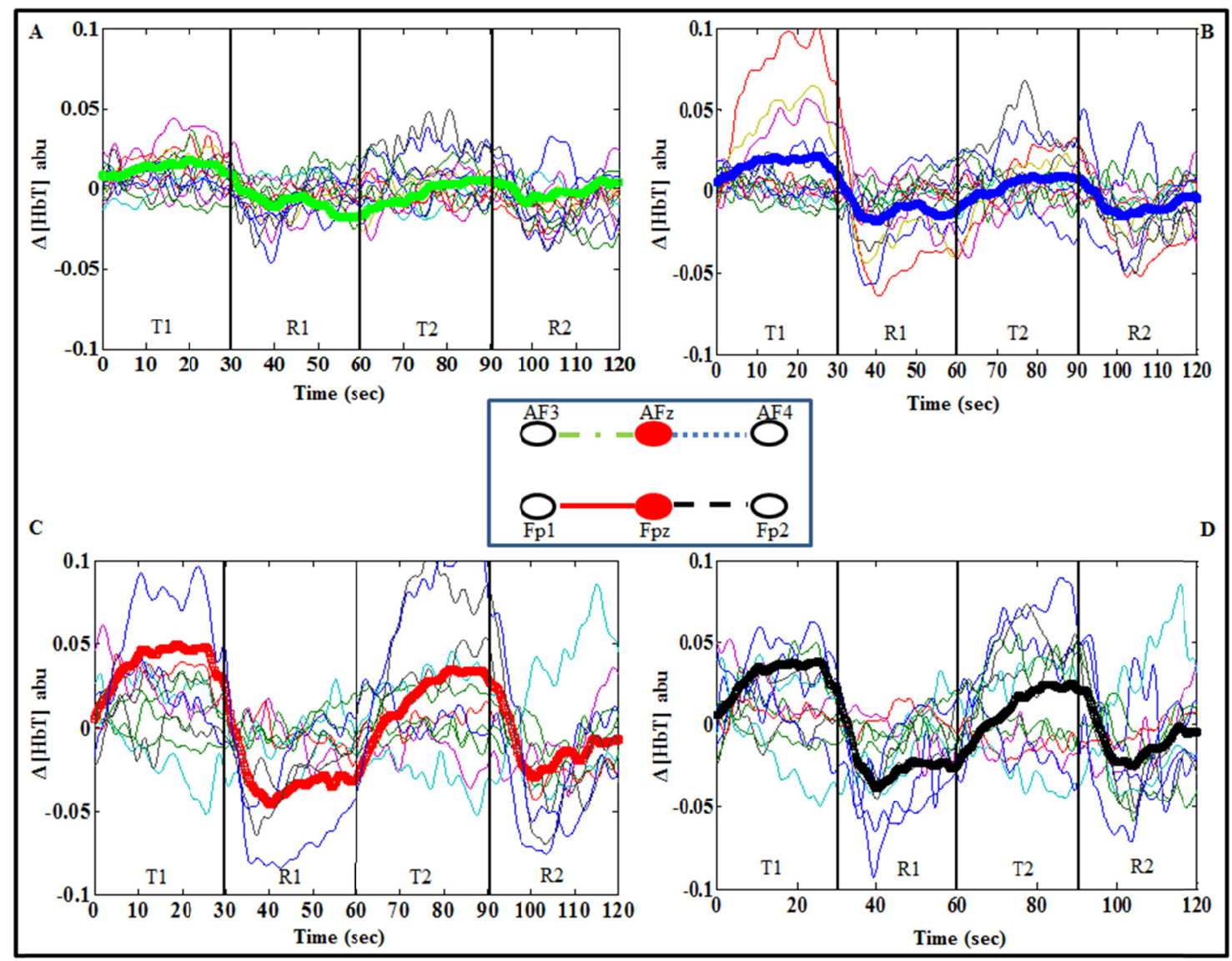

Figure A.5: Plot depicting the change in HbT across all the participants as well as the average of all the participants in the same plot corresponding to the dot placement finer motor control paradigm performed by participants using their dominant hand. The details of the figure are same as described in caption of Figure A.3. 
Appendix A.6

The plot shown below demonstrates the consistency of the data acquired from all the participants during dot placement finer motor control paradigm performed by participants using their non-dominant hand.

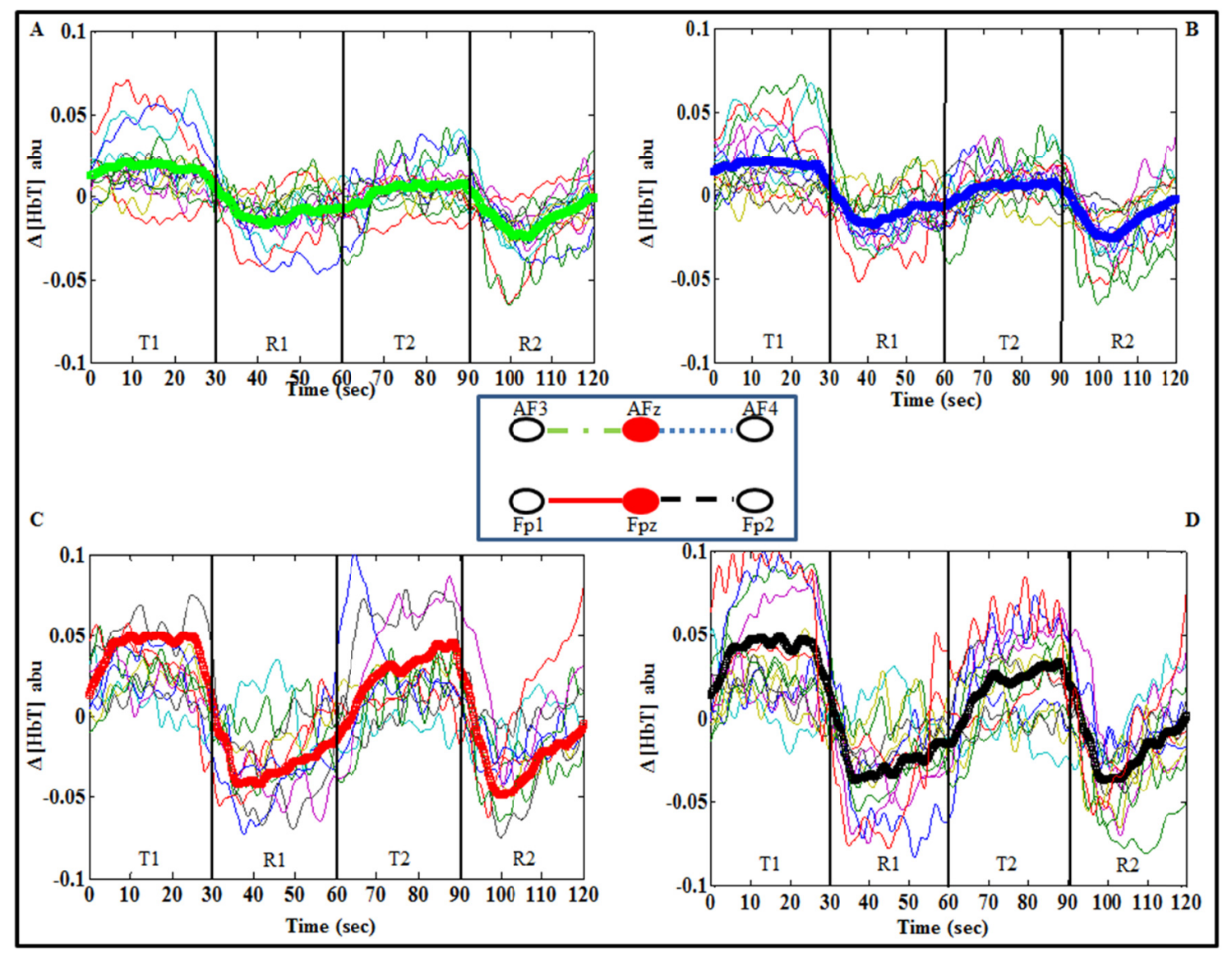

Figure A.6: Plot depicting the change in HbT across all the participants as well as the average of all the participants in the same plot corresponding to the dot placement finer motor control paradigm performed by participants using their non-dominant hand. The details of the figure are same as described in caption of Figure A.3. 


\section{Appendix A.7}

The Plot shown below demonstrates the consistency of the data acquired from all the participants during ball throw task paradigm performed by participants using their dominant and non-dominant hand.

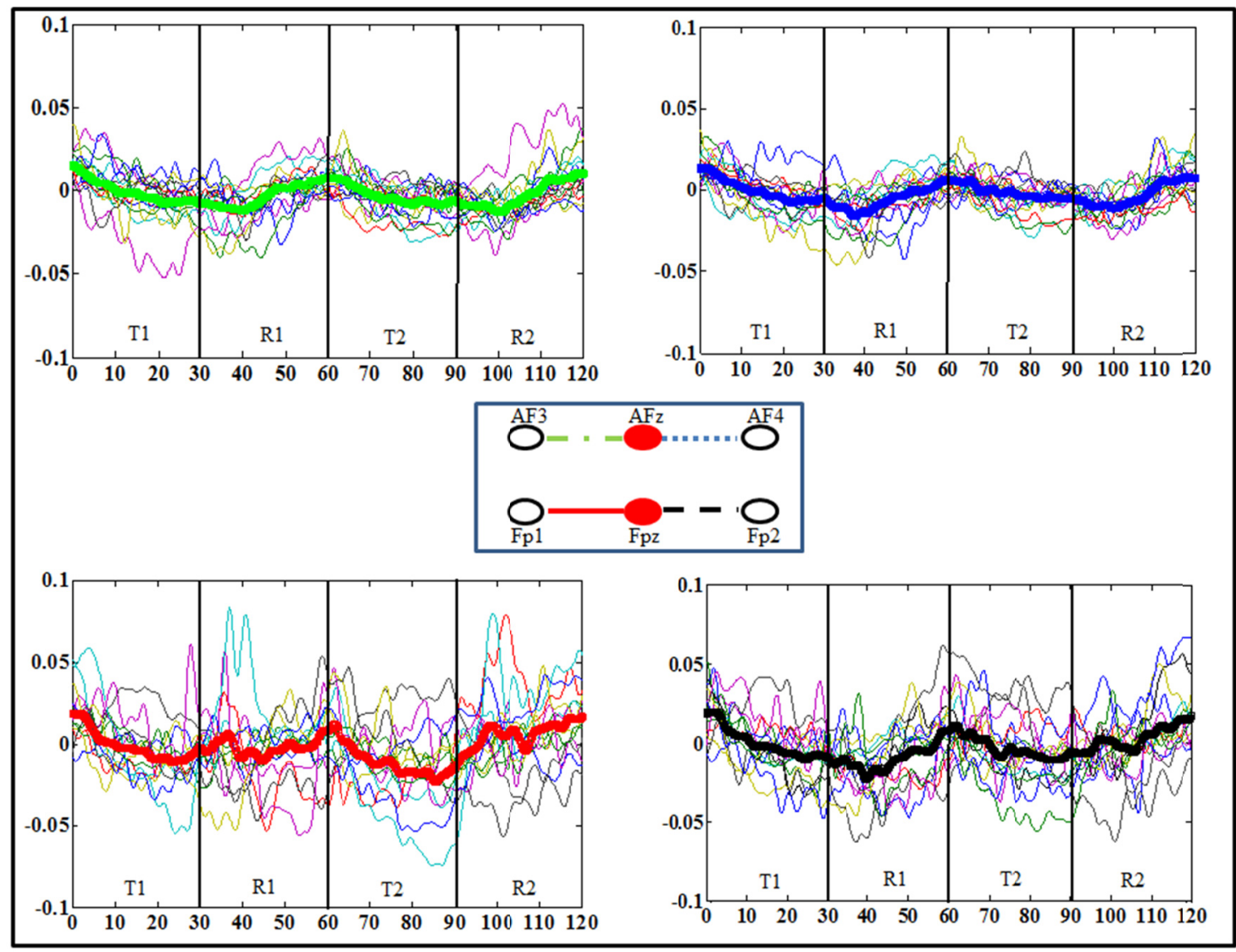

Figure A.7: Plot depicting the relative change in HbT across all the participants as well as the average of all the participants in the same plot corresponding to the ball throw task paradigm performed by participants using their dominant and non-dominant hand. The details of the figure are same as described in caption of Figure A.3. 


\section{UJWAL CHAUDHARY}

2008-2013 Graduate Researcher, Department of Biomedical Engineering, Florida International University, Miami, FL

2006-2008 Software Engineer, Infosys Technologies, India.

2002-2006 Bachelor of Technology, Biomedical Engineering, Sathyabama Deemed University, Chennai, India

\section{AWARDS}

1. Dissertation Year Fellowship, Florida International University's University Graduate School, Spring 2013 - Summer 2013.

2. Travel Award, Florida International University's Graduate Student Association, Fall 2012.

3. 2012 Kauffman Doctoral Student award, Pino Global Entrepreneurship Center and the Kauffman Foundation, Florida International University, Sept 122012.

4. Second place in Neuroscience Academic poster competition, Florida hospital for children, Orlando, May 32012.

5. Dissertation Evidence Acquisition Fellowship, Florida International University's University Graduate School, Spring 2012 - Summer 2012.

6. Summer Research Scholarship, Florida International Univeristy's MBRS-RISE, Summer 2011.

7. Travel Award, Florida International University's Graduate Student Association, Fall 2010.

8. Ranked first (Gold Medalist), Sathyabama Deemed University, B.Tech Biomedical Engineering, 2002-2006.

\section{PUBLICATIONS}

1) Ujwal Chaudhary, Michael Hall, Gustavo Rey and Anuradha Godavarty, "Language regions functional and effective connectivity using NIRS in response to language based stimuli", Submitted to Journal of Neurophysiology.

2) Cheng Zhang, Ujwal Chaudhary, Debrupa Lahiri, Anuradha Godavarty and Agarwal, Arvind, "Effect of Porosity on Photocatalytic Activity of Plasma Sprayed TiO2 Coating", Journal of Thermal Spray Technology (In Press), DOI: 10.1007/s11666-013-9964-1.

3) Cheng Zhang, Ujwal Chaudhary, Debrupa Lahiri, Anuradha Godavarty and Agarwal, Arvind, "Photo-catalytic Activity of Spark Plasma Sintered TiO2-Graphene Nanoplatelet Composite System”, Scripta Materialia 68, 719-722 (2013).

4) Michael Hall, Ujwal Chaudhary, Gustavo Rey and Anuradha Godavarty, "Temporal Mapping and Connectivity Using NIRS for Language Related Tasks", Journal of Neurolinguistics 26, 178-194 (2013). 
5) Ujwal Chaudhary, Michael Hall, Joe DeCerce, Gustavo Rey and Anuradha Godavarty, "Frontal activation and connectivity using near-infrared spectroscopy: Verbal fluency language study”, Brain Research Bulletin 84, 197-205 (2011).

6) Ujwal Chaudhary, Banghe Zhu, and Anuradha Godavarty," Frontal Cortical Connectivity and Lateralization of Joint Attention Experience Using Near-Infrared Spectroscopy", J. Near Infrared Spectrosc. 19, 105-116 (2011).

7) Ujwal Chaudhary, Young-Jin Jung, Bryant Thompson, Jean Gonzalez, Jennifer Davis, Patricia Gonzalez, Kyle Rice, Martha Bloyer, Leonard Elbaum, Anuradha Godavarty, "Investigation of Planning and Execution of Motor Skills in Healthy Adults using Simultaneous Near Infrared Spectroscopy and Kinematics Study", IFMBE Proceedings $29^{\text {th }}$ Southern Biomedical Engineering Conference 2013.

8) Ujwal Chaudhary, Bryant Thompson, Jean Gonzalez, Young-Jin Jung, Jennifer Davis, Patricia Gonzalez, Kyle Rice, Martha Bloyer, Leonard Elbaum, Anuradha Godavarty, "Simultaneous NIRS and kinematics study of planning and execution of motor skill task: towards cerebral palsy rehabilitation”, SPIE Photonics West Bios, San Francisco, CA, Feb. 2-7, 2013.

9) Ujwal Chaudhary, Michael Hall, Jean Gonzalez, Leonard Elbaum, Martha Bloyer and Anuradha Godavarty, "Motor Response Investigation in Individuals with Cerebral Palsy Using Near Infrared Spectroscopy", Optical Society of America, Miami, FL, Apr 29- May 2, 2012.

10) Michael Hall, Ujwal Chaudhary, Gustavo Rey and Anuradha Godavarty," Temporal Mapping and Connectivity Using NIRS for Language Related Tasks", Optical Society of America, Miami, FL, Apr 29- May 2, 2012.

11) Ujwal Chaudhary, Michael Hall, Anibal Gutierrez, Daniel Messinger, Gustavo Rey and Anuradha Godavarty," Activation in Frontal Cortex of Typically Developing and Children with Autism in Response to Joint Attention Skill using Near Infrared Spectroscopy", SPIE Photonics West Bios, San Francisco, CA, Jan. 21-26, 2011.

12) Michael Hall, Ujwal Chaudhary, Gustavo Rey and Anuradha Godavarty," Temporal Mapping and Connectivity Using NIRS for Language Related Tasks", SPIE Photonics West Bios, San Francisco, CA, Jan. 21-26, 2011.

13) Ujwal Chaudhary, Joesph DeCerce and Anuradha Godavarty," Brain Connectivity Study in Verbal Fluency Task using Near-Infrared Spectroscopy", Optical Society of America, Miami, FL, 2010.

14) Ujwal Chaudhary, Banghe Zhu, and Anuradha Godavarty," Brain Connectivity Study of Joint Attention Using Frequency-Domain Optical Imaging Technique", SPIE Photonics West Bios, San Francisco, CA, Jan. 22-27, 2010.

15) Ujwal Chaudhary, Banghe Zhu and Anuradha Godavarty," Brain Connectivity Studies of Joint Attention Using Frequency-Domain Diffuse Optical Imaging", IFMBE Proceedings $25^{\text {th }}$ Southern Biomedical Engineering Conference 2009.

Conference Presentations (not listed): 7 Oral, 10 Poster. 